\title{
Toxicity of Water and Sediment Samples Collected in the Vicinity of the Mixed Waste Management Facility, 1995 and 1996
}

by

W. L. Specht

Westinghouse Savannah River Company

Savannah River Site

Aiken, South Carolina 29808

DOE Contract No. DE-AC09-89SR18035

\section{RECEIVED \\ MAR 041998 \\ OSTI}

\section{MASTER}

This paper was prepared in connection with work done under the above contract number with the U. S. Department of Energy. By acceptance of this paper, the publisher and/or recipient acknowledges the U. S. Government's right to retain a nonexclusive, royalty-free license in and to any copyright covering this paper, along with the right to reproduce and to authorize others to reproduce all or part of the copyrighted paper. 


\section{DISCLAIMER}

This report was prepared as an account of work sponsored by an agency of the United States Government. Neither the United States Government nor any agency thereof, nor any of their employees, makes any warranty, express or implied, or assumes any legal liability or responsibility for the accuracy, completeness, or usefulness of any information, apparatus, product, or process disclosed, or represents that its use would not infringe privately owned rights. Reference herein to any specific commercial product, process, or service by trade name, trademark, manufacturer, or otherwise does not necessarily constitute or imply its endorsement, recommendation, or favoring by the United States Government or any agency thereof. The views and opinions of authors expressed herein do not necessarily state or reflect those of the United States Government or any agency thereof.

This report has been reproduced directly from the best available copy.

Available to DOE and DOE contractors from the Office of Scientific and Technical Information, P.O. Box 62, Oak Ridge, TN 37831; prices available from (615) 576-8401.

Available to the public from the National Technical Information Service, U.S. Department of Commerce, 5285 Port Royal Road, Springfield, VA 22161. 


\section{DISCLAIMER}

Portions of this document may be illegible electronic image products. Images are produced from the best available original document. 


\title{
TOXICITY OF WATER AND SEDIMENT SAMPLES COLLECTED IN THE VICINITY OF THE MIXED WASTE MANAGEMENT FACILITY, 1995-1996 (U)
}

\author{
by \\ WINONA L. SPECHT \\ SAVANNAH RIVER TECHNOLOGY CENTER
}

Approved by:

Dtmere-sheror

D.B. Moore-Shedrow, Section Manager

Environmental Sciences Section

Savannah River Technology Center

Publication Date: July 1996

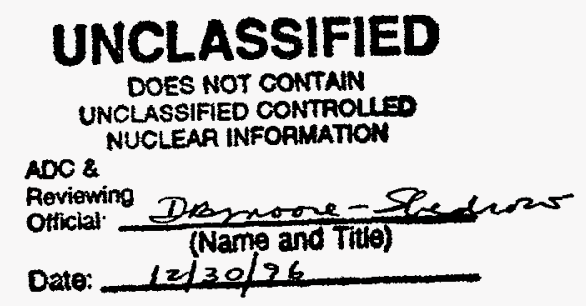

WESTINGHOUSE SAVANNAH RIVER COMPANY

SAVANNAH RIVER SITE

AIKEN, SC 29808

THIS DOCUMENT WAS PREPARED IN CONNECTION WITH WORK UNDER U.S. DEPARTMENT OF ENERGY CONTRACT DE-AC09-88SR18035. By acceptance of this paper, the publisher and/or recipient acknowledges the U.S. Government's right to retain a nonexclusive, royalty-free license in and to any copyright covering this paper, along with the right to reproduce and to authorize others to reproduce all or any part of the copyrighted paper. 


\section{Page}

1.0 EXECUTIVE SUMMARY

2.0 INTRODUCTION

3.0 SAMPLING LOCATIONS AND DATES 2

4.0 METHODS 6

4.1 Sample Collection 6

4.2 Water Toxicity 6

4.3 Sediment Toxicity 6

5.0 RESULTS

5.1 Water Chemistry 8

5.2 Water Toxicity 11

5.3 Sediment Toxicity 13

6.0 CONCLUSIONS 15

$\begin{array}{ll}7.0 \text { LITERATURE CITED } & 16\end{array}$

APPENDIX A - WATER TOXICITY RESULTS, JANUARY-FEBRUARY 1995

APPENDIX B - WATER TOXICITY RESULTS, AUGUST-SEPTEMBER 1995

APPENDIX C - WATER TOXICITY RESULTS, FEBRUARY-APRIL 1996

APPENDIX D - SEDIMENT TOXICITY RESULTS, AUGUST-SEPTEMBER 1995 


\section{LIST OF TABLES}

Page

Table 1. Maximum Tritium and Volatile Organic Compound Concentrations

in Shallow Wells and Bucket Samplers, March 1994

Table 2. Metal Concentrations at MWMF Sampling Locations, Aug.-Sept 1993

Table 3. Sampling Locations for Water and Sediment Toxicity Testing

Table 4. Test Conditions for Conducting a 7-day Definitive Water Toxicity Test with Ceriodaphnia dubia

Table 5. Test Conditions for Conducting a 10-day Sediment Toxicity Test with Hyalella azteca

Table 6. U.S. EPA Water Quality Criteria for Selected Metals

Table 7. Metal Concentrations at MWMF Sampling Locations, February 1996

Table 8. Metal Concentrations at MWMF Sampling Locations, April 1996

Table 9. Dissolved Oxygen, $\mathrm{pH}$, Hardness, Alkalinity, and Conductivity Measurements (Range) on Water Samples Used for Toxicity Testing

Table 10. No Observed Effect Concentrations (NOEC's) for MWMF Toxicity Testing Locations, 1995-1996

Table 11. Percent Mortality in Undiluted Water from the MWMF Sampling Locations, 1995-1996

Table 12. Results of 10-day Whole Sediment Hyalella azteca Toxicity Tests

\section{LIST OF FIGURES}

Figure 1. MWMF Sampling Locations

Figure 2. Background Sampling Locations 


\subsection{EXECUTIVE SUMMARY}

Three rounds of toxicity tests were conducted on water collected from eleven locations in the vicinity of the Mixed Waste Management Facility (MWMF) and four reference locations between January 1995 and April 1996. In addition, sediment toxicity tests were conducted at these same locations during the winter of 1996.

The results of the toxicity tests indicate that water from all of the sampling locations, including the four reference locations, was toxic in at least one of the three rounds of testing. No Observed Effect Concentrations (NOEC's) ranged from $<6.25 \%$ to $100 \%$. At most locations, the water was more toxic during the summer months. All of the sampling locations, including the reference sites, had potentially toxic concentrations of several metals, including aluminum, copper, iron, lead, and zinc. In addition some of the sampling locations had pH's that were low enough to cause toxicity. The observed toxicity is probably due to naturally elevated concentrations of one or more of these metals and/or low $\mathrm{pH}$. Toxicity Identification Evaluations (TIE's) are being performed at several sampling locations to identify the toxicants.

All but four of the sediment toxicity tests showed evidence of toxicity. Reference sites, as well as MWMF sampling sites were toxic, which suggests that the toxicity is due to naturally occurring conditions and is not related to MWMF contaminants. The toxicity may be due to anoxic conditions in the sediments.

In conclusion, the results of the water and sediment toxicity tests conducted at sampling locations in the vicinity of the MWMF and reference sites indicate that all of the locations are toxic to some extent. However, the toxicity appears to be due to naturally occurring toxicants, and not to MWMF contaminants.

\subsection{INTRODUCTION}

The Mixed Waste Management Facility (MWMF) occupies approximately 194 acres on a nearly flat divide between Upper Three Runs Creek to the north and Fourmile Branch to the south, and is bounded by Road 4 to the east and Road $C$ to the west. The MWMF was used to dispose of transuranic waste, low-level waste, and intermediate level radioactive waste. Inorganic chemical constituents known to be deposited in the MWMF include lead, mercury and cadmium. In addition, a number of organic compounds were stored in underground tanks in the MWMF or were present in buried waste and may be present in the groundwater beneath the MWMF. These compounds include naphthalene, toluene, tributylphosphate-kerosene, phenol, xylene, benzene, trimethylbenzene, and trichloroethylene.

Groundwater from the MWMF outcrops to the surface at numerous locations. Water quality surveys conducted in the vicinity of the MWMF in 1993 indicate elevated levels of tritium at numerous sampling locations and detectable concentrations of acetone, 1,2 dichloroethylene, methyl ethyl ketone, tetrachloroethylene, trichloroethylene and vinyl chloride in at least one shallow well or bucket sampling location (Dixon et al., 1994). The highest concentration of tritium or organics found during the 1994 sampling effort, as well as the location and EPA freshwater water quality criterion of each 
constituent is presented in Table 1. There presently are no freshwater water quality criteria for acetone, methyl ethyl ketone, vinyl chloride, or tritium. Of the remaining three volatile organic compounds that were found in detectable concentrations in the shallow wells or MWMF outcrops, none were found in concentrations that approach the EPA freshwater water quality criteria. Therefore, volatile organic compounds and tritium present in the groundwater outcrops from the MWMF would not be expected to be toxic to freshwater organisms living in the seeps and streams in the vicinity of the MWMF.

\section{Table 1. Maximum Tritium and Volatile Organic Compound Concentrations ${ }^{\mathrm{a}}$ in Shallow Wells and Bucket Samplers, March 1994}

\begin{tabular}{lcrcc} 
Compound & Well & Bucket & \multicolumn{2}{c}{$\begin{array}{c}\text { EPA FW WQC } \\
\text { Acute }\end{array}$} \\
$\begin{array}{lcccc}\text { Chronic } \\
1,2 \text { dichloroethylene }\end{array}$ & 368 & 102 & 11,600 & - \\
acetone & & & - & - \\
methyl ethyl ketone & 5.33 & - & 5,280 & - \\
tetrachloroethylene & 32.4 & 5.36 & 45,000 & 21,900 \\
trichloroethylene & - & 23 & - & - \\
vinyl chloride & 49,900 & 32,800 & - & - \\
tritium & & & &
\end{tabular}

${ }^{2}$ All concentrations are in $\mu \mathrm{g} / \mathrm{l}$, except tritium, which is reported in $\mathrm{pCi} / \mathrm{ml}$.

'U.S. EPA Freshwater Water Quality Criteria

Analyses for 13 metals (aluminum, arsenic, cadmium, chromium, copper, iron, lead, magnesium, manganese, mercury, selenium, silver, and zinc) were performed on samples collected from two background locations (BG-002 and BG-003) and five seep locations (FHB-013, FHB-014, FHB-015, FHB-018 and FHB-020 in August/September 1993 (Table 2). The results indicate that potentially toxic concentrations of iron, zinc, aluminum and/or chromium may occur at one or more sampling locations, including the uncontaminated reference locations.

In order to determine if the outcropping water poses a threat to aquatic life, toxicity tests were performed on water and sediment samples collected from 11 locations in the vicinity of the MWMF and Fourmile Branch as well as four uncontaminated reference locations.

\subsection{SAMPLING LOCATIONS AND DATES}

Water and sediment samples for toxicity testing were collected from a total of 15 locations, including seven seeps in the vicinity of the MWMF, three locations in the old F-Area effluent ditch, one location in Fourmile Branch, two background (uncontaminated reference) stream locations, and two background seep locations (Table 3; Figures 1 and 2). Toxicity tests on water samples were performed three times (January-February 1995, August-September 1995 and February-April 1996). It should be noted that all but four of the 1996 tests were conducted in late February. A series of rain events delayed completion of the toxicity tests at these four locations (FHB-012, FHB-013, FHB-018 and FHB-021) until mid-April. Sediment toxicity testing was conducted once at each sampling location during the winter of 1996. 
Table 2. Metal Concentrations at MWMF Sampling Locations, Aug-Sept 1993

$\begin{array}{lrrrrrr} & \text { BG-002 } & \text { BG-003 } & \text { FHB-013 } & \text { FHB-014 } & \text { FHB-018 } & \text { FHB-020 } \\ \mathrm{Al} & 6060 & 234 & <200 & <200 & <200 & <200 \\ \mathrm{As} & <10 & <10 & <10 & <10 & 10 & <10 \\ \mathrm{Cd} & <5 & <5 & <5 & <5 & <5 & <5 \\ \mathrm{Cr} & <10 & <10 & <10 & <10 & <10 & 16.8 \\ \mathrm{Cu} & <25 & <25 & <25 & <25 & <25 & <25 \\ \mathrm{Fe} & 7,690 & 580 & 5,025 & 565 & 47,100 & 4,510 \\ \mathrm{~Pb} & 4.6 & <3 & <3 & <3 & <3 & <3 \\ \mathrm{Mn} & 45 & 96 & 64 & <15 & 337 & 33 \\ \mathrm{Hg} & <0.2 & <0.2 & <0.2 & <0.2 & <0.2 & <0.2 \\ \mathrm{Se} & <5 & <5 & <5 & <5 & <5 & <5 \\ \mathrm{Ag} & <10 & <10 & <10 & <10 & <10 & <10 \\ \mathrm{Zn} & 84 & 22 & 28 & 36 & <20 & 57\end{array}$

All concentrations are in $\mu \mathrm{g} / \mathrm{l}$.

Numbers in bold indicate potential for toxicity

Table 3. Sampling Locations for Water and Sediment Toxicity Testing

\begin{tabular}{llcc} 
Location ID & Type & SRS North & SRS East \\
\hline BG-003 & Stream & 67633 & 59281 \\
BG-004 & Stream & 64723 & 68527 \\
FHB-012 & Stream & 73424 & 53878 \\
FHB-021 & Stream & 73113 & 53493 \\
FHB-022 & Stream & 72537 & 53372 \\
FMC-002H & Stream & 72200 & 43900 \\
BG-001 & Seep & 67208 & 59153 \\
BG-002 & Seep & 67229 & 59363 \\
FHB-013 & Seep & 73514 & 53840 \\
FHB-014 & Seep & 73571 & 53729 \\
FHB-018 & Seep & 73567 & 54024 \\
FHB-020 & Seep & 73431 & 54011 \\
FHB-023 & Seep & 73124 & 52954 \\
HSP-064 & Seep & 71846 & 54768 \\
HSP-092 & Seep & 72672 & 54129 \\
(FHB-006) & & &
\end{tabular}

$B G=$ background (reference)

$\mathrm{FHB}=$ near old $\mathrm{F}$-Area effluent ditch

$\mathrm{HSP}=$ along $\mathrm{H}$-Area seepline

$F M C=$ Fourmile Branch 


\section{Old F-Area Effluent Ditch}

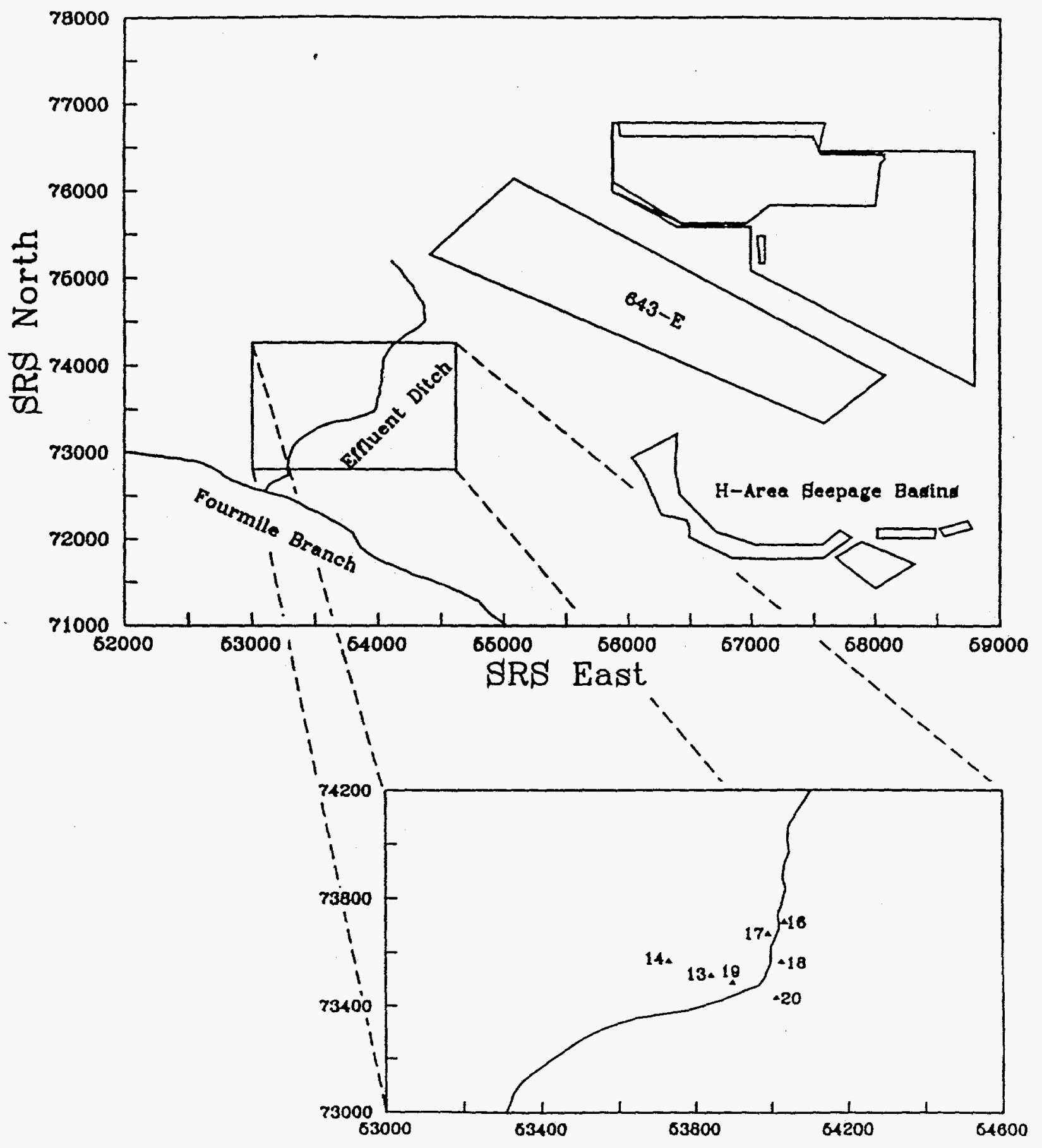

Figure 1. MWMF Sampling Locations 


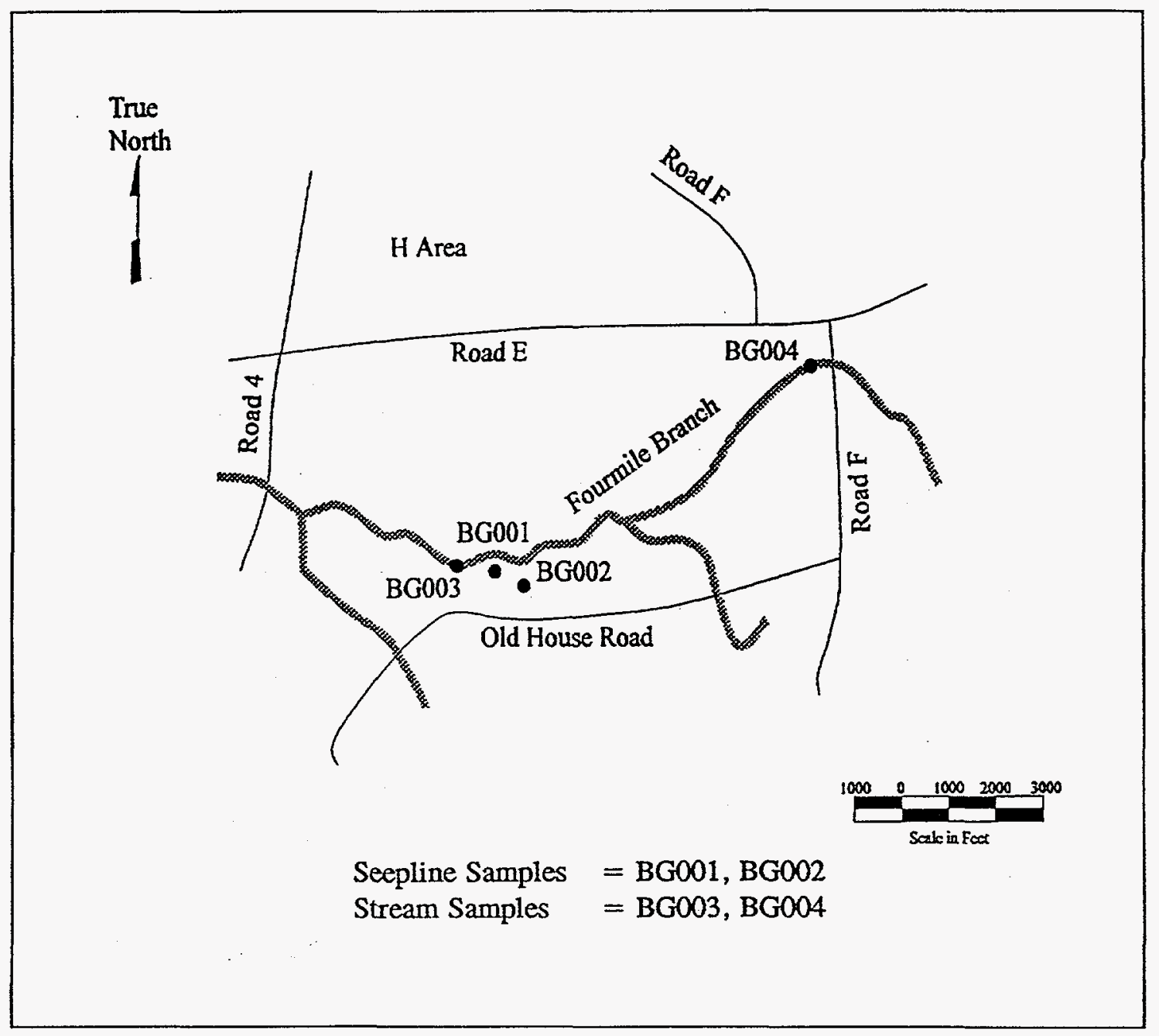

Figure 2. Background Sampling Locations 


\subsection{METHODS}

\subsection{Sample Collection}

Water - In accordance with EPA protocol, water samples were collected three times during a 7-day period at each location, such that the holding time of the water used for toxicity testing never exceeded 72 hours. For each collection, two 2 -liter plastic bottles of water were collected, placed on ice immediately and shipped to the testing laboratory via an express carrier.

Sediments - For the sediment toxicity tests, two liters of sediment were collected at each location. At three locations at each sampling site, a garden spade was forced into the sediment to a depth of approximately $25 \mathrm{~cm}$. A 1 liter sample was collected from the spade, taking care to collect sediment that had not contacted the metal blade of the spade. The three 1-liter samples were mixed together in a plastic tray and two liters of the representative sample were placed into a plastic jar, placed on ice, and shipped to the testing laboratory via an express carrier. At one of the reference sampling sites (BG004), excessive root mats that could not be cut by the spade precluded collection of a sediment sample, so no sediment toxicity test was performed for this site.

\subsection{Water Toxicity}

Definitive chronic toxicity tests were conducted on Ceriodaphnia dubia using EPA methods (Weber et al. 1989). Test conditions are specified in Table 4. Toxicity tests were performed on a series of dilutions, $(0,6.3 \%, 13 \%, 25 \%, 50 \%$ and $100 \%$ sample water), using 10 test organisms per concentration. At the end of the 7-day test the reproductive success (number of young produced) of organisms exposed to each dilution was compared statistically to that the control group $(0 \%)$ in order to determine if reproduction was impaired. The results of the tests were reported as NOEC's (No Observed Effect Concentration), which was the highest concentration tested that did not cause a significant reduction in reproduction. If there was no reproductive impact, the NOEC was $100 \%$. In addition, any mortality that occurs during the test was also recorded.

Water quality parameters routinely measured during the toxicity tests include temperature, $\mathrm{pH}$, hardness, alkalinity and specific conductance. In addition, analyses for selected metals were also conducted twice at each sampling location.

\subsection{Sediment Toxicity}

Ten-day whole sediment toxicity tests were conducted on the amphipod Hyalella azteca using EPA protocol (U.S. EPA, 1994). Test conditions are specified in Table 5. Water quality measurements routinely measured during the toxicity tests include hardness, alkalinity, conductivity, $\mathrm{pH}$, ammonia, temperature, and dissolved oxygen. 
Table 4. Test Conditions for Conducting a 7-day Definitive Water Toxicity Test with Ceriodaphnia dubia

\begin{tabular}{ll} 
Parameter & Conditions \\
\hline Test type & Static renewal \\
Temperature & $25 \pm 1^{\circ} \mathrm{C}$ \\
Light quality & Ambient laboratory illumination \\
Light intensity & $50-100$ foot candles \\
Photoperiod & $16 \mathrm{~L}: 8 \mathrm{D}$ \\
Test chamber size & $30 \mathrm{ml}$ \\
Test solution volume & $15 \mathrm{ml}$ \\
Renewal of test water & Daily \\
Age of test organisms & Less than $24 \mathrm{~h}$ at start of test; all released within a $12 \mathrm{~h}$ period \\
\# neonates/test chamber & 1 \\
\# Neonates/concentration & 10 \\
Feeding regime & $0.1 \mathrm{ml}$ YCT and algal suspension/test chamber/day \\
Aeration & None \\
Dilution water & Diluted mineral water \\
Number of dilutions & 5 and a control
\end{tabular}

Table 5. Test Conditions for Conducting a 10-day Sediment Toxicity Test with Hyalella azteca

\begin{tabular}{ll} 
Parameter & \multicolumn{1}{c}{ Conditions } \\
\hline Test type & Whole sediment toxicity test with renewal of overlying water \\
Temperature & $23 \pm 1^{\circ} \mathrm{C}$ \\
Light quality & Wide-spectrum fluorescent lights \\
Light intensity & 500 to $1000 \mathrm{lux}$ \\
Photoperiod & $16 \mathrm{~L}: 8 \mathrm{D}$ \\
Test chamber & $500 \mathrm{ml}$ beaker \\
Sediment volume & $100 \mathrm{ml}$ \\
Overlying water volume & $175 \mathrm{ml}$ \\
Water renewal & 2 volume additions/day \\
Age of organisms & 7 to 14 days at start of test \\
Number of organisms & 10 \\
per chamber & 8 \\
Number of replicates & \\
per treatment & YCT food, $1.5 \mathrm{ml} /$ day/test chamber \\
Feeding & None, unless DO drops below $40 \%$ of saturation \\
Aeration & Surface water \\
Overlying water & Hardness, alkalinity, conductivity, pH, and ammonia \\
Overlying water quality & at beginning and end of test; temperature and DO daily \\
parameters & 10 days \\
Test duration & Survival \\
Endpoint & Control survival $\geq 80 \%$ \\
Test acceptability &
\end{tabular}




\subsection{RESULTS}

\subsection{Water Chemistry}

Metals - The U.S. EPA (1991) has issued freshwater water quality criteria for many metals and organic compounds. The toxicity of most cationic metals is inversely related to water hardness. For a few metals, such as aluminum, toxicity is also $\mathrm{pH}$ dependent. Table 6 lists the EPA freshwater water quality criteria or other relevant criteria for metals that were sampled at the MWMF sampling locations. A hardness of $10 \mathrm{mg} / \mathrm{l}$ was used in calculating the criteria for metals for which the toxicity is hardness dependent.

Table 6. U.S. EPA Water Quality Criteria for Selected Metals

\begin{tabular}{ll} 
Metal & Criterion \\
Aluminum & $75^{\mathrm{a}}$ \\
Arsenic & $850^{\mathrm{b}}$ \\
Cadmium & $0.19^{\mathrm{c}}$ \\
Chromium & 11 \\
Copper & $1.65^{\mathrm{c}}$ \\
Iron & 1000 \\
Lead & $0.17^{\mathrm{c}}$ \\
Manganese & No criterion has been published. \\
Mercury & 0.102 \\
Selenium & 5 \\
Silver & $0.08^{\mathrm{c}}$ \\
Zinc & $15^{\mathrm{c}}$ \\
& \\
\hline all concentrations are in $\mu \mathrm{g} / \mathrm{l}$ & \\
asCDHEC criterion; U.S. EPA has not issued a criterion \\
bNo chronic value has been published; value is acute Lowest Observable Effect Level (LOEL) \\
'Criterion is based on a water hardness of $10 \mathrm{mg} / l$
\end{tabular}

Analyses for 12 metals were performed on samples collected from six of the MWMF toxicity testing locations in August-September 1993, as part of another sampling program (Table 2; Dixon, personal communication). These results indicate that potentially toxic concentrations of aluminum, iron, zinc, and lead may occur at some of the MWMF and reference locations.

In February 1996, samples were collected at 11 of the 15 MWMF sampling locations and analyzed for aluminum, copper, iron, lead, manganese, and zinc (Table 7). The results indicate that potentially toxic concentrations of aluminum were present at all but one of the sampling locations and that iron and zinc were measured at potentially toxic concentrations at four of the locations that were sampled. Copper was found at a potentially toxic concentration at one location (FHB-020).

In April 1996, samples were collected at all fifteen locations and analyzed for total and dissolved aluminum, total and dissolved iron, and total copper, lead, and zinc (Table 8). These results indicate that potentially toxic concentrations of aluminum were measured at all of the sampling locations and that potentially toxic concentrations of iron, copper, lead, and zinc were found at one or more locations. 
Table 7. Metal Concentrations at MWMF Sampling Locations, February 1996

$\begin{array}{lrrrrrr}\text { Location } & \mathrm{Al} & \mathrm{Cu} & \mathrm{Fe} & \mathrm{Pb} & \mathrm{Mn} & \mathrm{Zn} \\ \text { BG-001 } & 164 & <5 & 1,560 & <15 & 42 & 74 \\ \text { BG-002 } & 152 & <5 & 101 & <15 & 26 & <5 \\ \text { BG-003 } & \mathbf{8 6} & <5 & 510 & <15 & 42 & <5 \\ \text { BG-004 } & 407 & <5 & 873 & <15 & 68 & 11 \\ \text { FHB-006 } & 1,008 & <5 & 4,480 & <15 & 120 & 31 \\ \text { FHB-014 } & <50 & <5 & 216 & <15 & 6.2 & <5 \\ \text { FHB-020 } & \mathbf{2 , 3 6 0} & 18.1 & 10,900 & <15 & 63 & 27 \\ \text { FHB-022 } & 118 & <5 & 642 & <15 & 31 & 8 \\ \text { FHB-023 } & 154 & <5 & 1,500 & <15 & 53 & <5 \\ \text { HSP-064 } & 190 & <5 & 448 & <15 & 139 & 17 \\ \text { FMB-002H } & 85 & 9 & 844 & <15 & 34 & 8\end{array}$

All concentrations are in $\mu \mathrm{g} / \mathrm{l}$.

Numbers in bold indicate potential for toxicity

Table 8. Metal Concentrations at MWMF Sampling Locations, April 1996

$\begin{array}{lrrrrrrr}\text { Location } & \text { Total Al } & \text { Diss. Al } & \text { Cu } & \text { Total Fe } & \text { Diss. Fe } & \mathrm{Pb} & \mathrm{Zn} \\ \text { BG-001 } & 206 & 157 & <1 & \mathbf{2 , 7 3 0} & 796 & <3 & 3.2 \\ \text { BG-002 } & 2,390 & 96 & 3.1 & 835 & 82 & 3.5 & 7.5 \\ \text { BG-003 } & 140 & 97 & <1 & 744 & 399 & <3 & 1.6 \\ \text { BG-004 } & 243 & 161 & <1 & 1,230 & 685 & <3 & 2.5 \\ \text { FHB-006 } & 650 & 160 & <1 & 1,840 & 783 & <3 & 2.8 \\ \text { FHB-012 } & 291 & 32 & <1 & 655 & 89 & <3 & 3 \\ \text { FHB-013 } & 1,880 & 1,640 & 3.7 & 1,420 & 332 & <3 & 10 \\ \text { FHB-014 } & 128 & 21 & <1 & 105 & 50 & <3 & 2 \\ \text { FHB-018 } & 251 & 30 & 1.4 & 15,200 & 19,900 & <3 & 7 \\ \text { FHB-020 } & 840 & 274 & 3 & 12,600 & 8 & <3 & 6.9 \\ \text { FHB-021 } & 77 & 31 & <1 & 230 & 89 & <3 & 19 \\ \text { FHB-022 } & 120 & 61 & <1 & 1,010 & 582 & <3 & 6.9 \\ \text { FHB-023 } & 203 & 112 & <1 & 2,650 & 668 & <3 & 3.9 \\ \text { HSP-064 } & 94 & 52 & <1 & 314 & 165 & <3 & 4.5 \\ \text { FMB-002H } & 105 & 54 & 1 & 1,540 & 782 & <3 & 6.9\end{array}$

All concentrations are in $\mu \mathrm{g} / \mathrm{l}$.

Numbers in bold indicate potential for toxicity

Other Water Quality Parameters - Water quality parameters that are routinely performed in conjunction with the toxicity tests include $\mathrm{pH}$, total hardness, alkalinity, dissolved oxygen, and specific conductance (Table 9). The results indicate that all of the sampling locations had low hardness ( 2 to $20 \mathrm{mg} / \mathrm{l}$ as $\mathrm{CaCO}_{3}$ ), and most locations had low alkalinity $(0$ to $14 \mathrm{mg} / \mathrm{l})$ and conductivity $(<50 \mu \mathrm{mhos} / \mathrm{cm})$. However, several 
Table 9. Dissolved Oxygen, $\mathrm{pH}$, Hardness, Alkalinity, and Conductivity Measurements (Range) on Water Samples Used for Toxicity Testing

Location
BG-001
BG-002
BG-003
BG-004
FHB-006
FHB-012
FHB-013
FHB-014
FHB-018
FHB-020
FHB-021
FHB-022
FHB-023
HSP-064
FMC-002H

FMC- $002 \mathrm{H}$

Diss. Oxygen
$8.4-9.6$
$8.7-9.0$
$8.2-9.2$
$8.0-9.2$
$8.2-9.0$
$8.2-9.2$
dry
$8.5-9.1$
8.7
$8.6-9.4$
$8.0-9.4$
$8.2-9.2$
$8.4-8.7$
$8.4-9.2$
$8.4-9.2$

Location

BG-001

BG-002

BG-003

BG-004

FHB-006

FHB-012

FHB-013

FHB-014

FHB-018

FHB-020

FHB-021

FHB-022

FHB-023

HSP-064

FMC- $002 \mathrm{H}$

Diss. Oxygen
8.0
8.1
$8.2-10.0$
$8.0-10.0$
$8.0-10.0$
$8.5-9.4$
$8.4-9.6$
$7.9-9.3$
$8.2-9.0$
$7.8-10.0$
$8.5-9.7$
$8.2-9.3$
$7.8-9.4$
dry
$8.1-10.0$

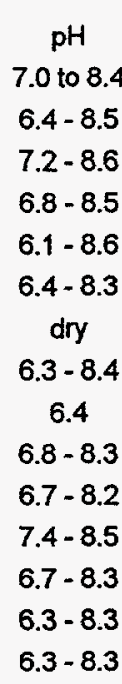

pH

5.5

5

$6.9-7.6$

$6.3-7.7$

$5.8-7.1$

$5.9-8.1$

$5.8-7.7$

$5.6-7.3$

$3.8-7.8$

$5.9-7.2$

$7.3-7.9$

$6.6-7.4$

$5.3-7.3$

dry

$6.4-7.9$
$\mathrm{pH}$

$4.9-5.0$

$6.2-7.2$

$7.5-8.2$

$5.8-7.2$

$5.5-6.8$

$5.6-6.4$

$5.4-6.0$

$5.2-5.5$

6.2

$6.1-6.8$

$6.4-7.3$

$7.3-8.2$

$5.8-7.3$

$6.1-7.9$

$7.4-7.9$

$\begin{array}{lc}\text { Location } & \text { Diss. Oxygen } \\ \text { BG-001 } & 8.0-8.2 \\ \text { BG-002 } & 8.2-8.5 \\ \text { BG-003 } & 8.4-9.0 \\ \text { BG-004 } & 8.0-9.0 \\ \text { FHB-006 } & 7.9-8.8 \\ \text { FHB-012 } & 8.4-8.8 \\ \text { FHB-013 } & 8.6-8.8 \\ \text { FHB-014 } & 8.2-8.6 \\ \text { FHB-018 } & 8.6 \\ \text { FHB-020 } & 7.6-8.8 \\ \text { FHB-021 } & 8.6-8.8 \\ \text { FHB-022 } & 8.4-8.6 \\ \text { FHB-023 } & 8.6 \\ \text { HSP-064 } & 8.2-8.8 \\ \text { FMC-002H } & 8.1-9.1\end{array}$

Jan - Feb 1995
Hardness
$4-11$
$2-4$
$4-11$
$6-10$
$4-6$
$8-10$
dry
$4-8$
$10-14$
$6-18$
$4-8$
$10-14$
$12-16$
$6-14$
$8-18$

Alkalinity

2

2

$2-4$

2- 4

4- 6

4- 6

dry

4-6

20- 47

4- 12

2-6

$6-8$

2- 4

4 - 28

$6-8$

Alkalinity
$<0.1-2$
$<0.1$
$4-8$
$8-14$
$4-8$
$4-6$
$6-8$
$6-8$
$4-33$
$4-12$
6
$8-31$
$2-12$
dry
$30-35$

$30-35$

Conductivity
16
$\begin{gathered}19-20 \\ 21-22 \\ 23 \\ 34-37 \\ 40 \\ \text { dry } \\ 32-33 \\ 57-112 \\ 25-42 \\ 43-44 \\ 48-55 \\ 28-33 \\ 25-28 \\ 44-51\end{gathered}$

Feb-Apr 1996

Hardness
$4-16$
$10-26$
$8-14$
$8-20$
$6-14$
$6-10$
$8-16$
$6-12$
$6-12$
10
$6-10$
$14-16$
$12-18$
$10-16$
$12-24$

$\begin{array}{cc}\text { Alkalinity } & \text { Conductivity } \\ 0.1-2 & 16-30 \\ <0.1-2 & 17-32 \\ 2 & 23-24 \\ 2-4 & 27-30 \\ 4-6 & 32-36 \\ - & - \\ - & - \\ 4-6 & 39 \\ - & - \\ 4-8 & 38-43 \\ - & - \\ 8-10 & 57-59 \\ 2 & 32-33 \\ 4-6 & 31-34 \\ 8-10 & 57-59\end{array}$

Conductivity

10- 14

$15-26$

$19-32$

$19-38$

$30-35$

$41-43$

$38-41$

$36-39$

$44-86$

$37-41$

$45-46$

$40-69$

$8-37$

dry

$40-68$ 
locations (FHB-018, FHB-022 and FMB-002H) usually had higher alkalinities (up to 47 $\mathrm{mg} / \mathrm{l}$ ) and conductivities (up to $112 \mu \mathrm{mhos} / \mathrm{cm}$ ) than the other sampling stations. In some instances, a high value for alkalinity or conductivity would occur in only one out of three samples from a location, which suggests intermittent influxes of substances with high alkalinity and/or conductivity.

Dissolved oxygen concentrations were always at least $7.6 \mathrm{mg} / \mathrm{l}$, which is greater than the $5 \mathrm{mg} / \mathrm{l}$ that is required to support most forms of aquatic life. $\mathrm{pH}$ was variable, ranging from 3.8 to 8.6. In samples with $\mathrm{pH}$ 's of less than 6 , high mortalities were often observed in the highest concentration (100\%) of sample. These results suggest that low $\mathrm{pH}$ may be responsible for at least part of the observed toxicity.

\subsection{Water Toxicity}

\subsubsection{General Overview}

Table 10 summarizes the results of the water toxicity tests that were performed during three time periods in 1995 and 1996, while Appendices A through C contain more detailed results of the tests.

Table 10. No Observed Effect Concentrations (NOEC's) for MWMF Toxicity Testing Locations, 1995-1996

TEST DATES JAN-FEB 1995 AUG-SEP 1995 FEB-APR 1996

\begin{tabular}{lccc} 
LOCATION & & & \\
BG-001 & $50 \%$ & $50 \%$ & $50 \%$ \\
BG-002 & $25 \%$ & $<6.25 \%$ & $50 \%$ \\
BG-003 & $50 \%$ & $6.25 \%$ & $25 \%$ \\
BG-004 & $50 \%$ & $12.50 \%$ & $50 \%$ \\
FHB-006 & $50 \%$ & $6.25 \%$ & $50 \%$ \\
FHB-012 & $50 \%$ & $50 \%$ & $25 \%$ \\
FHB-013 & Dry & $50 \%$ & $13 \%$ \\
FHB-014 & $100 \%$ & $<6.25 \%$ & $25 \%$ \\
FHB-018 & $25 \%$ & $12.50 \%$ & $6 \%$ \\
FHB-020 & $50 \%$ & $<6.25 \%$ & $50 \%$ \\
FHB-021 & $50 \%$ & $12.50 \%$ & $13 \%$ \\
FHB-022 & $50 \%$ & $6.25 \%$ & $100 \%$ \\
FHB-023 & $50 \%$ & $<6.25 \%$ & $50 \%$ \\
HSP-064 & $50 \%$ & 0.5 & $100 \%$ \\
FMC-002H & $100 \%$ & $<6.25 \%$ & $100 \%$ \\
\hline
\end{tabular}

The results indicate that most of the samples, including those from the reference locations (BG-001 through BG-004) were toxic to some extent, with NOEC's ranging from $<6.25 \%$ to $100 \%$. Of the 44 test results, only five showed no evidence of toxicity. The results also indicate substantial temporal variability. Eleven of the fifteen locations were more toxic in the summer than in either the winter of 1995 or 1996. This 
suggests that toxicity may be greatest during the low flow conditions that usually exist during the summer months, and reduced following periods of greater rainfall, which dilutes the seepwater directly, and also causes further dilution as it infiltrates into the shallow groundwater.

Approximately $30 \%$ of the undiluted $(100 \%)$ samples also resulted in complete mortality to the test organisms, and $73 \%$ of the undiluted samples caused at least partial mortality (Table 11).

Table 11. Percent Mortality in Undiluted Water from the MWMF Sampling Locations, 1995-1996

$\begin{array}{lccc}\text { LOCATION } & \text { Jan-Feb } 1995 & \text { Aug-Sept 1995 } & \text { Feb-Apr 1996 } \\ \text { BG-001 } & 90 & 100 & 100 \\ \text { BG-002 } & 100 & 100 & 100 \\ \text { BG-003 } & 20 & 40 & 0 \\ \text { BG-004 } & 30 & 50 & 10 \\ \text { FHB-006 } & 40 & 100 & 30 \\ \text { FHB-012 } & 50 & 0 & 10 \\ \text { FHB-013 } & \text { dry } & 90 & 0 \\ \text { FHB-014 } & 10 & 60 & 100 \\ \text { FHB-018 } & 100 & 100 & 100 \\ \text { FHB-020 } & 100 & 0 & 0 \\ \text { FHB-021 } & 20 & 0 & 0 \\ \text { FHB-022 } & 0 & 10 & 0 \\ \text { FHB-023 } & 100 & 40 & 100 \\ \text { HSP-064 } & 70 & \text { dry } & 0 \\ \text { FMC-002H } & 0 & 30 & 0\end{array}$

\subsubsection{Background Locations}

Uncontaminated reference locations included BG-001, BG-002, BG-003 and BG-004. BG-001 and BG-002 are located in uncontaminated groundwater outcrops and were selected as reference locations for the sampling sites adjacent to the Old F-Area Effluent ditch that are groundwater outcrops. BG-003 and BG-004 are located in uncontaminated flowing water and were selected as reference locations for the sampling sites in the Old F-Area Effluent Ditch.

As shown in Table 10, all of the toxicity tests conducted on water from the four reference locations were toxic to some extent during all three rounds of testing, with NOEC's ranging from $<6.25 \%$ to $50 \%$. BG-001 was consistently the least toxic, with NOEC's of $50 \%$ in all three sets of tests. However, BG-001 and BG-002 consistently had high mortality (90 to 100\%) in undiluted sample water. BG-002 through BG-004 were much more toxic in late summer (NOEC's of $<6.25$ to $12.5 \%$ ) than in winter (NOEC's of 25 to $50 \%$ ).

These results indicate that uncontaminated seeps and flowing water on the SRS may be somewhat toxic to Ceriodaphnia dubia. These results confirm earlier findings of 
toxicity at uncontaminated locations that were sampled as part of the $\mathrm{F} / \mathrm{H}$ seep toxicity testing program. In that study, it was determined that the toxicity was due to naturally occurring iron (ETT Environmental, 1994, 1995).

\subsubsection{MWMF Seeps}

Toxicity tests conducted on seeps in the vicinity of the MWMF (FHB-006, FHB-013, FHB-014, FHB-018, FHB-020, FHB-023 and HSP-064) had NOEC's ranging from $<6.25$ to $100 \%$ (Table 10). Most of the seeps were most toxic in late summer. However, the seeps that were tested in April 1996 (FHB-012, FHB-013, FHB-018 and FHB-021), rather than in February 1996 were considerably more toxic. Since their toxicity was comparable to the other locations in the earlier test periods, it is likely that the lower NOEC's in April 1996 were related to the degree to which the groundwater was diluted by precipitation. Further investigations may be needed to define temporal variability in toxicity at the MWMF sampling locations.

These results indicate that the MWMF seeps are no more toxic that the reference locations that were tested, which suggests that the toxicity is due to naturally occurring constituents, and not contaminants from the MWMF.

\subsubsection{Old F-Area Effluent Ditch}

Toxicity tests on samples collected from the old F-Area effluent ditch (FHB-012, FHB-021 and FHB-022) had NOEC values ranging from $12.5 \%$ to $100 \%$ (Table 10). Only one of the samples (FHB-022 in winter 1996) showed no evidence of toxicity. These results indicate that the sampling locations in the effluent ditch are no more toxic than the reference locations that were tested, which suggests that the toxicity is due to naturally occurring constituents, and not contaminants from the MWMF.

\subsubsection{Fourmile Branch}

Samples collected from Fourmile Branch (FMC-002H) showed no evidence of toxicity in the winters of 1995 or 1996, but the test conducted in late summer of 1995 had an NOEC of $<6.25 \%$, with $30 \%$ mortality in undiluted stream water (Table 10 ).

\subsection{Sediment Toxicity}

Sediment toxicity tests were performed on wetland soil or sediment samples collected from 14 of the 15 sampling locations. One location (BG-004) could not be sampled due to the presence of dense root mats, which precluded collection of a sediment sample. Of the fourteen locations that were tested, four passed the sediment toxicity test (percent survival did not significantly differ from that of the controls), while at the remaining ten locations survival was significantly lower than in the controls (Table 12).

Two of the three reference locations were toxic, while eight of the 11 test sites were toxic. Percent survival ranged from 0\% (FMC-002H) to 95\% (FHB-012). Toxic responses at both the reference and $\mathrm{FHB}$ locations suggests that the toxicity is due to natural conditions, rather than to the presence of toxicants from the MWMF. However, 
Table 12. Results of 10-day Whole Sediment Hyalella azteca Toxicity Tests

$\begin{array}{lcc}\text { LOCATION } & \text { PASS/FAIL } & \% \text { SURVIVAL } \\ \text { BG-001 } & \text { Pass } & 74 \% \\ \text { BG-002 } & \text { Fail } & 4 \% \\ \text { BG-003 } & \text { Fail } & 68 \% \\ \text { BG-004 } & \text { a } & a \\ \text { FHB-006 } & \text { Fail } & 3 \% \\ \text { FHB-012 } & \text { Pass } & 95 \% \\ \text { FHB-013 } & \text { Fail } & 55 \% \\ \text { FHB-014 } & \text { Pass } & 83 \% \\ \text { FHB-018 } & \text { Fail } & 50 \% \\ \text { FHB-020 } & \text { Pass } & 81 \% \\ \text { FHB-021 } & \text { Fail } & 73 \% \\ \text { FHB-022 } & \text { Fail } & 3 \% \\ \text { FHB-023 } & \text { Fail } & 20 \% \\ \text { HSP-064 } & \text { Fail } & 69 \% \\ \text { FMC-002H } & \text { Fail } & 0 \%\end{array}$

aNo sample was collected, due to dense root mats.

an examination of the supporting chemistry data for the overlying water (dissolved oxygen, conductivity, $\mathrm{pH}$, alkalinity, hardness, and ammonia) showed no clear reason for most of the failures. Some of the locations had relatively low pH $(\sim 5)$ and/or low hardness $(<20)$, but many of the sites that were toxic had $\mathrm{pH}$ and hardness values that should not have stressed the test organisms. Of particular concern is that the sample from Fourmile Branch was the most toxic of the 14 sampling locations, with $100 \%$ mortality by the end of the 10 day test period. Ammonia concentrations in the overlying water from the Foumile Branch test were $1.11 \mathrm{mg} / \mathrm{l}$ at the beginning of the test and 3.04 $\mathrm{mg} / \mathrm{l}$ at the end of the test (Appendix D). Dissolved oxygen concentrations were also less than $5 \mathrm{mg} / \mathrm{l}$ on three of the 10 test days. Ammonia concentrations in excess of 1 $\mathrm{mg} / \mathrm{l}$ and/or dissolved oxygen levels of less than $5 \mathrm{mg} / \mathrm{l}$ can often be toxic to many aquatic species. These results suggest that the Fourmile Branch test failed due to reduced (anaerobic) conditions, rather than to the presence of toxicants such as metals or organic compounds. Macroinvertebrate sampling of Fourmile Branch at Road C in 1993 indicated that this area of the stream supported a depauperate macroinvertebrate community and that the stream had very low concentrations of dissolved oxygen (Specht 1994). Further studies may be needed to determine the cause of the low oxygen levels in this portion of Fourmile Branch. Ammonia concentrations were also initially high (1.89 mg/l) in the test conducted on sediment collected from FHBO22. This site also exhibited very poor survival (3\%). The results of the sediment toxicity tests suggest that although most of the sediments were toxic to some extent, the toxicity is probably due to anaerobic conditions in the sediments and/or naturally occurring toxicants, since both the reference and FHB locations were toxic. 


\subsection{CONCLUSIONS}

The results of the toxicity tests indicate that water from all of the sampling locations, including the four reference locations, was toxic in at least one of the three rounds of testing. No Observed Effect Concentrations (NOEC's) ranged from $<6.25 \%$ to $100 \%$. At most locations, the water was more toxic during the summer months, and was probably due to reduced rainfall. Many of the sampling locations have naturally low pH's and potentially toxic concentrations of several metals, including aluminum, copper, iron, lead, and zinc have been measured at all of the sampling locations, including the reference locations. The observed toxicity is probably due to naturally elevated concentrations of one or more of these metals and/or low $\mathrm{pH}$. Toxicity Identification Evaluations (TIE's) are being performed at several sampling locations to identify the toxicants.

All but four of the sediment toxicity tests showed evidence of toxicity. Reference sites, as well as MWMF sampling sites were toxic, which suggests that the toxicity is due to naturally occurring conditions and is not related to MWMF contaminants. The toxicity may be due to anoxic conditions in the sediments.

In conclusion, the results of the water and sediment toxicity tests conducted at sampling locations in the vicinity of the MWMF and reference sites indicate that all of the locations are toxic to some extent. However, the toxicity appears to be due to naturally occurring toxicants, and not to MWMF contaminants. 


\subsection{LITERATURE CITED}

Dixon, K.L., C.L. Cummins, and V.A. Rogers. 1994. Quarterly Sampling of the Wetlands Along the Old F-Area Effluent Ditch: March 1994. WSRC-TR-94-0225

ETT Environmental, Inc. 1994. Chronic Toxicity Identification Evaluation, UTR-029 Seep at the Savannah River Site, November 1994.

ETT Environmental, Inc. 1995. Chronic Toxicity Identification Evaluation Phase II, UTR-029 Seep at the Savannah River Site, January 1995.

Specht, W.L. 1994. Results of Macroinvertebrate Sampling Conducted at 33 SRS Stream Locations, July-August 1993. WSRC-TR-95-0006.

U.S. Environmental Protection Agency. 1991. Water Quality Criteria Summary.

U.S. Environmental Protection Agency. 1994. Methods for Measuring Toxicity and Bioaccumulation of Sediment-associated Contaminants with Freshwater Invertebrates. EPA/600/R-94/024.

Weber, C.I, W.H. Peltier, T.J. Norberg-King, W.B. Horning II, F.A. Kessler, J.R. Menkedick, T.W. Neilheisel, P.A. Lewis, D.J. Klemm, Q.H. Pickering, E.L. Robinson, J.M. Lazorchak, L.J. Wymer, and R.W. Freyberg. 1989. Short-term Methods for Estimating the Chronic Toxicity of Effluents and Receiving Waters to Freshwater Organisms. EPA/600/4-89/001. 
APPENDIX A

WATER TOXICITY RESULTS, JANUARY-FEBRUARY 1995 
7 Day Ghronic Definitive Survival and Reproduction Bioassay

Test Organisms: Ceriodaphnia dubia

Method: EPA/600/4-89/001

\footnotetext{
Facility = Westimghouse savanmah Rivex company $\operatorname{Samp} 1 \in I D=B G O O 1$
}

Date: 2-2-95 
SAMPLING INFORMATION

\begin{tabular}{|c|c|c|c|c|c|c|c|c|}
\hline Dilution Water & $20 \% \mathrm{DMW}$ & $2-1-95$ & OH & 96.04 & 71.05 & 226 & 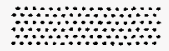 & \\
\hline Final Eff. 1 & Grab & $2-1-95$ & $08: 20 \mathrm{AM}$ & 3.92 & 2.03 & 16.4 & $<0.05$ & $3.0^{\circ} \mathrm{C}$ \\
\hline Einal Eff. 2 & Grab & $2-3-95$ & $08: 10 \mathrm{AM}$ & 11.1 & 2.03 & 15.6 & $<0.05$ & $3.2^{\circ} \mathrm{C}$ \\
\hline Final Eff. 3 & Grab & $2-6-95$ & $10: 10 \mathrm{AM}$ & 7.84 & 2.03 & 15.9 & $<0.05$ & $1.8^{\circ} \mathrm{C}$ \\
\hline
\end{tabular}


Results

\begin{tabular}{|l|l|l|l|l|}
\hline \multicolumn{2}{|l|}{ Client: WSRC } & Sample ID: BG001 & \\
\hline Log \#: T3524 & Start Date: $2-2-95$ & Time: & $4: 00$ PM \\
\hline
\end{tabular}

SURVIVAL EFFECTS

Contro1 Effluent

$\begin{array}{lllllll}\text { Effluent Conc. } & \text { SC.DMW } & 6.25 \% & 12.5 \% & 25 \% & 50 \% & 100 \%\end{array}$

\begin{tabular}{lllllll}
$48 \mathrm{hr}$. Mortality & $0 \%$ & $0 \%$ & $0 \%$ & $0 \%$ & $0 \%$ & $30 \%$ \\
7 Day Mortality & $0 \%$ & $10 \%$ & $0 \%$ & $0 \%$ & $0 \%$ & $90 \%$ \\
\hline
\end{tabular}

Method: Fishers Exact Test

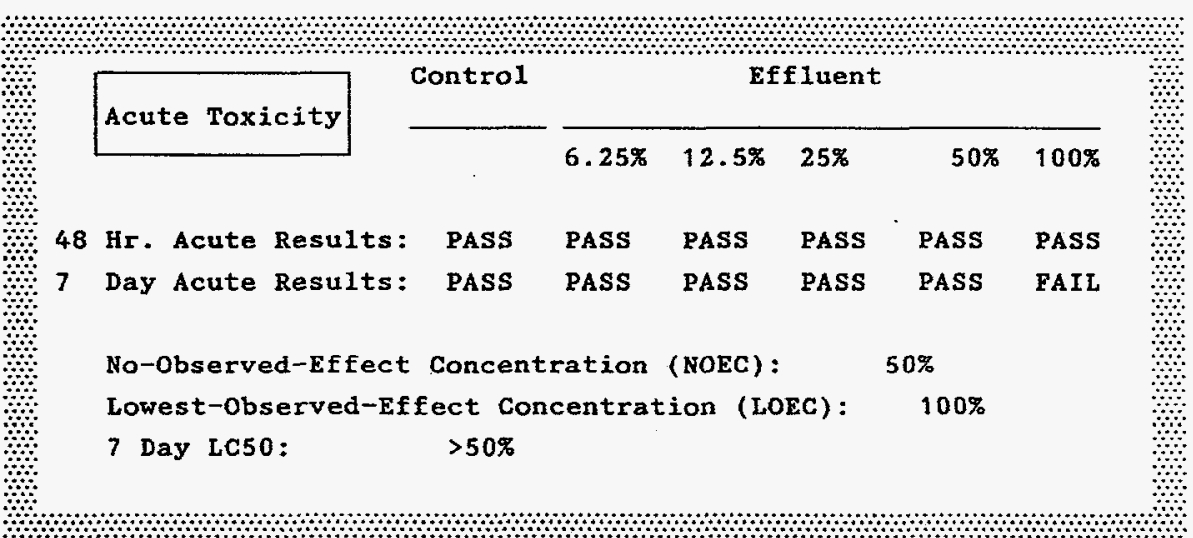

QUALITY CONTROL

Standard Toxicant: NaCI
Central Tendency:
2.04
$g / L$
Current Value:
1.9
$\mathrm{g} / \mathrm{L}$
Deviation:
0.58
Std Dev units. 
Results

\begin{tabular}{|l|l|l|l|l|}
\hline \multicolumn{2}{|l|}{ Client: WSRC } & Sample ID: BG001 & IWG: \\
\hline Log : : T3524 & Start Date: $2-2-95$ & Time: & $4: 00$ PM \\
\hline
\end{tabular}

CHRONIC EFFECTS

Control Effluent

TEST CONCENTRATION

Average young / female:

Standard Deviation:

$t=$

SC.DMW

\begin{tabular}{lllll}
\hline $6.25 \%$ & $12.5 \%$ & $25 \%$ & $50 \%$ & $100 \%$
\end{tabular}

$\begin{array}{rrrrrr}21.5 & 17.5 & 21.8 & 21.8 & 16.8 & 0.4 \\ 6.13 & 7.99 & 4.92 & 6.7 & 4.89 & 0.84 \\ & 1.43 & -0.1 & -0.1 & 1.68 & \text { NA }\end{array}$

$M S D=6.23$

Normality: Data Normal

$w=1$

Homogeneity: Data Homogeneous

$B=3.06$

Test Used: Dunnett's $t$ Test

Critical steel's Value: 75

Critical t value:

2.23

Chronic Toxicity

6.25\% No Chronic Toxicity

No-Observed-Effect Concentration (NOEC): $50 \%$

12.5\% No Chronic Toxicity Lowest-Observed-Effect Concentration (LOEC): $100 \%$ 7 Day EC50: $>50 \%$

$\begin{array}{ll}\text { 25\% } & \text { No Chronic Toxicity } \\ 50 \% & \text { No Chronic Toxicity } \\ 100 \% & \text { Chronically Toxic }\end{array}$


(803) 877-6942 - FAX (803) 877-6938

P.O. Box 16414, Greenville, SC 29606 - 6C Craftsman Court, Greer, SC 29650

7 Day Chronic Definitive Survival and Reproduction Bioassay

Test Organisms: Ceriodaphnia dubia

Method: EPA/600/4-89/001

\footnotetext{
Facility = Westinghouse Savannah River Company $\operatorname{SampIe} I D=$ BGOO2
}

Date: 2-2-95 
DEFINITIVE SURVIVAL AND REPRODUCTION

Gient:WSRC

Log : $:$ T3525

Sample ID: BG002
Start Time: $4: 00 \mathrm{PM}$

End Time: 1:15 PM IWC:
TEST CONCENTRATION: $\quad 0 \%$
Start Date: 2-2-95

End Date: 2-11-95

\begin{tabular}{|c|c|c|c|c|c|c|c|c|c|c|c|c|c|c|c|c|c|c|c|c|c|c|c|c|c|c|c|c|c|}
\hline Dey A & & B & C & D & $E$ & $\boldsymbol{F}$ & G & $\mathbf{H}$ & $\mathbf{Y}$ & $\boldsymbol{J}$ & old & new & old & new & Day & & B & $c$ & D & $\mathbf{E}$ & F & $\mathbf{G}$ & H & I & $\mathrm{J}$ & old & new & old & new \\
\hline 20 & & 0 & 0 & 0 & 0 & 0 & 0 & 0 & 0 & 0 & 翌單 & 8.6 & 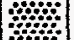 & 7.9 & 2 & 0 & 0 & 0 & 0 & 0 & 0 & 0 & 0 & 0 & 0 & 兹 & 8.6 & 器器 & 6.5 \\
\hline 30 & & 0 & 0 & 0 & 0 & 0 & 0 & 0 & 0 & 0 & 8.2 & 8.5 & 7.4 & 8.0 & 3 & 0 & 0 & 0 & 0 & 0 & 0 & 0 & 0 & 0 & 0 & 8.8 & 8.7 & 7.6 & 7.5 \\
\hline 40 & & 4 & 0 & 0 & 0 & 4 & 0 & 3 & 2 & 4 & 8.4 & 8.4 & 8.0 & 7.9 & 4 & 0 & 0 & 0 & 0 & 0 & 0 & 0 & 0 & 0 & 0 & 8.9 & 8.8 & 7.9 & 8.3 \\
\hline 57 & & 7 & 0 & 0 & 0 & 3 & 0 & 0 & 0 & 5 & 8.5 & 8.6 & 8.4 & 8.0 & & 0 & 5 & 0 & 2 & 0 & 3 & 4 & 0 & 0 & 4 & 8.5 & 8.6 & 8.3 & 8.2 \\
\hline 60 & & 0 & 0 & 0 & 0 & 0 & 0 & 0 & 0 & 0 & 9.2 & 8.6 & 8.5 & 8.2 & & 0 & 0 & 6 & 0 & 0 & 0 & 1 & 7 & 0 & 0 & 8.1 & 8.1 & 8.2 & 8.0 \\
\hline 712 & & 10 & 0 & 0 & 14 & 0 & 11 & 0 & 12 & 12 & 8.2 & 8.7 & 8.1 & 8.1 & 71 & 12 & 0 & 0 & 0 & 5 & 0 & 13 & 7 & 0 & 7 & 8.5 & 8.5 & 8.2 & 8.0 \\
\hline 80 & & 0 & 4 & 5 & 0 & 4 & 0 & 14 & 0 & 0 & 8.1 & 8.7 & $8.2 \mid$ & 8.1 & 8 & 4 & 2 & $\mathrm{x}$ & $x$ & 4 & 0 & 0 & 8 & 0 & 0 & 8.8 & 8.9 & 7.7 & 8.1 \\
\hline 913 & & 0 & 11 & 11 & 10 & 13 & 0 & 9 & 11 & 0 & 8.8 & 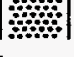 & 8.3 & 8 & 9 & 8 & 10 & $x$ & $x$ & 3 & 13 & 0 & 0 & 13 & 10 & | & 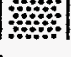 & 兹兹 & 蕠 \\
\hline ror32 & & 21 & 15 & 16 & 24 & 24 & 11 & 26 & 25 & 21 & 22 & & & & Tот2 & & 17 & 6 & 2 & 12 & 16 & 18 & 22 & 13 & 21 & 15 & & & \\
\hline TEST & & SONC & $\mathrm{NTR}$ & $4 \mathrm{TIO}$ & & 12. & & & & & $D .0$ & 0.0 & $\mathbf{p H}$ & $\mathrm{pH}$ & TES & & CONC & NTR & TION & & $25 \%$ & & & & & 0.0 & D.O & $\mathrm{pH}$ & $\mathrm{pH}$ \\
\hline Day & & B & c & D & $E$ & $F$ & $G$ & H & $I$ & $\mathbf{J}$ & old & new & $01 d$ & new & Day & A & B & $\mathrm{c}$ & D & $\mathbf{E}$ & $\mathbf{E}$ & G & H & $I$ & $\mathbf{J}$ & old & new & $01 d$ & new \\
\hline 2 & & 0 & 0 & 0 & 0 & 0 & 0 & 0 & 0 & 0 & 䎌器 & 8.5 & 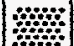 & 6.8 & 2 & 0 & 0 & 0 & 0 & 0 & 0 & 0 & 0 & 0 & 0 & $\mid$ & 8.9 & 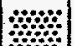 & 6.8 \\
\hline 3 & & 0 & 0 & 0 & 0 & 0 & 0 & 0 & 0 & 0 & B. 4 & B.2 & 7.6 & 7.6 & 3 & 0 & 0 & 0 & 0 & 0 & 0 & 0 & 0 & 0 & 0 & 8.4 & 8.4 & 7.7 & 7.6 \\
\hline 4 & & 3 & 2 & $\mathrm{x}$ & 0 & 0 & 3 & 0 & 4 & 2 & 8.8 & 8.7 & 8.0 & 8.4 & 4 & 4 & 1 & 0 & 0 & 1 & 0 & 1 & 0 & 4 & 0 & 8.7 & 8.6 & 8.1 & 8.4 \\
\hline 5 & & 8 & 0 & $\mathrm{x}$ & 0 & 3 & 7 & 0 & 6 & 6 & 8.7 & 8.6 & 8.5 & 8.3 & 5 & 3 & 5 & 0 & 8 & 2 & 0 & 2 & 0 & 8 & 0 & 8.5 & 8.4 & 8.5 & 8.4 \\
\hline 6 & & 0 & 6 & $\mathrm{x}$ & 0 & 0 & 0 & 7 & 0 & 0 & 8.5 & 8.0 & 8.3 & 8.0 & 6 & $x$ & 0 & 5 & 0 & 0 & 4 & 0 & 4 & 0 & 4 & 8.6 & 8.7 & 8.3 & 8.1 \\
\hline 7 & & 11 & 8 & $\mathrm{x}$ & 0 & 6 & 0 & 0 & 11 & 12 & 8.4 & 8.4 & 8.3 & 8.1 & 7 & $x$ & 12 & 8 & 10 & 9 & 6 & 6 & 10 & 0 & 2 & 8.5 & 8.4 & 8.3 & 8.0 \\
\hline 8 & & 0 & 0 & $\mathrm{x}$ & $\mathrm{x}$ & 3 & 4 & 0 & 0 & 0 & 8.8 & 8.8 & 8.1 & $(8.1)$ & 8 & $x$ & 0 & 0 & 6 & $\mathrm{x}$ & 0 & 0 & $x$ & 3 & 0 & 9.0 & $(9.0)$ & 8.2 & 7.9 \\
\hline 9 & & 0 & 0 & $x$ & $x$ & 7 & 8 & 12 & 0 & 0 & 器 & 器器 & 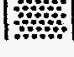 & 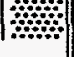 & 9 & $x$ & 0 & 8 & 0 & $x$ & 8 & 0 & $x$ & 5 & 10 & $\mid$ & $\mid$ & 获 & 获器 \\
\hline ror & 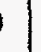 & 22 & 16 & 0 & 0 & 19 & 22 & 19 & 21 & 20 & 15 & & & & TOT & 7 & 18 & 21 & 24 & 12 & 18 & 9 & 14 & 20 & 16 & 16 & & & \\
\hline
\end{tabular}

TEST CONCENTRATION 50\%

\begin{tabular}{|l|l|l|ll}
\hline D.O & D.o & pH & pH & TEST CONCENTRATION $100 \%$
\end{tabular}

\begin{tabular}{ll|l|l}
\hline D.O & D.O & $\mathrm{pH}$ & $\mathrm{pH}$
\end{tabular}

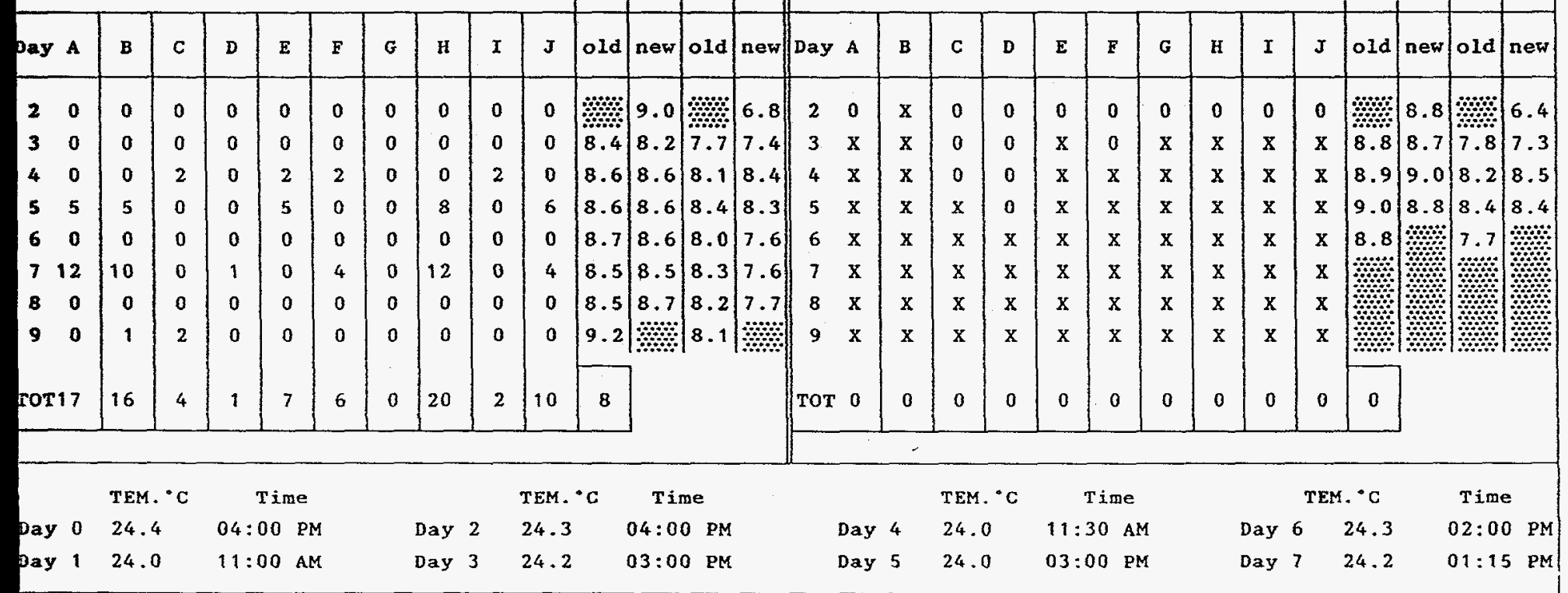


SAMPIING INFORMATION

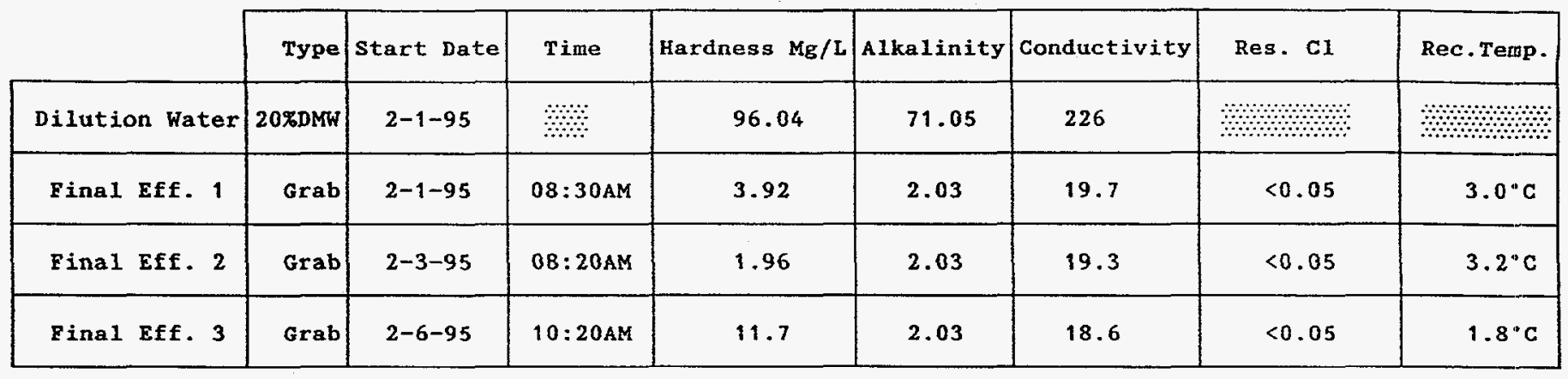


Resu1ts

\begin{tabular}{|l|l|l|l|l|}
\hline \multicolumn{2}{|l|}{ C1ient: WSRC } & Sample ID: BG002 & \\
\hline Log \#: T3525 & Start Date: $2-2-95$ & Time: & $4: 00$ PM \\
\hline
\end{tabular}

SURVIVAL EFFECTS

Control Effluent

$\begin{array}{lllllll}{ } } & \text { SC.DMW } & 6.25 \% & 12.5 \% & 25 \% & 50 \% & 100 \%\end{array}$

\begin{tabular}{llrrrrr}
$48 \mathrm{hr}$. Mortality & $0 \%$ & $0 \%$ & $0 \%$ & $0 \%$ & $0 \%$ & $10 \%$ \\
7 Day Mortality & $0 \%$ & $20 \%$ & $22 \%$ & $30 \%$ & $0 \%$ & $100 \%$ \\
\hline
\end{tabular}

Method: Fishers Exact Test

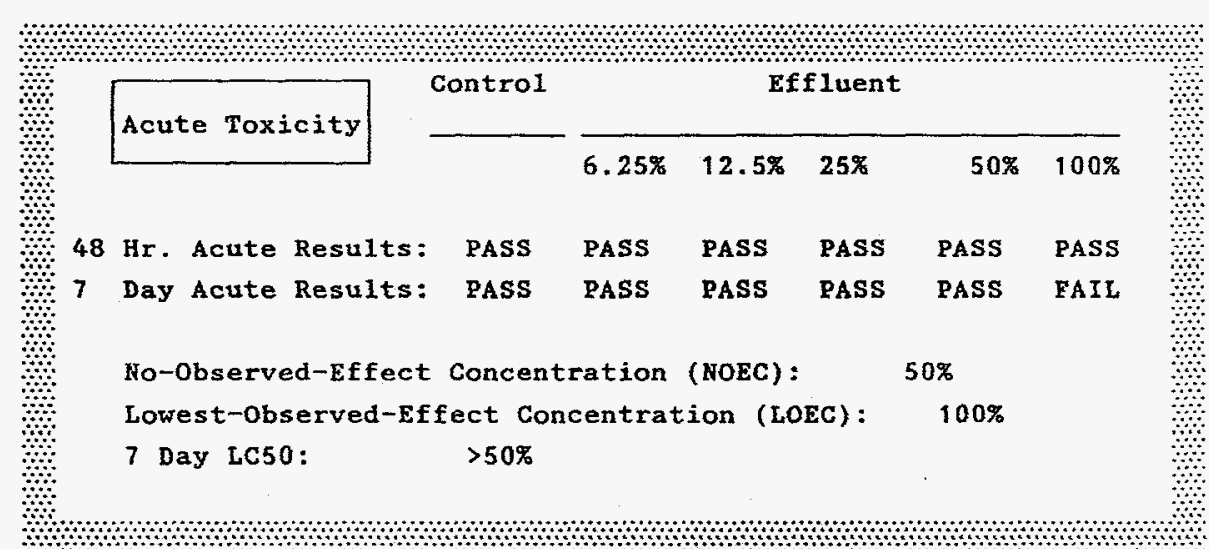

QUALITY CONTROL

Standard Toxicant: $\mathrm{NaCl}$

$\begin{array}{lccc}\text { Central Tendency: } & 2.04 & \mathrm{~g} / \mathrm{L} \\ \text { Current Value: } & 1.9 & \mathrm{~g} / \mathrm{L} \\ \text { Deviation: } & 0.58 & & \text { std Dev units. }\end{array}$


Results

\begin{tabular}{|l|l|l|l|l|}
\hline \multicolumn{2}{|l|}{ Client: WSRC } & Sample ID: BGO02 & IWC: \\
\hline Log \#: T3525 & Start Date: $2-2-95$ & & Time: & $4: 00$ PM \\
\hline
\end{tabular}

\section{CHRONIC EFFECTS}

Control Effluent

TEST CONCENTRATION

SC.DMW

\begin{tabular}{lllll}
\hline $6.25 \%$ & $12.5 \%$ & $25 \%$ & $50 \%$ & $100 \%$
\end{tabular}

Average young / female:

$\begin{array}{rrrrrr}21.5 & 15.1 & 15.4 & 15.9 & 8.3 & \text { NA } \\ 6.13 & 7.02 & 8.95 & 5.4 & 7.17 & \text { NA } \\ & 2.05 & 1.89 & 1.79 & 4.23 & \text { NA }\end{array}$

$$
t=
$$

tandard Deviation:

SC.DMW

$\begin{array}{lll}2.05 & 1.89 & 1.79 \quad 4.23\end{array}$

MSD $=7.01$

Normality: Data Normal

$w=0.96$

Homogeneity: Data Homogeneous

$\mathbf{B}=2.35$

Test Used: Dunnett's $t$ Test

Critical steel's value: 75

Critical $t$ value: 2.23

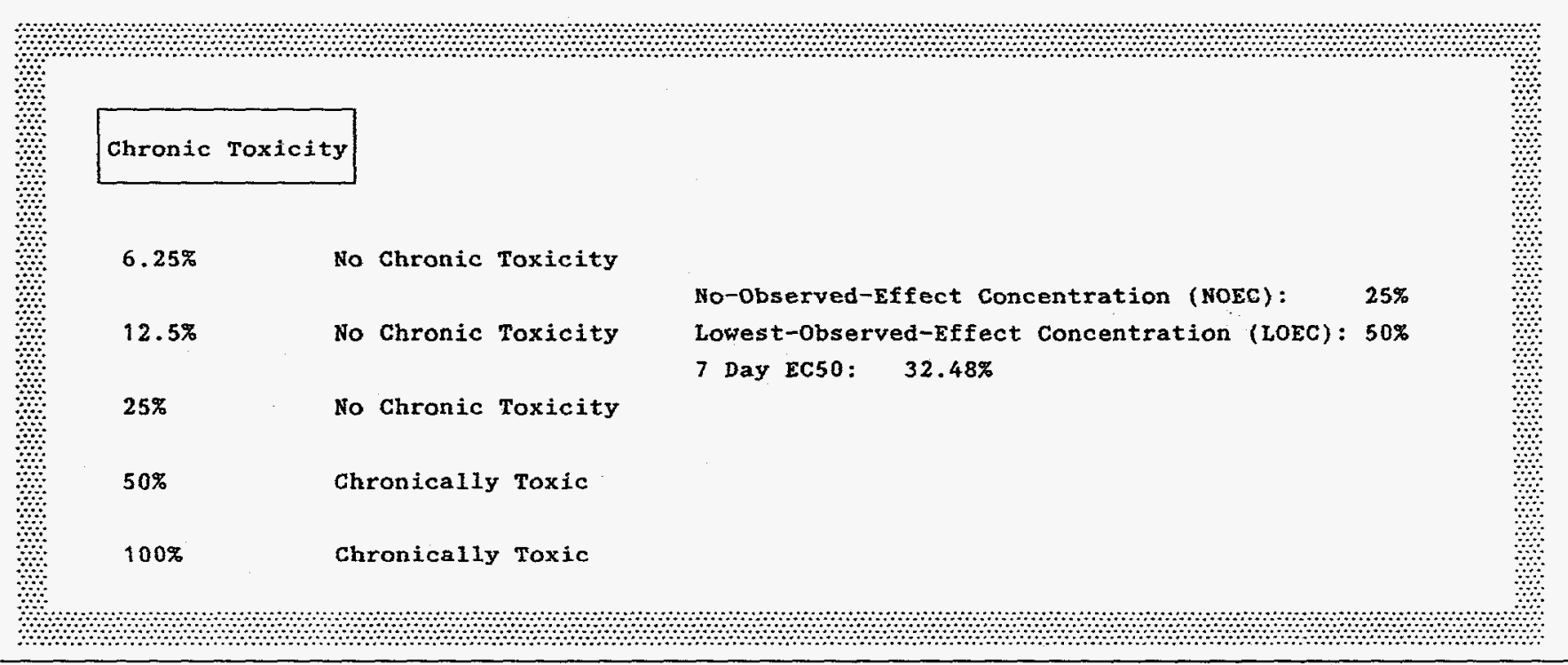


7 Day Ghromic Definitive Surviral and Reproduction Bioassay

Test Ofganisms: Ceriodaphnia dubia

Method: EPA/600/4-89/001
Facility: Westinghouse savanmah River Company Sample XD: BGOOB

pate: $2-2-95$ 
DEFINITIVE SURVIVAL AND REPRODUCTION

\begin{tabular}{|c|c|c|c|c|c|c|c|c|c|c|c|c|c|c|c|c|c|c|c|c|c|c|c|c|c|c|c|c|}
\hline \multicolumn{10}{|c|}{$\begin{array}{l}\text { Client:WSRC } \\
\text { Log \#: T3523 }\end{array}$} & \multicolumn{10}{|c|}{$\begin{array}{l}\text { Start Date: } 2-2-95 \\
\text { End Date: } 2-11-95\end{array}$} & \multicolumn{9}{|c|}{ Start Time: 3:00 PM } \\
\hline TEST & \multicolumn{4}{|c|}{ CONCENTRATION: } & \multicolumn{5}{|c|}{$0 \%$} & D.o & 0.0 & $\mathbf{p H}$ & $\mathrm{pH}$ & \multicolumn{6}{|c|}{ TESTY CONCENTRATION: } & \multicolumn{5}{|c|}{$6.25 \%$} & 0.0 & 0.0 & $\mathbf{p H}$ & \\
\hline Day A & B & C & D & E & $\mathbf{F}$ & G & H & $I$ & $\mathbf{J}$ & old & new & old & new & Day A & & & c & D & $E$ & $\boldsymbol{F}$ & $\mathbf{G}$ & H & I & $\mathbf{J}$ & $01 d$ r & new & old & \\
\hline 20 & 0 & 0 & 0 & 0 & 0 & 0 & 0 & 0 & 0 & 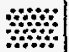 & 8.6 & 烈 & $7.9 \mid$ & 20 & & & 0 & 0 & 0 & 0 & 0 & 0 & 0 & 0 & 慗 & 8.7 & 器器 & \\
\hline 30 & 0 & 0 & 0 & 0 & 0 & 0 & 0 & 0 & 0 & 8.2 & 8.5 & 7.4 & $8.0\}$ & 30 & & & 0 & 0 & 0 & 0 & 0 & 0 & 0 & 0 & 8.6 & 8.6 & 7.5 & \\
\hline 44 & 3 & 3 & 3 & 3 & 0 & 4 & 2 & 3 & 3 & 8.4 & 8.4 & 8.0 & 7.9 & 44 & & & 4 & 4 & 4 & 0 & 5 & 4 & 0 & 0 & 9.2 & 9.2 & 8.0 & \\
\hline 50 & 4 & 0 & 6 & 6 & 5 & 5 & 6 & 0 & 0 & 8.5 & 8.6 & 8.4 & 8.0 & 5. 6 & & & 6 & 6 & 7 & $x$ & 7 & 0 & 9 & 1 & 8.8 & 8.8 & 8.5 & \\
\hline 60 & 5 & 0 & 0 & 0 & 8 & 0 & 0 & 0 & 0 & 9.2 & 8.6 & 8.5 & 8.2 & 60 & & & 0 & 0 & 0 & $\mathrm{x}$ & 0 & 3 & 0 & 0. & $9.2 \mid$ & 9.2 & $8.1 \mid$ & \\
\hline 79 & 0 & 12 & 8 & 0 & 0 & 8 & 10 & 6 & 2 & 8.2 & 8.7 & 8.1 & 8.1 & $7 \quad 12$ & & & 8 & 10 & 12 & $x$ & 10 & 9 & 0 & 0 & 8.8 & 8.6 & 7.2 & \\
\hline 80 & 8 & 16 & 10 & 0 & 10 & 6 & 0 & 0 & 0 & 8.1 & 8.7 & 8.2 & 8.1 & 80 & & & 0 & 0 & 0 & $x$ & 0 & 0 & 10 & 9 & 7.8 & 8.0 & 8.1 & \\
\hline 910 & 0 & 0 & 0 & 14 & 0 & 0 & 0 & 13 & 4 & 8.8 & \% & $|8.3|$ & 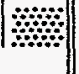 & 90 & & & 0 & 0 & 0 & $x$ & 0 & 0 & 0 & 0 & 落 & $\because$ & 获 & \\
\hline Tot23 & 20 & 31 & 27 & 23 & 23 & 23 & 18 & 22 & 9 & 22 & & & & тот 22 & & & 18 & 20 & 23 & 0 & 22 & 16 & 19 & 10 & 17 & & & \\
\hline TEST & conc & NTR & TIO & & 12. & & & & & 0.0 & D.o & $\mathrm{pH}$ & $\mathrm{pH}$ & TEST & & $C E !$ & & TION & & $25 \%$ & & & & & 0.0 & 0.0 & $\mathrm{pH}$ & \\
\hline Day A & B & c & D & $\mathbf{E}$ & $\mathbf{F}$ & G & H & I & $\mathbf{J}$ & $01 d$ & new & $01 d$ & new & Day A & & & $\mathrm{C}$ & D & $\mathbf{E}$ & $\mathbf{F}$ & G & H & I & $J$ & old & new & old & \\
\hline 20 & 0 & 0 & 0 & 0 & 0 & 0 & 0 & 0 & 0 & 垍蒴 & 8.8 & 前 & 7.1 & 20 & & & 0 & 0 & 0 & 0 & 0 & 0 & 0 & 0 & 模 & 9.1 & | & \\
\hline 30 & 0 & 0 & 0 & 0 & 0 & 0 & 0 & 0 & 0 & 8.5 & 8.6 & 7.5 & 7.5 & 30 & & & 0 & 0 & 0 & 0 & 0 & 0 & 0 & 0 & $8.6 \mid$ & 8.6 & 7.6 & \\
\hline 44 & 0 & 0 & 5 & 0 & 0 & 5 & 3 & 0 & 0 & 9.2 & $9.1 \mid$ & 8.1 & 8.3 & 4 & & & 6 & 5 & 5 & 0 & 3 & 0 & 0 & 0 & $|9.1|$ & 9.2 & 8.1 & \\
\hline 56 & 4 & 0 & 5 & 8 & 6 & 0 & 0 & 0 & 3 & 8.6 & 8.6 & 8.6 & 8.4 & 53 & & & 4 & 0 & 7 & 5 & 9 & 0 & 0 & 0 & 8.8 & 8.8 & 8.6 & \\
\hline 60 & 9 & 0 & 0 & 9 & 0 & 7 & 0 & 0 & 0 & 9.2 & 9.2 & 8.4 & $8.0 \mid$ & 60 & 1 & & 0 & 8 & 0 & 10 & 0 & 0 & 0 & 0 & 8.8 & 9.0 & 8.3 & \\
\hline 714 & 0 & 0 & 11 & 0 & 5 & 9 & 10 & 3 & 3 & 8.6 & 8.5 & 7.7 & 8.2 & 712 & & & 10 & 11 & 6 & 0 & 10 & 7 & 0 & 0 & 8.6 & 8.5 & 7.9 & \\
\hline 80 & 6 & 10 & 0 & 14 & 9 & 0 & 10 & 5 & 14 & 8.3 & 8.3 & 8,3 & $(8.1)$ & 80 & & & 0 & 0 & 14 & 10 & 0 & 9 & 12 & 0 & $8.2\}$ & 8.4 & 8.3 & \\
\hline 90 & 17 & 1 & 0 & 0 & 0 & 0 & 0 & 14 & 0 & 8.2 & $\mid$ & $|8.5|$ & $\mid$ & & & & 0 & 0 & 0 & 0 & 0 & 0 & 0 & 15 & 箱然 & | & 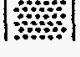 & \\
\hline TOT24 & 36 & 11 & 21 & 31 & 20 & 21 & 23 & 22 & 20 & 23 & & & & тот2 & & & 20 & 24 & 32 & 25 & 22 & 16 & 12 & 15 & 21 & & & \\
\hline
\end{tabular}

TEST CONCENTRATION 50\%

\begin{tabular}{ll|l|ll}
\hline D.O & D.O & PH & PH TEST CONCENTRATION & $100 \%$
\end{tabular}

$\begin{array}{llll}0.0 & 0.0 & \mathrm{pH} & \mathrm{pH}\end{array}$

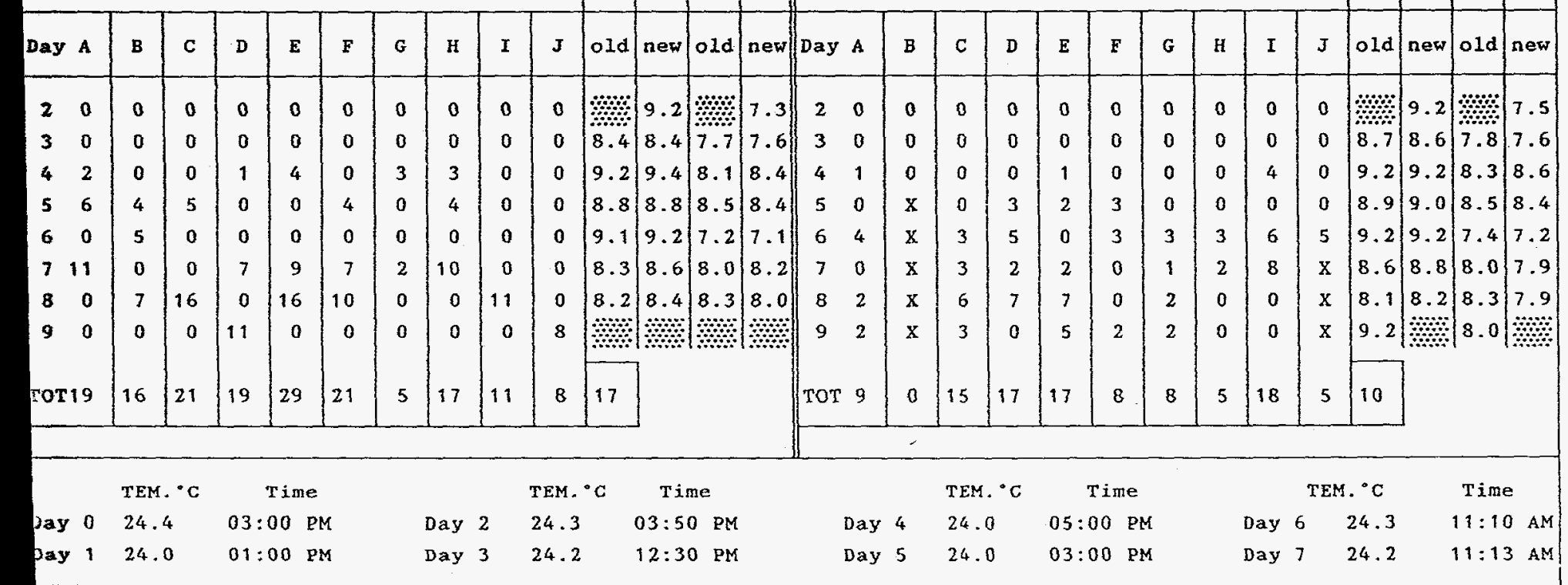




\section{SAMPLING INFORMATION}

\begin{tabular}{|c|c|c|c|c|c|c|c|c|}
\hline Dilution Water & $20 \% \mathrm{DMH}$ & $2-1-95$ & 列 & 96.04 & 71.05 & 226 & H. & \\
\hline Final Eff. 1 & Grab & $2-1-95$ & $08: 10 \mathrm{AM}$ & 3.92 & 2.03 & 21.2 & $<0.05$ & $3.0^{\circ} \mathrm{C}$ \\
\hline Final Eff. 2 & Grab & $2-3-95$ & $08: 00 \mathrm{AM}$ & 5.88 & 4.06 & 22.3 & $<0.05$ & $3.2^{\circ} \mathrm{C}$ \\
\hline Final Eff. 3 & Grab & $2-6-95$ & $10: 00 \mathrm{AM}$ & 11.7 & 2.03 & 20.6 & $<0.05$ & $1.8^{\circ} \mathrm{C}$ \\
\hline
\end{tabular}


Results

\begin{tabular}{|l|l|l|l|}
\hline Client: WSRC & Sample ID: BG003 & \\
\hline Log *: T3523 & Start Date: $2-2-95$ & Time: & $3: 00$ PM \\
\hline
\end{tabular}

SURVIVAL EFFECTS

Control Effluent

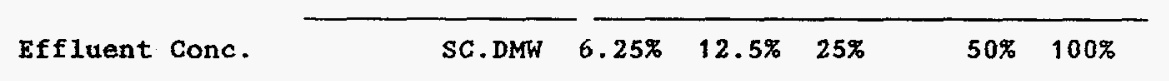

\begin{tabular}{lllllll}
48 hr. Mortality & $0 \%$ & $0 \%$ & $0 \%$ & $0 \%$ & $0 \%$ & $0 \%$ \\
7 Day Mortality & $0 \%$ & $10 \%$ & $0 \%$ & $0 \%$ & $0 \%$ & $20 \%$ \\
\hline
\end{tabular}

Method: Fishers Exact Test

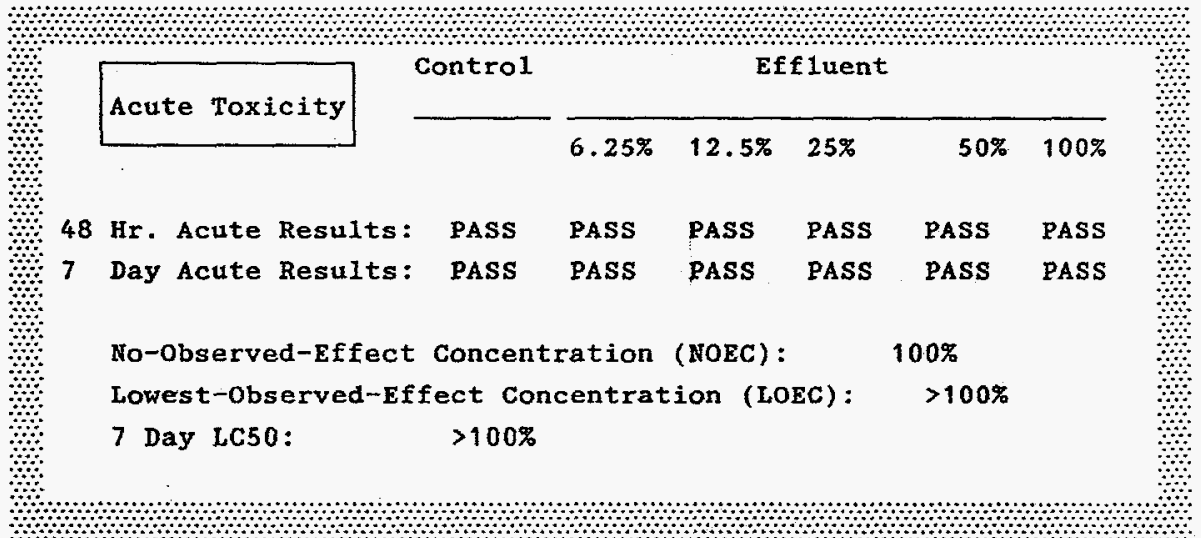

QUALITY CONTROL

\begin{tabular}{|c|c|c|c|c|}
\hline \multicolumn{2}{|c|}{ Standard Toxicant: } & \multicolumn{3}{|l|}{$\mathrm{NaCl}$} \\
\hline \multicolumn{3}{|c|}{ Central Tendency: } & 2.04 & $g / L$ \\
\hline & \multicolumn{2}{|c|}{ Current value: } & 1.9 & $g / L$ \\
\hline & Deviatio & 0.58 & & ev units. \\
\hline
\end{tabular}


Results

\begin{tabular}{|l|l|l|l|l|}
\hline \multicolumn{2}{|l|}{ Client: WSRC } & Sample ID: BG003 & IWC: \\
\hline Log \#: T3523 & Start Date: $2-2-95$ & Time: & $3: 00$ PM \\
\hline
\end{tabular}

CHRONIC EFFECTS

Control Effluent

TEST CONCENTRATION

Average young / female:

Standard Deviation:

$t=$

Steel's $=$

$\mathrm{MSD}=6.65$
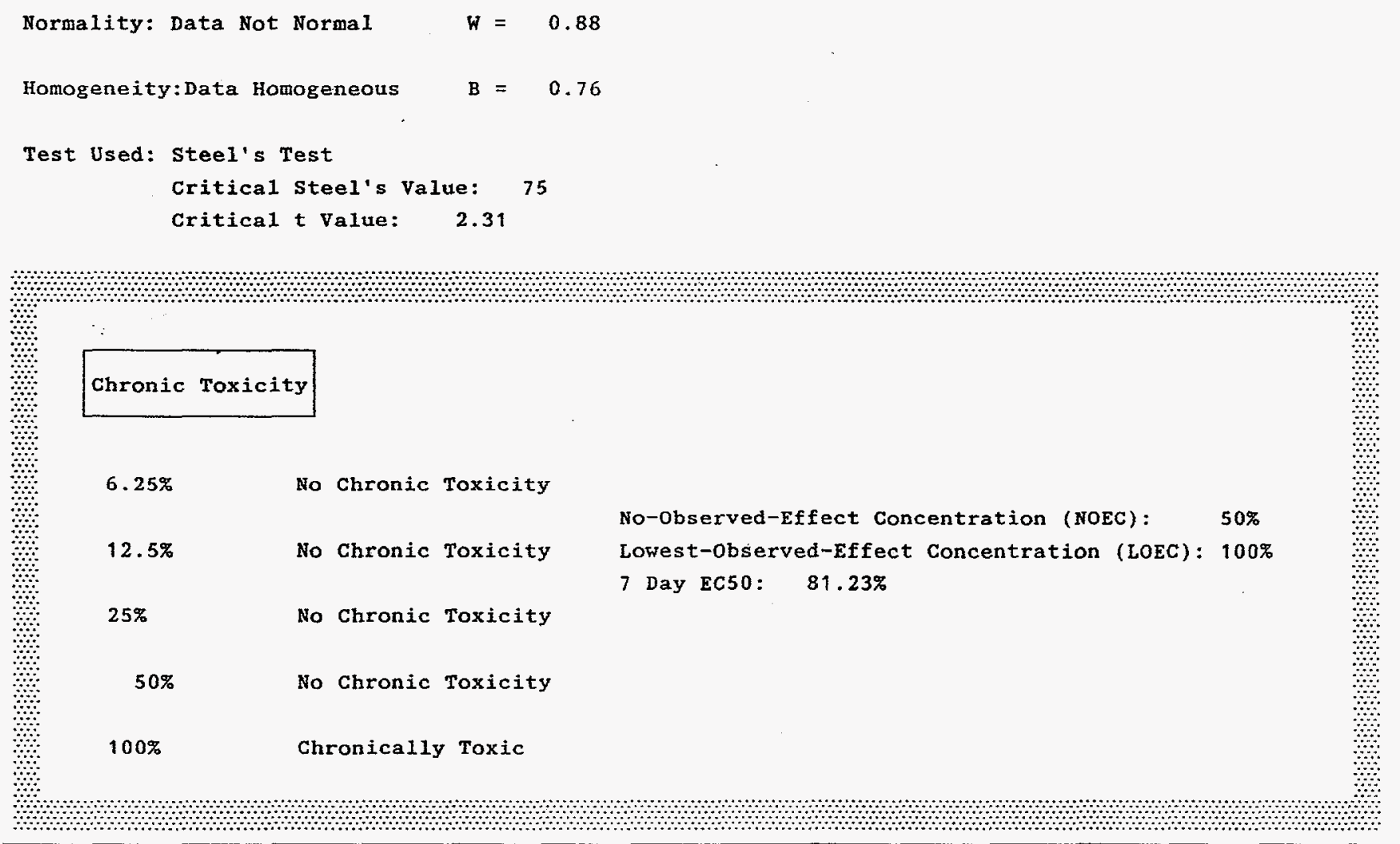
7 Day Chronic Definitive Survival and Reproduction Bioassay

Test Ofganisms: Ceriodaphnia dubia

Method: EPA/600/4-89/001

Date: $2-2-95$ 
DEFINITIVE SURVIVAL AND REPRODUCTION

Client:WSRC

Log \#: T3522

Sample ID: BG004
Start Date: 2-2-95

End Date: 2-11-95
Start Time: 2:45 PM

End Time: 1:00 PM IWC:

TEST CONCENTRATION: $0 \%$

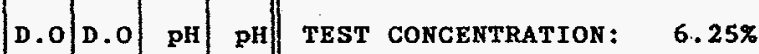

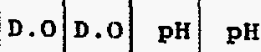

\begin{tabular}{|c|c|c|c|c|c|c|c|c|c|c|c|c|c|c|c|c|c|c|c|c|c|c|c|c|c|c|c|c|}
\hline Day & A & B & C & D & $\mathbf{E}$ & F & G & H & I & $\mathbf{J}$ & old & new & old & new & Day A & B & C & D & $\mathbf{E}$ & $\mathbf{F}$ & G & $\mathbf{H}$ & I & $\mathbf{J}$ & old & new & old & new \\
\hline 2 & 0 & 0 & 0 & 0 & 0 & 0 & 0 & 0 & 0 & 0 & 器器 & 8.6 & 䎌器 & $(7.9)$ & 0 & 0 & 0 & 0 & 0 & 0 & 0 & 0 & 0 & 0 & $\mid$ & 8.9 & 器 & 6.7 \\
\hline 3 & 0 & 0 & 0 & 0 & 0 & 0 & 0 & 0 & 0 & 0 & 8.2 & 8.5 & 7.4 & 8.0 & 30 & 0 & 0 & 0 & 0 & 0 & 0 & 0 & 0 & 0 & 8.0 & 8.3 & 7.5 & 7.6 \\
\hline 4 & 6 & 5 & 6 & 4 & 1 & 3 & 3 & 3 & 5 & 5 & 8.4 & 8.4 & 8.0 & 7.9 & 4 & 0 & 0 & 0 & 3 & 2 & 5 & 3 & 0 & 5 & 8.8 & 8.8 & 8.1 & 7.8 \\
\hline 5 & 4 & 9 & 0 & 0 & 6 & 5 & 10 & 0 & 0 & 0 & 8.5 & 8.6 & 8.4 & 8.0 & 8 & 4 & 5 & 5 & 9 & 7 & 8 & 0 & 5 & 8 & 8.4 & 8.6 & 8.6 & 8.2 \\
\hline 6 & 0 & 0 & 0 & 0 & 0 & 0 & 0 & 0 & 0 & 7 & 9.2 & 8.6 & 8.5 & 8.2 & 60 & 0 & 0 & 8 & 0 & 0 & 0 & 7 & 8 & 0 & 8.5 & 8.3 & 8.5 & 7.8 \\
\hline 7 & 10 & 10 & 13 & 8 & 0 & 11 & 10 & 7 & 9 & 11 & 8.2 & 8.7 & 8.1 & 8.1 & 711 & 12 & 12 & 0 & 9 & 10 & 9 & 12 & 13 & 11 & 8.5 & 8.4 & 7.4 & 7.6 \\
\hline 8 & 0 & 0 & 0 & 0 & 0 & 10 & 6 & 0 & 0 & 0 & 8.1 & 8.7 & 8.2 & 8.1 & 8 & 0 & 0 & 0 & 0 & 0 & 0 & 0 & 0 & 0 & 8.8 & 8.8 & 7.8 & 7.8 \\
\hline 9 & 0 & 0 & 4 & L & 13 & 0 & 0 & 14 & 12 & 0 & 8.8 & 器器 & 8.3 & 望融 & 9 & 11 & 12 & 12 & 0 & 0 & 0 & 0 & 0 & 0 & $\mid$ & : & 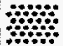 & טר \\
\hline TOT & & 24 & 23 & 12 & 20 & 29 & 29 & 24 & 26 & 23 & 24 & & & & TOT23 & 27 & 29 & 25 & 21 & 19 & 22 & 22 & 26 & 24 & 24 & & & \\
\hline
\end{tabular}

TEST CONCENTRATION $12.5 \%$

\begin{tabular}{l|l|l|l} 
D.O D.O & pH & pH & TEST CONGENTRATION 25\%
\end{tabular}

\begin{tabular}{|l|l|l|l|}
\hline D.O & D.O & $\mathrm{pH}$ & $\mathrm{pH}$ \\
\hline
\end{tabular}

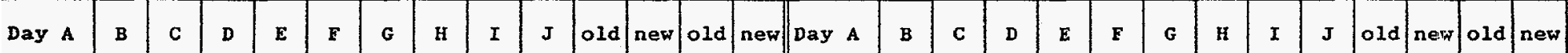

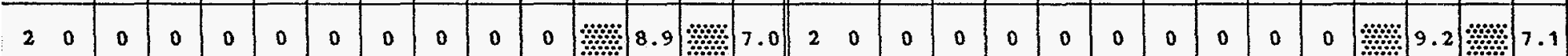

\begin{tabular}{lllllllllllllllllllllllll|l|l|l|l|l}
3 & 0 & 0 & 0 & 0 & 0 & 0 & 0 & 0 & 0 & 0 & 8.0 & 8.2 & 7.4 & 7.5 & 3 & 0 & 0 & 0 & 0 & 0 & 0 & 0 & 0 & 0 & 0 & 8.1 & 8.0 & 7.6 & 7.6
\end{tabular}

\begin{tabular}{ll|l|l|l|l|l|l|l|l|l|l|l|l|l|ll|l|l|l|l|l|l|l|l|l|l|l|l|l|}
4 & 5 & 5 & 3 & 0 & 4 & 4 & 1 & 4 & 0 & 5 & 9.0 & 9.0 & 8.1 & 8.1 & 4 & 6 & 5 & 3 & 0 & 4 & 1 & 5 & 5 & 0 & 4 & 9.2 & 9.2 & 8.2 & 8.2
\end{tabular}

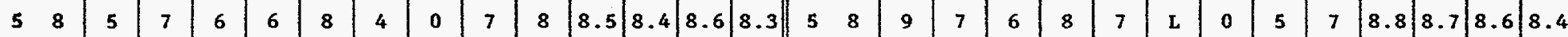

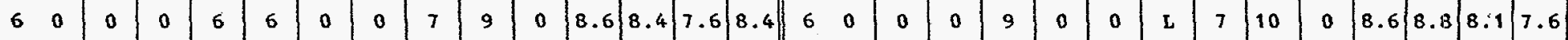

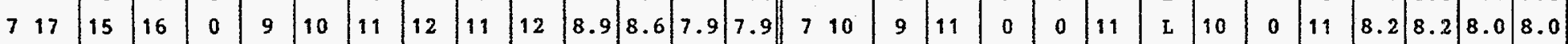

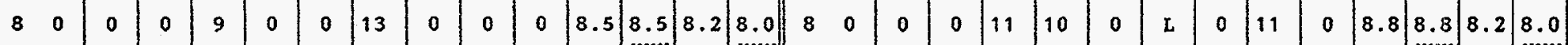

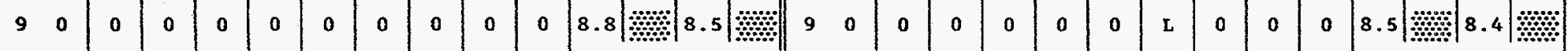

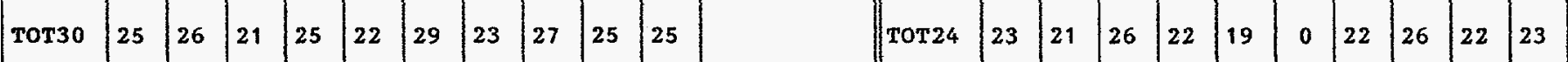

\begin{tabular}{|c|c|c|c|c|c|c|c|c|c|c|c|c|c|c|c|c|c|c|c|c|c|c|c|c|c|c|c|}
\hline TEST & \multicolumn{4}{|c|}{ CONCENTRATION } & \multicolumn{5}{|c|}{$50 \%$} & \multirow{2}{*}{\begin{tabular}{|c|}
.0 \\
old
\end{tabular}} & \multirow{2}{*}{\begin{tabular}{|c|}
0.0 \\
new
\end{tabular}} & \multirow{2}{*}{\begin{tabular}{|c|}
$\mathrm{pH}$ \\
old \\
\end{tabular}} & \multirow{2}{*}{$\begin{array}{c}\mathrm{pH} \\
\text { new } \\
\end{array}$} & \multicolumn{5}{|c|}{ TEST CONCENTRATION } & \multicolumn{5}{|c|}{$100 \%$} & \multirow{2}{*}{$\left|\begin{array}{l|}0.0 \\
010\end{array}\right|$} & \multirow{2}{*}{$\mid \begin{array}{l}0.0 \\
\text { new }\end{array}$} & \multirow{2}{*}{\begin{tabular}{|c|}
$\mathrm{pH}$ \\
old
\end{tabular}} & \multirow{2}{*}{\begin{tabular}{|l} 
pH \\
new
\end{tabular}} \\
\hline Day A & B & $c$ & D & $\mathbf{E}$ & $\mathbf{F}$ & G & $H$ & I & $\mathbf{J}$ & & & & & Day A & $B$ & C & D & $\mathbf{E}$ & $\mathbf{F}$ & G & H & I & $J$ & & & & \\
\hline 20 & 0 & 0 & 0 & 0 & 0 & 0 & 0 & 0 & 0 & \% & 9.4 & 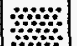 & 7.2 & 2 & 0 & 0 & 0 & 0 & 0 & 0 & 0 & 0 & 0 & 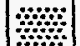 & 9.2 & 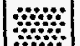 & 6.8 \\
\hline 30 & 0 & 0 & 0 & 0 & 0 & 0 & 0 & 0 & 0 & 8.6 & 8.6 & 7.7 & 7.6 & 30 & 0 & 0 & 0 & 0 & 0 & 0 & 0 & 0 & 0 & 8.4 & 8.4 & 7.9 & 7.5 \\
\hline 45 & 5 & 0 & 0 & 3 & 2 & 3 & $\mathbf{L}$ & 1 & 4 & 9.2 & 9.2 & 8.2 & 8.3 & 4 & 0 & 0 & $\mathrm{x}$ & 0 & 0 & 0 & 0 & 0 & 0 & 9.0 & 9.0 & 8.2 & 8.4 \\
\hline 58 & 4 & 6 & 8 & 8 & 5 & 8 & $\mathbf{L}$ & 8 & 9 & 8.6 & 8.6 & 8.5 & 8.1 & 5 & 3 & 1 & $\mathrm{x}$ & 0 & 2 & 0 & 0 & 0 & 0 & 8.8 & 8.7 & 8.5 & 8.5 \\
\hline 60 & 0 & 0 & 7 & 0 & 0 & 0 & $\mathbf{L}$ & 12 & 0 & 8.5 & 8.7 & 7.4 & 7.1 & 6 & 5 & 4 & $\mathrm{x}$ & 0 & 4 & 3 & 0 & 0 & 2 & 8.5 & 8.3 & 7.6 & 7.0 \\
\hline 712 & 14 & 9 & 0 & 10 & 8 & 11 & $\mathbf{L}$ & 0 & 13 & 8.2 & 8.2 & 8.1 & 7.9 & 7 & 0 & 0 & $\mathrm{x}$ & 3 & 0 & 0 & 3 & 0 & $x$ & 8.0 & 8.0 & 8.1 & 7.7 \\
\hline 80 & 0 & 7 & 11 & 0 & 0 & 0 & $\mathbf{L}$ & 0 & 0 & 8.6 & 8.7 & 8.2 & 7.9 & 8 & 4 & 1 & $\mathrm{x}$ & 1 & 3 & 3 & 5 & 2 & $x$ & 8.0 & 8.8 & 8.1 & 7.7 \\
\hline 90 & 0 & 0 & 0 & 0 & 0 & 0 & $\mathbf{L}$ & 0 & 0 & ن & | & $\mid$ & 谬 & 94 & $\mathrm{x}$ & 4 & $x$ & 0 & 5 & 4 & 1 & 0 & $x$ & 9.2 & \% & 8.2 & 1 \\
\hline тот25 & 23 & 22 & 26 & 21 & 15 & 22 & 0 & 21 & 26 & 22 & & & & тот11 & 12 & 10 & 0 & 4 & 14 & 10 & 9 & 2 & 2 & 7 & & & \\
\hline & & & & & & & & & & & & & & & - & & & & & & & & & & & & \\
\hline & \multicolumn{2}{|c|}{ TEM. ${ }^{\circ} \mathrm{C}$} & \multicolumn{3}{|c|}{ Time } & & & & \multicolumn{2}{|c|}{ TEM. ${ }^{\circ} \mathrm{C}$} & \multicolumn{2}{|c|}{ Time } & & & & TEY & & \multicolumn{3}{|c|}{ Time } & & \multicolumn{3}{|c|}{ TEM $^{\circ}{ }^{\circ} \mathrm{C}$} & \multicolumn{3}{|c|}{ Time } \\
\hline Day 0 & 24 & & 02 & & & & Day & 2 & 24.3 & & $03: 2 C$ & $0 \mathrm{PM}$ & & $\mathrm{Da}$ & y 4 & 24 & & $03:$ & $10 \mathrm{P}$ & & & Day & 6 & 24.3 & & $01: 30$ & $0 \mathrm{PM}$ \\
\hline Day 1 & 24 & & 11 & & & & Day & 3 & 24.2 & & $01: 00$ & 0 PM & & $\mathrm{Da}$ & y 5 & 24 & & $03:$ & $00 \mathrm{P}$ & & & Day & 7 & 24.2 & & $10: 15$ & $5 \mathrm{AM}$ \\
\hline
\end{tabular}


SAMPLING INFORMATION

\begin{tabular}{|c|c|c|c|c|c|c|c|c|}
\hline & Type & start Date & Time & Hardness $\mathrm{Mg} / \mathrm{L}$ & Alkalinity & Conductivity & Res. C1 & Rec.Temp. \\
\hline Dilution Water & $20 \% D M H$ & $2-1-95$ & 所 & 96.04 & 71.05 & 226 & H & $\ddot{\vdots}$ \\
\hline Final Eff. 1 & Grab & $2-1-95$ & $07: 40 \mathrm{AM}$ & 5.88 & 4.06 & 23.5 & $<0.05$ & $3.0^{\circ} \mathrm{C}$ \\
\hline Final Eff. 2 & Grab & $2-3-95$ & $07: 30 \mathrm{AM}$ & 5.88 & 4.06 & 23.0 & $<0.05$ & $3.2^{\circ} \mathrm{C}$ \\
\hline Final Eff. 3 & Grab & $2-6-95$ & $09: 30 \mathrm{AM}$ & 9.8 & 2.03 & 23.2 & $<0.05$ & $1.8^{\circ} \mathrm{C}$ \\
\hline
\end{tabular}


Results

\begin{tabular}{|l|l|l|l|}
\hline \multicolumn{2}{|l|}{} & Sample ID: BG004 & \\
\hline Log \#: T3522 & Start Date: $2-2-95$ & Time: & $2: 45$ PM \\
\hline
\end{tabular}

SURVIVAL EFEECTS

\begin{tabular}{|c|c|c|c|c|c|c|}
\hline \multirow[b]{2}{*}{ Effluent Conc. } & \multicolumn{2}{|c|}{ Contro1 } & \multicolumn{4}{|c|}{ Effluent } \\
\hline & SC.DMW & $6.25 \%$ & $12.5 \%$ & $25 \%$ & $50 \%$ & $100 \%$ \\
\hline $48 \mathrm{hr}$. Mortality & $0 \%$ & $0 \%$ & $0 \%$ & $0 \%$ & $0 \%$ & $0 \%$ \\
\hline 7 Day Mortality & $0 \%$ & $0 \%$ & $0 \%$ & $0 \%$ & $0 \%$ & $30 \%$ \\
\hline
\end{tabular}

Method: Fishers Exact Test

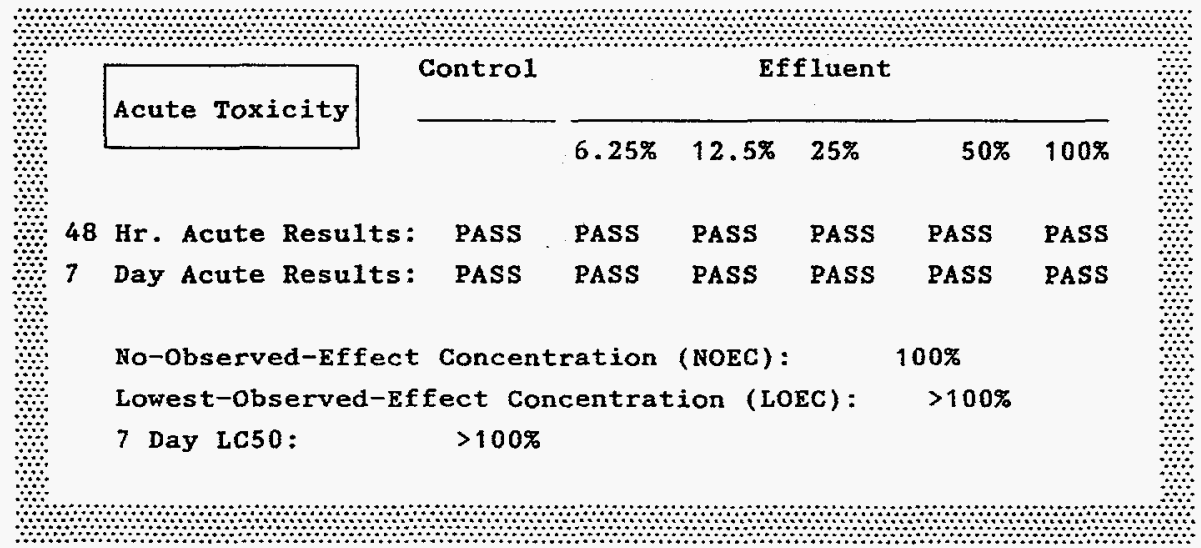

QUALITY CONTROL

Standard Toxicant: Nacl

Central Tendency:

Current Value:

Deviation:

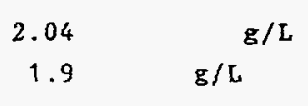

0.58 std Dev units. 
Resu1ts

\begin{tabular}{|l|l|l|l|}
\hline Client: WSRC & Sample ID: BG004 & IWC: \\
\hline Log \#: T3522 & Start Date: $2-2-95$ & Time: & $2: 45$ PM \\
\hline
\end{tabular}

CHRONIC EFFECTS

Control Effluent

TEST CONCENTRATION

SC. DMW

$6.25 \%$

$12.5 \% 25 \%$

$50 \%$

$100 \%$

Average young / female:

$\begin{array}{llllll}24.2 & 23.8 & 25.3 & 22.8 & 22.3 & 7.4\end{array}$

Standard Deviation:

3.31

3.01

2.87

$2.28 \quad 3.39$

4.93

$t=$

0.27

$-0.7$

$0.9 \quad 1.17$

10.72

Steel's $=$

107

121

$93.5 \quad 94.5$

64.5

$M S D=3.66$

Normality: Data Not Normal

$W=0.86$

Homogeneity: Data Homogeneous

$B=5.67$

Test Used: Steel's Test

Critical steel's value: 75

Critical t Value: 2.31

Chronic Toxicity
$6.25 \%$
No Chronic Toxicity
$12.5 \%$
No Chronic Toxicity
25\%
No Chronic Toxicity
$50 \%$
No Chronic Toxicity
$100 \%$
Chronically Toxic

No-Observed-Effect Concentration (NOEC): $50 \%$

Lowest-Observed-Effect Concentration (LOEC): $100 \%$

7 Day EC50: $70.71 \%$ 
(803) $877-6942$ - FAX (803) 877-6938

P.O. Box 16414, Greenville, SC $29606 \cdot 6$ C Craftsman Court, Greer, SC 29650

7 Day Chronic Definitive Survival and Reproduction Bioassay

Test Organisms: Ceriodaphnia dubia

Method: EPA/600/4-89/001

Facility = westinghouse savanmah

River Compary Sample ID: FHBOO6

Date: $\quad 2-2-95$ 


\section{DEFINITIVE SURVIVAL AND REPRODUCTION}

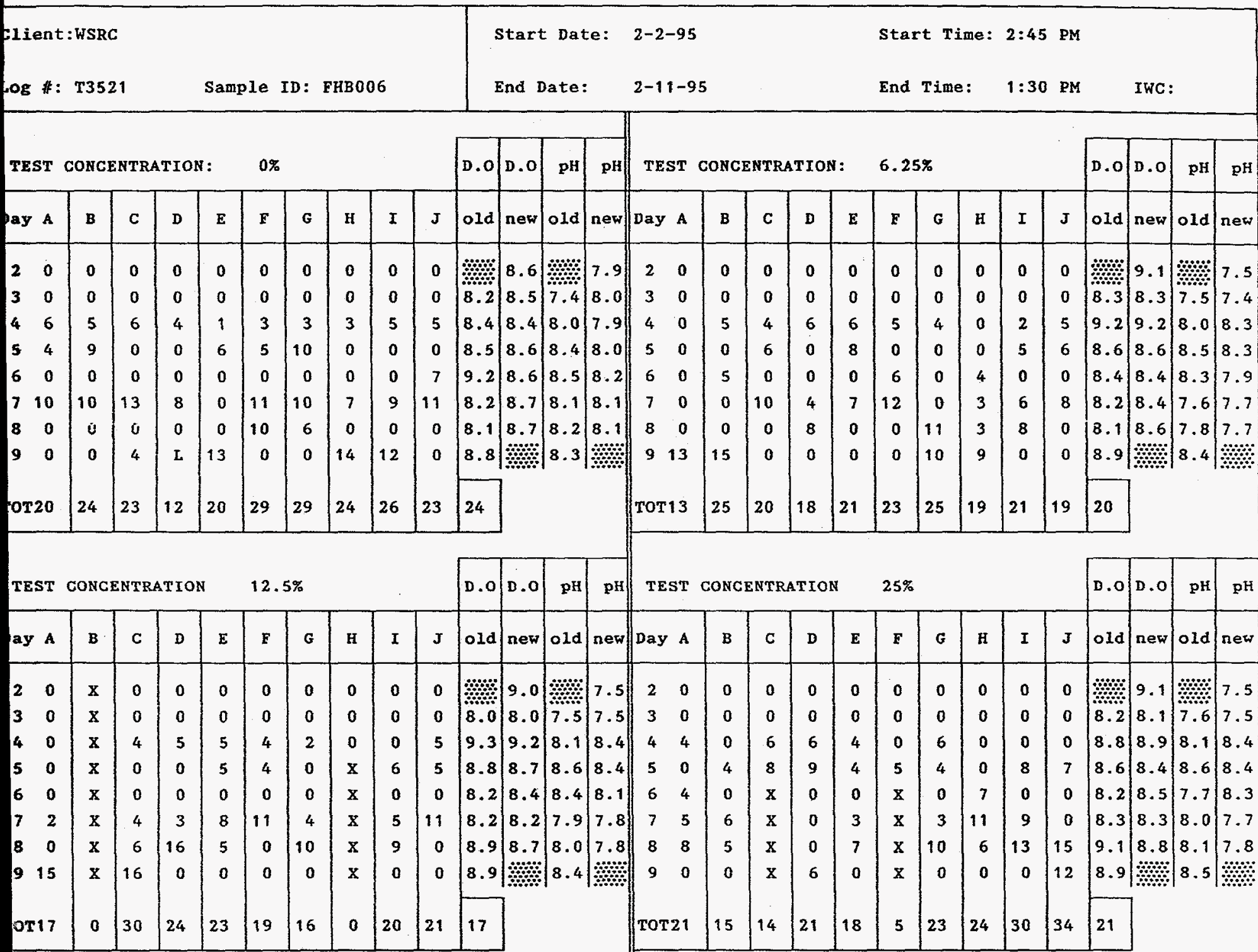

\begin{tabular}{ll|l|l|l|l|lll} 
TEST CONCENTRATION & $50 \%$ & 0.0 & 0.0 & pH & PH & TEST CONCENTRATION 100\%
\end{tabular}

D.O $0.00 \mathrm{pH} \quad \mathrm{pH}$

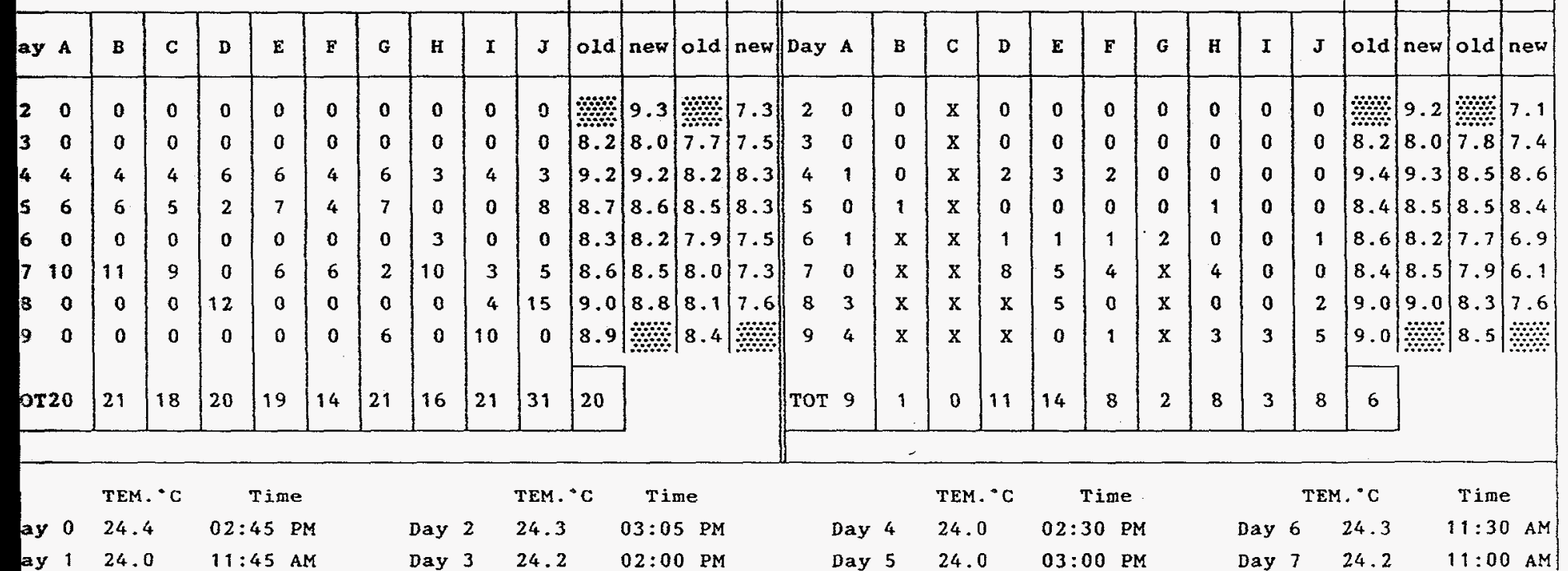


SAMPLING INFORMATION

\begin{tabular}{|c|c|c|c|c|c|c|c|c|}
\hline Dilution Water & 20\%DMW & $2-1-95$ & \% & 96.04 & 71.05 & 226 & $\because$ & 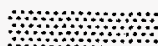 \\
\hline Final Eff. 1 & Grab & $2-1-95$ & $07: 40 \mathrm{AM}$ & 5.88 & 6.09 & 36.5 & $<0.05$ & $3.0^{\circ} \mathrm{C}$ \\
\hline Final Eff. 2 & Grab & $2-3-95$ & $07: 30 \mathrm{AM}$ & 3.92 & 4.06 & 34.5 & $<0.05$ & $3.2{ }^{\circ} \mathrm{C}$ \\
\hline Final Eff. 3 & Grab & $2-6-95$ & $09: 30 \mathrm{AM}$ & 3.92 & 4.06 & 36.8 & $<0.05$ & $1.8^{\circ} \mathrm{C}$ \\
\hline
\end{tabular}


Resu1ts

\begin{tabular}{|l|l|l|l|}
\hline Client: WSRC & Samp1e ID: FHB006 & \\
\hline Log \#: T3521 & Start Date: $2-2-95$ & Time: & $2: 45$ PM \\
\hline
\end{tabular}

SURVIVAL EFFECTS

\begin{tabular}{|c|c|c|c|c|c|c|}
\hline \multirow[b]{2}{*}{ Effluent Conc. } & \multicolumn{2}{|c|}{ Contro1 } & \multicolumn{4}{|c|}{ Effluent } \\
\hline & SC.DMW & $6.25 \%$ & $12.5 \%$ & $25 \%$ & $50 \%$ & $100 \%$ \\
\hline $48 \mathrm{hr}$. Mortality & $0 \%$ & $0 \%$ & $10 \%$ & $0 \%$ & $0 \%$ & $10 \%$ \\
\hline 7 Day Mortality & $0 \%$ & $0 \%$ & $20 \%$ & $20 \%$ & $0 \%$ & $40 \%$ \\
\hline
\end{tabular}

Method: Fishers Exact Test

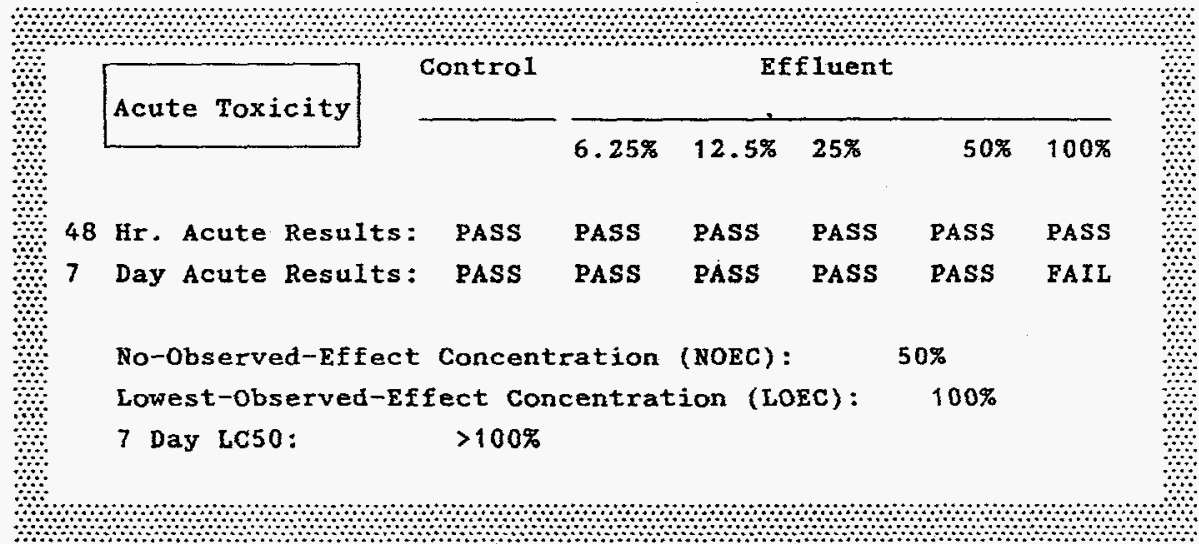

QUALITY CONTROL

\begin{tabular}{|llrc|}
\hline Standard Toxicant: & & & \\
& & & \\
& Central Tendency: & 2.04 & $\mathrm{~g} / \mathrm{L}$ \\
& Current Value: & 1.9 & $\mathrm{~g} / \mathrm{L}$ \\
Deviation: & 0.58 & Std Dev units. \\
\hline
\end{tabular}


Results

\begin{tabular}{|l|l|l|l|l|}
\hline Client: WSRC & Sample ID: FHB006 & IWC: \\
\hline Log $\$:$ T3521 & Start Date: $2-2-95$ & Time: & $2: 45$ PM \\
\hline
\end{tabular}

CHRONIC EFFECTS

Contro1 Effluent

TEST CONCENTRATION

$\begin{array}{llllll}\text { SC.DMW } & & & & & \end{array}$

Average young / female:

$\begin{array}{llllll}24.2 & 20.4 & 17 & 20.5 & 20.1 & 6.4\end{array}$

Standard Deviation:

$\mathbf{t}=$

3.31

3.57

9.79

8.24

4.48

4.65

1.34

2.53

1.3

1.44

6.24

Steel's $=$

85

84

95

82

64.5

MSI $=6.6$

Normality: Data Not Normal $\quad \mathrm{W}=0.85$

Homogeneity:Data Not Honogeneous $B=17.3$

Test Used: Steel's Test

Critical Steel's Value: 75

Critical $t$ Value: 2.31

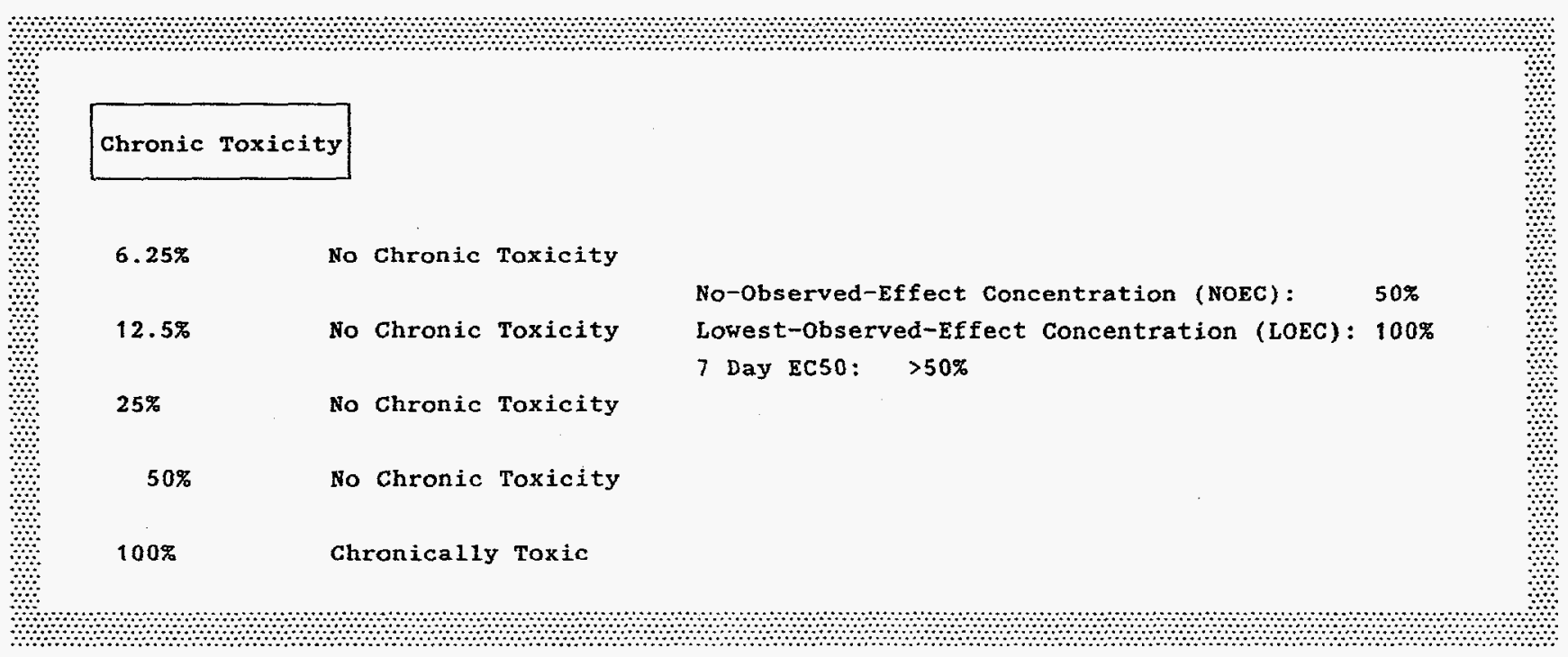


7 Day Chronic Definitive Surviva1 and Reproduction Bioassay

Test Organisms: Ceriodaphnia dubia

Method: EPA/600/4-89/001

\author{
Facility = Westinghouse savannah River Company \\ Sample ID: FXBO12 \\ Date: $\quad 2-23-95$
}


:1ient:WSRC

og \# : T3599
Sample ID: FHBO12
Start Date: 2-23-95

End Date: 3-3-95
Start Time: 3:00 PM

End Time: 4:00 PM IWC:

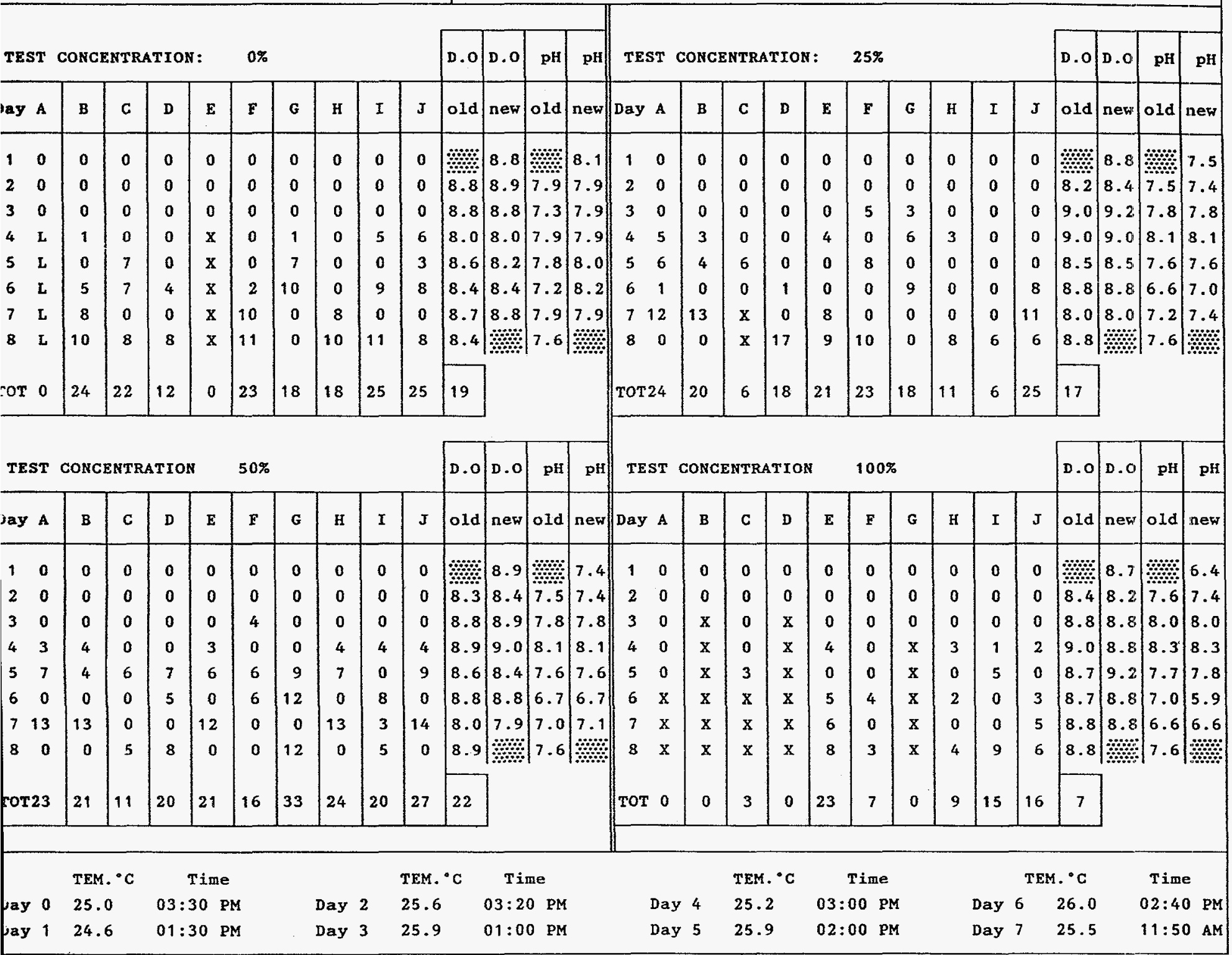


SAMPLING INFORMATION

\begin{tabular}{|c|c|c|c|c|c|c|c|c|}
\hline Dilution Water & $20 \% \mathrm{DMW}$ & $2-18-95$ & in & 100 & 60.9 & 184.8 & $\because n$ & \\
\hline Final Eff. 1 & Grab & $2-22-95$ & $09: 00 \mathrm{AM}$ & 8.0 & 4.06 & 40.2 & $<0.05$ & $0.9^{\circ} \mathrm{C}$ \\
\hline Final Eff. 2 & Grab & $2-24-95$ & $08: 30 \mathrm{AM}$ & 10.0 & 6.12 & 40.1 & $<0.05$ & $3.3^{\circ} \mathrm{C}$ \\
\hline Final Eff. 3 & Grab & $2-27-95$ & $08: 00 \mathrm{AM}$ & 8.0 & 4.08 & 39.9 & $<0.05$ & $1.6^{\circ} \mathrm{C}$ \\
\hline
\end{tabular}


Results

\begin{tabular}{|l|l|l|l|}
\hline \multicolumn{2}{|l|}{ Client: WSRC } & Sample ID: FHB012 & \\
\hline Log \#: T3599 & Start Date: $2-23-95$ & Time: & $3: 00$ PM \\
\hline
\end{tabular}

SURVIVAL EFFECTS

Control Effluent

Effluent Conc.

$\begin{array}{lrrrr}48 \text { hr. Mortality } & 0 \% & 0 \% & 0 \% & 20 \% \\ 7 \text { Day Mortality } & 11 \% & 10 \% & 0 \% & 50 \%\end{array}$

Method: Fishers Exact Test

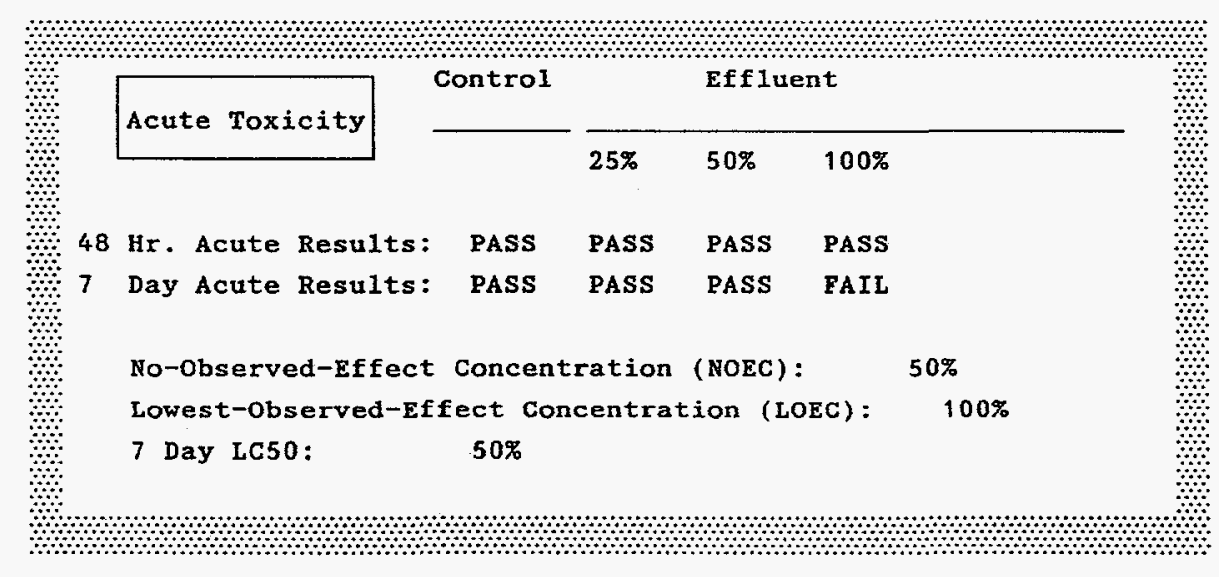

QUALITY CONTROL

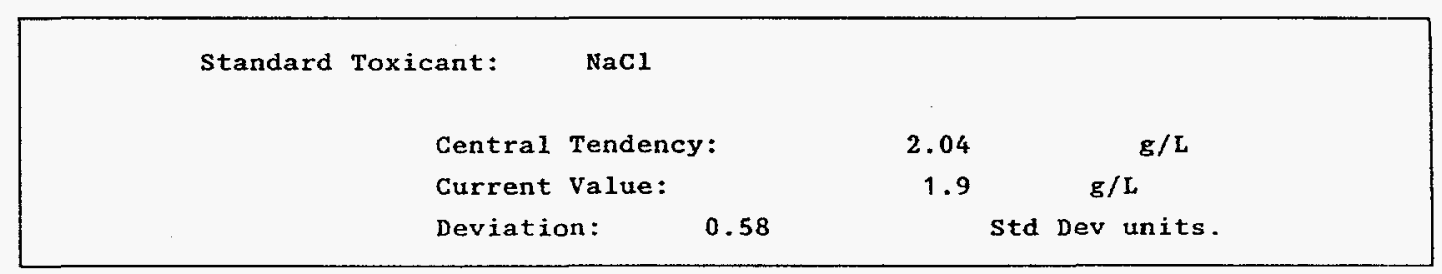


Results

\begin{tabular}{|l|l|l|l|}
\hline \multicolumn{2}{|l|}{ Client: WSRC } & Sample ID: FHB012 & IWC: \\
\hline Log \#: T3599 & Start Date: $2-23-95$ & Time: & $3: 00$ PM \\
\hline
\end{tabular}

CHRONIC EFFECTS

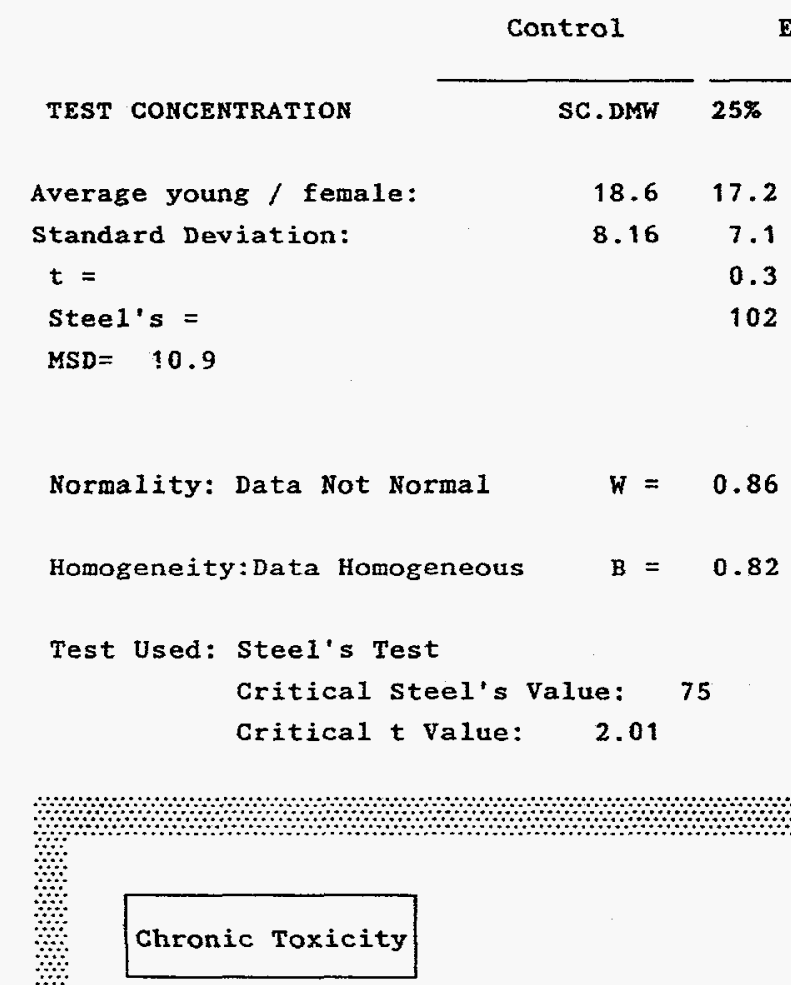

$25 \%$

No Chronic Toxicity

$50 \%$

No Chronic Toxicity

No-Observed-Effect Concentration (NOEC):

$50 \%$

$100 \%$

Chronically Toxic 


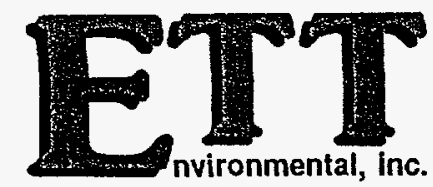

(803) 877-6942 - FAX (803) 877-6338

P.O. Box 16414, Greenville, SC $29606 \cdot 6$ C Craftsman Court, Greer, SC 29650

7 Day Chronic Definitive Survival and Reproduction Bioassay

Test Organisms: Ceriodaphnia dubia

Method: EPA/600/4-89/001

Facility = Westinghouse savanmah River company $\operatorname{Samp} 1 e \operatorname{ID}=\operatorname{FHBO} 4$

Date: $1-24-95$ 
DEFINITIVE SURVIVAL AND REPRODUCTION

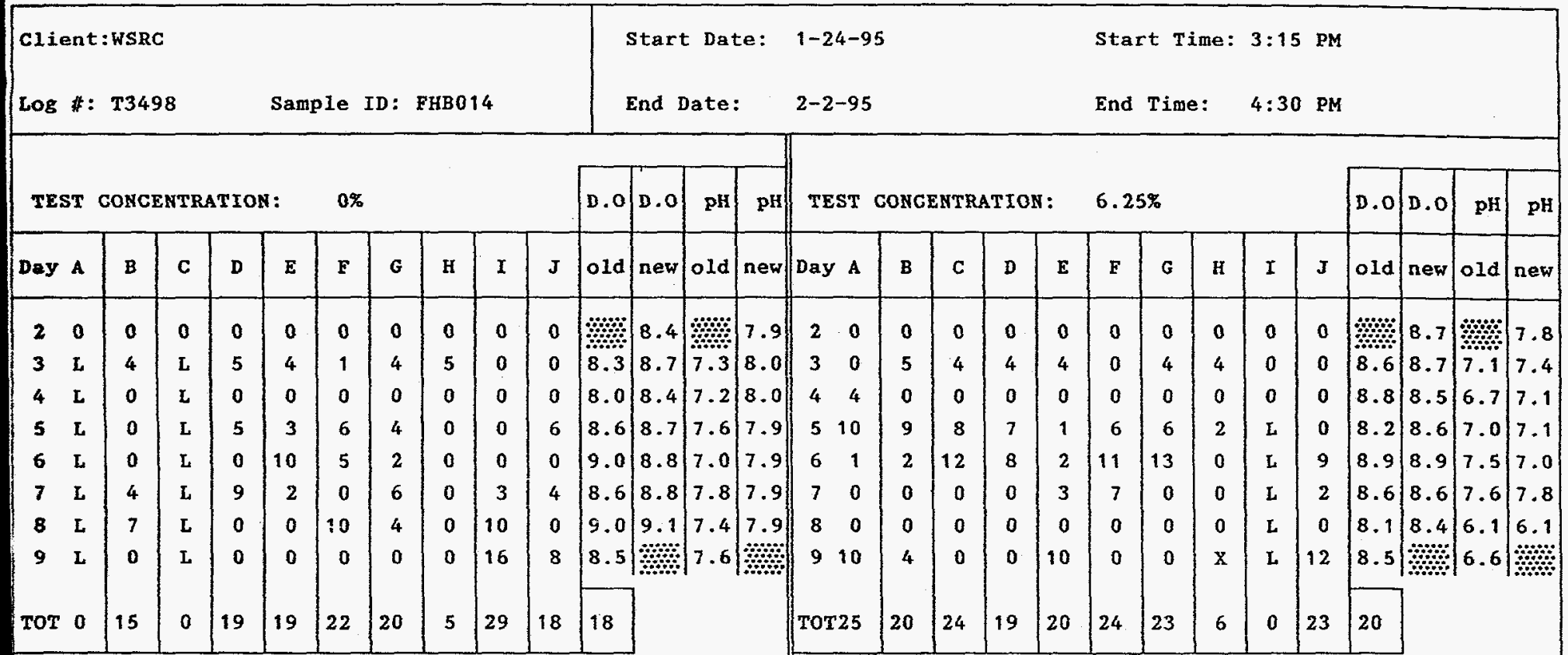

TEST CONCENTRATION $12.5 \%$

D.O D.O $\mathrm{pH}$ PH TEST CONCENTRATION $25 \%$

\begin{tabular}{|l|l|l|l|}
\hline 0.0 & 0.0 & $\mathrm{pH}$ & $\mathrm{pH}$ \\
\hline
\end{tabular}

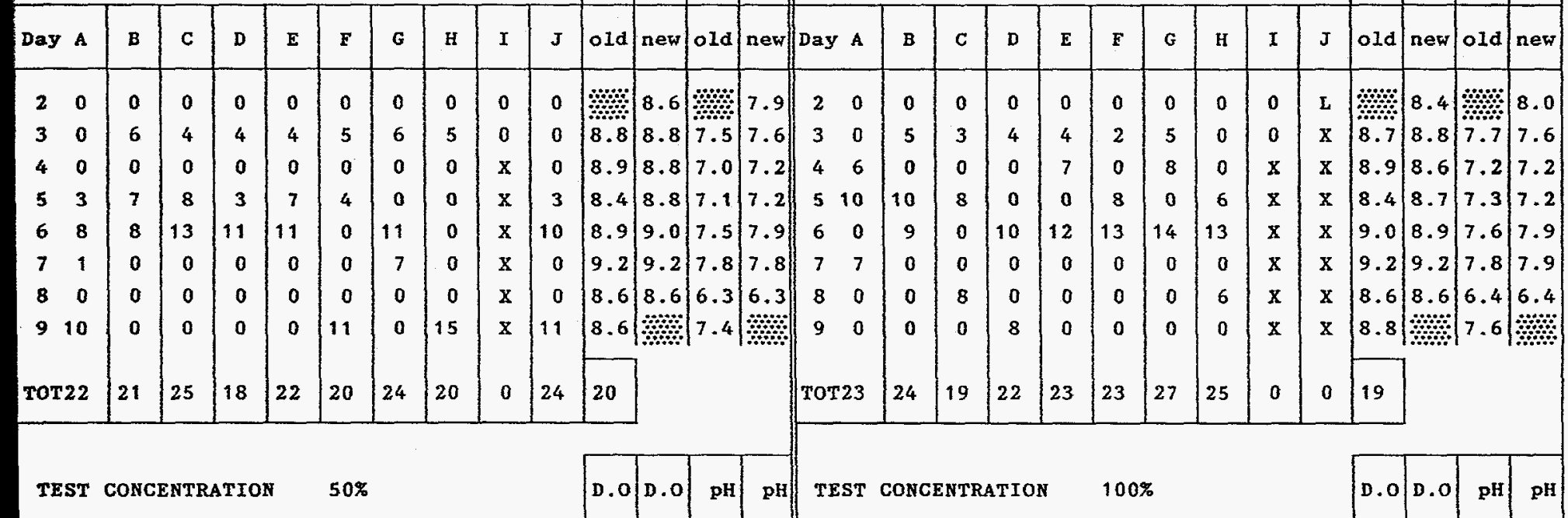

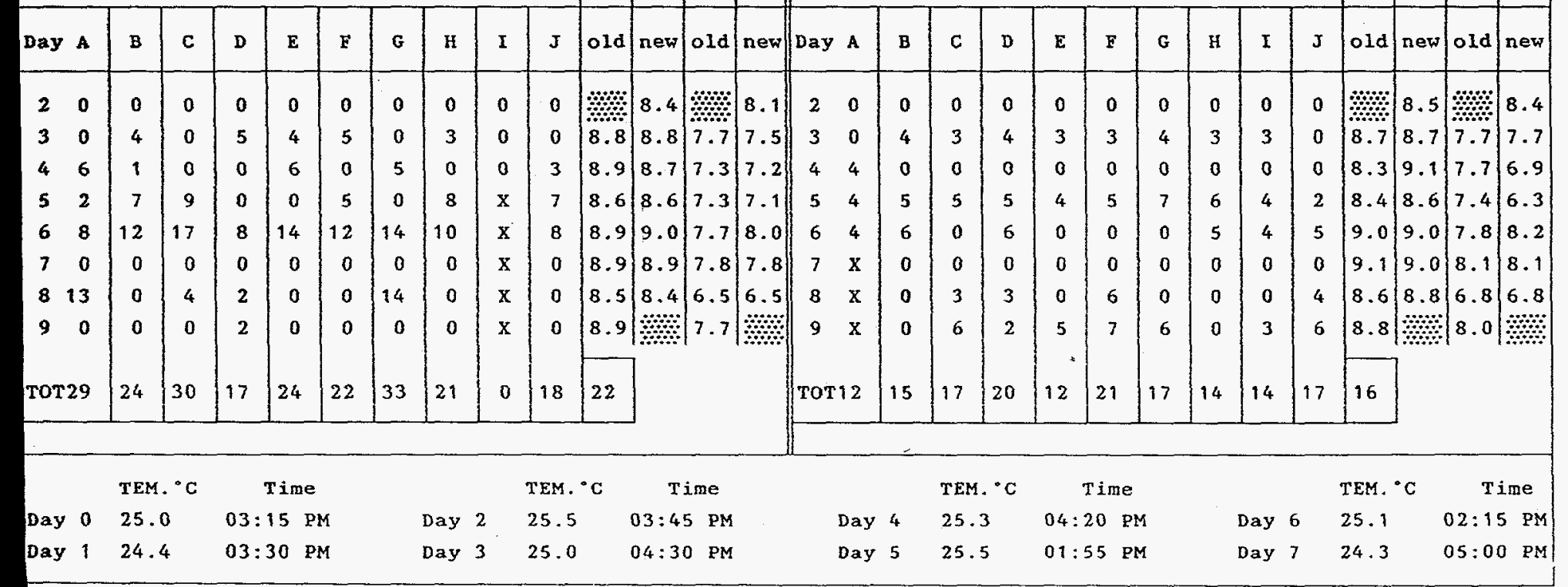


SAMPIING INFORMATION

\begin{tabular}{|c|c|c|c|c|c|c|c|c|}
\hline & Type & Start Date & Time & Hardness $\mathrm{Mg} / \mathrm{L}$ & Alkalinity & Conductivity & Res. C1 & Rec.Temp. \\
\hline Dilution Water & $20 \% \mathrm{DMW}$ & $1-24-95$ & 烈 & 96.0 & 75.1 & 223 & 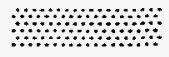 & \\
\hline Final Eff. 1 & Grab & $1-23-95$ & $09: 50 \mathrm{AM}$ & 4.0 & 4.06 & 31.8 & $<0.05$ & $2.0^{\circ} \mathrm{C}$ \\
\hline Final Eff. 2 & Grab & $1-25-95$ & $09: 45 \mathrm{AM}$ & 8.0 & 4.06 & 33.1 & $<0.05$ & $2.3^{\circ} \mathrm{C}$ \\
\hline Final Eff. 3 & Grab & $1-27-95$ & $09: 30 \mathrm{AM}$ & 6.0 & 6.09 & 32.1 & $<0.05$ & $2.3^{\circ} \mathrm{C}$ \\
\hline
\end{tabular}


Results

\begin{tabular}{|l|c|c|c|c|}
\hline \multicolumn{2}{|l|}{ Client: WSRC } & Sample ID: FHB014 & \\
\hline Log *: T3498 & Start Date: $1-24-95$ & Time: & $3: 15$ PM \\
\hline
\end{tabular}

SURVIVAL EFFECTS

Contro1 Effluent

$\begin{array}{lllllll}\text { Effluent Conc. } & \text { SC.DMW } & 6.25 \% & 12.5 \% & 25 \% & 50 \% & 100 \%\end{array}$

\begin{tabular}{lllllll}
$48 \mathrm{hr}$. Mortality & $0 \%$ & $0 \%$ & $10 \%$ & $20 \%$ & $0 \%$ & $0 \%$ \\
\hline Day Mortality & $0 \%$ & $11 \%$ & $10 \%$ & $20 \%$ & $10 \%$ & $10 \%$ \\
\hline
\end{tabular}

Method: Fishers Exact Test

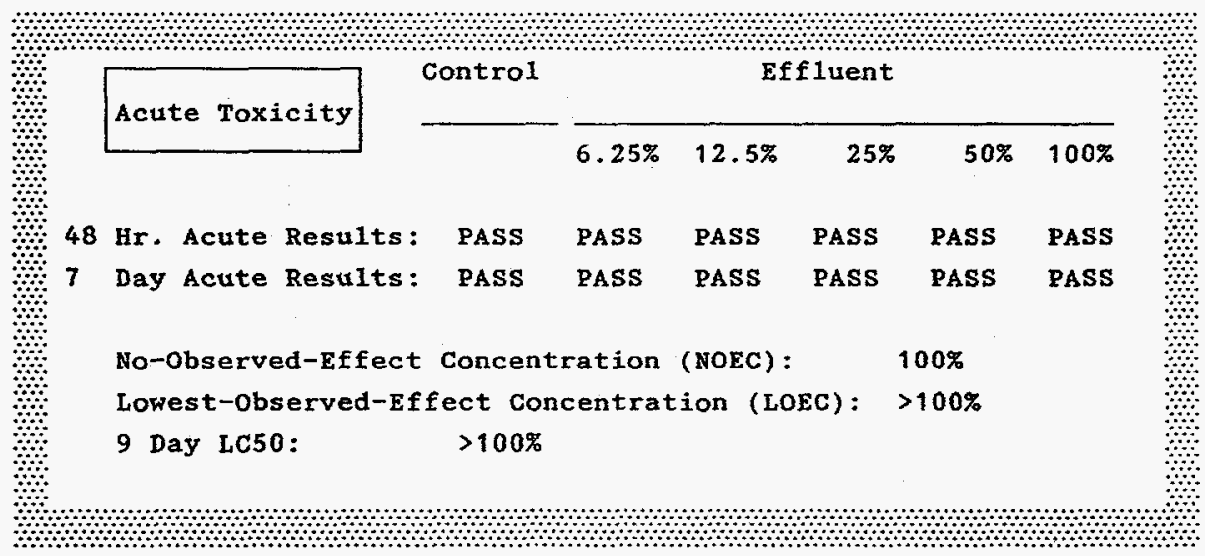

QUALTTY CONTROL

\begin{tabular}{|cccc|}
\hline Standard Toxicant: & Nacl & \\
& & & \\
& Central Tendency: & 2.04 & $\mathrm{~g} / \mathrm{L}$ \\
Current Value: & 1.9 & $\mathrm{~g} / \mathrm{L}$ \\
Deviation: & $\mathbf{0 . 5 8}$ & Std Dev units. \\
\hline
\end{tabular}


Results

\begin{tabular}{|l|l|l|l|l|}
\hline \multicolumn{2}{|l|}{ Client: WSRC } & Sample ID: FHB014 & IWC: \\
\hline Log \#: T3498 & Start Date: $1-24-95$ & Time: & $3: 15$ PM \\
\hline
\end{tabular}

CHRONIC EFFECTS

Control Effluent

TEST CONCENTRATION

SC.DMW

$6.25 \% \quad 12.5 \%$

$25 \%$

$50 \%$

$100 \%$

Average young / female:

$\begin{array}{llllll}18.4 & 20.4 & 19.6 & 18.6 & 21.8 & 15.9\end{array}$

Standard Deviation:

6.76

5.81

7.21

$10 \quad 9.24$

3.07

$\mathbf{t}=$

$-0.6-0.3$

$-0.06$

$-1$

0.70

steel's =

125

128 .

126

129

98

$M S D=8.18$

Normality: Data Not Normal $\mathrm{W}=0.75$

Homogeneity:Data Homogeneous

$\mathrm{B}=11.8$

Test Used: Steel's Test

Critical steel's Value: 75

Critical t value: 2.31

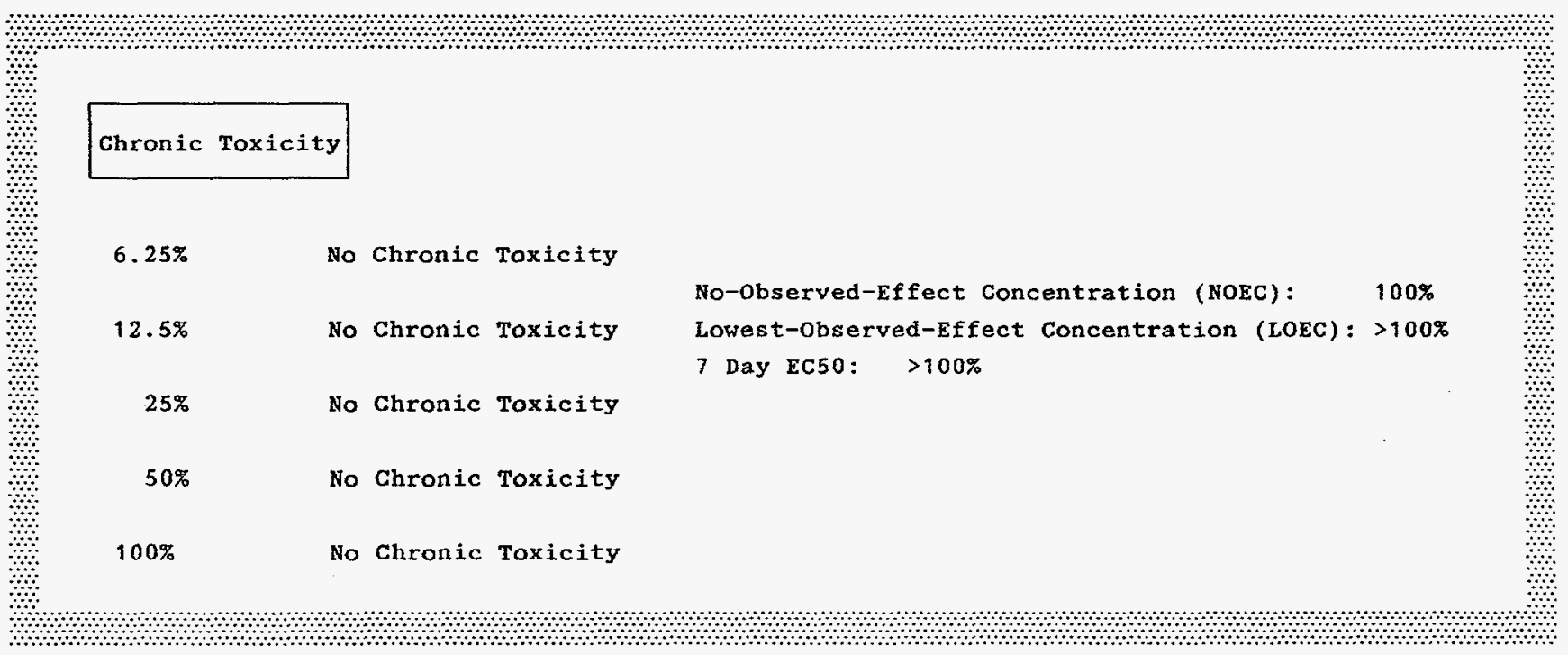


7 Day Chronic Definitive Survival and Reproduction Bioassay

Test Organisms: Ceriodaphnia dubia

Method: EPA/ $600 / 4-89 / 001$

\begin{abstract}
Facility : Westinghouse savannah Rivex Company Sample ID = FHBO18
\end{abstract}

Date: $\quad 2-23-95$ 
SAMPLING INEORMATION

\begin{tabular}{|c|c|c|c|c|c|c|c|c|}
\hline & Type & Start Date & Time & Hardness $\mathrm{Mg} / \mathrm{L}$ & Alkalinity & Conductivity & Res. C1 & Rec.Temp. \\
\hline Dilution Water & $20 \%$ DMW & $2-18-95$ & 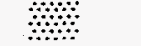 & 100 & 60.9 & 184.8 & 柇 & \\
\hline Final Eff. 1 & Grab & $2-22-95$ & $09: 40 \mathrm{AM}$ & 10.0 & 46.7 & 112.3 & $<0.05$ & $0.9^{\circ} \mathrm{C}$ \\
\hline Final Eff. 2 & Grab & $2-24-95$ & $08: 40 \mathrm{AM}$ & 14.0 & 32.6 & 81.7 & $<0.05$ & $3.3^{\circ} \mathrm{C}$ \\
\hline Final Eff. 3 & Grab & $2-27-95$ & $08: 40 \mathrm{AM}$ & 10.0 & 20.4 & 56.9 & $<0.05$ & $1.6^{\circ} \mathrm{C}$ \\
\hline
\end{tabular}


Results

\begin{tabular}{|l|l|l|l|l|}
\hline Client: WSRC & Sample ID: FHB018 & \\
\hline Log \#: T3600 & Start Date: $2-23-95$ & & Time: & $3: 00$ PM \\
\hline
\end{tabular}

SURVIVAL EFFECTS

Control Effluent

\begin{tabular}{lrrrrrrr}
\cline { 5 - 6 } & SC.DMW & $6.25 \%$ & $12.5 \%$ & $25 \%$ & $50 \%$ & $100 \%$ \\
\hline $48 \mathrm{hr}$. Mortality & $0 \%$ & $0 \%$ & $0 \%$ & $0 \%$ & $30 \%$ & $100 \%$ \\
7 Day Mortality & $11 \%$ & $11 \%$ & $30 \%$ & $0 \%$ & $40 \%$ & $100 \%$ \\
\hline
\end{tabular}

Method: $\quad$ ishers Exact Test

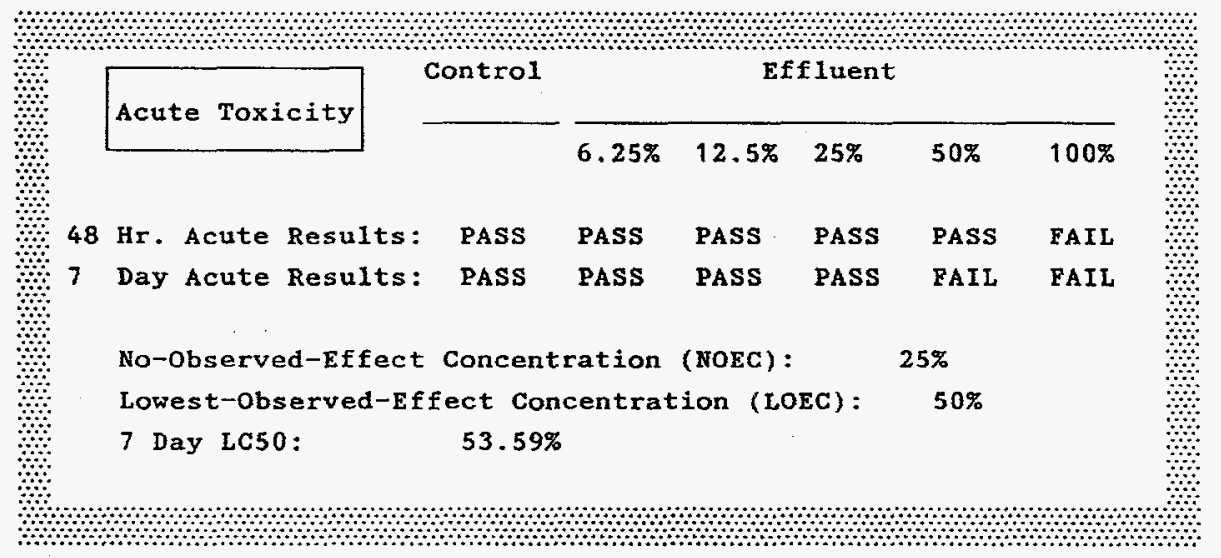

QUALITY CONTROL

\begin{tabular}{|c|c|c|c|c|}
\hline \multicolumn{2}{|c|}{ Standard Toxicant: } & $\mathrm{NaCl}$ & & \multirow[b]{2}{*}{$g / L$} \\
\hline \multicolumn{2}{|r|}{ Centra1 } & Tendency: & 2.04 & \\
\hline & \multicolumn{2}{|c|}{ Current Value: } & 1.9 & $g / L$ \\
\hline & Deviatio & 0.58 & & Dev units. \\
\hline
\end{tabular}


Results

\begin{tabular}{|l|l|l|l|l|}
\hline \multicolumn{2}{|l|}{ Glient: WSRC } & Sample ID: FHB018 & IWC: \\
\hline Log *: T3600 & Start Date: $2-23-95$ & Time: & $3: 00$ PM \\
\hline
\end{tabular}

CHRONIC EFFECTS

Control Effluent

TEST CONCENTRATION

Average young / female:

Standard Deviation:

$t=$

Steel's =

$\mathrm{MSD}=6.29$

Norma1ity: Data Not Norma1 $\quad W=0.87$

Homogeneity:Data Not Homogeneous $\mathrm{B}=\quad 19$

Test Used: Steel's Test

Critical steel's Value: 75

Critical t value: 2.23

$\begin{array}{rrrrrr}18.6 & 19.2 & 12.6 & 16.4 & 2.4 & \text { NA } \\ 8.16 & 9.15 & 5.4 & 2.72 & 2.76 & \text { NA } \\ -0.2 & 2.13 & 0.77 & 5.77 & \text { NA } \\ 109 & 81.5 & 90 & 71 & 65\end{array}$

Chronic Toxicity

6.25\% No Chronic Toxicity

No-Observed-Effect Concentration (NOEC): $25 \%$

12.5\% No Chronic Toxicity

Lowest-Observed-Effect Concentration (LOEC): $50 \%$

25\% No Chronic Toxicity

7 Day EC50: $24.16 \%$

50\% Chronically Toxic

$100 \% \quad$ Chronically Toxic 
7 Day Chronic Definitive Survival and Reproduction Bioassay

Test Organisms: Ceriodaphnia dubia

Method: EPA/600/4-89/001

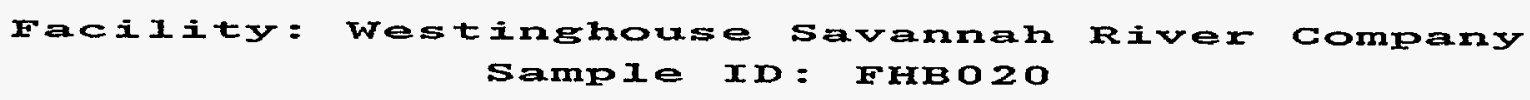

Date: $1-24-95$ 
SAMPLING INFORMATION

\begin{tabular}{|c|c|c|c|c|c|c|c|c|}
\hline Dilution Water & $20 \% \mathrm{DMW}$ & $1-24-95$ & m & 96.0 & 75.1 & 223 & $\vdots$ & \\
\hline Final Eff. 1 & Grab & $1-23-95$ & $09: 50 \mathrm{AM}$ & 6.0 & 4.06 & 25.4 & $<0.05$ & $2.0^{\circ} \mathrm{C}$ \\
\hline Final Eff. 2 & Grab & $1-25-95$ & $09: 40 \mathrm{AM}$ & 18.0 & 12.18 & 42.0 & $<0.05$ & $2.3^{*} \mathrm{C}$ \\
\hline Einal Eff. 3 & Grab & $1-27-95$ & $09: 20 \mathrm{AM}$ & 10.0 & 8.12 & 30.9 & $<0.05$ & $2 \cdot 3^{\circ} \mathrm{C}$ \\
\hline
\end{tabular}


Results

\begin{tabular}{|l|l|l|l|}
\hline \multicolumn{2}{|l|}{ Client: WSRC } & Sample ID: FHB020 & \\
\hline Log \#: T3497 & Start Date: $1-24-95$ & Time: & $3: 00$ PM \\
\hline
\end{tabular}

SURYIVAL EFFEGTS

Contro1 Effluent

$\begin{array}{lllllll}\text { Effluent Conc. } & \text { SC.DMW } & 6.25 \% & 12.5 \% & 25 \% & 50 \% & 100 \%\end{array}$

$\begin{array}{lllllll}48 \mathrm{hr} . \text { Mortality } & 0 \% & 10 \% & 0 \% & 11 \% & 0 \% & 60 \% \\ 7 \text { Day Mortality } & 0 \% & 10 \% & 0 \% & 11 \% & 0 \% & 100 \%\end{array}$

Method: Fishers Exact Test

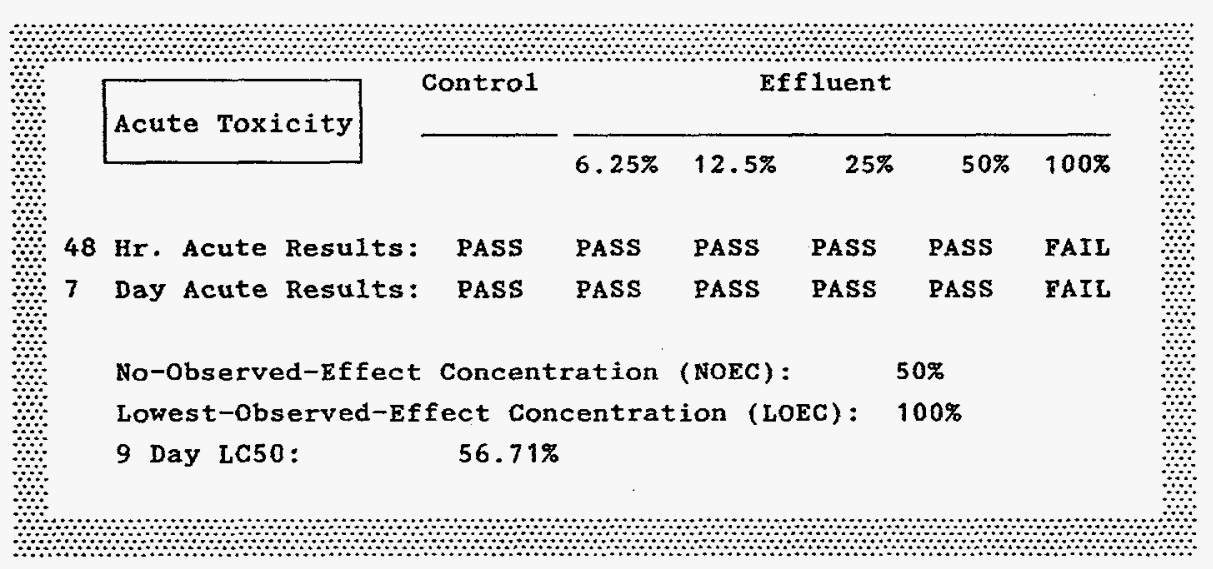

QUALITY CONTROL

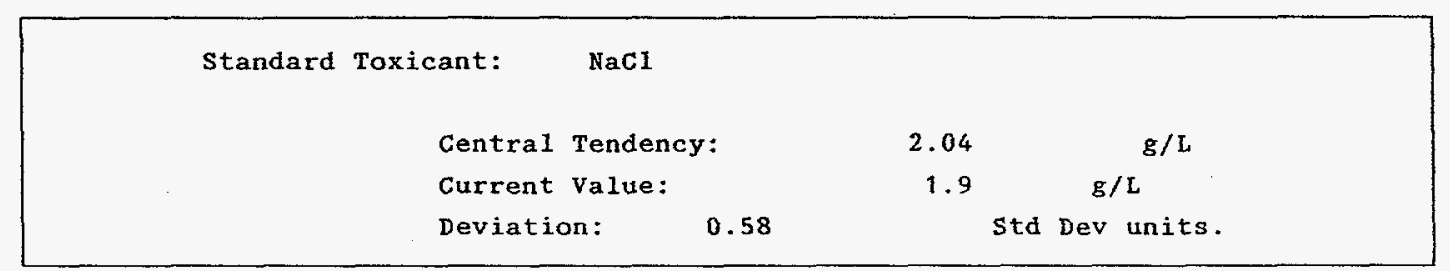


Resu1ts

\begin{tabular}{|l|l|l|l|l|}
\hline \multicolumn{2}{|l|}{ Client: WSRC } & Sample ID: FHB020 & IWC: \\
\hline Log : : T3497 & Start Date: $1-24-95$ & Time: & $3: 00$ PM \\
\hline
\end{tabular}

CHRONIC EFFECTS

Control Effluent

TEST CONCENTRATION

$\begin{array}{rrrrrr}\text { SC.DMW } & 6.25 \% & 12.5 \% & 25 \% & 50 \% & 100 \% \\ 18.4 & 19.4 & 24.8 & 19.4 & 23.5 & 0 \\ 6.76 & 7.47 & 3.6 & 8.59 & 4.3 & 0.00 \\ & -0.3 & -2.1 & -0.3 & -1.7 & 0.00 \\ & 55 & 55 & 105 & 86 & 55\end{array}$

Stee1's =

$\begin{array}{lllll}55 & 55 & 105 & 86 & 55\end{array}$

$M S D=6.86$

Normality: Data Not Normal $W=0.88$

Homogeneity:Data Homogeneous $\quad \mathrm{B}=7.8$

Test Used: Steel's Test

Critical steel's Value: 75

Critical $t$ Value: 2.23

Chronic Toxicity

6.25\% No Chronic Toxicity

No-Observed-Effect Concentration (NOEC): $50 \%$

12.5\% No Chronic Toxicity

Lowest-Observed-Effect Concentration (LOEC) : $100 \%$

7 Day EC50: $56.71 \%$

25\% No Chronic Toxicity

50\% No Chronic Toxicity

100\% Chronically Toxic 
(803) 877-6942 - FAX (803) 877-6938

P.O. Box 16414, Greenville, SC 29606 - 6C Craftsman Court, Greer, SC 29650

7 Day Chronic Definitive Surviva1 and Reproduction Bioassay

Test Organisms: Ceriodaphnia dubia

Method: EPA/ $600 / 4-89 / 001$

\footnotetext{
Facility : Westinghouse savannah Rivex Company Sample ID : FHBO21
}

Date: $2-23-95$ 
SAMPLING INEORMATION

\begin{tabular}{|c|c|c|c|c|c|c|c|c|}
\hline Dilution Water & $20 \% \mathrm{DMW}$ & $2-18-95$ & 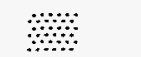 & 100 & 60.9 & 184.8 & & \\
\hline Final Eff. 1 & Grab & $2-22-95$ & $09: 40 \mathrm{AM}$ & 8.0 & 2.03 & 43.6 & $<0.05$ & $0.9^{\circ} \mathrm{C}$ \\
\hline Final Eff. 2 & Grab & $2-24-95$ & $08: 40 \mathrm{AM}$ & 4.0 & 6.12 & 43.4 & $<0.05$ & $3.3^{\circ} \mathrm{C}$ \\
\hline Einal Eff. 3 & Grab & $2-27-95$ & $08: 40 \mathrm{AM}$ & 8.0 & 4.08 & 44.1 & $<0.05$ & $1.6^{\circ} \mathrm{C}$ \\
\hline
\end{tabular}


Results

\begin{tabular}{|l|l|l|l|l|}
\hline \multicolumn{2}{|c|}{} & Sample ID: FHB021 & \\
\hline Log \#: T3601 & Start Date: $2-23-95$ & Time: & $5: 30$ PM \\
\hline
\end{tabular}

SURVIVAL EFPECTS

\begin{tabular}{|c|c|c|c|c|c|c|}
\hline \multirow[b]{2}{*}{ fffluent Conc. } & \multicolumn{2}{|l|}{ Control } & \multicolumn{4}{|c|}{ Effluent } \\
\hline & SC.DMW & $6.25 \%$ & $12.5 \%$ & $25 \%$ & $50 \%$ & $100 \%$ \\
\hline $48 \mathrm{hr}$. Mortality & $0 \%$ & $10 \%$ & $0 \%$ & $0 \%$ & $0 \%$ & $0 \%$ \\
\hline Day Mortality & $11 \%$ & $10 \%$ & $0 \%$ & $0 \%$ & $0 \%$ & $20 \%$ \\
\hline
\end{tabular}

Method: Fishers Exact Test

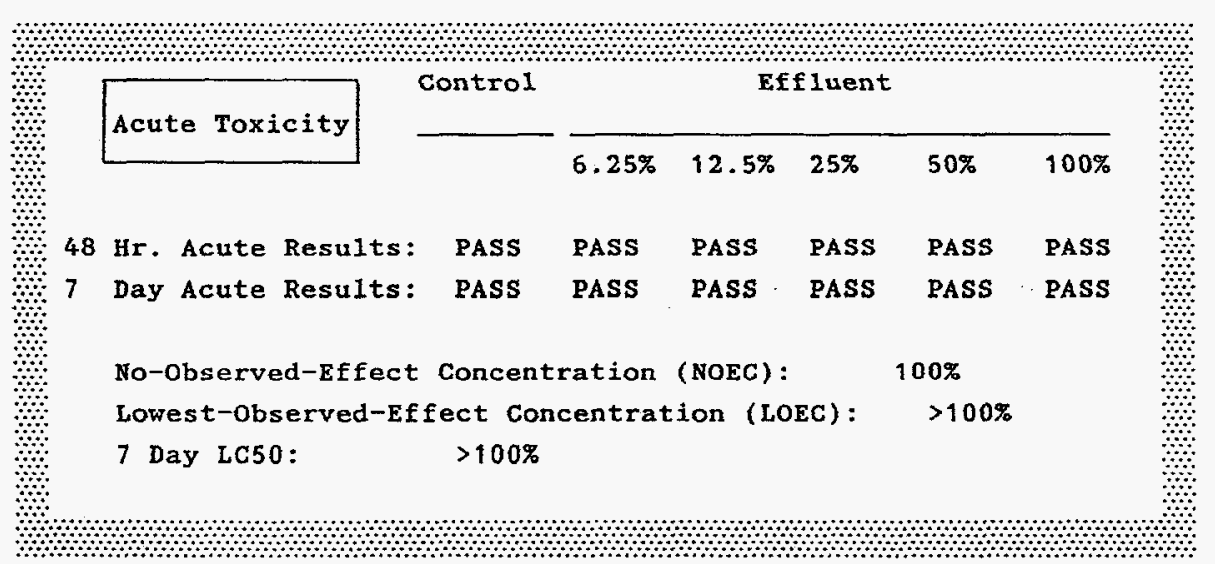

QUALITY CONTROL

\begin{tabular}{|cccc|}
\hline Standard Toxicant: & & \\
& & & \\
& Central Tendency: & 2.04 & $\mathrm{~g} / \mathrm{L}$ \\
Current Value: & 1.9 & $\mathrm{~g} / \mathrm{L}$ \\
Deviation: & 0.58 & Std Dev units. \\
\hline
\end{tabular}


Results

\begin{tabular}{|l|l|l|l|l|}
\hline \multicolumn{2}{|c|}{} & \multicolumn{2}{|c|}{ Sample ID: FHB021 } & IWC: \\
\hline Log \#: T3601 & Start Date: $2-23-95$ & Time: & $5: 30$ PM \\
\hline
\end{tabular}

CHRONIC EFFECTS

\begin{tabular}{|c|c|c|c|c|c|c|}
\hline \multirow[b]{2}{*}{ TEST CONGENTRATION } & \multirow{2}{*}{$\frac{\text { Sontrol }}{\text { SC.DMW }}$} & \multicolumn{4}{|c|}{ Effluent } & \multirow[b]{2}{*}{$100 \%$} \\
\hline & & $6.25 \%$ & $12.5 \%$ & $25 \%$ & $50 \%$ & \\
\hline Average young / female: & 18.6 & 17.8 & 18.7 & 18.5 & 19 & 10.2 \\
\hline Standard Deviation: & 8.16 & 7.22 & 2.98 & 3.06 & 3.5 & 6.01 \\
\hline$t=$ & & 0.3 & -0.06 & 0.02 & -0.2 & 3.29 \\
\hline steel's = & & 102 & 99.5 & 100 & 99.5 & 70 \\
\hline
\end{tabular}

MSD $=5.9$

Normality: Data Not Normal $W=0.83$

Homogeneity:Data Not Homogeneous $B=15.8$

Test Used: Steel's Test

Critical Steel's value: 75

Critical t value: 2.31

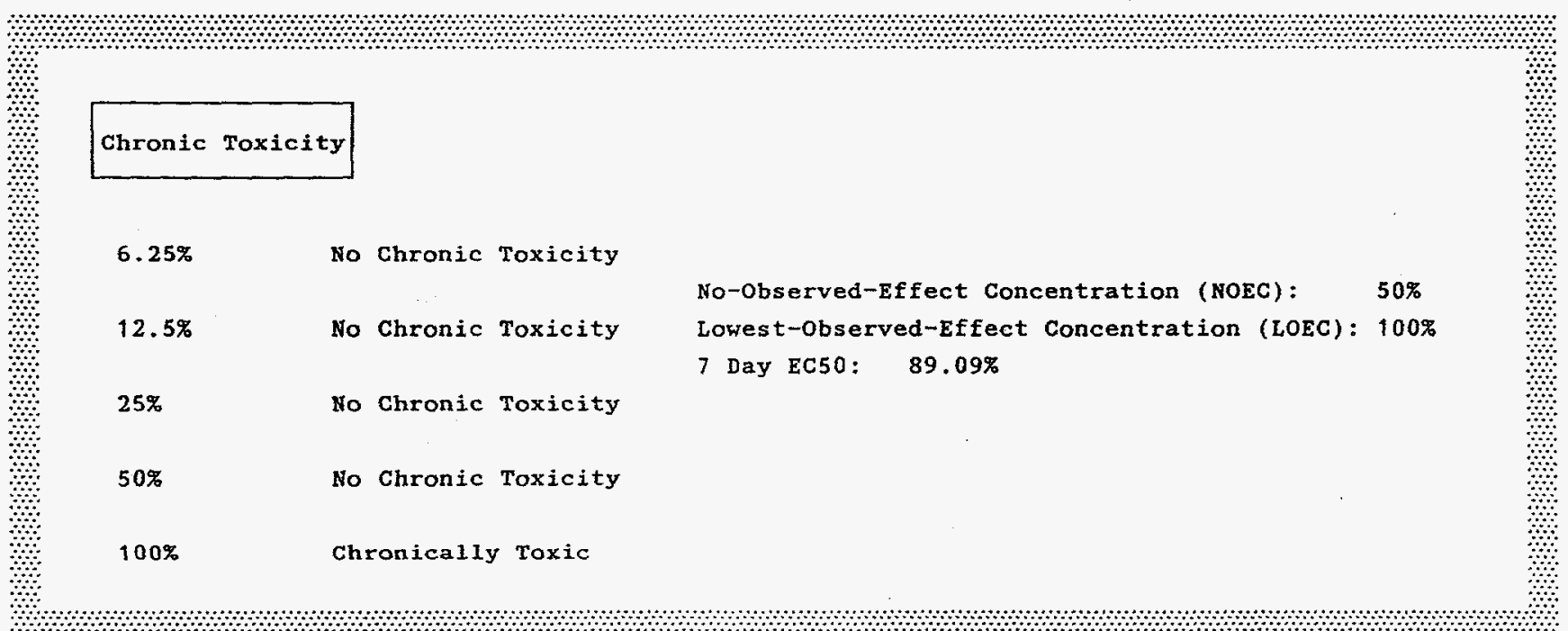


(803) 877-6942 - FAX (803) 877-6938

P.O. Box 16414, Greenville, SC 29606 - 6C Craftsman Court, Greer, SC 29650

7 Day Chronic Definitive Survival and Reproduction Bioassay

Test Organisms: Ceriodaphnia dubia

Method: EPA/600/4-89/001

Facility = Westinghouse savannah

River Company Sample ID: FHBO22

Date: 2-2-95 


\section{SAMPLING INFORMATION}

\begin{tabular}{|c|c|c|c|c|c|c|c|c|}
\hline Dilution Water & $20 \% D M W$ & $2-1-95$ & H. & 96.04 & 71.05 & 226 & 烈 & \\
\hline Final Eff. 1 & Grab & $2-1-95$ & $07: 30 \mathrm{AM}$ & 9.8 & 6.09 & 55.3 & $<0.05$ & $3.0^{\circ} \mathrm{C}$ \\
\hline Final Eff. 2 & Grab & $2-3-95$ & $07: 20 \mathrm{AM}$ & 11.8 & 8.12 & 52.9 & 0.08 & $3.2^{\circ} \mathrm{C}$ \\
\hline Final Eff. 3 & Grab & $2-6-95$ & $09: 00 \mathrm{AM}$ & 13.7 & 8.12 & 47.7 & $<0.05$ & $1.8^{\circ} \mathrm{C}$ \\
\hline
\end{tabular}


Results

\begin{tabular}{|l|l|l|l|l|}
\hline \multicolumn{2}{|l|}{ Client: WSRC } & Sample ID: FHB022 & \\
\hline Log \#: T3520 & Start Date: $2-2-95$ & & Time: \\
\hline
\end{tabular}

SURVIVAL EFFECTS

Control Effluent

Effluent Conc.

SC.DMW

$6.25 \%$

$12.5 \% 25 \%$

$50 \% 100 \%$

$48 \mathrm{hr}$. Mortality

$\begin{array}{llllll}0 \% & 0 \% & 0 \% & 0 \% & 0 \% & 0 \%\end{array}$

7 Day Mortality

$\begin{array}{llllll}0 \% & 0 \% & 10 \% & 10 \% & 10 \% & 0 \%\end{array}$

Method: Fishers Exact Test

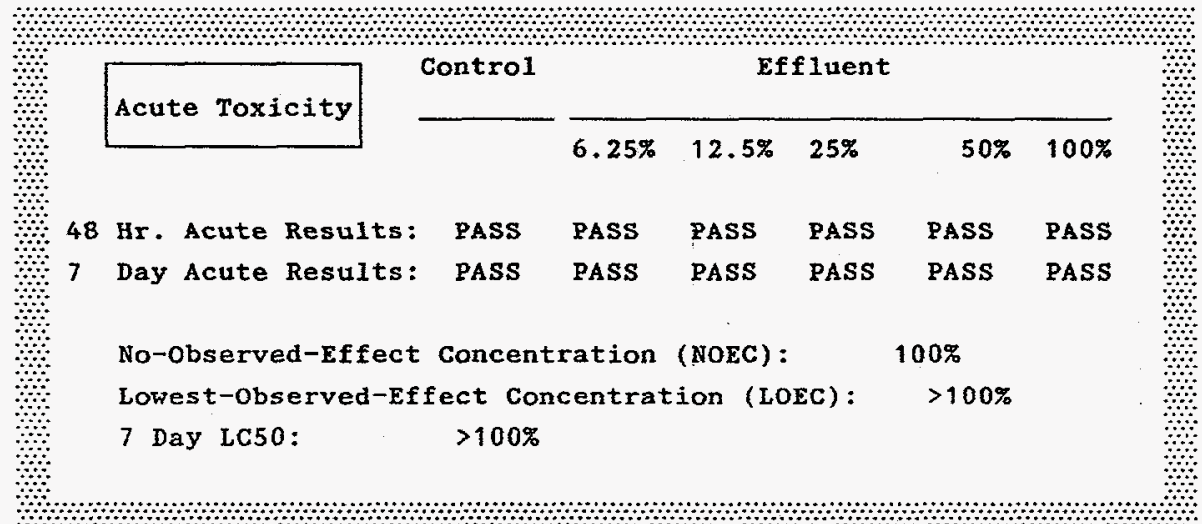

QUALITY CONTROL

Standard Toxicant: Nac1

Central Tendency:

Current value:

Deviation:

0.58
2.04
$g / L$
1.9
g/L

std Dev units. 
Results

\begin{tabular}{|l|l|l|l|l|}
\hline \multicolumn{2}{|l|}{ C1ient: WSRC } & Samp1e ID: FHB022 & IWC: \\
\hline Log \#: T3520 & Start Date: $2-2-95$ & Time: & $2: 30$ PM \\
\hline
\end{tabular}

\section{CHRONIC EFFECTS}

Control Effluent

TEST CONCENTRATION

Average young / female:

Standard Deviation:

$t=$

Steel's =

$M S D=6.29$

Normality: Data Not Normal

Homogeneity:Data Homogeneous

Test Used: Steel's Test

Critical Steel's Value: 75

Critical t value: 2.31
$\mathrm{W}=0.86$

$B=7.57$

$\begin{array}{rrrrrr} & & & & & \\ & 6.25 \% & 12.5 \% & 25 \% & 50 \% & 100 \% \\ 24.2 & 14.9 & 19 & 19.9 & 19.7 & 13.9 \\ 3.31 & 4.18 & 7.26 & 5.65 & 7.66 & 6.03 \\ & 3.42 & 1.92 & 1.59 & 1.66 & 3.79 \\ & 67 & 87 & 85.5 & 88.5 & 71\end{array}$

Chronic Toxicity

$6.25 \% \quad$ Ghronically Toxic **

No-Observed-Effect Concentration (NOEC): $\quad 50 \%$

12.5\% No Chronic Toxicity

Lowest-Observed-Effect Concentration (LOEC) : $100 \%$

7 Day EC50: $>50 \%$

257 No Chronic Toxicity

50\% No Chronic Toxicity

100\% Chronically Toxic

* $* 6.25 \%$ concentration mean production is regarded as an outlier 
7 Day Chxonic Definitive Survival and Reproduction Bioassay

Test Ofganisms: Ceriodaphnia dubia

Method: EPA/600/4-89/001

\footnotetext{
Facility : westinghouse savanman Rivex company Sample ID = FHBO23
}

Date: $\quad 1-24-95$ 
DEFINITIVE SURVIVAI AND REPRODUCTION

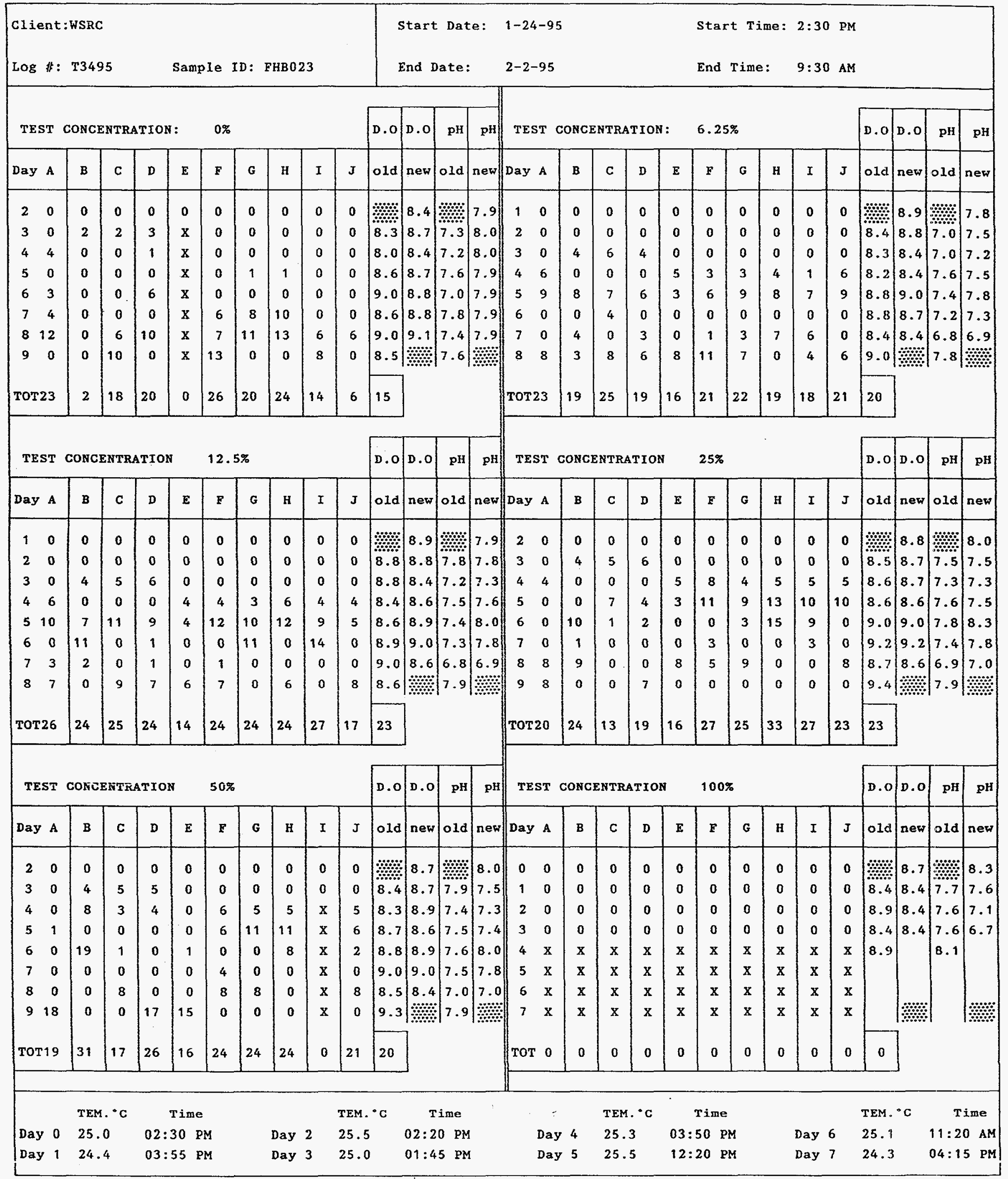




\section{SAMPIING INFORMATION}

\begin{tabular}{|c|c|c|c|c|c|c|c|c|}
\hline Dilution Water & $20 \% \mathrm{DMW}$ & $1-24-95$ & 所 & 96.0 & 75.1 & 223 & $\because$ & \\
\hline Final Eff. 1 & Grab & $1-23-95$ & $09: 30 \mathrm{AM}$ & 14.0 & 4.06 & 33.2 & $<0.05$ & $2.0^{\circ} \mathrm{C}$ \\
\hline Final Eff. 2 & Grab & $1-25-95$ & $09: 30 \mathrm{AM}$ & 16.0 & 2.03 & 31.1 & 0.08 & $2.3^{\circ} \mathrm{C}$ \\
\hline Final Eff. 3 & Grab & $1-27-95$ & $09: 00 \mathrm{AM}$ & 12.0 & 4.06 & 28.2 & $<0.05$ & $2.3^{\circ} \mathrm{C}$ \\
\hline
\end{tabular}


Results

\begin{tabular}{|l|l|l|l|}
\hline \multicolumn{2}{|l|}{ Client: WSRC } & Sample ID: FHB023 & \\
\hline Log \#: T3495 & Start Date: $1-24-95$ & Time: & $2: 30$ PM \\
\hline
\end{tabular}

SURVIVAL EFFECTS

\begin{tabular}{lcrrrrrr} 
& \multicolumn{6}{c}{ Control } & \multicolumn{6}{c}{ Effluent } \\
\cline { 2 - 3 } \cline { 6 - 8 } Effluent Conc. & SC.DMW & $6.25 \%$ & $12.5 \%$ & $25 \%$ & $50 \%$ & $100 \%$ \\
\hline & $10 \%$ & $0 \%$ & $0 \%$ & $0 \%$ & $10 \%$ & $0 \%$ \\
7 hr. Mortality & $10 \%$ & $0 \%$ & $0 \%$ & $0 \%$ & $10 \%$ & $100 \%$ \\
\hline
\end{tabular}

Method: Fishers Exact Test

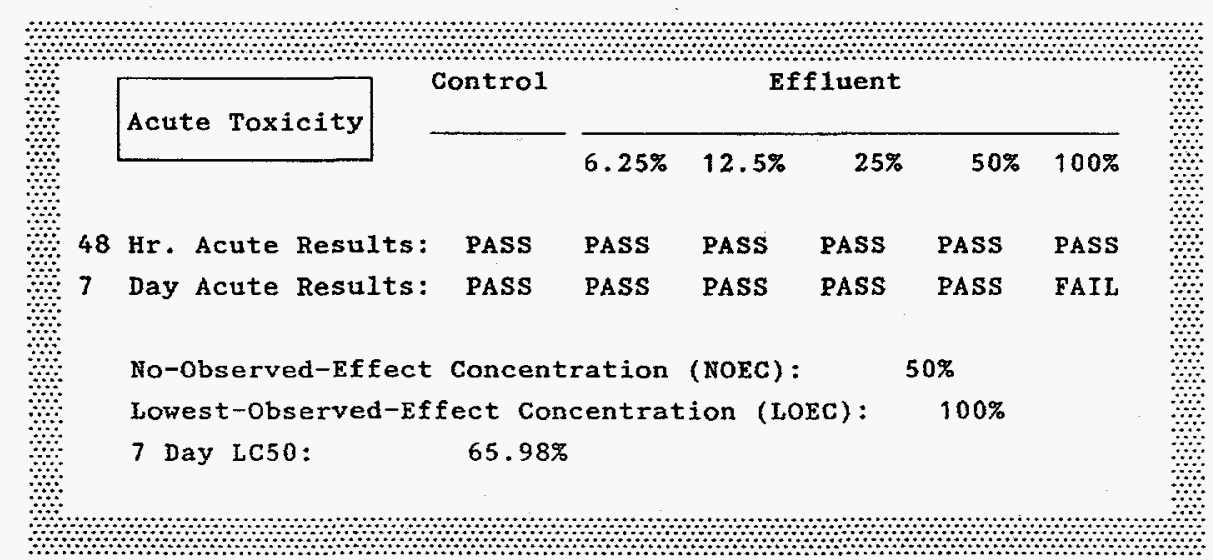

QUALITY CONTROL

\begin{tabular}{|cccc|}
\hline Standard Toxicant: & NaC1 & \\
& & & \\
& Central Tendency: & 2.04 & $\mathrm{~g} / \mathrm{L}$ \\
Current Value: & 1.9 & $\mathrm{~g} / \mathrm{L}$ \\
Deviation: & 0.58 & Std Dev units. \\
\hline
\end{tabular}


Results

\begin{tabular}{|l|l|l|l|}
\hline \multicolumn{2}{|l|}{ C1ient: WSRC } & Sample ID: FHB023 & IWC: \\
\hline Log 作: T3495 & Start Date: $1-24-95$ & Time: & $2: 30$ PM \\
\hline
\end{tabular}

CHRONIC EFEECTS

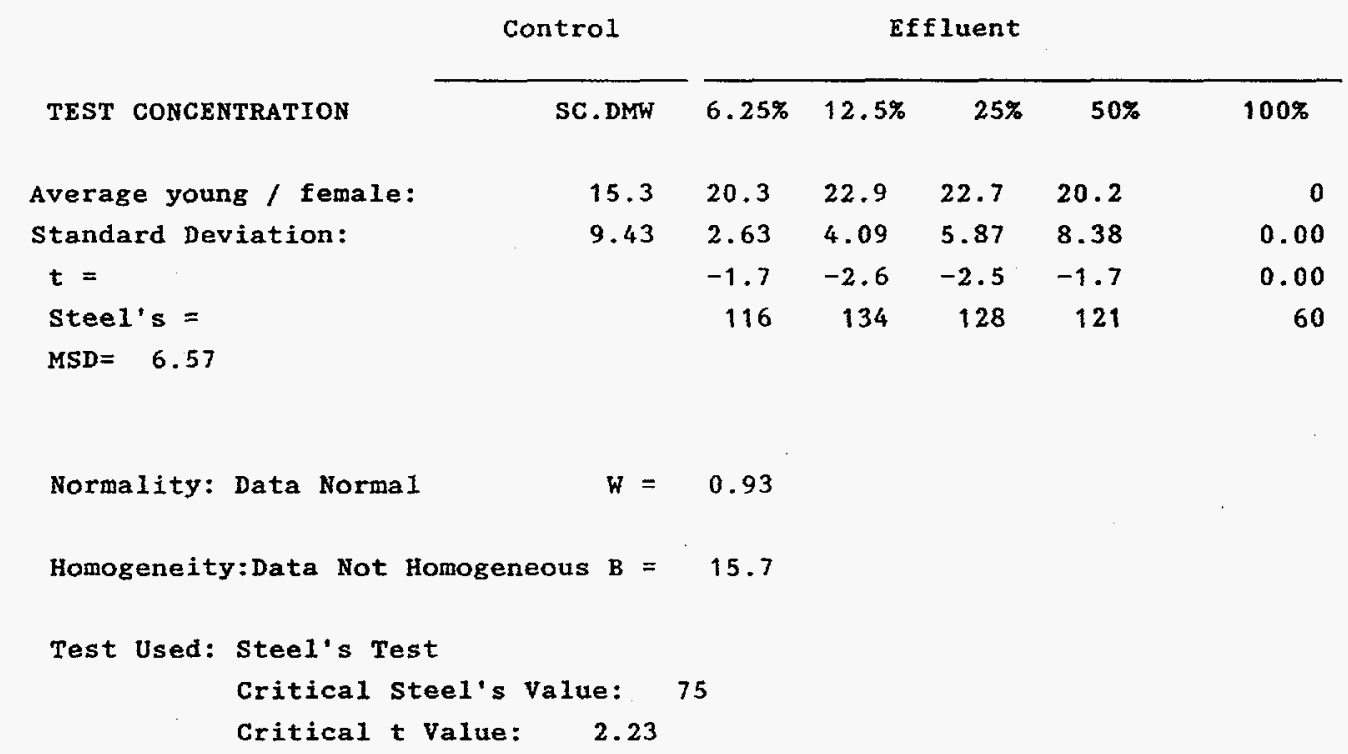


7 Day Chronic Definitive Survival and Reproduction Bioassay

Test Organisms: Ceriodaphnia dubia

Method: EPA/600/4-89/001

Facility: Westinghouse savannah River company $\operatorname{Sample} I D=\operatorname{HSPO64}$

pate: $\quad 1-24-95$ 
DEFINITIVE SURVIVAL AND REPRODUCTION

\begin{tabular}{|l|lll}
\hline Client:WSRC & Start Date: & $1-24-95$ & Start Time: $3: 30$ pM \\
Log \#: T3499 Sample ID: HSP064 & End Date: & $2-2-95$ & End Time: $5: 00$ PM \\
\hline
\end{tabular}

\begin{tabular}{|c|c|c|c|c|c|c|c|c|c|c|c|c|c|c|c|c|c|c|c|c|c|c|c|c|c|c|c|c|}
\hline TEST & \multicolumn{4}{|c|}{ CONCENTRATION: } & \multicolumn{5}{|c|}{$0 \%$} & \multirow{2}{*}{$\frac{0.0}{01 d}$} & \multirow{2}{*}{$\begin{array}{l}0.0 \\
\text { new }\end{array}$} & \multirow{2}{*}{$\mid$\begin{tabular}{c|}
$\mathrm{pH}$ \\
$\mathrm{old}$
\end{tabular}} & \multirow{2}{*}{ new } & \multicolumn{6}{|c|}{ TEST CONCENTRATION: } & \multicolumn{5}{|c|}{$6.25 \%$} & \multirow{2}{*}{$\frac{0.0}{01 d}$} & \multirow{2}{*}{ D.o } & \multirow{2}{*}{$\underbrace{\mathbf{p H}}_{0.1 d}$} & \multirow{2}{*}{$\frac{\mathrm{pH}}{\text { new }}$} \\
\hline Day A & B & C & D & $\mathbf{E}$ & $\mathbf{F}$ & G & H & I & $\mathrm{J}$ & & & & & Day & $A$ & B & c & D & & $\mathbf{F}$ & G & H & & & & & & \\
\hline 20 & 0 & 0 & 0 & 0 & 0 & 0 & 0 & 0 & 0 & \% & 8.4 & 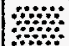 & 7.9 & 2 & 0 & $\mathbf{0}$ & 0 & c & & 0 & 0 & 0 & & & 和器 & 8.1 & \% & 7.9 \\
\hline 30 & 0 & 3 & 4 & 4 & $\mathbf{0}$ & 3 & 0 & 4 & 2 & 8.3 & 8.7 & 7.3 & 8.0 & 3 & 2 & 5 & 4 & 5 & & 0 & 0 & 0 & & & 8.8 & 8.9 & 7.3 & 7.8 \\
\hline 43 & 3 & 0 & 0 & 0 & 4 & 0 & 2 & 0 & 0 & 8.0 & 8.4 & 7.2 & 8.0 & 4 & 0 & 0 & 0 & 0 & & 0 & 0 & 0 & & & 8.4 & 8.4 & 6.7 & 7.2 \\
\hline 50 & 2 & 4 & 0 & 3 & 2 & 3 & 4 & 3 & 3 & 8.6 & 8.7 & 7.6 & 7.9 & 5 & 8 & 10 & 9 & 8 & & 0 & 0 & 0 & & & 8.2 & 8.4 & 6.7 & 6.9 \\
\hline 60 & 0 & 0 & 0 & 0 & 0 & 0 & 0 & 0 & 0 & 9.0 & 8.8 & 7.0 & 7.9 & 6 & 0 & 2 & 2 & 1 & & 0 & 0 & 0 & & & 8.6 & 8.7 & 6.7 & 7.0 \\
\hline 70 & 0 & 0 & 0 & 0 & 0 & 0 & 0 & 0 & $\mathrm{X}$ & 8.6 & 8.8 & 7.8 & 7.9 & 7 & 0 & 0 & c & c & & 0 & 0 & 0 & & & 9.2 & 9.2 & 7.6 & 7.6 \\
\hline $\begin{array}{ll}8 & 13\end{array}$ & 0 & 0 & 0 & 0 & 16 & 0 & 0 & o & $\mathrm{x}$ & 9.0 & 9.1 & 7.4 & 7.9 & 8 & 8 & 6 & 6 & $\varepsilon$ & & 6 & 9 & 7 & & & 8.2 & 8.4 & 6.0 & 6.1 \\
\hline $\begin{array}{ll}9 & 8\end{array}$ & 10 & 9 & 12 & 13 & 0 & 8 & 4 & 11 & $\mathrm{x}$ & 8.5 & 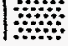 & 7.6 & $\mid$ & 9 & 0 & 0 & 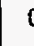 & $c$ & & 24 & 21 & 13 & & & 9.2 & 嵫 & 7.9 & \\
\hline TOT 24 & 15 & 16 & 16 & 20 & 22 & 14 & 10 & 18 & 5 & 16 & & & & Tot & & 23 & 21 & 22 & & 30 & 30 & 20 & & & 22 & & & \\
\hline
\end{tabular}

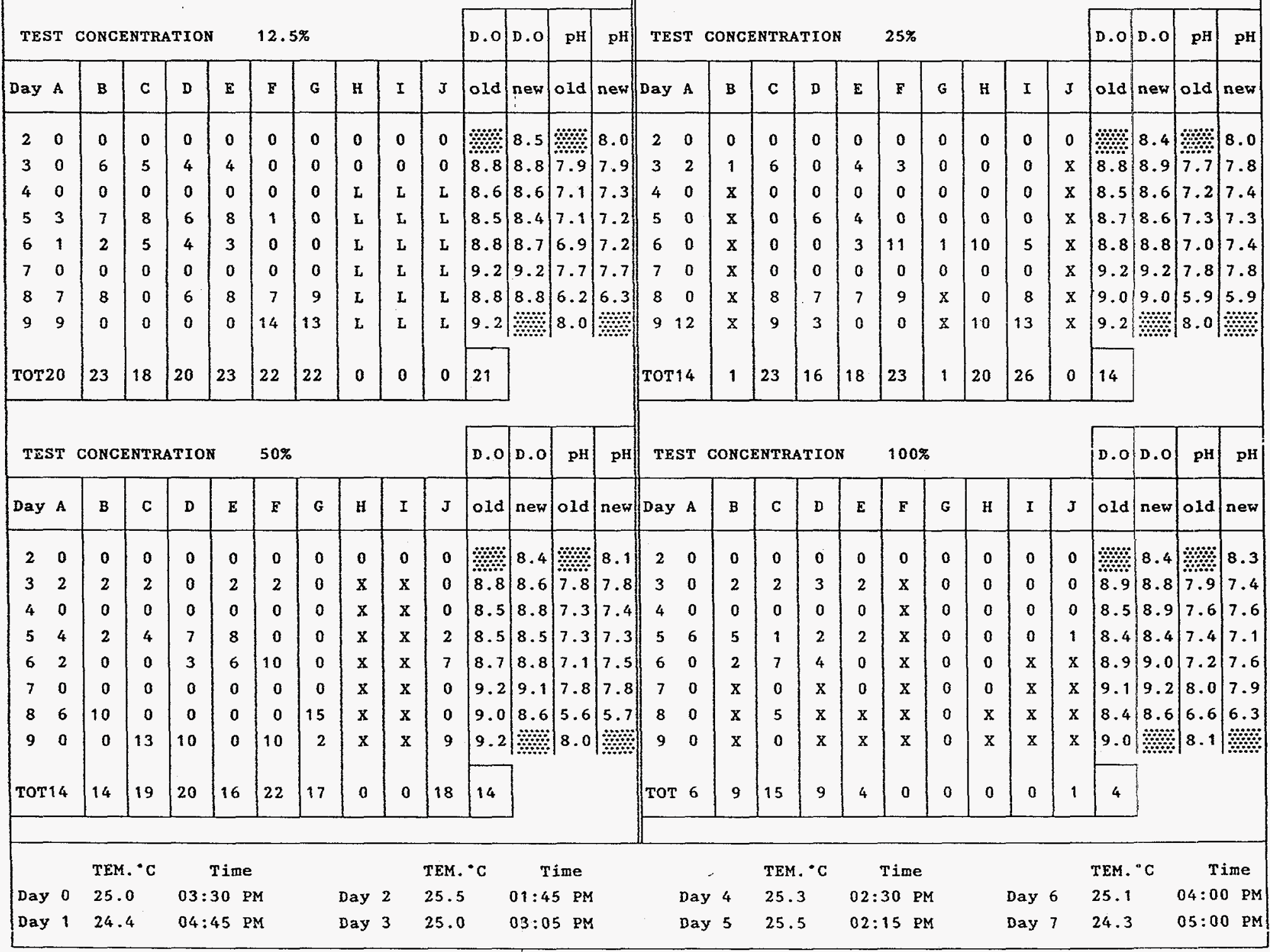


SAMPLING INEORMATION

\begin{tabular}{|c|c|c|c|c|c|c|c|c|}
\cline { 2 - 7 } & Type & Start Date & Time & Hardness Mg/L & Alkalinity & Conductivity & Res. C1 & Rec. Temp. \\
\hline Dilution Water & $20 \%$ DM & $1-24-95$ & $\ldots \ldots$ & 96.0 & 75.1 & 223 & $\vdots$ \\
\hline Final Eff. 1 & Grab & $1-23-95$ & $10: 20 \mathrm{AM}$ & 6.0 & 4.06 & 24.7 & $<0.05$ \\
\hline Final Eff. 2 & Grab & $1-25-95$ & $10: 00 \mathrm{AM}$ & 14.0 & 28.4 & 28.1 & $2.0^{\circ} \mathrm{C}$ \\
\hline Final Eff. 3 & Grab & $1-27-95$ & $09: 40 \mathrm{AM}$ & 6.0 & 4.06 & 26.7 & $2.3^{\circ} \mathrm{C}$ \\
\hline
\end{tabular}


Results

\begin{tabular}{|l|l|l|l|l|}
\hline \multicolumn{2}{|l|}{ Client: WSRC } & Sample ID: HSP064 & \\
\hline Log \# : T3499 & Start Date: $1-24-95$ & Time: & $3: 30$ PM \\
\hline
\end{tabular}

SURVIVAL EFFECTS

Contro1 Effluent

Effluent Conc.

SC. DMW $\quad 6.25 \% \quad 12.5 \% \quad 25 \% \quad 50 \% \quad 100 \%$

$48 \mathrm{hr}$. Mortality

$\begin{array}{llllll}0 \% & 0 \% & 0 \% & 20 \% & 20 \% & 10 \%\end{array}$

7 Day Mortality

$\begin{array}{llllll}10 \% & 0 \% & 0 \% & 30 \% & 20 \% & 70 \%\end{array}$

Method: Fishers Exact Test

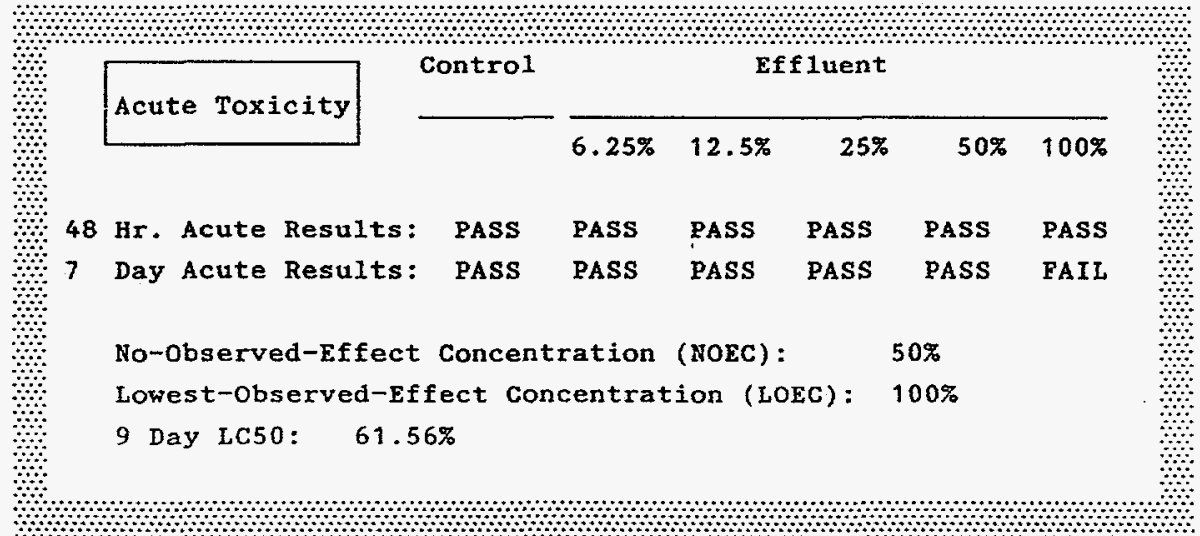

QUALITY CONTROL

\begin{tabular}{|llrc|}
\hline Standard Toxicant: NaC1 & & \\
& & & \\
& & & \\
& Central Tendency: & 2.04 & $\mathrm{~g} / \mathrm{L}$ \\
Current Value: & 1.9 & $\mathrm{~g} / \mathrm{L}$ \\
Deviation: & 0.58 & \multicolumn{2}{c|}{ Std Dev units. } \\
\hline
\end{tabular}


Results

\begin{tabular}{|l|l|l|l|}
\hline C1ient: WSRC & Sample ID: HSP064 & IWC: \\
\hline Log \#: T3499 & Start Date: $1-24-95$ & Time: & $3: 30$ PM \\
\hline
\end{tabular}

CHRONIC EFFECTS

Control Effluent

TEST CONCENTRATION

SC.DMW

\begin{tabular}{lllll}
\hline $6.25 \%$ & $12.5 \%$ & $25 \%$ & $50 \%$ & $100 \%$
\end{tabular}

Average young / female:

$\begin{array}{llllll}16 & 22.1 & 21.1 & 14.2 & 14 & 4.4\end{array}$

Standard Deviation:

5.6

$\begin{array}{lll}4.93 & 1.86 \quad 9.98\end{array}$

7.79

5.23

$-1.8$

$-1.4$

0.53

0.59

0.00

Stee1's =

134

112

105

101

61.5

$\mathrm{MSD}=7.74$

Normality: Data Not Normal $\quad W=0.81$

Homogeneity:Data Not Homogeneous $B=15.2$

Test Used: Steel's Test

Critical Steel's Value: 75

Critical $t$ Value: 2.23

Chronic Toxicity

6.25\% No Chronic Toxicity

12.5\% No Chronic Toxicity

No-Observed-Effect Concentration (NOEC):

Lowest-Observed-Effect Concentration (LOEC): $100 \%$

7 Day EC50: $61.56 \%$

$25 \%$

No Chronic Toxicity

50\% No Chronic Toxicity

$100 \% \quad$ Chronica11y Toxic 
(803) 877-6942 - FAX (803) 877-6938

P.O. Box 16414, Greenville, SC 29606 - 6C Craftsman Court, Greer, SC 29650

7 Day Chronic Definitive Survival and Reproduction Bioassay

Test Organisms: Ceriodaphnia dubia

Method: EPA/600/4-89/001

\author{
Facility = Westinghouse savannah River company \\ Sample ID = FMCOO2H \\ Date: $1-24-95$
}


DEFINITIVE SURVIVAL AND REPRODUCTION

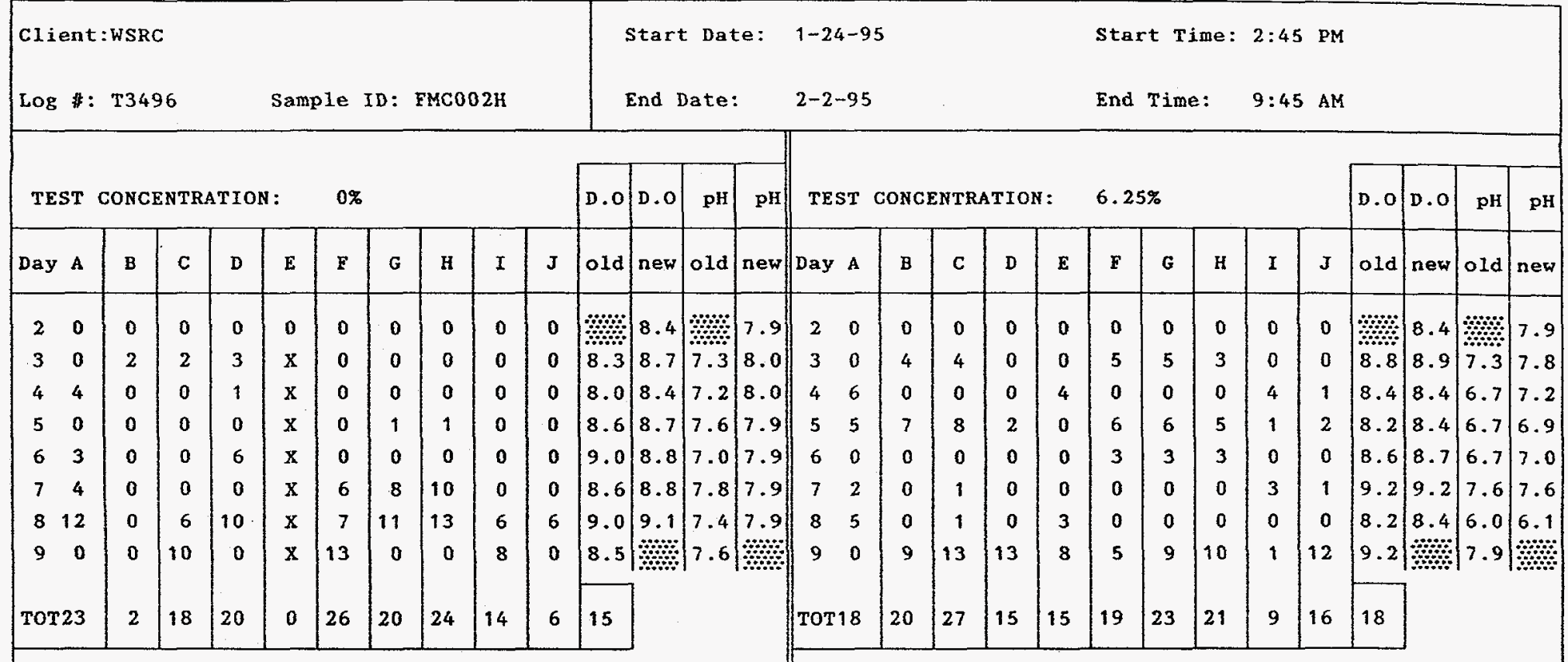

\begin{tabular}{|c|c|c|c|c|c|c|c|c|c|c|c|c|c|c|c|c|c|c|c|c|c|c|c|c|c|c|c|c|}
\hline TEST & \multicolumn{5}{|c|}{ CONCENTRATION } & \multicolumn{5}{|c|}{$12.5 \%$} & \multirow{2}{*}{\begin{tabular}{|l|}
0.0 \\
$01 \mathrm{~d}$
\end{tabular}} & \multirow{2}{*}{\begin{tabular}{|l|} 
D.o \\
new
\end{tabular}} & \multirow{2}{*}{$\mid \begin{array}{l}\mathrm{pH} \\
\mathrm{old}\end{array}$} & \multirow{2}{*}{\begin{tabular}{|c|}
$\mathrm{PH}$ \\
new
\end{tabular}} & \multicolumn{5}{|c|}{ TEST CONCENTRATION } & \multicolumn{5}{|l|}{$25 \%$} & \multirow{2}{*}{\begin{tabular}{|l|}
0.0 \\
$01 d$
\end{tabular}} & \multirow{2}{*}{ new } & \multirow{2}{*}{\begin{tabular}{|c|}
$\mathrm{pH}$ \\
$01 \mathrm{~d}$
\end{tabular}} & \multirow{2}{*}{\begin{tabular}{|c} 
pH \\
new
\end{tabular}} \\
\hline Day A & & B & $\mathbf{C}$ & D & $\mathbf{E}$ & $\mathbf{F}$ & $\mathbf{G}$ & H & I & $\mathbf{J}$ & & & & & Day A & $\mathbf{B}$ & c & D & $\boldsymbol{E}$ & $\mathbf{F}$ & G & H & I & $\mathbf{J}$ & & & & \\
\hline 20 & & 0 & 0 & 0 & $\mathbf{0}$ & 0 & 0 & 0 & 0 & 0 & 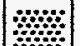 & 8.5 & 前 & 8.0 & 20 & 0 & 0 & o & 0 & 0 & 0 & 0 & 0 & 0 & Ha & 8.4 & 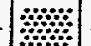 & 8.0 \\
\hline 30 & & 4 & 5 & 5 & 0 & 0 & 0 & 0 & 0 & 0 & 8.8 & 8.8 & 7.9 & 7.9 & 30 & 6 & $\mathbf{0}$ & 4 & 0 & 0 & 0 & 0 & o & 0 & 8.8 & 8.9 & 7.7 & 7.8 \\
\hline 43 & & 0 & 0 & 0 & 4 & 6 & 7 & 5 & 4 & 4 & 8.6 & 8.6 & 7.1 & 7.3 & 44 & 1 & 3 & 0 & 0 & 4 & 4 & 6 & 4 & 5 & 8.5 & 8.6 & 7.2 & 7.4 \\
\hline 59 & & 8 & 0 & 3 & 7 & 0 & 7 & 7 & 1 & 0 & 8.5 & 8.4 & 7.1 & 7.2 & $\begin{array}{ll}5 & 10\end{array}$ & $\mathrm{x}$ & 7 & 9 & 4 & 5 & 8 & 10 & 4 & 6 & 8.7 & 8.6 & 7.3 & 7.3 \\
\hline 61 & & 0 & 3 & 0 & 0 & 0 & 0 & 0 & 0 & 0 & 8.8 & $8.7 \mid$ & 6.9 & $7.2 \mid$ & 60 & $\mathrm{x}$ & 2 & 8 & 0 & 0 & 0 & 0 & 0 & 0 & 8.8 & 8.8 & 7.0 & 3.4 \\
\hline 78 & & 1 & 0 & 1 & 0 & 6 & 8 & 7 & 0 & 0 & 9.2 & 9.2 & 7.7 & 7.7 & 73 & $x$ & 0 & 0 & 0 & 0 & 1 & 0 & 0 & 1 & 9.2 & 9.2 & 7.8 & 7.8 \\
\hline 80 & & 0 & 0 & 1 & 3 & 5 & 6 & 7 & 6 & 9 & 8.8 & 8.8 & 6.2 & 6.3 & 85 & $\mathrm{x}$ & 3 & 0 & 6 & 5 & 7 & 2 & 3 & 6 & 9.0 & 9.0 & 5.9 & 5.9 \\
\hline 90 & & 8 & 10 & 15 & 5 & 0 & 0 & 0 & 10 & 11 & $|9.2|$ & | & 8.0 & 翌 & 90 & $\mathrm{x}$ & 12 & 0 & 9 & 6 & 0 & 11 & 7 & 0 & $|9.2|$ & $\mid$ & $\mid 8.0$ & 胼 \\
\hline тот 21 & & 21 & 18 & 25 & 19 & 17 & 28 & 26 & 21 & 24 & 22 & & & & тот 22 & 7 & 27 & 21 & 19 & 20 & 20 & 29 & 18 & 18 & 20 & & & \\
\hline \multicolumn{2}{|c|}{ TEST } & conc & ENTR & TIOI & & $50 \%$ & & & & & D.o & D.o & $\mathrm{pH}$ & $\mathrm{pH}$ & \multicolumn{5}{|c|}{ TEST CONCENTRATION } & \multicolumn{5}{|c|}{$100 \%$} & 0.0 & 0.0 & $\mathrm{pH}$ & $\mathrm{pH}$ \\
\hline Day A & & B & c & $\mathbf{D}$ & $\mathbf{E}$ & $\mathbf{E}$ & $G$ & $\mathbf{H}$ & $\mathbf{I}$ & $\mathbf{J}$ & old & new & $01 d$ & new & Day A & B & $\mathrm{C}$ & D & $\mathbf{E}$ & $\boldsymbol{F}$ & c & н & I & $\mathbf{J}$ & $01 d$ & new & $01 d$ & new \\
\hline 20 & & 0 & 0 & $\mathbf{0}$ & 0 & 0 & 0 & 0 & $\mathbf{0}$ & 0 & 前 & 8.4 & 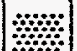 & 8.1 & 20 & 0 & 0 & 0 & 0 & 0 & 0 & 0 & 0 & 0 & \% & 8.4 & $\mid$ & 8.3 \\
\hline 3 & & 4 & 5 & 5 & 0 & 0 & 0 & o & 0 & 0 & 8.8 & 8.6 & 7.8 & 7.8 & 30 & 4 & 2 & o & 0 & 0 & 0 & 0 & 0 & 0 & 8.9 & 8.8 & 7.9 & 7.4 \\
\hline 4 & & 0 & 0 & 0 & 0 & 4 & 4 & 4 & 3 & 0 & 8.5 & 8.8 & 7.3 & 7.4 & 4 & 0 & 0 & 0 & 4 & 3 & 2 & 5 & 3 & 0 & 8.5 & 8.9 & 7.6 & 7.6 \\
\hline 58 & 3 & 8 & 5 & 7 & 7 & 6 & 6 & 9 & 9 & 0 & 8.5 & 8.5 & 7.3 & 7.3 & 5 & 8 & 7 & 7 & 6 & 7 & 8 & 6 & 6 & 0 & 8.4 & 8.4 & 7.4 & 7.1 \\
\hline 60 & 0 & 6 & 4 & 5 & 2 & 0 & 0 & 0 & 9 & 0 & 8.7 & 8.8 & 7.1 & 7.5 & 60 & 5 & 3 & 6 & 1 & 0 & 0 & 0 & 6 & 0 & 8.9 & 9.0 & 7.2 & 7.6 \\
\hline 70 & 0 & 0 & 0 & 0 & 0 & 0 & 0 & 0 & 0 & 0 & 9.2 & 9.1 & 7.8 & 7.8 & 70 & 0 & 0 & 0 & 0 & 8 & 0 & 4 & 0 & 0 & 9.1 & 9.2 & 8.0 & 7.9 \\
\hline 84 & 4 & 0 & 0 & 0 & 1 & 4 & 13 & 8 & 0 & 4 & 9.0 & 8.6 & 5.6 & 5.7 & 80 & 0 & 0 & 0 & 0 & 0 & 0 & 0 & 13 & 6 & 8.4 & 8.6 & 56.6 & 6.3 \\
\hline 94 & 4 & 0 & 11 & 0 & 7 & 1 & 0 & 0 & 0 & 10 & 9.2 & $\mid$ & 8.0 & | & 910 & 11 & 13 & 7 & 16 & 0 & 12 & 7 & 0 & 8 & 9.0 & 醇 & $|8.1|$ & 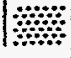 \\
\hline \multicolumn{2}{|c|}{ тот22 } & 18 & 25 & 17 & 17 & 15 & 23 & 21 & 21 & 14 & 19 & & & & Tor 21 & 28 & 25 & 20 & 27 & 18 & 22 & 22 & 28 & 14 & 23 & & & \\
\hline \multirow{3}{*}{$\begin{array}{l}\text { Day } \\
\text { Day }\end{array}$} & \multicolumn{3}{|c|}{ TEM. $\cdot \mathrm{C}$} & \multicolumn{4}{|c|}{ Time } & \multicolumn{4}{|c|}{ TEM. ${ }^{\circ} \mathrm{C}$} & & ime & & & & TEM & $\therefore \mathrm{c}$ & & Time & & & & & TEM. ${ }^{\circ}$ & & & ime \\
\hline & & 25 . & & $02:$ & $45 \mathrm{P}$ & & & Day & 2 & 25.5 & & $03: 15$ & 5 PM & & $\mathrm{Da}$ & y 4 & 25. & & $03:$ & $25 \mathrm{PM}$ & & & Day & 6 & 25.1 & & $11: 45$ & $5 \mathrm{AM}$ \\
\hline & 1 & 24. & & 04: & $20 \mathrm{P}$ & & & Day & 3 & 25.0 & & $02: 15$ & $5 \mathrm{PM}$ & & Da & ay 5 & 25. & & $01:$ & $00 \mathrm{PM}$ & & & Day & 7 & 24.3 & & $04: 30$ & $0 \mathrm{PM}$ \\
\hline
\end{tabular}


SAMPLING INFORMATION

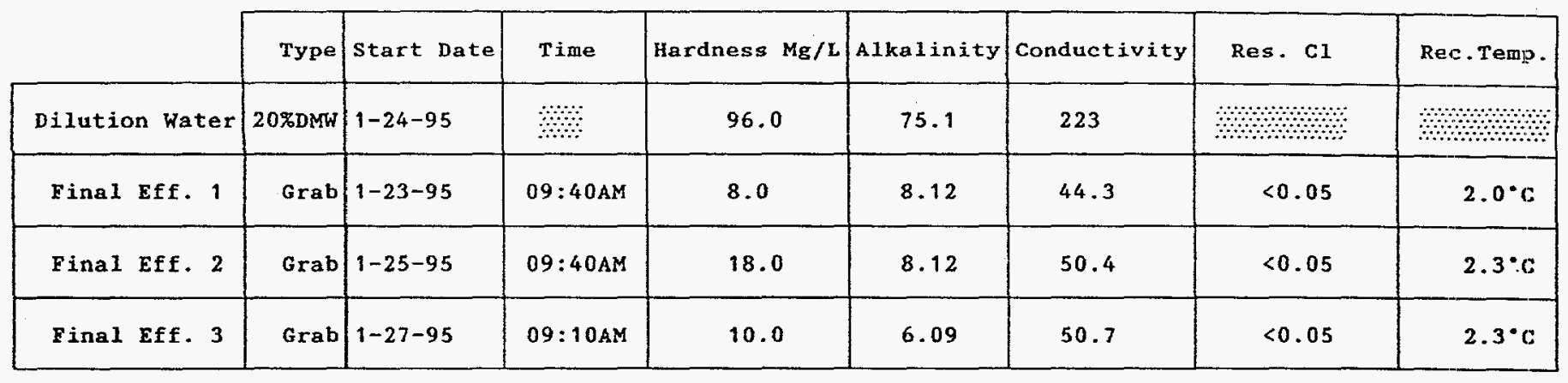


Results

\begin{tabular}{|l|l|l|l|}
\hline \multicolumn{2}{|l|}{ Cient: WSRC } & Sample ID: FMC002H & \\
\hline Log \#: T3496 & Start Date: $1-24-95$ & Time: & $2: 45$ PM \\
\hline
\end{tabular}

SURVIVAL EFFECTS

\begin{tabular}{|c|c|c|c|c|c|c|}
\hline \multirow[b]{2}{*}{ Effluent Conc. } & \multicolumn{2}{|c|}{ Control } & \multicolumn{4}{|c|}{ Effluent } \\
\hline & SC.DMW & $6.25 \%$ & $12.5 \%$ & $25 \%$ & $50 \%$ & $100 \%$ \\
\hline $48 \mathrm{hr}$. Mortality & $10 \%$ & $0 \%$ & $0 \%$ & $0 \%$ & $0 \%$ & $0 \%$ \\
\hline 7 Day Mortality & $10 \%$ & $0 \%$ & $0 \%$ & $10 \%$ & $0 \%$ & $0 \%$ \\
\hline
\end{tabular}

Method: Fishers Exact Test

Acute Toxicity
Control

QUALITY CONTROL

Standard Toxicant: NaC1

Central Tendency:

Current Value:

Deviation:
2.04

1.9

0.58
g/L

g/L

Std Dev units. 
Results

\begin{tabular}{|l|l|l|l|}
\hline \multicolumn{2}{|l|}{ Client: WSRC } & Sample ID: FMC002H & IWC: \\
\hline Log F: T3496 & Start Date: $1-24-95$ & Time: & $2: 45$ PM \\
\hline
\end{tabular}

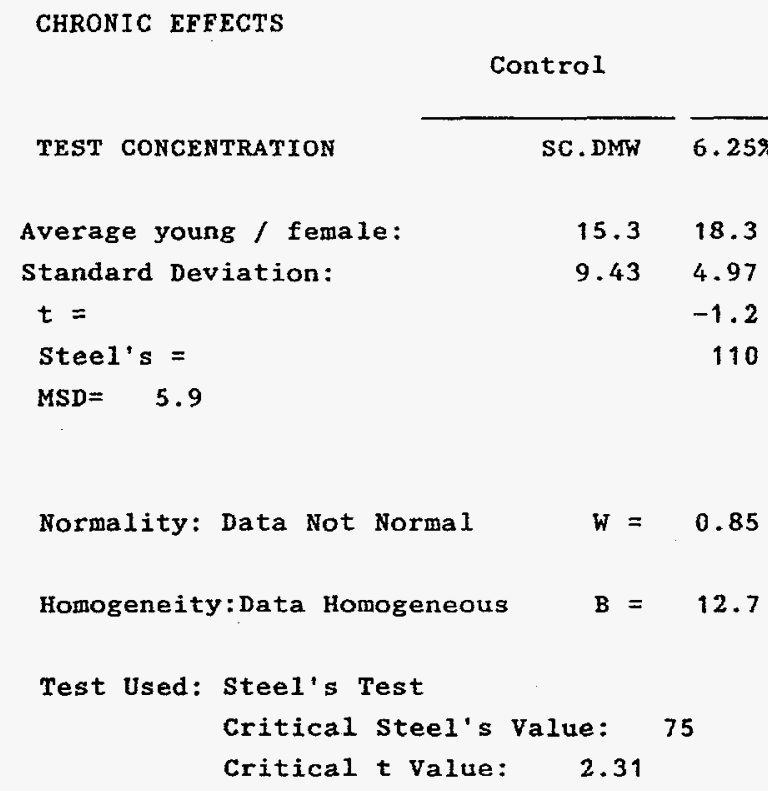


APPENDIX B

WATER TOXICITY RESULTS, AUGUST-SEPTEMBER 1995 
7 Day Chronic Definitive Survival and Reproduction Bioassay

Test Organisms: Ceriodaphnia dubia

Method: EPA/600/4-89/001

Facility = Westinghouse savannah Rivex Company Sample ID: BG OOI EFEluent

Date: $8-17-95$ 
DEFINITIVE SURVIVAL AND REPRODUCTION

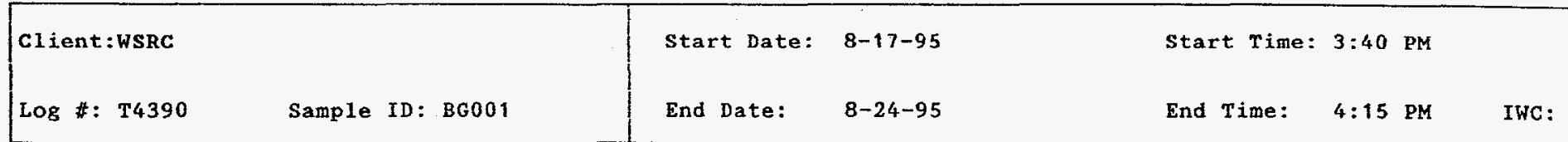

\begin{tabular}{|c|c|c|c|c|c|c|c|c|c|c|c|c|c|c|c|c|c|c|c|c|c|c|c|c|c|c|c|c|}
\hline TEST & \multicolumn{5}{|c|}{ CONCENTRATION: } & \multicolumn{5}{|c|}{$0 \%$} & \multirow{2}{*}{$\left|\begin{array}{l}0.0 \\
01 d\end{array}\right|$} & \multirow{2}{*}{\begin{tabular}{|l|}
0.0 \\
new
\end{tabular}} & \multirow{2}{*}{\begin{tabular}{|c|}
$\mathrm{pH}$ \\
$\mathrm{Old}$ \\
\end{tabular}} & \multirow{2}{*}{\begin{tabular}{|c|}
$\mathrm{pH}$ \\
new
\end{tabular}} & \multicolumn{5}{|c|}{ TEST CONCENTRATION: } & \multicolumn{5}{|c|}{$6.25 \%$} & \multirow{2}{*}{$|0.0|$} & \multirow{2}{*}{$\frac{\text { D.o }}{\text { new }}$} & \multirow{2}{*}{ pH } & \multirow{2}{*}{$\mathrm{pH}_{\text {new }}^{\mathrm{pH}}$} \\
\hline Day A & $\mathbf{A}$ & $\mathbf{B}$ & $\mathbf{c}$ & D & $\mathbf{E}$ & $\mathbf{F}$ & G & $\mathbf{H}$ & I & $\mathbf{J}$ & & & & & Day A & $\mathbf{B}$ & c & D & $\mathbf{E}$ & $\mathbf{F}$ & G & $\mathbf{H}$ & I & $\mathbf{J}$ & & & & \\
\hline 00 & 0 & 0 & 0 & 0 & 0 & 0 & 0 & 0 & 0 & 0 & 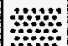 & 9.1 & 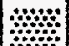 & 7.9 & $0 \quad 0$ & 0 & 0 & 0 & 0 & 0 & 0 & 0 & 0 & 0 & 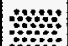 & 8.1 & 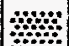 & 7.5 \\
\hline 10 & 0 & 0 & 0 & 0 & 0 & 0 & 0 & 0 & 0 & 0 & 8.8 & 8.9 & 7.9 & 7.9 & 10 & 0 & 0 & 0 & 0 & 0 & 0 & 0 & 0 & 0 & 8.3 & 8.4 & 7.3 & 7.3 \\
\hline 20 & 0 & 0 & 0 & 0 & 0 & 0 & 0 & 0 & 0 & 0 & 8.4 & 8.6 & 7.9 & 7.9 & 20 & 0 & 0 & 0 & 0 & 0 & 0 & 0 & 0 & 0 & 8.7 & 8.8 & 8.1 & 8.2 \\
\hline 30 & 0 & 0 & 0 & 0 & 0 & 0 & 0 & 4 & 0 & 0 & 8.5 & 8.5 & 8.2 & 8.0 & 30 & 0 & 0 & 0 & 0 & 0 & 0 & 0 & 0 & 0 & 8.4 & 8.4 & 7.8 & 7.7 \\
\hline 44 & 4 & 3 & 2 & 3 & 3 & 2 & 4 & 0 & 4 & 3 & 9.2 & 8.7 & 7.7 & 8.0 & 4 & 6 & 6 & 0 & 0 & 0 & 4 & 5 & 3 & 4 & 9.7 & 9.8 & 7.5 & 7.5 \\
\hline 50 & 0 & 0 & 0 & 7 & 0 & 6 & 7 & 8 & 0 & 7 & 8.1 & 8.8 & 7.6 & 8.2 & 5 & 0 & 0 & 5 & 5 & 4 & 9 & 0 & 7 & 6 & 9.4 & 9.6 & 7.7 & 7.6 \\
\hline 69 & 9 & 7 & 9 & 9 & 6 & 9 & 0 & 0 & 6 & 0 & 10 & 9.3 & 8.0 & 8.3 & 6 & 7 & 6 & 7 & 6 & 0 & 0 & 8 & 0 & 0 & 10. & 10. & 7.8 & 7.5 \\
\hline 77 & 7 & 7 & 8 & 0 & 10 & 0 & 10 & 10 & 10 & 11 & 9.2 & 望 & 8.0 & 兹 & $\begin{array}{l}79 \\
7\end{array}$ & 11 & 9 & 9 & 8 & 11 & 8 & 10 & 10 & 10 & 9.2 & $\mid$ & 7.5 & 兹器 \\
\hline TOT20 & & 17 & 19 & 19 & 19 & 17 & 21 & 22 & 20 & 21 & 20 & & & & ToT20 & 24 & 21 & 21 & 19 & 15 & 21 & 23 & 20 & 20 & 20 & & & \\
\hline
\end{tabular}

\begin{tabular}{|c|c|c|c|c|c|c|c|c|c|c|c|}
\hline TEST CONCENTRATION & $12.5 \%$ & D.o & $|\mathbf{b} .0|$ & $\mathrm{pH}$ & $\mathrm{pH}$ & TEST CONGENTRATION & $25 \%$ & D.o & $0.0 \mid$ & $\mathrm{pH}$ & pH \\
\hline
\end{tabular}

\begin{tabular}{|c|c|c|c|c|c|c|c|c|c|c|c|c|c|c|c|c|c|c|c|c|c|c|c|c|c|c|c|c|c|}
\hline Day A & B & C & D & $E$ & $\mathbf{P}$ & G & $\mathbf{H}$ & I & $\mathrm{J}$ & 1010 & ner & 1010 & new & Day & & & & D & $\Sigma$ & $F$ & $G$ & H & I & $\mathrm{J}$ & & $1 d \mid n$ & naw & 1010 & ew \\
\hline
\end{tabular}

\begin{tabular}{|c|c|c|c|c|c|c|c|c|c|c|c|c|c|c|c|c|c|c|c|c|c|c|c|c|c|c|c|}
\hline & & & & & & & & & & & & & & & & & & & & & & & & & & & \\
\hline 0 & 0 & 0 & 0 & 0 & 0 & 0 & 0 & 0 & 0 & 前型 & 8.1 & 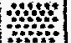 & $|7.3|$ & 00 & 0 & 0 & 0 & 0 & 0 & 0 & 0 & 0 & 0 & 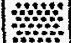 & 8.0 & W & 7.4 \\
\hline 10 & 0 & 0 & 0 & 0 & 0 & 0 & 0 & 0 & 0 & 8.5 & 8.4 & 7.5 & $7.4 \mid$ & 10 & 0 & 0 & 0 & 0 & 0 & 0 & 0 & 0 & 0 & 8.4 & 8.4 & 7.6 & $|7.4|$ \\
\hline 20 & 0 & 0 & 0 & 0 & 0 & 0 & 0 & 0 & 0 & 8.8 & 8.8 & $8.0 \mid$ & 8.2 & 20 & 0 & $x$ & 0 & 0 & 0 & 0 & 0 & 0 & 0 & 8.7 & 8.8 & $|8.0|$ & 8.1 \\
\hline 30 & 0 & 0 & 0 & 0 & 0 & 0 & 4 & 0 & 0 & 8.4 & 8.5 & 7.8 & 7.8 & 30 & 0 & $x$ & 0 & 0 & 0 & 0 & 0 & 0 & 0 & 8.4 & 8.5 & 7.8 & 7.8 \\
\hline 43 & 3 & 4 & 0 & 0 & 0 & 4 & 0 & 4 & 3 & 9.9 & 10 & 7.5 & $|7.7|$ & 43 & 3 & $\mathrm{x}$ & 2 & 0 & 0 & 0 & 0 & 2 & 4 & 9.8 & 10 & 7.6 & 7.6 \\
\hline 50 & 0 & 7 & 4 & 5 & 3 & 6 & 7 & 0 & 7 & 9.6 & 9.6 & $|7.8|$ & $7.6 \mid$ & 57 & 0 & $\mathrm{x}$ & 6 & 6 & 4 & 6 & 8 & 7 & 8 & 9.5 & $9.5 \mid$ & 7.8 & 7.4 \\
\hline 68 & 8 & 0 & 6 & 6 & 3 & 0 & 0 & 6 & 0 & 10 & 10 & 7.8 & 7.4 & 60 & 6 & $x$ & 7 & 6 & 6 & 0 & 0 & 0 & 0 & 10 & 10 & 7.6 & 7.2 \\
\hline 76 & 9 & 11 & 10 & 0 & 6 & 12 & 10 & 7 & 10 & 9.4 & 娄 & $|7.5|$ & h & 714 & 10 & $x$ & 9 & 11 & 8 & 9 & 13 & 9 & 7 & 9.4 & & $7.5 \mid$ & 兹率 \\
\hline тот17 & 20 & 22 & 20 & 11 & 12 & 22 & 21 & 17 & 20 & 18 & & & & Tот24 & 19 & 0 & 24 & 23 & 18 & 15 & 21 & 18 & 19 & 18 & & & \\
\hline
\end{tabular}

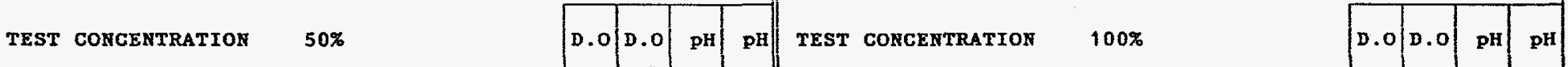

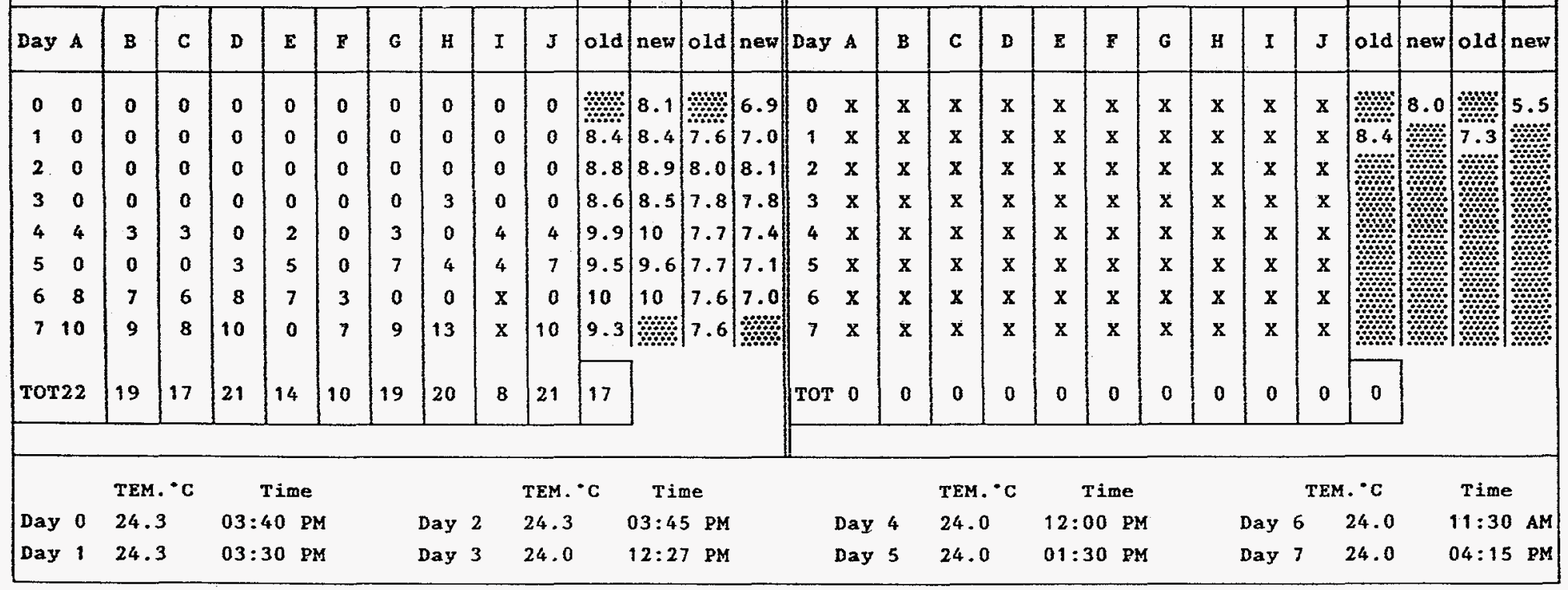


SAMPLING INFORMATION

\begin{tabular}{|c|c|c|c|c|c|c|c|c|}
\hline Dilution Water & $20 \% \mathrm{DMW}$ & $8-16-95$ & H & 84.0 & 66.9 & 220 & i & \\
\hline Final Eff. 1 & Comp & $8-16-95$ & $7: 10 \mathrm{AM}$ & 6.0 & 2.03 & 10 & $<0.05$ & $0.8^{\circ} \mathrm{C}$ \\
\hline Final Eff. 2 & Comp & $8-18-95$ & $8: 50 \mathrm{AM}$ & 8.0 & 2.04 & 11.5 & $<0.05$ & $0.4^{\circ} \mathrm{C}$ \\
\hline Final Eff. 3 & Comp & $8-21-95$ & $7: 30 \mathrm{AM}$ & 10.0 & $<0.1$ & 13.5 & $<0.05$ & $0.7^{\circ} \mathrm{C}$ \\
\hline
\end{tabular}


Results

\begin{tabular}{|l|l|l|l|}
\hline Client: WSRC & Sample ID: BG001 & \\
\hline Log \#: T4390 & Start Date: $8-17-95$ & Time: & $3: 40$ PM \\
\hline
\end{tabular}

SURVIVAL EFFECTS

Control Effluent

\begin{tabular}{llllllll}
\hline Effluent Conc. & SC.DMW & $6.25 \%$ & $12.5 \%$ & $25 \%$ & $50 \%$ & $100 \%$
\end{tabular}

$48 \mathrm{hr}$. Mortality

$0 \% \quad 0 \% \quad 0 \% \quad 10 \% \quad 0 \% \quad 100 \%$

$\begin{array}{lllllll}7 \text { Day Mortality } & 0 \% & 0 \% & 0 \% & 10 \% & 10 \% & 100 \%\end{array}$

Method: Fishers Exact Test

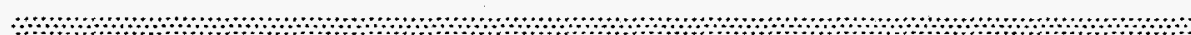

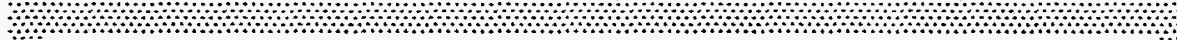

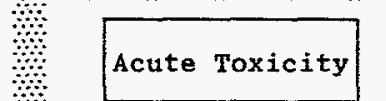

Control

Effluent

Acute Toxicity

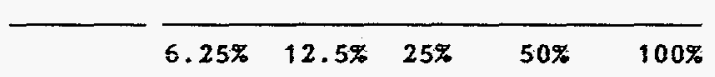

$48 \mathrm{Hr}$. Acute Results:

PASS

PASS

PASS PASS FAIL

PASS PASS FAIL

No-Observed-Effect Concentration (NOEC):

$50 \%$

Lowest-Observed-Effect Concentration (LOEC) :

$100 \%$

7 Day LC50:

$61.56 \%$

QUALITY CONTROL

\begin{tabular}{|ccc|}
\hline Standard Toxicant: & & \\
& & \\
Central Tendency: & 1.98 & $\mathrm{~g} / \mathrm{L}$ \\
Current Value: & 1.91 & $\mathrm{~g} / \mathrm{L}$ \\
Deviation: & \multicolumn{2}{c|}{ Std Dev units. } \\
\hline
\end{tabular}


Results

\begin{tabular}{|l|l|l|l|}
\hline \multicolumn{2}{|l|}{ Client: WSRC } & Sample ID: BG00t & IWC: \\
\hline Log \#: T4390 & Start Date: $8-17-95$ & Time: & $3: 40$ PM \\
\hline
\end{tabular}

\section{CHRONIC EFFECTS}

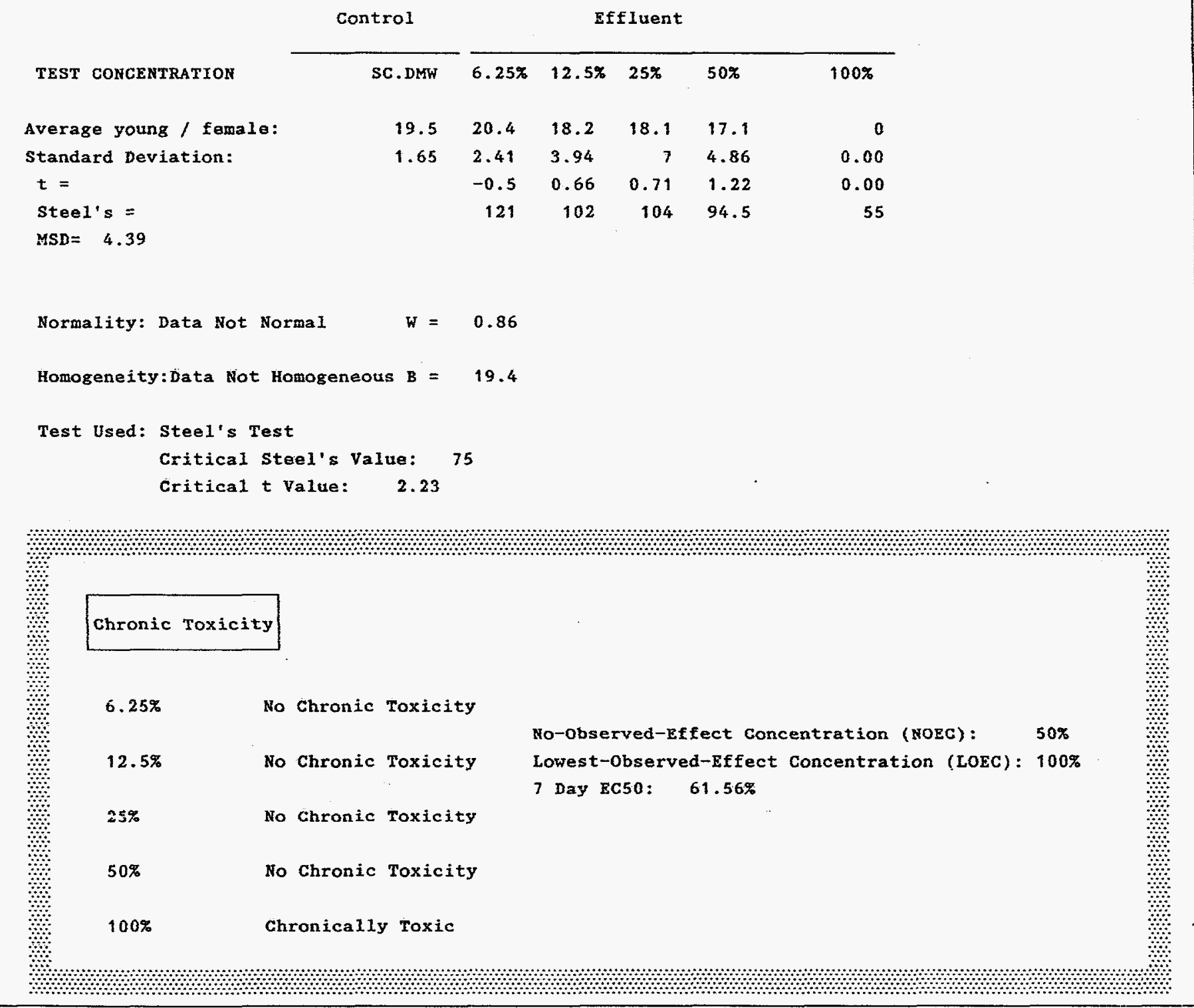


7 Day Chronic Definitive Survival and Reproduction Bioassay

Test Organisms: Ceriodaphnia dubia

Method: EPA/600/4-89/001

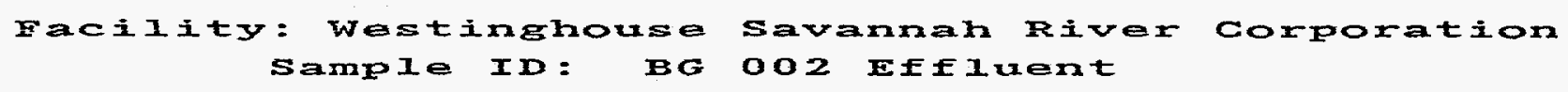

Date: $8-17-95$ 
DEFINITIVE SURVIVAL AND REPRODUCTION

\begin{tabular}{|c|c|c|c|c|c|c|c|c|c|c|c|c|c|c|c|c|c|c|c|c|c|c|c|c|c|c|c|c|}
\hline \multicolumn{10}{|c|}{$\begin{array}{l}\text { Client: WSRC } \\
\text { Log \#: T4391 }\end{array}$} & \multicolumn{4}{|c|}{$\begin{array}{l}\text { Start Date: } \\
\text { End Date: }\end{array}$} & \multicolumn{6}{|c|}{$\begin{array}{l}8-17-95 \\
8-24-95\end{array}$} & \multicolumn{8}{|c|}{ Start Time: $4: 00 \mathrm{PM}$} & \\
\hline TEST & \multicolumn{4}{|c|}{ CONCENTRATION : } & \multicolumn{5}{|c|}{$0 \%$} & D.o 1 & 0.0 & $\mathrm{pH}$ & $\mathrm{pH}$ & \multicolumn{6}{|c|}{ TEST CONCENTRATION: } & \multicolumn{5}{|c|}{$6.25 \%$} & D.o & D.o & $\mathrm{pH}$ & $\mathrm{pH}$ \\
\hline Day A & B & c & $D$ & $\mathbf{E}$ & $\mathbf{F}$ & G & $\mathbf{H}$ & $\mathbf{I}$ & $\mathbf{J}$ & old & new & old & new & Day A & & $\mathbf{B}$ & $\mathrm{c}$ & D & $\mathbf{E}$ & $\boldsymbol{F}$ & G & $\mathbf{H}$ & $\mathbf{I}$ & $J$ & old & пеш & old & new \\
\hline 00 & 0 & 0 & 0 & 0 & 0 & 0 & 0 & 0 & D & \% & 9.1 & $\ldots$ & 7.9 & 00 & & 0 & 0 & 0 & 0 & 0 & 0 & 0 & 0 & 0 & 垍: & $8.1:$ & 橭: & 7.3 \\
\hline 10 & 0 & 0 & 0 & 0 & 0 & 0 & $\mathbf{0}$ & 0 & 0 & 8.8 & 8.9 & 7.9 & 7.9 & 10 & 0 & 0 & 0 & 0 & 0 & 0 & 0 & 0 & 0 & 0 & 8.0 & 8.2 & 7.4 & 7.4 \\
\hline 20 & 0 & 0 & 0 & 0 & 0 & 0 & 0 & 0 & 0 & 8.48 & 8.6 & 7.9 & 7.9 & 20 & 0 & 0 & 0 & 0 & 0 & 0 & 0 & 0 & 0 & 0 & 8.6 & 8.6 & 8.0 & 8.0 \\
\hline 30 & 0 & 0 & 0 & 0 & 0 & 0 & 2 & 0 & 0 & $8.5 \mid 8$ & 8.5 & 8.2 & 8.0 & 30 & 0 & 0 & 0 & 0 & 0 & 0 & 0 & 2 & 0 & 0 & 8.6 & 8.6 & 7.9 & 7.6 \\
\hline 43 & 3 & 3 & 2 & 3 & 2 & 4 & 3 & 4 & 3 & $|9.2|=$ & 8.7 & 7.7 & 8.0 & 40 & 0 & 0 & 0 & 0 & 0 & 0 & 0 & 0 & 0 & 0 & 9.8 & 9.7 & $7.7 \mid$ & 6.9 \\
\hline 56 & 3 & 2 & 2 & 0 & 6 & 0 & 7 & 8 & 0 & $8.1 \mid 8$ & 8.8 & 7.6 & 8.2 & $5 \quad 5$ & 5 & 4 & 3 & 4 & 1 & 4 & 5 & 5 & 4 & 4 & 9.6 & 9.5 & 7.5 & 7.2 \\
\hline 69 & 6 & 8 & 4 & 5 & 0 & 6 & 0 & 0 & 5 & 10 & 9.3 & 8.0 & 8.3 & 67 & 7 & 0 & 0 & 0 & 10 & 0 & 0 & 0 & 0 & 0 & 10 & 10 & 7.5 & 7.4 \\
\hline 70 & 9 & 7 & 11 & 10 & 9 & 9 & 10 & 9 & 9 & $|9.2|$ & 前 & 8.0 & ma & 75 & 5 & 7 & 7 & 10 & 3 & 7 & 9 & 7 & 8 & 7 & 9.3 & $\mid$ & 7.5 & 要 \\
\hline тот18 & 21 & 20 & 19 & 18 & 17 & 19 & 22 & 21 & 17 & 19 & & & & \multicolumn{2}{|c|}{ тот17 } & 11 & 10 & 14 & 14 & 11 & 14 & 14 & 12 & 11 & 13 & & & \\
\hline \multicolumn{5}{|c|}{ TEST CONCENTRATION } & 12.5 & & & & & D.o 1 & D.o & $\mathrm{pH}$ & $\mathrm{pH}$ & \multicolumn{6}{|c|}{ TEST CONCENTRATION } & \multicolumn{5}{|l|}{$25 \%$} & 0.0 & D.o & $\mathrm{pH}$ & $\mathbf{p H}$ \\
\hline Day A & $\mathbf{B}$ & c & $\mathbf{D}$ & $\mathbf{E}$ & $\mathbf{F}$ & $G$ & H & $\mathbf{I}$ & $\mathbf{J}$ & old & new & old & new & Day A & & B & c & $\mathbf{D}$ & 5 & F & $\mathbf{G}$ & H & I & $\mathbf{J}$ & old & new & old & new \\
\hline $0 \quad 0$ & 0 & 0 & 0 & 0 & 0 & 0 & 0 & 0 & 0 & 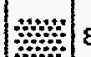 & 8.1 & 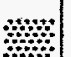 & 7.1 & 00 & 0 & 0 & 0 & 0 & 0 & 0 & 0 & 0 & 0 & 0 & 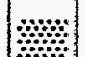 & 8.1 & 垍垍 & 7.2 \\
\hline 10 & 0 & 0 & 0 & 0 & 0 & 0 & 0 & 0 & 0 & $8.2 \mid$ & 8.4 & 7.5 & 7.2 & 10 & 0 & 0 & 0 & 0 & 0 & 0 & 0 & 0 & 0 & 0 & 8.0 & 8.0 & 7.5 & 7.2 \\
\hline 20 & 0 & 0 & 0 & 0 & 0 & 0 & 0 & 0 & 0 & 8.8 & 8.8 & 8.0 & 8.1 & 20 & 0 & 0 & 0 & 0 & 0 & 0 & 0 & 0 & 0 & 0 & 8.7 & 8.8 & 8.0 & 8.1 \\
\hline 30 & 0 & 0 & 0 & 0 & 0 & 0 & 0 & 0 & 0 & 8.6 & 8.6 & 7.9 & 7.6 & 30 & 0 & 0 & 0 & 0 & 0 & 0 & 0 & 0 & 0 & 0 & 8.4 & 8.5 & 7.9 & 7.5 \\
\hline 40 & 0 & 0 & 1 & 1 & 0 & 0 & 0 & 0 & 0 & $9.8 \mid$ & 9.8 & 7.6 & 7.1 & 40 & 0 & 0 & 0 & 0 & 2 & 0 & 1 & 1 & 4 & 0 & 9.8 & 9.7 & $9.5 \mid$ & 6.9 \\
\hline $\begin{array}{ll}5 & 2\end{array}$ & 4 & 3 & 4 & 0 & 2 & 3 & 0 & 0 & 0 & 9.4 & 9.6 & 7.7 & 7.4 & 56 & 6 & 3 & 2 & 2 & 0 & 3 & 2 & 5 & 4 & 0 & 9.5 & 9.5 & 9.7 & 7.3 \\
\hline 60 & 6 & 0 & 0 & 3 & 0 & 0 & 6 & 3 & 3 & 10 & 10 & 7.7 & 7.5 & 60 & 0 & 5 & 0 & 0 & 4 & 0 & 0 & 0 & 0 & 5 & 10 & 10 & 7.8 & 7.5 \\
\hline 75 & 0 & 7 & 9 & 5 & 6 & 5 & 5 & 7 & 5 & $9.4:$ & $\mid$ & 7.5 & 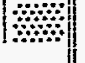 & 73 & 3 & 0 & 4 & 4 & 0 & 5 & 5 & 7 & 8 & 7 & 9.4 & $\mid$ & $|7,3|$ & 前 \\
\hline TOT 7 & 10 & 10 & 14 & 9 & 8 & 8 & 11 & 10 & 8 & 10 & & & & ToT 9 & 9 & 8 & 6 & 6 & 6 & 8 & 8 & 13 & 16 & 12 & 9 & & & \\
\hline \multicolumn{5}{|l|}{ TEST } & $50 \%$ & & & & & 0.0 & 0.0 & $\mathrm{pH}$ & $\mathrm{pH}$ & \multicolumn{11}{|c|}{ TEST CONCENTRATION } & 0.0 & 0.0 & $\mathrm{pH}$ & $\mathrm{pH}$ \\
\hline Day A & B & $\mathrm{c}$ & $D$ & s & $\mathbf{F}$ & G & H & $\mathbf{I}$ & $\mathbf{J}$ & old & new & old & new & Day A & A & $\mathbf{B}$ & C & $\mathbf{D}$ & E & $\mathbf{F}$ & $\mathbf{G}$ & H & $\mathbf{I}$ & $J$ & $01 d$ & new & old & new \\
\hline 00 & 0 & 0 & 0 & 0 & 0 & 0 & 0 & 0 & 0 & 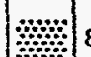 & 8.1 & ․․․․․․․ & 6.8 & $0 x$ & $\mathrm{x}$ & $\mathrm{x}$ & $\mathbf{x}$ & $\mathbf{x}$ & $\mathbf{x}$ & $\mathbf{x}$ & $x$ & $\mathrm{x}$ & $\mathrm{x}$ & $\mathbf{x}$ & 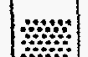 & 8.1 & \% & 5.0 \\
\hline 10 & 0 & 0 & 0 & 0 & 0 & 0 & 0 & 0 & 0 & 8.4 & 8.4 & 7.6 & 7.0 & $1 x$ & $x$ & $x$ & $x$ & $\mathrm{x}$ & $x$ & $\mathbf{x}$ & $\mathrm{x}$ & $x$ & $\mathrm{x}$ & $x$ & 8.0 & 前获 & 6.4 & 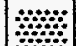 \\
\hline 20 & 0 & 0 & 0 & 0 & 0 & 0 & 0 & 0 & 0 & 8.6 & 8.6 & 7.9 & $8.0 \mid$ & $2 x$ & $x$ & $x$ & $\mathbf{x}$ & $\mathbf{x}$ & $x$ & $x$ & $\mathbf{x}$ & $x$ & $x$ & $x$ & 垍 & $1 \%$ & 前 & \% \\
\hline 30 & 0 & 0 & 0 & 0 & 0 & 0 & 0 & 0 & 0 & 8.4 & 8.4 & 7.8 & 7.1 & $3 x$ & $\mathrm{x}$ & $\mathbf{x}$ & $x$ & $x$ & $\mathbf{x}$ & $\mathrm{x}$ & $x$ & $\mathrm{x}$ & $\mathbf{x}$ & $x$ & 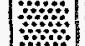 & 染器 & \% & 栗 \\
\hline 40 & 0 & 0 & 0 & 2 & 0 & 1 & 1 & 4 & 0 & 10 & 10 & 7.1 & 6.5 & $4 x$ & $\mathrm{x}$ & $\mathbf{x}$ & $\mathbf{x}$ & $x$ & $\mathbf{x}$ & $x$ & $\mathbf{x}$ & $x$ & $x$ & $x$ & 票 & 器 & | & 前 \\
\hline 54 & 3 & 3 & 2 & 0 & 2 & 5 & 6 & 5 & 0 & 9.3 & 9.5 & 7.8 & 7.1 & 5 & $\mathrm{x}$ & $\mathrm{x}$ & $\mathbf{x}$ & $\mathrm{x}$ & $\mathrm{x}$ & $x$ & $x$ & $x$ & $\mathbf{x}$ & $x$ & 慗 & 臸 & $\mid$ & \% \\
\hline 66 & 0 & 0 & 0 & 5 & 0 & 0 & 0 & $\mathbf{x}$ & 3 & 10 & 10 & 7.8 & 7.2 & $6 x$ & $x$ & $\mathbf{x}$ & $\mathbf{x}$ & $\mathbf{x}$ & $\mathbf{x}$ & $x$ & $x$ & $x$ & $\mathbf{x}$ & $x$ & \% & $\mid$ & 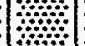 & G \\
\hline 70 & 6 & 5 & 3 & 6 & 4 & 9 & 0 & $x$ & 4 & 9.3 & $\mid$ & $|7.2|$ & 前 & $7 x$ & $\mathrm{x}$ & $\mathbf{x}$ & $x$ & $x$ & $x$ & $\mathrm{x}$ & $\mathbf{x}$ & $x$ & $\mathrm{x}$ & $\mathrm{x}$ & 政 & \% & : & $\mid$ \\
\hline тот10 & 9 & 8 & 5 & 13 & 6 & 15 & 7 & 9 & 7 & 9 & & & & TOT & 0 & 0 & 0 & 0 & 0 & 0 & 0 & 0 & 0 & 0 & 0 & & & \\
\hline & TEM & ${ }^{\circ} \cdot{ }^{\circ} \mathrm{C}$ & & Time & & & & & TEM. & ${ }^{\circ} \mathrm{C}$ & Tim & & & & & & TEM. & $\cdot \mathrm{C}$ & & Time & & & & TEM & $1 .{ }^{\circ} \mathrm{C}$ & & Time & \\
\hline Day 0 & 24 & & $03:$ & $40 \mathrm{P}$ & & & Day & 2 & 24.3 & & $03: 45$ & $P M$ & & & bay & 4 & 24.0 & & $12: 0$ & $00 \mathrm{PM}$ & & & Day 6 & 6 & 24.0 & & $11: 30$ & 0 AM \\
\hline Day 1 & 24. & & $03:$ & $30 \mathrm{P}$ & & & Day & 3 & 24.0 & & $12: 27$ & $\mathrm{PM}$ & & & aý & 5 & 24.0 & & $01: 3$ & $30 \mathrm{PM}$ & & & Day & 7 & 24.0 & & $04: 15$ & $5 \mathrm{PM}$ \\
\hline
\end{tabular}


SAMPLING INFORMATION

\begin{tabular}{|c|c|c|c|c|c|c|c|c|}
\hline Dilution Water & 20\%DMW & $8-16-95$ & m & 84.0 & 66.9 & 220 & 然 & \\
\hline Final Eff. 1 & Comp & $8-16-95$ & $7: 20 \mathrm{AM}$ & 6.0 & $<0.1$ & 15 & $<0.05$ & $0.8^{\circ} \mathrm{C}$ \\
\hline Final Eff. 3 & Comp & $8-21-95$ & $7: 40 \mathrm{AM}$ & 16.0 & $<0.1$ & 25.7 & $<0.05$ & $0.7^{\circ} \mathrm{C}$ \\
\hline
\end{tabular}


Results

\begin{tabular}{|l|l|l|l|}
\hline \multicolumn{2}{|c|}{} & Sample ID: BG002 & \\
\hline Log \#: T4391 & Start Date: $8-17-95$ & Time: & $4: 00$ PM \\
\hline
\end{tabular}

SURVIVAL EFFECTS

Control Effluent

$\begin{array}{lllllll}\text { Effluent Conc. } & \text { SC.DMW } & 6.25 \% & 12.5 \% & 25 \% & 50 \% & 100 \%\end{array}$

\begin{tabular}{lllllll}
$48 \mathrm{hr}$. Mortality & $0 \%$ & $0 \%$ & $0 \%$ & $0 \%$ & $0 \%$ & $100 \%$ \\
7 Day Mortality & $0 \%$ & $0 \%$ & $0 \%$ & $0 \%$ & $10 \%$ & $100 \%$ \\
\hline
\end{tabular}

Method: Fishers Exact Test

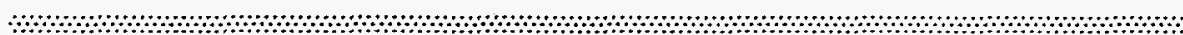

Acute Toxicity

QUALITY CONTROL

\begin{tabular}{|cccc|}
\hline Standard Toxicant: Nac1 & & \\
& & & \\
Central Tendency: & 1.98 & $\mathrm{~g} / \mathrm{L}$ \\
Current Value: & 1.91 & $\mathrm{~g} / \mathrm{L}$ \\
Deviation: & 0.29 & & Std Dev units. \\
\hline
\end{tabular}


Results

\begin{tabular}{|l|l|l|l|}
\hline \multicolumn{2}{|l|}{ Client: WSRC } & Sample ID: BG002 & IWC: \\
\hline Log \#: T4391 & Start Date: $8-17-95$ & Time: & $4: 00$ PM \\
\hline
\end{tabular}

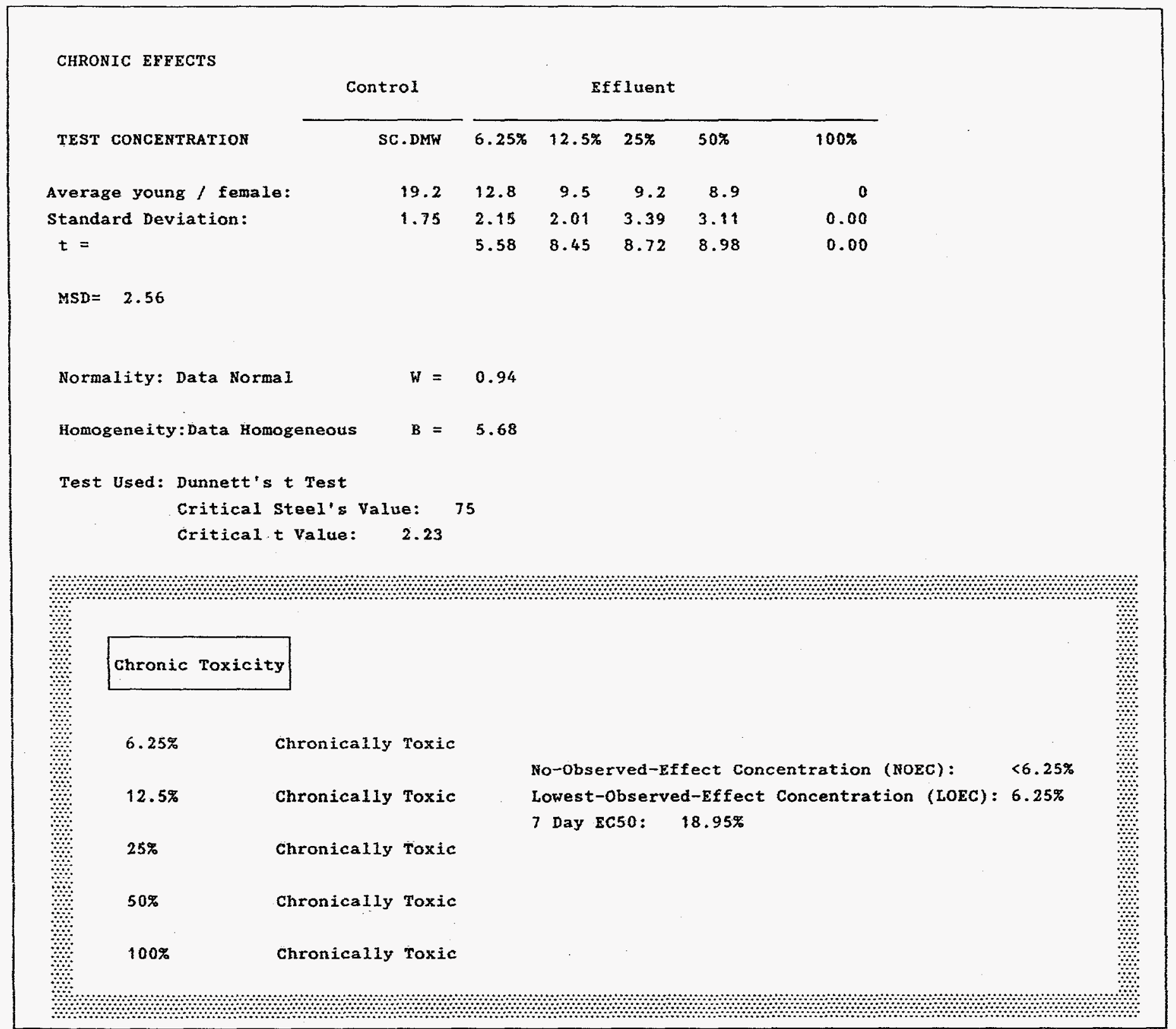


P.O. Box 16414, Greenville, SC 29606 : 4 Craftsman Court, Greer, SC 29650

7 Day Chronic Definitive Survival and Reproduction Bioassay

Test Organisms: Ceriodaphnia dubia

Method: EPA/600/4-89/001

\footnotetext{
Facility :
}

Westinghouse

Savannah

River

Corporation

Sample ID =

BG 003

Date: $8-17-95$ 
DEFINITIVE SURVIVAL AND REPRODUCTION

\begin{tabular}{|c|c|c|c|c|c|c|c|c|c|c|c|c|c|c|c|c|c|c|c|c|c|c|c|c|c|c|c|}
\hline \multicolumn{10}{|c|}{$\begin{array}{l}\text { Client:WSRC } \\
\text { Log \#: T4392 }\end{array}$} & \multicolumn{4}{|c|}{$\begin{array}{l}\text { Start Date: } \\
\text { End Date: }\end{array}$} & \multicolumn{5}{|c|}{$\begin{array}{l}8-17-95 \\
8-24-95\end{array}$} & \multicolumn{9}{|c|}{ Start Time: $4: 00 \mathrm{PM}$} \\
\hline \multicolumn{5}{|c|}{ TEST CONCENTRATION: } & \multicolumn{2}{|l|}{$0 \%$} & & & & D.o 1 & 0.0 & $\mathrm{pH}$ & $\mathbf{p H}$ & \multicolumn{5}{|c|}{ TEST CONCENTRATION: } & \multicolumn{5}{|c|}{$6.25 \%$} & 0.0 & D.o & $\mathbf{p H}$ & $\mathrm{pH}$ \\
\hline Day A & B & c & $\mathrm{D}$ & B & $\mathbf{F}$ & G & H & $I$ & $\mathrm{~J}$ & old & new & old & new & Day A & $\mathfrak{B}$ & c & $\mathrm{D}$ & $\mathbf{E}$ & $F$ & G & H & I & $\mathbf{J}$ & $01 d$ & new & old & new \\
\hline 00 & 0 & 0 & 0 & 0 & 0 & 0 & 0 & 0 & 0 & \% & 9.1 & 前 & 7.9 & 00 & 0 & 0 & 0 & 0 & 0 & 0 & 0 & 0 & 0 & 象 & 8.1 & \% & 7.7 \\
\hline 10 & $\mathbf{0}$ & 0 & 0 & 0 & o & 0 & 0 & 0 & 0 & 8.8 & 8.9 & 7.9 & 7.9 & 10 & 0 & 0 & 0 & 0 & 0 & 0 & 0 & 0 & 0 & 8.4 & 8.4 & 7.4 & 7.5 \\
\hline 20 & 0 & 0 & 0 & 0 & 0 & 0 & 0 & 0 & 0 & 8.4 & 8.6 & 7.9 & 7.9 & 20 & 0 & 0 & 0 & 0 & 0 & 0 & 0 & 0 & 0 & 8.6 & 8.7 & 7.9 & 8.0 \\
\hline 30 & 3 & 0 & 0 & 0 & 0 & 0 & 0 & 0 & 0 & 8.5 & 8.5 & 8.2 & 8.0 & 30 & 0 & 0 & 0 & 0 & 0 & 0 & 0 & 0 & 0 & 8.5 & 8.6 & 7.8 & 7.6 \\
\hline 44 & 6 & 2 & 3 & 3 & 5 & 3 & 3 & 2 & 3 & 9.2 & 8.7 & 7.7 & 8.0 & 42 & 3 & 1 & 3 & 0 & 0 & 0 & 3 & 0 & 0 & $9.9 \mid$ & 9.9 & 7.4 & 7.1 \\
\hline 50 & 0 & 6 & 6 & 3 & 0 & 0 & 0 & 0 & 4 & 8.1 & 8.8 & 7.6 & 8.2 & 57 & 6 & 2 & 8 & 1 & 2 & 3 & 0 & 4 & 4 & 9.4 & 9.4 & 7.8 & 7.7 \\
\hline 68 & 0 & 0 & 0 & 0 & 6 & 8 & 5 & 5 & 0 & 10 & 9.3 & 8.0 & 8.3 & 60 & 0 & 5 & 0 & 5 & 0 & 0 & 9 & 0 & 0 & 10. & 10. & 7.6 & 7.6 \\
\hline 710 & 9 & 8 & 9 & 11 & 9 & 9 & 8 & 10 & 11 & $9.21:$ & 前 & $|8.0|$ & | & 78 & 8 & 9 & 10 & 10 & 8 & 8 & 9 & 6 & 10 & $|9.2|$ & $|9.2|$ & $|7.6|$ & $\mid$ \\
\hline Tor 22 & 18 & 16 & 18 & 17 & 20 & 20 & 16 & 17 & 18 & 18 & & & & TOF17 & 17 & 17 & 21 & 16 & 10 & 11 & 21 & 10 & 14 & 15 & & & \\
\hline TEST & CONCE & NTRA & ATION & & 12.5 & & & & & 0.0 & 0.0 & $\mathbf{p H}$ & $\mathrm{pH}$ & \multicolumn{5}{|c|}{ TEST CONCENTRATION } & \multicolumn{5}{|l|}{$25 \%$} & D.o & 0.0 & $\mathrm{pH}$ & $\mathrm{pH}$ \\
\hline Day A & B & $\mathrm{C}$ & D & $\mathbf{E}$ & $\mathbf{F}$ & G & H & $I$ & $\mathbf{J}$ & old & new & old & new & Day A & $\mathbf{B}$ & c & $\mathrm{b}$ & $\mathbf{E}$ & F & G & H & I & $J$ & old. & new & old & new \\
\hline 00 & 0 & 0 & 0 & 0 & 0 & 0 & 0 & 0 & 0 & $\mid$ & 8.2 & W... & 7.5 & 00 & 0 & 0 & 0 & 0 & 0 & 0 & 0 & 0 & 0 & 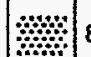 & 8.2 : & Ha & 7.5 \\
\hline 10 & 0 & 0 & 0 & 0 & 0 & 0 & 0 & 0 & 0 & $8.3 \mid$ & 8.2 & 7.5 & 7.6 & 10 & 0 & 0 & 0 & 0 & 0 & 0 & 0 & 0 & 0 & $8.3 \mid$ & 8.4 & 7.4 & 7.6 \\
\hline 20 & 0 & 0 & 0 & 0 & 0 & 0 & 0 & 0 & 0 & 8.6 & 8.7 & 8. & 8.0 & 20 & 0 & 0 & 0 & 0 & 0 & 0 & 0 & 0 & 0 & 8.6 & 8.7 & 7.9 & 8.0 \\
\hline 30 & 3 & 0 & 0 & 0 & 0 & 0 & 0 & 0 & 0 & 8.4 & 8.5 & 7.8 & 7.7 & $\begin{array}{ll}30 \\
0\end{array}$ & 0 & 0 & 0 & 0 & 0 & 0 & 0 & 0 & 0 & 8.4 & 8.6 & 7.8 & 7.7 \\
\hline 44 & 0 & 0 & 2 & 3 & 2 & 0 & 0 & 0 & 0 & 9.9 & 10. & 7.6 & 7.5 & 43 & 4 & 3 & 3 & 3 & 3 & 2 & 3 & 3 & 3 & $9.9 \mid$ & 9.9 & 7.7 & 7.5 \\
\hline 56 & 6 & 0 & 6 & 0 & 0 & 4 & 2 & 3 & 4 & 9.5 & 9.4 & 7.8 & $7.7 \mid$ & 56 & 6 & 3 & 8 & 7 & 0 & 0 & 0 & 1 & 0 & 9.5 & 9.5 .7 & 7.8 & 7.6 \\
\hline 60 & 0 & 3 & 0 & 8 & 8 & 0 & 0 & 0 & 0 & 10. & 10. & 7.6 & 7.6 & $\begin{array}{ll}6 & 0\end{array}$ & 0 & 0 & 0 & 0 & 5 & 8 & 7 & 8 & 6 & 10. & 10 & 7.7 & 7.6 \\
\hline 76 & 0 & 0 & 6 & 9 & 10 & 6 & 8 & 7 & 6 & $|9.3|$ & 翟 & $|7.6|$ & m & 711 & 0 & 0 & 5 & 5 & 5 & 8 & 9 & 6 & 1 & $9.3:$ & 垍 & 7.7 & 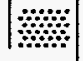 \\
\hline тот16 & 9 & 3 & 14 & 20 & 20 & 10 & 10 & 10 & 10 & 12 & & & & тот 20 & 10 & 6 & 16 & 15 & 13 & 18 & 19 & 18 & 10 & 15 & & & \\
\hline \multicolumn{5}{|c|}{ TEST CONCENTRATION } & $50 \%$ & & & & & D.o & D.o & $\mathrm{pH}$ & $\mathbf{p H}$ & \multicolumn{10}{|c|}{ TEST CONCENTRATION } & 0.015 & 0.0 & $\mathrm{pH}$ & $\mathrm{pH}$ \\
\hline Day A & $\mathbf{B}$ & c & $\mathfrak{D}$ & $\mathbf{E}$ & $F$ & G & $\mathbf{H}$ & $I$ & $\mathbf{J}$ & old & new & $01 d$ & new & Day A & $\mathbf{B}$ & c & $\mathbf{D}$ & $\mathbf{E}$ & $\mathbf{F}$ & G & H & I & $\mathfrak{J}$ & old $n$ & new & old & new \\
\hline 00 & 0 & 0 & 0 & 0 & 0 & 0 & 0 & 0 & 0 & $\mid$ & 8.2 & 前 & 7.2 & 00 & 0 & 0 & 0 & 0 & 0 & 0 & 0 & 0 & 0 & 器 & $8.2:$ & 前 & 7.6 \\
\hline 10 & 0 & 0 & 0 & 0 & 0 & 0 & 0 & 0 & 0 & $8.3 \mid$ & 8.3 & 6.9 & 7.7 & 10 & 0 & 0 & 0 & 0 & 0 & 0 & 0 & 0 & 0 & 8.4 & 8.3 & 6.2 & 7.4 \\
\hline 20 & 0 & 0 & $\mathrm{x}$ & 0 & 0 & $x$ & 0 & 0 & 0 & 8.6 & 8.6 & 7.9 & $7.9 \mid$ & 20 & 0 & 0 & $\mathbf{x}$ & $x$ & 0 & $\mathrm{x}$ & 0 & 0 & $\mathrm{x}$ & $8.5 \mid$ & $8.6 \mid$ & 7.5 & 7.6 \\
\hline 30 & 0 & 0 & $x$ & 0 & 0 & $\mathbf{x}$ & 0 & 0 & 0 & 8.4 & 8.6 & 7.8 & 7.6 & 30 & 2 & 0 & $\mathrm{x}$ & $\mathrm{x}$ & 0 & $x$ & 0 & 0 & $\mathrm{x}$ & 8.4 & 8.4 & 7.3 & 6.9 \\
\hline 44 & 3 & 0 & $\mathrm{x}$ & 3 & 0 & $\mathbf{x}$ & 3 & 0 & 0 & 10 & 10. & 7.7 & 7.5 & 40 & 0 & 0 & $\mathrm{x}$ & $x$ & 0 & $x$ & 0 & 0 & $x$ & 9.9 & 10 & 7.7 & 7.2 \\
\hline 57 & 0 & 1 & $x$ & 0 & 0 & $\mathrm{x}$ & 0 & 6 & 0 & 9.4 & 9.5 & 7.8 & 7.5 & 50 & 3 & 3 & $\mathrm{x}$ & $x$ & 0 & $\mathrm{X}$ & 0 & 3 & $x$ & 9.4 & 9.5 & 7.7 & 7.2 \\
\hline 60 & 5 & 2 & $\mathbf{x}$ & 5 & 2 & $\mathbf{x}$ & 6 & 7 & 6 & 10. & 10. & 7.7 & 7.5 & 60 & 0 & 0 & $\mathrm{x}$ & $\mathrm{x}$ & 5 & $\mathbf{x}$ & 1 & 0 & $x$ & 10 & 10 & 7.2 & 6.9 \\
\hline 710 & 8 & 4 & $x$ & 0 & 3 & $\mathbf{x}$ & 8 & 0 & 6 & $9.3 \mid$ & 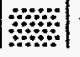 & $|7.6|$ & 前 & 73 & 0 & 3 & $\mathrm{x}$ & $\mathrm{x}$ & 2 & $\mathrm{x}$ & 4 & 3 & $\mathrm{x}$ & $9.31 \%$ & | & 7.6 & | \\
\hline TOT21 & 16 & 7 & 0 & 8 & 5 & 0 & 17 & 13 & 12 & 9 & & & & TOT 3 & 5 & 6 & 0 & 0 & 7 & 0 & 5 & 6 & 0 & 3 & & & \\
\hline \multirow{3}{*}{$\begin{array}{ll}\text { Day } & 0 \\
\text { Day } & 1\end{array}$} & \multicolumn{2}{|c|}{ TEM. ${ }^{\bullet} \mathrm{C}$} & \multicolumn{4}{|c|}{ Time } & & & TEM. & & Time & & & - & & TEM & $\cdot^{\circ} \mathrm{C}$ & & Time & & & & TEM & $1 \cdot{ }^{\circ} \mathrm{c}$ & & Time & \\
\hline & 24 & & $04:$ & $00 \mathrm{P}$ & & & Day & 2 & 24.0 & & $04: 30$ & PM & & Day & y 4 & 24. & & $02: 3$ & $30 \mathrm{PM}$ & & & Day 6 & & 24.0 & & $1: 00$ & $0 \mathrm{PM}$ \\
\hline & 24 & & $04:$ & $00 \mathrm{P}$ & & & Day & 3 & 24.0 & & $01: 00$ & PM & & Day & y 5 & 24. & & $04: 0$ & $00 \mathrm{PN}$ & & & Day & & 24.0 & & $3: 45$ & $5 \mathrm{PM}$ \\
\hline
\end{tabular}


SAMPIING INEORMATION

\begin{tabular}{|c|c|c|c|c|c|c|c|c|}
\hline Dilution Water & 20\%DMW & $8-16-95$ & h & 84.0 & 66.9 & 220 & 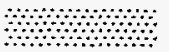 & \\
\hline Final Eff. 1 & Grab & $8-16-95$ & $7: 00 \mathrm{AM}$ & 8.0 & 6.09 & 19.0 & $<0.05$ & $0.8^{\circ} \mathrm{C}$ \\
\hline Final Eff. 2 & Grab & $8-18-95$ & $8: 50 \mathrm{AM}$ & 8.0 & 4.08 & 29.3 & $<0.05$ & $0.4^{\circ} \mathrm{C}$ \\
\hline Final Eff. 3 & Grab & $8-21-95$ & $7: 30 \mathrm{AM}$ & 6.0 & 8.16 & 32.4 & $<0.05$ & $0.7^{\circ} \mathrm{C}$ \\
\hline
\end{tabular}


Results

\begin{tabular}{|l|l|l|l|}
\hline \multicolumn{2}{|l|}{ Client: WSRC } & Sample ID: BG003 & \\
\hline Log \#: T4392 & Start Date: $8-17-95$ & Time: & $4: 00$ PM \\
\hline
\end{tabular}

SURVIVAL EFFECTS

\begin{tabular}{|c|c|c|c|c|c|c|}
\hline \multirow[b]{2}{*}{ Effluent Conc. } & \multicolumn{2}{|c|}{ Control } & \multicolumn{4}{|c|}{ Effiuent } \\
\hline & SC. .DMW & $6.25 \%$ & $12.5 \%$ & $25 \%$ & $50 \%$ & $100 \%$ \\
\hline $48 \mathrm{hr}$. Mortality & $0 \%$ & $0 \%$ & $0 \%$ & $\mathbf{0} \%$ & $20 \%$ & $40 \%$ \\
\hline 7 Day Mortality & $0 \%$ & $0 \%$ & $0 \%$ & $0 \%$ & $20 \%$ & $40 \%$ \\
\hline
\end{tabular}

Method: Fishers Exact Test

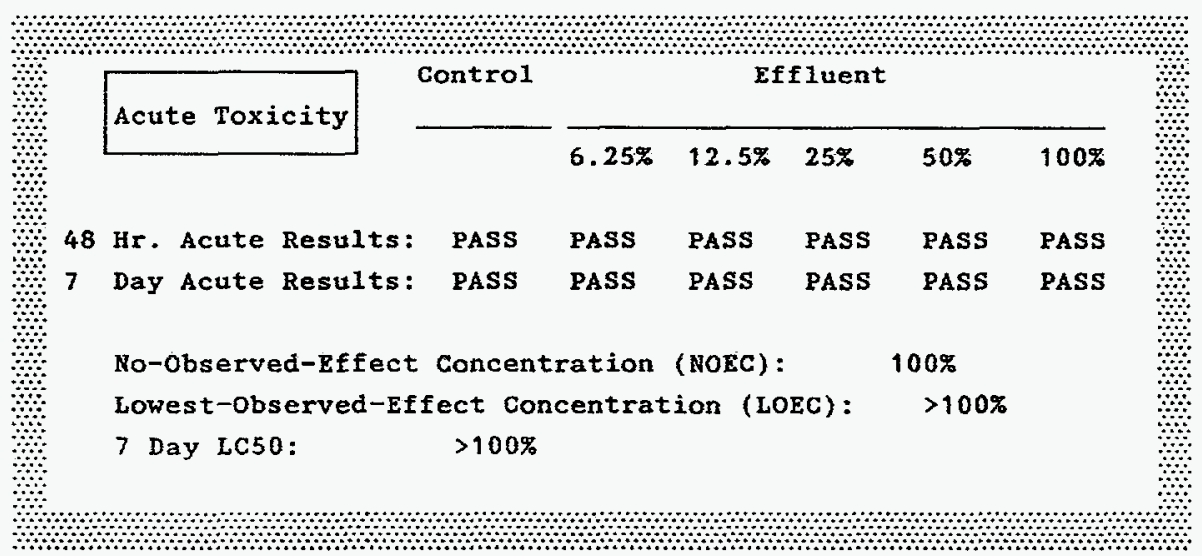

QUALITY CONTROL

\begin{tabular}{|cccc|}
\hline Standard Toxicant: Nac1 & & \\
& & & \\
& Central Tendency: & 1.98 & $\mathrm{~g} / \mathrm{L}$ \\
Current Value: & 1.91 & $\mathrm{~g} / \mathrm{L}$ \\
Deviation: & 0.29 & \multicolumn{2}{c|}{ Std Dev units. } \\
\hline
\end{tabular}


Results

\begin{tabular}{|l|l|l|l|}
\hline \multicolumn{2}{|l|}{ Client: WSRC } & Sample ID: BG003 & IWC: \\
\hline Log \#: T4392 & Start Date: $8-17-95$ & Time: & $4: 00$ PM \\
\hline
\end{tabular}

CHRONIC EFFECTS

$$
\text { Control Effluent }
$$

TEST CONCENTRATION

\begin{tabular}{lrrrrrr} 
& & & & & & \\
\cline { 5 - 7 } TEST CONCENTRATION & SC.DMW & $6.25 \%$ & $12.5 \%$ & $25 \%$ & $50 \%$ & $100 \%$ \\
Average young / female: & 18.2 & 15.4 & 12.2 & 14.5 & 9.9 & 3.2 \\
Standard Deviation: & 1.93 & 4.09 & 5.31 & 4.62 & 7.13 & 2.94 \\
$t=$ & & 1.35 & 2.89 & 1.78 & 4 & 7.22 \\
Steel's $=$ & & 83 & 72 & 82 & 68 & 55
\end{tabular}

MSD $=4.8$

Normality: Data Not Normal $W=0.86$

Homogeneity:Data Not Homogeneous $B=15.6$

Test Used: Steel's Test

Critical Steel's value: 75

Critical t value: 2.31

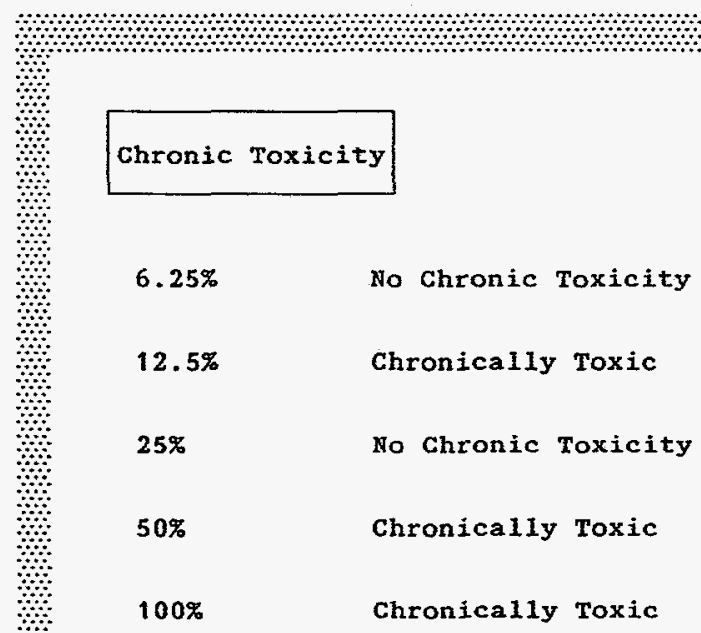

No-Observed-Effect Concentration (NORC): $\quad 6.25 \%$ Lowest-Observed-Effect Concentration (LOEC): $12.5 \%$ 7 Day EC50: 40.61 
7 Day Chronic Definitive Survival and Reproduction Bioassay

Test Organisms: Ceriodaphnia dubia

Method: EPA/600/4-89/001

Facixity :

westinghoxse SampIe ID:
Savaninan BG 004

Date: $\quad 8-17-95$ 
DEFINITIVE SURVIVAL AND REPRODUCTION

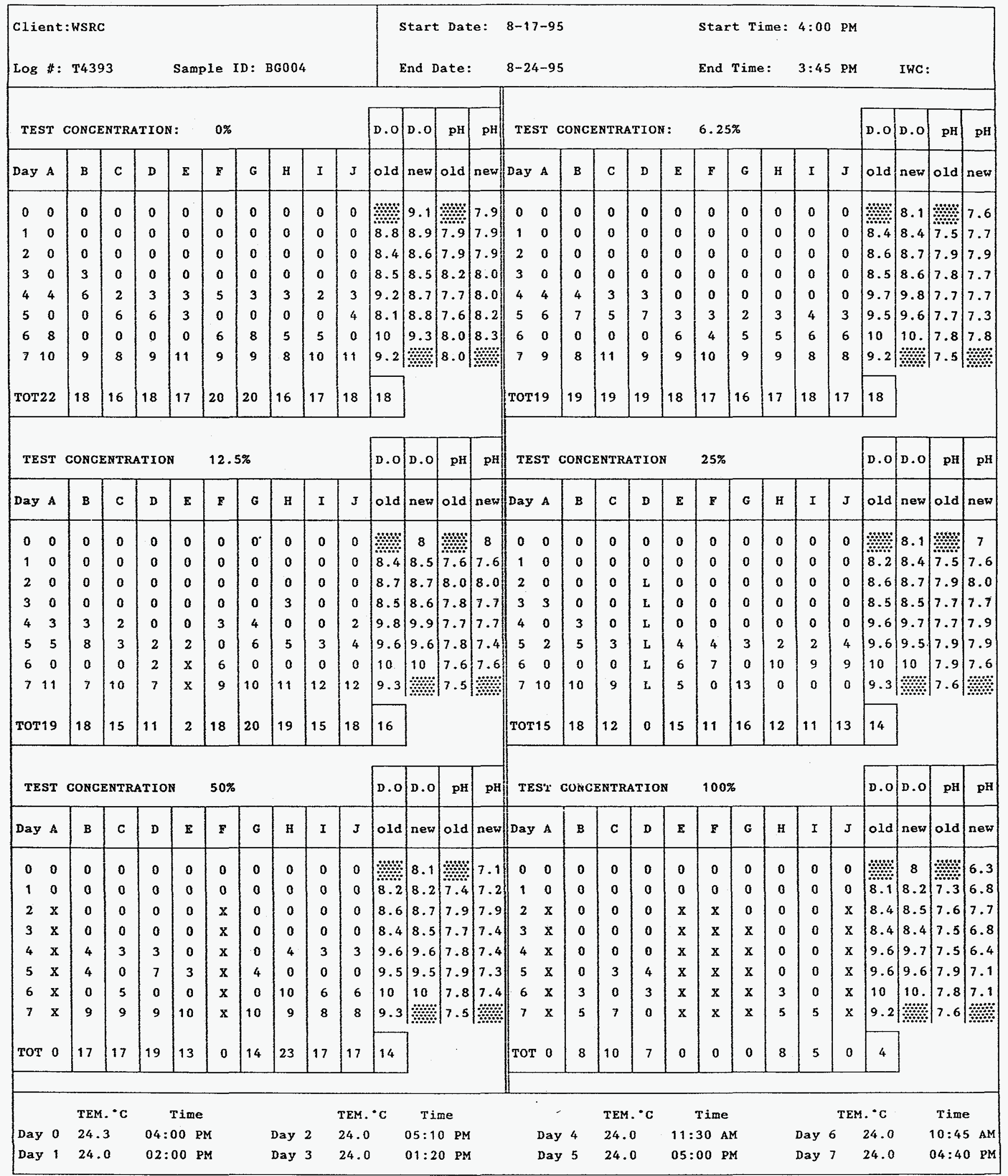


SAMPLING INEORMATION

\begin{tabular}{|c|c|c|c|c|c|c|c|c|}
\hline Dilution Water & $20 \% \mathrm{DMW}$ & $8-16-95$ & H & 84.0 & 66.9 & 220 & 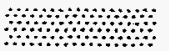 & \\
\hline Final Eff. 1 & Grab & $8-16-95$ & $7: 40 \mathrm{AM}$ & 10.0 & 8.12 & 19.0 & $<0.05$ & $0.8^{\circ} \mathrm{C}$ \\
\hline Einal Eff. 2 & Grab & $8-18-95$ & $8: 20 \mathrm{AM}$ & 14.0 & 10.2 & 34.2 & $<0.05$ & $0.4^{\circ} \mathrm{C}$ \\
\hline Final Eff. 3 & Grab & $8-21-95$ & $8: 00 \mathrm{AM}$ & 14.0 & 14.28 & 38.1 & $<0.05$ & $0.7^{\circ} \mathrm{C}$ \\
\hline
\end{tabular}


Results

\begin{tabular}{|l|l|l|l|}
\hline \multicolumn{2}{|l|}{ C1ient: WSRC } & \multicolumn{1}{|c|}{ Sample ID: BG004 } & \\
\hline Log \#: T4393 & Start Date: $8-17-95$ & Time: & $4: 00$ PM \\
\hline
\end{tabular}

SURVIVAL EFFECTS

\begin{tabular}{|c|c|c|c|c|c|c|}
\hline \multirow[b]{2}{*}{ Effluent Conc. } & \multicolumn{2}{|c|}{ Control } & \multicolumn{4}{|c|}{ Effluent } \\
\hline & SC.DMW & $6.25 \%$ & $12.5 \%$ & $25 \%$ & $50 \%$ & $100 \%$ \\
\hline $48 \mathrm{hr}$. Mortality & $0 \%$ & $0 \%$ & $0 \%$ & $0 \%$ & $20 \%$ & $40 \%$ \\
\hline 7 Day Mortality & $0 \%$ & $0 \%$ & $10 \%$ & $0 \%$ & $20 \%$ & $50 \%$ \\
\hline
\end{tabular}

Method: Fishers Exact Test

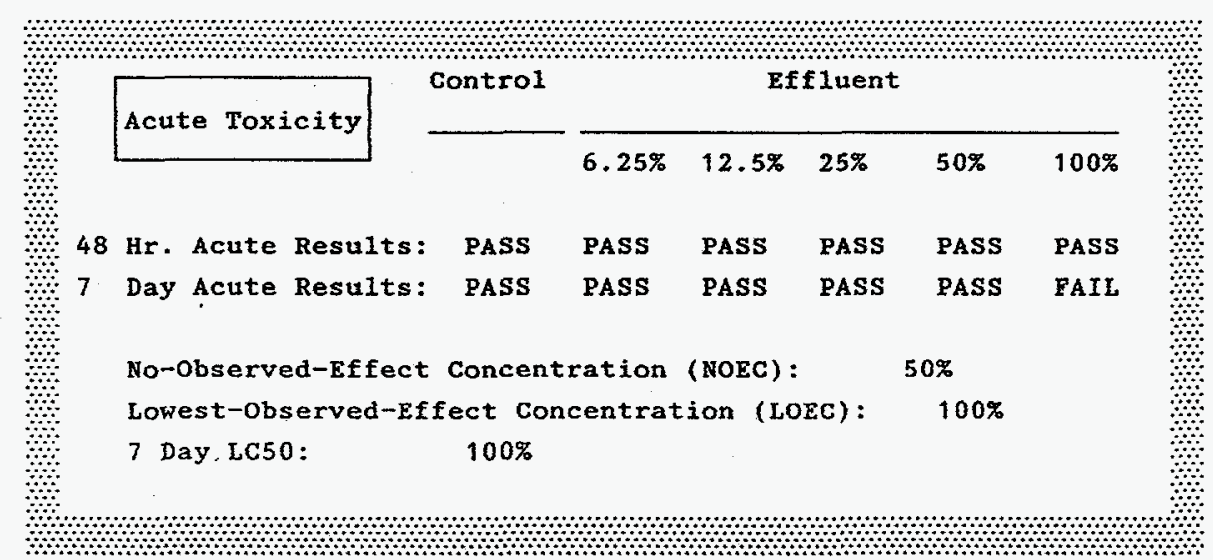

QUALITY CONTROL

\begin{tabular}{|cccc|}
\hline Standard Toxicant: Nac1 & & \\
& & & \\
& Central Tendency: & 1.98 & $\mathrm{~g} / \mathrm{L}$ \\
Current Value: & 1.91 & $\mathrm{~g} / \mathrm{L}$ \\
Deviation: & 0.29 & \multicolumn{2}{c|}{ Std Dev units. } \\
\hline
\end{tabular}


Results

\begin{tabular}{|c|c|c|c|c|}
\hline Client: WSRC & & Sample ID: BG004 & & \\
\hline Log \# : T4393 & Start Date: & $8-17-95$ & Time: & 4:00 PM \\
\hline
\end{tabular}

CHRONIC EFFECTS

\begin{tabular}{|c|c|c|c|c|c|c|}
\hline \multirow[b]{2}{*}{ TEST CONCENTRATION } & \multirow{2}{*}{$\frac{\text { Control }}{\text { SC.DMW }}$} & \multicolumn{4}{|c|}{ Effluent } & \multirow[b]{2}{*}{$100 \%$} \\
\hline & & $6.25 \%$ & $12.5 \%$ & $25 \%$ & $50 \%$ & \\
\hline Average young / female: & 18.2 & 17.9 & 15.5 & 13.7 & 13.7 & 3.8 \\
\hline Standard Deviation: & 1.93 & 1.1 & 5.44 & 2.45 & 7.7 & 4.18 \\
\hline$t=$ & & 0.13 & 1.16 & 1.89 & 1.93 & 0.00 \\
\hline steel's = & & 104 & 93.5 & 61.5 & 84 & 55 \\
\hline
\end{tabular}

$M S D=5.23$

Normality: Data Not Normal $W=0.7$

Homogeneity:Data Not Homogeneous $B=35.7$

Test Used: Steel's Test

Critical Steel's Value: 75

Critical t value: 2.23

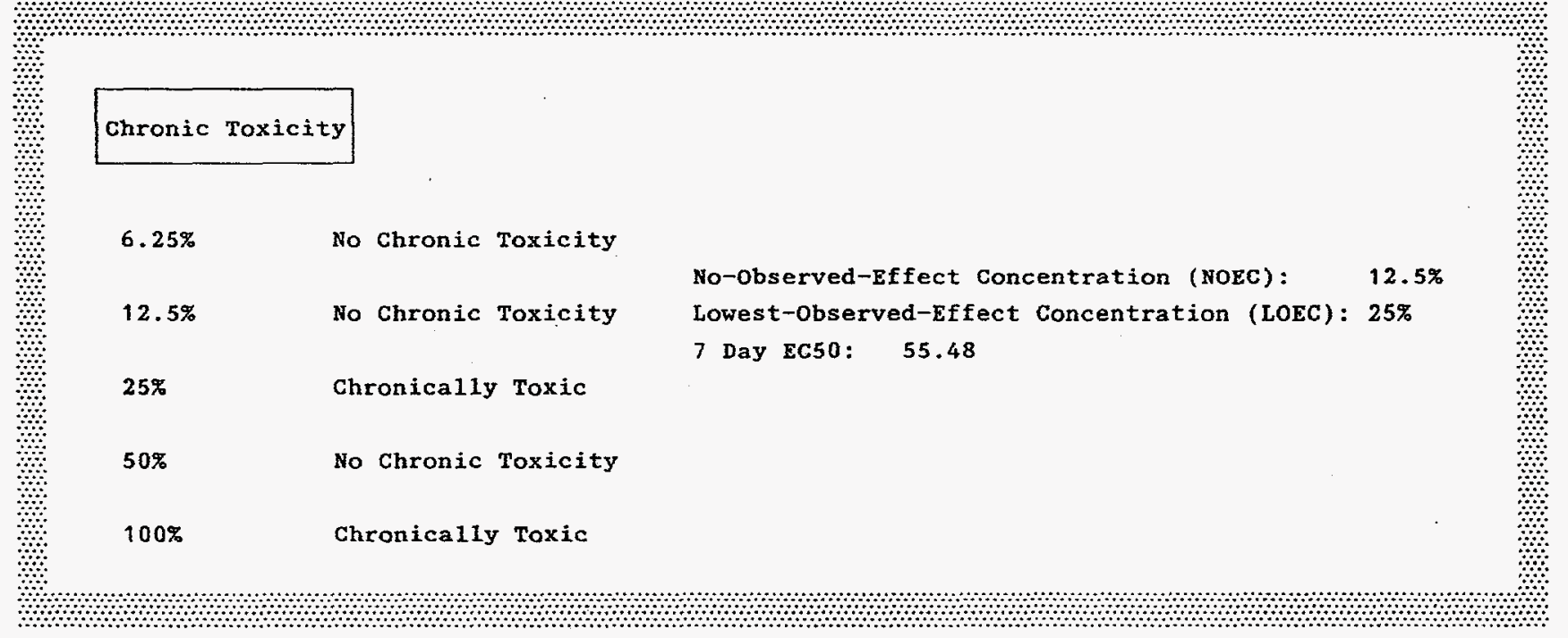


7 Day Chronic Definitive Survival and Reproduction Bioassay

Test Organisms: Ceriodaphnia dubia

Method: EPA/600/4-89/001

\footnotetext{
Facility: Westinghouse savannah Rivex company Sample ID $=$ FHB-OOG EfFluent
}

Date: $8-24-95$ 


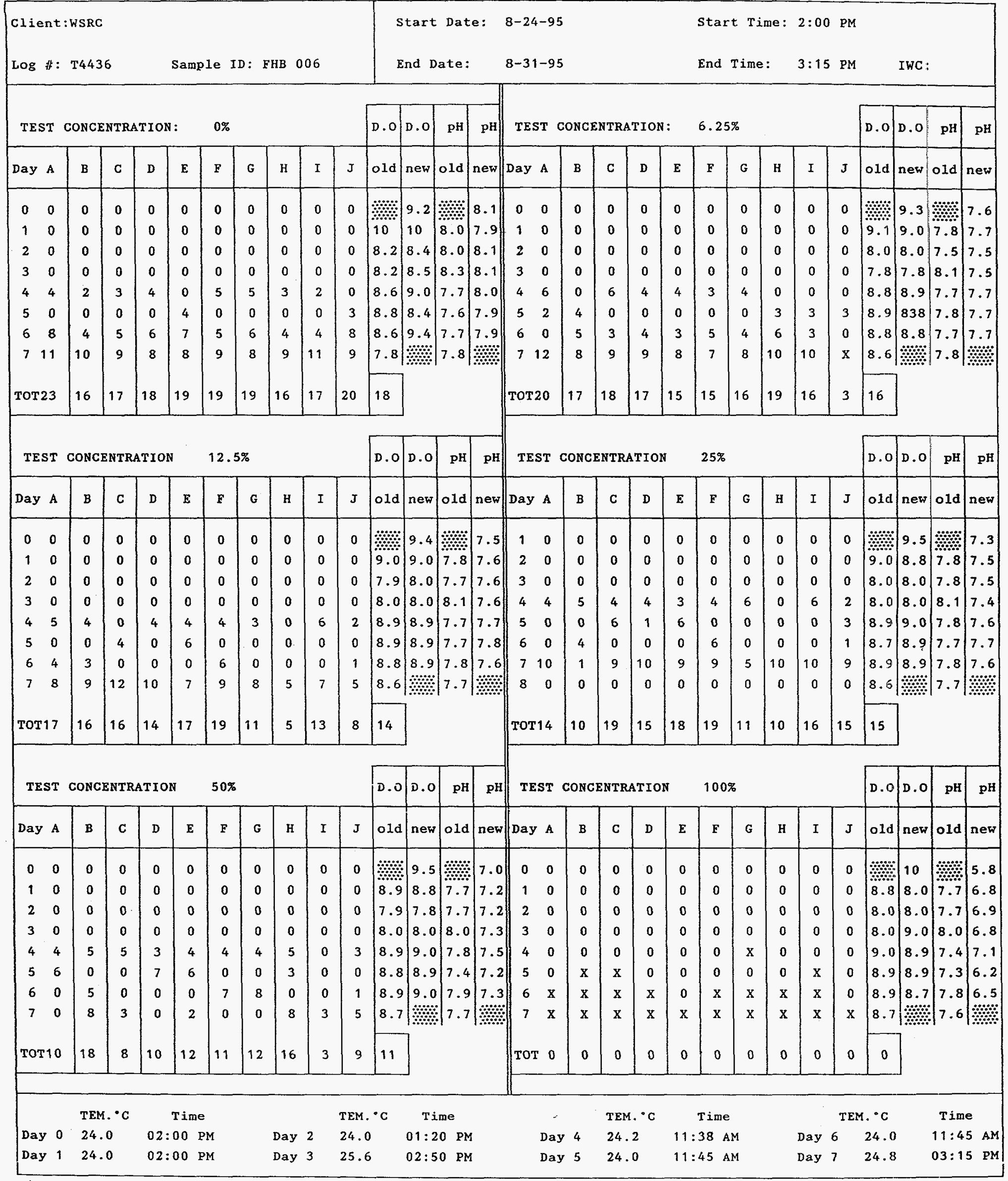


SAMPLING INFORMATION

\begin{tabular}{|c|c|c|c|c|c|c|c|c|}
\hline & Type & Start Date & Time & Hardness $\mathrm{Mg} / \mathrm{L}$ & Alkalinity & Conductivity & Res. $\mathrm{C} 1$ & Rec.Temp. \\
\hline Dilution Water & $20 \%$ DMW & $08-24-95$ & $\because$ & 90 & 73.4 & 194.3 & m & \\
\hline Final Eff. 1 & Grab & $8-23-95$ & $08: 40 \mathrm{AM}$ & 6.0 & 8.16 & 34.5 & $<0.05$ & $0.9^{\circ} \mathrm{C}$ \\
\hline Final Eff. 2 & Grab & $8-25-95$ & $09: 50 \mathrm{AM}$ & 4.0 & 8.16 & 35.0 & $<0.05$ & $0.9^{\circ} \mathrm{C}$ \\
\hline Final Eff. 3 & Grab & $8-28-95$ & $08: 40 \mathrm{AM}$ & 8.0 & 4.08 & 29.9 & $<0.05$ & $0.9^{\circ} \mathrm{C}$ \\
\hline
\end{tabular}


Results

\begin{tabular}{|l|l|l|l|l|}
\hline \multicolumn{2}{|l|}{ Client: WSRC } & Sample ID: FHB 006 & \\
\hline Log : T4436 & Start Date: $8-24-95$ & & Time: & $2: 00$ PM \\
\hline
\end{tabular}

SURVIVAL EFFECTS

\begin{tabular}{|c|c|c|c|c|c|c|}
\hline \multirow[b]{2}{*}{ Effluent Conc. } & \multicolumn{2}{|c|}{ Control } & \multicolumn{4}{|c|}{ Effluent } \\
\hline & SC.DMW & $6.25 \%$ & $12.5 \%$ & $25 \%$ & $50 \%$ & $100 \%$ \\
\hline $48 \mathrm{hr}$. Mortality & $0 \%$ & $0 \%$ & $0 \%$ & $0 \%$ & $0 \%$ & $0 \%$ \\
\hline 7 Day Mortality & $0 \%$ & $10 \%$ & $0 \%$ & $0 \%$ & $0 \%$ & $100 \%$ \\
\hline
\end{tabular}

Method: Fishers Exact Test

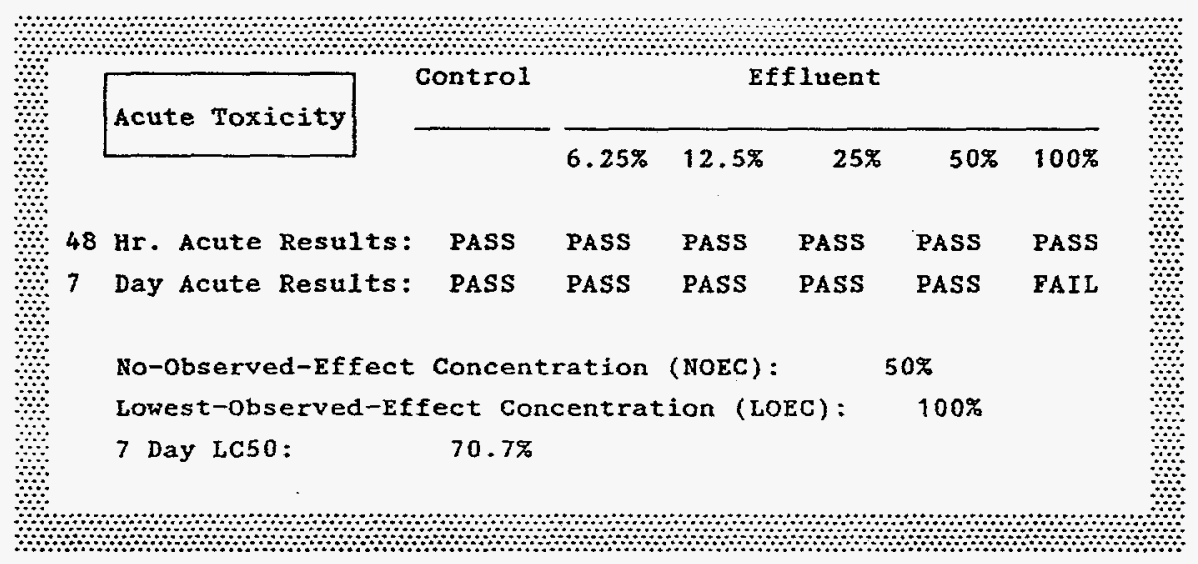

QUALITY CONTROL

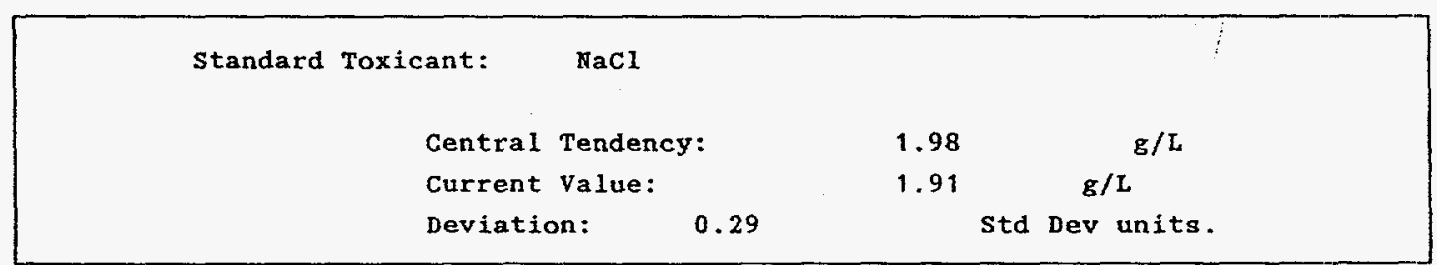


Results

\begin{tabular}{|l|l|l|l|l|}
\hline \multicolumn{2}{|l|}{ Client: WSRC } & Sample ID: FHB 006 & IWC: \\
\hline Log $:$ T4436 & Start Date: $8-24-95$ & Time: & $2: 00$ PM \\
\hline
\end{tabular}

CHRONIC EFFECTS

\begin{tabular}{|c|c|c|c|c|c|c|}
\hline \multirow[b]{2}{*}{ TEST CONCENTRATION } & \multirow{2}{*}{$\frac{\text { Control }}{\text { SC.DMW }}$} & \multicolumn{4}{|c|}{ Effluent } & \multirow[b]{2}{*}{$100 \%$} \\
\hline & & $6.25 \%$ & $12.5 \%$ & $25 \%$ & $50 \%$ & \\
\hline Average young / female: & 18.4 & 15.6 & 13.6 & 14.7 & 10.9 & 0 \\
\hline Standard Deviation: & 2.12 & 4.72 & 4.43 & 3.47 & 4.15 & 0.00 \\
\hline$t=$ & & 1.61 & 2.76 & 2.13 & 4.31 & N/A \\
\hline
\end{tabular}

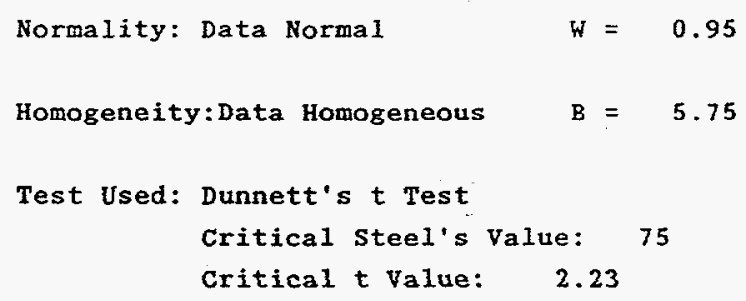




\section{Day Chxonic Definitive Survival} and Reproduction Bioassay

Test Organisms: Ceriodaphnia dubia

Method: EPA/600/4-89/001

Facility: WSRC

Sample ID $=$ FHB-O12

$$
\text { Date: } \quad 9-7-95
$$


DEFINITIVE SURVIVAL AND REPRODUCTION

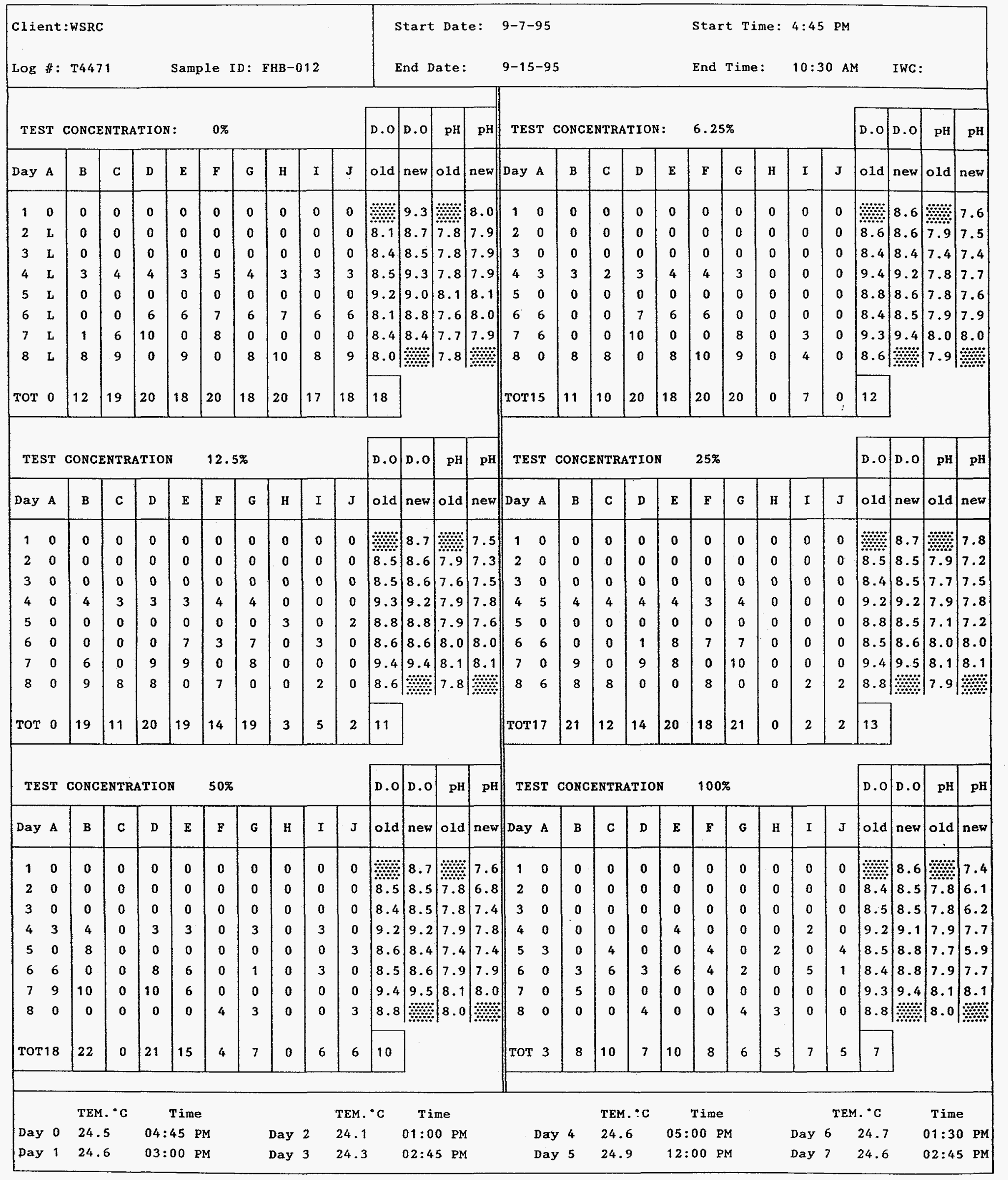




\section{SAMPIING INFORMATION}

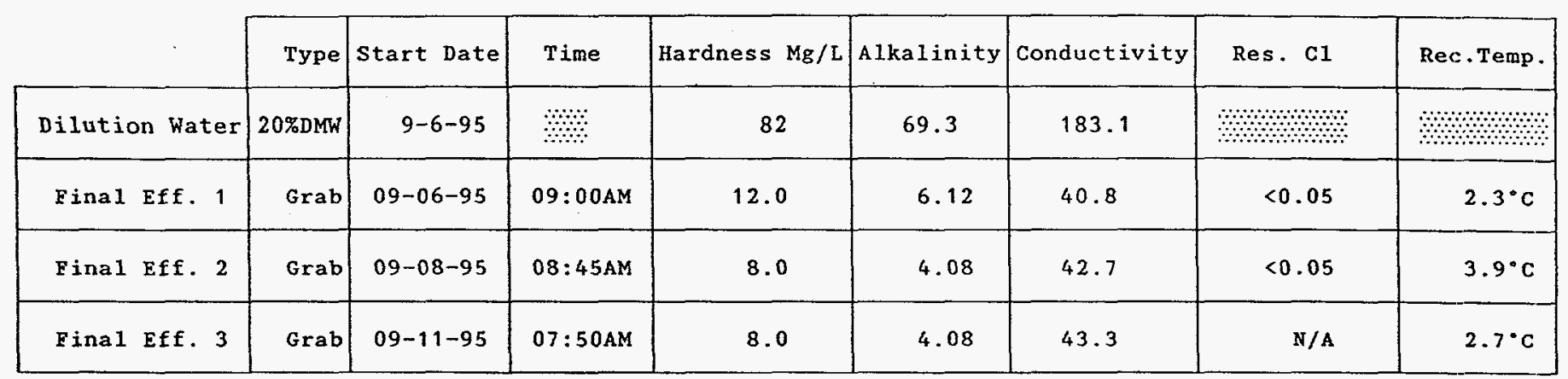


Results

\begin{tabular}{|l|l|l|l|l|}
\hline \multicolumn{2}{|l|}{ Client: WSRC } & Sample ID: FHB-012 & \\
\hline Log \#: T4471 & Start Date: $9-7-95$ & Time: & $4: 45$ PM \\
\hline
\end{tabular}

SURVIVAL EFFECTS

\begin{tabular}{|c|c|c|c|c|c|c|}
\hline \multirow[b]{2}{*}{ Effluent Conc } & \multicolumn{2}{|c|}{ Control } & \multicolumn{4}{|c|}{ Effluent } \\
\hline & SC. DMW & $6.25 \%$ & $12.5 \%$ & $25 \%$ & $50 \%$ & $100 \%$ \\
\hline $48 \mathrm{hr}$. Mortality & $0 \%$ & $0 \%$ & $0 \%$ & $0 \%$ & $0 \%$ & $0 \%$ \\
\hline 7 Day Mortality & $0 \%$ & $0 \%$ & $0 \%$ & $0 \%$ & $0 \%$ & $0 \%$ \\
\hline
\end{tabular}

Method: Fishers Exact Test

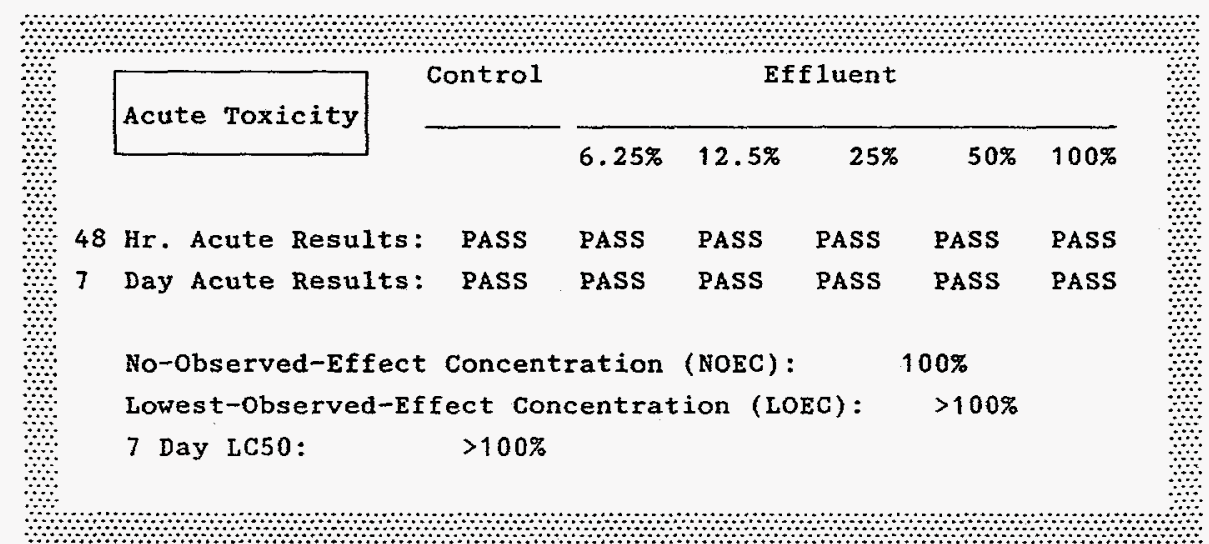

QUALITY CONTROL

\begin{tabular}{|cccc|}
\hline Standard Toxicant: NaCl & & \\
& & & \\
& Central Tendency: & 1.98 & $\mathrm{~g} / \mathrm{L}$ \\
Gurrent Value: & 1.91 & $\mathrm{~g} / \mathrm{L}$ \\
Deviation: & 0.29 & \multicolumn{2}{c|}{ Std Dev units. } \\
\hline
\end{tabular}


Resu1ts

\begin{tabular}{|l|l|l|l|l|}
\hline \multicolumn{2}{|l|}{ Client: WSRC } & Sample ID: FHB-012 & IWC: \\
\hline Log \#: T4471 & Start Date: $9-7-95$ & & Time: & $4: 45$ PM \\
\hline
\end{tabular}

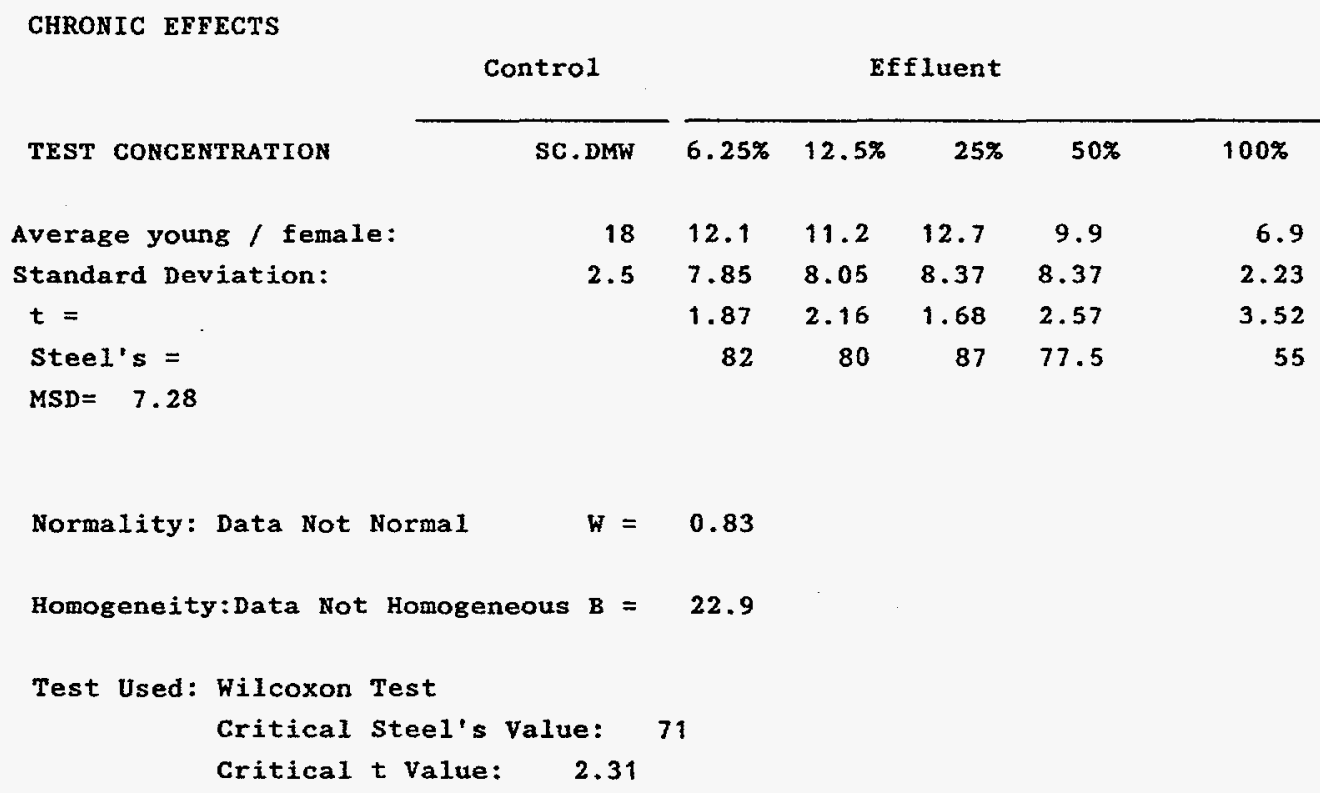

8.37

2.23

1.87

$\begin{array}{lll}2.16 & 1.68 & 2.57\end{array}$

$\begin{array}{lllll}82 & 80 & 87 & 77.5 & 55\end{array}$

3.52

Normality: Data Not Norma1 $W=0.83$

Homogeneity:Data Not Homogeneous $\mathbf{B}=\mathbf{2 2 . 9}$

Test Used: Wilcoxon Test

Critical Steel's value: 71

Critical t value: 2.31 
7 Day Chronic Definitive Survival and Repxoduction Bioassay

Test Organisms: Ceriodaphnia dubia

Method: EPA/ $600 / 4-89 / 001$

Facility : WSRC

Sample ID: FHB-O13

Date: $\quad 09-07-95$ 
SAMPIING INFORMATION

\begin{tabular}{|c|c|c|c|c|c|c|c|c|}
\hline & Type & Start Date & Time & Hardness $\mathrm{Mg} / \mathrm{L}$ & Alkalinity & Conductivity & Res. $\mathrm{C} 1$ & Rec. Temp. \\
\hline Dilution Water & $20 \% \mathrm{DMW}$ & $09-06-95$ & H & 82 & 69.3 & 183.1 & in & \\
\hline Final Eff. 1 & Grab & $09-06-95$ & $09: 30 \mathrm{AM}$ & 14.0 & 6.12 & 41.4 & $<0.05$ & $2.3^{\circ} \mathrm{C}$ \\
\hline Final Eff. 2 & Grab & $09-08-95$ & 08:00AM & 8.0 & 6.12 & 38.3 & $<0.05$ & $3.9^{\circ} \mathrm{C}$ \\
\hline Final Eff. 3 & Grab & $09-11-95$ & $08: 00 \mathrm{AM}$ & 6.0 & 8.16 & 38.3 & $\mathrm{~N} / \mathrm{A}$ & $2.7^{\circ} \mathrm{C}$ \\
\hline
\end{tabular}


Results

\begin{tabular}{|l|l|l|l|}
\hline \multicolumn{2}{|l|}{ C1ient: WSRC } & Sample ID: FHB-013 & \\
\hline Log \#: T4472 & Start Date: $09-07-95$ & Time: & $5: 00$ PM \\
\hline
\end{tabular}

SURVIVAL EFFECTS

Contro1 Effluent

\begin{tabular}{lccccccc}
\cline { 3 - 6 } Effluent Conc. & SC.DMW & $6.25 \%$ & $12.5 \%$ & $25 \%$ & $50 \%$ & $100 \%$ \\
\hline $48 \mathrm{hr}$. Mortality & $0 \%$ & $0 \%$ & $0 \%$ & $0 \%$ & $0 \%$ & $20 \%$ \\
7 Day Mortality & $0 \%$ & $0 \%$ & $0 \%$ & $0 \%$ & $0 \%$ & $90 \%$ \\
\hline
\end{tabular}

Method: Fishers Exact Test

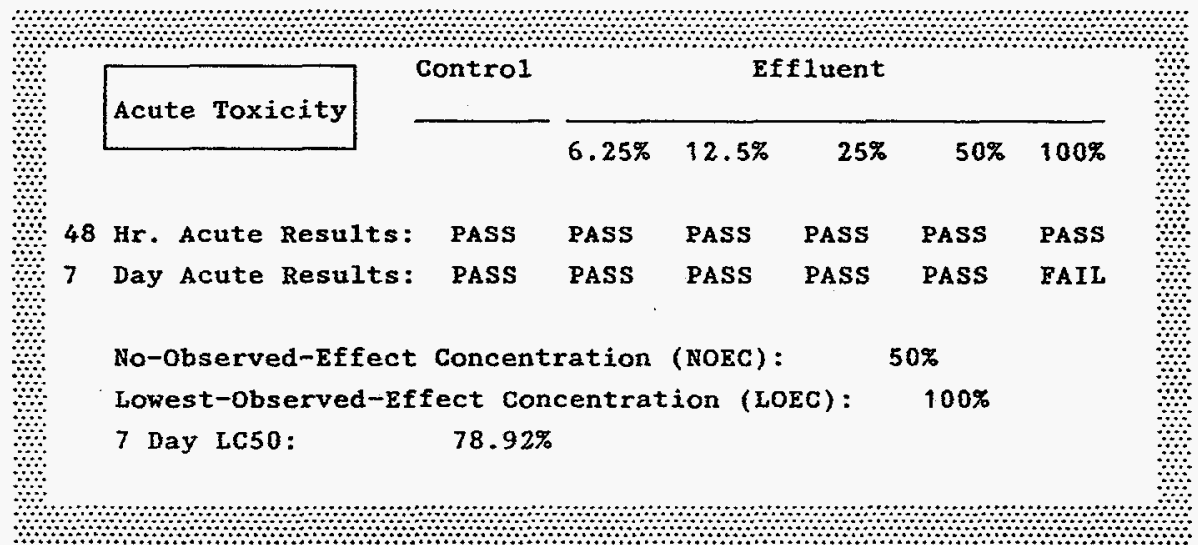

QUALITY CONTROL

\begin{tabular}{|llll|}
\hline Standard Toxicant: & & \\
& & & \\
& Central Tendency: & 1.98 & $\mathrm{~g} / \mathrm{L}$ \\
Current Value: & & 1.91 & $\mathrm{~g} / \mathrm{L}$ \\
Deviation: & 0.29 & \multicolumn{2}{c|}{ Std Dev units. } \\
\hline
\end{tabular}


Results

\begin{tabular}{|l|l|l|l|l|}
\hline \multicolumn{2}{|l|}{ C1ient: WSRC } & \multicolumn{1}{|c|}{ Sample ID: FHB-013 } & IWC: \\
\hline Log \#: T4472 & Start Date: $09-07-95$ & Time: & $5: 00$ PM \\
\hline
\end{tabular}

\section{CHRONIC EFFECTS}

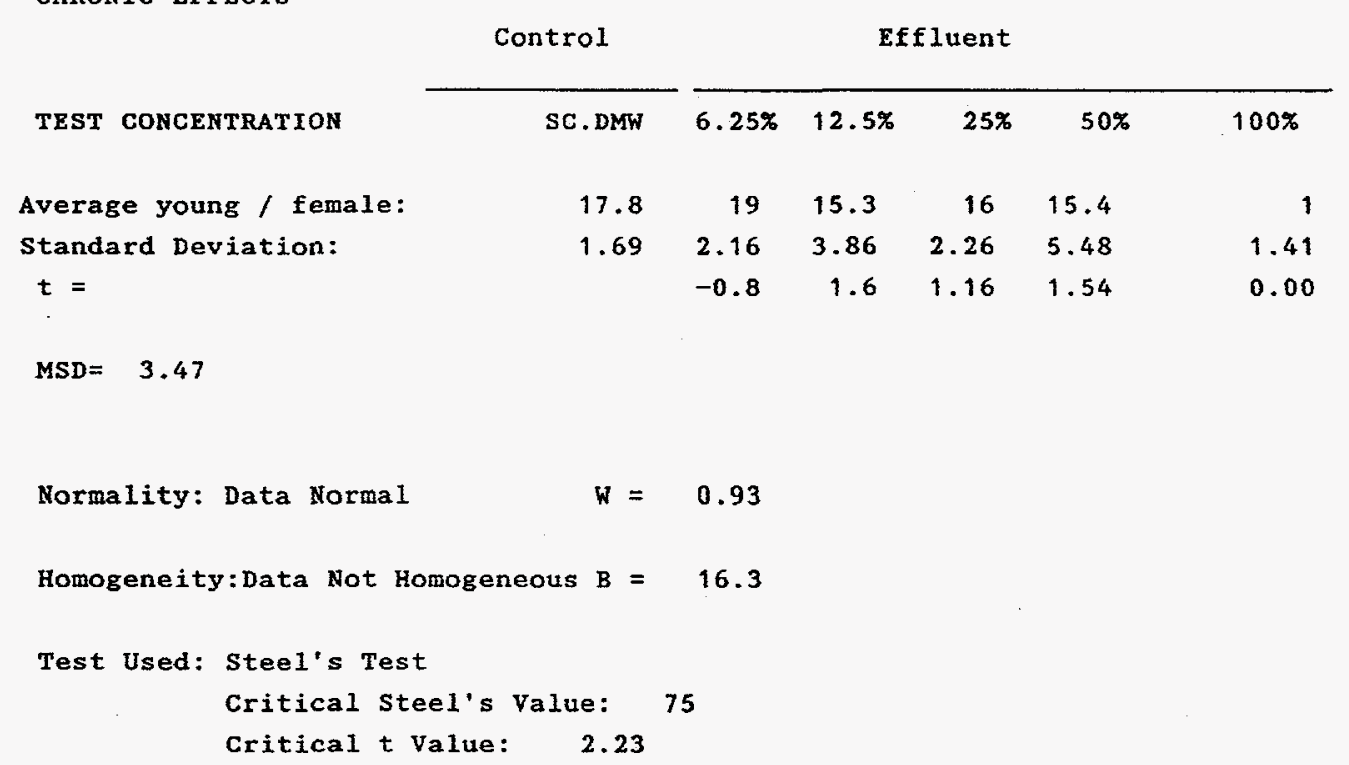

$\begin{array}{rrrrrr}17.8 & 19 & 15.3 & 16 & 15.4 & 1 \\ 1.69 & 2.16 & 3.86 & 2.26 & 5.48 & 1.41 \\ & -0.8 & 1.6 & 1.16 & 1.54 & 0.00\end{array}$
Standard Deviation: $t=$

0.00

$M S D=3.47$

Normality: Data Normal $W=0.93$

Homogeneity: Data Not Homogeneous $B=16.3$

Test Used: Stee1's Test

Critical Steel's Value: 75

Critical t value: 2.23

Chronic Toxicity
$6.25 \%$
No Chronic Toxicity
12.5\% No Chronic Toxicity
$25 \%$
No Chronic Toxicity
$50 \%$
No Chronic Toxicity
$100 \%$
Chronically Toxic

No-Observed-Effect Concentration (NOEC):

$50 \%$ Lowest-Observed-Effect Concentration (LOEC): $100 \%$

7 Day EC50: $57.43 \%$ 
7 Day Chronic Definitive Survivai and Reproduction Bioassay

Test Organisms: Ceriodaphnia dubia

Method: EPA/600/4-89/001

\footnotetext{
Facility = Westinghouse Savannah Rivex Company SampIe ID: FHP-OI4 EIIIuent
}

Date: $\quad 8-24-95$ 


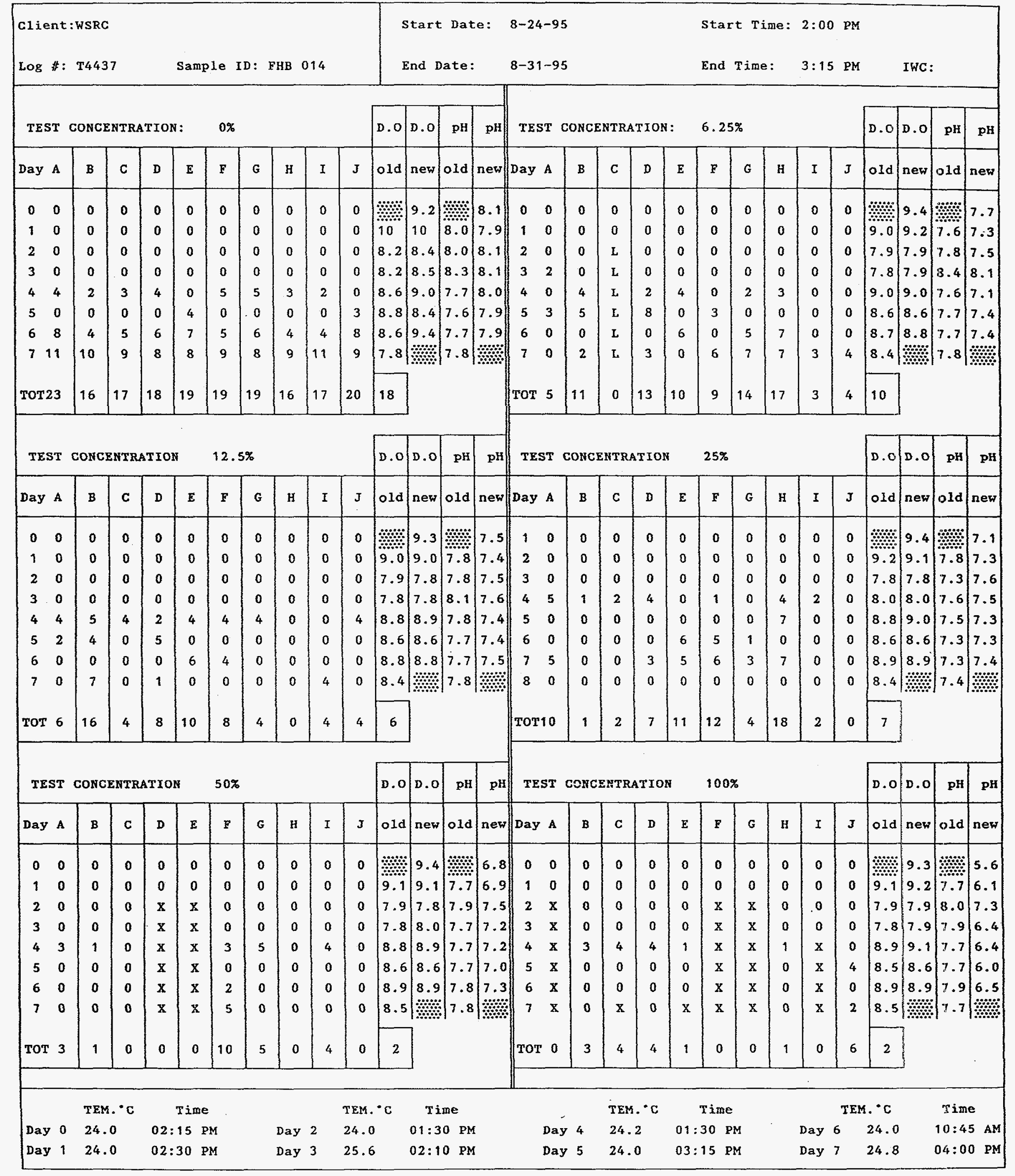


SAMPLING INFORMATION

\begin{tabular}{|c|c|c|c|c|c|c|c|c|}
\hline Dilution water & $20 \% \mathrm{DMW}$ & $08-24-95$ & O & 90 & 73.4 & 194.3 & m & \\
\hline Final Eff. 1 & Grab & $8-23-95$ & $08: 30 \mathrm{AM}$ & 4.0 & 8.16 & 39.1 & $<0.05$ & $0.9^{\circ} \mathrm{C}$ \\
\hline Final Eff. 3 & Grab & $8-28-95$ & $08: 20 \mathrm{AM}$ & 4.0 & 6.12 & 35.8 & $<0.05$ & $0.9^{\circ} \mathrm{C}$ \\
\hline
\end{tabular}


Results

\begin{tabular}{|l|l|l|l|l|}
\hline \multicolumn{2}{|l|}{} & Sample ID: FHB 014 & \\
\hline Log \#: T4437 & Start Date: $8-24-95$ & Time: & $2: 00$ PM \\
\hline
\end{tabular}

SURVIVAL EFPECTS

Contro1 Effluent

\begin{tabular}{lrrrrrrr}
\cline { 2 - 4 } Effluent Conc. & SC.DMW & $6.25 \%$ & $12.5 \%$ & $25 \%$ & $50 \%$ & $100 \%$ \\
\hline $48 \mathrm{hr}$. Mortality & $0 \%$ & $0 \%$ & $0 \%$ & $0 \%$ & $20 \%$ & $30 \%$ \\
7 Day Mortality & $0 \%$ & $0 \%$ & $0 \%$ & $0 \%$ & $20 \%$ & $60 \%$
\end{tabular}

Method: Fishers Exact Test

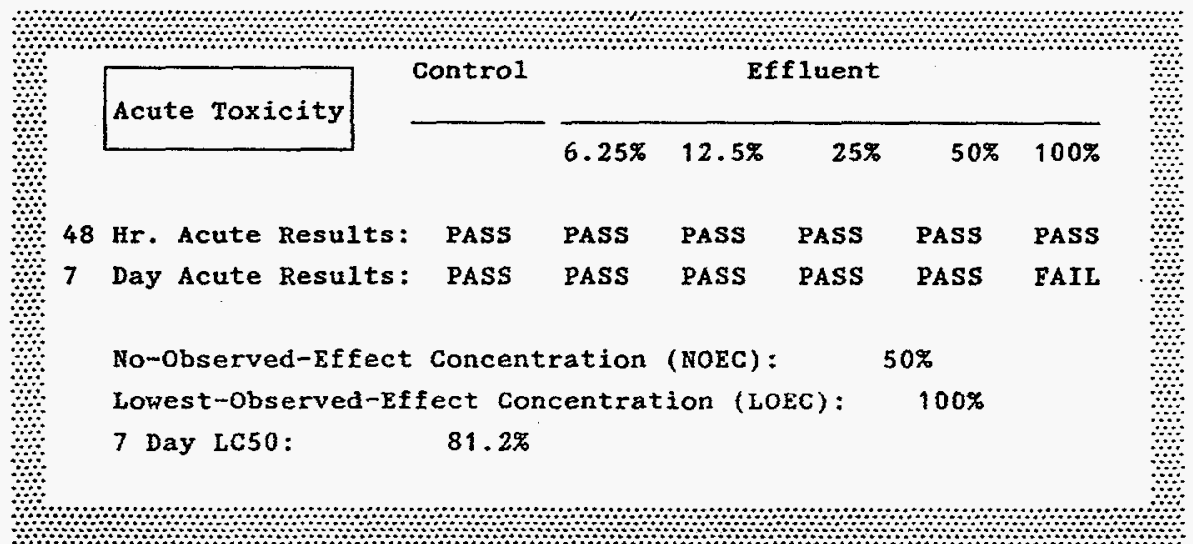

QUALITY CONTROL

\begin{tabular}{|cccc|}
\hline Standard Toxicant: & & \\
& & & \\
& Central Tendency: & 1.98 & $\mathrm{~g} / \mathrm{L}$ \\
Current Value: & 1.91 & $\mathrm{~g} / \mathrm{L}$ \\
Deviation: & 0.29 & \multicolumn{2}{c|}{ Std Dev units. } \\
\hline
\end{tabular}


Results

\begin{tabular}{|l|l|l|l|l|}
\hline \multicolumn{2}{|l|}{ Client: WSRC } & Sample ID: FHB 014 & IWC: \\
\hline Log $::$ T4437 & Start Date: $8-24-95$ & Time: & $2: 00$ PM \\
\hline
\end{tabular}

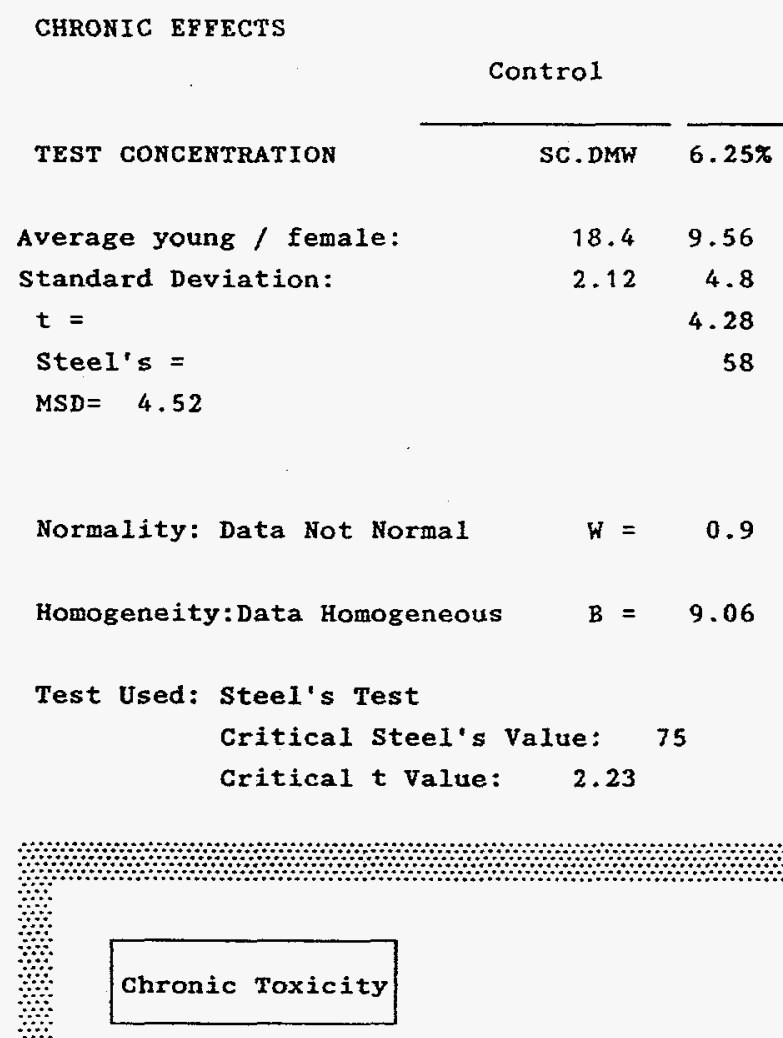

CHRONIC EFFECTS

TEST CONCENTRATION

Control

58

Normality: Data Not Normal

Effluent

2.18

0.00 
7 Day Chxonic Definitive Survival and Reproduction Bioassay

Test Organisms: Ceriodaphnia dubia

Method: EPA/600/4-89/001

Facility : WSRC

Sample ID: FHB-O18

Date: $\quad 09-07-95$ 
DEFINITIVE SURVIVAL AND REPRODUCTION

\begin{tabular}{|c|c|c|c|c|c|c|c|c|c|c|c|c|c|c|c|c|c|c|c|c|c|c|c|c|c|c|c|c|}
\hline \multicolumn{11}{|c|}{$\begin{array}{l}\text { C1ient: WSRC } \\
\text { Log \#\#: T4473 }\end{array}$} & \multicolumn{4}{|c|}{$\begin{array}{l}\text { Start Date: } \\
\text { End Date: }\end{array}$} & \multicolumn{5}{|c|}{$\begin{array}{l}09-07-95 \\
09-15-95\end{array}$} & \multicolumn{6}{|c|}{$\begin{array}{l}\text { Start Time: } 5: 20 \mathrm{pM} \\
\text { End Time: } \quad 10: 50 \mathrm{AM}\end{array}$} & \multicolumn{3}{|l|}{ IWC: } \\
\hline \multicolumn{2}{|c|}{ TEST } & \multicolumn{4}{|c|}{ CONCENTRATION : } & \multicolumn{5}{|l|}{$0 \%$} & 0.0 & 0.0 & $\mathrm{pH}$ & $\mathrm{pH}$ & \multicolumn{5}{|c|}{ TEST CONCENTRATION: } & \multicolumn{5}{|c|}{$6.25 \%$} & D.o & D.0 & $\mathrm{pH}$ & $\mathrm{pH}$ \\
\hline Day A & & B & c & D & $\mathrm{E}$ & $\mathbf{F}$ & G & $\mathbf{H}$ & I & $\mathrm{J}$ & $01 \mathrm{~d}$ & new & old & new & Day A & B & $\mathrm{c}$ & D & $\mathbf{E}$ & $F$ & G & $\mathbf{H}$ & I & $\mathbf{J}$ & old & new & $01 d$ & new \\
\hline 10 & & 0 & 0 & 0 & 0 & 0 & 0 & 0 & 0 & 0 & 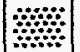 & 9.3 & 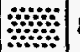 & 8.0 & 10 & 0 & 0 & 0 & 0 & 0 & 0 & 0 & 0 & 0 & $\mid$ & 8.6 & ........ & $|7.6|$ \\
\hline 20 & & 0 & 0 & 0 & 0 & 0 & 0 & 0 & 0 & 0 & 8.1 & 8.7 & 7.8 & 7.9 & 20 & 0 & 0 & 0 & 0 & 0 & 0 & 0 & 0 & 0 & 8.2 & 8.2 & 7.5 & 7.1 \\
\hline 30 & & 0 & 0 & 0 & 0 & 0 & 0 & 0 & 0 & 0 & 8.4 & 8.5 & 7.8 & 7.9 & 30 & 0 & 0 & 0 & 0 & 0 & 0 & 0 & 0 & 0 & $8.2 \mid$ & 8.2 & 7.6 & 7.2 \\
\hline 43 & & 3 & 2 & 3 & 4 & 4 & 5 & 3 & 4 & 4 & 8.5 & 9.3 & 7.8 & 7.9 & 43 & 0 & 3 & 0 & 0 & 0 & 3 & 3 & 0 & 1 & 9.2 & 9.2 & $7.2 \mid$ & 7.6 \\
\hline 50 & & 0 & 1 & 3 & 0 & 0 & 0 & 0 & 0 & 0 & 9.2 & 9.0 & 8.1 & $(8.1)$ & 50 & 0 & 0 & 2 & 5 & 3 & 0 & 0 & 4 & 4 & 8.4 & $8.5 \mid$ & $7.6 \mid$ & 7.2 \\
\hline 60 & 0 & 6 & 4 & 6 & 7 & 0 & 7 & 6 & 4 & 0 & 8.1 & 8.8 & 7.6 & 8.0 & 66 & 4 & 4 & 6 & 6 & 5 & 6 & 6 & 6 & 0 & 8.5 & 8.6 & 7.2 & 7.9 \\
\hline 70 & 0 & 0 & 6 & 0 & 8 & 0 & 8 & 8 & 8 & 7 & 8.4 & 8.4 & $7.7 \mid$ & 7.9 & 78 & 8 & 7 & 0 & 8 & 0 & 8 & 8 & 8 & 7 & 9.2 & 9.3 & $7.9 \mid$ & 8.0 \\
\hline 88 & 8 & 9 & 6 & 7 & 0 & 8 & 0 & 0 & 0 & 9 & $|8.0|$ & $\mid$ & $|7.8|:$ & 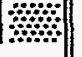 & 80 & 4 & 3 & 9 & 0 & 9 & 0 & 0 & 0 & 9 & 8.6 & $\mid$ & $|8.0|$ & $\mid$ \\
\hline \multicolumn{2}{|c|}{ Tot11 } & 18 & 19 & 19 & 19 & 12 & 20 & 17 & 16 & 20 & 17 & & & & тот17 & 16 & 17 & 17 & 19 & 17 & 17 & 17 & 18 & 21 & 18 & & & \\
\hline \multicolumn{2}{|c|}{ TEST } & CONC & ENTR & ATION & & 12.5 & & & & & D. O & D.o & $\mathrm{pH}$ & $\mathrm{pH}$ & \multicolumn{5}{|c|}{ TEST CONCENTRATION } & \multicolumn{5}{|l|}{$25 \%$} & 0.0 & 0.0 & $\mathbf{p H}$ & $\mathrm{pH}$ \\
\hline Day A & & B & c & D & $\varepsilon$ & $F$ & G & $\mathbf{H}$ & $I$ & $J$ & $01 d$ & new & $01 d$ & new & Day A & B & $c$ & D & $E$ & $F$ & $G$ & и & I & $J$ & old & new & old & new \\
\hline 10 & 0 & 0 & 0 & 0 & 0 & 0 & 0 & 0 & 0 & 0 & 器器 & 8.6 & 前器 & 7.4 & 10 & 0 & 0 & 0 & 0 & 0 & 0 & 0 & 0 & 0 & : & 8.6 & $\mid$ & 7.7 \\
\hline 20 & 0 & 0 & 0 & 0 & 0 & 0 & 0 & 0 & 0 & 0 & 8.5 & 8.3 & 7.6 & 7.2 & 20 & 0 & 0 & 0 & 0 & 0 & 0 & 0 & 0 & 0 & 8.2 & 8.3 & 7.7 & 7.1 \\
\hline 30 & 0 & 0 & 0 & 0 & 0 & 0 & 0 & 0 & 0 & 0 & 8.2 & 8.3 & 7.7 & 7.4 & 30 & 0 & 0 & 0 & 0 & 0 & 0 & 0 & 0 & 0 & 8.3 & 8.3 & 7.7 & 7.4 \\
\hline 44 & 4 & 2 & 0 & 0 & 5 & 0 & 3 & 4 & 0 & 4 & 9.3 & 9.2 & 7.8 & 7.7 & 44 & 3 & 4 & 0 & 0 & 0 & 4 & 3 & 4 & 3 & $|9.2|$ & $9.2 \mid$ & 7.9 & 7.8 \\
\hline 50 & 0 & 0 & 3 & 3 & 2 & 0 & 0 & 1 & 0 & 0 & 8.5 & 8.5 & 7.8 & 7.2 & 50 & 0 & 0 & 0 & 0 & 0 & 0 & 0 & 0 & 0 & 8.4 & $8.5 \mid$ & 7.8 & 7.0 \\
\hline 66 & 6 & 5 & 6 & 6 & 6 & 3 & 5 & 6 & 7 & 0 & 8.6 & 8.7 & 7.9 & 7.9 & 63 & 4 & 3 & 2 & 3 & 2 & 0 & 4 & 4 & $L$ & 8.6 & 8.7 & 8.0 & 8.0 \\
\hline 78 & 8 & 8 & 7 & 0 & 8 & 0 & 8 & 0 & 6 & 8 & 9.2 & 9.3 & $8.0 \mid$ & 8.1 & 78 & 0 & 0 & 0 & 0 & 0 & 0 & 8 & 8 & L & 9.3 & 9.3 & $8.1 \mid$ & 8.1 \\
\hline 80 & 0 & 0 & 0 & 6 & 0 & 7 & 0 & 0 & 0 & 0 & 8.8 & $\mid$ & $|7.8|$ & 前 & 80 & 4 & 0 & 4 & 0 & 1 & 0 & 0 & 0 & I. & 8.6 & $\mid$ & $|8.0|$ & | \\
\hline \multicolumn{2}{|c|}{ TOT18 } & 15 & 16 & 15 & 21 & 10 & 16 & 11 & 13 & 12 & 15 & & & & ToT15 & 11 & 7 & 6 & 3 & 3 & 4 & 15 & 16 & 3 & 9 & & & \\
\hline TEST & & CONC & ENTR & ATION & & $50 \%$ & & & & & 0.0 & 0.0 & $\mathrm{pH}$ & $\mathbf{p H}$ & TEST & CONC & ENTR & ATION & & 1008 & & & & & 0.0 & D.o & $\mathrm{pH}$ & $\mathrm{pH}$ \\
\hline Day A & A & B & $\mathrm{C}$ & D & $\mathbf{E}$ & $F$ & G & H & I & $J$ & old & new & old & new & Day A & $\mathbf{B}$ & c & D & $\mathbf{E}$ & $\mathbf{F}$ & G & H & I & $\mathrm{J}$ & old & new & $01 d$ & new \\
\hline 10 & 0 & 0 & 0 & 0 & 0 & 0 & 0 & 0 & 0 & 0 & 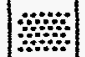 & 8.6 & 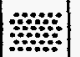 & 7.5 & 10 & 0 & 0 & 0 & 0 & 0 & 0 & o & 0 & 0 & 器器 & 8.6 & 前器 & 3.8 \\
\hline 20 & 0 & 0 & 0 & 0 & 0 & 0 & 0 & 0 & 0 & 0 & 8.4 & 8.4 & 7.7 & 6.9 & 20 & $\mathrm{x}$ & 0 & 0 & 0 & $\mathrm{x}$ & 0 & 0 & 0 & 0 & 8.3 & 8.2 & 7.6 & 5.5 \\
\hline 30 & 0 & 0 & 0 & 0 & 0 & 0 & 0 & 0 & 0 & 0 & 8.3 & 8.4 & 7.7 & 7.2 & 30 & $\mathbf{x}$ & 0 & $\mathrm{x}$ & 0 & $x$ & 0 & 0 & 0 & 0 & 8.2 & 8.3 & 7.8 & 6.4 \\
\hline 43 & 3 & 2 & 0 & 0 & 0 & 0 & 3 & 3 & 4 & 3 & 9.2 & 9.1 & 7.9 & 7.8 & $4 X$ & $x$ & $\mathrm{x}$ & $x$ & $x$ & $\mathrm{x}$ & $x$ & $\mathbf{x}$ & $\mathrm{x}$ & $x$ & 9.0 & 9.0 & 7.8 & 7.8 \\
\hline 50 & 0 & 0 & 0 & 0 & 0 & 0 & 0 & 0 & 0 & 0 & 8.4 & 8.3 & 7.9 & 6.8 & $5 x$ & $x$ & $x$ & $x$ & $x$ & $x$ & $x$ & $x$ & $x$ & $x$ & 8.4 & 前器 & 6.9 & 前 \\
\hline 60 & 0 & 0 & 0 & 0 & 0 & 0 & 0 & 0 & 0 & 0 & $8.6 \mid$ & $8.6 \mid$ & 8.0 & 7.9 & $6 x$ & $x$ & $\mathrm{X}$ & $x$ & $\mathrm{x}$ & $\mathrm{x}$ & $x$ & $x$ & $x$ & $x$ & 受器 & (즘 & 器器 & O \\
\hline 70 & 0 & 0 & 0 & 0 & 0 & 0 & 0 & 0 & 0 & 0 & 9.4 & 9.4 & 8.0 & 8.0 & $7 x$ & $x$ & $x$ & $\mathrm{x}$ & $x$ & $\mathrm{x}$ & $x$ & $x$ & $\mathbf{x}$ & $\mathrm{x}$ & \% & (1) & 営器 & 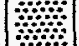 \\
\hline 80 & 0 & 0 & 0 & 0 & 0 & 0 & 0 & 0 & 0 & 0 & $8.8 \mid$ & $\mid$ & $|7.9|$ & 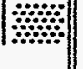 & $8 x$ & $x$ & $x$ & $x$ & $\mathrm{x}$ & $\mathrm{x}$ & $x$ & $x$ & $\mathrm{x}$ & $x$ & 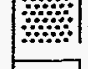 & 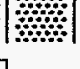 & $\mid$ & $\mid$ \\
\hline TOT & 3 & 2 & 0 & 0 & 0 & 0 & 3 & 3 & 4 & 3 & 2 & & & & Tот 0 & 0 & 0 & 0 & 0 & 0 & 0 & 0 & 0 & 0 & 0 & & & \\
\hline & & TEY & $1 .{ }^{\circ} \mathrm{C}$ & & Time & & & & & TEM. & $\cdot c$ & Tim & & & - & & TEM & $1 .{ }^{\bullet} \mathrm{C}$ & & Time & & & & TEM & $1 . \cdot \mathrm{C}$ & & Time & \\
\hline Day & 0 & 24. & & $05: 0$ & $00 \mathrm{P}$ & & & Day & 2 & 24.1 & & $01: 50$ & PM & & Day & 44 & 24. & & $05:$ & $30 \mathrm{P}$ & & & Day 6 & 6 & 24.7 & & $02: 40$ & $0 \mathrm{PM}$ \\
\hline Day & 1 & 24. & & $04: 0$ & $00 \mathrm{P}$ & & & Day & 3 & 24.3 & & $03: 45$ & $5 \mathrm{PM}$ & & Day & 5 & 24. & & 02: & $20 \mathrm{P}$ & & & Day 7 & 7 & 24.6 & & $10: 50$ & $0 \mathrm{AM}$ \\
\hline
\end{tabular}


SAMPLING INFORMATION

\begin{tabular}{|c|c|c|c|c|c|c|c|c|}
\hline Dilution Water & $20 \% \mathrm{DMW}$ & $09-06-95$ & H & 82 & 69.3 & 183.1 & & \\
\hline Final EfE. 1 & Grab & $09-06-95$ & $09: 45 \mathrm{AM}$ & 8.0 & 4.08 & 44.1 & $<0.05$ & $2.3^{\circ} \mathrm{C}$ \\
\hline Final Eff. 2 & Grab & $09-08-95$ & $09: 15 \mathrm{AM}$ & 10.0 & 32.6 & 86.5 & $<0.05$ & $3.9^{\circ} \mathrm{C}$ \\
\hline Final Eff. 3 & Grab & $09-11-95$ & $08: 10 \mathrm{AM}$ & 6.0 & 8.16 & 48.7 & N/A & $2.7^{\circ} \mathrm{C}$ \\
\hline
\end{tabular}


Results

\begin{tabular}{|l|l|l|l|}
\hline \multicolumn{2}{|l|}{ Client: WSRC } & Sample ID: FHB-018 & \\
\hline Log \#: T4473 & Start Date: $09-07-95$ & Time: & $5: 20$ PM \\
\hline
\end{tabular}

SURVIVAL EFFECTS

Control Effluent

$\begin{array}{lllllll}\text { Effluent Conc. } & \text { SC.DMW } & 6.25 \% & 12.5 \% & 25 \% & 50 \% & 100 \%\end{array}$

$\begin{array}{lllllll}48 \mathrm{hr} \text {. Mortality } & 0 \% & 0 \% & 0 \% & 0 \% & 0 \% & 30 \%\end{array}$

$\begin{array}{lllllll}7 \text { Day Mortality } & 0 \% & 0 \% & 0 \% & 0 \% & 0 \% & 100 \%\end{array}$

Method: Fishers Exact Test

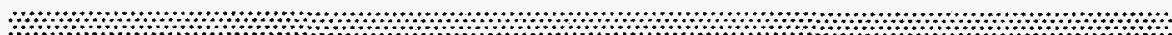

\begin{tabular}{l} 
Acute Toxicity \\
\cline { 5 - 7 }
\end{tabular}

QUALITY CONTROL.

\begin{tabular}{|cccc|}
\hline Standard Toxicant: NaCl & & \\
& & & \\
Central Tendency: & 1.98 & $\mathrm{~g} / \mathrm{L}$ \\
Current Value: & 1.91 & $\mathrm{~g} / \mathrm{L}$ \\
Deviation: & 0.29 & Std Dev units. \\
\hline
\end{tabular}


Results

\begin{tabular}{|l|l|l|l|}
\hline \multicolumn{2}{|l|}{ Client: WSRC } & Sample ID: FHB-018 & IWC: \\
\hline Log \#: T4473 & Start Date: $09-07-95$ & Time: & $5: 20$ PM \\
\hline
\end{tabular}

CHRONIC EFFECTS

TEST CONCENTRATION

Average young / female:

Standard Deviation:

$t=$

Stee1's

$M S D=3.28$

\begin{tabular}{|c|c|c|c|c|c|}
\hline \multirow{2}{*}{$\frac{\text { Control }}{\text { SC.DMW }}$} & \multicolumn{4}{|c|}{ Effluent } & \multirow[b]{2}{*}{$100 \%$} \\
\hline & $6.25 \%$ & $12.5 \%$ & $25 \%$ & $50 \%$ & \\
\hline 17.1 & 17.6 & 14.7 & 8.89 & 1.8 & 0 \\
\hline \multirow[t]{3}{*}{3.21} & 1.43 & 3.33 & 5.42 & 1.62 & 0.00 \\
\hline & -0.3 & 1.64 & 5.47 & 10.5 & 0.00 \\
\hline & 99.5 & 82.5 & 62 & 55 & \\
\hline
\end{tabular}

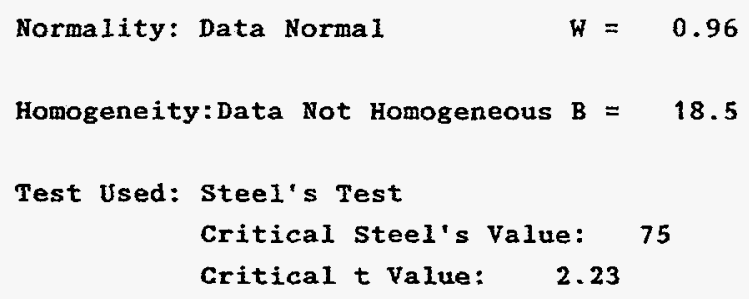




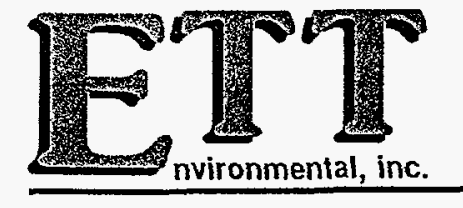

(803) 877-6942 - FAX (803) 877-6938

P.O. Box 16414, Greenville, SC 29606 - 4 Craftsman Court, Greer, SC 29650

7 Day Chronic Definitive Survival and Reproduction Bioassay

Test Organisms: Ceriodaphnia dubia

Method: EPA/600/4-89/001

Facility $=$ westinghouse savannah River company
Sample ID: Frib-o20 Effluent

Date: $8-24-95$ 


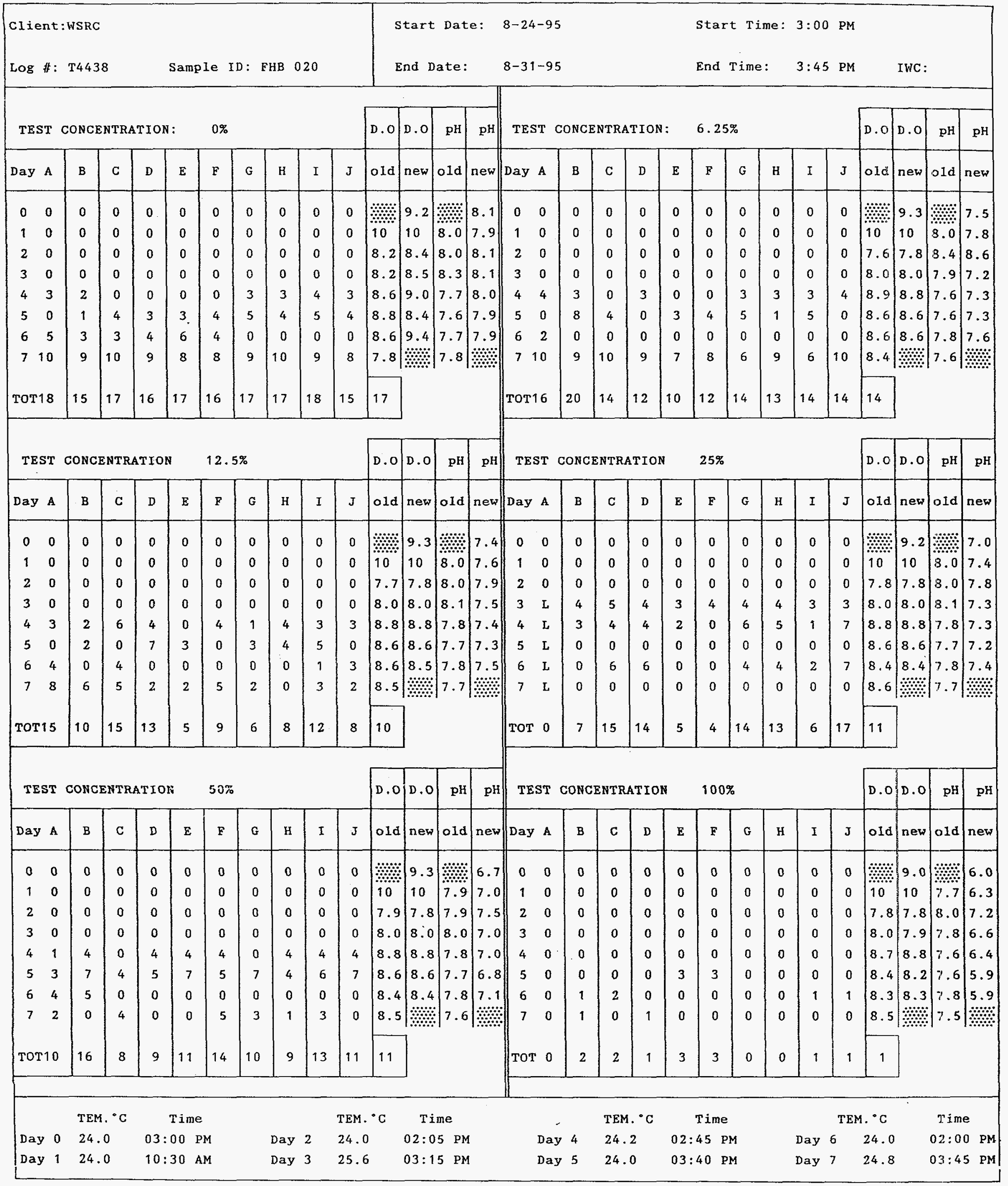


SAMPLING INFORMATION

\begin{tabular}{|c|c|c|c|c|c|c|c|c|}
\hline & Type & Start Date & Time & Hardness $\mathrm{Mg} / \mathrm{L}$ & Alkalinity & Conductivity & Res. Cl & Rec. Temp. \\
\hline Dilution Water & $20 \% \mathrm{DMW}$ & $08-24-95$ & 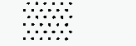 & 90 & 73.4 & 194.3 & & \\
\hline Final Eff. 1 & Grab & $8-23-95$ & $08: 20 \mathrm{AM}$ & 6.0 & 12.24 & 37.7 & $<0.05$ & $0.9^{\circ} \mathrm{C}$ \\
\hline Final Eff. 2 & Grab & $8-25-95$ & & 6.0 & 12.24 & 41.1 & $<0.05$ & $0.9^{\circ} \mathrm{C}$ \\
\hline Final Eff. 3 & Grab & $8-28-95$ & $08: 10 \mathrm{AM}$ & 10.0 & 4.08 & 36.7 & $<0.05$ & $0.9^{\circ} \mathrm{C}$ \\
\hline
\end{tabular}


Results

\begin{tabular}{|l|l|l|l|}
\hline \multicolumn{2}{|l|}{ Client: WSRC } & Sample ID: FHB 020 & \\
\hline Log \#: T4438 & Start Date: $8-24-95$ & Time: & $3: 00$ PM \\
\hline
\end{tabular}

SURVIVAL EFFECTS

Control Effluent

Effluent Conc.

SC. DMW

$6.25 \% \quad 12.5 \%$

$25 \%$

$50 \% \quad 100 \%$

$48 \mathrm{hr}$. Mortality

$\begin{array}{llllll}0 \% & 0 \% & 0 \% & 0 \% & 0 \% & 0 \%\end{array}$

7 Day Mortality

$\begin{array}{llllll}0 \% & 0 \% & 0 \% & 0 \% & 0 \% & 0 \%\end{array}$

Method: Fishers Exact Test

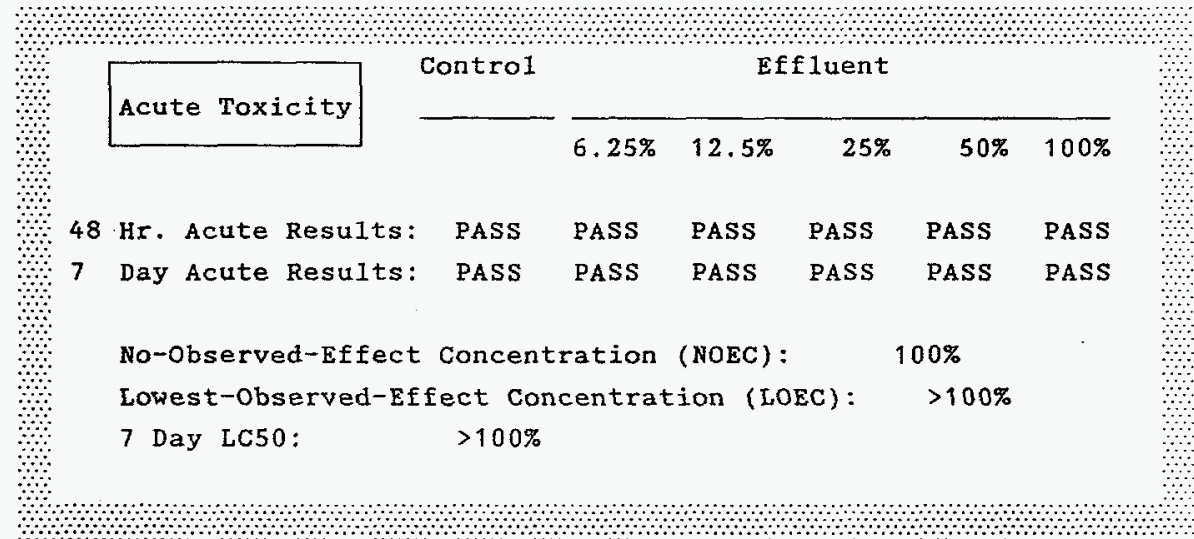

QUALITY CONTROL

\begin{tabular}{|cccc|}
\hline Standard Toxicant: & NaCl & \\
& & & \\
Central Tendency: & 1.98 & $\mathrm{~g} / \mathrm{L}$ \\
Current Value: & 1.91 & $\mathrm{~g} / \mathrm{L}$ \\
Deviation: & 0.29 & Std Dev units. \\
\hline
\end{tabular}


Results

\begin{tabular}{|l|l|l|l|l|}
\hline \multicolumn{2}{|l|}{ Client: WSRC } & Sample ID: FHB 020 & IWC: \\
\hline Log \#: T4438 & Start Date: $8-24-95$ & Time: & $3: 00$ PM \\
\hline
\end{tabular}

CHRONIC EFFECTS

Control Effluent

TEST CONCENTRATION

SC.DMW

\begin{tabular}{lllll}
\hline $6.25 \%$ & $12.5 \%$ & $25 \%$ & $50 \%$ & $100 \%$
\end{tabular}

Average young / female:

$\begin{array}{llll}13.9 & 10.1 & 10.6 & 11.1\end{array}$

standard Deviation:

$\begin{array}{rrrrrr}16.6 & 13.9 & 10.1 & 10.6 & 11.1 & 1.3 \\ 1.07 & 2.69 & 3.54 & 4.98 & 2.51 & 1.16 \\ & 2.06 & 4.96 & 4.49 & 4.2 & 11.67 \\ 68 & 57 & 62 & 58 & 55\end{array}$

Stee1's =

MSD $=3.05$

Normality: Data Not Normal $W=0.87$

Homogeneity: Data Not Homogeneaus $B=26.1$

Test Used: Steel's Test

Critical Steel's Value: 75

Critical t value: 2.31

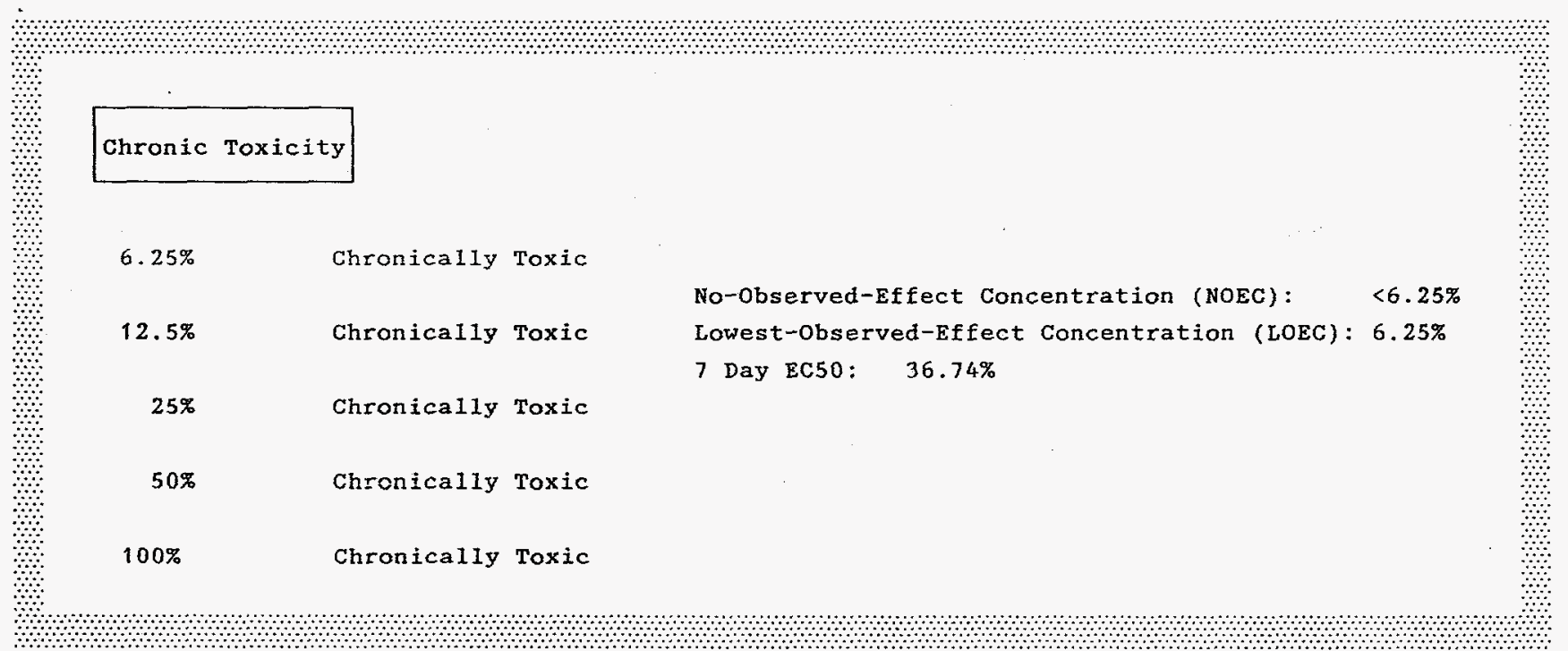


7 Day Ghronic Definitive Survival and Reproduction Bioassay

Test Organisms: Ceriodaphnia dubia

Method: EPA/600/4-89/001

Facility = WSRC

Sample ID = FHB-O21

Date: $\quad 09-07-95$ 
DEFINITIVE SURVIVAL AND REPRODUCTION

\begin{tabular}{|c|c|c|c|c|c|}
\hline Client:WSRC & & Start Date: & $09-07-95$ & Start Time: & $5: 40 \mathrm{PM}$ \\
\hline Log \#: T4474 & Sample ID: FHB-021 & End Date: & $09-15-95$ & End Time: & $11: 00 \mathrm{AM}$ \\
\hline
\end{tabular}

\begin{tabular}{|c|c|c|c|c|c|c|c|c|c|c|c|c|c|c|c|c|c|c|c|c|c|c|c|c|c|c|c|c|}
\hline TEST & \multicolumn{4}{|c|}{ CONCENTRATION : } & \multicolumn{5}{|c|}{$0 \%$} & \multirow{2}{*}{\begin{tabular}{|c|}
0.0 \\
$01 \mathrm{~d}$
\end{tabular}} & \multirow{2}{*}{ D.o } & \multirow{2}{*}{\begin{tabular}{|c|}
$\mathrm{pH}$ \\
$01 \mathrm{~d}$
\end{tabular}} & \multirow{2}{*}{$\begin{array}{c}\mathrm{pH} \\
\text { new }\end{array}$} & \multicolumn{6}{|c|}{ TEST CONCENTRATION: } & \multicolumn{5}{|c|}{$6.25 \%$} & \multirow{2}{*}{$\frac{0.0}{010}$} & \multirow{2}{*}{$\frac{0.0}{\text { new }}$} & \multirow{2}{*}{\begin{tabular}{|c|}
$\mathrm{pH}$ \\
$\mathrm{old}$
\end{tabular}} & \multirow{2}{*}{\begin{tabular}{|l}
$\mathrm{pH}$ \\
new
\end{tabular}} \\
\hline Day A & B & $\mathrm{C}$ & D & $\mathbf{E}$ & $\mathbf{F}$ & G & $\mathbf{H}$ & I & $\mathbf{J}$ & & & & & Day & $A$ & B & $\mathrm{C}$ & $\mathrm{D}$ & $\mathbf{E}$ & $\mathbf{F}$ & $\mathbf{G}$ & $\mathbf{H}$ & $\mathbf{I}$ & $\mathbf{J}$ & & & & \\
\hline 10 & $\mathbf{0}$ & 0 & 0 & 0 & 0 & 0 & 0 & 0 & 0 & \% & 9.3 & 藍 & 8.0 & 1 & 0 & 0 & 0 & 0 & 0 & 0 & 0 & 0 & 0 & 0 & 望 & 8.5 & 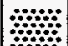 & 7.5 \\
\hline 20 & $\mathbf{0}$ & 0 & 0 & 0 & $\mathbf{0}$ & 0 & 0 & $\mathbf{0}$ & 0 & 8.1 & 8.7 & 7.8 & 7.9 & 2 & 0 & 0 & 0 & 0 & 0 & 0 & 0 & 0 & 0 & 0 & 8.3 & 8.3 & 7.5 & 7.2 \\
\hline 30 & 0 & 0 & 0 & 0 & 0 & 0 & 0 & 0 & 0 & 8.4 & 8.5 & 7.8 & 7.9 & 3 & 0 & 0 & 0 & 0 & 0 & 0 & 0 & 0 & 0 & 0 & 8.4 & 8.5 & 7.7 & 7.4 \\
\hline 43 & 3 & 2 & 3 & 4 & 3 & 5 & 3 & 3 & 4 & 8.5 & 9.3 & 7.8 & 7.9 & 4 & 4 & 5 & 4 & 3 & 2 & 2 & 3 & 2 & 3 & 2 & 9.0 & 9.1 & 7.9 & 7.7 \\
\hline 50 & 0 & 3 & 3 & 0 & 0 & 0 & 0 & 6 & 0 & 9.2 & 9.0 & 8.1 & 8.1 & 5 & 0 & 0 & 0 & 0 & 0 & 0 & 0 & 0 & 0 & 0 & 9.2 & 9.2 & 7.8 & 7.7 \\
\hline 67 & 0 & 0 & 4 & 6 & 1 & 6 & 4 & 0 & 5 & 8.1 & 8.8 & 7.6 & 8.0 & 6 & 5 & 6 & 7 & 0 & 0 & 6 & 2 & 0 & 0 & 0 & 8.5 & 8.4 & 7.8 & 7.8 \\
\hline 70 & 8 & 7 & 0 & 0 & 0 & 8 & 8 & 9 & 8 & 8.4 & 8.4 & 7.7 & 7.9 & 7 & 0 & 0 & 0 & 4 & 7 & 0 & 0 & 0 & 6 & 0 & 9.0 & 9.2 & 8.0 & 8.0 \\
\hline 88 & 8 & 4 & 8 & 8 & 7 & 0 & 0 & 0 & 0 & 8.0 & 翌器 & 7.8 & 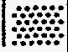 & 8 & 8 & 7 & 7 & 8 & 3 & 8 & 0 & 8 & 8 & 9 & 8.6 & 徨哭 & 8.0 & 前 \\
\hline TOT18 & \begin{tabular}{l|l}
8 & 19
\end{tabular} & 16 & 18 & 18 & 11 & 19 & 15 & 18 & 17 & 17 & & & & TOT & & 18 & 18 & 15 & 12 & 16 & 5 & 10 & 17 & 11 & 14 & & & \\
\hline
\end{tabular}

\begin{tabular}{|c|c|c|c|c|c|c|c|c|c|c|c|}
\hline TEST CONCENTRATION & $12.5 \%$ & D.0 & D.o & $\mathrm{pH}$ & $\mathbf{p H}$ & TEST CONCENTRATION & $25 \%$ & D.o & D.o & ph & $\mathrm{pH}$ \\
\hline
\end{tabular}

\begin{tabular}{|c|c|c|c|c|c|c|c|c|c|c|c|c|c|c|c|c|c|c|c|c|c|c|c|c|c|c|c|c|c|}
\hline Day & $A$ & $\mathbf{B}$ & $\mathrm{C}$ & D & $\mathbf{E}$ & $\boldsymbol{F}$ & G & H & I & $\mathrm{J}$ & old & new & $01 \mathrm{~d}$ & new & Day & $\mathbf{A}$ & $B$ & c & D & $\mathbf{E}$ & $F$ & G & $\mathbf{H}$ & I & $\mathbf{J}$ & $01 d$ & new & old & new \\
\hline 1 & 0 & 0 & 0 & 0 & 0 & 0 & 0 & 0 & 0 & 0 & 器器 & 8.5 & 望然 & 7.4 & 1 & 0 & 0 & 0 & 0 & 0 & 0 & 0 & 0 & 0 & 0 & 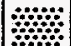 & 8.4 & 前 & 7.5 \\
\hline 2 & 0 & 0 & 0 & 0 & 0 & 0 & 0 & 0 & 0 & 0 & 8.3 & 8.4 & 7.6 & 7.4 & 2 & 0 & 0 & 0 & 0 & 0 & 0 & 0 & 0 & 0 & 0 & 8.4 & 8.4 & 7.6 & 7.4 \\
\hline 3 & 0 & 0 & 0 & 0 & 0 & 0 & 0 & 0 & 0 & 0 & 8.4 & 8.5 & 7.8 & 7.6 & 3 & 0 & 0 & 0 & 0 & 0 & 0 & 0 & 0 & 0 & 0 & 8.4 & 8.4 & 7.8 & 7.6 \\
\hline 4 & 3 & 4 & 3 & 4 & 6 & 3 & 3 & 3 & 4 & 3 & 9.2 & 9.1 & 7.9 & 7.8 & 4 & 0 & 3 & 2 & 3 & $\sigma$ & 5 & 0 & 4 & 3 & 0 & 9.2 & 9.1 & 7.9 & 7.8 \\
\hline 5 & 0 & 0 & 7 & 0 & 0 & 0 & 0 & 6 & 7 & 0 & 9.1 & 9.2 & 7.9 & 7.8 & 5 & 7 & 0 & 0 & 0 & 0 & 1 & 3 & 0 & 0 & 0 & 9.3 & 9.2 & 8.0 & 7.8 \\
\hline 6 & 5 & 5 & 0 & 0 & 0 & 6 & 6 & 0 & 0 & 7 & 8.5 & 8.6 & 7.9 & 7.9 & 6 & 0 & 3 & 0 & 0 & 0 & 2 & 5 & 5 & 4 & 6 & 8.6 & 8.6 & 7.9 & 7.9 \\
\hline 7 & 0 & 0 & 8 & 8 & 0 & 0 & 0 & 8 & 8 & 9 & 9.1 & 9.2 & 8.0 & 8.1 & 7 & 0 & 0 & 0 & 0 & 0 & 0 & 0 & 0 & 8 & 8 & 9.2 & 9.2 & 8.0 & 8.1 \\
\hline 8 & 0 & 7 & 0 & 0 & 7 & 0 & 0 & 0 & 0 & 0 & 8.8 & 前解 & 7.8 & ant & 8 & 4 & 6 & 3 & 4 & 0 & 0 & 0 & 0 & 0 & 0 & 8.6 & 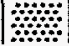 & 7.8 & H \\
\hline
\end{tabular}

\begin{tabular}{|l|l|l|l|l|l|l|l|l|l|l|} 
TOT 8 & 16 & 18 & 12 & 13 & 9 & 9 & 17 & 19 & 19 & 14 \\
\hline
\end{tabular}

\begin{tabular}{|l|l|l|l|l|l|l|l|l|l|l|}
\hline TOT11 & 12 & 5 & 7 & 0 & 8 & 8 & 9 & 15 & 14 & 9 \\
\hline
\end{tabular}

TEST CONCENTRATION 50\%

\begin{tabular}{|l|l|l|l|l|l|l|l|}
\hline D. & D.O & $\mathrm{PH}$ & $\mathrm{PH}$ & TEST CONGENTRATION & 100\%
\end{tabular}

\begin{tabular}{|l|l|l|l|}
\hline D.o & D.O & $\mathrm{pH}$ & $\mathrm{pH}$ \\
\hline
\end{tabular}

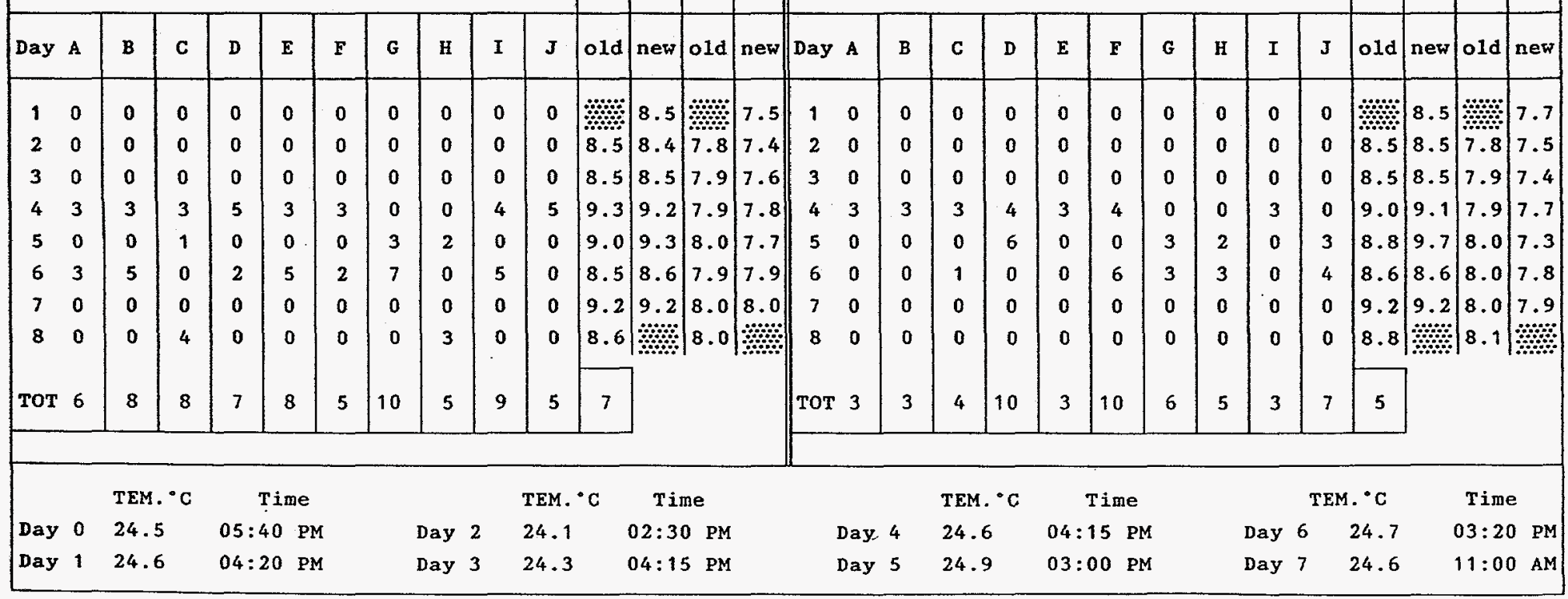


SAMPLING INFORMATION

\begin{tabular}{|c|c|c|c|c|c|c|c|c|}
\hline Dilution Water & $20 \% \mathrm{DMW}$ & $09-06-95$ & H & 82 & 69.3 & 183.1 & & 4 \\
\hline Final Eff. 1 & Grab & $09-06-95$ & $10: 15 \mathrm{AM}$ & 4.0 & 6.12 & 46.1 & $<0.05$ & $2.3^{\circ} \mathrm{C}$ \\
\hline Final Eff. 3 & Grab & $09-11-95$ & $08: 30 \mathrm{AM}$ & 6.0 & 6.12 & 45.4 & $\mathbf{N} / \mathbf{A}$ & $2.7^{\circ} \mathrm{C}$ \\
\hline
\end{tabular}


Results

\begin{tabular}{|l|l|l|l|l|}
\hline \multicolumn{2}{|l|}{ Client: WSRC } & Sample ID: FHB-021 & \\
\hline Log \#: T4474 & Start Date: $09-07-95$ & Time: & $5: 40$ PM \\
\hline
\end{tabular}

SURVIVAL EFFECTS

\begin{tabular}{|c|c|c|c|c|c|c|}
\hline \multirow[b]{2}{*}{ Effluent Conc. } & \multicolumn{2}{|c|}{ Control } & \multicolumn{4}{|c|}{ Effluent } \\
\hline & SC.DMW & $6.25 \%$ & $12.5 \%$ & $25 \%$ & $50 \%$ & $100 \%$ \\
\hline $48 \mathrm{hr}$. Mortality & $0 \%$ & $0 \%$ & $0 \%$ & $0 \%$ & $0 \%$ & $0 x$ \\
\hline 7 Day Mortality & $0 \%$ & $0 \%$ & $0 \%$ & $0 \%$ & $0 \%$ & $0 \%$ \\
\hline
\end{tabular}

Method: Fishers Exact Test

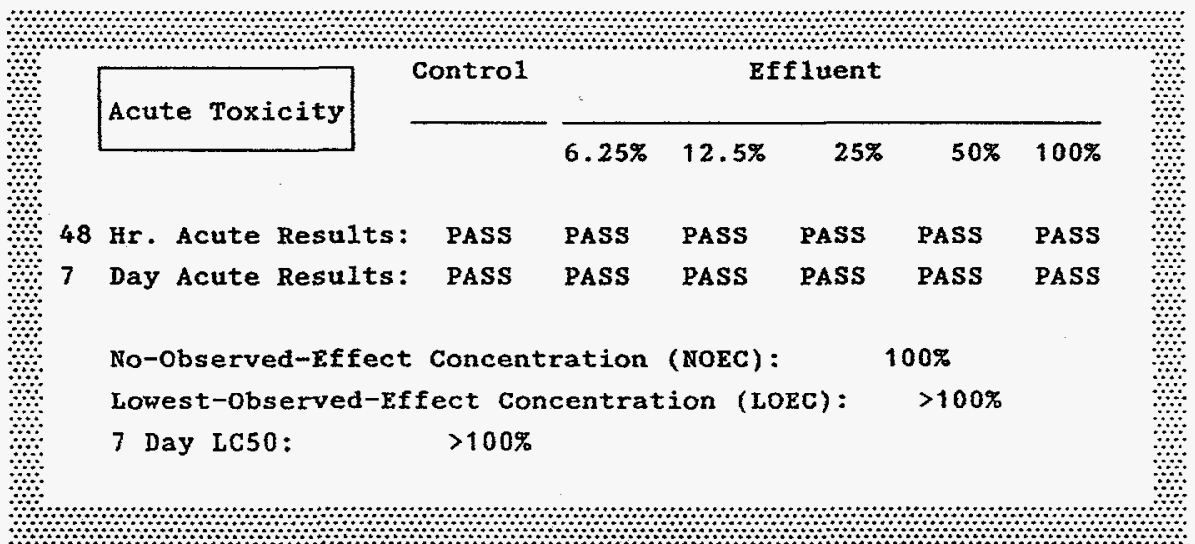

QUALITY CONTROL

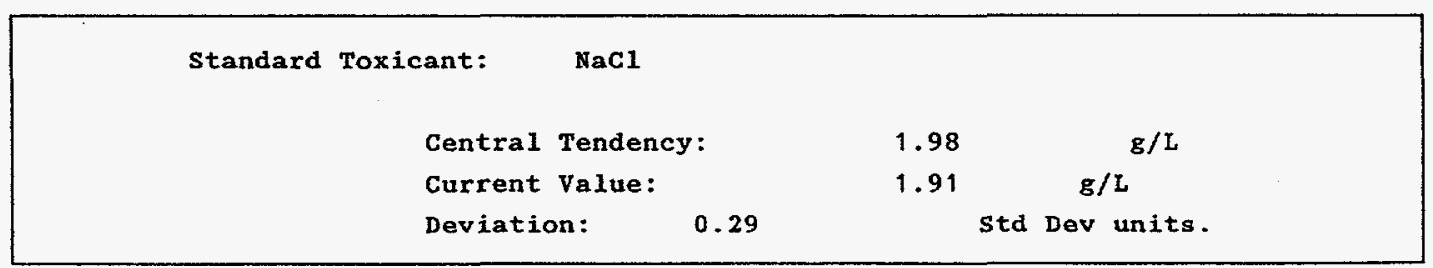


Results

\begin{tabular}{|l|l|l|l|l|}
\hline \multicolumn{2}{|l|}{ Client: WSRC } & Sample ID: FHB-021 & IWC: \\
\hline Log \#: T4474 & Start Date: $09-07-95$ & Time: & $5: 40$ PM \\
\hline
\end{tabular}

CHRONIC EFFECTS

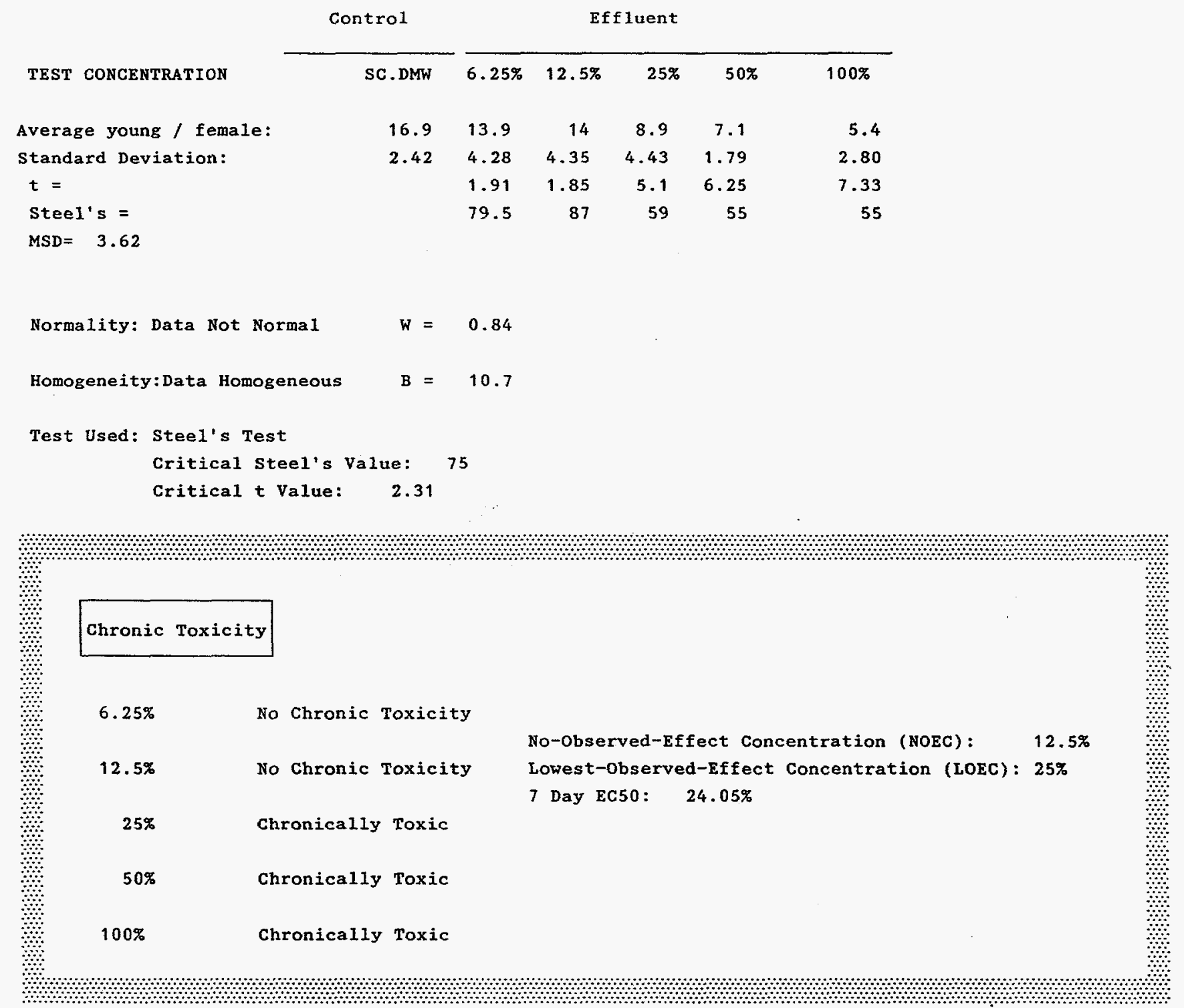




\section{Day Chronic Definitive Survival and Reproduction Bioassay \\ Method: EPA/600/4-89/001}

Test Organism: Ceriodaphnia dubia

Facility:Westinghouse Savannah River Company

Sample ID:FHB-022

Date: $\quad 8 / 24 / 95$ 


\section{CHRONIC DEFINITIVE SURVIVAL AND REPRODUCTION TEST Survival and Reproduction Results}

Client: Westinghouse Savannah River Company

Sample Identification: FHB-022

Test Date: $\quad 8 / 24 / 95$

\begin{tabular}{|c|c|c|c|c|c|c|}
\hline \multicolumn{3}{|c|}{ Reproduction at } & $0.0 \%$ & \multicolumn{4}{c|}{ Concentration } \\
\hline Rep. & Day 3/4 & Day 5 & Day 6 & Day 7 & Day 8 & Total \\
\hline 1 & 3 & 0 & 4 & 0 & 10 & 17 \\
\hline 2 & 0 & 4 & 5 & 0 & 9 & 18 \\
\hline 3 & 0 & 4 & 5 & 0 & 9 & 18 \\
\hline 4 & 1 & 3 & 6 & 0 & 9 & 19 \\
\hline 5 & 0 & 3 & 7 & 0 & 8 & 18 \\
\hline 6 & 0 & 4 & 6 & 9 & 0 & 19 \\
\hline 7 & 2 & 3 & 5 & 7 & 0 & 17 \\
\hline 8 & 4 & 1 & 6 & 0 & 9 & 20 \\
\hline 9 & 0 & 7 & 3 & 9 & 0 & 19 \\
\hline 10 & 4 & 5 & 0 & 7 & 0 & 16 \\
\hline
\end{tabular}

\begin{tabular}{|c|c|c|c|c|c|c|}
\hline \multicolumn{3}{|c|}{ Reproduction at } & $6.25 \%$ & \multicolumn{4}{c|}{ Concentration } \\
\hline Rep. & Day 3/4 & Day 5 & Day 6 & Day 7 & Day 8 & Total \\
\hline 1 & 2 & 1 & 0 & 7 & 13 & 23 \\
\hline 2 & 0 & 3 & 2 & 5 & 9 & 19 \\
\hline 3 & 0 & 3 & 0 & 8 & 8 & 19 \\
\hline 4 & 4 & 0 & 4 & 0 & 6 & 14 \\
\hline 5 & 4 & 0 & 0 & 0 & 5 & 9 \\
\hline 6 & 2 & 0 & 3 & 0 & 10 & 15 \\
\hline 7 & 4 & 5 & 0 & 0 & 9 & 18 \\
\hline 8 & 4 & 3 & 0 & 5 & 8 & 20 \\
\hline 9 & 3 & 4 & 0 & 0 & 8 & 15 \\
\hline 10 & 3 & 0 & 0 & 6 & 11 & 20 \\
\hline
\end{tabular}

\begin{tabular}{|c|c|c|c|c|c|c|}
\hline \multicolumn{3}{|c|}{ Reproduction at } & \multicolumn{1}{|c|}{$12.5 \%$} & \multicolumn{3}{c|}{ Concentration } \\
\hline Rep. & Day 3/4 & Day 5 & Day 6 & Day 7 & Day 8 & Total \\
\hline 1 & 1 & 3 & 0 & 0 & 7 & 11 \\
\hline 2 & 0 & 4 & 0 & 0 & 5 & 9 \\
\hline 3 & 0 & 3 & 0 & 0 & 2 & 5 \\
\hline 4 & 0 & 3 & 0 & 0 & 6 & 9 \\
\hline 5 & 0 & 4 & 0 & 0 & 7 & 11 \\
\hline 6 & 0 & 3 & 0 & 8 & 9 & 20 \\
\hline 7 & 4 & 2 & 0 & 0 & 6 & 12 \\
\hline 8 & 4 & $\mathrm{X}$ & $\mathrm{X}$ & $\mathrm{X}$ & $\mathrm{X}$ & 4 \\
\hline 9 & 4 & 0 & 0 & 0 & 7 & 11 \\
\hline 10 & 3 & 0 & 0 & 0 & 5 & 8 \\
\hline
\end{tabular}

\begin{tabular}{|c|c|c|c|c|c|c|}
\hline \multicolumn{4}{|c|}{ Reproduction at } & $25.0 \%$ & \multicolumn{3}{c|}{ Concentration } \\
\hline Rep. & Day 3/4 & Day 5 & Day 6 & Day 7 & Day 8 & Total \\
\hline 1 & 2 & 2 & 0 & 0 & 6 & 10 \\
\hline 2 & 4 & 0 & 8 & 0 & 2 & 14 \\
\hline 3 & 4 & 0 & 0 & 0 & 0 & 4 \\
\hline 4 & 3 & 1 & 0 & 0 & 6 & 10 \\
\hline 5 & 3 & 0 & 0 & 0 & 0 & 3 \\
\hline 6 & 0 & 0 & 0 & 4 & 4 & 8 \\
\hline 7 & 5 & 3 & 0 & 5 & 2 & 15 \\
\hline 8 & 0 & 4 & 0 & 0 & 0 & 4 \\
\hline 9 & 5 & 0 & 2 & 0 & 1 & 8 \\
\hline 10 & 4 & 0 & 0 & 0 & 7 & 11 \\
\hline
\end{tabular}

\begin{tabular}{|c|c|c|c|c|c|c|}
\hline \multicolumn{4}{|c|}{ Reproduction at } & \multicolumn{4}{c|}{$50 \%$} & \multicolumn{4}{c|}{ Concentration } \\
\hline Rep. & Day 3/4 & Day 5 & Day 6 & Day 7 & Day 8 & Total \\
\hline 1 & 0 & 0 & 0 & 0 & 0 & 0 \\
\hline 2 & 0 & 3 & 7 & 0 & 2 & 12 \\
\hline 3 & 5 & 1 & 0 & 5 & 7 & 18 \\
\hline 4 & 1 & 3 & 0 & 0 & 5 & 9 \\
\hline 5 & 0 & 3 & 0 & 0 & 6 & 9 \\
\hline 6 & 0 & 4 & 0 & 0 & 1 & 5 \\
\hline 7 & 5 & 0 & 0 & 0 & 0 & 5 \\
\hline 8 & 4 & 1 & 0 & 5 & 1 & 11 \\
\hline 9 & $\mathrm{X}$ & $\mathrm{X}$ & $\mathrm{X}$ & $\mathrm{X}$ & $\mathrm{X}$ & 0 \\
\hline 10 & 3 & 2 & 0 & 3 & 4 & 12 \\
\hline
\end{tabular}

\begin{tabular}{|c|c|c|c|c|c|c|}
\hline \multicolumn{9}{|c|}{ Reproduction at } & \multicolumn{1}{|c|}{$100.0 \%$} & \multicolumn{3}{c|}{ Concentration } \\
\hline Rep. & Day 3/4 & Day 5 & Day 6 & Day 7 & Day 8 & Total \\
\hline 1 & 0 & 4 & 0 & 0 & 0 & 4 \\
\hline 2 & 4 & 0 & 6 & 8 & 0 & 18 \\
\hline 3 & 2 & 5 & 0 & 5 & 3 & 15 \\
\hline 4 & 2 & 3 & 0 & 4 & 3 & 12 \\
\hline 5 & 0 & 3 & 5 & 5 & 0 & 13 \\
\hline 6 & 3 & 0 & 0 & 5 & 3 & 11 \\
\hline 7 & 0 & 0 & 4 & 5 & 5 & 14 \\
\hline 8 & 3 & 2 & 0 & 4 & 3 & 12 \\
\hline 9 & & $X$ & $X$ & $X$ & $X$ & 0 \\
\hline 10 & 2 & 2 & 0 & 2 & 0 & 6 \\
\hline
\end{tabular}




\section{CHRONIC DEFINITIVE SURVIVAL AND REPRODUCTION TEST \\ Statistical Analyses}

Client: Westinghouse Savannah River Company

Sample Identification: FHB-022

Test Date: $\quad 8 / 24 / 95$

\begin{tabular}{|c|c|}
\hline \multicolumn{2}{|l|}{ Test for Normality } \\
\hline Test Used: Shapiro Wilks & $\begin{array}{rr}\mathrm{W}= & 0.976 \\
\text { critical } & 0.93\end{array}$ \\
\hline The data are nc & distribution \\
\hline
\end{tabular}

Test for Homogeneity of Variance

Test Used: Bartlett's Test

$\mathrm{B}=$ critical

17.63

15.1

The data are not homogeneous in variance

\section{Effluent Conc. vs. Reproduction}

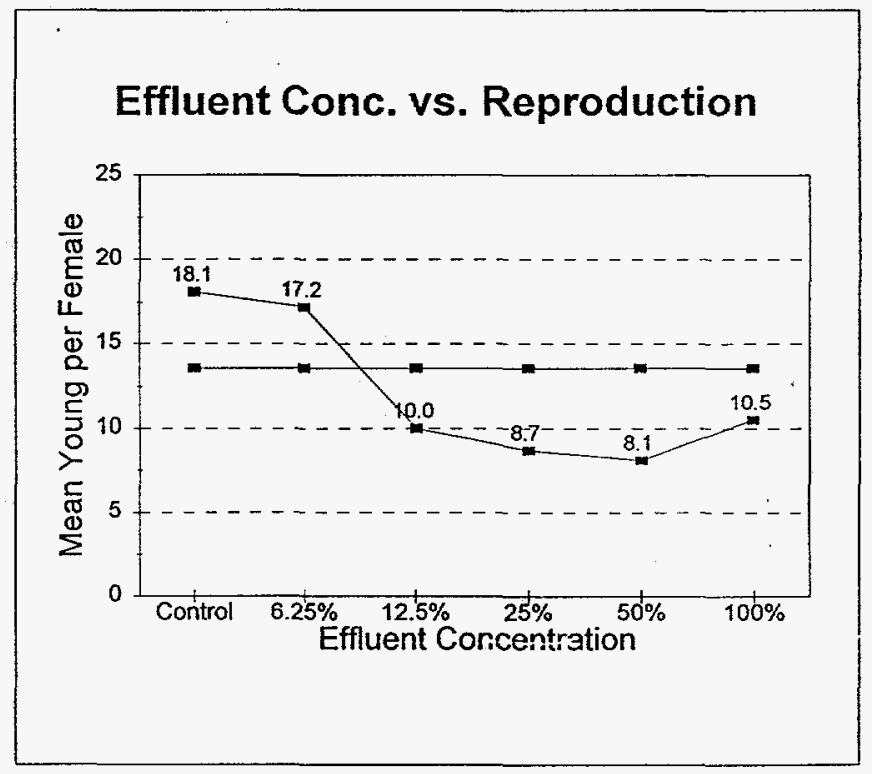

\begin{tabular}{|c|c|c|c|c|c|}
\hline \multicolumn{6}{|c|}{ Test for Differences in Reproduction } \\
\hline \multicolumn{2}{|l|}{ Test Type Used: } & \multicolumn{4}{|c|}{ Wilcoxon's Test } \\
\hline t Test & \multicolumn{5}{|c|}{ Test Concentration } \\
\hline Control & $6.25 \%$ & $12.5 \%$ & $25 \%$ & $50 \%$ & $100 \%$ \\
\hline t Value & 0.46 & 4.15 & 4.82 & 5.13 & 3.90 \\
\hline critical & 2.33 & 2.33 & 2.33 & 2.33 & \\
\hline mean & 17.2 & 10 & 8.7 & 8.1 & 10.5 \\
\hline \multicolumn{6}{|l|}{$\mathrm{MSD}=4.54$} \\
\hline \multirow[t]{2}{*}{ Wilcoxon's Test } & \multicolumn{5}{|c|}{ Test Concentration } \\
\hline & $6.25 \%$ & $12.5 \%$ & $25 \%$ & $50 \%$ & $100 \%$ \\
\hline Rank Sum & 103.5 & 64.5 & 55 & & \\
\hline critical & 75 & 75 & 75 & & \\
\hline
\end{tabular}

\section{Summary}

The No Observed Effect Concentration (NOEC) was $6.25 \%$

The Lowest Observed Effect Concentration (LOEC) was $12.5 \%$

The Chronic Value (ChV) was $8.8 \%$ 


\section{CHRONIC DEFINITIVE SURVIVAL AND REPRODUCTION TEST Water Chemistry}

Client: Westinghouse Savannah River Company

Sample Identification: FHB-022

Test Date: $\quad 8 / 24 / 95$

\section{pH (After Renewal)}

\begin{tabular}{|l|r|r|r|r|r|r|}
\hline \multicolumn{7}{|c|}{ Test Concentration } \\
\hline & Control & $6.25 \%$ & $12.5 \%$ & $25 \%$ & $50 \%$ & $100 \%$ \\
\hline Initial & 8.12 & 7.74 & 7.61 & 7.53 & 7.30 & 6.65 \\
\hline Day 1 & 7.97 & 7.77 & 7.71 & 7.51 & 7.46 & 7.32 \\
\hline Day 2 & 8.19 & 7.48 & 7.62 & 7.63 & 7.57 & 7.44 \\
\hline Day 3 & 8.15 & 7.17 & 7.46 & 7.51 & 7.40 & 7.11 \\
\hline Day 4 & 8.01 & 7.68 & 7.68 & 7.58 & 7.29 & 6.91 \\
\hline Day 5 & 7.95 & 7.53 & 7.59 & 7.59 & 7.11 & 7.10 \\
\hline Day 6 & 7.96 & 7.66 & 7.66 & 7.58 & 7.50 & 7.20 \\
\hline
\end{tabular}

\section{Dissolved Oxygen (After Renewal)}

\begin{tabular}{|l|r|r|r|r|r|r|}
\hline & \multicolumn{7}{|c|}{ Test Concentration } \\
\hline Initial & 9.2 & 9.3 & 9.3 & 9.3 & 9.4 & 9.3 \\
\hline Day 1 & 7.5 & 8.4 & 7.8 & 8.5 & 8.5 & 8.5 \\
\hline Day 2 & 8.4 & 7.7 & 7.8 & 7.8 & 7.9 & 7.9 \\
\hline Day 3 & 8.5 & 7.8 & 8.6 & 7.8 & 7.8 & 7.9 \\
\hline Day 4 & 9.0 & 8.5 & 8.4 & 8.6 & 8.8 & 9.3 \\
\hline Day 5 & 8.4 & 8.5 & 8.4 & 8.4 & 8.2 & 8.4 \\
\hline Day 6 & 9.4 & 8.4 & 8.5 & 8.2 & 8.3 & 8.2 \\
\hline
\end{tabular}

Dilution Water \& Effluent Parameters

\begin{tabular}{|l|r|r|r|r|r|}
\hline & Res Cl. & Alkalin & Hard. & Cond. & \\
\hline & $\mathrm{mg} / \mathrm{L}$ & $\mathrm{mg} / \mathrm{L}$ & $\mathrm{mg} / \mathrm{L}$ & $\mu \mathrm{mhos}$ & \\
\hline Eff. Sample 1 & $<0.05$ & 28.56 & 20 & 69.3 & \\
\hline Eff. Sample 2 & $<0.05$ & 30.6 & 20 & 67.6 & \\
\hline Eff. Sample 3 & $<0.05$ & 8.16 & 10 & 39.9 & \\
\hline & & & & & \\
\hline Dilution Water & & 66.9 & 100 & 189.2 & \\
\hline & & & & & \\
\hline & & & & & \\
\hline
\end{tabular}

\section{$\mathrm{pH}$ (Before Renewal)}

\begin{tabular}{|l|r|r|r|r|r|r|}
\hline \multicolumn{7}{|c|}{ Test Concentration } \\
\hline & Control & $6.25 \%$ & $12.5 \%$ & $25 \%$ & $50 \%$ & $100 \%$ \\
\hline Day 1 & 8.08 & 7.85 & 7.93 & 7.95 & 7.95 & 7.82 \\
\hline Day 2 & 8.05 & 7.73 & 7.86 & 7.91 & 7.92 & 7.92 \\
\hline Day 3 & 8.31 & 7.93 & 8.07 & 8.10 & 8.09 & 8.03 \\
\hline Day 4 & 7.73 & 7.77 & 7.90 & 7.93 & 7.88 & 7.82 \\
\hline Day 5 & 7.66 & 7.74 & 7.79 & 7.81 & 7.55 & 7.58 \\
\hline Day 6 & 7.73 & 7.72 & 7.79 & 7.81 & 7.82 & 7.74 \\
\hline Final & 7.86 & 8.12 & 7.98 & 8.14 & 8.01 & 7.98 \\
\hline
\end{tabular}

Dissolved Oxygen (Before Renewall)

\begin{tabular}{|l|r|r|r|r|r|r|}
\hline \multicolumn{7}{|c|}{ Test Concentration } \\
\hline & Control & $6.25 \%$ & $12.5 \%$ & $25 \%$ & $50 \%$ & $100 \%$ \\
\hline Day 1 & 8.0 & 8.4 & 8.4 & 8.5 & 8.4 & 8.5 \\
\hline Day 2 & 8.2 & 7.6 & 7.7 & 7.8 & 7.8 & 7.9 \\
\hline Day 3 & 8.2 & 7.8 & 7.8 & 7.8 & 7.8 & 7.8 \\
\hline Day 4 & 8.6 & 8.6 & 8.6 & 8.6 & 8.6 & 8.7 \\
\hline Day 5 & 8.8 & 8.5 & 8.6 & 8.4 & 8.3 & 8.4 \\
\hline Day 6 & 8.6 & 8.4 & 8.4 & 8.3 & 8.3 & 8.3 \\
\hline Final & 7.8 & 8.4 & 8.4 & 8.2 & 8.4 & 8.1 \\
\hline
\end{tabular}

\section{Temperature}

\begin{tabular}{|l|r|}
\hline \multicolumn{2}{|c|}{ Incubator ${ }^{\circ} \mathrm{C}$} \\
\hline Initial & 24.0 \\
\hline Day 1 & 24.0 \\
\hline Day 2 & 24.0 \\
\hline Day 3 & 25.6 \\
\hline Day 4 & 24.2 \\
\hline Day 5 & 24.0 \\
\hline Day 6 & 24.0 \\
\hline Final & 24.8 \\
\hline
\end{tabular}


P.O. Box 16414, Greenville, SC 29606 • 4 Craftsman Court, Greer, SC 29650

7 Day Chronic Definitive Survival and Reproduction Bioassay

Test Organisms: Ceriodaphnia dubia

Method: EPA/600/4-89/001

Facility : Westinghouse savannah River company Sample ID: FHB-OZ3 Effluent

Date: $\quad 8-24-95$ 


\begin{tabular}{|c|c|c|c|c|c|c|c|c|c|c|c|c|c|c|c|c|c|c|c|c|c|c|c|c|c|c|}
\hline \multicolumn{4}{|c|}{$\begin{array}{l}\text { Client:WSRC } \\
\text { Log \#: T4440 }\end{array}$} & \multicolumn{2}{|c|}{ Sample } & \multicolumn{4}{|c|}{ ID: $\mathrm{FHB} \quad 023$} & \multicolumn{4}{|c|}{$\begin{array}{l}\text { Start Date: } \\
\text { End Date: }\end{array}$} & \multicolumn{5}{|c|}{$\begin{array}{l}8-24-95 \\
8-31-95\end{array}$} & \multicolumn{5}{|c|}{$\begin{array}{l}\text { Start Time: } 4: 30 \mathrm{PM} \\
\text { End Time: } \quad 2: 00 \mathrm{PM}\end{array}$} & \multicolumn{3}{|l|}{ IWC : } \\
\hline TEST & CONCr & NTRA & TION & & $0 \%$ & & & & & $D .0$ & 0.0 & $\mathrm{pH}$ & $\mathrm{pH} \|$ & \multicolumn{5}{|c|}{ TEST CONCENTRATION : } & \multicolumn{4}{|l|}{$6.25 \%$} & D.o & D.o & $\mathrm{pH}$ & $\mathrm{pH}$ \\
\hline Day A & B & $\mathrm{c}$ & $\mathbf{D}$ & $E$ & $\mathbf{F}$ & G & $\mathrm{H}$ & I & $\mathrm{J}$ & old & new & $01 \mathrm{~d}$ & new & Day A & B & c & $\mathrm{D}$ & $\mathbf{E}$ & $\mathrm{F}$ & H & I & $\mathrm{J}$ & old & new & $0.1 \mathrm{~d}$ & new \\
\hline 00 & 0 & 0 & 0 & 0 & 0 & 0 & 0 & 0 & 0 & 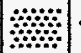 & 9.2 & 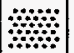 & 8.1 & & 0 & 0 & 0 & 0 & 0 & 0 & 0 & 0 & 前 & 9.3 & 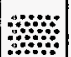 & 7.6 \\
\hline 10 & 0 & 0 & 0 & 0 & 0 & 0 & 0 & 0 & 0 & 10 & 10 & 8.0 & 7.9 & & 0 & 0 & 0 & 0 & 0 & 0 & 0 & 0 & 8.9 & $|9.0| 7$ & 7.6 & 6.8 \\
\hline 20 & 0 & 0 & 0 & 0 & 0 & 0 & 0 & 0 & 0 & $8.2 \mid$ & $8.4 \mid$ & 8.0 & 8.1 & & 0 & 0 & 0 & 0 & 0 & 0 & 0 & 0 & $7.9 \mid$ & 7.9 & B.1 & $|7.9|$ \\
\hline 30 & 0 & 0 & 0 & 0 & 0 & 0 & 0 & 0 & 0 & $|8.2|$ & 8.5 & 8.3 & 8.1 & 30 & 0 & 0 & 0 & 0 & 0 & 0 & 0 & 0 & $8.0 \mid$ & 8.0 & B. & 7.5 \\
\hline 43 & 0 & 0 & 3 & 3 & 4 & 4 & 4 & 5 & 4 & $|8.6|$ & 9.0 & 7.7 & 8.0 & 44 & 2 & 0 & 0 & 0 & 4 & 4 & 3 & 3 & 8.6 & \begin{tabular}{|l|l|}
8.5 &
\end{tabular} & 7.9 & 7.7 \\
\hline $\begin{array}{ll}5 & 5\end{array}$ & 3 & 4 & 5 & 5 & 0 & 0 & 0 & 5 & 0 & $|8.8| 8$ & 8.4 & 7.6 & 7.9 & 50 & 4 & 3 & 4 & 3 & 0 & 0 & 0 & 0 & 8.7 & $|8.8| 7$ & 7.7 & 7.5 \\
\hline 60 & 4 & 6 & 9 & 5 & 6 & 7 & 6 & 2 & 6 & $|8.6| 5$ & $|9.4|$ & 7.7 & 7.9 & 65 & 10 & 0 & 1 & 6 & 6 & 6 & 6 & 5 & 8.4 & 8.4 & 7.5 & 7.3 \\
\hline 710 & 9 & 9 & 0 & 6 & 8 & 9 & 8 & 10 & 9 & 7.8 & | & 7.81 & 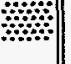 & 77 & 0 & 12 & 7 & 6 & 5 & 8 & 7 & 10 & $8.4:$ & $\mid$ & $7.5 \%$ & | \\
\hline ToT18 & 16 & 19 & 17 & 19 & 18 & 20 & 18 & 22 & 19 & 19 & & & & тот16 & 16 & 15 & 12 & 15 & $15 \quad 17$ & 18 & 16 & 18 & 16 & & & \\
\hline \multicolumn{5}{|c|}{ TEST CONCENTRATION } & $12.5 \%$ & & & & & D.o & D.o & $\mathrm{pH}$ & $\mathrm{pH}$ & \multicolumn{5}{|c|}{ TEST CONCENTRATION } & \multicolumn{4}{|l|}{$25 \%$} & 0.0 & D.o & $\mathrm{pH}$ & $\mathrm{pH}$ \\
\hline Day A & B & $\mathrm{c}$ & $\mathrm{D}$ & $\mathbf{E}$ & $\mathbf{F}$ & $G$ & H & $I$ & $\mathrm{~J}$ & $01 d \mathrm{r}$ & new & old & new & Day A & B & C & D & E & $\mathrm{F}$ & H & I & $\mathbf{J}$ & $01 d \mid$ & new & old & new \\
\hline 00 & 0 & 0 & 0 & 0 & 0 & 0 & 0 & 0 & 0 & : & 9.3 & 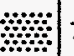 & 7.5 & $\begin{array}{ll}0 & 0\end{array}$ & 0 & 0 & 0 & 0 & 0 & 0 & 0 & 0 & 前 & 9.4 & 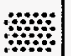 & 7.4 \\
\hline 10 & 0 & 0 & 0 & 0 & 0 & 0 & 0 & 0 & 0 & $|9.0| 5$ & $|9.0|$ & 7.7 & 7.4 & 10 & 0 & 0 & 0 & 0 & 0 & 0 & 0 & 0 & 9.0 & 9.0 & 7.7 & 7.4 \\
\hline 20 & 0 & 0 & 0 & 0 & 0 & 0 & 0 & 0 & 0 & $|7.9|$ & $|7.9|$ & 8.0 & $7.7 \mid$ & 20 & 0 & 0 & 0 & 0 & 0 & 0 & 0 & 0 & 7.8 & 7.9 & 7.4 & 7.5 \\
\hline 30 & 0 & o & 0 & 0 & 0 & 0 & 0 & 0 & 0 & $\left.8.0\right|^{8}$ & $8.0 \mid 8$ & 8.1 & $7.7 \mid$ & 30 & 0 & 0 & 0 & 0 & 0 & 0 & 0 & 0 & 8.1 & $|8.0|$ & 7.5 & 7.7 \\
\hline 44 & 3 & 0 & 5 & 0 & 3 & 4 & 5 & 4 & 5 & $\left.8.4\right|^{8}$ & 8.5 & 7.8 & $7.7 \mid$ & 44 & 0 & 1 & 5 & 0 & 4 & 4 & 3 & 0 & 8.5 & 8.5 & 7.7 & 7.6 \\
\hline 50 & 0 & 4 & 0 & 3 & 0 & 0 & 0 & 0 & 0 & $|8.7|$ & 8.8 & 7.7 & 7.5 & 56 & 2 & 2 & 0 & 3 & 0 & 0 & 0 & 3 & 8.8 & 8.8 & 7.6 & 7.2 \\
\hline 60 & 7 & 0 & 2 & 0 & 4 & 0 & 0 & 5 & 0 & $|8.4|$ & 8.4 & 7.6 & $7.3 \mid$ & 60 & 3 & 0 & 2 & 0 & 3 & 0 & 4 & 2 & 8.4 & 8.3 & 7.2 & 7.2 \\
\hline 712 & 5 & 9 & 10 & 11 & 7 & 7 & 9 & 0 & 8 & $8.4:$ & . & $7.3 \mid$ & & 34 & 5 & 6 & 4 & 7 & 6 & 7 & 5 & 0 & 8.3 & 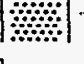 & 7.2 & 前 \\
\hline TOT16 & 15 & 13 & 17 & 14 & 14 & 11 & 14 & 9 & 13 & 14 & & & & TOT14 & 10 & 9 & 11 & 10 & $1 3 \longdiv { 1 2 }$ & 211 & 12 & 5 & 11 & & & \\
\hline \multicolumn{5}{|c|}{ TEST CONCENTRATION } & $50 \%$ & & & & & D.o 1 & D.o & $\mathrm{pH}$ & $\mathrm{pH}$ & \multicolumn{5}{|c|}{ TEST CONCENTRATIUN } & \multicolumn{3}{|l|}{$100 \%$} & & D.o & $D .0$ & $\mathrm{pH}$ & $\mathrm{pH}$ \\
\hline Day A & B & $\mathrm{c}$ & D & $\mathrm{E}$ & $\mathbf{F}$ & G & H & I & $\mathrm{J}$ & $01 d$ & new & $01 d \mathrm{r}$ & new & Day A & B & c & D & $E$ & F & H & I & $\mathrm{J}$ & o1d & new & old & new \\
\hline $\begin{array}{ll}0 & 0\end{array}$ & 0 & 0 & 0 & 0 & 0 & 0 & 0 & 0 & 0 & 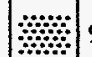 & 9.4 & ו. & 7.2 & $\begin{array}{ll}0 & 0\end{array}$ & 0 & 0 & 0 & 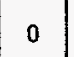 & 0 & 0 & 0 & 0 & . & $|9.4|$ & । & 7.0 \\
\hline 10 & 0 & 0 & 0 & 0 & 0 & 0 & 0 & 0 & 0 & 8.8 & 8.9 & 737 & $7.1 \|$ & 10 & 0 & 0 & 0 & 0 & 0 & $\mathbf{0}$ & 0 & 0 & {$[8.9$} & $\left.8.9\right|^{\circ}$ & 7.7 & 7.2 \\
\hline 20 & 0 & 0 & 0 & 0 & 0 & 0 & 0 & 0 & 0 & 7.9 & $8.0\}$ & 8.0 & 7.1 & 20 & 0 & 0 & 0 & 0 & 0 & 0 & 0 & 0 & 7.7 & 7.8 & 7.2 & 7.3 \\
\hline 30 & 0 & 0 & 0 & 0 & 0 & 0 & 0 & 0 & 0 & 8.1 & 8.2 & 8.1 & 7.6 & $\begin{array}{ll}3 & 0\end{array}$ & 0 & 0 & 0 & 0 & 0 & 0 & 0 & 0 & 7.8 & 8.0 & 7.3 & 7.2 \\
\hline $4 \quad 2$ & 0 & 2 & 4 & 3 & 4 & 4 & 4 & 4 & 4 & 8.4 & 8.6 & 7.7 & 7.3 & 43 & 4 & 4 & 4 & 3 & 3 & 2 & 3 & 4 & 8.4 & 8.8 & 7.2 & 6.9 \\
\hline 50 & 2 & 0 & 0 & 0 & 0 & 0 & 0 & 0 & 0 & $8.7 \mid$ & $8.7 \mid$ & 7.7 & 6.9 & 50 & 0 & 0 & 0 & 0 & 0 & 0 & 0 & 0 & 8.7 & 8.8 & | 5.9 & 5.3 \\
\hline 60 & 6 & 2 & 8 & 4 & 7 & 0 & 0 & 10 & 0 & $8.4 \mid$ & 8.4 & 7.7 & 7.0 & 60 & 6 & 0 & 7 & 4 & 0 & 0 & 0 & 0 & 8.1 & 8.0 & 5.3 & 6.0 \\
\hline 76 & 2 & 3 & 0 & 5 & 0 & 8 & 7 & 0 & 9 & 8.4 & | & 7.6 & 兹 & $7 x$ & 0 & $\mathrm{x}$ & 3 & 5 & $\mathrm{x}$ & 5 & 6 & $\mathrm{x}$ & 8.4 & & $|6.0|$ & \\
\hline \multirow[t]{2}{*}{ TOT 8} & 10 & 7 & 12 & 12 & 11 & 12 & 11 & 14 & 13 & 11 & & & & TOT 3 & 10 & 4 & 14 & 12 & 3 & 7 & 9 & 4 & 7 & & & \\
\hline & TEM & $\cdot^{\circ} \mathrm{c}$ & & Time & & & & & TEM. & & Time & & & & 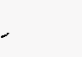 & TEM & $\therefore^{\circ} \mathrm{c}$ & & rime & & & TEM & $1 .{ }^{\circ} \mathrm{C}$ & & Time & \\
\hline Day 0 & 24. & & $04: 3$ & $30 \mathrm{PV}$ & & & Day 2 & & 24.0 & & $2: 50$ & $\mathrm{PM}$ & & Day & & 24 & & $04: 0$ & $00 \mathrm{PM}$ & & Day 6 & & 24.0 & & $03: 50$ & $0 \mathrm{PM}$ \\
\hline Day 1 & 24. & & $01: 0$ & $00 \mathrm{Pr}$ & $M$ & & Day 3 & & 25.6 & & $01: 40$ & PM & & Day & y 5 & 24.0 & & $02: 3$ & $30 \mathrm{PM}$ & & Day 7 & & 24.8 & & $32: 00$ & 0 PM \\
\hline
\end{tabular}


SAMPLING INFORMATION

\begin{tabular}{|c|c|c|c|c|c|c|c|c|}
\hline & Type & Start Date & Time & Hardness $\mathrm{Mg} / \mathrm{L}$ & Alkalinity & Conductivity & Res. Cl & Rec. Temp. \\
\hline Dilution Water & $20 \% \mathrm{DMW}$ & $08-24-95$ & 所 & 90 & 73.4 & 194.3 & ओ & \\
\hline Final Ef $f .1$ & Grab & $8-23-95$ & $09: 00 \mathrm{AM}$ & 10.0 & 12.24 & 37.3 & $<0.05$ & $0.9^{\circ} \mathrm{C}$ \\
\hline Final Eff. 2 & Grab & $8-25-95$ & $09: 25 \mathrm{AM}$ & 10.0 & 8.16 & 8.16 & $<0.05$ & $0.9^{\circ} \mathrm{C}$ \\
\hline Final Eff. 3 & Grab & $8-28-95$ & $07: 50 \mathrm{AM}$ & 12.0 & 2.04 & 29.4 & $<0.05$ & $0.9^{\circ} \mathrm{C}$ \\
\hline
\end{tabular}


Results

\begin{tabular}{|l|l|l|l|l|}
\hline \multicolumn{2}{|l|}{ Client: WSRC } & Sample ID: FHB 023 & \\
\hline Log \#: T4440 & Start Date: $8-24-95$ & Time: & $4: 30$ PM \\
\hline
\end{tabular}

SURVIVAL EFFECTS

Control Effluent

$\begin{array}{lllllll}\text { Effluent Conc. } & \text { SC.DMW } & 6.25 \% & 12.5 \% & 25 \% & 50 \% & 100 \%\end{array}$

$\begin{array}{lllllll}48 \mathrm{hr} \text {. Mortality } & 0 \% & 0 \% & 0 \% & 0 \% & 0 \% & 0 \% \\ 7 \text { Day Mortality } & 0 \% & 0 \% & 0 \% & 0 \% & 0 \% & 40 \%\end{array}$

Method: Fishers Exact Test

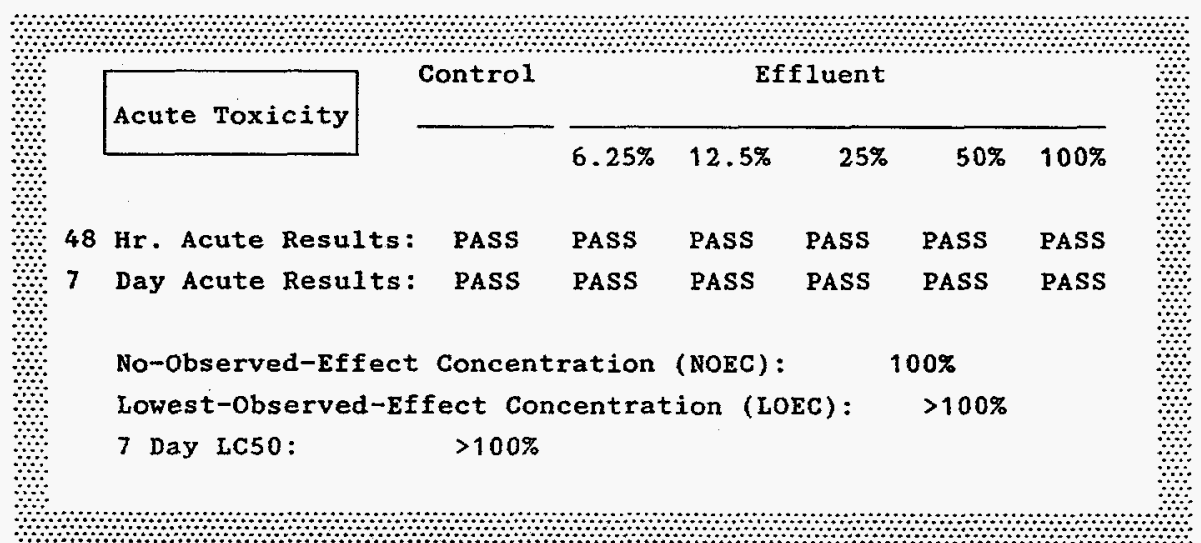

QUALITY CONTROL

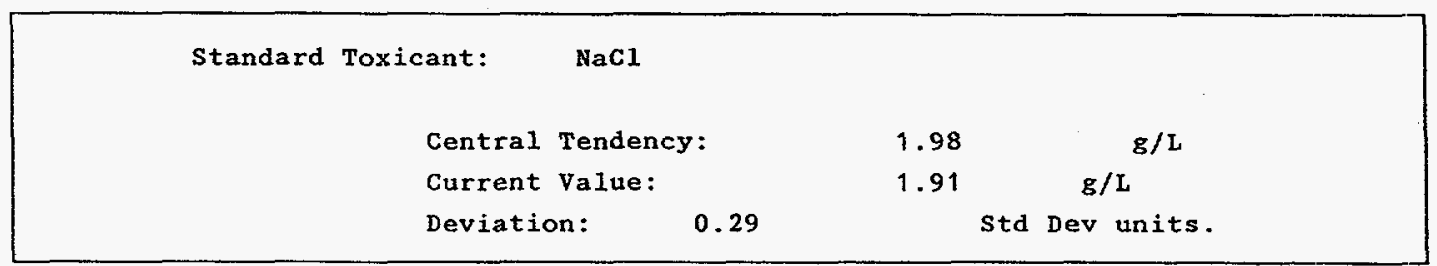


Results

\begin{tabular}{|l|l|l|l|}
\hline \multicolumn{2}{|l|}{ Client: WSRC } & Sample ID: FHB 023 & IWC: \\
\hline Log \#: T4440 & Start Date: $8-24-95$ & Time: & $4: 30$ PM \\
\hline
\end{tabular}

CHRONIC EFFECTS

Control Effluent

TEST CONCENTRATION

$\begin{array}{llllll}\text { SC.DMW } & 6.25 \% & 12.5 \% & 25 \% & 50 \% & 100 \%\end{array}$

$\begin{array}{lrrrrrr}\text { Average young / female: } & 18.6 & 15.8 & 13.6 & 10.7 & 11 & 6.9 \\ \text { Standard Deviation: } & 1.65 & 1.75 & 2.32 & 2.5 & 2.16 & 4.12 \\ \text { t }= & & 2.45 & 4.38 & 6.92 & 6.66 & 10.26 \\ \text { Steel's = } & & 65 & 57 & 55 & 55 & 55 \\ \text { MSD = 2.64 } & & & & & & \end{array}$

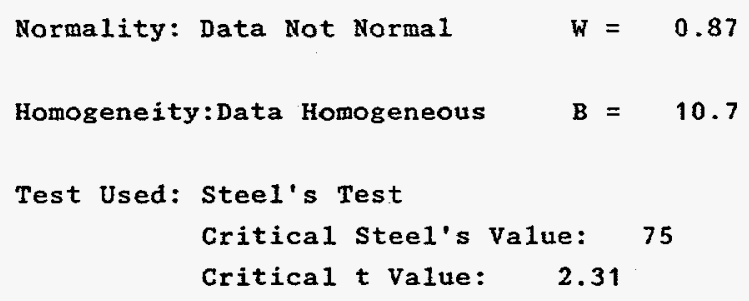


7 Day Chxomic Definitive Survival and Reproduction Bioassay

Test Organisms: Ceriodaphnia dubia

Method: EPA/600/4-89/001

$$
\text { Sample ID: FMC 002H }
$$

Date $=8-17-95$ 
DEFINITIVE SURVIVAL AND REPRODUCTION

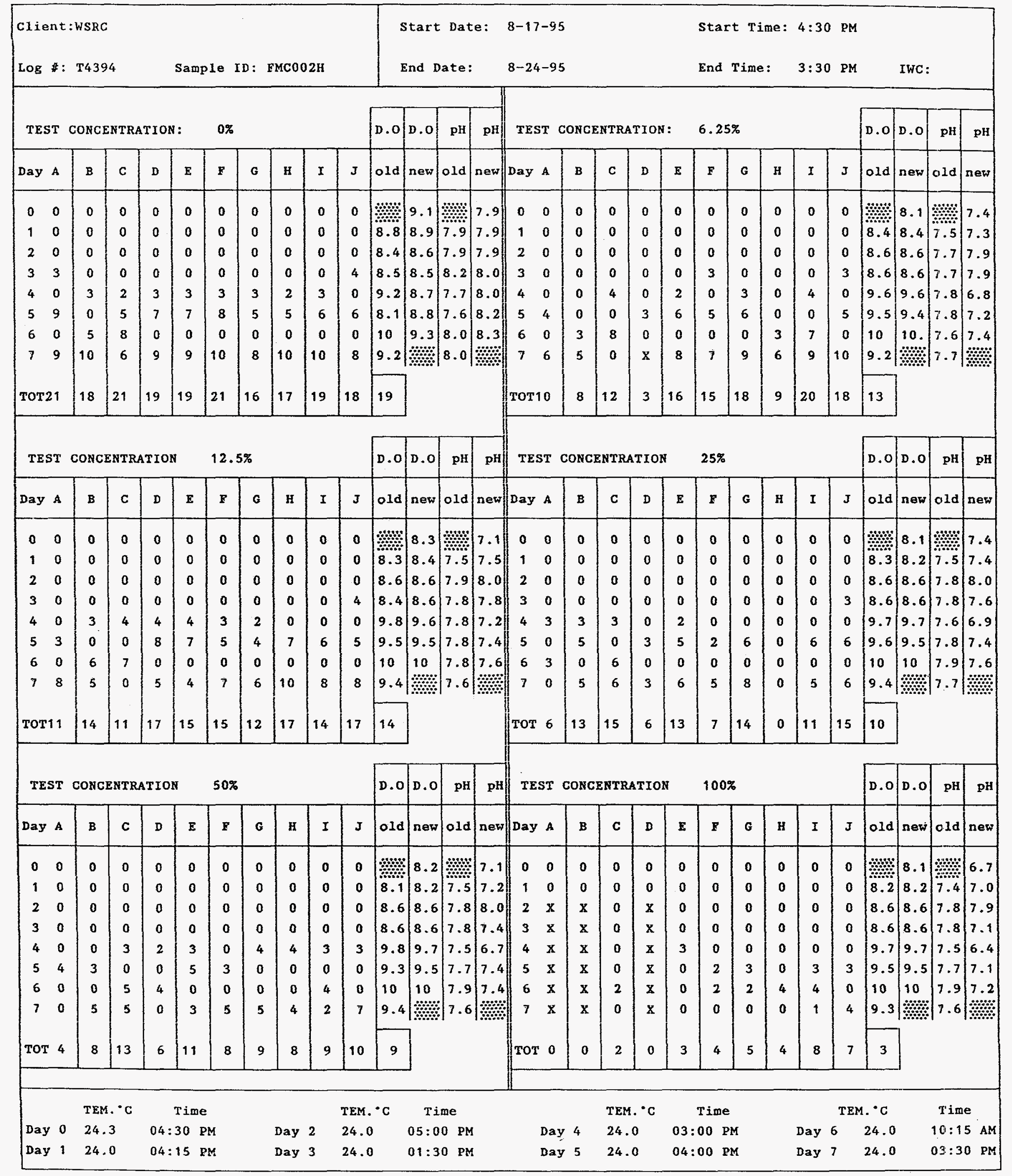


SAMPLING INFORMATION

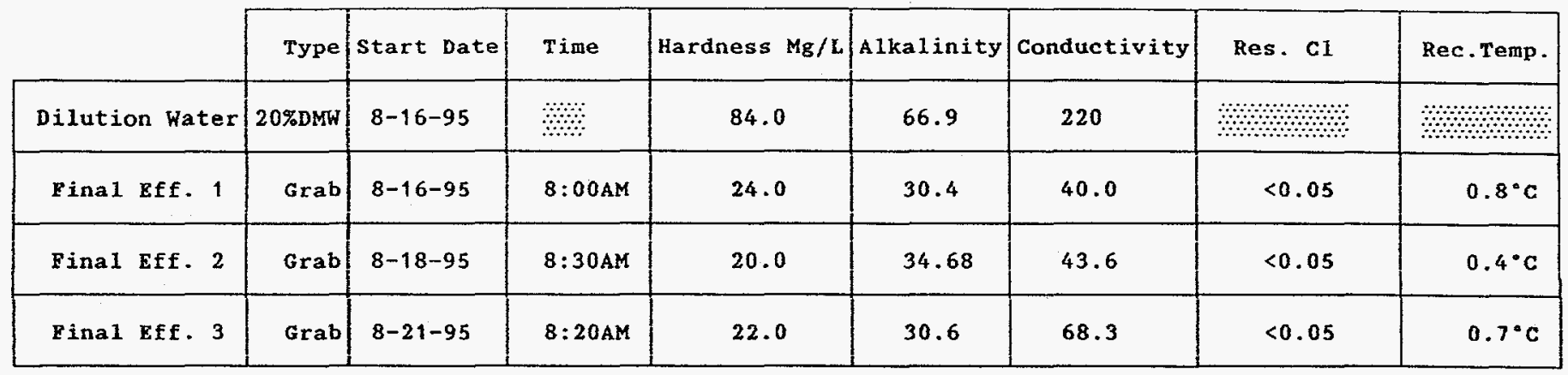


Results

\begin{tabular}{|l|l|l|l|}
\hline \multicolumn{2}{|c|}{ Client: WSRC } & Sample ID: FMC002H & \\
\hline Log \#: T4394 & Start Date: $8-17-95$ & Time: & $4: 30$ PM \\
\hline
\end{tabular}

SURVIVAL EFFECTS

Control Effluent

$\begin{array}{lllllll}\text { Effluent Conc. } & \text { SC.DMW } & 6.25 \% & 12.5 \% & 25 \% & 50 \% & 100 \%\end{array}$

\begin{tabular}{lllllll}
48 hr. Mortality & $0 \%$ & $0 \%$ & $0 \%$ & $0 \%$ & $0 \%$ & $30 \%$ \\
7 Day Mortality & $0 \%$ & $10 \%$ & $0 \%$ & $0 \%$ & $0 \%$ & $30 \%$ \\
\hline
\end{tabular}

Method: Fishers Exact Test

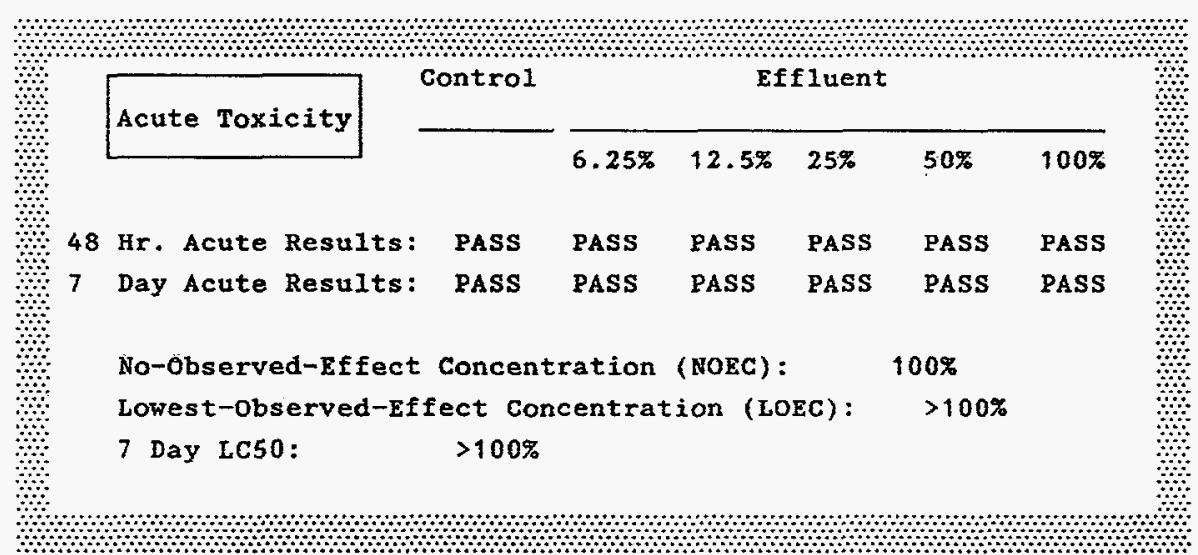

QUALITY CONTROL

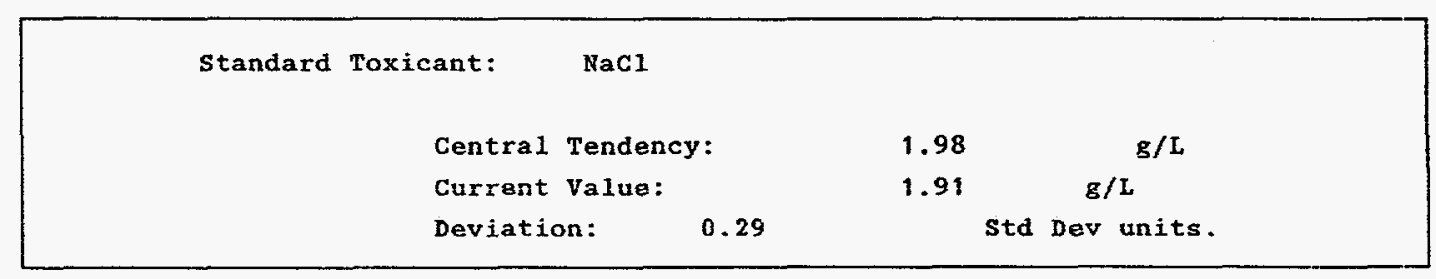


Results

\begin{tabular}{|l|l|l|l|}
\hline \multicolumn{2}{|l|}{ Client: WSRC } & Sample ID: FMC002H & IWC: \\
\hline Log \#: T4394 & Start Date: $8-17-95$ & Time: & $4: 30$ PM \\
\hline
\end{tabular}

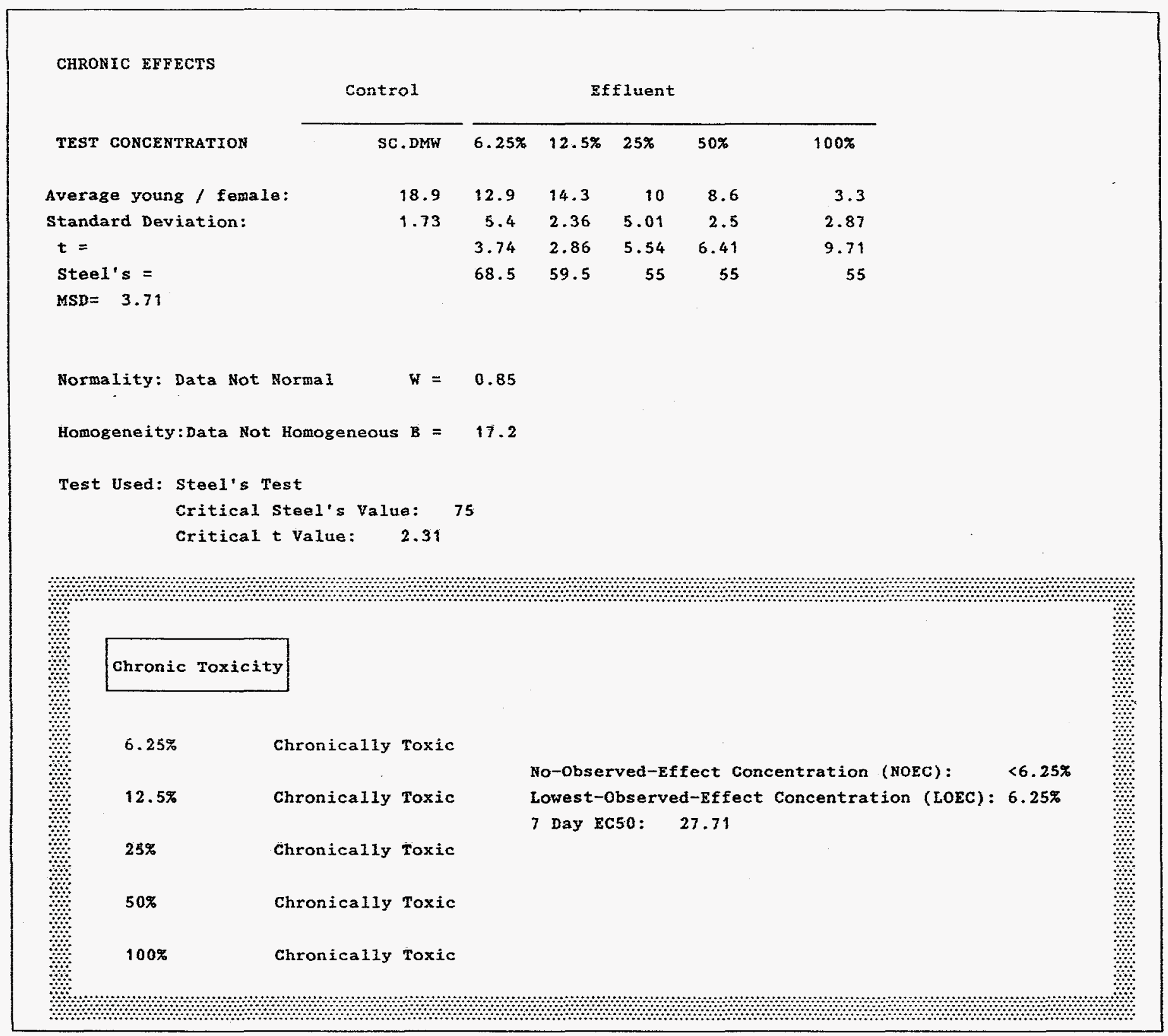


APPENDIX C

WATER TOXICITY RESULTS, FEBRUARY-APRIL 1996 
(864) 877-6942 - FAX (864) 877-6938

P.O. Box 16414, Greenville, SC 29606 - 4 Craftsman Court, Greer, SC 29650

\section{Day Chronic Definitive Survival and Reproduction Bioassay \\ Method: EPA/600/4-89/001}

Test Organism: $\quad$ Ceriodaphnia dubia

Facility:Westinghouse Savannah River Company

Sample ID:BG001

Date: 2/22/96 


\section{CHRONIC DEFINITIVE SURVIVAL AND REPRODUCTION TEST Survival and Reproduction Results}

Client: Westinghouse Savannah River Company

Sample Identification: BG001

Test Date: $\quad 2 / 22 / 96$

\begin{tabular}{|c|c|c|c|c|c|c|}
\hline \multicolumn{2}{|c|}{ Reproduction at } & \multicolumn{3}{c|}{$0 \%$} & \multicolumn{3}{c|}{ Concentration } \\
\hline Rep. & Day 3/4 & Day 5 & Day 6 & Day 7 & Day 8 & Total \\
\hline 1 & 0 & 5 & 0 & 9 & 11 & 25 \\
\hline 2 & 6 & 3 & 8 & 0 & 0 & 17 \\
\hline 3 & 5 & 8 & 8 & 0 & 0 & 21 \\
\hline 4 & 4 & 7 & 8 & 0 & 0 & 19 \\
\hline 5 & 3 & 0 & 9 & 12 & 0 & 24 \\
\hline 6 & 5 & 0 & 8 & 0 & 14 & 27 \\
\hline 7 & 5 & 0 & 7 & 11 & 0 & 23 \\
\hline 8 & 5 & 6 & 9 & 0 & 0 & 20 \\
\hline 9 & 5 & 8 & 0 & 9 & 0 & 22 \\
\hline 10 & 5 & 8 & 0 & 10 & 0 & 23 \\
\hline
\end{tabular}

\begin{tabular}{|c|c|c|c|c|c|c|}
\hline \multicolumn{3}{|c|}{ Reproduction at } & \multicolumn{2}{c|}{$6.25 \%$} & \multicolumn{3}{c|}{ Concentration } \\
\hline Rep. & Day 3/4 & Day 5 & Day 6 & Day 7 & Day 8 & Total \\
\hline 1 & 5 & 6 & 0 & 0 & 13 & 24 \\
\hline 2 & 4 & 7 & 8 & 0 & 0 & 19 \\
\hline 3 & 5 & 6 & 9 & 0 & 0 & 20 \\
\hline 4 & 6 & 6 & 10 & 0 & 0 & 22 \\
\hline 5 & 4 & 8 & 10 & 0 & 0 & 22 \\
\hline 6 & 5 & 0 & 8 & 12 & 0 & 25 \\
\hline 7 & 5 & 0 & 9 & 0 & 12 & 26 \\
\hline 8 & 6 & 8 & 10 & 0 & 0 & 24 \\
\hline 9 & 5 & 9 & 0 & 12 & 0 & 26 \\
\hline 10 & 5 & 8 & 0 & 11 & 0 & 24 \\
\hline
\end{tabular}

\begin{tabular}{|c|c|c|c|c|c|c|}
\hline \multicolumn{2}{|c|}{ Reproduction at } & \multicolumn{1}{c|}{$1.25 \%$} & \multicolumn{3}{c|}{ Concentration } \\
\hline Rep. & Day 3/4 & Day 5 & Day 6 & Day 7 & Day 8 & Total \\
\hline 1 & 5 & 10 & 0 & 8 & 0 & 23 \\
\hline 2 & 3 & 6 & 8 & 0 & 0 & 17 \\
\hline 3 & 5 & 9 & 10 & 0 & 0 & 24 \\
\hline 4 & 4 & 8 & 8 & 0 & 0 & 20 \\
\hline 5 & 5 & 0 & 9 & 12 & 0 & 26 \\
\hline 6 & 4 & 7 & 8 & 0 & 0 & 19 \\
\hline 7 & 4 & 0 & 9 & 0 & 10 & 23 \\
\hline 8 & 4 & 0 & 9 & 0 & 8 & 21 \\
\hline 9 & 5 & 8 & 0 & 12 & 0 & 25 \\
\hline 10 & 4 & $\mathrm{~L}$ & $\mathrm{~L}$ & $\mathrm{~L}$ & $\mathrm{~L}$ & $\mathrm{~L}$ \\
\hline
\end{tabular}

\begin{tabular}{|c|c|c|c|c|c|c|}
\hline \multicolumn{3}{|c|}{ Reproduction at } & \multicolumn{4}{|c|}{$25.0 \%$ Concentration } \\
\hline Rep. & Day $3 / 4$ & Day 5 & Day 6 & Day 7 & Day 8 & Total \\
\hline 1 & 5 & 8 & 0 & 13 & 0 & 26 \\
\hline 2 & 4 & 4 & 9 & 0 & 0 & 17 \\
\hline 3 & 5 & 6 & 7 & $\overline{0}$ & 0 & 18 \\
\hline 4 & 5 & 4 & 0 & 12 & 0 & 21 \\
\hline 5 & 4 & 0 & 10 & 12 & 0 & 26 \\
\hline 6 & 4 & 0 & 0 & 6 & 14 & 24 \\
\hline 7 & 4 & 0 & 9 & 0 & 10 & 23 \\
\hline 8 & 4 & 6 & 9 & 0 & 0 & 19 \\
\hline 9 & 3 & 8 & 0 & 10 & 0 & 21 \\
\hline 10 & 4 & 8 & 0 & 11 & 0 & 23 \\
\hline
\end{tabular}

\begin{tabular}{|c|c|c|c|c|c|c|}
\hline \multicolumn{2}{|c|}{ Reproduction at } & \multicolumn{4}{c|}{$50.0 \%$} & \multicolumn{3}{c|}{ Concentration } \\
\hline Rep. & Day 3/4 & Day 5 & Day 6 & Day 7 & Day 8 & Total \\
\hline 1 & 4 & 7 & 10 & 0 & 0 & 21 \\
\hline 2 & 5 & 7 & 7 & 0 & 0 & 19 \\
\hline 3 & 4 & 9 & 10 & 0 & 0 & 23 \\
\hline 4 & 4 & 5 & 0 & 10 & 0 & 19 \\
\hline 5 & 3 & 0 & 12 & 11 & 0 & 26 \\
\hline 6 & 4 & 0 & 0 & 7 & 0 & 11 \\
\hline 7 & 4 & 0 & 0 & 0 & 7 & 11 \\
\hline 8 & 2 & 6 & 6 & 0 & 10 & 24 \\
\hline 9 & 2 & 5 & 0 & 8 & 0 & 15 \\
\hline 10 & 4 & 9 & 0 & 10 & 0 & 23 \\
\hline
\end{tabular}

\begin{tabular}{|c|c|c|c|c|c|c|}
\hline \multicolumn{6}{|c|}{$100.0 \%$} & \multicolumn{3}{c|}{ Concentration } \\
\hline Reproduction at & Day $3 / 4$ & Day 5 & Day 6 & Day 7 & Day 8 & Total \\
\hline 1 & $\mathrm{X}$ & $\mathrm{X}$ & $\mathrm{X}$ & $\mathrm{X}$ & $\mathrm{X}$ & 0 \\
\hline 2 & $\mathrm{X}$ & $\mathrm{X}$ & $\mathrm{X}$ & $\mathrm{X}$ & $\mathrm{X}$ & 0 \\
\hline 3 & $\mathrm{X}$ & $\mathrm{X}$ & $\mathrm{X}$ & $\mathrm{X}$ & $\mathrm{X}$ & 0 \\
\hline 4 & $\mathrm{X}$ & $\mathrm{X}$ & $\mathrm{X}$ & $\mathrm{X}$ & $\mathrm{X}$ & 0 \\
\hline 5 & $\mathrm{X}$ & $\mathrm{X}$ & $\mathrm{X}$ & $\mathrm{X}$ & $\mathrm{X}$ & 0 \\
\hline 6 & $\mathrm{X}$ & $\mathrm{X}$ & $\mathrm{X}$ & $\mathrm{X}$ & $\mathrm{X}$ & 0 \\
\hline 7 & $\mathrm{X}$ & $\mathrm{X}$ & $\mathrm{X}$ & $\mathrm{X}$ & $\mathrm{X}$ & 0 \\
\hline 8 & $\mathrm{X}$ & $\mathrm{X}$ & $\mathrm{X}$ & $\mathrm{X}$ & $\mathrm{X}$ & 0 \\
\hline 9 & $\mathrm{X}$ & $\mathrm{X}$ & $\mathrm{X}$ & $\mathrm{X}$ & $\mathrm{X}$ & 0 \\
\hline 10 & $\mathrm{X}$ & $\mathrm{X}$ & $\mathrm{X}$ & $\mathrm{X}$ & $\mathrm{X}$ & 0 \\
\hline
\end{tabular}




\section{CHRONIC DEFINITIVE SURVIVAL AND REPRODUCTION TEST Statistical Analyses}

Client: Westinghouse Savannah River Company

Sample Identification: BG001

Test Date: $\quad$ 2/22/96

\begin{tabular}{|lrr}
\hline Test for Normality & & \\
& $\mathrm{W}=$ & 0.973 \\
Test Used: Shapiro Wilks & critical & 0.93
\end{tabular}

The data are normal in distribution
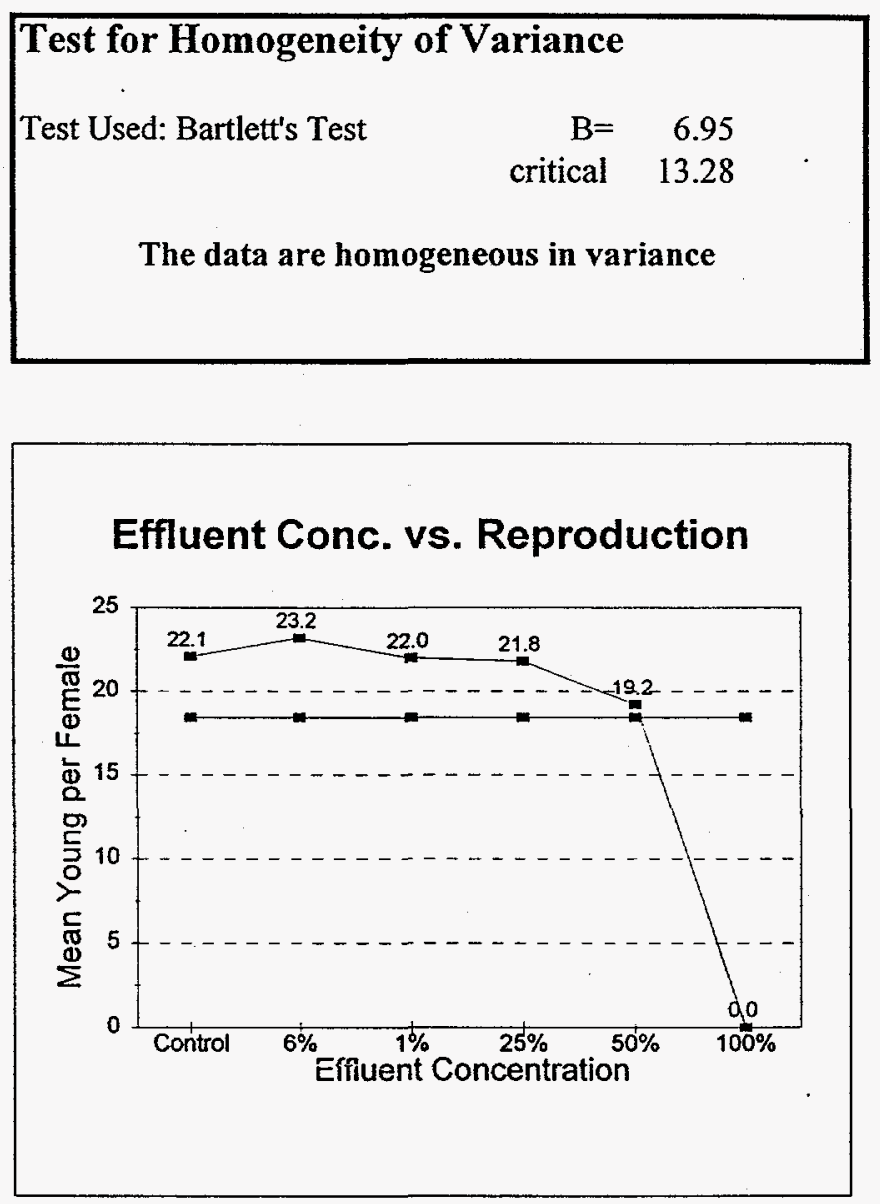

Summary

Sample $\mathrm{pH}$ was acidic; toxicity may be due to acidity 


\section{CHRONIC DEFINITIVE SURVIVAL AND REPRODUCTION TEST Water Chemistry}

Client: Westinghouse Savannah River Company

Sample Identification: BG001

Test Date: $\quad$ 2/22/96

pH (After Renewal)

\begin{tabular}{|l|r|r|r|r|r|r|}
\hline \multicolumn{7}{|c|}{ Test Concentration } \\
\hline & Control & $6.25 \%$ & $1.25 \%$ & $25 \%$ & $50.0 \%$ & $100 \%$ \\
\hline Initial & 8.04 & 6.79 & 7.55 & 6.23 & 7.06 & 4.97 \\
\hline Day 1 & 8.09 & 7.19 & 7.36 & 7.31 & 6.98 & 4.90 \\
\hline Day 2 & 8.00 & 6.87 & 7.46 & 7.18 & 6.97 & 4.99 \\
\hline Day 3 & 8.02 & 7.89 & 7.98 & 8.03 & 8.01 & \\
\hline Day 4 & 8.03 & 7.73 & 7.58 & 7.31 & 6.71 & \\
\hline Day 5 & 8.08 & 7.69 & 7.47 & 7.34 & 6.81 & \\
\hline Day 6 & 8.11 & 7.83 & 7.61 & 7.41 & 7.20 & \\
\hline
\end{tabular}

Dissolved Oxygen (After Renewal)

\begin{tabular}{|l|r|r|r|r|r|r|}
\hline \multicolumn{7}{|c|}{ Test Concentration } \\
\hline & Control & $6.25 \%$ & $1.25 \%$ & $25 \%$ & $50.0 \%$ & $100 \%$ \\
\hline Initial & 8.6 & 8.4 & 8.6 & 8.4 & 8.4 & 8.2 \\
\hline Day 1 & 8.4 & 8.6 & 8.4 & 8.4 & 84.0 & 8.0 \\
\hline Day 2 & 8.6 & 8.4 & 8.4 & 8.3 & 8.3 & 8.1 \\
\hline Day 3 & 8.5 & 8.5 & 8.5 & 8.6 & 8.6 & \\
\hline Day 4 & 8.7 & 8.6 & 8.6 & 8.5 & 8.6 & \\
\hline Day 5 & 8.2 & 8.6 & 8.4 & 8.5 & 8.6 & \\
\hline Day 6 & 8.9 & 8.4 & 8.4 & 8.6 & 8.4 & \\
\hline
\end{tabular}

Dilution Water \& Effluent Parameters

\begin{tabular}{|l|r|r|r|r|r|}
\hline & Res Cl. & Alkalin & Hard. & Cond. & \\
\hline & $\mathrm{mg} / \mathrm{L}$ & $\mathrm{mg} / \mathrm{L}$ & $\mathrm{mg} / \mathrm{L}$ & $\mu \mathrm{mhos}$ & \\
\hline Eff. Sample 1 & 0.2 & 0.01 & 16 & 29.5 & \\
\hline Eff. Sample 2 & $<.05$ & 2.03 & 10 & 16.0 & \\
\hline Eff. Sample 3 & $<.05$ & 2.03 & 4 & 15.9 & \\
\hline & & & & & \\
\hline Dilution Water & & 100 & 69 & 209 & \\
\hline & & & & & \\
\hline & & & & & \\
\hline
\end{tabular}

pH (Before Renewal)

\begin{tabular}{|l|r|r|r|r|r|r|}
\hline \multicolumn{7}{|c|}{ Test Concentration } \\
\hline & Control & $6.25 \%$ & $1.25 \%$ & $25 \%$ & $50.0 \%$ & $100 \%$ \\
\hline Day 1 & 7.93 & 7.48 & 7.61 & 7.65 & 7.71 & 7.02 \\
\hline Day 2 & 8.14 & 7.37 & 7.86 & 7.73 & 7.80 & 6.98 \\
\hline Day 3 & 8.26 & 8.12 & 8.17 & 8.16 & 8.02 & \\
\hline Day 4 & 7.73 & 8.01 & 8.06 & 8.10 & 7.96 & \\
\hline Day 5 & 8.07 & 7.96 & 8.01 & 7.99 & 7.89 & \\
\hline Day 6 & 8.05 & 8.02 & 7.93 & 7.90 & 7.88 & \\
\hline Final & 8.20 & 8.31 & 8.24 & 8.35 & 8.10 & \\
\hline
\end{tabular}

Dissolved Oxygen (Before Renewal)

\begin{tabular}{|l|r|r|r|r|r|r|}
\hline \multicolumn{7}{|c|}{ Test Concentration } \\
\hline & Control & $6.25 \%$ & $1.25 \%$ & $25 \%$ & $50.0 \%$ & $100 \%$ \\
\hline Day 1 & 8.3 & 8.3 & 8.4 & 8.2 & 8.3 & 8.0 \\
\hline Day 2 & 8.2 & 8.4 & 8.3 & 8.0 & 8.2 & 8.0 \\
\hline Day 3 & 8.5 & 8.6 & 8.6 & 8.6 & 8.5 & \\
\hline Day 4 & 8.2 & 8.4 & 8.4 & 8.2 & 8.3 & \\
\hline Day 5 & 8.4 & 8.3 & 8.2 & 8.3 & 8.4 & \\
\hline Day 6 & 8.5 & 8.2 & 8.0 & 8.2 & 8.1 & \\
\hline Final & 8.6 & 8.1 & 8.0 & 8.2 & 8.0 & \\
\hline
\end{tabular}

Temperature
\begin{tabular}{|l|r|}
\hline Incubator ${ }^{\circ} \mathrm{C}$ \\
\hline Initial & 26.0 \\
\hline Day 1 & 25.8 \\
\hline Day 2 & 24.8 \\
\hline Day 3 & 24.1 \\
\hline Day 4 & 26.0 \\
\hline Day 5 & 24.0 \\
\hline Day 6 & 25.1 \\
\hline Final & 24.4 \\
\hline
\end{tabular}


(864) 877-6942 - FAX (864) 877-6938

P.O. Box 16414, Greenville, SC 29606 - 4 Craftsman Court, Greer, SC 29650

\section{Day Chronic Definitive Survival and Reproduction Bioassay \\ Method: EPA/600/4-89/001}

Test Organism: Ceriodaphnia dubia

Facility:Westinghouse Savannah River Company

Sample ID:BG-002

Date: $\quad 2 / 22 / 96$ 


\section{CHRONIC DEFINITIVE SURVIVAL AND REPRODUCTION TEST Survival and Reproduction Results}

Client: Westinghouse Savannah River Company

Sample Identification: BG-002

Test Date: $\quad 2 / 22 / 96$

\begin{tabular}{|c|c|c|c|c|c|c|}
\hline \multicolumn{3}{|c|}{ Reproduction at } & $0.0 \%$ & \multicolumn{3}{c|}{ Concentration } \\
\hline Rep. & Day 3/4 & Day 5 & Day 6 & Day 7 & Day 8 & Total \\
\hline 1 & 5 & 0 & 0 & 10 & 12 & 27 \\
\hline 2 & 4 & 6 & 0 & 11 & 0 & 21 \\
\hline 3 & 5 & 7 & 8 & 0 & 0 & 20 \\
\hline 4 & 4 & 7 & 0 & 9 & 0 & 20 \\
\hline 5 & 3 & 0 & 8 & 0 & 8 & 19 \\
\hline 6 & 4 & 5 & 8 & 0 & 0 & 17 \\
\hline 7 & 4 & 0 & 0 & 12 & 10 & 26 \\
\hline 8 & 5 & 0 & 0 & 0 & 9 & 14 \\
\hline 9 & 4 & 0 & 0 & 6 & 10 & 20 \\
\hline 10 & 6 & 7 & 8 & 0 & 0 & 21 \\
\hline
\end{tabular}

\begin{tabular}{|c|c|c|c|c|c|c|}
\hline \multicolumn{9}{|c|}{ Reproduction at } & $6.25 \%$ & \multicolumn{4}{c|}{ Concentration } \\
\hline Rep. & Day 3/4 & Day 5 & Day 6 & Day 7 & Day 8 & Total \\
\hline 1 & 4 & 9 & 8 & 0 & 0 & 21 \\
\hline 2 & 5 & 6 & 0 & 11 & 0 & 22 \\
\hline 3 & 5 & 0 & 8 & 14 & 0 & 27 \\
\hline 4 & 4 & 7 & 0 & 8 & 0 & 19 \\
\hline 5 & 6 & 0 & 10 & 10 & 0 & 26 \\
\hline 6 & 5 & 9 & 10 & 0 & 0 & 24 \\
\hline 7 & 5 & 3 & 0 & 13 & 0 & 21 \\
\hline 8 & 5 & 0 & 8 & 10 & 0 & 23 \\
\hline 9 & 4 & 0 & 0 & 8 & 0 & 12 \\
\hline 10 & 6 & 8 & 9 & 0 & 0 & 23 \\
\hline
\end{tabular}

\begin{tabular}{|c|c|c|c|c|c|c|}
\hline \multicolumn{9}{|c|}{ Reproduction at } & \multicolumn{4}{|c|}{$12.5 \%$} & \multicolumn{4}{c|}{ Concentration } \\
\hline Rep. & Day 3/4 & Day 5 & Day 6 & Day 7 & Day 8 & Total \\
\hline 1 & 5 & 7 & 0 & 12 & 0 & 24 \\
\hline 2 & 5 & 0 & 0 & 13 & 0 & 18 \\
\hline 3 & 5 & 0 & 8 & 14 & 0 & 27 \\
\hline 4 & 4 & 4 & 0 & 13 & 0 & 21 \\
\hline 5 & 5 & 0 & 10 & 0 & 0 & 15 \\
\hline 6 & 6 & 6 & 8 & 0 & 0 & 20 \\
\hline 7 & 5 & 0 & 0 & 8 & 0 & 13 \\
\hline 8 & 4 & 0 & 7 & 12 & 0 & 23 \\
\hline 9 & 5 & 0 & 0 & 13 & 6 & 24 \\
\hline 10 & 6 & 8 & 0 & 0 & 0 & 14 \\
\hline
\end{tabular}

\begin{tabular}{|c|c|c|c|c|c|c|}
\hline \multicolumn{3}{|c|}{ Reproduction at } & $25.0 \%$ & \multicolumn{3}{c|}{ Concentration } \\
\hline Rep. & Day 3/4 & Day 5 & Day 6 & Day 7 & Day 8 & Total \\
\hline 1 & 4 & 6 & 0 & 13 & 0 & 23 \\
\hline 2 & 5 & 0 & 0 & 12 & 0 & 17 \\
\hline 3 & 5 & 0 & 10 & 12 & 0 & 27 \\
\hline 4 & 4 & 4 & 10 & 0 & 0 & 18 \\
\hline 5 & 6 & 0 & 10 & 10 & 0 & 26 \\
\hline 6 & 3 & 9 & 8 & 0 & 0 & 20 \\
\hline 7 & 4 & 5 & 0 & 14 & 0 & 23 \\
\hline 8 & 5 & 0 & $x$ & $x$ & $x$ & 5 \\
\hline 9 & 5 & 7 & 0 & 10 & 0 & 22 \\
\hline 10 & 5 & 7 & 7 & 0 & 0 & 19 \\
\hline
\end{tabular}

\begin{tabular}{|c|c|c|c|c|c|c|}
\hline \multicolumn{3}{|c|}{ Reproduction at } & \multicolumn{3}{c|}{$5 \%$} & \multicolumn{3}{c|}{ Concentration } \\
\hline Rep. & Day 3/4 & Day 5 & Day 6 & Day 7 & Day 8 & Total \\
\hline 1 & 4 & 4 & 8 & 0 & 0 & 16 \\
\hline 2 & 5 & 6 & 0 & 24 & 0 & 35 \\
\hline 3 & 4 & 0 & 0 & 16 & 0 & 20 \\
\hline 4 & 4 & 6 & 0 & 18 & 0 & 28 \\
\hline 5 & 5 & 1 & 6 & 10 & 0 & 22 \\
\hline 6 & 4 & 8 & 7 & 0 & 0 & 19 \\
\hline 7 & 3 & 0 & 0 & 10 & 0 & 13 \\
\hline 8 & 4 & 5 & 0 & 14 & 0 & 23 \\
\hline 9 & 4 & 6 & 8 & 0 & 0 & 18 \\
\hline 10 & 6 & 6 & 0 & 0 & 0 & 12 \\
\hline
\end{tabular}

\begin{tabular}{|c|c|c|c|c|c|c|}
\hline \multicolumn{9}{|c|}{ Reproduction at $100.0 \%$} & \multicolumn{3}{c|}{ Concentration } \\
\hline Rep. & Day 3/4 & Day 5 & Day 6 & Day 7 & Day 8 & Total \\
\hline 1 & 5 & $\mathrm{x}$ & $\mathrm{x}$ & $\mathrm{x}$ & $\mathrm{x}$ & 5 \\
\hline 2 & 0 & $\mathrm{x}$ & $\mathrm{x}$ & $\mathrm{x}$ & $\mathrm{x}$ & 0 \\
\hline 3 & 4 & $\mathrm{x}$ & $\mathrm{x}$ & $\mathrm{x}$ & $\mathrm{x}$ & 4 \\
\hline 4 & 0 & $\mathrm{x}$ & $\mathrm{x}$ & $\mathrm{x}$ & $\mathrm{x}$ & 0 \\
\hline 5 & 5 & $\mathrm{x}$ & $\mathrm{x}$ & $\mathrm{x}$ & $\mathrm{x}$ & 5 \\
\hline 6 & 4 & $\mathrm{x}$ & $\mathrm{x}$ & $\mathrm{x}$ & $\mathrm{x}$ & 4 \\
\hline 7 & 0 & $\mathrm{x}$ & $\mathrm{x}$ & $\mathrm{x}$ & $\mathrm{x}$ & 0 \\
\hline 8 & 0 & $\mathrm{x}$ & $\mathrm{x}$ & $\mathrm{x}$ & $\mathrm{x}$ & 0 \\
\hline 9 & 0 & $\mathrm{x}$ & $\mathrm{x}$ & $\mathrm{x}$ & $\mathrm{x}$ & 0 \\
\hline 10 & 6 & $\mathrm{x}$ & $\mathrm{x}$ & $\mathrm{x}$ & $\mathrm{x}$ & 6 \\
\hline
\end{tabular}




\section{CHRONIC DEFINITIVE SURVIVAL AND REPRODUCTION TEST Statistical Analyses}

Client: Westinghouse Savannah River Company

Sample Identification: BG-002

Test Date: $\quad$ 2/22/96

\begin{tabular}{|lrr|}
\hline Test for Normality & & \\
& $\mathrm{W}=$ & 0.984 \\
Test Used: Shapiro Wilks & critical & 0.93 \\
& & \\
& \\
The data are normal in distribution
\end{tabular}

\begin{tabular}{|c|c|c|}
\hline \multicolumn{3}{|c|}{ Test for Homogeneity of Variance } \\
\hline Test Used: Bartlett's Test & $\begin{array}{r}\mathrm{B}= \\
\text { critical }\end{array}$ & $\begin{array}{r}10.24 \\
15.1\end{array}$ \\
\hline \multicolumn{3}{|c|}{ The data are homogeneous in variance } \\
\hline
\end{tabular}

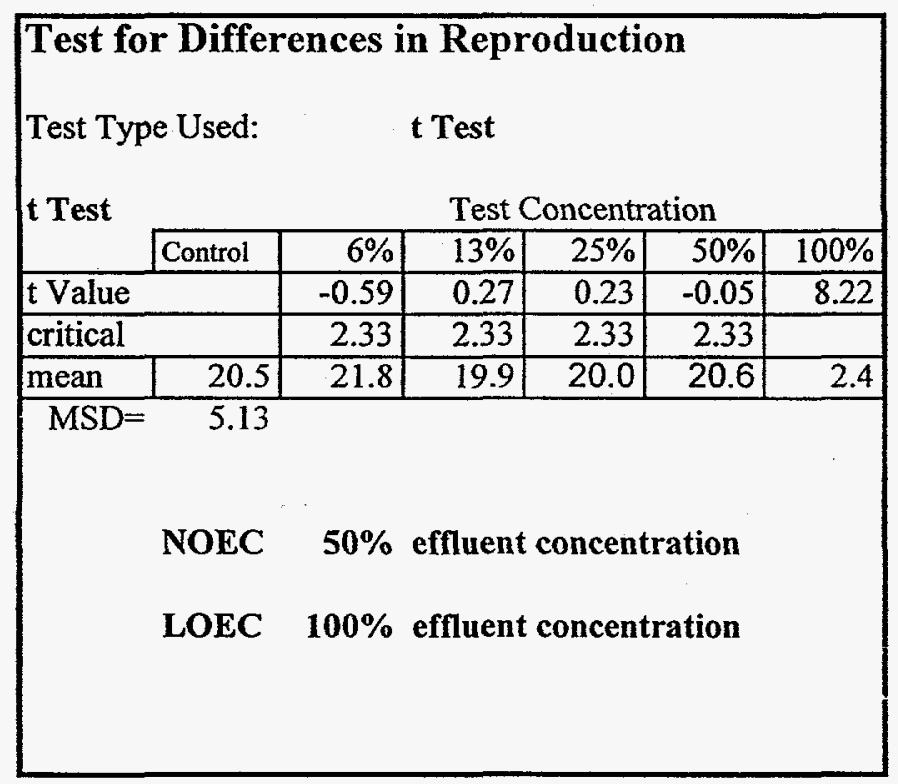

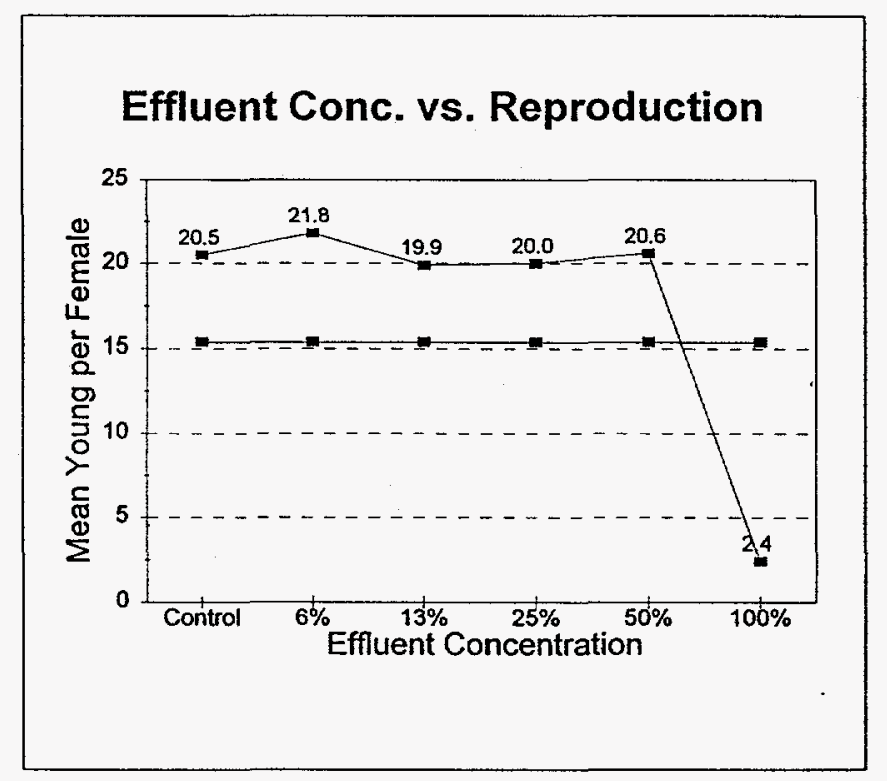

\section{Summary}




\section{CHRONIC DEFINITIVE SURVIVAL AND REPRODUCTION TEST Water Chemistry}

Client: Westinghouse Savannah River Company

Sample Identification: BG-002

Test Date: $\quad$ 2/22/96

\section{pH (After Renewal)}

\begin{tabular}{|l|r|r|r|r|r|r|}
\hline \multicolumn{7}{|c|}{ Test Concentration } \\
\hline & Control & $6.25 \%$ & $12.5 \%$ & $25.0 \%$ & $50.0 \%$ & $100 \%$ \\
\hline Initial & 8.04 & 7.77 & 7.04 & 7.55 & 7.14 & 6.52 \\
\hline Day 1 & 8.09 & 7.78 & 7.81 & 7.77 & 7.71 & 6.38 \\
\hline Day 2 & 8.00 & 7.69 & 7.70 & 7.65 & 7.61 & 6.19 \\
\hline Day 3 & 8.02 & 8.06 & 8.12 & 8.12 & 8.15 & 7.23 \\
\hline Day 4 & 8.03 & 8.01 & 8.01 & 8.05 & 8.06 & \\
\hline Day 5 & 8.08 & 7.93 & 7.84 & 7.97 & 7.81 & \\
\hline Day 6 & 8.11 & 8.04 & 7.98 & 8.07 & 7.93 & \\
\hline
\end{tabular}

Dissolved Oxygen (After Renewal)

\begin{tabular}{|l|r|r|r|r|r|r|}
\hline \multicolumn{7}{|c|}{ Test Concentration } \\
\hline & Control & $6.25 \%$ & \multicolumn{1}{|c|}{$12.5 \%$} & $25.0 \%$ & $50.0 \%$ & $100 \%$ \\
\hline Initial & 8.6 & 8.4 & 8.4 & 8.4 & 8.4 & 8.4 \\
\hline Day 1 & 8.4 & 8.2 & 8.4 & 8.4 & 8.4 & 8.2 \\
\hline Day 2 & 8.6 & 8.4 & 8.4 & 8.4 & 8.4 & 8.4 \\
\hline Day 3 & 8.5 & 8.4 & 8.4 & 8.4 & 8.5 & 8.5 \\
\hline Day 4 & 8.7 & 8.6 & 8.3 & 8.6 & 8.6 & \\
\hline Day 5 & 8.2 & 8.5 & 8.4 & 8.1 & 8.4 & \\
\hline Day 6 & 8.9 & 8.4 & 8.2 & 8.4 & 8.6 & \\
\hline
\end{tabular}

Dilution Water \& Effluent Parameters

\begin{tabular}{|l|c|r|r|r|r|}
\hline & Res Cl. & Alkalin & Hard. & Cond. & \\
\hline & $\mathrm{mg} / \mathrm{L}$ & $\mathrm{mg} / \mathrm{L}$ & $\mathrm{mg} / \mathrm{L}$ & $\mu \mathrm{mhos}$ & \\
\hline Eff. Sample 1 & 0.08 & 2.03 & 18 & 17.2 & \\
\hline Eff. Sample 2 & $<.05$ & $<.01$ & 26 & 30.4 & \\
\hline Eff. Sample 3 & $<.05$ & $<.01$ & 10 & 31.6 & \\
\hline & & & & & \\
\hline Dilution Water & & 69 & 100 & 209 & \\
\hline & & & & & \\
\hline & & & & & \\
\hline
\end{tabular}

\section{$\mathrm{pH}$ (Before Renewal)}

\begin{tabular}{|l|r|r|r|r|r|r|}
\hline \multicolumn{7}{|c|}{ Test Concentration } \\
\hline & Control & $6.25 \%$ & $12.5 \%$ & $25.0 \%$ & $50.0 \%$ & $100 \%$ \\
\hline Day 1 & 7.93 & 8.15 & 8.23 & 8.41 & 8.47 & 7.48 \\
\hline Day 2 & 8.14 & 8.03 & 8.17 & 8.46 & 8.39 & 7.53 \\
\hline Day 3 & 8.26 & 8.23 & 8.20 & 8.19 & 8.14 & 7.22 \\
\hline Day 4 & 7.73 & 8.17 & 8.14 & 8.09 & 8.07 & \\
\hline Day 5 & 8.07 & 8.31 & 8.26 & 8.11 & 8.19 & \\
\hline Day 6 & 8.05 & 8.19 & 8.24 & 8.26 & 8.23 & \\
\hline Final & 8.20 & 8.28 & 8.27 & 8.16 & 8.29 & \\
\hline
\end{tabular}

\section{Dissolved Oxygen (Before Renewal)}

\begin{tabular}{|l|r|r|r|r|r|r|}
\hline \multicolumn{7}{|c|}{ Test Concentration } \\
\hline & Control & $6.25 \%$ & $12.5 \%$ & $25.0 \%$ & $50.0 \%$ & $100 \%$ \\
\hline Day 1 & 8.3 & 8.6 & 8.6 & 8.7 & 8.7 & .8 .8 \\
\hline Day 2 & 8.2 & 8.3 & 8.4 & 8.2 & 8.4 & 8.4 \\
\hline Day 3 & 8.5 & 8.5 & 8.5 & 8.5 & 8.5 & 8.5 \\
\hline Day 4 & 8.2 & 8.4 & 8.4 & 8.4 & 8.4 & \\
\hline Day 5 & 8.4 & 8.3 & 8.2 & 8.3 & 8.4 & \\
\hline Day 6 & 8.5 & 8.2 & 8.0 & 8.4 & 8.4 & \\
\hline Final & 8.6 & 8.3 & 8.2 & 8.2 & 8.2 & \\
\hline
\end{tabular}

Temperature
\begin{tabular}{|l|r|}
\hline \multicolumn{2}{|c|}{ Incubator ${ }^{\circ} \mathrm{C}$} \\
\hline Initial & 26.0 \\
\hline Day 1 & 25.8 \\
\hline Day 2 & 24.8 \\
\hline Day 3 & 24.1 \\
\hline Day 4 & 26.0 \\
\hline Day 5 & 24.0 \\
\hline Day 6 & 25.1 \\
\hline Final & 24.4 \\
\hline
\end{tabular}


(864) 877-6942 - FAX (864) 877-6938

P.O. Box 16414, Greenville, SC 29606 - 4 Craftsman Court, Greer, SC 29650

\section{Day Chronic Definitive Survival and Reproduction Bioassay}

Method: EPA/600/4-89/001

Test Organism: $\quad$ Ceriodaphnia dubia

Facility: Westinghouse Savannah River Company

Sample ID:BG-003

Date: $\quad 2 / 22 / 96$ 


\section{CHRONIC DEFINITIVE SURVIVAL AND REPRODUCTION TEST Survival and Reproduction Results}

Client: Westinghouse Savannah River Company

Sample Identification: BG-003

Test Date: $\quad$ 2/22/96 T5100

\begin{tabular}{|c|c|c|c|c|c|c|}
\hline \multicolumn{2}{|c|}{ Reproduction at } & \multicolumn{3}{c|}{$0 \%$} & \multicolumn{3}{c|}{ Concentration } \\
\hline Rep. & Day 3/4 & Day 5 & Day 6 & Day 7 & Day 8 & Total \\
\hline 1 & 5 & 6 & 10 & 0 & 0 & 21 \\
\hline 2 & 6 & 8 & 10 & 0 & 0 & 24 \\
\hline 3 & 6 & 5 & 0 & 13 & 0 & 24 \\
\hline 4 & 5 & 6 & 0 & 0 & 13 & 24 \\
\hline 5 & 5 & 8 & 0 & 0 & 13 & 26 \\
\hline 6 & 5 & 9 & 0 & 13 & 0 & 27 \\
\hline 7 & 0 & 0 & 0 & 2 & 10 & 12 \\
\hline 8 & 4 & 0 & 0 & 0 & 12 & 16 \\
\hline 9 & 13 & 0 & 0 & 0 & 11 & 24 \\
\hline 10 & 3 & 5 & 0 & 0 & 10 & 18 \\
\hline
\end{tabular}

\begin{tabular}{|c|c|c|c|c|c|c|}
\hline \multicolumn{3}{|c|}{ Reproduction at } & \multicolumn{4}{|c|}{$6.25 \%$ Concentration } \\
\hline Rep. & Day $3 / 4$ & Day 5 & Day 6 & Day 7 & Day 8 & Total \\
\hline 1 & 3 & $\overline{6}$ & 5 & 0 & 0 & $\overline{14}$ \\
\hline 2 & 6 & 9 & 12 & 0 & 0 & 27 \\
\hline 3 & 5 & 6 & 0 & 7 & 0 & 18 \\
\hline 4 & 6 & 0 & 0 & 5 & 11 & 22 \\
\hline 5 & 1 & 0 & 0 & 9 & 15 & 25 \\
\hline 6 & 5 & 7 & 0 & 13 & 0 & 25 \\
\hline 7 & 0 & 0 & 3 & 0 & $\overline{11}$ & 14 \\
\hline 8 & 5 & 0 & 0 & 5 & 10 & 20 \\
\hline 9 & 10 & 0 & 0 & 0 & 12 & 22 \\
\hline 10 & 3 & 0 & 0 & 0 & 10 & 13 \\
\hline
\end{tabular}

\begin{tabular}{|c|c|c|c|c|c|c|}
\hline \multicolumn{2}{|c|}{ Reproduction at } & \multicolumn{4}{c|}{$12.5 \%$} & \multicolumn{3}{c|}{ Concentration } \\
\hline Rep. & Day 3/4 & Day 5 & Day 6 & Day 7 & Day 8 & Total \\
\hline 1 & 5 & 0 & 6 & 0 & 12 & 23 \\
\hline 2 & 3 & 6 & 0 & 0 & 10 & 19 \\
\hline 3 & 0 & 5 & 0 & 9 & 10 & 24 \\
\hline 4 & 4 & 6 & 0 & 2 & 0 & 12 \\
\hline 5 & 1 & 8 & 0 & 11 & 0 & 20 \\
\hline 6 & 4 & 5 & 0 & 9 & 0 & 18 \\
\hline 7 & 4 & 6 & 0 & 4 & 0 & 14 \\
\hline 8 & 0 & 0 & 0 & 3 & 11 & 14 \\
\hline 9 & 10 & 0 & 0 & 0 & 10 & 20 \\
\hline 10 & 4 & 0 & 4 & 0 & 11 & 19 \\
\hline
\end{tabular}

\begin{tabular}{|c|c|c|c|c|c|c|}
\hline \multicolumn{3}{|c|}{ Reproduction at } & \multicolumn{3}{c|}{$25.0 \%$} & \multicolumn{3}{c|}{ Concentration } \\
\hline Rep. & Day 3/4 & Day 5 & Day 6 & Day 7 & Day 8 & Total \\
\hline 1 & 6 & 4 & 10 & 0 & 0 & 20 \\
\hline 2 & 4 & 7 & $x$ & 0 & 0 & 11 \\
\hline 3 & 3 & 6 & 0 & 10 & 0 & 19 \\
\hline 4 & 4 & 0 & 0 & 0 & 12 & 16 \\
\hline 5 & 2 & 7 & 0 & 12 & 0 & 21 \\
\hline 6 & 2 & 3 & 0 & 0 & 12 & 17 \\
\hline 7 & 3 & 3 & 0 & 0 & 13 & 19 \\
\hline 8 & 5 & 0 & 0 & 0 & 11 & 16 \\
\hline 9 & 14 & 0 & 0 & 10 & 0 & 24 \\
\hline 10 & 1 & 3 & 0 & 2 & 13 & 19 \\
\hline
\end{tabular}

\begin{tabular}{|c|c|c|c|c|c|c|}
\hline \multicolumn{3}{|c|}{ Reproduction at } & \multirow{2}{*}{$\frac{50.0 \%}{\text { Day } 5}$} & \multicolumn{3}{|c|}{ Concentration } \\
\hline Rep. & Day $2 / 3$ & Day 4 & & Day 6 & Day 7 & Total \\
\hline 1 & 6 & 6 & 6 & 0 & 0 & 18 \\
\hline 2 & 2 & 1 & 9 & 0 & $\overline{0}$ & 12 \\
\hline 3 & 3 & 4 & 0 & 9 & 0 & 16 \\
\hline 4 & 3 & 0 & 6 & 0 & 9 & 18 \\
\hline 5 & 3 & 2 & 0 & 4 & 0 & 9 \\
\hline 6 & 5 & 6 & 0 & 1 & 6 & 18 \\
\hline 7 & 2 & 0 & 0 & 0 & 10 & 12 \\
\hline 8 & 4 & 0 & 0 & 3 & 9 & 16 \\
\hline 9 & 9 & 0 & 5 & 0 & 0 & 14 \\
\hline 10 & 0 & 6 & 0 & 0 & 7 & 13 \\
\hline
\end{tabular}

\begin{tabular}{|c|c|c|c|c|c|c|}
\hline \multicolumn{6}{|c|}{ Reproduction at } & \multicolumn{5}{c|}{$100 \%$} & \multicolumn{3}{c|}{ Concentration } \\
\hline Rep. & Day 2/3 & Day 4 & Day 5 & Day 6 & Day 7 & Total \\
\hline 1 & 5 & 5 & 0 & 6 & 0 & 16 \\
\hline 2 & 3 & 0 & 0 & 3 & 12 & 18 \\
\hline 3 & 2 & 0 & 4 & 4 & 0 & 10 \\
\hline 4 & 1 & 0 & 2 & 7 & 0 & 10 \\
\hline 5 & 0 & 0 & 0 & 4 & 5 & 9 \\
\hline 6 & 2 & 2 & 6 & 7 & 0 & 17 \\
\hline 7 & 2 & 4 & 0 & 5 & 0 & 11 \\
\hline 8 & 5 & 0 & 6 & 5 & 0 & 16 \\
\hline 9 & 2 & 6 & 7 & 0 & 0 & 15 \\
\hline 10 & 0 & 2 & 6 & 0 & 0 & 8 \\
\hline
\end{tabular}




\section{CHRONIC DEFINITIVE SURVIVAL AND REPRODUCTION TEST \\ Statistical Analyses}

Client: Westinghouse Savannah River Company

Sample Identification: BG-003

Test Date: $\quad 2 / 22 / 96$

\begin{tabular}{|lrr|}
\hline Test for Normality & & \\
& W & 0.928 \\
Test Used: Shapiro Wilks & critical & 0.93 \\
& & \\
& \\
The data are not normal in distribution \\
\end{tabular}

\section{Test for Homogeneity of Variance}

Test Used: Bartlett's Test

$\begin{array}{rr}\mathrm{B}= & 3.23 \\ \text { critical } & 15.1\end{array}$

The data are homogeneous in variance

\begin{tabular}{|c|c|c|c|c|c|c|}
\hline \multicolumn{7}{|c|}{ Test for Differences in Reproduction } \\
\hline \multicolumn{3}{|c|}{ Test Type Used: } & \multicolumn{4}{|c|}{ t Test } \\
\hline \multirow[t]{2}{*}{ t Test } & \multicolumn{6}{|c|}{ Test Concentration } \\
\hline & Control & $6 \%$ & $13 \%$ & $25 \%$ & $50 \%$ & $100 \%$ \\
\hline \multicolumn{2}{|l|}{ t Value } & 0.88 & 1.82 & 1.88 & 3.87 & 4.75 \\
\hline \multicolumn{2}{|l|}{ critical } & 2.33 & 2.33 & 2.33 & 2.33 & \\
\hline mean & 21.6 & 20.0 & 18.3 & 18.2 & 14.6 & 13.0 \\
\hline \multicolumn{7}{|l|}{$\overline{\mathrm{MSD}}=$} \\
\hline & NOEC & \multicolumn{5}{|c|}{$25 \%$ effluent concentration } \\
\hline & LOEC & \multicolumn{5}{|c|}{$50 \%$ effluent concentration } \\
\hline
\end{tabular}

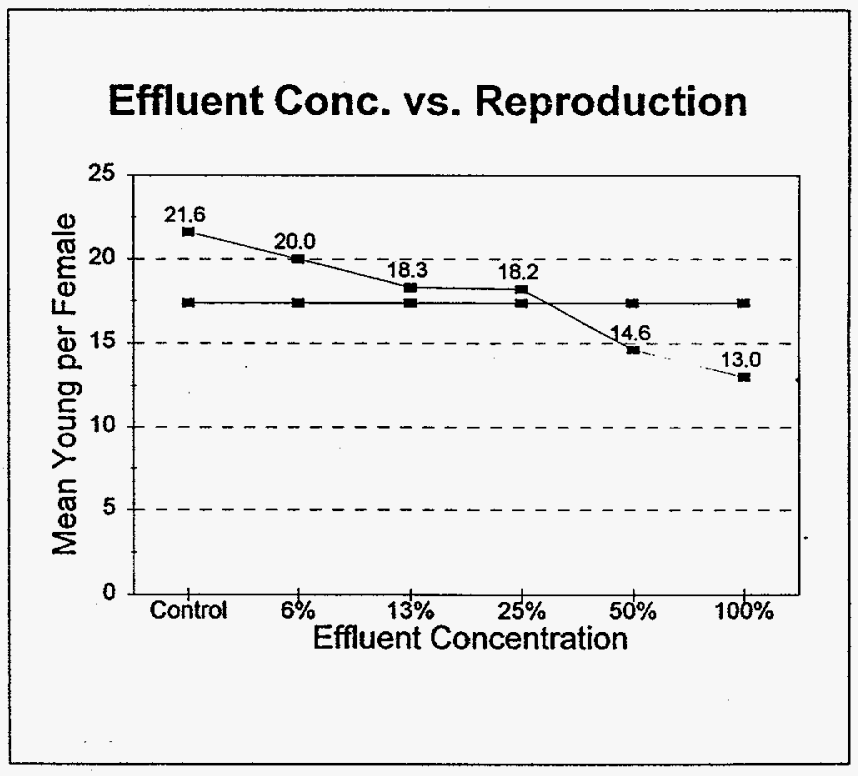

\section{Summary}




\section{CHRONIC DEFINITIVE SURVIVAL AND REPRODUCTION TEST Water Chemistry}

Client: Westinghouse Savannah River Company

Sample Identification: BG-003

Test Date: $\quad$ 2/22/96

pH (After Renewal)

\begin{tabular}{|l|r|r|r|r|r|r|}
\hline \multicolumn{7}{|c|}{ Test Concentration } \\
\hline & Control & $6.25 \%$ & \multicolumn{1}{|c|}{$12.5 \%$} & \multicolumn{1}{|c|}{$25.0 \%$} & $50.0 \%$ & $100 \%$ \\
\hline Initial & 8.04 & 7.27 & 7.59 & 7.36 & 7.62 & 7.51 \\
\hline Day 1 & 8.09 & 7.78 & 7.82 & 7.81 & 7.81 & 7.86 \\
\hline Day 2 & 8.00 & 7.68 & 7.91 & 7.76 & 7.69 & 7.94 \\
\hline Day 3 & 8.02 & 7.64 & 7.77 & 7.90 & 7.95 & 8.01 \\
\hline Day 4 & 8.03 & 7.75 & 7.82 & 7.85 & 7.88 & 8.15 \\
\hline Day 5 & 8.08 & 7.74 & 7.81 & 7.84 & 7.86 & 7.84 \\
\hline Day 6 & 8.11 & 7.85 & 8.01 & 8.03 & 7.88 & 7.85 \\
\hline
\end{tabular}

Dissolved Oxygen (After Renewal)

\begin{tabular}{|l|r|r|r|r|r|r|}
\hline \multicolumn{7}{|c|}{ Test Concentration } \\
\hline & Control & $6.25 \%$ & $12.5 \%$ & $25.0 \%$ & $50.0 \%$ & $100 \%$ \\
\hline Initial & 8.6 & 8.6 & 8.6 & 8.6 & 8.6 & 8.6 \\
\hline Day 1 & 8.4 & 8.8 & 8.8 & 8.9 & 8.9 & 9.0 \\
\hline Day 2 & 8.6 & 8.6 & 8.6 & 8.6 & 8.5 & 9.0 \\
\hline Day 3 & 8.5 & 8.6 & 8.6 & 8.5 & 8.5 & 8.4 \\
\hline Day 4 & 8.7 & 8.4 & 8.4 & 8.4 & 8.4 & 8.4 \\
\hline Day 5 & 8.2 & 8.4 & 8.4 & 8.4 & 8.4 & 8.4 \\
\hline Day 6 & 8.9 & 8.1 & 8.2 & 8.3 & 8.4 & 8.4 \\
\hline
\end{tabular}

Dilution Water \& Effluent Parameters

\begin{tabular}{|l|c|r|r|r|r|}
\hline & Res Cl. & Alkalin & Hard. & Cond. & \\
\hline & $\mathrm{mg} / \mathrm{L}$ & $\mathrm{mg} / \mathrm{L}$ & $\mathrm{mg} / \mathrm{L}$ & $\mu$ mhos & \\
\hline Eff. Sample 1 & 0.06 & 2.03 & 8 & 24.1 & \\
\hline Eff. Sample 2 & $<.05$ & 2.03 & 14 & 24.3 & \\
\hline Eff. Sample 3 & $<.05$ & 2.03 & 8 & 23.3 & \\
\hline & & & & & \\
\hline Dilution Water & & 69 & 100 & 209 & \\
\hline & & & & & \\
\hline & & & & & \\
\hline
\end{tabular}

$\mathrm{pH}$ (Before Renewal)

\begin{tabular}{|l|r|r|r|r|r|r|}
\hline \multicolumn{7}{|c|}{ Test Concentration } \\
\hline & Control & $6.25 \%$ & $12.5 \%$ & $25.0 \%$ & $50.0 \%$ & $100 \%$ \\
\hline Day 1 & 7.93 & 7.99 & 8.12 & 8.14 & 8.17 & 8.26 \\
\hline Day 2 & 8.14 & 8.06 & 8.09 & 8.21 & 8.30 & 8.46 \\
\hline Day 3 & 8.26 & 8.16 & 8.17 & 8.14 & 8.03 & 7.04 \\
\hline Day 4 & 7.73 & 8.12 & 8.12 & 8.10 & 8.07 & 8.13 \\
\hline Day 5 & 8.07 & 8.25 & 8.28 & 8.19 & 8.16 & 7.44 \\
\hline Day 6 & 8.05 & 8.20 & 8.15 & 8.16 & 7.87 & 7.24 \\
\hline Final & 8.20 & & & & & \\
\hline
\end{tabular}

Dissolved Oxygen (Before Renewal)

\begin{tabular}{|l|r|r|r|r|r|r|}
\hline \multicolumn{7}{|c|}{ Test Concentration } \\
\hline & Control & $6.25 \%$ & $12.5 \%$ & $25.0 \%$ & $50.0 \%$ & $100 \%$ \\
\hline Day 1 & 8.3 & 8.9 & 8.9 & 9.0 & 9.1 & 9.1 \\
\hline Day 2 & 8.2 & 8.6 & 8.4 & 8.4 & 8.4 & 8.6 \\
\hline Day 3 & 8.5 & 8.5 & 8.4 & 8.2 & 8.2 & 8.2 \\
\hline Day 4 & 8.2 & 8.4 & 8.4 & 8.4 & 8.4 & 8.4 \\
\hline Day 5 & 8.4 & 8.4 & 8.4 & 8.4 & 8.4 & 8.4 \\
\hline Day 6 & 8.5 & 8.2 & 8.2 & 8.3 & 8.4 & 8.4 \\
\hline Final & 8.6 & & & & & \\
\hline
\end{tabular}

Temperature

\begin{tabular}{|l|r|}
\hline \multicolumn{2}{|c|}{ Incubator ${ }^{\circ} \mathrm{C}$} \\
\hline Initial & 26.0 \\
\hline Day 1 & 25.8 \\
\hline Day 2 & 24.0 \\
\hline Day 3 & 24.1 \\
\hline Day 4 & 26.0 \\
\hline Day 5 & 24.0 \\
\hline Day 6 & 25.1 \\
\hline Final & 24.4 \\
\hline
\end{tabular}




\title{
7 Day Chronic Definitive Survival and Reproduction Bioassay
} Method: EPA/600/4-89/001

\author{
Test Organism: \\ Ceriodaphnia dubia
}

Facility:Westinghouse Savannah River Company

Sample ID:BG-004

Date: $\quad 2 / 29 / 96$ 


\section{CHRONIC DEFINITIVE SURVIVAL AND REPRODUCTION TEST Survival and Reproduction Results}

Client: Westinghouse Savannah River Company

Sample Identification: BG-004

Test Date: $\quad$ 2/29/96 T5145

\begin{tabular}{|c|c|c|c|c|c|c|}
\hline \multicolumn{3}{|c|}{ Reproduction at } & \multirow{2}{*}{$\begin{array}{l}0.0 \% \\
\text { Day } 5\end{array}$} & \multicolumn{3}{|c|}{ Concentration } \\
\hline Rep. & Day $2 / 3$ & Day 4 & & Day 6 & Day 7 & Total \\
\hline 1 & $\overline{0}$ & 6 & $\overline{9}$ & $\overline{0}$ & 10 & 25 \\
\hline 2 & 0 & 4 & 10 & $\overline{9}$ & $\overline{0}$ & 23 \\
\hline 3 & 6 & 0 & 8 & 15 & 0 & 29 \\
\hline 4 & 0 & 3 & 7 & 14 & 0 & 24 \\
\hline 5 & 4 & 0 & 10 & 8 & 0 & 22 \\
\hline 6 & 4 & 0 & 10 & 13 & 0 & 27 \\
\hline 7 & 0 & 5 & 9 & 12 & 0 & 26 \\
\hline 8 & 4 & 0 & 10 & 7 & 0 & 21 \\
\hline 9 & 0 & 7 & 0 & 8 & 0 & 15 \\
\hline 10 & 0 & 4 & 0 & 9 & 11 & 24 \\
\hline
\end{tabular}

\begin{tabular}{|c|c|c|c|c|c|c|}
\hline \multicolumn{3}{|c|}{ Reproduction at } & $6.25 \%$ & \multicolumn{4}{c|}{ Concentration } \\
\hline Rep. & Day 2/3 & Day 4 & Day 5 & Day 6 & Day 7 & Total \\
\hline 1 & 0 & 6 & 9 & 0 & 10 & 25 \\
\hline 2 & 0 & 6 & 8 & 11 & 0 & 25 \\
\hline 3 & 6 & 0 & 10 & 12 & 0 & 28 \\
\hline 4 & 0 & 5 & 8 & 13 & 0 & 26 \\
\hline 5 & 5 & 0 & 10 & 10 & 0 & 25 \\
\hline 6 & 4 & 0 & 8 & 9 & 0 & 21 \\
\hline 7 & 0 & 4 & 0 & 8 & 9 & 21 \\
\hline 8 & 4 & 0 & 11 & 12 & 0 & 27 \\
\hline 9 & 4 & 0 & 11 & 0 & 9 & 24 \\
\hline 10 & 0 & 5 & 8 & 0 & 10 & 23 \\
\hline
\end{tabular}

\begin{tabular}{|c|c|c|c|c|c|c|}
\hline \multicolumn{3}{|c|}{ Reproduction at } & \multicolumn{1}{|c|}{$12.5 \%$} & \multicolumn{3}{c|}{ Concentration } \\
\hline Rep. & Day 2/3 & Day 4 & Day 5 & Day 6 & Day 7 & Total \\
\hline 1 & 0 & 5 & 10 & 0 & 11 & 26 \\
\hline 2 & 4 & 0 & 9 & 12 & 0 & 25 \\
\hline 3 & 6 & 0 & 8 & 11 & 0 & 25 \\
\hline 4 & 4 & 0 & 8 & 12 & 0 & 24 \\
\hline 5 & 4 & 9 & 0 & 10 & 0 & 23 \\
\hline 6 & 5 & 0 & 8 & 16 & 0 & 29 \\
\hline 7 & 0 & 5 & 10 & 11 & 0 & 26 \\
\hline 8 & 4 & 0 & 9 & 10 & 0 & 23 \\
\hline 9 & 0 & 5 & 8 & 0 & 10 & 23 \\
\hline 10 & 0 & 4 & 2 & 11 & 0 & 17 \\
\hline
\end{tabular}

\begin{tabular}{|c|c|c|c|c|c|c|}
\hline \multicolumn{3}{|c|}{ Reproduction at } & \multicolumn{3}{c|}{$2.0 \%$} & \multicolumn{4}{c|}{ Concentration } \\
\hline Rep. & Day 2/3 & Day 4 & Day 5 & Day 6 & Day 7 & Total \\
\hline 1 & 0 & 4 & 10 & 12 & 0 & 26 \\
\hline 2 & 4 & 0 & 8 & 9 & 0 & 21 \\
\hline 3 & 6 & 0 & 12 & 12 & 0 & 30 \\
\hline 4 & 0 & 6 & 10 & 11 & 0 & 27 \\
\hline 5 & 6 & 9 & 0 & 10 & 0 & 25 \\
\hline 6 & 4 & 0 & 11 & 11 & 0 & 26 \\
\hline 7 & 0 & 1 & 9 & 10 & 0 & 20 \\
\hline 8 & 4 & 0 & 10 & 10 & 0 & 24 \\
\hline 9 & 0 & 5 & 3 & 0 & 8 & 16 \\
\hline 10 & 0 & 5 & 8 & 0 & 10 & 23 \\
\hline
\end{tabular}

\begin{tabular}{|c|c|c|c|c|c|c|}
\hline \multicolumn{3}{|c|}{ Reproduction at } & \multicolumn{5}{c|}{$50 \%$} & \multicolumn{3}{c|}{ Concentration } \\
\hline Rep. & Day 2/3 & Day 4 & Day 5 & Day 6 & Day 7 & Total \\
\hline 1 & 0 & 5 & 11 & 13 & 0 & 29 \\
\hline 2 & 0 & 5 & 9 & 10 & 0 & 24 \\
\hline 3 & 0 & 5 & 9 & 13 & 0 & 27 \\
\hline 4 & 4 & 0 & 8 & 13 & 0 & 25 \\
\hline 5 & 4 & 0 & 8 & 12 & 0 & 24 \\
\hline 6 & 5 & 0 & 9 & 11 & 0 & 25 \\
\hline 7 & 0 & 1 & 8 & 0 & 9 & 18 \\
\hline 8 & 4 & 0 & 9 & 13 & 0 & 26 \\
\hline 9 & 4 & 0 & 9 & 10 & 0 & 23 \\
\hline 10 & 0 & 5 & 8 & 10 & 0 & 23 \\
\hline
\end{tabular}

\begin{tabular}{|c|c|c|c|c|c|c|}
\hline \multicolumn{3}{|c|}{ Reproduction at } & $100.0 \%$ & Concentration \\
\hline Rep. & Day 2/3 & Day 4 & Day 5 & Day 6 & Day 7 & Total \\
\hline 1 & 0 & 4 & 0 & 0 & 2 & 6 \\
\hline 2 & 0 & 3 & 0 & 0 & 0 & 3 \\
\hline 3 & 0 & 4 & 3 & 0 & 4 & 11 \\
\hline 4 & 0 & 3 & 4 & 0 & 4 & 11 \\
\hline 5 & 2 & 0 & 4 & 6 & 0 & 12 \\
\hline 6 & 0 & 4 & 5 & 0 & 0 & 9 \\
\hline 7 & 0 & 4 & 0 & 0 & 1 & 5 \\
\hline 8 & 0 & 0 & 4 & 4 & 0 & 8 \\
\hline 9 & 0 & 0 & 1 & 0 & 0 & 1 \\
\hline 10 & 0 & 4 & 4 & 0 & $X$ & 8 \\
\hline
\end{tabular}




\section{CHRONIC DEFINITIVE SURVIVAL AND REPRODUCTION TEST \\ Statistical Analyses}

Client: Westinghouse Savannah River Company

Sample Identification: BG-004

Test Date: $\quad 2 / 29 / 96$

\begin{tabular}{|lrr|}
\hline Test for Normality & & \\
Test Used: Shapiro Wilks & $\mathrm{W}=$ & 0.955 \\
& critical & 0.93 \\
& & \\
The data are normal in distribution \\
\end{tabular}

\begin{tabular}{|c|c|c|}
\hline \multicolumn{3}{|c|}{ Test for Homogeneity of Variance } \\
\hline Test Used: Bartlett's Test & $\begin{array}{r}\mathrm{B}= \\
\text { critical }\end{array}$ & $\begin{array}{l}3.43 \\
15.1\end{array}$ \\
\hline \multicolumn{3}{|c|}{ The data are homogeneous in variance } \\
\hline
\end{tabular}

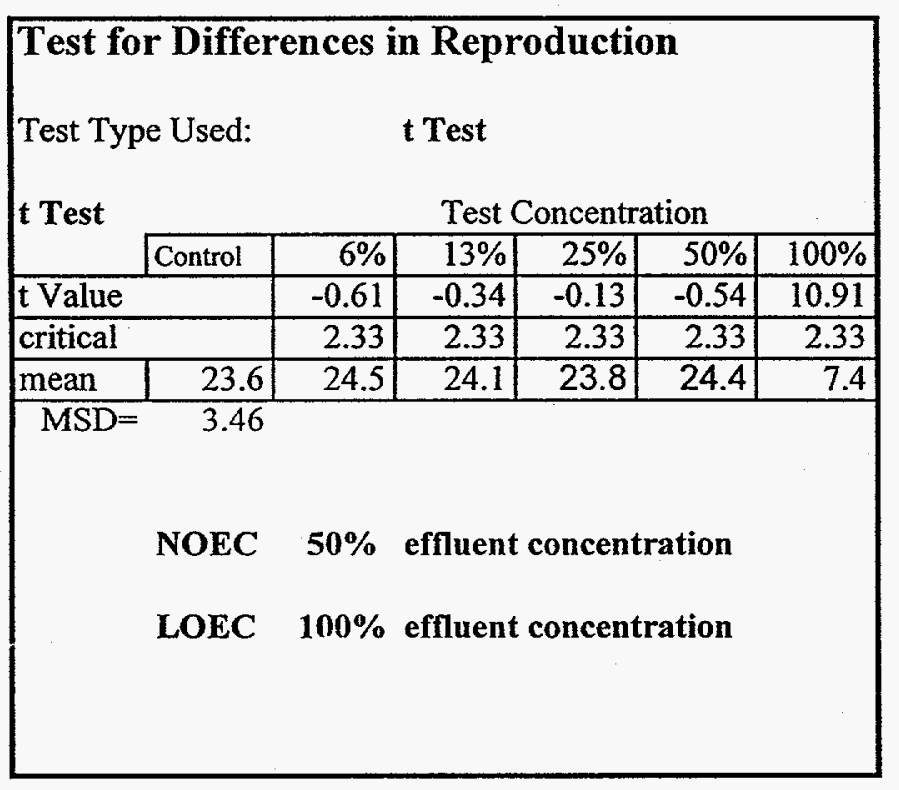

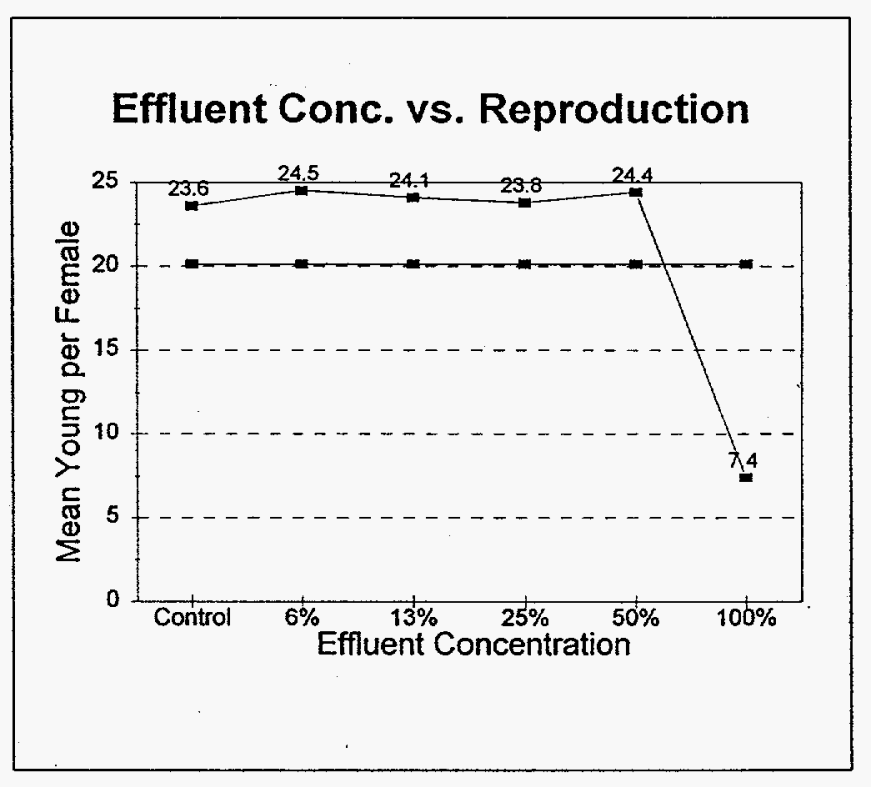

Summary 


\section{CHRONIC DEFINITIVE SURVIVAL AND REPRODUCTION TEST Water Chemistry}

Client: Westinghouse Savannah River Company

Sample Identification: BG-004

Test Date: 2/29/96

$\mathrm{pH}$ (After Renewal)

\begin{tabular}{|l|r|r|r|r|r|r|}
\hline \multicolumn{7}{|c|}{ Test Concentration } \\
\hline & Control & $6.25 \%$ & $12.5 \%$ & $25 \%$ & $50 \%$ & $100 \%$ \\
\hline Initial & 8.12 & 8.14 & 8.13 & 8.15 & 7.95 & 6.26 \\
\hline Day 1 & 7.98 & 8.10 & 7.87 & 7.66 & 7.29 & 7.19 \\
\hline Day 2 & 7.97 & 8.01 & 7.85 & 7.65 & 7.34 & 6.74 \\
\hline Day 3 & 7.98 & 8.11 & 7.97 & 7.91 & 7.65 & 6.17 \\
\hline Day 4 & 8.06 & 7.88 & 7.73 & 7.65 & 7.35 & 6.71 \\
\hline Day 5 & 8.05 & 8.03 & 7.96 & 7.75 & 7.53 & 6.06 \\
\hline Day 6 & 8.14 & 8.12 & 7.99 & 7.88 & 7.37 & 5.84 \\
\hline
\end{tabular}

Dissolved Oxygen (After Renewal)

\begin{tabular}{|l|r|r|r|r|r|r|}
\hline \multicolumn{7}{|c|}{ Test Concentration } \\
\hline & Control & $6.25 \%$ & $12.5 \%$ & $25 \%$ & $50 \%$ & $100 \%$ \\
\hline Initial & 8.6 & 8.4 & 8.2 & 8.4 & 8.3 & 8.0 \\
\hline Day 1 & 8.6 & 8.8 & 8.8 & 8.9 & 8.9 & 9.0 \\
\hline Day 2 & 8.5 & 8.8 & 8.6 & 8.8 & 8.4 & 8.8 \\
\hline Day 3 & 8.6 & 8.4 & 8.4 & 8.6 & 8.6 & 8.7 \\
\hline Day 4 & 8.8 & 8.2 & 8.2 & 8.3 & 8.3 & 8.4 \\
\hline Day 5 & 8.9 & 8.6 & 8.6 & 8.6 & 8.7 & 8.7 \\
\hline Day 6 & 8.7 & 8.6 & 8.6 & 8.6 & 8.8 & 8.8 \\
\hline
\end{tabular}

Dilution Water \& Effluent Parameters

\begin{tabular}{|l|c|r|r|r|l|}
\hline & Res Cl. & Alkalin & Hard. & Cond. & \\
\hline & $\mathrm{mg} / \mathrm{L}$ & $\mathrm{mg} / \mathrm{L}$ & $\mathrm{mg} / \mathrm{L}$ & $\mu \mathrm{mhos}$ & \\
\hline Eff. Sample 1 & $<0.05$ & 4.06 & 14 & 29.9 & \\
\hline Eff. Sample 2 & $<0.05$ & 2.03 & 8 & 27.8 & \\
\hline Eff. Sample 3 & $<0.05$ & 4.06 & 20 & 27.1 & \\
\hline & & & & & \\
\hline Dilution Water & & 71.1 & 84 & 222 & \\
\hline & & & & & \\
\hline & & & & & \\
\hline
\end{tabular}

pH (Before Renewal)

\begin{tabular}{|l|r|r|r|r|r|r|}
\hline \multicolumn{7}{|c|}{ Test Concentration } \\
\hline & Control & $6.25 \%$ & $12.5 \%$ & $25 \%$ & $50 \%$ & $100 \%$ \\
\hline Day 1 & 8.10 & 8.12 & 7.49 & 8.12 & 7.87 & 7.35 \\
\hline Day 2 & 8.27 & 8.16 & 8.08 & 8.00 & 7.93 & 8.27 \\
\hline Day 3 & 8.44 & 8.38 & 8.41 & 8.45 & 8.41 & 6.76 \\
\hline Day 4 & 8.09 & 8.17 & 8.19 & 8.21 & 8.03 & 7.18 \\
\hline Day 5 & 8.33 & 8.38 & 8.34 & 8.22 & 7.81 & 6.51 \\
\hline Day 6 & 8.30 & 8.19 & 8.20 & 7.90 & 7.72 & 7.56 \\
\hline Final & 8.21 & 8.28 & 8.22 & 8.19 & 8.14 & 7.35 \\
\hline
\end{tabular}

Dissolved Oxygen (Before Renewal)

\begin{tabular}{|l|r|r|r|r|r|r|}
\hline \multicolumn{7}{|c|}{ Test Concentration } \\
\hline & Control & $6.25 \%$ & $12.5 \%$ & $25 \%$ & $50 \%$ & $100 \%$ \\
\hline Day 1 & 8.3 & 8.9 & 8.9 & 9.0 & 9.0 & 9.1 \\
\hline Day 2 & 8.2 & 8.6 & 8.4 & 8.6 & 8.7 & 8.8 \\
\hline Day 3 & 8.8 & 8.4 & 8.5 & 8.5 & 8.6 & 8.6 \\
\hline Day 4 & 8.6 & 8.4 & 8.4 & 8.6 & 8.6 & 8.8 \\
\hline Day 5 & 8.5 & 8.4 & 8.4 & 8.4 & 8.4 & 8.5 \\
\hline Day 6 & 8.6 & 8.8 & 8.8 & 8.9 & 9.0 & 9.0 \\
\hline Final & 8.3 & 8.0 & 8.0 & 8.0 & 8.2 & 8.2 \\
\hline
\end{tabular}

Temperature
\begin{tabular}{|l|r|}
\hline Incubator ${ }^{\circ} \mathrm{C}$ \\
\hline Initial & 24.4 \\
\hline Day 1 & 24.3 \\
\hline Day 2 & 25.7 \\
\hline Day 3 & 25.1 \\
\hline Day 4 & 24.2 \\
\hline Day 5 & 25.7 \\
\hline Day 6 & 25.1 \\
\hline Final & 25.3 \\
\hline
\end{tabular}


(864) 877-6942 - FAX (864) 877-6938

P.O. Box 16414, Greenville, SC $29606 \cdot 4$ Craftsman Court, Greer, SC 29650

\section{Day Chronic Definitive Survival and Reproduction Bioassay \\ Method: EPA/600/4-89/001}

Test Organism: Ceriodaphnia dubia

Facility: Westinghouse Savannah River Company

Sample ID:FHB-006

Date: $\quad 2 / 29 / 96$ 


\section{CHRONIC DEFINITIVE SURVIVAL AND REPRODUCTION TEST Survival and Reproduction Results}

Client: Westinghouse Savannah River Company

Sample Identification: FHB-006

Test Date: $\quad$ 2/29/96 T5141

\begin{tabular}{|c|c|c|c|c|c|c|}
\hline \multicolumn{3}{|c|}{ Reproduction at } & \multicolumn{3}{c|}{ Concentration } \\
\hline Rep. & Day $2 / 3$ & Day 4 & Day 5 & Day 6 & Day 7 & Total \\
\hline 1 & 0 & 5 & 7 & 9 & 0 & 21 \\
\hline 2 & 0 & 5 & 8 & 0 & 12 & 25 \\
\hline 3 & 0 & 6 & 7 & 12 & 0 & 25 \\
\hline 4 & 0 & 7 & 9 & 0 & 10 & 26 \\
\hline 5 & 3 & 7 & 0 & 13 & 0 & 23 \\
\hline 6 & 5 & 7 & 0 & 10 & 0 & 22 \\
\hline 7 & 6 & 0 & 11 & 13 & 0 & 30 \\
\hline 8 & 0 & 6 & 9 & 10 & 0 & 25 \\
\hline 9 & 5 & 0 & 9 & 12 & 0 & 26 \\
\hline 10 & 0 & 8 & 7 & 10 & 0 & 25 \\
\hline
\end{tabular}

\begin{tabular}{|c|c|c|c|c|c|c|}
\hline \multicolumn{3}{|c|}{ Reproduction at } \\
\hline Rep. & Day 2/3 & Day 4 & Day 5 & Day 6 & Day 7 & Total \\
\hline 1 & 0 & 5 & 7 & 0 & 0 & 12 \\
\hline 2 & 5 & 0 & 8 & 9 & 0 & 22 \\
\hline 3 & 0 & 6 & 7 & 10 & 0 & 23 \\
\hline 4 & 0 & 6 & 0 & 0 & 12 & 18 \\
\hline 5 & 0 & 6 & 10 & 10 & 0 & 26 \\
\hline 6 & 5 & 10 & 0 & 5 & 8 & 28 \\
\hline 7 & 6 & 0 & 7 & 6 & 0 & 19 \\
\hline 8 & 0 & 4 & 0 & 0 & 10 & 14 \\
\hline 9 & 6 & 0 & 11 & 10 & 0 & 27 \\
\hline 10 & 0 & 1 & 8 & 0 & 12 & 21 \\
\hline
\end{tabular}

\begin{tabular}{|c|c|c|c|c|c|c|}
\hline \multicolumn{3}{|c|}{ Reproduction at } & $12.50 \%$ & \multicolumn{3}{c|}{ Concentration } \\
\hline Rep. & Day 2/3 & Day 4 & Day 5 & Day 6 & Day 7 & Total \\
\hline 1 & 4 & 0 & 9 & 10 & 0 & 23 \\
\hline 2 & 5 & 0 & 8 & 9 & 0 & 22 \\
\hline 3 & 0 & 5 & 8 & 14 & 0 & 27 \\
\hline 4 & 5 & 0 & 9 & 11 & 0 & 25 \\
\hline 5 & 5 & 7 & 0 & 11 & 0 & 23 \\
\hline 6 & 6 & 7 & 0 & 9 & 0 & 22 \\
\hline 7 & 0 & 0 & 3 & 0 & 0 & 3 \\
\hline 8 & 0 & 6 & 9 & 0 & 10 & 25 \\
\hline 9 & 0 & 7 & 8 & 0 & 8 & 23 \\
\hline 10 & 4 & 0 & 9 & 10 & 0 & 23 \\
\hline
\end{tabular}

\begin{tabular}{|c|c|c|c|c|c|c|}
\hline \multicolumn{3}{|c|}{ Reproduction at } & \multicolumn{3}{c|}{$25.0 \%$} & \multicolumn{3}{c|}{ Concentration } \\
\hline Rep. & Day 2/3 & Day 4 & Day 5 & Day 6 & Day 7 & Total \\
\hline 1 & 4 & 0 & 9 & 8 & 0 & 21 \\
\hline 2 & 5 & 0 & 8 & 12 & 0 & 25 \\
\hline 3 & 0 & 4 & 8 & 0 & 2 & 14 \\
\hline 4 & 0 & 7 & 7 & 0 & 0 & 14 \\
\hline 5 & 4 & 6 & 0 & 11 & 0 & 21 \\
\hline 6 & 6 & 8 & 0 & 10 & 0 & 24 \\
\hline 7 & 5 & 0 & 8 & 10 & 0 & 23 \\
\hline 8 & 0 & 4 & 2 & 0 & 10 & 15 \\
\hline 9 & 0 & 5 & 5 & 0 & 8 & 18 \\
\hline 10 & 4 & 0 & 10 & 10 & 0 & 24 \\
\hline
\end{tabular}

\begin{tabular}{|c|c|c|c|c|c|c|}
\hline \multicolumn{3}{|c|}{ Reproduction at } & \multicolumn{3}{c|}{$50.0 \%$} & \multicolumn{3}{c|}{ Concentration } \\
\hline Rep. & Day 2/3 & Day 4 & Day 5 & Day 6 & Day 7 & Total \\
\hline 1 & 4 & 0 & 8 & 11 & 0 & 23 \\
\hline 2 & 5 & 0 & 9 & 5 & 0 & 19 \\
\hline 3 & 0 & 5 & 5 & 3 & 0 & 13 \\
\hline 4 & 0 & 5 & 7 & 0 & 6 & 18 \\
\hline 5 & 3 & 0 & 6 & 5 & 0 & 14 \\
\hline 6 & 4 & 7 & 0 & 13 & 0 & 24 \\
\hline 7 & 4 & 0 & 11 & 14 & 0 & 29 \\
\hline 8 & 0 & 7 & 0 & 8 & 0 & 15 \\
\hline 9 & 0 & 0 & 8 & 2 & 0 & 10 \\
\hline 10 & 0 & 0 & 2 & 0 & 8 & 10 \\
\hline
\end{tabular}

\begin{tabular}{|c|c|c|c|c|c|c|}
\hline \multicolumn{3}{|c|}{ Reproduction at } \\
\hline Rep. & Day 2/3 & Day 4 & Day 5 & Day 6 & Day 7 & Total \\
\hline 1 & 0 & 4 & 3 & 0 & 4 & 11 \\
\hline 2 & 3 & $\mathrm{X}$ & $\mathrm{X}$ & $\mathrm{X}$ & $\mathrm{X}$ & 3 \\
\hline 3 & 0 & 2 & 0 & 0 & 3 & 5 \\
\hline 4 & 0 & 4 & 0 & 0 & 0 & 4 \\
\hline 5 & 0 & 0 & 3 & $\mathrm{X}$ & $\mathrm{X}$ & 3 \\
\hline 6 & 0 & 0 & 0 & $\mathrm{X}$ & $\mathrm{X}$ & 0 \\
\hline 7 & 0 & 3 & 0 & 0 & 2 & 5 \\
\hline 8 & $\mathrm{~L}$ & $\mathrm{~L}$ & $\mathrm{~L}$ & $\mathrm{~L}$ & $\mathrm{~L}$ & $\mathrm{~L}$ \\
\hline 9 & 0 & 2 & 4 & 0 & 0 & 6 \\
\hline 10 & 0 & 3 & 4 & 0 & 4 & 11 \\
\hline
\end{tabular}




\section{CHRONIC DEFINITIVE SURVIVAL AND REPRODUCTION TEST Statistical Analyses}

Client: Westinghouse Savannah River Company

Sample Identification: FHB-006

Test Date: $\quad 2 / 29 / 96$

\begin{tabular}{|lrr|}
\hline Test for Normality & \\
Test Used: Shapiro Wilks & $\mathrm{W}=$ & 0.962 \\
& critical & 0.93 \\
& \\
The data are normal in distribution
\end{tabular}

\begin{tabular}{|lrr|}
\hline Test for Homogeneity of Variance & \\
Test Used: Bartlett's Test & $\mathrm{B}=$ & 10.22 \\
& critical & 15.1 \\
& - & \\
The data are homogeneous in variance \\
\hline
\end{tabular}

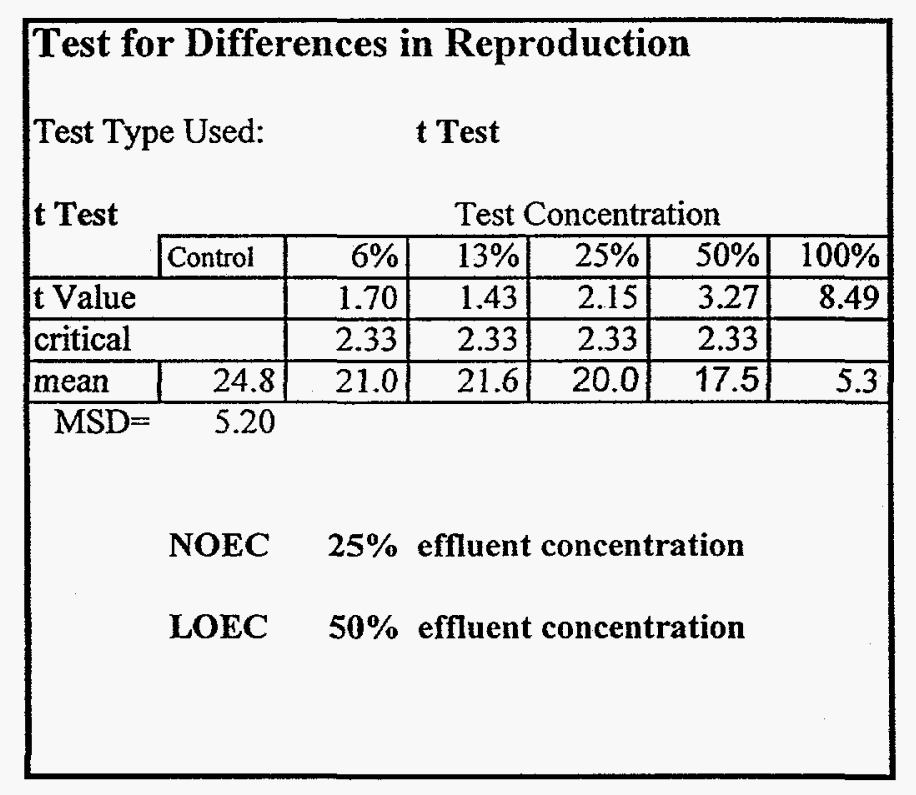

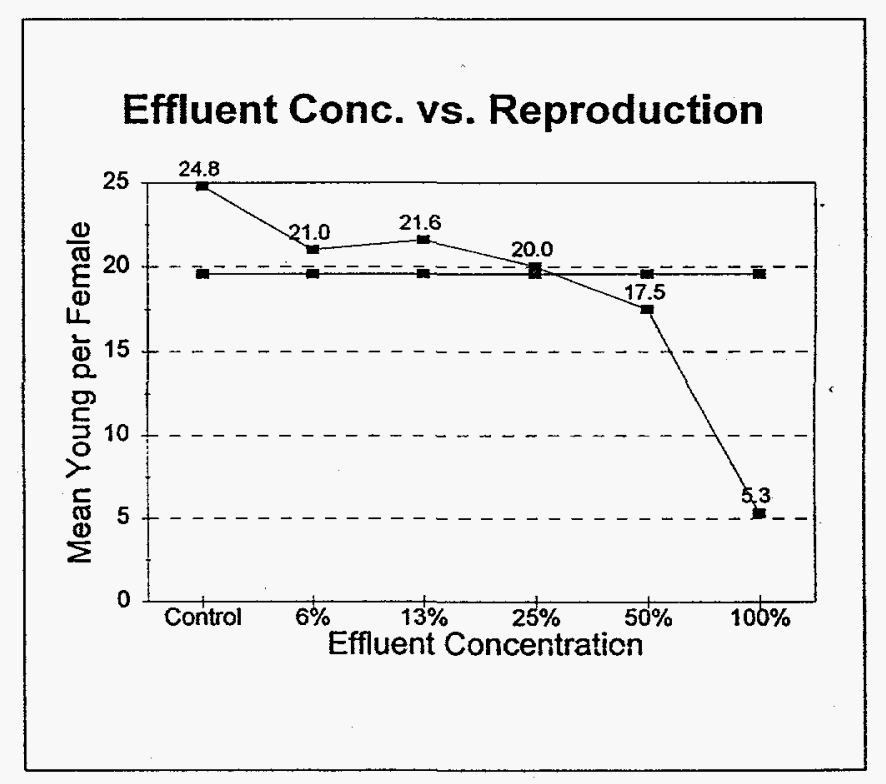

Summary 


\section{CHRONIC DEFINITIVE SURVIVAL AND REPRODUCTION TEST Water Chemistry}

Client: Westinghouse Savannah River Company

Sample Identification: FHB-006

Test Date: $\quad$ 2/29/96

\section{pH (After Renewal)}

\begin{tabular}{|l|r|r|r|r|r|r|}
\hline \multicolumn{7}{|c|}{ Test Concentration } \\
\hline & Control & $6.25 \%$ & $12.5 \%$ & $25 \%$ & $50 \%$ & $100 \%$ \\
\hline Initial & 8.12 & 7.83 & 7.57 & 7.06 & 6.76 & 5.79 \\
\hline Day 1 & 7.98 & 7.88 & 7.84 & 7.52 & 6.91 & 6.58 \\
\hline Day 2 & 7.97 & 7.93 & 7.81 & 7.50 & 7.08 & 6.77 \\
\hline Day 3 & 7.98 & 7.97 & 7.92 & 7.53 & 7.04 & 5.83 \\
\hline Day 4 & 8.06 & 7.89 & 7.70 & 7.67 & 7.11 & 6.11 \\
\hline Day 5 & 8.05 & 7.99 & 7.78 & 7.48 & 6.87 & 5.54 \\
\hline Day 6 & 8.14 & 7.89 & 7.67 & 7.33 & 6.89 & 5.71 \\
\hline
\end{tabular}

Dissolved Oxygen (After Renewal)

\begin{tabular}{|l|r|r|r|r|r|r|}
\hline \multicolumn{7}{|c|}{ Test Concentration } \\
\hline & Control & $6.25 \%$ & $12.5 \%$ & $25 \%$ & $50 \%$ & $100 \%$ \\
\hline Initial & 8.6 & 8.2 & 8.3 & 8.2 & 8.2 & 7.9 \\
\hline Day 1 & 8.6 & 8.2 & 8.2 & 8.3 & 8.3 & 8.5 \\
\hline Day 2 & 8.5 & 8.4 & 8.3 & 8.4 & 8.6 & 8.4 \\
\hline Day 3 & 8.6 & 8.4 & 8.4 & 8.5 & 8.6 & 8.8 \\
\hline Day 4 & 8.8 & 8.2 & 8.2 & 8.4 & 8.4 & 8.5 \\
\hline Day 5 & 8.9 & 8.4 & 8.2 & 8.3 & 8.4 & 8.6 \\
\hline Day 6 & 8.7 & 8.4 & 8.3 & 8.4 & 8.6 & 8.5 \\
\hline
\end{tabular}

Dilution Water \& Effluent Parameters

\begin{tabular}{|l|c|r|r|r|r|}
\hline & Res Cl. & Alkalin & Hard. & Cond. & \\
\hline & $\mathrm{mg} / \mathrm{L}$ & $\mathrm{mg} / \mathrm{L}$ & $\mathrm{mg} / \mathrm{L}$ & $\mu \mathrm{mhos}$ & \\
\hline Eff. Sample 1 & $<0.05$ & 4.06 & 14 & 34.3 & \\
\hline Eff. Sample 2 & $<0.05$ & 4.06 & 6 & 32.2 & \\
\hline Eff. Sample 3 & $<0.05$ & 6.09 & 14 & 35.8 & \\
\hline & & & & & \\
\hline Dilution Water & & 71.1 & 84 & 222 & \\
\hline & & & & & \\
\hline & & & & & \\
\hline
\end{tabular}

pH (Before Renewal)

\begin{tabular}{|l|r|r|r|r|r|r|}
\hline \multicolumn{7}{|c|}{ Test Concentration } \\
\hline & Control & $6.25 \%$ & $12.5 \%$ & $25 \%$ & $50 \%$ & $100 \%$ \\
\hline Day 1 & 8.10 & 8.28 & 8.24 & 8.21 & 7.76 & 7.27 \\
\hline Day 2 & 8.27 & 8.16 & 7.83 & 7.68 & 7.21 & 7.40 \\
\hline Day 3 & 8.44 & 8.48 & 8.49 & 8.52 & 7.86 & 7.50 \\
\hline Day 4 & 8.09 & 8.31 & 8.39 & 8.44 & 7.89 & 7.76 \\
\hline Day 5 & 8.33 & 8.29 & 8.16 & 8.18 & 7.85 & 6.88 \\
\hline Day 6 & 8.30 & 8.37 & 8.18 & 8.05 & 7.86 & 7.89 \\
\hline Final & 8.21 & 7.92 & 8.14 & 8.00 & 8.11 & 8.19 \\
\hline
\end{tabular}

Dissolved Oxygen (Before Renewal)

\begin{tabular}{|l|r|r|r|r|r|r|}
\hline \multicolumn{7}{|c|}{ Test Concentration } \\
\hline & Control & $6.25 \%$ & $12.5 \%$ & $25 \%$ & $50 \%$ & $100 \%$ \\
\hline Day 1 & 8.3 & 8.4 & 8.4 & 8.5 & 8.5 & 8.6 \\
\hline Day 2 & 8.2 & 8.0 & 8.1 & 8.2 & 8.0 & 8.1 \\
\hline Day 3 & 8.8 & 8.2 & 8.2 & 8.4 & 8.7 & 9.0 \\
\hline Day 4 & 8.6 & 8.0 & 8.2 & 8.3 & 8.2 & 8.3 \\
\hline Day 5 & 8.5 & 8.1 & 8.0 & 8.2 & 8.4 & 8.4 \\
\hline Day 6 & 8.6 & 8.2 & 8.1 & 8.3 & 8.3 & 8.2 \\
\hline Final & 8.3 & 8.2 & 8.0 & 8.3 & 8.2 & 8.4 \\
\hline
\end{tabular}


(864) 877-6942 - FAX (864) 877-6938

P.O. Box 16414, Greenville, SC 29606 • 4 Craftsman Court, Greer, SC 29650

\section{Day Chronic Definitive Survival and Reproduction Bioassay}

Method: EPA/600/4-89/001

Test Organism:

Ceriodaphnia duibia

Facility: Westinghouse Savannah River Company

Sample ID: FHB-012

Date: $\quad 4 / 11 / 96$ 


\section{CHRONIC DEFINITIVE SURVIVAL AND REPRODUCTION TEST Survival and Reproduction Results}

Client: $\quad$ WSRC

Sample Identification: FHB-012

Test Date: $\quad$ 4/11/96 $\quad$ T5364

\begin{tabular}{|c|c|c|c|c|c|c|}
\hline \multicolumn{3}{|c|}{ Reproduction at } & $0.0 \%$ & \multicolumn{3}{c|}{ Concentration } \\
\hline Rep. & Day 3/4 & Day 5 & Day 6 & Day 7 & Day 8 & Total \\
\hline 1 & 4 & 1 & 6 & 9 & 0 & 20 \\
\hline 2 & 5 & 9 & 7 & 0 & 0 & 21 \\
\hline 3 & 4 & 6 & 0 & 9 & 0 & 19 \\
\hline 4 & 5 & 4 & 0 & 6 & 0 & 15 \\
\hline 5 & 0 & 3 & 6 & 4 & 0 & 13 \\
\hline 6 & 0 & 7 & 8 & 10 & 0 & 25 \\
\hline 7 & 4 & 6 & 0 & 6 & 0 & 16 \\
\hline 8 & 3 & 5 & 0 & 10 & 0 & 18 \\
\hline 9 & 0 & 5 & 7 & 8 & 0 & 20 \\
\hline 10 & 0 & 8 & 0 & 9 & 0 & 17 \\
\hline
\end{tabular}

\begin{tabular}{|c|c|c|c|c|c|c|}
\hline \multicolumn{3}{|c|}{ Reproduction at } & \multicolumn{3}{c|}{ Co\% } & \multicolumn{3}{c|}{ Concentration } \\
\hline Rep. & Day 3/4 & Day 5 & Day 6 & Day 7 & Day 8 & Total \\
\hline 1 & 4 & 4 & 0 & 9 & 0 & 17 \\
\hline 2 & 4 & 11 & 9 & 0 & 0 & 24 \\
\hline 3 & 3 & 0 & 7 & 8 & 0 & 18 \\
\hline 4 & 2 & 3 & 7 & 8 & 0 & 20 \\
\hline 5 & 4 & 2 & 0 & 7 & 0 & 13 \\
\hline 6 & 0 & 7 & 0 & 8 & 0 & 15 \\
\hline 7 & 0 & 6 & 9 & 11 & 0 & 26 \\
\hline 8 & 3 & 8 & 0 & 9 & 0 & 20 \\
\hline 9 & 4 & 6 & 0 & 8 & 0 & 18 \\
\hline 10 & 3 & 5 & 0 & 7 & 0 & 15 \\
\hline
\end{tabular}

\begin{tabular}{|c|c|c|c|c|c|c|}
\hline \multicolumn{3}{|c|}{ Reproduction at } & \multirow{2}{*}{$\frac{12.0 \%}{\text { Day } 6}$} & \multicolumn{3}{|c|}{ Concentration } \\
\hline Rep. & Day 3/4 & Day 5 & & Day 7 & Day 8 & Total \\
\hline 1 & 3 & 5 & $\overline{0}$ & 7 & 0 & 15 \\
\hline 2. & 5 & 7 & 9 & .0 & 0 & 21 \\
\hline 3 & 4 & 0 & 10 & 6 & 0 & 20 \\
\hline 4 & 0 & 8 & 0 & 9 & 0 & 17 \\
\hline 5 & $\overline{0}$ & 5 & 7 & 8 & $\overline{0}$ & 20 \\
\hline 6 & L & $\bar{L}$ & $\bar{L}$ & $L$ & 0 & $\mathbf{L}$ \\
\hline 7 & 5 & 5 & 0 & 10 & 0 & 20 \\
\hline 8 & 4 & 6 & 0 & $\overline{9}$ & 0 & 19 \\
\hline 9 & 0 & 6 & 8 & $\overline{0}$ & 0 & 14 \\
\hline 10 & 3 & 4 & 0 & 8 & 0 & 15 \\
\hline
\end{tabular}

\begin{tabular}{|c|c|c|c|c|c|c|}
\hline \multicolumn{3}{|c|}{ Reproduction at } \\
\hline Rep. & Day 3/4 & Day 5 & Day 6 & Day 7 & Day 8 & Total \\
\hline 1 & 4 & 7 & 0 & 9 & 0 & 20 \\
\hline 2 & 4 & 7 & 0 & 7 & 0 & 18 \\
\hline 3 & 3 & 0 & 6 & 6 & 0 & 15 \\
\hline 4 & 5 & 5 & 0 & 8 & 0 & 18 \\
\hline 5 & 3 & 0 & 6 & 9 & 0 & 18 \\
\hline 6 & 2 & 4 & 0 & 8 & 0 & 14 \\
\hline 7 & 0 & 4 & 6 & 0 & 0 & 10 \\
\hline 8 & 1 & 6 & 0 & 6 & 0 & 13 \\
\hline 9 & 4 & 0 & 7 & 8 & 0 & 19 \\
\hline 10 & 4 & 6 & 0 & 9 & 0 & 19 \\
\hline
\end{tabular}

\begin{tabular}{|c|c|c|c|c|c|c|}
\hline \multicolumn{3}{|c|}{ Reproduction at } & \multicolumn{3}{c|}{$50 \%$} & \multicolumn{3}{c|}{ Concentration } \\
\hline Rep. & Day 3/4 & Day 5 & Day 6 & Day 7 & Day 8 & Total \\
\hline 1 & 4 & 7 & 1 & 0 & 0 & 12 \\
\hline 2 & 3 & 2 & 6 & 0 & 0 & 11 \\
\hline 3 & 3 & 0 & 6 & 4 & 0 & 13 \\
\hline 4 & 4 & 2 & 4 & 3 & 0 & 13 \\
\hline 5 & 4 & 0 & 0 & 6 & 0 & 10 \\
\hline 6 & 0 & 2 & 4 & 0 & 0 & 6 \\
\hline 7 & 0 & 5 & 5 & 0 & 0 & 10 \\
\hline 8 & 0 & 4 & 8 & 0 & 0 & 12 \\
\hline 9 & 0 & 4 & 6 & 4 & 0 & 14 \\
\hline 10 & 4 & 0 & 7 & 0 & 0 & 11 \\
\hline
\end{tabular}

\begin{tabular}{|c|c|c|c|c|c|c|}
\hline \multicolumn{9}{|c|}{$100.0 \%$} & Concentration \\
\hline Reproduction at & Day 3/4 & Day 5 & Day 6 & Day 7 & Day 8 & Total \\
\hline 1 & 4 & 4 & 0 & 3 & 0 & 11 \\
\hline 2 & 4 & 5 & 4 & 0 & 0 & 13 \\
\hline 3 & 3 & 0 & 6 & 2 & 0 & 11 \\
\hline 4 & 0 & 3 & 0 & 1 & 0 & 4 \\
\hline 5 & 1 & 2 & 0 & $X$ & 0 & 3 \\
\hline 6 & 2 & 2 & 0 & 5 & 0 & 9 \\
\hline 7 & 0 & 3 & 0 & 4 & 0 & 7 \\
\hline 8 & 3 & 4 & 8 & 0 & 0 & 15 \\
\hline 9 & 0 & 1 & 0 & 3 & 0 & 4 \\
\hline 10 & 4 & 0 & 0 & 3 & 0 & 7 \\
\hline
\end{tabular}




\section{CHRONIC DEFINITIVE SURVIVAL AND REPRODUCTION TEST Statistical Analyses}

Client: WSRC

Sample Identification:

FHB-012

Test Date:

$4 / 11 / 96$

\begin{tabular}{|lrr}
\hline Test for Normality. & & \\
& & \\
Test Used: Shapiro Wilks & W= & 0.934 \\
& critical & 0.93
\end{tabular}

The data are normal in distribution

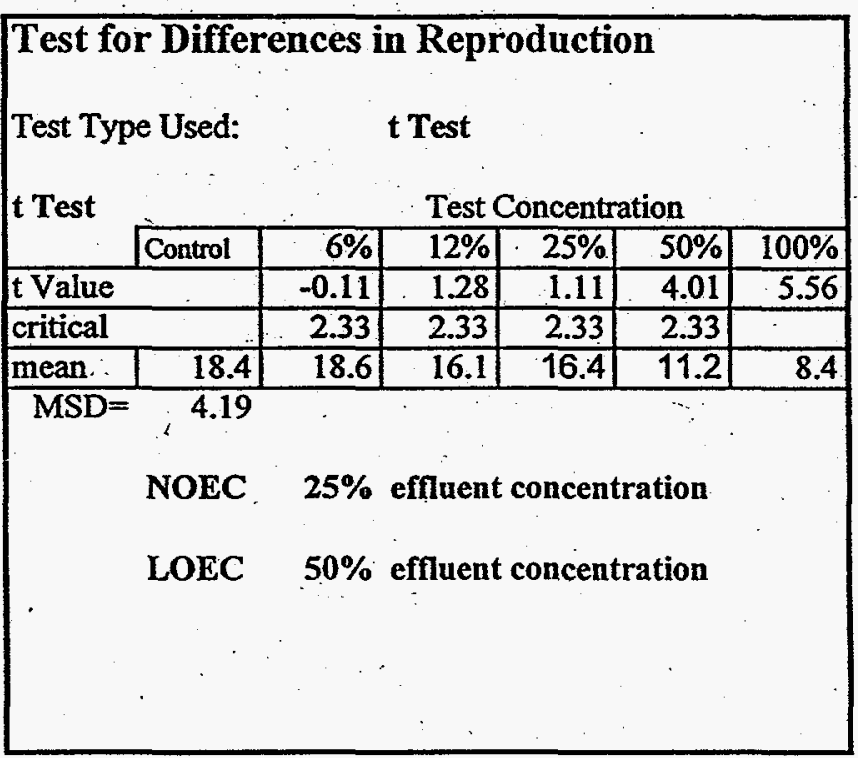
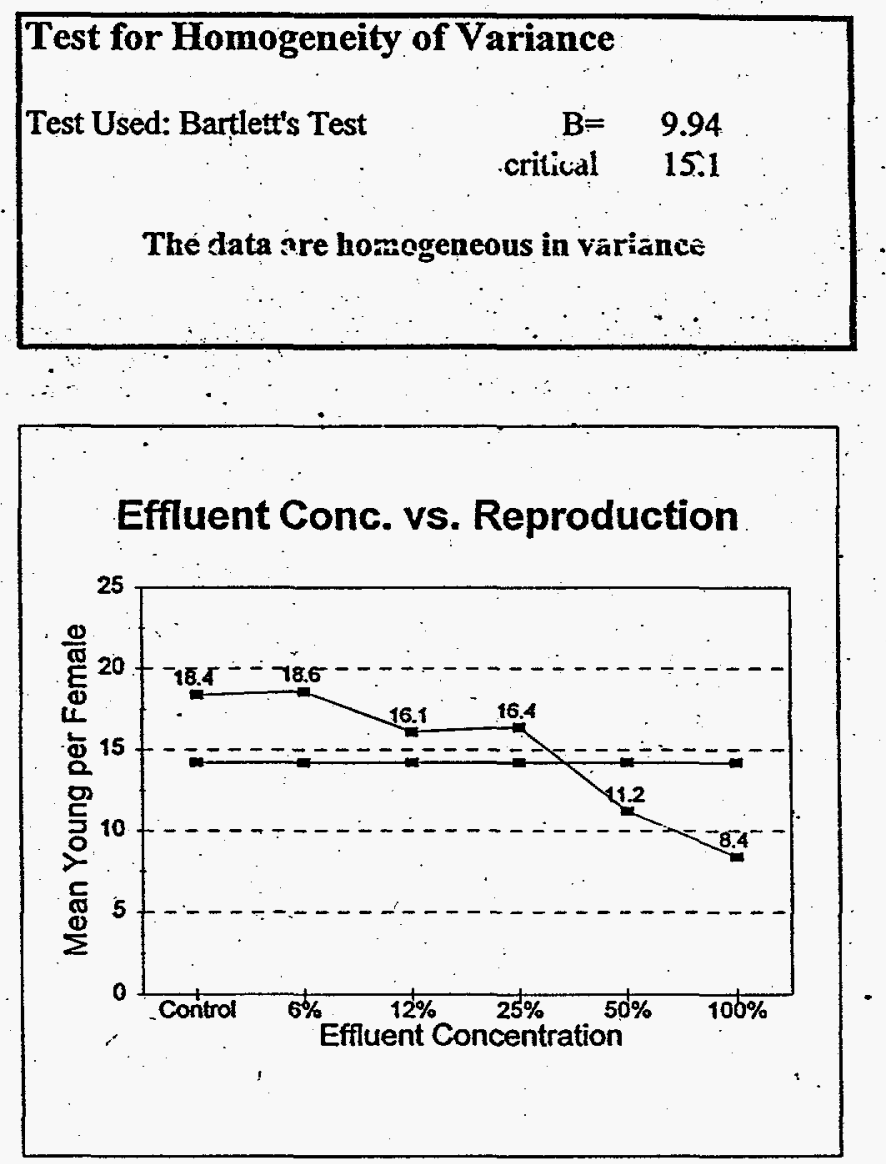

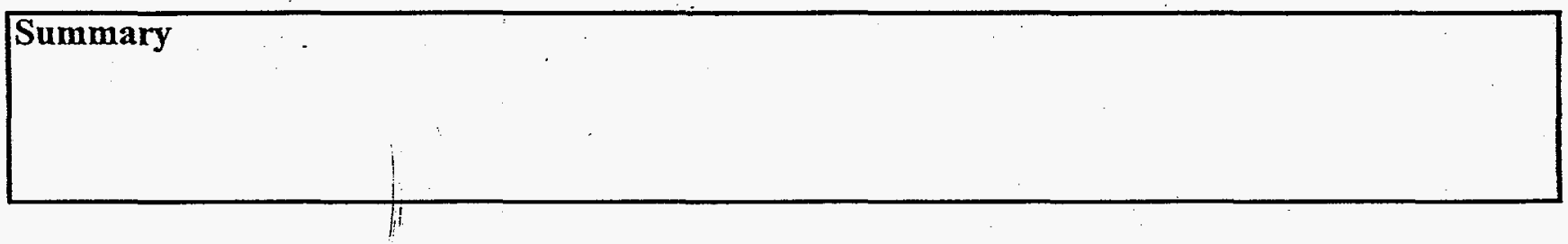




\section{CHRONIC DEFINITIVE SURVIVAL AND REPRODUCTION TEST Water Chemistry}

Client: Westinghouse Savannah River Company

Sample Identification: FHB-012

Test Date: $\quad$ 4/11/96

$\mathrm{pH}$ (After Renewal)

\begin{tabular}{|l|r|r|r|r|r|r|}
\hline \multicolumn{7}{|c|}{ Test Concentration } \\
\hline & Control & $6 \%$ & $12 \%$ & $25 \%$ & $50 \%$ & $100 \%$ \\
\hline Initial & 8.30 & 6.97 & 6.43 & 6.29 & 6.17 & 6.08 \\
\hline Day 1 & 8.10 & 7.96 & 7.23 & 6.99 & 6.37 & 6.14 \\
\hline Day 2 & 7.95 & 7.02 & 7.00 & 6.48 & 6.27 & 6.18 \\
\hline Day 3 & 8.03 & 7.35 & 7.10 & 6.89 & 6.57 & 6.39 \\
\hline Day 4 & 8.09 & 7.89 & 7.54 & 6.79 & 6.08 & 5.59 \\
\hline Day 5 & 8.07 & 7.04 & 7.19 & 7.23 & 7.13 & 5.77 \\
\hline Day 6 & 8.08 & 7.91 & 7.65 & 7.24 & 6.57 & 5.59 \\
\hline
\end{tabular}

Dissolved Oxygen (After Renewal)

\begin{tabular}{|l|r|r|r|r|r|r|}
\hline \multicolumn{7}{|c|}{ Test Concentration } \\
\hline & Control & $6 \%$ & $12 \%$ & $25 \%$ & $50 \%$ & $100 \%$ \\
\hline Initial & 8.7 & 8.4 & 8.5 & 8.4 & 8.3 & 8.4 \\
\hline Day 1 & 8.8 & 8.5 & 8.5 & 8.5 & 8.6 & 8.6 \\
\hline Day 2 & 8.7 & 8.2 & 8.4 & 8.4 & 8.6 & 8.8 \\
\hline Day 3 & 8.6 & 8.7 & 8.6 & 8.7 & 8.6 & 8.8 \\
\hline Day 4 & 8.5 & 8.4 & 8.4 & 8.5 & 8.5 & 8.7 \\
\hline Day 5 & 8.7 & 8.4 & 8.5 & 8.5 & 8.6 & 8.6 \\
\hline Day 6 & 8.7 & 8.4 & 8.4 & 8.6 & 8.6 & 8.8 \\
\hline
\end{tabular}

Dilution Water \& Effluent Parameters

\begin{tabular}{|l|r|r|r|r|r|}
\hline & Res Cl. & Alkalin & Hard. & Cond. & \\
\hline & $\mathrm{mg} / \mathrm{L}$ & $\mathrm{mg} / \mathrm{L}$ & $\mathrm{mg} / \mathrm{L}$ & $\mu$ mhos & \\
\hline Eff. Sample 1 & $<0.05$ & no data & 10 & no data & \\
\hline Eff. Sample 2 & & & 10 & & \\
\hline Eff. Sample 3 & & & 6 & & \\
\hline & & & & & \\
\hline Dilution Water & & 69 & 86 & 178 & \\
\hline & & & & & \\
\hline & & & & & \\
\hline
\end{tabular}

pH (Before Renewal)

\begin{tabular}{|l|r|r|r|r|r|r|}
\hline \multicolumn{7}{|c|}{ Test Concentration } \\
\hline & Control & $6 \%$ & $12 \%$ & $25 \%$ & $50 \%$ & $100 \%$ \\
\hline Day 1 & 8.18 & 7.79 & 7.48 & 7.24 & 7.19 & 7.04 \\
\hline Day 2 & 8.14 & 7.97 & 7.71 & 7.53 & 7.31 & 7.11 \\
\hline Day 3 & 8.20 & 7.47 & 7.27 & 7.10 & 6.79 & 6.53 \\
\hline Day 4 & 8.26 & 7.97 & .7 .56 & 7.22 & 6.84 & 6.18 \\
\hline Day 5 & 8.33 & 7.81 & 7.50 & 7.10 & 6.67 & 6.03 \\
\hline Day 6 & 8.11 & 7.79 & 7.47 & 7.34 & 6.81 & 6.13 \\
\hline Final & 8.07 & 7.73 & 7.58 & 7.31 & 6.71 & 6.12 \\
\hline
\end{tabular}

Dissolved Oxygen (Before Renewal)

\begin{tabular}{|l|r|r|r|r|r|r|}
\hline \multicolumn{7}{|c|}{ Test Concentration } \\
\hline & Control & $6 \%$ & $12 \%$ & $25 \%$ & $50 \%$ & $100 \%$ \\
\hline Day 1 & 8.6 & 8.5 & 8.5 & 8.6 & 8.6 & 8.8 \\
\hline Day 2 & 8.5 & 8.3 & 8.4 & 8.4 & 8.6 & 8.7 \\
\hline Day 3 & 8.2 & 8.4 & 8.6 & 8.5 & 8.6 & $\mathbf{8 . 4}$ \\
\hline Day 4 & 8.6 & 8.4 & 8.4 & 8.5 & 8.5 & 8.5 \\
\hline Day 5 & 8.6 & 8.5 & 8.5 & 8.5 & 8.6 & 8.6 \\
\hline Day 6 & 8.5 & 8.4 & 8.4 & 8.5 & 8.6 & .8 .7 \\
\hline Final & 8.6 & 8.2 & 8.2 & 8.6 & 8.6 & 8.8 \\
\hline
\end{tabular}

Temperature
\begin{tabular}{|l|r|}
\hline Incubator ${ }^{\circ} \mathrm{C}$ \\
\hline Initial & 24.5 \\
\hline Day 1 & 24.5 \\
\hline Day 2 & 25.3 \\
\hline Day 3 & 24.9 \\
\hline Day 4 & 25.5 \\
\hline Day 5 & 25.0 \\
\hline Day 6 & 26.0 \\
\hline Final & 24.8 \\
\hline
\end{tabular}




\section{Day Chronic Definitive Survival and Reproduction Bioassay Method: EPA/600/4-89/001}

Test Organism:

Ceriodaphnia dubia

Facility: Westinghouse Savannah River Company

Sample ID: FHB-013

Date: $\quad 4 / 11 / 96$ 


\section{CHRONIC DEFINITIVE SURVIVAL AND REPRODUCTION TEST Survival and Reproduction Results}

Client: WSRC

Sample Identification: FHB-013

Test Date: $\quad 4 / 11 / 96 \quad$ T5365

\begin{tabular}{|c|c|c|c|c|c|c|}
\hline \multicolumn{3}{|c|}{ Reproduction at } & $0.0 \%$ & \multicolumn{3}{c|}{ Concentration } \\
\hline Rep. & Day 3/4 & Day 5 & Day 6 & Day 7 & Day 8 & Total \\
\hline 1 & 3 & 0 & 7 & 9 & 0 & 19 \\
\hline 2 & 0 & 4 & 0 & 7 & 10 & 21 \\
\hline 3 & 0 & 4 & 0 & 8 & 6 & 18 \\
\hline 4 & 3 & 6 & 0 & 10 & 0 & 19 \\
\hline 5 & 3 & 6 & 0 & 8 & 0 & 17 \\
\hline 6 & 2 & 0 & 6 & 10 & 0 & 18 \\
\hline 7 & 0 & 4 & 6 & 0 & 9 & 19 \\
\hline 8 & 0 & 4 & 5 & 0 & 8 & 17 \\
\hline 9 & 0 & 3 & 7 & 0 & 10 & 20 \\
\hline 10 & 4 & 0 & 6 & 10 & 0 & 20 \\
\hline
\end{tabular}

\begin{tabular}{|c|c|c|c|c|c|c|}
\hline \multicolumn{4}{|c|}{ Reproduction at } & $6.0 \%$ & \multicolumn{3}{c|}{ Concentration } \\
\hline Rep. & Day 3/4 & Day 5 & Day 6 & Day 7 & Day 8 & Total \\
\hline 1 & 0 & 5 & 0 & 6 & 12 & 23 \\
\hline 2 & 0 & 0 & 0 & 5 & 6 & 11 \\
\hline 3 & 4 & 6 & 4 & 0 & 12 & 26 \\
\hline 4 & 2 & 5 & 0 & 10 & 0. & 17 \\
\hline 5 & 4 & 5 & 0 & 9 & 0 & 18 \\
\hline 6 & 0 & 0 & 0 & 6 & 11 & 17 \\
\hline 7 & 0 & 4 & 7 & 0 & 6 & 17 \\
\hline 8 & 0 & 5 & 8 & 0 & 7 & 20 \\
\hline 9 & 3 & 4 & 0 & 10 & 0 & 17 \\
\hline 10 & 0 & 2 & 7 & 0 & 12 & 21 \\
\hline
\end{tabular}

\begin{tabular}{|c|c|c|c|c|c|c|}
\hline \multicolumn{3}{|c|}{ Reproduction at } & $12.0 \%$ & \multicolumn{3}{c|}{ Concentration } \\
\hline Rep. & Day 3/4 & Day 5 & Day 6 & Day 7 & Day 8 & Total \\
\hline 1 & 0 & 0 & 3 & 6 & 0 & 9 \\
\hline 2 & 0 & 0 & 4 & 7 & 0 & 11 \\
\hline 3 & 0 & 3 & 0 & 8 & 8 & 19 \\
\hline 4 & 0 & 8 & 4 & 0 & 10 & 22 \\
\hline 5 & 0 & 7 & 0 & 6 & 12 & 25 \\
\hline 6 & 0 & 0 & 8 & 0 & 14 & 22 \\
\hline 7 & 0 & 3 & 0 & 5 & 0 & 8 \\
\hline 8 & 0 & 0 & 4 & 0 & 7 & 11 \\
\hline 9 & 0 & 4 & 7 & 0 & 8 & 19 \\
\hline 10 & 0 & 4 & 8 & 0 & 12 & 24 \\
\hline
\end{tabular}

\begin{tabular}{|c|c|c|c|c|c|c|}
\hline \multicolumn{3}{|c|}{ Reproduction at } \\
\hline Rep. & Day 3/4 & Day 5 & Day 6 & Day 7 & Day 8 & Total \\
\hline 1 & 0 & 1 & 0 & 3 & 0 & 4 \\
\hline 2 & 4 & 2 & 0 & 4 & 3 & 13 \\
\hline 3 & 3 & 1 & 0 & 0 & 1 & 5 \\
\hline 4 & 0 & 1 & 3 & 0 & 0 & 4 \\
\hline 5 & 2 & 3 & 0 & 0 & 9 & -14 \\
\hline 6 & 4 & 6 & 0 & 0 & $X$ & 10 \\
\hline 7 & 0 & 0 & 3 & 0 & 6 & 9 \\
\hline 8 & 0 & 0 & 2 & 3 & 5 & 10 \\
\hline 9 & 0 & 4 & 4 & 0 & 0 & 8 \\
\hline 10 & 0 & 2 & 6 & 2 & 4 & 14 \\
\hline
\end{tabular}

\begin{tabular}{|c|c|c|c|c|c|c|}
\hline \multicolumn{3}{|c|}{ Reproduction at } & $50.0 \%$ & \multicolumn{4}{c|}{ Concentration } \\
\hline Rep. & Day 3/4 & Day 5 & Day 6 & Day 7 & Day 8 & Total \\
\hline 1 & 0 & 4 & 0 & 0 & 3 & 7 \\
\hline 2 & 0 & 4 & 0 & 3 & 0 & 7 \\
\hline 3 & 0 & 3 & 2 & 0 & 2 & 7 \\
\hline 4 & 0 & 0 & 3 & 0 & 1 & 4 \\
\hline 5 & 2 & 3 & 0 & 4 & 0 & 9 \\
\hline 6 & 3 & 3 & 0 & 0 & 4 & 10 \\
\hline 7 & 0 & 0 & 0 & 3 & 0 & 3 \\
\hline 8 & 0 & 0 & 3 & 0 & 1 & 4 \\
\hline 9 & 0 & 1 & 0 & 0 & 0 & 1 \\
\hline 10 & 0 & 3 & 0 & 4 & 3 & 10 \\
\hline
\end{tabular}

\begin{tabular}{|c|c|c|c|c|c|c|}
\hline \multicolumn{3}{|c|}{ Reproduction at } & $100.0 \%$ & \multicolumn{3}{c|}{ Concentration } \\
\hline Rep. & Day 3/4 & Day 5 & Day 6 & Day 7 & Day 8 & Total \\
\hline 1 & 0 & 2 & 0 & 0 & 3 & 5 \\
\hline 2 & 0 & 0 & 0 & 0 & 0 & 0 \\
\hline 3 & 0 & 0 & 0 & 0 & 2 & 2 \\
\hline 4 & 0 & 4 & 0 & 0 & 1 & 5 \\
\hline 5 & L & L & L & L & L & L \\
\hline 6 & 0 & 3 & 0 & 0 & 0 & 3 \\
\hline 7 & 0 & 2 & 0 & 0 & 3 & 5 \\
\hline 8 & 0 & 0 & 0 & 0 & 0 & 0 \\
\hline 9 & 0 & 0 & 0 & 0 & 0 & 0 \\
\hline 10 & 0 & 4 & 0 & 0 & 2 & 6 \\
\hline
\end{tabular}




\section{CHRONIC DEFINITIVE SURVIVAL AND REPRODUCTION TEST \\ Statistical Analyses}

Client: WSRC

Sample Identification: FHB-013

Test Date: $\quad 4 / 11 / 96$

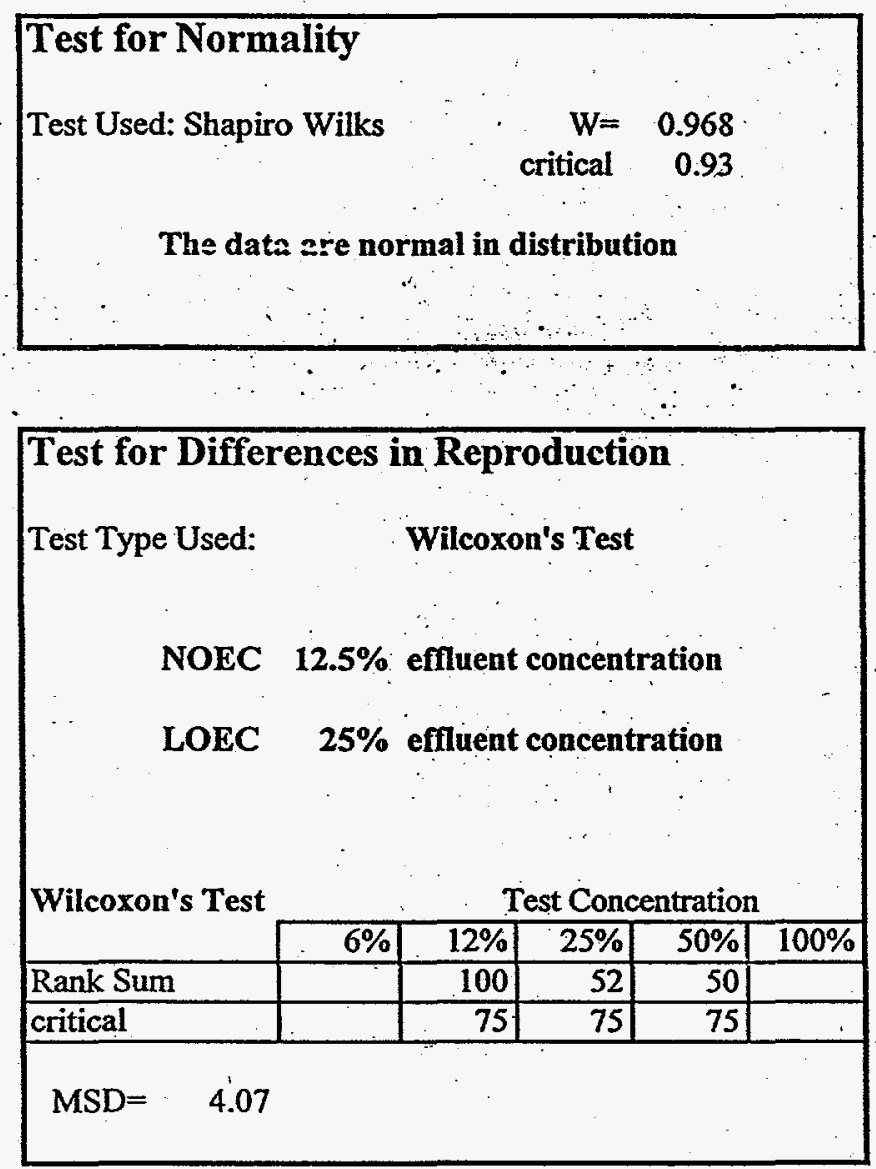
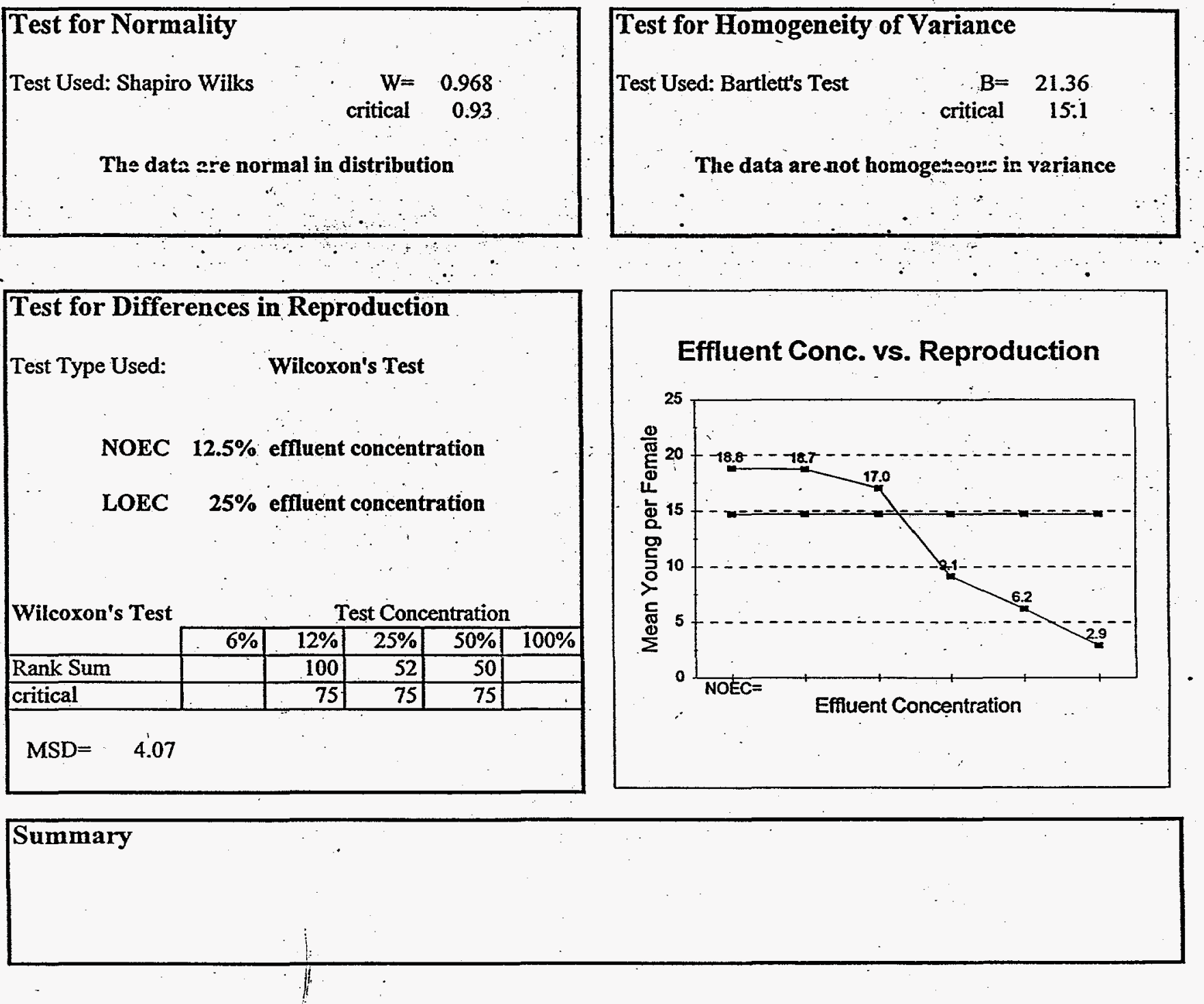


\section{CHRONIC DEFINITIVE SURVIVAL AND REPRODUCTION TEST Water Chemistry}

Client: Westinghouse Savannah River Company

Sample Identification: FHB-013

Test Date: $\quad$ 4/11/96

\section{pH (After Renewal)}

\begin{tabular}{|l|r|r|r|r|r|r|}
\hline \multicolumn{7}{|c|}{ Test Concentration } \\
\hline & Control & $6 \%$ & $12 \%$ & $25 \%$ & $50 \%$ & $100 \%$ \\
\hline Initial & 8.30 & 7.02 & 6.53 & 6.14 & 5.81 & 5.63 \\
\hline Day 1 & 8.10 & 7.54 & 6.97 & 6.35 & 6.27 & 6.04 \\
\hline Day 2 & 7.95 & no data & no data & no data & no data & no data \\
\hline Day 3 & 8.03 & 7.48 & 7.66 & 7.14 & 6.90 & 5.79 \\
\hline Day 4 & 8.09 & 7.09 & 6.98 & 6.81 & 6.40 & 5.58 \\
\hline Day 5 & 8.07 & 7.10 & 7.25 & 6.93 & 6.28 & 5.39 \\
\hline Day 6 & 8.08 & 7.06 & 7.31 & 7.04 & 6.19 & 5.43 \\
\hline
\end{tabular}

\section{Dissolved Oxygen (After Renewal)}

\begin{tabular}{|l|r|r|r|r|r|r|}
\hline \multicolumn{7}{|c|}{ Test Concentration } \\
\hline & Control & $6 \%$ & $12 \%$ & $25 \%$ & $50 \%$ & $100 \%$ \\
\hline Initial & 8.7 & 8.4 & 8.5 & 8.6 & 8.4 & 8.6 \\
\hline Day 1 & 8.8 & 8.6 & 8.6 & 8.6 & 8.8 & 8.8 \\
\hline Day 2 & 8.7 & no data & no data & no data & no data & no data \\
\hline Day 3 & 8.6 & 8.6 & 8.7 & 8.6 & 8.7 & 8.7 \\
\hline Day 4 & 8.5 & 8.6 & 8.6 & 8.4 & 8.8 & 8.6 \\
\hline Day 5 & 8.7 & 8.7 & 8.7 & 8.6 & 8.7 & 8.6 \\
\hline Day 6 & 8.7 & 8.6 & 8.6 & 8.7 & 8.6 & 8.6 \\
\hline
\end{tabular}

\section{Dilution Water \& Effluent Parameters}

\begin{tabular}{|l|r|r|r|r|l|}
\hline & Res Cl. & Alkalin & Hard. & Cond. & \\
\hline & $\mathrm{mg} / \mathrm{L}$ & $\mathrm{mg} / \mathrm{L}$ & $\mathrm{mg} / \mathrm{L}$ & $\mu$ mhos & \\
\hline Eff. Sample 1 & $<0.05$ & no data & 16 & no data & \\
\hline Eff. Sample 2 & & & 10 & & \\
\hline Eff. Sample 3 & & & 8 & & \\
\hline & & & & & \\
\hline Dilution Water & & 69 & 86 & 178 & \\
\hline & & & & & \\
\hline & & & & & \\
\hline
\end{tabular}

\section{pH (Before Renewal)}

\begin{tabular}{|l|r|r|r|r|r|r|}
\hline \multicolumn{7}{|c|}{ Test Concentration } \\
\hline & Control & $6 \%$ & $12 \%$ & $25 \%$ & $50 \%$ & $100 \%$ \\
\hline Day 1 & 8.18 & 7.59 & 7.03 & 6.48 & 6.25 & 6.07 \\
\hline Day 2 & 8.14 & no data & no data & no data & no data & no data \\
\hline Day 3 & 8.20 & 7.85 & 7.88 & 7.68 & 7.43 & 6.34 \\
\hline Day 4 & 8.26 & 7.56 & 7.39 & 7.18 & 7.21 & 6.14 \\
\hline Day 5 & 8.33 & 7.44 & 7.63 & 6.98 & 6.73 & 6.19 \\
\hline Day 6 & 8.11 & 7.40 & 7.68 & 7.04 & 6.68 & 5.97 \\
\hline Final & 8.07 & 7.41 & 7.73 & 6.96 & 6.18 & 6.14 \\
\hline
\end{tabular}

Dissolved Oxygen (Before Renewal)

\begin{tabular}{|l|r|r|r|r|r|r|}
\hline \multicolumn{7}{|c|}{ Test Concentration } \\
\hline & Control & $6 \%$ & $12 \%$ & $25 \%$ & $50 \%$ & $100 \%$ \\
\hline Day 1 & 8.6 & 8.4 & 8.4 & 8.4 & 8.5 & 8.6 \\
\hline Day 2 & 8.5 & no data & no data & no data & no data & no data \\
\hline Day 3 & 8.2 & 8.4 & 8.4 & 8.5 & 8.4 & 8.5 \\
\hline Day 4 & 8.6 & -8.5 & 8.4 & 8.6 & 8.4 & 8.6 \\
\hline Day 5 & 8.6 & 8.3 & 8.6 & 8.4 & 8.6 & 8.4 \\
\hline Day 6 & 8.5 & 8.4 & 8.4 & 8.5 & 8.6 & 8.6 \\
\hline Final & 8.6 & 8.2 & 8.3 & 8.2 & 8.4 & 8.4 \\
\hline
\end{tabular}

\section{Temperature}

\begin{tabular}{|l|r|}
\hline \multicolumn{2}{|c|}{ Incubator ${ }^{\circ} \mathrm{C}$} \\
\hline Initial & 24.5 \\
\hline Day 1 & 24.5 \\
\hline Day 2 & 25.3 \\
\hline Day 3 & 24.9 \\
\hline Day 4 & 25.5 \\
\hline Day 5 & 25.0 \\
\hline Day 6 & 26.0 \\
\hline Final & 24.8 \\
\hline
\end{tabular}


(864) 877-6942 - FAX (864) 877-6938

P.O. Box 16414, Greenville, SC 29606 - 4 Craftsman Court, Greer, SC 29650

\title{
7 Day Chronic Definitive Survival and Reproduction Bioassay
}

Method: EPA/600/4-89/001

\author{
Test Organism: Ceriodaphnia dubia
}

Facility:Westinghouse Savannah River Company

Sample ID:FHB-014

Date: $\quad 2 / 29 / 96$ 


\section{CHRONIC DEFINITIVE SURVIVAL AND REPRODUCTION TEST Survival and Reproduction Results}

Client: Westinghouse Savannah River Company

Sample Identification: FHB-014

Test Date: $\quad$ 2/29/96 T5142

\begin{tabular}{|c|c|c|c|c|c|c|}
\hline \multicolumn{3}{|c|}{ Reproduction at } & \multicolumn{3}{c|}{$0 \%$} & \multicolumn{4}{c|}{ Concentration } \\
\hline Rep. & Day 2/3 & Day 4 & Day 5 & Day 6 & Day 7 & Total \\
\hline 1 & 0 & 6 & 10 & 11 & 0 & 27 \\
\hline 2 & 0 & 5 & 8 & 13 & 0 & 26 \\
\hline 3 & 4 & 0 & 6 & 12 & 0 & 22 \\
\hline 4 & 0 & 7 & 9 & 11 & 0 & 27 \\
\hline 5 & 5 & 7 & 0 & 11 & 0 & 23 \\
\hline 6 & 5 & 10 & 0 & 12 & 0 & 27 \\
\hline 7 & 0 & 6 & 5 & 0 & 8 & 19 \\
\hline 8 & 0 & 7 & 7 & 0 & 8 & 22 \\
\hline 9 & 0 & 0 & 7 & 12 & 10 & 29 \\
\hline 10 & 5 & 0 & 9 & 0 & 10 & 24 \\
\hline
\end{tabular}

\begin{tabular}{|c|c|c|c|c|c|c|}
\hline \multicolumn{3}{|c|}{ Reproduction at } & \multicolumn{3}{c|}{$6.25 \%$} & \multicolumn{3}{c|}{ Concentration } \\
\hline Rep. & Day $2 / 3$ & Day 4 & Day 5 & Day 6 & Day 7 & Tolal \\
\hline 1 & 0 & 8 & 8 & 0 & 12 & 28 \\
\hline 2 & 0 & 7 & 9 & 12 & 0 & 28 \\
\hline 3 & 5 & 0 & 8 & 10 & 0 & 23 \\
\hline 4 & 5 & 1 & 5 & 0 & 0 & 11 \\
\hline 5 & 5 & 0 & 8 & 9 & 0 & 22 \\
\hline 6 & 6 & 10 & 0 & 10 & 0 & 26 \\
\hline 7 & 0 & 6 & 6 & 0 & 0 & 12 \\
\hline 8 & 0 & 9 & 6 & 10 & 0 & 25 \\
\hline 9 & 4 & 0 & 8 & 0 & 6 & 18 \\
\hline 10 & 4 & 0 & 8 & 10 & 0 & 22 \\
\hline
\end{tabular}

\begin{tabular}{|c|c|c|c|c|c|c|}
\hline \multicolumn{2}{|c|}{ Reproduction at } & \multicolumn{3}{c|}{$12.5 \%$} & \multicolumn{3}{c|}{ Concentration } \\
\hline Rep. & Day $2 / 3$ & Day 4 & Day 5 & Day 6 & Day 7 & Total \\
\hline 1 & 0 & 8 & 8 & 0 & 6 & 22 \\
\hline 2 & 0 & 7 & 8 & 10 & 0 & 25 \\
\hline 3 & 0 & 4 & 7 & 12 & 0 & 23 \\
\hline 4 & 6 & 0 & 9 & 10 & 0 & 25 \\
\hline 5 & 0 & 7 & 6 & 11 & 0 & 24 \\
\hline 6 & 3 & 9 & 0 & 12 & 0 & 24 \\
\hline 7 & 0 & 3 & 7 & 10 & 0 & 20 \\
\hline 8 & 0 & 6 & 8 & 0 & 0 & 14 \\
\hline 9 & 0 & 6 & 0 & 0 & 10 & 16 \\
\hline 10 & 5 & 0 & 8 & 11 & 0 & 24 \\
\hline
\end{tabular}

\begin{tabular}{|c|c|c|c|c|c|c|}
\hline \multicolumn{3}{|c|}{ Reproduction at } & \multicolumn{4}{|c|}{$25.0 \%$ Concentration } \\
\hline Rep. & Day $2 / 3$ & $\overline{\text { Day } 4}$ & Day 5 & Day 6 & Day 7 & Total \\
\hline 1 & 0 & $\frac{1}{6}$ & 8 & 0 & 8 & 22 \\
\hline 2 & 0 & 7 & 9 & 0 & 0 & 16 \\
\hline 3 & 5 & $\overline{0}$ & 10 & 10 & 0 & 25 \\
\hline 4 & 5 & 0 & 7 & 13 & $\overline{0}$ & 25 \\
\hline 5 & 5 & 6 & $\overline{0}$ & 12 & 0 & 23 \\
\hline 6 & 6 & 8 & 0 & 10 & 0 & 24 \\
\hline 7 & 0 & 3 & 9 & 9 & 0 & 21 \\
\hline 8 & 0 & 5 & 8 & 11 & $\overline{0}$ & 24 \\
\hline 9 & 0 & $\overline{0}$ & 7 & 10 & 0 & 17 \\
\hline 10 & 4 & 0 & 8 & 10 & 0 & 22 \\
\hline
\end{tabular}

\begin{tabular}{|c|c|c|c|c|c|c|}
\hline \multicolumn{2}{|c}{ Reproduction at } & \multicolumn{3}{c|}{$50.0 \%$} & \multicolumn{4}{c|}{ Concentration } \\
\hline Rep. & Day 2/3 & Day 4 & Day 5 & Day 6 & Day 7 & Total \\
\hline 1 & 0 & 6 & 9 & 0 & 0 & 15 \\
\hline 2 & 0 & 5 & 8 & 0 & 0 & 13 \\
\hline 3 & 5 & 0 & 8 & 10 & 0 & 23 \\
\hline 4 & 0 & 2 & 9 & 11 & 0 & 22 \\
\hline 5 & 6 & 7 & 0 & 11 & 0 & 24 \\
\hline 6 & 4 & 8 & 0 & 9 & 0 & 21 \\
\hline 7 & 0 & 7 & 8 & 10 & 0 & 25 \\
\hline 8 & 0 & 3 & 6 & 0 & 0 & 9 \\
\hline 9 & 0 & 0 & 8 & 0 & 0 & 8 \\
\hline 10 & 0 & 0 & 9 & 0 & 0 & 9 \\
\hline
\end{tabular}

\begin{tabular}{|c|c|c|c|c|c|c|}
\hline \multicolumn{6}{|c|}{ Reproduction at } & \multicolumn{5}{|c|}{$100.0 \%$} & \multicolumn{3}{c|}{ Concentration } \\
\hline Rep. & Day $2 / 3$ & Day 4 & Day 5 & Day 6 & Day 7 & Total \\
\hline 1 & $\mathrm{X}$ & $\mathrm{X}$ & $\mathrm{X}$ & $\mathrm{X}$ & $\mathrm{X}$ & 0 \\
\hline 2 & $\mathrm{X}$ & $\mathrm{X}$ & $\mathrm{X}$ & $\mathrm{X}$ & $\mathrm{X}$ & 0 \\
\hline 3 & $\mathrm{X}$ & $\mathrm{X}$ & $\mathrm{X}$ & $\mathrm{X}$ & $\mathrm{X}$ & 0 \\
\hline 4 & $\mathrm{X}$ & $\mathrm{X}$ & $\mathrm{X}$ & $\mathrm{X}$ & $\mathrm{X}$ & 0 \\
\hline 5 & $\mathrm{X}$ & $\mathrm{X}$ & $\mathrm{X}$ & $\mathrm{X}$ & $\mathrm{X}$ & 0 \\
\hline 6 & $\mathrm{X}$ & $\mathrm{X}$ & $\mathrm{X}$ & $\mathrm{X}$ & $\mathrm{X}$ & 0 \\
\hline 7 & $\mathrm{X}$ & $\mathrm{X}$ & $\mathrm{X}$ & $\mathrm{X}$ & $\mathrm{X}$ & 0 \\
\hline 8 & $\mathrm{X}$ & $\mathrm{X}$ & $\mathrm{X}$ & $\mathrm{X}$ & $\mathrm{X}$ & 0 \\
\hline 9 & $\mathrm{X}$ & $\mathrm{X}$ & $\mathrm{X}$ & $\mathrm{X}$ & $\mathrm{X}$ & 0 \\
\hline 10 & $\mathrm{X}$ & $\mathrm{X}$ & $\mathrm{X}$ & $\mathrm{X}$ & $\mathrm{X}$ & 0 \\
\hline
\end{tabular}




\section{CHRONIC DEFINITIVE SURVIVAL AND REPRODUCTION TEST Water Chemistry}

Client: Westinghouse Savannah River Company

Sample Identification: FHB-014

Test Date: $\quad$ 2/29/96

pH (After Renewal)

\begin{tabular}{|l|r|r|r|r|r|r|}
\hline \multicolumn{7}{|c|}{ Test Concentration } \\
\hline & Control & $6.25 \%$ & $12.5 \%$ & $25 \%$ & $50 \%$ & $100 \%$ \\
\hline Initial & 8.12 & 7.89 & 7.55 & 7.32 & 6.58 & 5.24 \\
\hline Day 1 & 7.98 & 7.81 & 7.69 & 7.31 & 7.65 & 5.46 \\
\hline Day 2 & 7.97 & 7.97 & 7.81 & 7.67 & 7.29 & \\
\hline Day 3 & 7.98 & 7.99 & 7.52 & 7.31 & 6.84 & \\
\hline Day 4 & 8.06 & 7.84 & 7.62 & 7.52 & 8.94 & \\
\hline Day 5 & 8.05 & 7.86 & 7.41 & 7.12 & 6.64 & \\
\hline Day 6 & 8.14 & 7.79 & 7.59 & 7.16 & 6.80 & \\
\hline
\end{tabular}

Dissolved Oxygen (After Renewal)

\begin{tabular}{|l|r|r|r|r|r|r|}
\hline \multicolumn{7}{|c|}{ Test Concentration } \\
\hline & Control & $6.25 \%$ & $12.5 \%$ & $25 \%$ & $50 \%$ & $100 \%$ \\
\hline Initial & 8.6 & 8.2 & 8.4 & 8.3 & 8.4 & 8.2 \\
\hline Day 1 & 8.6 & 8.3 & 8.4 & 8.2 & 8.2 & 8.4 \\
\hline Day 2 & 8.5 & 8.4 & 8.4 & 8.4 & 8.6 & 8.6 \\
\hline Day 3 & 8.6 & 8.4 & 8.4 & 8.5 & 8.6 & \\
\hline Day 4 & 8.8 & 8.2 & 8.2 & 8.4 & 8.4 & \\
\hline Day 5 & 8.9 & 8.4 & 8.4 & 8.3 & 8.6 & \\
\hline Day 6 & 8.7 & 8.5 & 8.4 & 8.4 & 8.6 & \\
\hline
\end{tabular}

Dilution Water \& Effluent Parameters

\begin{tabular}{|l|c|r|r|r|r|}
\hline & Res Cl. & Alkalin & Hard. & Cond. & \\
\hline & $\mathrm{mg} / \mathrm{L}$ & $\mathrm{mg} / \mathrm{L}$ & $\mathrm{mg} / \mathrm{L}$ & $\mu \mathrm{mhos}$ & \\
\hline Eff. Sample 1 & $<0.05$ & 6.09 & 12 & 38.8 & \\
\hline Eff. Sample 2 & $<0.05$ & 4.06 & 6 & 38.7 & \\
\hline Eff. Sample 3 & $<0.05$ & 6.09 & 6 & 39.4 & \\
\hline & & & & & \\
\hline Dilution Water & & 71.1 & 84 & 222 & \\
\hline & & & & & \\
\hline & & & & & \\
\hline
\end{tabular}

pH (Before Renewal)
\begin{tabular}{|l|r|r|r|r|r|r|}
\hline \multicolumn{7}{|c|}{ Test Concentration } \\
\hline & Control & $6.25 \%$ & $12.5 \%$ & $25 \%$ & $50 \%$ & $100 \%$ \\
\hline Day 1 & 8.10 & 7.73 & 7.80 & 7.69 & 8.03 & 8.31 \\
\hline Day 2 & 8.27 & 8.28 & 8.26 & 8.22 & 8.29 & 8.23 \\
\hline Day 3 & 8.44 & 8.41 & 8.31 & 8.54 & 8.48 & \\
\hline Day 4 & 8.09 & 8.11 & 8.27 & 8.21 & 8.23 & \\
\hline Day 5 & 8.33 & 8.31 & 8.32 & 8.07 & 8.21 & \\
\hline Day 6 & 8.30 & 8.36 & 8.34 & 8.30 & 8.34 & \\
\hline Final & 8.21 & 7.89 & 8.36 & 8.28 & 8.26 & \\
\hline
\end{tabular}

Dissolved Oxygen (Before Renewal)

\begin{tabular}{|l|r|r|r|r|r|r|}
\hline \multicolumn{7}{|c|}{ Test Concentration } \\
\hline & Control & $6.25 \%$ & $12.5 \%$ & $25 \%$ & $50 \%$ & $100 \%$ \\
\hline Day 1 & 8.3 & 8.0 & 8.2 & 8.3 & 8.2 & 8.3 \\
\hline Day 2 & 8.2 & 8.1 & 8.3 & 8.2 & 8.0 & 8.2 \\
\hline Day 3 & 8.8 & 8.6 & 8.6 & 8.8 & 8.9 & \\
\hline Day 4 & 8.6 & 8.4 & 8.4 & 8.6 & 8.6 & \\
\hline Day 5 & 8.5 & 8.0 & 8.2 & 8.0 & 8.1 & \\
\hline Day 6 & 8.6 & 8.2 & 8.3 & 8.3 & 8.2 & \\
\hline Final & 8.3 & 8.4 & 8.4 & 8.4 & 8.0 & \\
\hline
\end{tabular}

Temperature

\begin{tabular}{|l|r|}
\hline \multicolumn{2}{|c|}{ Incubator ${ }^{\circ} \mathrm{C}$} \\
\hline Initial & 24.4 \\
\hline Day 1 & 24.3 \\
\hline Day 2 & 25.7 \\
\hline Day 3 & 25.1 \\
\hline Day 4 & 24.2 \\
\hline Day 5 & 25.7 \\
\hline Day 6 & 25.1 \\
\hline Final & 25.3 \\
\hline
\end{tabular}




\section{CHRONIC DEFINITIVE SURVIVAL AND REPRODUCTION TEST \\ Statistical Analyses}

Client: Westinghouse Savannah River Company

Sample Identification: FHB-014

Test Date: $\quad$ 2/29/96

\begin{tabular}{|lrr}
\hline Test for Normality & & \\
Test Used: Shapiro Wilks & $W=$ & 0.949 \\
& critical & 0.93
\end{tabular}

The data are normal in distribution
NOEC $\quad 25 \%$ effluent concentration

LOEC $\quad 50 \%$ effluent concentration

\begin{tabular}{|c|c|c|c|c|c|c|}
\hline Test fo & r Diffel & nces & n Repr & ducti & & \\
\hline Test Typ & e Used: & & t Test & & & \\
\hline$t$ Test & & & Test & incentr & & \\
\hline & Control & $\overline{6 \%}$ & $13 \%$ & $25 \%$ & $50 \%$ & $100 \%$ \\
\hline t Value & & 1.43 & 1.34 & 1.24 & 3.55 & N/A \\
\hline critical & & 2.33 & 2.33 & 2.33 & 2.33 & \\
\hline mean & 24.6 & 21.5 & 21.7 & 21.9 & 16.9 & $\overline{0}$ \\
\hline $\mathrm{MSD}=$ & 5.06 & & & & & \\
\hline & NOEC & $25 \%$ & effluent & oncent & ation & \\
\hline & LOEC & $50 \%$ & effluent & oncent & tion & \\
\hline
\end{tabular}

\begin{tabular}{|crr|}
\hline Test for Homogeneity of Variance & \\
Test Used: Bartlett's Test & $\mathrm{B}=$ & 9.80 \\
& critical & 13.28 \\
The data are homogeneous in variance
\end{tabular}

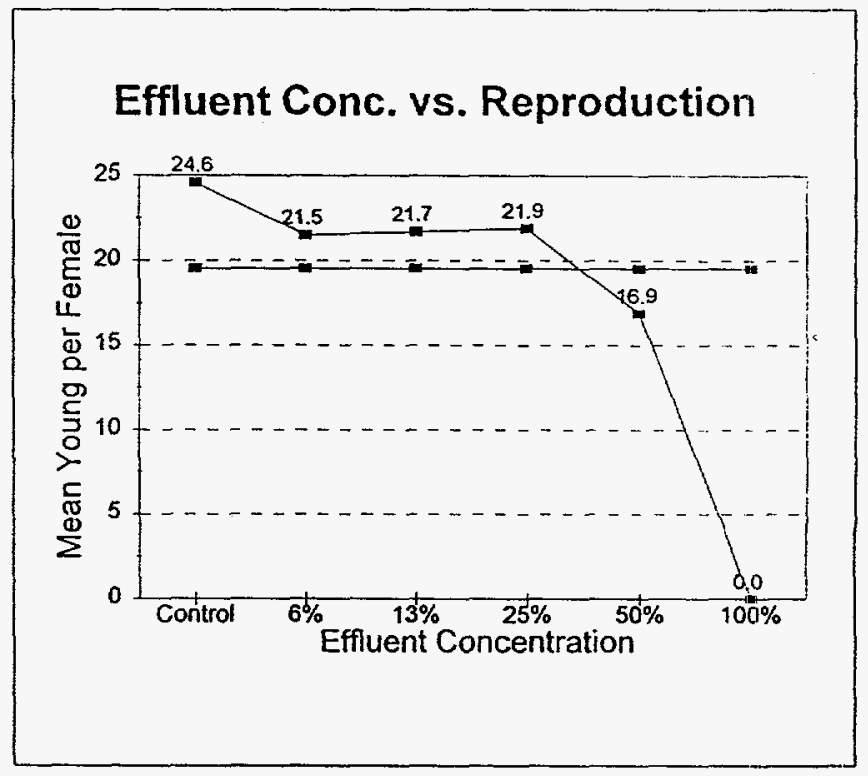

Comments:

The $\mathrm{pH}$ of the sample was acidic; mortality may have been due to acidity. Alkalinity and hardness were low. 


\section{Day Chronic Definitive Survival and Reproduction Bioassay Method: EPA/600/4-89/001}

Test Organism: Ceriodaphnia dubia

Facility: Westinghouse Savannah River Company

Sample ID: FHB-018

Date: $\quad 4 / 11 / 96$ 


\section{CHRONIC DEFINITIVE SURVIVAL AND REPRODUCTION TEST Survival and Reproduction Results}

Client: $\quad$ WSRC

Sample Identification: FHB-018

Test Date: $\quad 4 / 11 / 96$

\begin{tabular}{|c|c|c|c|c|c|c|}
\hline \multicolumn{3}{|c|}{ Reproduction at } & $0.0 \%$ & \multicolumn{3}{c|}{ Concentration } \\
\hline Rep. & Day 3/4 & Day 5 & Day 6 & Day 7 & Day 8 & Total \\
\hline 1 & 4 & 1 & 6 & 9 & 0 & 20 \\
\hline 2 & 5 & 9 & 7 & 0 & 0 & 21 \\
\hline 3 & 4 & 6 & 0 & 9 & 0 & 19 \\
\hline 4 & 5 & 4 & 0 & 6 & 0 & 15 \\
\hline 5 & 0 & 3 & 6 & 4 & 0 & 13 \\
\hline 6 & 0 & 7 & 8 & 10 & 0 & 25 \\
\hline 7 & 4 & 6 & 0 & 6 & 0 & 16 \\
\hline 8 & 3 & 5 & 0 & 10 & 0 & 18 \\
\hline 9 & 0 & 5 & 7 & 8 & 0 & 20 \\
\hline 10 & 0 & 8 & 0 & 9 & 0 & 17 \\
\hline
\end{tabular}

\begin{tabular}{|c|c|c|c|c|c|c|}
\hline \multicolumn{3}{|l|}{ Reproduction at } & $6.0 \%$ & \multicolumn{4}{c|}{ Concentration } \\
\hline Rep. & Day 3/4 & Day 5 & Day 6 & Day 7 & Day 8 & Total \\
\hline 1 & 3 & 0 & 7 & 8 & 0 & 18 \\
\hline 2 & 0 & 0 & 9 & 10 & 0 & 19 \\
\hline 3 & 0 & 0 & 5 & 10 & 0 & 15 \\
\hline 4 & 0 & 6 & 9 & 6 & 0 & 21 \\
\hline 5 & 0 & 3 & 6 & 9 & 0 & 18 \\
\hline 6 & 2 & 0 & 7 & 11 & 0 & 20 \\
\hline 7 & 0 & 0 & 0 & 10 & 0 & 10 \\
\hline 8 & 0 & 4 & 8 & 12 & 0 & 24 \\
\hline 9 & 0 & 4 & 0 & 11 & 0 & 15 \\
\hline 10 & 0 & 0 & 6 & 12 & 0 & 18 \\
\hline
\end{tabular}

\begin{tabular}{|c|c|c|c|c|c|c|}
\hline \multicolumn{3}{|c|}{ Reproduction at } & \multicolumn{3}{c|}{$12.0 \%$} & \multicolumn{3}{c|}{ Concentration } \\
\hline Rep. & Day 3/4 & Day 5 & Day 6 & Day 7 & Day 8 & Total \\
\hline 1 & 0 & 0 & 9 & 5 & 0 & 14 \\
\hline 2 & 1 & 3 & 0 & 7 & 0 & 11 \\
\hline 3 & 0 & 3 & 6 & 4 & 0 & 13 \\
\hline 4 & 0 & 5 & 0 & 9 & 0 & 14 \\
\hline 5 & 3 & 4 & 0 & 7 & 0 & 14 \\
\hline 6 & 0 & 3 & 5 & 6 & 0 & 14 \\
\hline 7 & 0 & 4 & 6 & 7 & 0 & 17 \\
\hline 8 & 0 & 2 & 7 & 6 & 0 & 15 \\
\hline 9 & 4 & 6 & 0 & 7 & 0 & 17 \\
\hline 10 & 0 & 0 & 7 & 8 & 0 & 15 \\
\hline
\end{tabular}

\begin{tabular}{|c|c|c|c|c|c|c|}
\hline \multicolumn{3}{|c|}{ Reproduction at } & \multicolumn{4}{|c|}{$25.0 \%$ Concentration } \\
\hline Rep. & Day 3/4 & Day 5 & Day 6 & Day 7 & Day 8 & Total \\
\hline $\mathrm{I}$ & 0 & $\overline{0}$ & $\overline{0}$ & $\overline{2}$ & 0 & 2 \\
\hline 2 & $\overline{0}$ & 3 & $\overline{0}$ & 4 & $\overline{0}$ & 7 \\
\hline 3 & $\bar{X}$ & $\mathrm{X}$ & $\bar{x}$ & $\mathrm{X}$ & $\mathrm{X}$ & 0 \\
\hline 4 & $\overline{0}$ & $\overline{0}$ & $\overline{0}$ & 3 & 0 & 3 \\
\hline 5 & $\overline{0}$ & 1 & 0 & 4 & 0 & 5 \\
\hline 6 & 0 & 3 & 2 & 5 & 0 & 10 \\
\hline 7 & 0 & 3 & 0 & 6 & 0 & 9 \\
\hline 8 & 0 & 0 & 0 & 4 & 0 & 4 \\
\hline 9 & $\overline{0}$ & 0 & $0^{-}$ & 4 & 0 & 4 \\
\hline 10 & 0 & 0 & 0 & 5 & $\overline{0}$ & 5 \\
\hline
\end{tabular}

\begin{tabular}{|c|c|c|c|c|c|c|}
\hline \multicolumn{6}{|c|}{ Reproduction at $\because$} \\
\hline Rep. & Day 3/4 & Day 5 & Day 6 & Day 7 & Day 8 & Total \\
\hline 1 & $\mathrm{X}$ & $\mathrm{X}$ & $\mathrm{X}$ & $\mathrm{X}$ & $\mathrm{X}$ & 0 \\
\hline 2 & 0 & 0 & 0 & 0 & 0 & 0 \\
\hline 3 & $\mathrm{X}$ & $\mathrm{X}$ & $\mathrm{X}$ & $\mathrm{X}$ & $\mathrm{X}$ & 0 \\
\hline 4 & 0 & 0 & 0 & 0 & 0 & 0 \\
\hline 5 & $\mathrm{X}$ & $\mathrm{X}$ & $\mathrm{X}$ & $\mathrm{X}$ & $\mathrm{X}$ & 0 \\
\hline 6 & $\mathrm{X}$ & $\mathrm{X}$ & $\mathrm{X}$ & $\mathrm{X}$ & $\mathrm{X}$ & 0 \\
\hline 7 & 0 & 0 & 0 & 0 & 0 & 0 \\
\hline 8 & $\mathrm{X}$ & $\mathrm{X}$ & $\mathrm{X}$ & $\mathrm{X}$ & $\mathrm{X}$ & 0 \\
\hline 9 & 0 & 0 & 0 & 0 & 0 & 0 \\
\hline 10 & 0 & 0 & 0 & 0 & 0 & 0 \\
\hline
\end{tabular}

\begin{tabular}{|c|c|c|c|c|c|c|}
\hline \multicolumn{7}{|c|}{ Reproduction at $100.0 \%$ Concentration } \\
\hline Rep. & Day 3/4 & Day 5 & Day 6 & Day 7 & Day 8 & Total \\
\hline 1 & $\mathrm{X}$ & $\mathrm{X}$ & $\mathrm{X}$ & $\mathrm{X}$ & $\mathrm{X}$ & 0 \\
\hline 2 & $\mathrm{X}$ & $\mathrm{X}$ & $\mathrm{X}$ & $\mathrm{X}$ & $\mathrm{X}$ & 0 \\
\hline 3 & $\mathrm{X}$ & $\mathrm{X}$ & $\mathrm{X}$ & $\mathrm{X}$ & $\mathrm{X}$ & 0 \\
\hline 4 & $\mathrm{X}$ & $\mathrm{X}$ & $\mathrm{X}$ & $\mathrm{X}$ & $\mathrm{X}$ & 0 \\
\hline 5 & $\mathrm{X}$ & $\mathrm{X}$ & $\mathrm{X}$ & $\mathrm{X}$ & $\mathrm{X}$ & 0 \\
\hline 6 & $\mathrm{X}$ & $\mathrm{X}$ & $\mathrm{X}$ & $\mathrm{X}$ & $\mathrm{X}$ & 0 \\
\hline 7 & $\mathrm{X}$ & $\mathrm{X}$ & $\mathrm{X}$ & $\mathrm{X}$ & $\mathrm{X}$ & 0 \\
\hline 8 & $\mathrm{X}$ & $\mathrm{X}$ & $\mathrm{X}$ & $\mathrm{X}$ & $\mathrm{X}$ & 0 \\
\hline 9 & $\mathrm{X}$ & $\mathrm{X}$ & $\mathrm{X}$ & $\mathrm{X}$ & $\mathrm{X}$ & 0 \\
\hline 10 & $\mathrm{X}$ & $\mathrm{X}$ & $\mathrm{X}$ & $\mathrm{X}$ & $\mathrm{X}$ & 0 \\
\hline
\end{tabular}




\section{CHRONIC DEFINITIVE SURVIVAL AND REPRODUCTION TEST \\ Statistical Analyses}

Client: WSRC

Sample Identification: FHB-018

Test Date: $\quad 4 / 11 / 96 \quad$ T5266

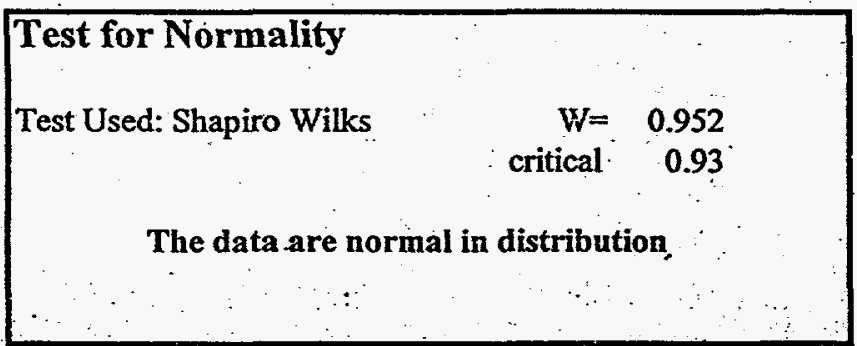

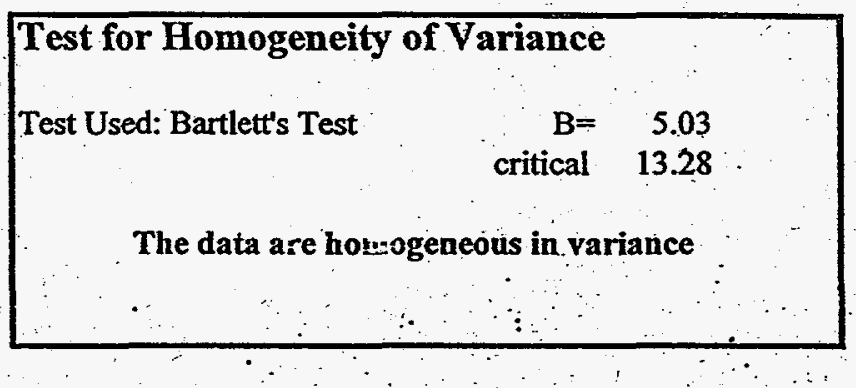

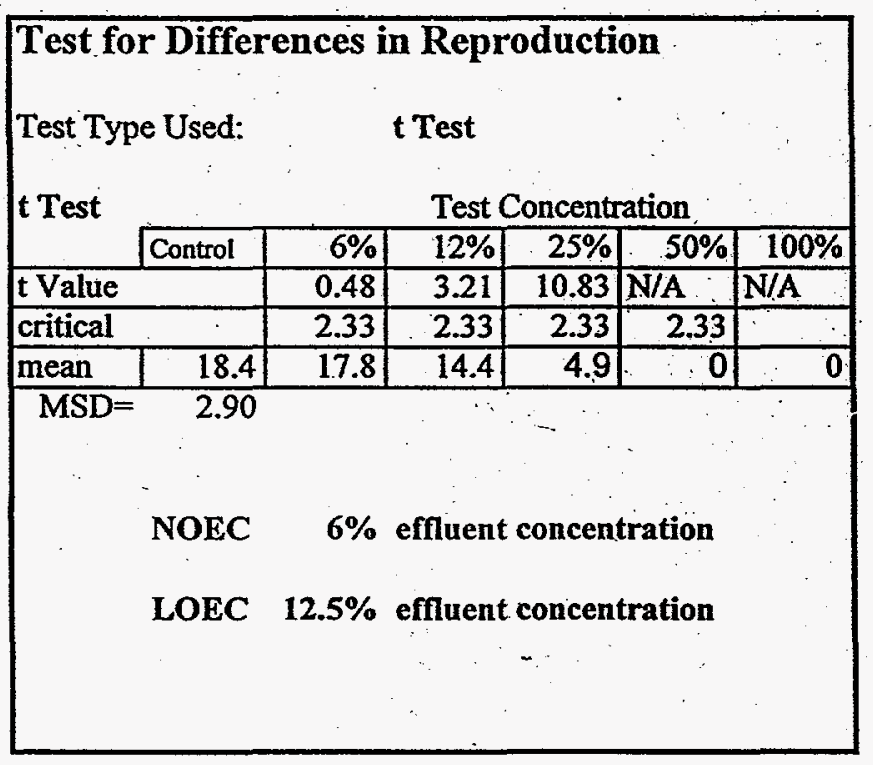

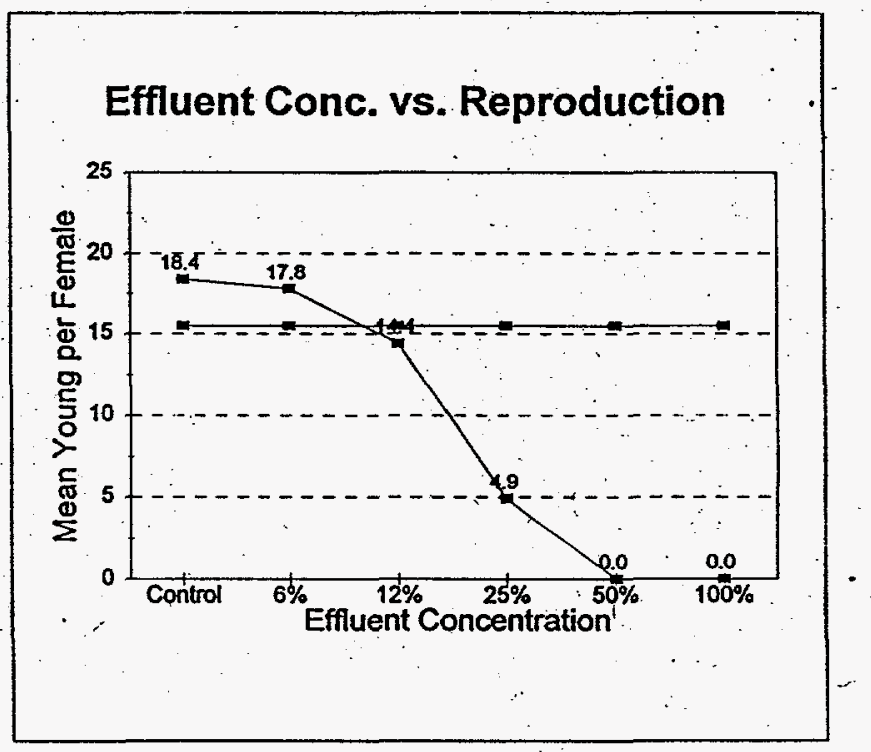

Summary 


\section{CHRONIC DEFINITIVE SURVIVAL AND REPRODUCTION TEST Water Chemistry}

Client: Westinghouse Savannah River Company

Sample Identification: FHB-018

Test Date: $\quad$ 4/11/96

\section{pH (After Renewal)}

\begin{tabular}{|c|c|c|c|c|c|c|}
\hline & \multicolumn{6}{|c|}{ Test Concentration. } \\
\hline & Control & $6 \%$ & $12 \%$ & $25 \%$ & $50 \%$ & $100 \%$ \\
\hline Initial & 8.30 & 7.17 & 7.46 & 7.78 & 6.21 & 6.16 \\
\hline Day 1 & 8.10 & 7.96 & 7.54 & 7.48 & 6.79 & * \\
\hline Day 2 & 7.95 & 7.73 & 7.58 & 7.31 & 6.71 & \\
\hline Day 3 & 8.03 & 7.01 & 7.57 & 7.16 & 6.56 & \\
\hline Day 4 & 8.09 & 7.87 & 7.21 & 6.58 & 6.19 & \\
\hline Day 5 & 8.07 & 7.31 & 7.13 & 6.82 & 6.51 & $\because$ \\
\hline Day 6 & 8.08 & 7.69 & 7.47 & 7.34 & .6 .81 & \\
\hline
\end{tabular}

Dissolved Oxygen (After Renewal)

\begin{tabular}{|l|r|r|r|r|r|r|}
\hline \multicolumn{7}{|c|}{ Test Concentration } \\
\hline & Control & $6 \%$ & $12 \%$ & $25 \%$ & $50 \%$ & $100 \%$ \\
\hline Initial & 8.7 & 8.6 & 8.5 & 8.6 & 8.5 & 8.6 \\
\hline Day 1 & 8.8 & 8.4 & 8.4 & 8.5 & 8.5 & $*$ \\
\hline Day 2 & 8.7 & 8.2 & 8.2 & 8.4 & 8.4 & \\
\hline Day 3 & 8.6 & 8.6 & 8.7 & 8.7 & 8.6 & \\
\hline Day 4 & 8.5 & 8.4 & 8.4 & 8.5 & 8.6 & \\
\hline Day 5 & 8.7 & 8.3 & 8.3 & 8.3 & 8.4 & \\
\hline Day 6 & 8.7 & 8.2 & 8.3 & 8.4 & 8.4 & \\
\hline
\end{tabular}

Dilution Water \& Effluent Parameters

\begin{tabular}{|l|r|r|r|r|r|}
\hline & Res Cl. & Alkalin & Hard. & Cond. & \\
\hline & $\mathrm{mg} / \mathrm{L}$ & $\mathrm{mg} / \mathrm{L}$ & $\mathrm{mg} / \mathrm{L}$ & $\mu \mathrm{mhos}$ & \\
\hline Eff. Sample 1 & $<0.05$ & no data & 10 & no data & \\
\hline Eff. Sample 2 & & & 12 & & \\
\hline Eff. Sample 3 & & & 6 & & \\
\hline & & & & & \\
\hline Dilution Water & & 69 & 86 & 178 & \\
\hline & & & & & \\
\hline & & & & & \\
\hline
\end{tabular}

pH (Before Renewal).

\begin{tabular}{|l|r|r|r|r|r|r|}
\hline \multicolumn{7}{|c|}{ Test Concentration } \\
\hline & Control & $6 \%$ & $12 \%$ & $25 \%$ & $50 \%$ & $100 \%$ \\
\hline Day 1 & 8.18 & 7.81 & 7.59 & 7.43 & 7.22 & 6.90 \\
\hline Day 2 & 8.14 & 7.96 & 7.71 & 7.65 & 6.97 & $*$ \\
\hline Day 3 & 8.20 & 7.16 & 7.42 & 7.10 & 6.95 & \\
\hline Day 4 & 8.26 & 7.94 & 7.43 & 6.99 & 6.25 & \\
\hline Day 5 & 8.33 & 7.99 & 7.71 & 7.59 & 7.22 & \\
\hline Day 6 & 8.11 & 8.04 & 7.74 & 7.56 & 7.24 & \\
\hline Final & 8.07 & 7.89 & 7.67 & 7.44 & 7.19 & \\
\hline \multicolumn{7}{|c}{ All test organisms dead } \\
\hline
\end{tabular}

Dissolved Oxygen (Before Renewal)

\begin{tabular}{|l|r|r|r|r|r|r|}
\hline \multicolumn{7}{|c|}{ Test Concentration } \\
\hline & Control & $6 \%$ & $12 \%$ & $25 \%$ & $50 \%$ & $100 \%$ \\
\hline Day 1 & 8.6 & 8.4 & 8.4 & 8.6 & 8.6 & 8.6 \\
\hline Day 2 & 8.5 & 8.4 & 8.5 & 8.6 & 8.6 & $*$ \\
\hline Day 3 & 8.2 & 8.3 & 8.4 & 8.5 & 8.4 & \\
\hline Day 4 & 8.6 & 8.4 & 8.4 & 8.5 & 8.5 & \\
\hline Day 5 & 8.6 & 8.4 & 8.4 & 8.4 & 8.4 & \\
\hline Day 6 & 8.5 & 8.4 & 8.4 & 8.6 & 8.8 & \\
\hline Final & 8.6 & 8.2 & 8.2 & 8.4 & 8.6 & \\
\hline
\end{tabular}

\section{Temperature}

\begin{tabular}{|l|r|}
\hline \multicolumn{2}{|c|}{ Incubator ${ }^{\circ} \mathrm{C}$} \\
\hline Initial & 24.5 \\
\hline Day 1 & 24.5 \\
\hline Day 2 & 25.3 \\
\hline Day 3 & 24.9 \\
\hline Day 4 & 25.5 \\
\hline Day 5 & 25.0 \\
\hline Day 6 & 26.0 \\
\hline Final & 24.8 \\
\hline
\end{tabular}


(864) 877-6942 - FAX (864) 877-6938

P.O. Box 16414, Greenville, SC $29606 \cdot 4$ Craftsman Court, Greer, SC 29650

\section{Day Chronic Definitive Survival and Reproduction Bioassay}

Method: EPA/600/4-89/001

Test Organism: Ceriodaphnia dubia

Facility:Westinghouse Savannah River Company

Sample ID:FHB-020

Date: $\quad 2 / 29 / 96$ 


\section{CHRONIC DEFINITIVE SURVIVAL AND REPRODUCTION TEST Survival and Reproduction Results}

Client: Westinghouse Savannah River Company

Sample Identification: FHB-020

Test Date: $\quad 2 / 29 / 96 \quad$ T5143

\begin{tabular}{|c|c|c|c|c|c|c|}
\hline \multicolumn{3}{|c|}{ Reproduction at } & \multicolumn{3}{c|}{$0 \%$} & \multicolumn{3}{c|}{ Concentration } \\
\hline Rep. & Day 3/4 & Day 5 & Day 6 & Day 7 & Day 8 & Total \\
\hline 1 & 5 & 10 & 10 & 0 & 0 & 25 \\
\hline 2 & 5 & 9 & 7 & 0 & 0 & 21 \\
\hline 3 & 5 & 0 & 0 & 0 & 11 & 16 \\
\hline 4 & 4 & 10 & 0 & 0 & 10 & 24 \\
\hline 5 & 5 & 8 & 0 & 0 & 8 & 21 \\
\hline 6 & 4 & 9 & 2 & 0 & 10 & 25 \\
\hline 7 & 5 & 10 & 5 & 0 & 0 & 20 \\
\hline 8 & 6 & 3 & 0 & 0 & 11 & 20 \\
\hline 9 & 5 & 10 & 10 & 0 & 0 & 25 \\
\hline 10 & 4 & 12 & 0 & 0 & 10 & 26 \\
\hline
\end{tabular}

\begin{tabular}{|c|c|c|c|c|c|c|}
\hline \multicolumn{3}{|c|}{ Reproduction at } \\
\hline Rep. & Day 3/4 & Day 5 & Day 6 & Day 7 & Day 8 & Total \\
\hline 1 & 5 & 9 & 13 & 0 & 0 & 27 \\
\hline 2 & 5 & 0 & 0 & 0 & 10 & 15 \\
\hline 3 & 4 & 8 & 0 & 7 & 0 & 19 \\
\hline 4 & 6 & 8 & 0 & 5 & 0 & 19 \\
\hline 5 & 0 & 10 & 9 & 0 & 0 & 19 \\
\hline 6 & 13 & 0 & 12 & 0 & 0 & 25 \\
\hline 7 & 4 & 10 & 14 & 0 & 0 & 28 \\
\hline 8 & 4 & 9 & 11 & 0 & 0 & 24 \\
\hline 9 & 6 & 10 & 10 & 0 & 0 & 26 \\
\hline 10 & 3 & 8 & 0 & 0 & 12 & 23 \\
\hline
\end{tabular}

\begin{tabular}{|c|c|c|c|c|c|c|}
\hline \multicolumn{4}{|c|}{ Reproduction at } & $12.5 \%$ & \multicolumn{3}{c|}{ Concentration } \\
\hline Rep. & Day 3/4 & Day 5 & Day 6 & Day 7 & Day 8 & Total \\
\hline 1 & 7 & 9 & 0 & 13 & 0 & 29 \\
\hline 2 & 0 & 4 & 14 & 0 & 0 & 18 \\
\hline 3 & 4 & 7 & 0 & 3 & 10 & 24 \\
\hline 4 & 12 & 0 & 0 & 0 & 10 & 22 \\
\hline 5 & 0 & 9 & 12 & 0 & 0 & 21 \\
\hline 6 & 14 & 0 & 16 & 0 & 0 & 30 \\
\hline 7 & 6 & 8 & 17 & 0 & 0 & 31 \\
\hline 8 & 4 & 9 & 3 & 0 & 8 & 24 \\
\hline 9 & 4 & 11 & 9 & 0 & 0 & 24 \\
\hline 10 & 2 & 12 & 8 & 0 & 0 & 22 \\
\hline
\end{tabular}

\begin{tabular}{|c|c|c|c|c|c|c|}
\hline \multicolumn{3}{|c|}{ Reproduction at } & \multicolumn{3}{c|}{$25.0 \%$} & \multicolumn{3}{c|}{ Concentration } \\
\hline Rep. & Day 3/4 & Day 5 & Day 6 & Day 7 & Day 8 & Total \\
\hline 1 & 3 & 9 & 13 & 0 & 0 & 25 \\
\hline 2 & 0 & 4 & 12 & 12 & 0 & 28 \\
\hline 3 & 3 & 2 & 8 & 4 & 0 & 17 \\
\hline 4 & 7 & 9 & 0 & 12 & 0 & 28 \\
\hline 5 & 4 & 9 & 11 & 0 & 0 & 24 \\
\hline 6 & 9 & 0 & 8 & 0 & 0 & 17 \\
\hline 7 & 5 & 12 & 0 & 0 & 0 & 17 \\
\hline 8 & 5 & 7 & 12 & 0 & 0 & 24 \\
\hline 9 & 5 & 10 & 14 & 0 & 0 & 29 \\
\hline 10 & 0 & 10 & 13 & 7 & 0 & 30 \\
\hline
\end{tabular}

\begin{tabular}{|c|c|c|c|c|c|c|}
\hline \multicolumn{3}{|c|}{ Reproduction at } & \multicolumn{4}{|c|}{$50.0 \%$ Concentration } \\
\hline Rep. & Day 3/4 & Day 5 & $\begin{array}{l}\text { Day } 6 \\
\end{array}$ & Day 7 & Day 8 & Total \\
\hline 1 & 6 & 8 & $\overline{0}$ & 0 & $\overline{0}$ & 14 \\
\hline 2 & $\overline{0}$ & 8 & 9 & 10 & 0 & 27 \\
\hline 3 & $\overline{0}$ & 5 & 12 & 0 & 0 & 17 \\
\hline 4 & 8 & 11 & 0 & 17 & 0 & 36 \\
\hline 5 & 4 & 8 & 9 & 0 & 0 & 21 \\
\hline 6 & 6 & 8 & 13 & 0 & 0 & 27 \\
\hline 7 & 3 & 9 & 11 & 0 & 0 & 23 \\
\hline 8 & 5 & 7 & 0 & 14 & 0 & 26 \\
\hline 9 & 2 & 8 & 13 & 0 & 0 & 23 \\
\hline 10 & 2 & 9 & 9 & 0 & 0 & 20 \\
\hline
\end{tabular}

\begin{tabular}{|c|c|c|c|c|c|c|}
\hline \multicolumn{3}{|c|}{ Reproduction at } & \multicolumn{4}{|c|}{$100.0 \%$ Concentration } \\
\hline Rep. & Day 3/4 & Day 5 & Day 6 & Day 7 & Day 8 & Total \\
\hline 1 & 3 & 5 & 7 & 0 & $\overline{0}$ & 15 \\
\hline 2 & 2 & 3 & 7 & 0 & 0 & 12 \\
\hline 3 & 4 & 4 & 1 & 11 & $\overline{0}$ & 20 \\
\hline 4 & 3 & 0 & 4 & 5 & 0 & 12 \\
\hline 5 & 4 & 8 & 6 & 0 & 0 & 18 \\
\hline 6 & 2 & 5 & 6 & 5 & 0 & 18 \\
\hline 7 & 5 & 4 & 11 & 0 & 0 & 20 \\
\hline 8 & 2 & 4 & 10 & 0 & $\overline{0}$ & 16 \\
\hline 9 & 3 & 5 & 12 & 0 & 0 & 20 \\
\hline 10 & 5 & 6 & 10 & 0 & 0 & 21 \\
\hline
\end{tabular}




\section{CHRONIC DEFINITIVE SURVIVAL AND REPRODUCTION TEST Statistical Analyses}

Client: Westinghouse Savannah River Company

Sample Identification: FHB-020

Test Date: $\quad$ 2/29/96

\section{Test for Normality}

Test Used: Shapiro Wilks

$$
\begin{array}{rr}
W= & 0.948 \\
\text { critical } & 0.93
\end{array}
$$

The data are normal in distribution

\section{Test for Homogeneity of Variance}

Test Used: Bartlett's Test

$\mathrm{B}=$

critical

5.65

15.1

\begin{tabular}{|c|c|c|c|c|c|c|}
\hline \multicolumn{7}{|c|}{ Test for Differences in Reproduction } \\
\hline \multicolumn{2}{|c|}{ Test Type Used: } & \multicolumn{2}{|r|}{ t Test } & & & \\
\hline & \multicolumn{6}{|c|}{ Test Concentration } \\
\hline & Control & $6 \%$ & $13 \%$ & $25 \%$ & $50 \%$ & $100 \%$ \\
\hline \multicolumn{2}{|c|}{ t Value } & -0.10 & -1.10 & -0.80 & -0.55 & 2.55 \\
\hline \multicolumn{2}{|l|}{ critical } & 2.33 & 2.33 & 2.33 & 2.33 & 2.33 \\
\hline mean & 22.3 & 22.5 & 24.5 & 23.9 & 23.4 & 17.2 \\
\hline \multicolumn{7}{|l|}{$\mathrm{MSD}=$} \\
\hline & $\begin{array}{l}\text { NOEC } \\
\text { LOEC }\end{array}$ & \multicolumn{5}{|c|}{$\begin{array}{l}50 \% \text { effluent concentration } \\
100 \% \text { effluent concentration }\end{array}$} \\
\hline \multicolumn{7}{|c|}{$\begin{array}{l}\text { The reduction in reproduction at } 100 \% \text { concentration } \\
\text { was only marginally significant. }\end{array}$} \\
\hline
\end{tabular}

The data are homogeneous in variance

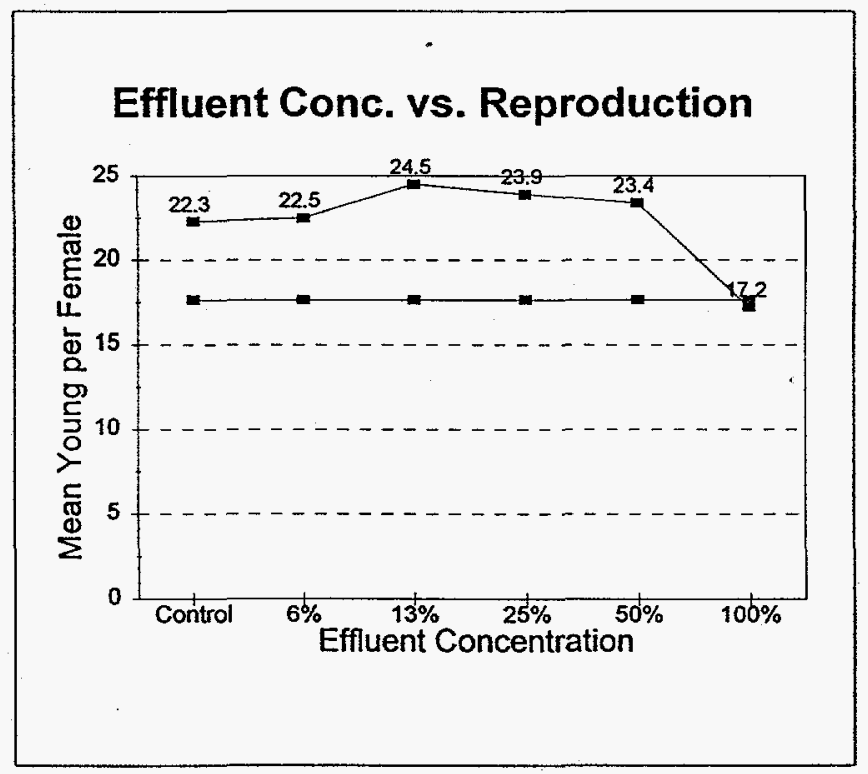

Summary 


\section{CHRONIC DEFINITIVE SURVIVAL AND REPRODUCTION TEST Water Chemistry}

Client: Westinghouse Savannah River Company

Sample Identification: FHB-020

Test Date: $\quad$ 2/29/96

pH (After Renewal)

\begin{tabular}{|l|r|r|r|r|r|r|}
\hline \multicolumn{7}{|c|}{ Test Concentration } \\
\hline & Control & $6.25 \%$ & $12.5 \%$ & $25 \%$ & $50 \%$ & $100 \%$ \\
\hline Initial & 8.12 & 7.77 & 7.71 & 7.72 & 7.21 & 6.32 \\
\hline Day 1 & 7.98 & 7.82 & 7.68 & 7.55 & 7.13 & 6.55 \\
\hline Day 2 & 7.97 & 8.03 & 7.93 & 7.69 & 7.27 & 6.23 \\
\hline Day 3 & 7.98 & 8.18 & 7.77 & 7.72 & 7.09 & 6.75 \\
\hline Day 4 & 8.06 & 7.70 & 7.75 & 7.71 & 7.45 & 6.82 \\
\hline Day 5 & 8.05 & 7.85 & 7.75 & 7.51 & 7.17 & 6.09 \\
\hline Day 6 & 8.14 & 7.76 & 7.68 & 7.50 & 7.29 & 6.31 \\
\hline
\end{tabular}

Dissolved Oxygen (After Renewal)

\begin{tabular}{|l|r|r|r|r|r|r|}
\hline \multicolumn{7}{|c|}{ Test Concentration } \\
\hline & Control & $6.25 \%$ & $12.5 \%$ & $25 \%$ & $50 \%$ & $100 \%$ \\
\hline Initial & 8.6 & 8.6 & 8.6 & 8.6 & 8.7 & 8.7 \\
\hline Day 1 & 8.6 & 8.6 & 8.6 & 8.7 & 8.8 & 8.8 \\
\hline Day 2 & 8.5 & 8.7 & 8.4 & 8.6 & 8.6 & 8.6 \\
\hline Day 3 & 8.6 & 8.4 & 8.4 & 8.5 & 8.6 & 8.8 \\
\hline Day 4 & 8.8 & 8.3 & 8.4 & 8.6 & 8.6 & 8.6 \\
\hline Day 5 & 8.9 & 8.5 & 8.5 & 8.6 & 8.4 & 7.6 \\
\hline Day 6 & 8.7 & 8.7 & 8.6 & 8.6 & 8.3 & 7.8 \\
\hline
\end{tabular}

Dilution Water \& Effluent Parameters

\begin{tabular}{|l|c|r|r|r|r|}
\hline & Res Cl. & Alkalin & Hard. & Cond. & \\
\hline & $\mathrm{mg} / \mathrm{L}$ & $\mathrm{mg} / \mathrm{L}$ & $\mathrm{mg} / \mathrm{L}$ & $\mu \mathrm{mhos}$ & \\
\hline Eff. Sample 1 & $<0.05$ & 4.06 & 10 & 38.2 & \\
\hline Eff. Sample 2 & $<0.05$ & 8.12 & 10 & 42.4 & \\
\hline Eff. Sample 3 & $<0.05$ & 8.12 & 10 & 43 & \\
\hline & & & & & \\
\hline Dilution Water & & 71.1 & 84 & 222 & \\
\hline & & & & & \\
\hline & & & & & \\
\hline
\end{tabular}

$\mathrm{pH}$ (Before Renewal)

\begin{tabular}{|l|r|r|r|r|r|r|}
\hline \multicolumn{7}{|c|}{ Test Concentration } \\
\hline & Control & $6.25 \%$ & $12.5 \%$ & $25 \%$ & $50 \%$ & $100 \%$ \\
\hline Day 1 & 8.10 & 7.99 & 6.97 & 8.11 & 7.34 & 7.74 \\
\hline Day 2 & 8.27 & 8.25 & 8.19 & 8.15 & 7.97 & 8.66 \\
\hline Day 3 & 8.44 & 8.41 & 8.43 & 8.53 & 8.42 & 7.94 \\
\hline Day 4 & 8.09 & 8.24 & 8.37 & 8.17 & 8.08 & 7.51 \\
\hline Day 5 & 8.33 & 8.25 & 8.19 & 8.11 & 8.00 & 7.44 \\
\hline Day 6 & 8.30 & 8.02 & 8.10 & 8.07 & 7.97 & 7.40 \\
\hline Final & 8.21 & 8.12 & 8.17 & 8.14 & 8.02 & 7.71 \\
\hline
\end{tabular}

Dissolved Oxygen (Before Renewal)

\begin{tabular}{|l|r|r|r|r|r|r|}
\hline \multicolumn{7}{|c|}{ Test Concentration } \\
\hline & Control & $6.25 \%$ & $12.5 \%$ & $25 \%$ & $50 \%$ & $100 \%$ \\
\hline Day 1 & 8.3 & 8.8 & 8.8 & 8.9 & 9.0 & 9.1 \\
\hline Day 2 & 8.2 & 8.4 & 8.6 & 8.5 & 8.4 & 8.6 \\
\hline Day 3 & 8.8 & 8.4 & 8.6 & 8.6 & 8.8 & 9.1 \\
\hline Day 4 & 8.6 & 8.4 & 8.6 & 8.8 & 8.9 & 8.8 \\
\hline Day 5 & 8.5 & 8.3 & 8.4 & 8.3 & 8.1 & 8.0 \\
\hline Day 6 & 8.6 & 8.5 & 8.5 & 8.2 & 8.2 & 8.1 \\
\hline Final & 8.3 & 8.4 & 8.6 & 8.2 & 8.2 & 8.0 \\
\hline
\end{tabular}

Temperature
\begin{tabular}{|l|r|}
\hline Incubator ${ }^{\circ} \mathrm{C}$ \\
\hline Initial & 24.4 \\
\hline Day 1 & 24.3 \\
\hline Day 2 & 25.7 \\
\hline Day 3 & 25.1 \\
\hline Day 4 & 24.2 \\
\hline Day 5 & 25.7 \\
\hline Day 6 & 25.1 \\
\hline Final & 25.3 \\
\hline
\end{tabular}


(864) 877-6942 - FAX (864) 877-6938

P.O. Box 16414, Greenville, SC 29606 - 4 Craftsman Court, Greer, SC 29650

\section{Day Chronic Definitive Survival and Reproduction Bioassay Method: EPA/600/4-89/001.}

Test Organism: $\quad$ Ceriodaphnia dubia

Facility: Westinghouse Savannah River Company

Sample ID: FHB-021

Date: 4/11/96 


\section{CHRONIC DEFINITIVE SURVIVAL AND REPRODUCTION TEST Survival and Reproduction Results}

Client: WSRC

Sample Identification: FHB-021

Test Date: $\quad 4 / 11 / 96$

\begin{tabular}{|c|c|c|c|c|c|c|}
\hline \multicolumn{3}{|c|}{ Reproduction at } & $0.0 \%$ & \multicolumn{3}{c|}{ Concentration } \\
\hline Rep. & Day 3/4 & Day 5 & Day 6 & Day 7 & Day 8 & Total \\
\hline 1 & 3 & 0 & 7 & 9 & 0 & 19 \\
\hline 2 & 0 & 4 & 0 & 7 & 10 & 21 \\
\hline 3 & 0 & 4 & 0 & 8 & 6 & 18 \\
\hline 4 & 3 & 6 & 0 & 10 & 0 & 19 \\
\hline 5 & 3 & 6 & 0 & 8 & 0 & 17 \\
\hline 6 & 2 & 0 & 6 & 10 & 0 & 18 \\
\hline 7 & 0 & 4 & 6 & 0 & 9 & 19 \\
\hline 8. & 0 & 4 & 5 & 0 & 8 & 17 \\
\hline 9 & 0. & 3 & 7 & 0 & 10 & 20 \\
\hline 10 & 4 & 0 & 6 & 10 & 0 & 20 \\
\hline
\end{tabular}

\begin{tabular}{|c|c|c|c|c|c|c|}
\hline \multicolumn{3}{|c|}{ Reproduction at } \\
\hline Rep. & Day 3/4 & Day 5 & Day 6 & Day 7 & Day 8 & Total \\
\hline 1 & 0 & 3 & 0 & 7 & 12 & 22 \\
\hline 2 & 0 & 4 & 4 & 0 & 11 & 19 \\
\hline 3 & 2 & 1 & 0 & 8 & 6 & 17 \\
\hline 4 & 0 & 5 & 7 & 0 & 9 & 21 \\
\hline 5 & 3 & 0 & 2 & 10 & 4 & 19 \\
\hline 6 & 0 & 3 & 0 & 7 & 9 & 19 \\
\hline 7 & 0 & 0 & 1 & 8 & 4 & 13 \\
\hline 8 & 0 & 0 & 7 & 8 & 2 & 17 \\
\hline 9 & 0 & 0 & 0 & 6 & 0 & 6 \\
\hline 10 & 3 & 3 & 0 & 8 & 7 & 21 \\
\hline
\end{tabular}

\begin{tabular}{|c|c|c|c|c|c|c|}
\hline \multicolumn{3}{|c|}{ Reproduction at } & $12.0 \%$ & \multicolumn{3}{c|}{ Concentration } \\
\hline Rep. & Day 3/4 & Day 5 & Day 6 & Day 7 & Day 8 & Total \\
\hline 1 & 0 & 3 & 0 & 8 & 4 & 15 \\
\hline 2 & 0 & 3 & 0 & 7 & 12 & 22 \\
\hline 3 & 0 & 4 & 0 & 10 & 7 & 21 \\
\hline 4 & 0 & 3 & 7 & 0 & 12 & 22 \\
\hline 5 & 0 & 3 & 8 & 0 & 10 & 21 \\
\hline 6 & 0 & 1 & 0 & 8 & 0 & 9 \\
\hline 7 & 0 & 0 & 0 & 6 & 6 & 12 \\
\hline 8 & 0 & 3 & 0 & 7 & 10 & 20 \\
\hline 9 & 3 & 7 & 0 & 4 & 4 & 18 \\
\hline 10 & 0 & 1 & 0 & 6 & 6 & 13 \\
\hline
\end{tabular}

\begin{tabular}{|c|c|c|c|c|c|c|}
\hline \multicolumn{3}{|c|}{ Reproduction at } \\
\hline Rep. & Day 3/4 & Day 5 & Day 6 & Day 7 & Day 8 & Total \\
\hline 1 & 2 & 0 & 0 & 5 & 3 & 10 \\
\hline 2 & 0 & 3 & 4 & 0 & 10 & 17 \\
\hline 3 & 0 & 2 & 6 & 0 & 12 & 20 \\
\hline 4 & 0 & 4 & 6 & 0 & 10 & 20 \\
\hline 5 & 1 & 4 & 0 & 0 & 10 & 15 \\
\hline 6 & 0 & 0 & 0 & 4 & 12 & 16 \\
\hline 7 & 0 & 3 & 0 & 4 & 10 & 17 \\
\hline 8 & 0 & 0 & 0 & 3 & 6 & 9 \\
\hline 9 & 0 & 0 & 0 & 5 & 12 & 17 \\
\hline 10 & 0 & 0 & 4 & 6 & 4 & 14 \\
\hline
\end{tabular}

\begin{tabular}{|c|c|c|c|c|c|c|}
\hline \multicolumn{3}{|c|}{ Reproduction at } & $50.0 \%$ & \multicolumn{3}{c|}{ Concentration } \\
\hline Rep. & Day 3/4 & Day 5 & Day 6 & Day 7 & Day 8 & Total \\
\hline 1 & 0 & 1 & 0 & 4 & 6 & 11 \\
\hline 2 & 0 & 1 & 0 & 5 & 4 & 10 \\
\hline 3 & 0 & 0 & 0 & 6 & 3 & 9 \\
\hline 4 & 0 & 0 & 0 & 7 & 5 & 12 \\
\hline 5 & 0 & 0 & 0 & 0 & 0 & 0 \\
\hline 6 & 0 & 0 & 0 & 4 & 0 & 4 \\
\hline 7 & 0 & 0 & 0 & 0 & 9 & 9 \\
\hline 8 & 0 & 0 & 0 & 0 & 3 & 3 \\
\hline 9 & 0 & 0 & 0 & 5 & 0 & 5 \\
\hline 10 & 0 & 0 & 0 & 4 & 6 & 10 \\
\hline
\end{tabular}

\begin{tabular}{|c|c|c|c|c|c|c|}
\hline \multicolumn{3}{|c|}{ Reproduction at } & $100.0 \%$ & \multicolumn{3}{c|}{ Concentration } \\
\hline Rep. & Day 3/4 & Day 5 & Day 6 & Day 7 & Day 8 & Total \\
\hline 1 & 0 & 0 & 0 & 3 & 2 & 5 \\
\hline 2 & 0 & 4 & 0 & 6 & 0 & 10 \\
\hline 3 & 0 & 1 & 0 & 0 & 4 & 5 \\
\hline 4 & 0 & 3 & 0 & 4 & 4 & 1 \\
\hline 5 & 0 & 0 & 0 & 3 & 4 & 7 \\
\hline 6 & 0 & 0 & 0 & 5 & 3 & 8 \\
\hline 7 & 0 & 0 & 0 & 0 & 0 & 0 \\
\hline 8 & 0 & 3 & 0 & 3 & 0 & 6 \\
\hline 9 & 0 & 0 & 0 & 2 & 1 & 3 \\
\hline 10 & 0 & 0 & 0 & 3 & 4 & 7 \\
\hline
\end{tabular}




\section{CHRONIC DEFINITIVE SURVIVAL AND REPRODUCTION TEST \\ Statistical Analyses}

Client:

WSRC

Sample Identification:

FHB-021

Test Date: $\quad 4 / 11 / 96$

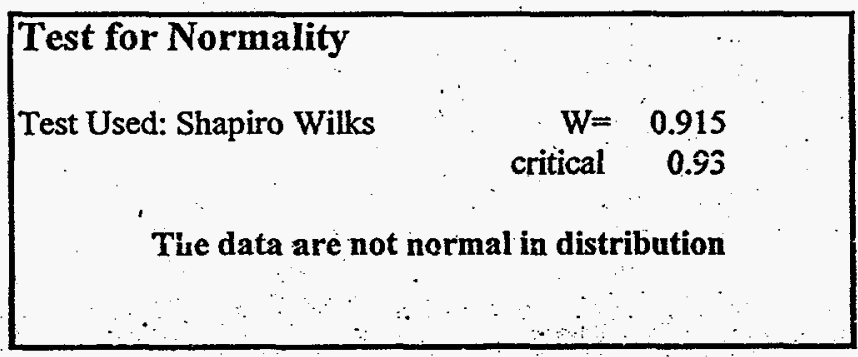

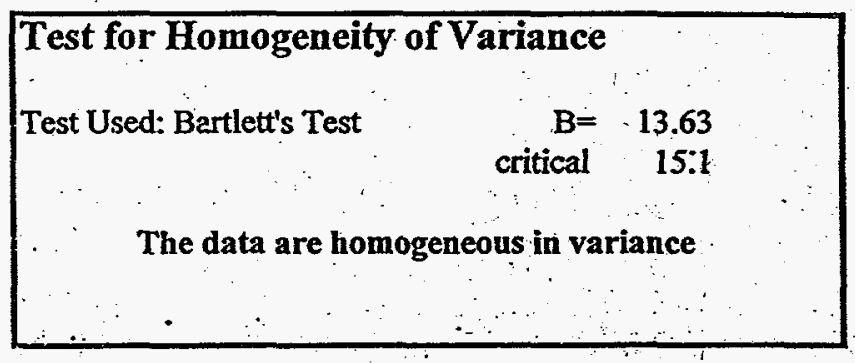

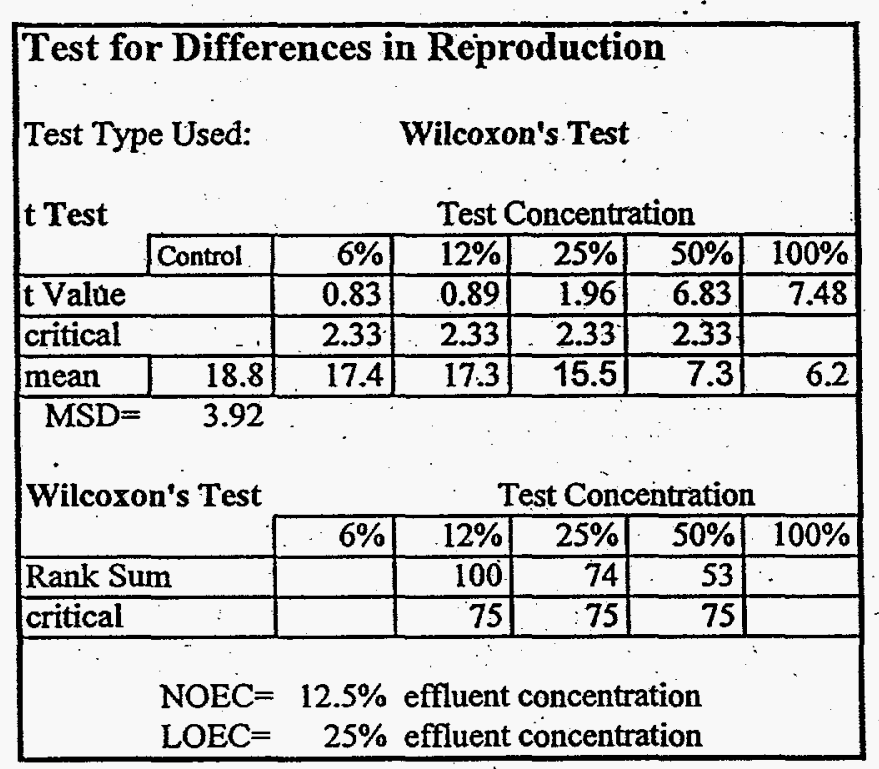

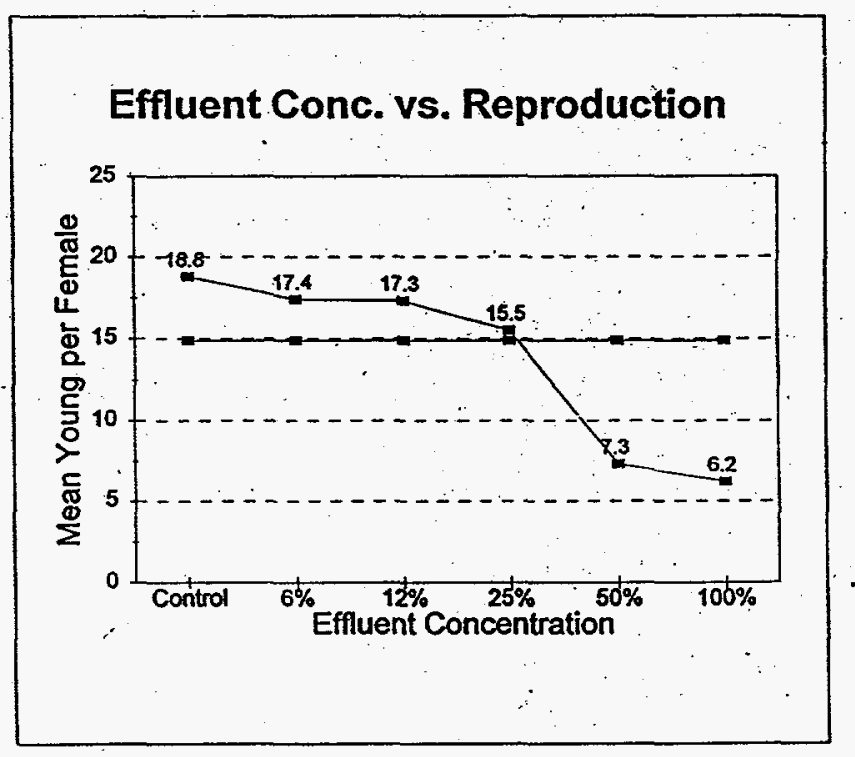

\section{Comments}

The toxicity at $25 \%$ effluent concentration was marginal. 


\section{CHRONIC DEFINITIVE SURVIVAL AND REPRODUCTION TEST Water Chemistry}

Client: Westinghouse Savannah River Company

Sample Identification: FHB-021

Test Date: $\quad 4 / 11 / 96$

pH (After Renewal)

\begin{tabular}{|l|r|r|r|r|r|r|}
\hline \multicolumn{7}{|c|}{ Test Concentration } \\
\hline & Control & $6 \%$ & $12 \%$ & $25 \%$ & $50 \%$ & $100 \%$ \\
\hline Initial & 8.28 & 7.48 & 7.01 & 6.93 & 6.87 & 6.75 \\
\hline Day 1 & 8.12 & 7.87 & 7.66 & 7.57 & 7.25 & 7.03 \\
\hline Day 2 & 7.95 & no data & no data & no data & no data & no data \\
\hline Day 3 & 8.03 & 7.97 & 7.96 & 7.79 & 7.57 & 7.26 \\
\hline Day 4 & 8.09 & 7.94 & 7.89 & 7.74 & 7.57 & 6.85 \\
\hline Day 5 & .8 .07 & 7.82 & 7.80 & 7.53 & 6.95 & 6.51 \\
\hline Day 6 & 8.08 & 7.93 & 7.76 & 7.48 & 6.87 & 6.39 \\
\hline
\end{tabular}

\section{Dissolved Oxygen (After Renewal)}

\begin{tabular}{|l|r|r|r|r|r|r|}
\hline \multicolumn{7}{|c|}{ Test Concentration } \\
\hline & Control & $6 \%$ & $12 \%$ & $25 \%$ & $50 \%$ & $100 \%$ \\
\hline Initial & 8.7 & 8.6 & 8.5 & 8.6 & 8.6 & 8.7 \\
\hline Day' 1 & 8.8 & 8.4 & 8.4 & 8.6 & 8.6 & 8.7 \\
\hline Day 2 & 8.7 & no data & no data & no data & no data & no data \\
\hline Day 3 & 8.6 & 8.6 & 8.6 & 8.5 & 8.6 & 8.8 \\
\hline Day 4 & 8.5 & 8.6 & 8.6 & 8.4 & 8.4 & 8.7 \\
\hline Day 5 & 8.7 & 8.7 & 8.6 & 8.6 & 8.4 & 8.6 \\
\hline Day 6 & 8.7 & 8.6 & 8.7 & 8.6 & 8.6 & 8.6 \\
\hline
\end{tabular}

Dilution Water \& Effluent Parameters

\begin{tabular}{|l|r|r|r|r|l|}
\hline & Res Cl. & Alkalin & Hard. & Cond. & \\
\hline & $\mathrm{mg} / \mathrm{L}$ & $\mathrm{mg} / \mathrm{L}$ & $\mathrm{mg} / \mathrm{L}$ & $\mu \mathrm{mhos}$ & \\
\hline Eff. Sample 1 & $<0.05$ & no data & 6 & no data &. \\
\hline Eff. Sample 2 & & & 10 & & \\
\hline Eff. Sample 3 & & & 10 & & \\
\hline & & & & & \\
\hline Dilution Water & & 69 & 86 & 178 & \\
\hline & & & & & \\
\hline & & 1 & & & \\
\hline
\end{tabular}

\section{pH (Before Renewal)}

\begin{tabular}{|l|r|r|r|r|r|r|}
\hline \multicolumn{7}{|c|}{ Test Concentration } \\
\hline & Control & $6 \%$ & $12 \%$ & $25 \%$ & $50 \%$ & $100 \%$ \\
\hline Day 1 & 8.18 & 7.94 & 7.87 & 7.58 & 7.23 & 7.11 \\
\hline Day 2 & 8.14 & no data & no data & no data & no data & no data \\
\hline Day 3 & 8.20 & 7.95 & 7.95 & 7.82 & 7.64 & 7.15 \\
\hline Day 4 & 8.26 & 7.97 & 8.11 & 8.00 & 8.00 & 7.03 \\
\hline Day 5 & 8.33 & .8 .08 & 8.03 & 7.86 & 7.65 & 6.94 \\
\hline Day 6 & 8.11 & 8.01 & 8.07 & 7.23 & 7.57 & 6.94 \\
\hline Final & 8.07 & 8.14 & .8 .21 & 7.98 & 7.73 & 7.14 \\
\hline
\end{tabular}

\section{Dissolved Oxygen (Before Renewall)}

\begin{tabular}{|l|r|r|r|r|r|r|}
\hline \multicolumn{7}{|c|}{ Test Concentration } \\
\hline & Control & $6 \%$ & $12 \%$ & $25 \%$ & $50 \%$ & $100 \%$ \\
\hline Day 1 & 8.6 & 8.4 & 8.4 & 8.8 & 8.8 & 9.0 \\
\hline Day 2 & 8.5 & no data & no data & no data & no data & no data \\
\hline Day 3 & 8.2 & 8.2 & 8.4 & 8.4 & 8.3 & 8.4 \\
\hline Day 4 & 8.6 & 8.3 & 8.3 & 8.4 & 8.4 & 8.6 \\
\hline Day 5 & 8.6 & 8.4 & 8.3 & 8.4 & 8.4 & 8.4 \\
\hline Day 6 & 8.5 & 8.4 & 8.3 & 8.4 & 8.4 & 8.3 \\
\hline Final & 8.6 & 8.3 & 8.4 & 8.2 & 8.4 & 8.4 \\
\hline
\end{tabular}




\section{Day Chronic Definitive Survival and Reproduction Bioassay}

Method: EPA/600/4-89/001

Test Organism: Ceriodaphnia dubia

Facility:Westinghouse Savannah River Company

Sample ID:FHB-022

Date: $\quad 2 / 22 / 96$ 


\section{CHRONIC DEFINITIVE SURVIVAL AND REPRODUCTION TEST Survival and Reproduction Results}

Client: Westinghouse Savannah River Company

Sample Identification: FHB-022

Test Date: $\quad$ 2/22/96

\begin{tabular}{|c|c|c|c|c|c|c|}
\hline \multicolumn{3}{|c|}{ Reproduction at } & \multirow{2}{*}{$\begin{array}{l}0.0 \% \\
\text { Day } 5\end{array}$} & \multicolumn{3}{|c|}{ Concentration } \\
\hline Rep. & Day $2 / 3$ & Day 4 & & Day 6 & Day 7 & Total \\
\hline 1 & 0 & 6 & 8 & 0 & 14 & 28 \\
\hline 2 & 4 & 0 & 4 & 11 & 0 & 19 \\
\hline 3 & 4 & 7 & 0 & 8 & 0 & 19 \\
\hline 4 & 4 & 6 & 0 & 0 & 14 & 24 \\
\hline 5 & $\overline{2}$ & 4 & 7 & 10 & 0 & 23 \\
\hline 6 & 4 & 0 & 0 & 8 & 10 & 22 \\
\hline 7 & 4 & 0 & 8 & 10 & 0 & 22 \\
\hline 8 & 3 & 6 & 2 & 10 & 0 & 21 \\
\hline 9 & 0 & 5 & 8 & 0 & 9 & 22 \\
\hline 10 & 0 & 3 & 10 & 0 & 13 & 26 \\
\hline
\end{tabular}

\begin{tabular}{|c|c|c|c|c|c|c|}
\hline \multicolumn{4}{|c|}{ Reproduction at } & $6.25 \%$ & \multicolumn{4}{c|}{ Concentration } \\
\hline Rep. & Day 2/3 & Day 4 & Day 5 & Day 6 & Day 7 & Total \\
\hline 1 & 0 & 7 & 9 & 0 & 10 & 26 \\
\hline 2 & 4 & 0 & 9 & 10 & 0 & 23 \\
\hline 3 & 5 & 6 & 0 & 9 & 0 & 20 \\
\hline 4 & 4 & 6 & 0 & 0 & 8 & 18 \\
\hline 5 & 4 & 0 & 8 & 10 & 0 & 22 \\
\hline 6 & 4 & 1 & 7 & 0 & 9 & 21 \\
\hline 7 & 4 & 0 & 8 & 10 & 0 & 22 \\
\hline 8 & 0 & 6 & 7 & 11 & 0 & 24 \\
\hline 9 & 0 & 2 & 7 & 0 & 9 & 18 \\
\hline 10 & 0 & 4 & 8 & 0 & 10 & 22 \\
\hline
\end{tabular}

\begin{tabular}{|c|c|c|c|c|c|c|}
\hline \multicolumn{3}{|c|}{ Reproduction at } & \multicolumn{5}{|c|}{$12.5 \%$} & \multicolumn{3}{c|}{ Concentration } \\
\hline Rep. & Day 2/3 & Day 4 & Day 5 & Day 6 & Day 7 & Total \\
\hline 1 & 0 & 2 & 9 & 10 & 0 & 21 \\
\hline 2 & 0 & 4 & 7 & 10 & 0 & 21 \\
\hline 3 & 4 & 6 & 0 & 9 & 0 & 19 \\
\hline 4 & 4 & 5 & 7 & 3 & 0 & 19 \\
\hline 5 & 4 & 0 & 8 & 10 & 0 & 22 \\
\hline 6 & 4 & 0 & 7 & 0 & 13 & 24 \\
\hline 7 & 5 & 0 & 10 & 9 & 0 & 24 \\
\hline 8 & 4 & 0 & 9 & 10 & 0 & 23 \\
\hline 9 & 0 & 4 & 8 & 0 & 11 & 23 \\
\hline 10 & 0 & 6 & 9 & 0 & 10 & 25 \\
\hline
\end{tabular}

\begin{tabular}{|c|c|c|c|c|c|c|}
\hline \multicolumn{3}{|c|}{ Reproduction at } & \multicolumn{5}{c|}{$25.0 \%$} & \multicolumn{4}{c|}{ Concentration } \\
\hline Rep. & Day 2/3 & Day 4 & Day 5 & Day 6 & Day 7 & Total \\
\hline 1 & 0 & 1 & 8 & 10 & 0 & 19 \\
\hline 2 & 4 & 0 & 8 & 0 & 11 & 23 \\
\hline 3 & 4 & 6 & 0 & 8 & 0 & 18 \\
\hline 4 & 6 & 4 & 0 & 0 & 11 & 21 \\
\hline 5 & 0 & 4 & 7 & 0 & 10 & 21 \\
\hline 6 & 4 & 0 & 9 & 0 & 0 & 13 \\
\hline 7 & 5 & 0 & 9 & 11 & 0 & 25 \\
\hline 8 & 0 & 4 & 10 & 9 & 0 & 23 \\
\hline 9 & 0 & 6 & 7 & 10 & 0 & 23 \\
\hline 10 & 0 & 2 & 8 & 0 & 12 & 22 \\
\hline
\end{tabular}

\begin{tabular}{|c|c|c|c|c|c|c|}
\hline \multicolumn{3}{|c|}{ Reproduction at } \\
\hline Rep. & Day $2 / 3$ & Day 4 & Day 5 & Day 6 & Day 7 & Total \\
\hline 1 & 0 & 2 & 8 & 0 & 11 & 21 \\
\hline 2 & 0 & 7 & 7 & 10 & 0 & 24 \\
\hline 3 & 4 & 8 & 0 & 9 & 0 & 21 \\
\hline 4 & 5 & 5 & 0 & 0 & 10 & 20 \\
\hline 5 & 5 & 0 & 8 & 10 & 0 & 23 \\
\hline 6 & 4 & 0 & 8 & 9 & 0 & 21 \\
\hline 7 & 4 & 0 & 7 & 9 & 0 & 20 \\
\hline 8 & 3 & 0 & 9 & 10 & 0 & 22 \\
\hline 9 & 0 & 3 & 0 & 8 & 9 & 20 \\
\hline 10 & 0 & 2 & 9 & 0 & 8 & 19 \\
\hline
\end{tabular}

\begin{tabular}{|c|c|c|c|c|c|c|}
\hline \multicolumn{9}{|c|}{ Reproduction at } \\
\hline Rep. & Day 2/3 & Day 4 & Day 5 & Day 6 & Day 7 & Total \\
\hline 1 & 0 & 4 & 6 & 0 & 10 & 20 \\
\hline 2 & 4 & 0 & 0 & 12 & 9 & 25 \\
\hline 3 & 3 & 6 & 0 & 8 & 0 & 17 \\
\hline 4 & 4 & 6 & 0 & 0 & 9 & 19 \\
\hline 5 & 4 & 1 & 8 & 11 & 0 & 24 \\
\hline 6 & 3 & 0 & 7 & 13 & 0 & 23 \\
\hline 7 & 4 & 0 & 8 & 10 & 0 & 22 \\
\hline 8 & 3 & 4 & 4 & 8 & 0 & 19 \\
\hline 9 & 0 & 3 & 9 & 0 & 10 & 22 \\
\hline 10 & 0 & 4 & 8 & 0 & 11 & 23 \\
\hline
\end{tabular}




\section{CHRONIC DEFINITIVE SURVIVAL AND REPRODUCTION TEST \\ Statistical Analyses}

Client: Westinghouse Savannah River Company

Sample Identification: FHB-022

Test Date: $\quad$ 2/22/96

\begin{tabular}{|lrr|}
\hline Test for Normality & \\
Test Used: Shapiro Wilks & $\mathrm{W}=$ & 0.974 \\
& critical & 0.93 \\
& & \\
The data are normal in distribution
\end{tabular}

\section{Test for Homogeneity of Variance}

Test Used: Bartlett's Test

$\mathrm{B}=$

critical

6.25

15.1

The data are homogeneous in variance

\section{Effluent Conc. vs. Reproduction}

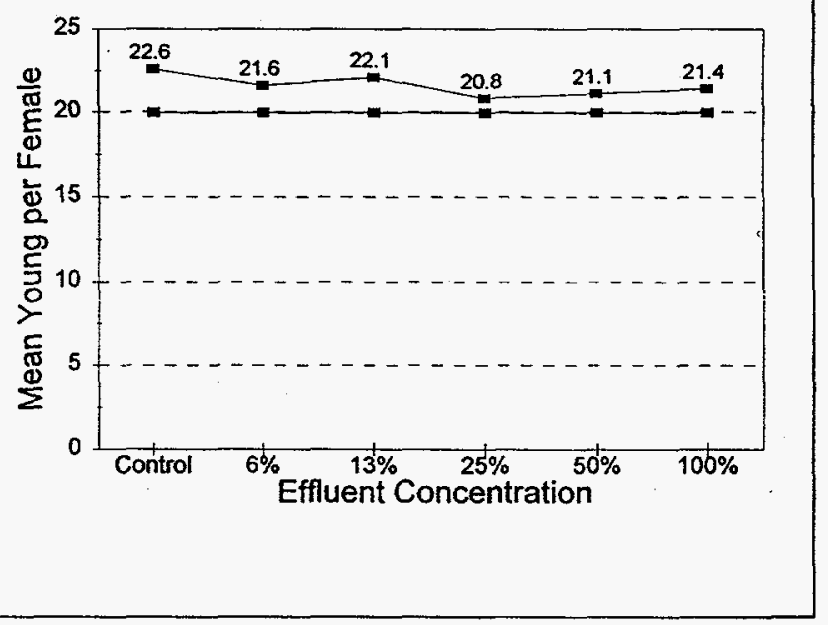

Summary 


\section{CHRONIC DEFINITIVE SURVIVAL AND REPRODUCTION TEST Water Chemistry}

Client: Westinghouse Savannah River Company

Sample Identification: FHB-022

Test Date: $\quad$ 2/22/96

pH (After Renewal)

\begin{tabular}{|l|r|r|r|r|r|r|}
\hline \multicolumn{7}{|c|}{ Test Concentration } \\
\hline & Control & $6.25 \%$ & $12.5 \%$ & $25 \%$ & $50 \%$ & $100 \%$ \\
\hline Initial & 8.04 & 7.58 & 7.87 & 7.84 & 7.88 & 7.82 \\
\hline Day 1 & 8.09 & 7.73 & 7.76 & 7.80 & 7.83 & 7.85 \\
\hline Day 2 & 8.00 & 7.66 & 7.82 & 7.91 & 7.86 & 7.99 \\
\hline Day 3 & 8.02 & 7.85 & 7.91 & 7.95 & 8.07 & 8.20 \\
\hline Day 4 & 8.03 & 7.69 & 7.94 & 7.95 & 7.98 & 8.14 \\
\hline Day 5 & 8.08 & 7.98 & 7.60 & 7.91 & 7.68 & 7.38 \\
\hline Day 6 & 8.11 & 7.70 & 7.82 & 7.89 & 7.93 & 8.09 \\
\hline
\end{tabular}

Dissolved Oxygen (After Renewal)

\begin{tabular}{|l|r|r|r|r|r|r|}
\hline \multicolumn{7}{|c|}{ Test Concentration } \\
\hline & Control & $6.25 \%$ & $12.5 \%$ & $25 \%$ & $50 \%$ & $100 \%$ \\
\hline Initial & 8.6 & 8.6 & 8.6 & 8.6 & 8.6 & 8.6 \\
\hline Day 1 & 8.4 & 8.4 & 8.4 & 8.4 & 8.4 & 8.4 \\
\hline Day 2 & 8.6 & 8.6 & 8.6 & 8.4 & 8.4 & 8.6 \\
\hline Day 3 & 8.5 & 8.4 & 8.4 & 8.5 & 8.5 & 8.5 \\
\hline Day 4 & 8.7 & 8.2 & 8.2 & 8.3 & 8.3 & 8.4 \\
\hline Day 5 & 8.2 & 8.4 & 8.5 & 8.4 & 8.4 & 8.5 \\
\hline Day 6 & 8.9 & 8.3 & 8.3 & 8.4 & 8.5 & 8.5 \\
\hline
\end{tabular}

Dilution Water \& Effluent Parameters

\begin{tabular}{|l|c|r|r|r|r|}
\hline & Res Cl. & Alkalin & Hard. & Cond. & \\
\hline & $\mathrm{mg} / \mathrm{L}$ & $\mathrm{mg} / \mathrm{L}$ & $\mathrm{mg} / \mathrm{L}$ & $\mu \mathrm{mhos}$ & \\
\hline Eff. Sample 1 & $<0.05$ & 8.12 & 16 & 57.3 & \\
\hline Eff. Sample 2 & $<0.05$ & 10.2 & 16 & 59.1 & \\
\hline Eff. Sample 3 & $<0.05$ & 10.2 & 14 & 57.3 & \\
\hline & & & & & \\
\hline Dilution Water & & 69 & 100 & 209 & \\
\hline & & & & & \\
\hline & & & & & \\
\hline
\end{tabular}

pH (Before Renewal)

\begin{tabular}{|l|r|r|r|r|r|r|}
\hline \multicolumn{7}{|c|}{ Test Concentration } \\
\hline & Control & $6.25 \%$ & $12.5 \%$ & $25 \%$ & $50 \%$ & $100 \%$ \\
\hline Day 1 & 7.93 & 8.14 & 8.27 & 8.27 & 8.18 & 7.97 \\
\hline Day 2 & 8.14 & 8.20 & 8.34 & 8.29 & 8.07 & 7.68 \\
\hline Day 3 & 8.26 & 8.14 & 8.14 & 8.16 & 8.08 & 7.79 \\
\hline Day 4 & 7.73 & 8.05 & 8.10 & 8.18 & 8.17 & 8.14 \\
\hline Day 5 & 8.07 & 7.24 & 7.98 & 7.79 & 7.95 & 8.12 \\
\hline Day 6 & 8.05 & 8.29 & 8.42 & 8.42 & 8.27 & 8.04 \\
\hline Final & 8.20 & 8.20 & 8.16 & 8.19 & 8.14 & 8.00 \\
\hline
\end{tabular}

Dissolved Oxygen (Before Renewal)

\begin{tabular}{|l|r|r|r|r|r|r|}
\hline \multicolumn{7}{|c|}{ Test Concentration } \\
\hline & Control & $6.25 \%$ & $12.5 \%$ & $25 \%$ & $50 \%$ & $100 \%$ \\
\hline Day 1 & 8.3 & 8.7 & 8.7 & 8.7 & 8.7 & 8.7 \\
\hline Day 2 & 8.2 & 8.4 & 8.4 & 8.3 & 8.4 & 8.2 \\
\hline Day 3 & 8.5 & 8.3 & 8.4 & 8.4 & 8.4 & 8.4 \\
\hline Day 4 & 8.2 & 8.4 & 8.4 & 8.5 & 8.5 & 8.3 \\
\hline Day 5 & 8.4 & 8.0 & 8.1 & 8.2 & 8.1 & 8.2 \\
\hline Day 6 & 8.5 & 8.2 & 8.3 & 8.5 & 8.5 & 8.6 \\
\hline Final & 8.6 & 8.6 & 8.7 & 8.7 & 8.8 & 8.8 \\
\hline
\end{tabular}

Temperature

\begin{tabular}{|l|r|}
\hline \multicolumn{2}{|c|}{ Incubator ${ }^{\circ} \mathrm{C}$} \\
\hline Initial & 26.0 \\
\hline Day 1 & 25.8 \\
\hline Day 2 & 24.8 \\
\hline Day 3 & 24.1 \\
\hline Day 4 & 26.0 \\
\hline Day 5 & 24.0 \\
\hline Day 6 & 25.1 \\
\hline Final & 24.4 \\
\hline
\end{tabular}


(864) 877-6942 - FAX (864) 877-6938

P.O. Box 16414, Greenville, SC 29606 - 4 Craftsman Court, Greer, SC 29650

\section{Day Chronic Definitive Survival and Reproduction Bioassay}

Method: EPA/600/4-89/001

Test Organism: Ceriodaphnia dubia

Facility:Westinghouse Savannah River Company

Sample ID:FHB-023

Date: $2 / 22 / 96$ 


\section{CHRONIC DEFINITIVE SURVIVAL AND REPRODUCTION TEST Survival and Reproduction Results}

Client: Westinghouse Savannah River Company

Sample Identification: FHB-023

Test Date: $\quad$ 2/22/96

\begin{tabular}{|c|c|c|c|c|c|c|}
\hline \multicolumn{3}{|c|}{ Reproduction at } & $0.0 \%$ & \multicolumn{4}{c|}{ Concentration } \\
\hline Rep. & Day $2 / 3$ & Day 4 & Day 5 & Day 6 & Day 7 & Total \\
\hline 1 & 0 & 5 & 10 & 0 & 13 & 28 \\
\hline 2 & 0 & 6 & 8 & 12 & 0 & 26 \\
\hline 3 & 4 & 0 & 9 & 10 & 0 & 23 \\
\hline 4 & 4 & 0 & 8 & 0 & 10 & 22 \\
\hline 5 & 4 & 8 & 0 & 12 & 0 & 24 \\
\hline 6 & 0 & 3 & 5 & 0 & 10 & 18 \\
\hline 7 & 3 & 6 & 0 & 8 & 0 & 17 \\
\hline 8 & 5 & 0 & 7 & 5 & 0 & 17 \\
\hline 9 & 0 & 5 & 7 & 0 & 9 & 21 \\
\hline 10 & 4 & 0 & 7 & 8 & 0 & 19 \\
\hline
\end{tabular}

\begin{tabular}{|c|c|c|c|c|c|c|}
\hline \multicolumn{3}{|c|}{ Reproduction at } & $6.25 \%$ & \multicolumn{3}{c|}{ Concentration } \\
\hline Rep. & Day 2/3 & Day 4 & Day 5 & Day 6 & Day 7 & Total \\
\hline 1 & 0 & 4 & 6 & 0 & 12 & 22 \\
\hline 2 & 0 & 5 & 8 & 11 & 0 & 24 \\
\hline 3 & 6 & 0 & 8 & 10 & 0 & 24 \\
\hline 4 & 0 & 5 & 7 & 10 & 0 & 22 \\
\hline 5 & 4 & 7 & 0 & 0 & 9 & 20 \\
\hline 6 & 0 & 5 & 7 & L & L & L \\
\hline 7 & 4 & 7 & 0 & 0 & 11 & 22 \\
\hline 8 & 3 & 7 & 0 & 0 & 9 & 19 \\
\hline 9 & 0 & 5 & 9 & 10 & 0 & 24 \\
\hline 10 & 3 & 0 & 5 & 0 & 10 & 18 \\
\hline
\end{tabular}

\begin{tabular}{|c|c|c|c|c|c|c|}
\hline \multicolumn{3}{|c|}{ Reproduction at } & \multirow{2}{*}{$\begin{array}{l}12.5 \% \\
\text { Day } 5\end{array}$} & \multicolumn{2}{|c|}{ Concentration } & \multirow[b]{2}{*}{ Total } \\
\hline Rep. & Day $2 / 3$ & Day 4 & & Day 6 & Day 7 & \\
\hline 1 & 0 & 5 & 7 & $\overline{0}$ & 8 & 20 \\
\hline 2 & 0 & 2 & 6 & 10 & 0 & 18 \\
\hline 3 & 4 & 0 & 6 & 10 & 0 & 20 \\
\hline 4 & 5 & 0 & 7 & 10 & 0 & 22 \\
\hline 5 & 4 & 9 & 0 & 8 & 0 & 21 \\
\hline 6 & 0 & 5 & 5 & 10 & 0 & 20 \\
\hline 7 & 3 & 8 & 0 & 11 & 0 & 22 \\
\hline 8 & 0 & 5 & 7 & 0 & 9 & 21 \\
\hline 9 & 0 & 5 & 8 & 10 & 0 & 23 \\
\hline 10 & 4 & 8 & 0 & 11 & 0 & 23 \\
\hline
\end{tabular}

\begin{tabular}{|c|c|c|c|c|c|c|}
\hline \multicolumn{3}{|c|}{ Reproduction at } & $25.0 \%$ & \multicolumn{3}{c|}{ Concentration } \\
\hline Rep. & Day $2 / 3$ & Day 4 & Day 5 & Day 6 & Day 7 & Total \\
\hline 1 & 0 & 5 & 7 & 0 & 14 & 26 \\
\hline 2 & 0 & 6 & 9 & 0 & 10 & 25 \\
\hline 3 & 0 & 3 & 5 & 11 & 0 & 19 \\
\hline 4 & 4 & 0 & 7 & 9 & 0 & 20 \\
\hline 5 & 5 & 8 & 0 & 0 & 8 & 21 \\
\hline 6 & 0 & 3 & 5 & 0 & 10 & 18 \\
\hline 7 & 0 & 4 & 6 & 0 & 9 & 19 \\
\hline 8 & 3 & 7 & 9 & 0 & 0 & 19 \\
\hline 9 & 0 & 6 & 8 & 0 & 10 & 24 \\
\hline 10 & 4 & 7 & 0 & 12 & 0 & 23 \\
\hline
\end{tabular}

\begin{tabular}{|c|c|c|c|c|c|c|}
\hline \multicolumn{3}{|c|}{ Reproduction at } & $50.0 \%$ & \multicolumn{3}{c|}{ Concentration } \\
\hline Rep. & Day 2/3 & Day 4 & Day 5 & Day 6 & Day 7 & Total \\
\hline 1 & 0 & 1 & 5 & 0 & 10 & 16 \\
\hline 2 & 0 & 5 & 7 & 0 & 12 & 24 \\
\hline 3 & 0 & 4 & 8 & 10 & 0 & 22 \\
\hline 4 & 0 & 2 & 5 & 0 & 12 & 19 \\
\hline 5 & 4 & 8 & 0 & 11 & 0 & 23 \\
\hline 6 & 0 & 4 & 6 & 0 & 8 & 18 \\
\hline 7 & 5 & 0 & 7 & 11 & 0 & 23 \\
\hline 8 & 5 & 7 & 0 & 0 & 10 & 22 \\
\hline 9 & 0 & 4 & 7 & 0 & 12 & 23 \\
\hline 10 & 0 & 3 & 7 & 10 & 0 & 20 \\
\hline
\end{tabular}

\begin{tabular}{|c|c|c|c|c|c|c|}
\hline \multicolumn{9}{|c|}{ Reproduction at } & $100.0 \%$ & Concentration \\
\hline Rep. & Day $2 / 3$ & Day 4 & Day 5 & Day 6 & Day 7 & Total \\
\hline 1 & 0 & $\mathrm{X}$ & $\mathrm{X}$ & $\mathrm{X}$ & $\mathrm{X}$ & 0 \\
\hline 2 & $\mathrm{X}$ & $\mathrm{X}$ & $\mathrm{X}$ & $\mathrm{X}$ & $\mathrm{X}$ & 0 \\
\hline 3 & $\mathrm{X}$ & $\mathrm{X}$ & $\mathrm{X}$ & $\mathrm{X}$ & $\mathrm{X}$ & 0 \\
\hline 4 & $\mathrm{X}$ & $\mathrm{X}$ & $\mathrm{X}$ & $\mathrm{X}$ & $\mathrm{X}$ & 0 \\
\hline 5 & $\mathrm{X}$ & $\mathrm{X}$ & $\mathrm{X}$ & $\mathrm{X}$ & $\mathrm{X}$ & 0 \\
\hline 6 & 0 & $\mathrm{X}$ & $\mathrm{X}$ & $\mathrm{X}$ & $\mathrm{X}$ & 0 \\
\hline 7 & $\mathrm{X}$ & $\mathrm{X}$ & $\mathrm{X}$ & $\mathrm{X}$ & $\mathrm{X}$ & 0 \\
\hline 8 & $\mathrm{X}$ & $\mathrm{X}$ & $\mathrm{X}$ & $\mathrm{X}$ & $\mathrm{X}$ & 0 \\
\hline 9 & 0 & $\mathrm{X}$ & $\mathrm{X}$ & $\mathrm{X}$ & $\mathrm{X}$ & 0 \\
\hline 10 & $\mathrm{X}$ & $\mathrm{X}$ & $\mathrm{X}$ & $\mathrm{X}$ & $\mathrm{X}$ & 0 \\
\hline
\end{tabular}




\section{CHRONIC DEFINITIVE SURVIVAL AND REPRODUCTION TEST \\ Statistical Analyses}

Client: Westinghouse Savannah River Company

Sample Identification: FHB-023

Test Date: $\quad$ 2/22/96

\begin{tabular}{|lrr}
\hline Test for Normality & & \\
& & \\
Test Used: Shapiro Wilks & critical & 0.978 \\
& 0.93
\end{tabular}

The data are normal in distribution
Test for Homogeneity of Variance

Test Used: Bartlett's Test

$\mathrm{B}=$

critical $\quad 13.28$

The data are homogeneous in variance

Effluent Conc. vs. Reproduction

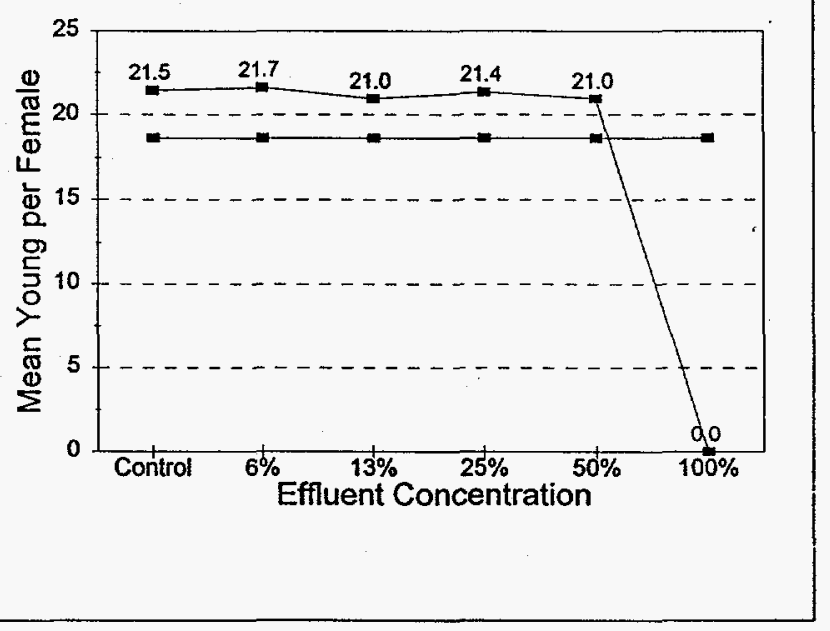

Summary 


\section{CHRONIC DEFINITIVE SURVIVAL AND REPRODUCTION TEST \\ Water Chemistry}

Client: Westinghouse Savannah River Company

Sample Identification: FHB-023

Test Date: $\quad$ 2/22/96

pH (After Renewal)

\begin{tabular}{|l|r|r|r|r|r|r|}
\hline \multicolumn{7}{|c|}{ Test Concentration } \\
\hline & Control & $6.25 \%$ & $12.5 \%$ & $25 \%$ & $50 \%$ & $100 \%$ \\
\hline Initial & 8.04 & 7.81 & 7.46 & 7.42 & 7.39 & 6.03 \\
\hline Day 1 & 8.09 & 7.58 & 7.60 & 7.42 & 7.12 & 5.84 \\
\hline Day 2 & 8.00 & 7.70 & 7.69 & 7.51 & 7.06 & 6.07 \\
\hline Day 3 & 8.02 & 8.12 & 8.15 & 8.16 & 8.15 & 7.28 \\
\hline Day 4 & 8.03 & 7.77 & 7.77 & 7.71 & 7.65 & \\
\hline Day 5 & 8.08 & 7.89 & 7.74 & 7.47 & 7.11 & \\
\hline Day 6 & 8.11 & 7.82 & 7.85 & 7.83 & 7.64 & \\
\hline
\end{tabular}

Dissolved Oxygen (After Renewal)

\begin{tabular}{|l|r|r|r|r|r|r|}
\hline \multicolumn{7}{|c|}{ Test Concentration } \\
\hline & Control & $6.25 \%$ & $12.5 \%$ & $25 \%$ & $50 \%$ & $100 \%$ \\
\hline Initial & 8.6 & 8.8 & 8.8 & 8.6 & 8.6 & 8.6 \\
\hline Day 1 & 8.4 & 8.6 & 8.6 & 8.8 & 8.5 & 8.6 \\
\hline Day 2 & 8.6 & 8.8 & 8.7 & 8.6 & 8.4 & 8.6 \\
\hline Day 3 & 8.5 & 8.3 & 8.3 & 8.5 & 8.5 & 8.6 \\
\hline Day 4 & 8.7 & 8.4 & 8.4 & 8.4 & 8.2 & \\
\hline Day 5 & 8.2 & 8.0 & 8.2 & 8.4 & 8.4 & \\
\hline Day 6 & 8.9 & 8.2 & 8.2 & 8.2 & 8.5 & \\
\hline
\end{tabular}

Dilution Water \& Effluent Parameters

\begin{tabular}{|l|l|r|r|r|r|}
\hline & Res Cl. & Alkalin & Hard. & Cond. & \\
\hline & $\mathrm{mg} / \mathrm{L}$ & $\mathrm{mg} / \mathrm{L}$ & $\mathrm{mg} / \mathrm{L}$ & $\mu \mathrm{mhos}$ & \\
\hline Eff. Sample 1 & .06 & 2.03 & 12 & 32.9 & \\
\hline Eff. Sample 2 & $<0.05$ & 2.03 & 16 & 32.6 & \\
\hline Eff. Sample 3 & $<0.05$ & 2.03 & 18 & 31.9 & \\
\hline & & & & & \\
\hline Dilution Water & & 69 & 100 & 209 & \\
\hline & & & & & \\
\hline & & & & & \\
\hline
\end{tabular}

pH (Before Renewal)

\begin{tabular}{|l|r|r|r|r|r|r|}
\hline \multicolumn{7}{|c|}{ Test Concentration } \\
\hline & Control & $6.25 \%$ & $12.5 \%$ & $25 \%$ & $50 \%$ & $100 \%$ \\
\hline Day 1 & 7.93 & 8.35 & 8.32 & 8.28 & 7.81 & 8.06 \\
\hline Day 2 & 8.14 & 8.30 & 8.21 & 8.40 & 7.91 & 8.10 \\
\hline Day 3 & 8.26 & 8.16 & 8.20 & 8.20 & 8.10 & 8.15 \\
\hline Day 4 & 7.73 & 8.18 & 8.20 & 8.15 & 8.16 & 7.97 \\
\hline Day 5 & 8.07 & 7.85 & 7.99 & 8.07 & 8.12 & \\
\hline Day 6 & 8.05 & 8.29 & 8.26 & 8.28 & 8.27 & \\
\hline Final & 8.20 & 8.21 & 8.18 & 8.12 & 8.10 & \\
\hline
\end{tabular}

Dissolved Oxygen (Before Renewal)

\begin{tabular}{|l|r|r|r|r|r|r|}
\hline \multicolumn{7}{|c|}{ Test Concentration } \\
\hline & Control & $6.25 \%$ & $12.5 \%$ & $25 \%$ & $50 \%$ & $100 \%$ \\
\hline Day 1 & 8.3 & 8.4 & 8.6 & 8.5 & 8.6 & 8.4 \\
\hline Day 2 & 8.2 & 8.2 & 8.2 & 8.4 & 8.4 & 8.4 \\
\hline Day 3 & 8.5 & 8.5 & 8.5 & 8.5 & 8.5 & 8.5 \\
\hline Day 4 & 8.2 & 8.3 & 8.3 & 8.4 & 8.4 & 8.5 \\
\hline Day 5 & 8.4 & 8.4 & 8.5 & 8.4 & 8.5 & \\
\hline Day 6 & 8.5 & 8.4 & 8.4 & 8.4 & 8.5 & \\
\hline Final & 8.6 & 8.2 & 8.2 & 8.4 & 8.4 & \\
\hline
\end{tabular}

Temperature

\begin{tabular}{|l|r|}
\hline \multicolumn{2}{|c|}{ Incubator ${ }^{\circ} \mathrm{C}$} \\
\hline Initial & 26.0 \\
\hline Day 1 & 25.8 \\
\hline Day 2 & 24.8 \\
\hline Day 3 & 24.1 \\
\hline Day 4 & 26.0 \\
\hline Day 5 & 24.0 \\
\hline Day 6 & 25.1 \\
\hline Final & 24.4 \\
\hline
\end{tabular}


(864) $877-6942$ - FAX (864) 877-6938

P.O. Box 16414, Greenville, SC 29606 - 4 Craftsman Court, Greer, SC 29650

\title{
7 Day Chronic Definitive Survival and Reproduction Bioassay Method: EPA/600/4-89/001
}

\author{
Test Organism: $\quad$ Ceriodaphnia dubia
}

Facility:Westinghouse Savannah River Company

Sample ID:HSP-064

Date: $\quad 2 / 29 / 96$ 


\section{CHRONIC DEFINITIVE SURVIVAL AND REPRODUCTION TEST Survival and Reproduction Results}

Client: $\quad$ Westinghouse Savannah River Company

Sample Identification: HSP-064

Test Date: $\quad 2 / 29 / 96 \quad$ T5144

\begin{tabular}{|c|c|c|c|c|c|c|}
\hline \multicolumn{2}{|c|}{ Reproduction at } & \multicolumn{3}{c|}{ Co } & \multicolumn{3}{c|}{ Concentration } \\
\hline Rep. & Day 2/3 & Day 4 & Day 5 & Day 6 & Day 7 & Total \\
\hline 1 & 0 & 6 & 10 & 14 & 0 & 30 \\
\hline 2 & 0 & 0 & 4 & 4 & 9 & 17 \\
\hline 3 & 0 & 5 & 8 & 0 & 13 & 26 \\
\hline 4 & 5 & 0 & 9 & 6 & 5 & 25 \\
\hline 5 & 4 & 0 & 9 & 3 & 4 & 20 \\
\hline 6 & 5 & 9 & 0 & 10 & 0 & 24 \\
\hline 7 & 4 & 4 & 6 & 0 & 7 & 21 \\
\hline 8 & 0 & 5 & 7 & 6 & 3 & 21 \\
\hline 9 & 5 & 0 & 10 & 10 & 0 & 25 \\
\hline 10 & 0 & 3 & 11 & 9 & 0 & 23 \\
\hline
\end{tabular}

\begin{tabular}{|c|c|c|c|c|c|c|}
\hline \multicolumn{3}{|c|}{ Reproduction at } & \multicolumn{5}{l|}{$6.25 \%$} & \multicolumn{3}{c|}{ Concentration } \\
\hline Rep. & Day $2 / 3$ & Day 4 & Day 5 & Day 6 & Day 7 & Total \\
\hline 1 & 0 & 6 & 11 & 13 & 0 & 30 \\
\hline 2 & 0 & 0 & 8 & 11 & 0 & 19 \\
\hline 3 & 0 & 5 & 5 & 0 & 7 & 17 \\
\hline 4 & 2 & 0 & 7 & 7 & 0 & 16 \\
\hline 5 & 1 & 0 & 6 & 12 & 0 & 19 \\
\hline 6 & 5 & 8 & 0 & 11 & 0 & 24 \\
\hline 7 & 0 & 7 & 10 & 9 & 0 & 26 \\
\hline 8 & 0 & 6 & 7 & 11 & 0 & 24 \\
\hline 9 & 5 & 0 & 8 & 10 & 0 & 23 \\
\hline 10 & 5 & 0 & 8 & 7 & 0 & 20 \\
\hline
\end{tabular}

\begin{tabular}{|c|c|c|c|c|c|c|}
\hline \multicolumn{2}{|c|}{ Reproduction at } & \multicolumn{1}{l|}{$12.5 \%$} & \multicolumn{4}{c|}{ Concentration } \\
\hline Rep. & Day 2/3 & Day 4 & Day 5 & Day 6 & Day 7 & Total \\
\hline 1 & 0 & 6 & 8 & 14 & 0 & 28 \\
\hline 2 & 0 & 0 & 2 & 11 & 10 & 23 \\
\hline 3 & 0 & 5 & 7 & 4 & 0 & 16 \\
\hline 4 & 3 & 0 & 8 & 12 & 0 & 23 \\
\hline 5 & 0 & 0 & 9 & 0 & 9 & 18 \\
\hline 6 & 4 & 0 & 7 & 8 & 0 & 19 \\
\hline 7 & 4 & 0 & 8 & 11 & 0 & 23 \\
\hline 8 & 0 & 6 & 9 & 0 & 7 & 22 \\
\hline 9 & 0 & 6 & 5 & 5 & 0 & 16 \\
\hline 10 & 4 & 0 & 8 & 0 & 4 & 16 \\
\hline
\end{tabular}

\begin{tabular}{|c|c|c|c|c|c|c|}
\hline \multicolumn{3}{|c|}{ Reproduction at } & \multicolumn{3}{c|}{$25.0 \%$} & \multicolumn{3}{c|}{ Concentration } \\
\hline Rep. & Day 2/3 & Day 4 & Day 5 & Day 6 & Day 7 & Total \\
\hline 1 & 0 & 5 & 10 & 9 & 0 & 24 \\
\hline 2 & 0 & 0 & 6 & 4 & 3 & 13 \\
\hline 3 & 0 & 0 & 3 & 6 & 12 & 21 \\
\hline 4 & 6 & 0 & 8 & 3 & 7 & 24 \\
\hline 5 & 3 & 5 & 0 & 1 & 8 & 17 \\
\hline 6 & 4 & 0 & 7 & 7 & 5 & 23 \\
\hline 7 & 4 & 0 & 12 & 7 & 0 & 23 \\
\hline 8 & 0 & 5 & 8 & 10 & 0 & 23 \\
\hline 9 & 0 & 6 & 8 & 0 & 0 & 14 \\
\hline 10 & 1 & 0 & 9 & 13 & 0 & 23 \\
\hline
\end{tabular}

\begin{tabular}{|c|c|c|c|c|c|c|}
\hline \multicolumn{2}{|c|}{ Reproduction at } & \multicolumn{3}{c|}{$50.0 \%$} & \multicolumn{3}{c|}{ Concentration } \\
\hline Rep. & Day 2/3 & Day 4 & Day 5 & Day 6 & Day 7 & Total \\
\hline 1 & 0 & 4 & 9 & 11 & 0 & 24 \\
\hline 2 & 0 & 0 & 4 & 9 & 0 & 13 \\
\hline 3 & 0 & 5 & 0 & 0 & 0 & 5 \\
\hline 4 & 0 & 8 & 7 & 0 & 0 & 15 \\
\hline 5 & 1 & 0 & 2 & 11 & 0 & 14 \\
\hline 6 & 5 & 5 & 0 & 3 & 4 & 17 \\
\hline 7 & 3 & 0 & 6 & 11 & 0 & 20 \\
\hline 8 & 0 & 4 & 7 & 0 & 8 & 19 \\
\hline 9 & 0 & 0 & 10 & 11 & 0 & 21 \\
\hline 10 & 4 & 0 & 8 & 8 & 0 & 20 \\
\hline
\end{tabular}

\begin{tabular}{|c|c|c|c|c|c|c|}
\hline \multicolumn{3}{|c|}{ Reproduction at } & \multicolumn{5}{|c|}{$100.0 \%$} & \multicolumn{3}{c|}{ Concentration } \\
\hline Rep. & Day 2/3 & Day 4 & Day 5 & Day 6 & Day 7 & Total \\
\hline 1 & 0 & 4 & 6 & 10 & 0 & 20 \\
\hline 2 & 0 & 0 & 4 & 6 & 12 & 22 \\
\hline 3 & 0 & 4 & L & L & L & L \\
\hline 4 & 0 & 0 & 6 & 7 & 9 & 22 \\
\hline 5 & 4 & 0 & 6 & 8 & 0 & 18 \\
\hline 6 & 3 & 0 & 5 & 11 & 0 & 19 \\
\hline 7 & 0 & 4 & 8 & 9 & 0 & 21 \\
\hline 8 & 0 & 2 & 3 & 0 & 11 & 16 \\
\hline 9 & 0 & 5 & 8 & 9 & 0 & 22 \\
\hline 10 & 0 & 6 & 9 & 9 & 0 & 24 \\
\hline
\end{tabular}




\section{CHRONIC DEFINITIVE SURVIVAL AND REPRODUCTION TEST \\ Statistical Analyses}

Client: Westinghouse Savannah River Company

Sample Identification: HSP-064

Test Date: $\quad$ 2/29/96

\section{Test for Normality}

Test Used: Shapiro Wilks

$$
\begin{array}{rr}
W= & 0.948 \\
\text { critical } & 0.93
\end{array}
$$

The data are normal in distribution
Test for Homogeneity of Variance

Test Used: Bartlett's Test

$$
\begin{array}{r}
B= \\
\text { critical }
\end{array}
$$

15.1

The data are homogeneous in variance

\section{Effluent Conc. vs. Reproduction}

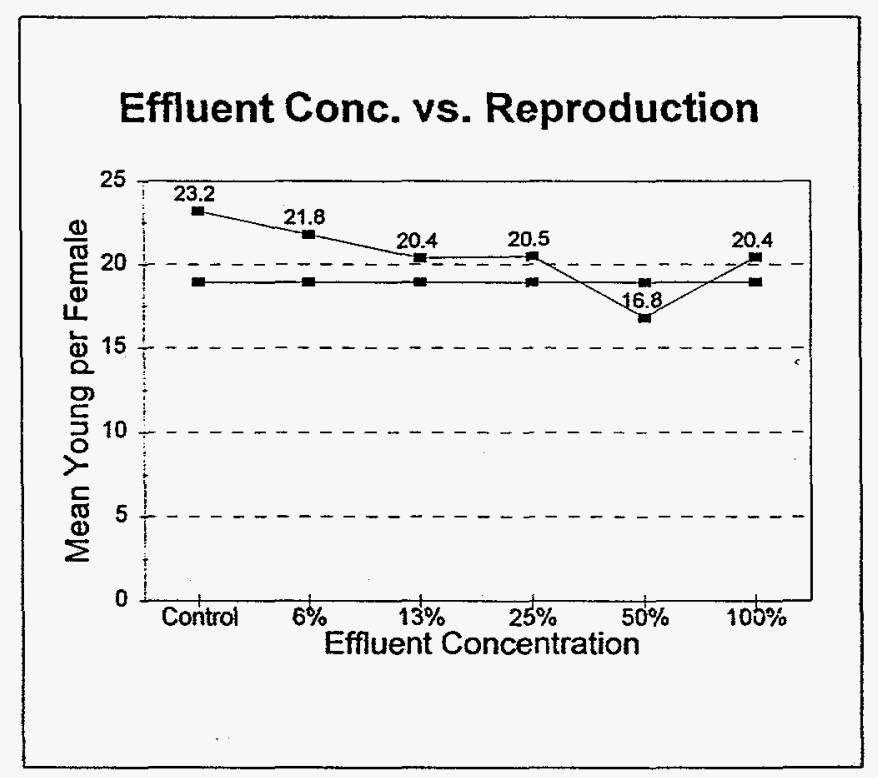

The sample is not acutely or chronically toxic Results at $50 \%$ concentration are considered anomalous.

Summary 


\section{CHRONIC DEFINITIVE SURVIVAL AND REPRODUCTION TEST \\ Water Chemistry}

Client: Westinghouse Savannah River Company

Sample Identification: HSP-064

Test Date: $\quad 2 / 29 / 96$

pH (After Renewal)

\begin{tabular}{|l|r|r|r|r|r|r|}
\hline \multicolumn{7}{|c|}{ Test Concentration } \\
\hline & Control & $6.25 \%$ & $12.5 \%$ & $25 \%$ & $50 \%$ & $100 \%$ \\
\hline Initial & 8.12 & 7.69 & 7.83 & 7.71 & 7.48 & 6.15 \\
\hline Day 1 & 7.98 & 7.77 & 7.83 & 7.65 & 7.34 & 6.31 \\
\hline Day 2 & 7.97 & 8.10 & 8.00 & 7.87 & 7.45 & 6.52 \\
\hline Day 3 & 7.98 & 8.20 & 7.98 & 7.93 & 7.43 & 7.04 \\
\hline Day 4 & 8.06 & 8.07 & 7.85 & 7.77 & 7.33 & 6.12 \\
\hline Day 5 & 8.05 & 7.97 & 7.98 & 8.01 & 7.99 & 7.94 \\
\hline Day 6 & 8.14 & 8.16 & 8.11 & 8.04 & 7.91 & 7.25 \\
\hline
\end{tabular}

Dissolved Oxygen (After Renewal)

\begin{tabular}{|l|r|r|r|r|r|r|}
\hline \multicolumn{7}{|c|}{ Test Concentration } \\
\hline & Control & $6.25 \%$ & $12.5 \%$ & $25 \%$ & $50 \%$ & $100 \%$ \\
\hline Initial & 8.6 & 8.5 & 8.6 & 8.7 & 8.7 & 8.8 \\
\hline Day 1 & 8.6 & 8.6 & 8.7 & 8.6 & 8.6 & 8.6 \\
\hline Day 2 & 8.5 & 8.6 & 8.8 & 8.4 & 8.6 & 8.6 \\
\hline Day 3 & 8.6 & 8.4 & 8.5 & 8.5 & 8.6 & 8.7 \\
\hline Day 4 & 8.8 & 8.6 & 8.6 & 8.6 & 8.6 & 8.6 \\
\hline Day 5 & 8.9 & 8.4 & 8.4 & 8.3 & 8.3 & 8.2 \\
\hline Day 6 & 8.7 & 8.6 & 8.6 & 8.5 & 8.3 & 8.3 \\
\hline
\end{tabular}

Dilution Water \& Effluent Parameters

\begin{tabular}{|l|r|r|r|r|r|}
\hline & Res Cl. & Alkalin & Hard. & Cond. & \\
\hline & $\mathrm{mg} / \mathrm{L}$ & $\mathrm{mg} / \mathrm{L}$ & $\mathrm{mg} / \mathrm{L}$ & $\mu$ mhos & \\
\hline Eff. Sample 1 & $<0.05$ & 4.06 & 10 & 32.1 & \\
\hline Eff. Sample 2 & $<0.05$ & 6.09 & 10 & 33.7 & \\
\hline Eff. Sample 3 & $<0.05$ & 6.09 & 16 & 30.8 & \\
\hline & & & & & \\
\hline Dilution Water & & 71.1 & 84 & 222 & \\
\hline & & & & & \\
\hline & & & & & \\
\hline
\end{tabular}

$\mathrm{pH}$ (Before Renewal)

\begin{tabular}{|l|r|r|r|r|r|r|}
\hline \multicolumn{7}{|c|}{ Test Concentration } \\
\hline & Control & $6.25 \%$ & $12.5 \%$ & $25 \%$ & $50 \%$ & $100 \%$ \\
\hline Day 1 & 8.10 & 8.16 & 8.24 & 8.28 & 8.31 & 8.49 \\
\hline Day 2 & 8.27 & 8.25 & 8.18 & 8.07 & 7.93 & 8.10 \\
\hline Day 3 & 8.44 & 8.46 & 8.50 & 8.53 & 8.44 & 8.24 \\
\hline Day 4 & 8.09 & 8.34 & 8.27 & 8.23 & 8.17 & 7.59 \\
\hline Day 5 & 8.33 & 8.20 & 8.17 & 8.16 & 8.03 & 7.58 \\
\hline Day 6 & 8.30 & 8.27 & 8.21 & 8.22 & 8.07 & 7.64 \\
\hline Final & 8.21 & 8.28 & 8.24 & 8.16 & 8.16 & 7.57 \\
\hline
\end{tabular}

Dissolved Oxygen (Before Renewal)

\begin{tabular}{|l|r|r|r|r|r|r|}
\hline \multicolumn{7}{|c|}{ Test Concentration } \\
\hline & Control & $6.25 \%$ & $12.5 \%$ & $25 \%$ & $50 \%$ & $100 \%$ \\
\hline Day 1 & 8.3 & 8.2 & 8.0 & 8.3 & 8.4 & 8.2 \\
\hline Day 2 & 8.2 & 8.3 & 8.2 & 8.2 & 8.5 & 8.0 \\
\hline Day 3 & 8.8 & 8.6 & 8.6 & 8.8 & 8.9 & 9.0 \\
\hline Day 4 & 8.6 & 8.5 & 8.5 & 8.6 & 8.7 & 8.7 \\
\hline Day 5 & 8.5 & 8.2 & 8.2 & 8.2 & 8.1 & 8.1 \\
\hline Day 6 & 8.6 & 8.4 & 8.4 & 8.4 & 8.4 & 8.3 \\
\hline Final & 8.3 & 8.4 & 8.4 & 8.5 & 8.5 & 8.6 \\
\hline
\end{tabular}

Temperature

\begin{tabular}{|l|r|}
\hline \multicolumn{2}{|c|}{ Incubator ${ }^{\circ} \mathrm{C}$} \\
\hline Initial & 24.4 \\
\hline Day 1 & 24.3 \\
\hline Day 2 & 25.7 \\
\hline Day 3 & 25.1 \\
\hline Day 4 & 24.2 \\
\hline Day 5 & 25.7 \\
\hline Day 6 & 25.1 \\
\hline Final & 25.3 \\
\hline
\end{tabular}




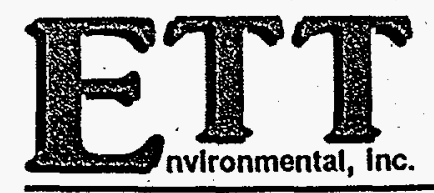

(864) 877-6942 - FAX (864) 877-6938

P.O. Box 16414, Greenville, SC 29606 - 4 Craftsman Court, Greer, SC 29650

\title{
7 Day Chronic Definitive Survival and Reproduction Bioassay \\ Method: EPA/600/4-89/001
}

\author{
Test Organism: $\quad$ Ceriodaphnid dubia
}

Facility: Westinghouse Savannah River Company

Sample ID: FMC-002H

Date: $\quad 2 / 22 / 96$ 


\section{CHRONIC DEFINITIVE SURVIVAL AND REPRODUCTION TEST Survival and Reproduction Results}

Client: Westinghouse Savannah River Company

Sample Identification: FMC-002H

Test Date: $\quad 2 / 22 / 96 \quad$ T5101

\begin{tabular}{|c|c|c|c|c|c|c|}
\hline \multicolumn{3}{|c|}{ Reproduction at } & \multicolumn{4}{|c|}{$0.0 \%$ Concentration } \\
\hline Rep. & Day $3 / 4$ & Day 5 & Day 6 & Day 7 & Day 8 & Total \\
\hline$i$ & 13 & 0 & 4 & 0 & 0 & 17 \\
\hline 2 & 4 & 5 & 7 & 0 & 0 & 16 \\
\hline 3 & 12 & 0 & 7 & 0 & 0 & 19 \\
\hline 4 & 4 & 2 & 0 & $\overline{0}$ & 13 & 19 \\
\hline 5 & 2 & 6 & 8 & 0 & 0 & 16 \\
\hline 6 & 4 & 8 & 12 & 0 & $\theta$ & 24 \\
\hline 7. & 6 & 4 & 0 & 0 & 10 & 20 \\
\hline 8 & 2 & 0 & 1. & 0 & 12 & 15 \\
\hline 9 & 16 & 0 & 0 & 0 & 9 & 25 \\
\hline 10 & 5 & 8 & 0 & 8 & 0 & 21 \\
\hline
\end{tabular}

\begin{tabular}{|c|c|c|c|c|c|c|}
\hline \multicolumn{2}{|c|}{ Reproduction at } & \multicolumn{4}{|c|}{$6.25 \%$} & \multicolumn{3}{|c|}{ Concentration } \\
\hline Rep. & Day 3/4 & Day 5 & Day 6 & Day 7 & Day 8 & Total \\
\hline 1 & 12 & 0 & 1 & 0 & 12 & 25 \\
\hline 2 & 4 & 10 & 1 & 0 & 14 & 29 \\
\hline 3 & 13 & 0 & 7 & 0 & 0 & 20 \\
\hline 4 & 0 & 1 & 0 & 8 & 9 & 18 \\
\hline 5 & 4 & 7 & 6 & 0 & 0 & 17 \\
\hline 6 & 2 & 6 & 7 & 0 & 0 & 15 \\
\hline 7 & 0 & 2 & 3 & 0 & 10 & -15 \\
\hline 8 & 0 & 0 & 0 & 9 & 11 & 20 \\
\hline 9 & 15 & 0 & 0 & 0 & 10 & 25 \\
\hline 10 & 4 & 9 & 0 & 8 & 0 & 21 \\
\hline
\end{tabular}

\begin{tabular}{|c|c|c|c|c|c|c|}
\hline \multicolumn{3}{|c|}{ Reproduction at } & \multirow{2}{*}{$\begin{array}{l}12.5 \% \\
\text { Day } 6\end{array}$} & \multicolumn{2}{|c|}{ Concentration } & \\
\hline Rep. & Day 3/4 & Day 5 & & Day 7 & Day 8 & Total \\
\hline 1 & 5 & $\overline{0}$ & 11 & $\overline{0}$ & $\overline{0}$ & 16 \\
\hline 2 & 5 & 7 & 0 & 0 & .10 & 22 \\
\hline 3 & 11 & 0 & 8 & 0 & 0 & 19. \\
\hline 4 & 2 & 3 & 0 & 11 & $\overline{0}$ & 16 \\
\hline 5 & 6 & 4 & 0 & 7 & 0 & 17 \\
\hline 6 & 4 & 5 & 11 & 0 & $\overline{0}$ & 20 \\
\hline 7 & 4 & 6 & 4 & 0 & 8 & 22 \\
\hline 8. & 0 & 0 & 0 & 7 & 13 & 20 \\
\hline 9 & 12 & 0 & 0 & 0 & 11. & 23 \\
\hline 10 & 2 & 4 & 0 & 3 & 7. & 16 \\
\hline
\end{tabular}

\begin{tabular}{|c|c|c|c|c|c|c|}
\hline \multicolumn{3}{|c|}{ Reproduction at } & \multicolumn{3}{c|}{$25.0 \%$} & \multicolumn{3}{c|}{ Concentration } \\
\hline Rep. & Day 3/4 & Day 5 & Day 6 & Day 7 & Day 8 & Total \\
\hline 1 & 9 & 0 & 6 & 12 & 0 & 27 \\
\hline 2 & 4 & 6 & 2 & 0 & 9 & 21 \\
\hline 3 & 8 & 0 & 9 & 0 & 0 & 17 \\
\hline 4 & 0 & 0 & 0 & 8 & 10 & 18 \\
\hline 5 & 2 & 6 & 0 & 12 & 0 & 20 \\
\hline 6 & 6 & 6 & 9 & 0 & 0 & 21 \\
\hline 7 & 3 & 8 & 5 & 0 & 0 & 16 \\
\hline 8 & 0 & 0 & 5 & 7 & 11 & 23 \\
\hline 9 & 3 & 7 & 2 & 0 & 9 & 21 \\
\hline 10 & 4 & 9 & 6 & 2 & 0 & 21 \\
\hline
\end{tabular}

\begin{tabular}{|c|c|c|c|c|c|c|}
\hline \multicolumn{6}{|c|}{ Reproduction at } & \multicolumn{6}{c|}{$50.0 \%$} & \multicolumn{3}{c|}{ Concentration } \\
\hline Rep. & Day 3/4 & Day 5 & Day 6 & Day 7 & Day 8 & Total \\
\hline 1 & 12 & 0 & 0 & 1 & 10 & 23 \\
\hline 2 & 4 & 10 & 10 & 0 & 0 & 24 \\
\hline 3 & 10 & 0 & 8 & 0 & 0 & 18 \\
\hline 4 & 0 & 2 & 0 & 14 & 17 & 33 \\
\hline 5 & 1 & 4 & 7 & 0 & 11 & 23 \\
\hline 6 & 3 & 3 & 10 & 0 & 11 & 27 \\
\hline 7 & 0 & 0 & 1 & 0 & 15 & 16 \\
\hline 8 & 0 & 0 & 1 & 7 & 10 & 18 \\
\hline 9 & 11 & 0 & 0 & 0 & 9 & 20 \\
\hline 10 & 6 & 7 & 0 & 12 & 0 & 25 \\
\hline
\end{tabular}

\begin{tabular}{|c|c|c|c|c|c|c|}
\hline \multicolumn{6}{|c|}{$100.0 \%$} & \multicolumn{3}{c|}{ Concentration } \\
\hline Reproduction at & Day 3/4 & Day 5 & Day 6 & Day 7 & Day 8 & Total \\
\hline 1 & 4 & 8 & 0 & 10 & 0 & 22 \\
\hline 2 & 4 & 4 & 3 & 0 & 10 & 21 \\
\hline 3 & 9 & 0 & 5 & 5 & 0 & 19 \\
\hline 4 & 1 & 3 & 0 & 7 & 11 & 22 \\
\hline 5 & 3 & 6 & 0 & 3 & 13 & 25 \\
\hline 6 & 1 & 9 & 9 & 0 & 0 & 19 \\
\hline 7 & 3 & 3 & 12 & 0 & 0 & 18 \\
\hline 8 & 0 & 1 & 7 & 0 & 13 & 21 \\
\hline 9 & 5 & 6 & 10 & 0 & 0 & 21 \\
\hline 10 & 6 & 10 & 0 & 12 & 0 & 28 \\
\hline
\end{tabular}




\section{CHRONIC DEFINITIVE SURVIVAL AND REPRODUCTION TEST Statistical Analyses}

Client: Westinghouse Savannah River Company

Sample Identification: FMC-002H

Test Date: $\quad 2 / 22 / 96$

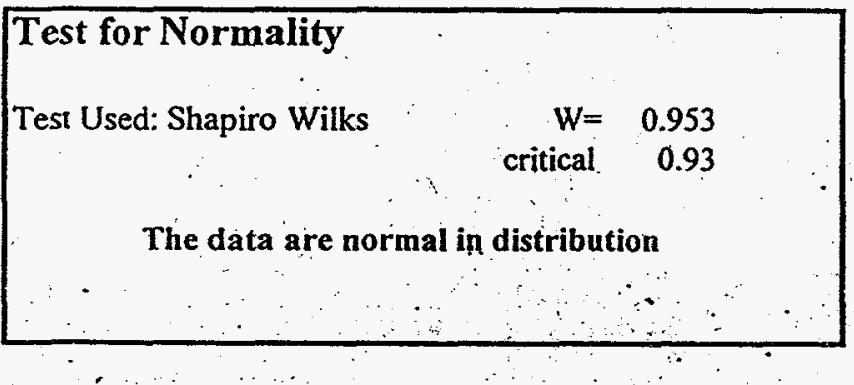

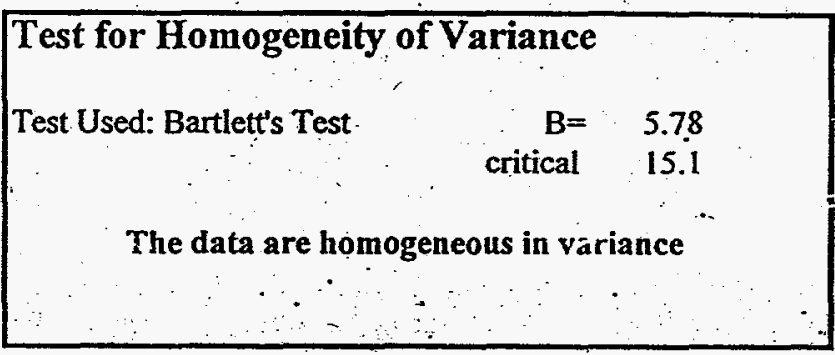

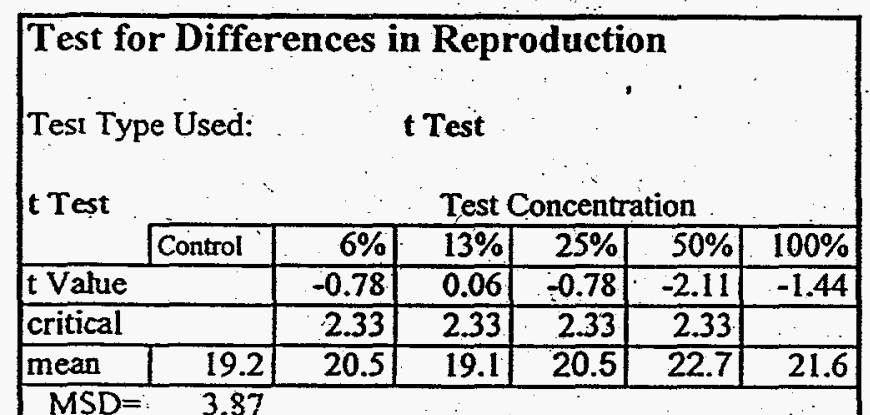

NOEC $>100 \%$ effluent concentration

LOEC $>100 \%$ effluent concentration

The sample is not acutely or chronically toxic.

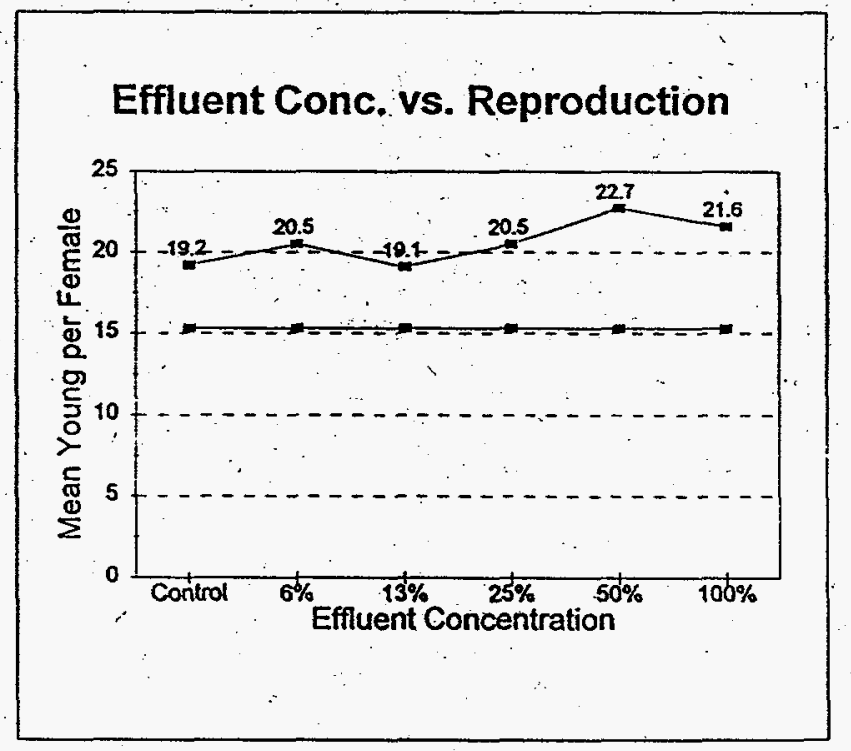

Summary 


\section{CHRONIC DEFINITIVE SURVIVAL AND REPRODUCTION TEST \\ Water Chemistry}

Client: Westinghouse Savannah River Company

Sample Identification: FMC-002H

Test Date: $\quad$ 2/22/96

pH (After Renewal)
\begin{tabular}{|l|r|r|r|r|r|r|}
\hline \multicolumn{7}{|c|}{ Test Concentration } \\
\hline & Control & $6.25 \%$ & $12.5 \%$ & $25 \%$ & $50 \%$ & $100 \%$ \\
\hline Initial & 8.04 & 7.86 & 7.24 & 7.80 & 7.35 & 7.37 \\
\hline Day 1 & 8.09 & 7.76 & 7.79 & 7.80 & 7.78 & 7.56 \\
\hline Day 2 & 8.00 & 7.81 & 7.69 & 7.86 & 7.90 & 7.39 \\
\hline Day 3 & 8.02 & 7.62 & 7.76 & 7.89 & 8.00 & 7.57 \\
\hline Day 4 & 8.03 & 7.91 & 7.99 & 8.01 & 7.22 & 7.64 \\
\hline Day 5 & 8.08 & 7.79 & 7.86 & 7.88 & 7.83 & 7.88 \\
\hline Day 6 & 8.11 & 8.05 & 8.06 & 8.05 & 8.02 & 7.54 \\
\hline
\end{tabular}

pH (Before Renewal)
\begin{tabular}{|l|r|r|r|r|r|r|}
\hline \multicolumn{7}{|c|}{ Test Concentration } \\
\hline & Control & $6.25 \%$ & $12.5 \%$ & $25 \%$ & $50 \%$ & $100 \%$ \\
\hline Day 1 & 7.93 & 7.94 & 8.05 & 8.12 & 8.15 & 8.30 \\
\hline Day 2 & 8.14 & 7.86 & 8.01 & 8.19 & 8.26 & 8.41 \\
\hline Day 3 & 8.26 & 8.04 & 8.19 & 8.18 & 8.03 & 7.99 \\
\hline Day 4 & 7.73 & 8.16 & 8.14 & 8.11 & 8.00 & 7.49 \\
\hline Day 5 & 8.07 & 8.18 & 8.26 & 8.17 & 8.12 & 7.73 \\
\hline Day 6 & 8.05 & 8.23 & 8.17 & 8.19 & 8.07 & 7.48 \\
\hline Final & 8.20 & & & & & \\
\hline
\end{tabular}

Dissolved Oxygen (After Renewal)

\begin{tabular}{|l|r|r|r|r|r|r|}
\hline \multicolumn{7}{|c|}{ Test Concentration } \\
\hline & Control & $6.25 \%$ & $12.5 \%$ & $25 \%$ & $50 \%$ & $100 \%$ \\
\hline Initial & 8.6 & 8.8 & 8.8 & 8.8 & 8.6 & 8.6 \\
\hline Day 1 & 8.4 & 8.7 & 8.8 & 9.0 & 9.1 & 9.1 \\
\hline Day 2 & 8.6 & 8.8 & 8.6 & 8.8 & 9.0 & 8.6 \\
\hline Day 3 & 8.5 & .7 .8 & 8.1 & 8.0 & 8.1 & 8.1 \\
\hline Day 4 & 8.7 & 8.1 & 8.1 & 8.2 & 8.2 & 8.2 \\
\hline Day 5 & 8.2 & 8.2 & 8.4 & 8.4 & 8.5 & 8.5 \\
\hline Day 6 & 8.9 & 8.4 & 8.4 & 8.4 & 8.4 & 8.3 \\
\hline
\end{tabular}

Dissolved Oxygen (Before Renewal)

\begin{tabular}{|r|r|r|r|r|r|r|}
\hline \multicolumn{7}{|c|}{ Test Concentration } \\
\hline & Control & $6.25 \%$ & $12.5 \%$ & $25 \%$ & $50 \%$ & $100 \%$ \\
\hline Day 1 & 8.3 & 8.4 & 8.6 & 8.6 & 8.8 & 8.8 \\
\hline Day 2 & 8.2 & 8.6 & 8.4 & 8.3 & 8.8 & 8.6 \\
\hline Day 3 & 8.5 & 7.0 & 8.2 & 8.1 & 8.2 & 8.1 \\
\hline Day 4 & 8.2 & 8.2 & 8.4 & 8.4 & 8.4 & 8.4 \\
\hline Day 5 & 8.4 & 8.2 & 8.3 & 8.4 & 8.4 & 8.4 \\
\hline Day 6 & 8.5 & 8.3 & 8.3 & 8.4 & 8.4 & 8.2 \\
\hline Final & 8.6 & & & & & \\
\hline
\end{tabular}

Dilution Water \& Effluent Parameters

\begin{tabular}{|l|r|r|r|r|r|}
\hline & Res Cl. & Alkalin & Hard. & Cond. & \\
\hline & $\mathrm{mg} / \mathrm{L}$ & $\mathrm{mg} / \mathrm{L}$ & $\mathrm{mg} / \mathrm{L}$ & $\mu \mathrm{mhos}$ & \\
\hline Eff. Sample 1 & 0.06 & 10.2 & $\mathrm{I2}$ & 56.9 & \\
\hline Eff. Sample 2 & $<.05$ & 10.2 & 24 & 58.8 & \\
\hline Eff. Sample 3 & $<.05$ & 8.12 & 14 & 57.2 & \\
\hline & & & & & \\
\hline Dilution Water & & 68 & 82 & 196.3 & \\
\hline & & & & & \\
\hline & & & & & \\
\hline
\end{tabular}

Temperature

\begin{tabular}{|l|r|}
\hline \multicolumn{2}{|c|}{ Incubator ${ }^{\circ} \mathrm{C}$} \\
\hline Initial & 26.0 \\
\hline Day 1 & 25.8 \\
\hline Day 2 & 24.0 \\
\hline Day 3 & 24.1 \\
\hline Day 4 & 26.0 \\
\hline Day 5 & 24.0 \\
\hline Day 6 & 25.1 \\
\hline Final & 24.4 \\
\hline
\end{tabular}


APPENDIX D

SEDIMENT TOXICITY RESULTS, AUGUST-SEPTEMBER 1995 
P.O. Box 16414, Greenville, SC $29606 \cdot 4$ Craftsman Court, Greer, SC 29650

\title{
10 DAY AMPHIPOD SEDIMENT TOXICITY TEST
}

\author{
EPA/600/R-94/024 Method 100.1 \\ Test Organism: Hyallela azteca
}

Client: Westinghouse Savannah River Company Sample Identification: WSRC BG 001

Test Start Date: $\quad 8-29-95$ 


\section{DAY AMPHIPOD SEDIMENT TOXICITY TEST}

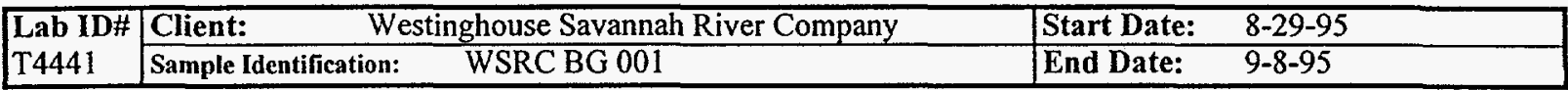

Test System

EPA/600/R-94/024 Method 100.1

The test was set as a Pass/Fail test with a control treatment and one sample treatment. Each treatment replicate consisted of $100 \mathrm{~g}$ of sediment and $175 \mathrm{~mL}$ of dilution water. There were eight replicates for each treatment. Ten test organisms were placed in each replicate. Sediment from a local creek was used in the control treatment. Test organisms were fed with YCT food. The test was conducted at a temperature of $23^{\circ} \mathrm{C}$ with ambient laboratory light conditions. Test vessels were $500 \mathrm{~mL}$ plastic beakers placed in a constant temperature incubator room.

Test Organisms
Order: Amphipoda
Species: Hyallela azteca
Source: Aquatic Biosystems, CO
Life Stage: $\quad 2-3 \mathrm{~mm}$
\% Mortality During 48 Hr Prior to Test::
Taxonomic Verification: RWK $8 / 28 / 95$
Culturing: $\quad$ as received

$\begin{aligned} & \text { Observations: } \quad \text { Cultures healthy } \\ & \text { Acclimation: } \quad \text { None }\end{aligned}$

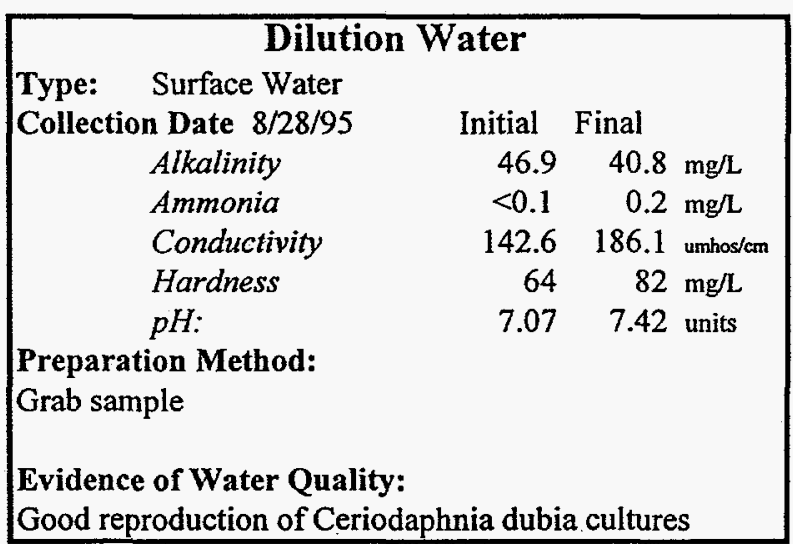

Control Sediment
Source: Resurrection Creek (Greer S C)
Collection Date: $8-28-95$
Preservation: kept at $0-4^{\circ} \mathrm{C}$ in plastic containers
Date/Time Added to Test Chambers: 8-28-95
Observations: organic
Collection Method: grab
Disposal:

\begin{tabular}{|l|}
\hline \multicolumn{1}{|c|}{ Test Sediment } \\
Source: WSRC BG 001 \\
Collection Date $8 / 22 / 95 \quad$ Homogenized?: yes \\
Preservation: kept at $0-4^{\circ} \mathrm{C}$ in plastic containers \\
Date/Time Added to Test Chambers: $8 / 28 / 95$ \\
Observations: organic \\
Collection Method: grab \\
Shipment: Overnight at $4^{\circ} \mathrm{C}$
\end{tabular}

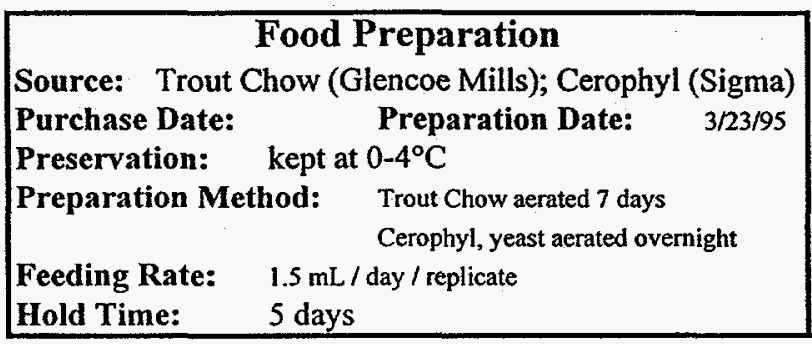

\begin{tabular}{l} 
Test Chambers \\
Type: Plastic $500 \mathrm{~mL}$ beakers \\
Sediment Volume: $\quad 100 \mathrm{~mL}$ \\
Sediment Depth $4 \mathrm{~cm}$ \\
Overlying Water Volume: $\quad 175 \mathrm{~mL}$ \\
Overlying Water Depth: $\quad 5 \mathrm{~cm}$ \\
Aeration: none \\
\hline
\end{tabular}




\section{DAY AMPHIPOD SEDIMENT TOXICITY TEST}

\begin{tabular}{|l|ll|ll|}
\hline Lab ID\# & Client: & \multicolumn{1}{|c|}{ Westinghouse Savannah River Company } & Start Date: & 8-29-95 \\
\cline { 2 - 5 } T4441 & Sample Identification: & WSRC BG 001 & End Date: & 9-8-95 \\
\hline
\end{tabular}

\section{WATER CHEMISTRY TEST METHODS}

Temperature: SM 18th Ed. 2550

Dissolved Oxygen: SM 18th Ed. 4500-O

pH: SM 18th Ed. 4500-H+
Alkalinity: SM 18th Ed, 2320

Hardness: SM 18th Ed. 2340

Conductivity: SM 18th Ed. 2510

WATER CHEMISTRY TEST DATA - OVERLYING WATER

CONTROL TREATMENT

\begin{tabular}{|c|r|r|r|r|}
\hline Test Day & $\begin{array}{c}\text { Temp. } \\
\text { C }\end{array}$ & $\begin{array}{c}\text { pH } \\
\text { units }\end{array}$ & $\begin{array}{c}\text { D.O. } \\
\text { mg/L }\end{array}$ & $\begin{array}{c}\text { D.O. } \\
\% \text { sat. }\end{array}$ \\
\hline Start & 23.3 & 7.07 & 7.8 & $91 \%$ \\
\hline 1 & 22.1 & & 7.0 & $80 \%$ \\
\hline 2 & 21.9 & & 6.0 & $67 \%$ \\
\hline 3 & 21.8 & & 5.6 & $63 \%$ \\
\hline 4 & 22.0 & & 5.3 & $61 \%$ \\
\hline 5 & 23.0 & & 5.8 & $67 \%$ \\
\hline 6 & 22.5 & & 5.5 & $63 \%$ \\
\hline 7 & 22.3 & & 5.6 & $64 \%$ \\
\hline 8 & 22.0 & & 5.3 & $61 \%$ \\
\hline 9 & 22.0 & & 5.2 & $60 \%$ \\
\hline 10 & 22.1 & 7.42 & 4.7 & $54 \%$ \\
\hline
\end{tabular}

SAMPLE TREATMENT

\begin{tabular}{|c|c|c|c|c|c|c|c|c|}
\hline $\begin{array}{c}\text { Test } \\
\text { Day }\end{array}$ & $\begin{array}{c}\text { Cond. } \\
\text { umhos/cm }\end{array}$ & $\begin{array}{c}\text { NH3 } \\
\mathrm{mg} / \mathrm{L}\end{array}$ & $\begin{array}{c}\text { Temp. } \\
\mathrm{C}\end{array}$ & $\begin{array}{c}\mathrm{pH} \\
\text { units }\end{array}$ & $\begin{array}{c}\text { D.O. } \\
\mathrm{mg} / \mathrm{L}\end{array}$ & $\begin{array}{c}\text { D.O. } \\
\% \text { sat. }\end{array}$ & $\begin{array}{c}\text { Alk. } \\
\mathrm{mg} / \mathrm{L}\end{array}$ & $\begin{array}{c}\text { Hard. } \\
\mathrm{mg} / \mathrm{L}\end{array}$ \\
\hline Start & 73 & 0.597 & 23.0 & 6.31 & 6.8 & $79 \%$ & 14.3 & 30.0 \\
\hline 1 & & & 22.0 & & 6.2 & $71 \%$ & & \\
\hline 2 & & & 21.9 & & 5.4 & $61 \%$ & & \\
\hline 3 & & & 22.0 & & 5.4 & $62 \%$ & & \\
\hline 4 & & & 22.0 & & 5.3 & $61 \%$ & & \\
\hline 5 & & & 22.6 & & 5.0 & $57 \%$ & & \\
\hline 6 & & & 22.4 & & 5.4 & $62 \%$ & & \\
\hline 7 & & & 22.1 & & 5.6 & $64 \%$ & & \\
\hline 8 & & & 22.1 & & 5.0 & $57 \%$ & & \\
\hline 9 & & & 22.0 & & 5.1 & $59 \%$ & & \\
\hline 10 & 133 & & 22.2 & 6.85 & 4.8 & $55 \%$ & 46.9 & 56.0 \\
\hline
\end{tabular}

\section{SURVIVAL AND GROWTH RESULTS}

CONTROL TREATMENT

SAMPLE TREATMENT

\begin{tabular}{|c|c|c|c|c|c|c|c|c|c|c|c|c|c|}
\hline & \multicolumn{3}{|c|}{ \# Organisms } & \multirow[b]{2}{*}{$\begin{array}{c}\% \\
\text { Survival }\end{array}$} & \multirow{2}{*}{$\begin{array}{c}\text { Mean } \\
\text { Length } \\
(\mathrm{mm})\end{array}$} & \multirow{2}{*}{$\begin{array}{r}\text { Mean } \\
\text { Length } \\
(\mathrm{mm})\end{array}$} & & \multicolumn{3}{|c|}{ \# Organisms } & \multirow[b]{2}{*}{$\begin{array}{c}\% \\
\text { Survival }\end{array}$} & \multirow{2}{*}{\begin{tabular}{|l} 
Mean \\
Length \\
$(\mathrm{mm})$
\end{tabular}} & \multirow{2}{*}{$\begin{array}{l}\text { Mean } \\
\text { Length } \\
\text { (mm) }\end{array}$} \\
\hline & Initial & $\begin{array}{l}\text { Live at } \\
\text { End }\end{array}$ & \begin{tabular}{|c|} 
Dead \\
at End
\end{tabular} & & & & & Initial & $\begin{array}{c}\text { Live at } \\
\text { End }\end{array}$ & $\begin{array}{l}\text { Dead } \\
\text { at End }\end{array}$ & & & \\
\hline$\overline{\mathrm{A}}$ & 10 & 6 & 1 & $60 \%$ & 2.4 & 2.40 & $\mathrm{~A}$ & 10 & 7 & 0 & $70 \%$ & 2.52 & 2.52 \\
\hline $\mathrm{B}$ & 10 & $\mathrm{NA}$ & $\mathrm{NA}$ & $\mathrm{NA}$ & $\mathrm{NA}$ & $\mathrm{NA}$ & $B$ & 10 & 9 & 0 & $90 \%$ & 2.44 & 2.44 \\
\hline C & 10 & 7 & 0 & $70 \%$ & 2.18 & 2.18 & C & 10 & 6 & 0 & $60 \%$ & 2.44 & 2.44 \\
\hline $\mathrm{D}$ & 10 & 7 & 0 & $70 \%$ & 2.18 & 2.18 & $\mathrm{D}$ & 10 & 5 & 0 & $50 \%$ & 2.41 & 2.41 \\
\hline $\mathrm{E}$ & 10 & 10 & 0 & $100 \%$ & 2.17 & 2.17 & $\mathrm{E}$ & 10 & 10 & 0 & $100 \%$ & 2.24 & 2.24 \\
\hline $\mathrm{F}$ & 10 & 8 & 2 & $80 \%$ & 2.12 & 2.12 & $\mathrm{~F}$ & 10 & 6 & 0 & $60 \%$ & 2.33 & 2.33 \\
\hline $\mathrm{G}$ & 10 & 9 & 0 & $90 \%$ & 2.13 & 2.13 & $G$ & 10 & 8 & 0 & $80 \%$ & 2.26 & 2.26 \\
\hline $\mathrm{H}$ & 10 & 9 & 0 & $90 \%$ & 2.2 & 2.15 & $\mathrm{H}$ & 10 & 8 & 0 & $80 \%$ & 2.3 & 2.34 \\
\hline Mean & 10.0 & & & $80 \%$ & & 2.19 & Mean & 10.0 & & & $74 \%$ & & 2.37 \\
\hline
\end{tabular}




\section{- 10 DAY AMPHIPOD SEDIMENT TOXICITY TEST}

\begin{tabular}{|l|l|l|l|}
\hline \multirow{2}{*}{$\begin{array}{l}\text { Lab ID\# } \\
\text { T4441 }\end{array}$} & Client: $\quad$ Westinghouse Savannah River Company & Start Date: & 8-29-95 \\
\cline { 2 - 4 } & Sample Identification: WSRC BG 001 & End Date: & $9-8-95$ \\
\hline
\end{tabular}

\section{RESULTS}

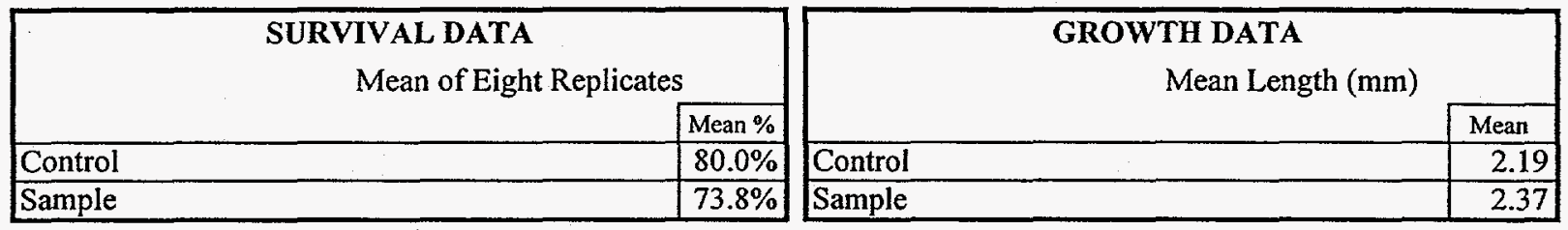

\section{STATISTICAL ANALYSES}

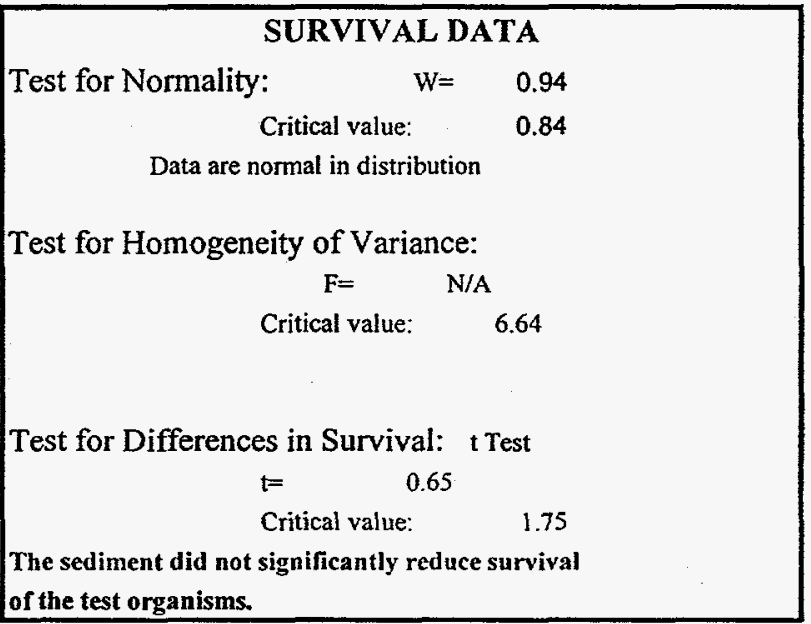

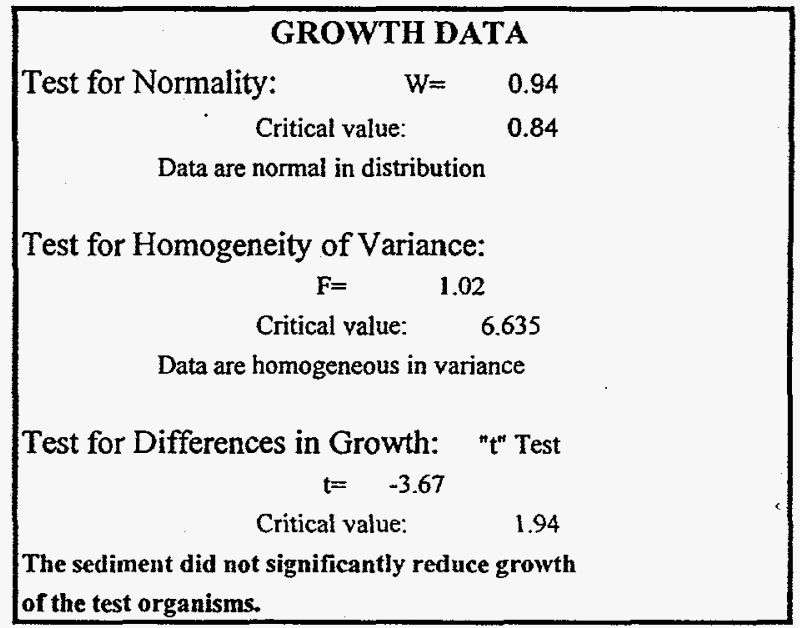

\section{COMMENTS}

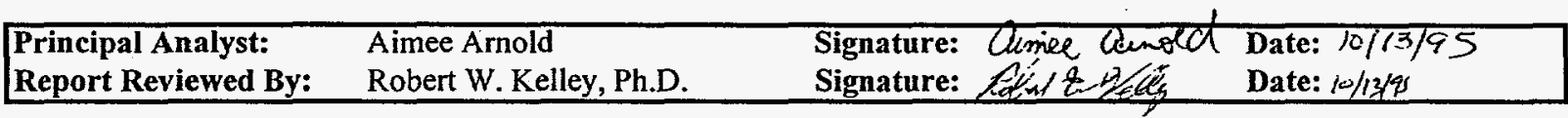

\section{CONCLUSIONS}

The test sediment does not adversely affect the survival and growth of freshwater amphipods. 


\title{
10 DAY AMPHIPOD SEDIMENT TOXICITY TEST
}

\author{
EPA/600/R-94/024 Method 100.1
}

Test Organism: Hyallela azteca

Client: Westinghouse Savannah River Company Sample Identification: WSRC BG 002

Test Start Date: $\quad 9-30-95$ 


\section{DAY AMPHIPOD SEDIMENT TOXICITY TEST}

\begin{tabular}{|c|c|c|c|}
\hline \multirow{2}{*}{$\begin{array}{l}\text { Lab ID \# } \\
\text { T4442 }\end{array}$} & Client: & Jestinghouse Savannah River Company & Start Date: $\quad 9-30-95$ \\
\hline & Sample Identification: & WSRC BG 002 & End Date: \\
\hline
\end{tabular}

\section{Test System}

EPA/600/R-94/024 Method 100.1

The test was set as a Pass/Fail test with a control treatment and one sample treatment. Each treatment replicate consisted of $100 \mathrm{~g}$ of sediment and $175 \mathrm{~mL}$ of dilution water. There were eight replicates for each treatment. Ten test organisms were placed in each replicate. Sediment from a local river was used in the control treatment. Test organisms were fed with YCT food. The test was conducted at a temperature of $23^{\circ} \mathrm{C}$ with ambient laboratory light conditions. Test vessels were $500 \mathrm{~mL}$ plastic beakers placed in a constant temperature incubator room.

\begin{tabular}{|c|c|c|}
\hline \multicolumn{3}{|c|}{ Test Organisms } \\
\hline $\begin{array}{l}\text { Order: Ampl } \\
\text { Species: Hyall } \\
\text { Source: Aqua } \\
\text { Life Stage: } \\
\text { \% Mortality D } \\
\text { Taxonomic Ver } \\
\text { Culturing: }\end{array}$ & $\begin{array}{l}\text { oda } \\
\text { a azteca } \\
\text { Research Organisms, NH } \\
2-3 \mathrm{~mm} \\
\text { ing } 48 \text { Hr Prior to Test:: } \\
\text { ication: RWK } 9 / 29 / 95 \\
\text { as received }\end{array}$ & $0 \%$ \\
\hline $\begin{array}{l}\text { Observations: } \\
\text { Acclimation: }\end{array}$ & $\begin{array}{l}\text { Cultures healthy } \\
\text { None }\end{array}$ & \\
\hline
\end{tabular}

Control Sediment
Source: South Tyger river @ Hwy. 14
Collection Date: 9-19-95
Preservation: kept at $0-4^{\circ} \mathrm{C}$ in plastic containers
Daterime Added to Test Chambers: 9-29-95
Observations: silt
Collection Method: grab
Disposal:

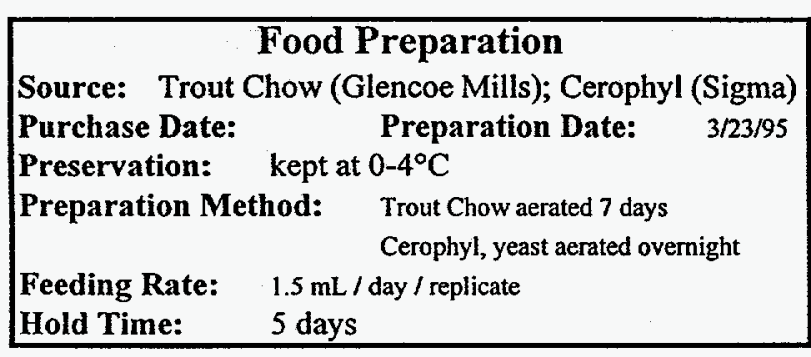

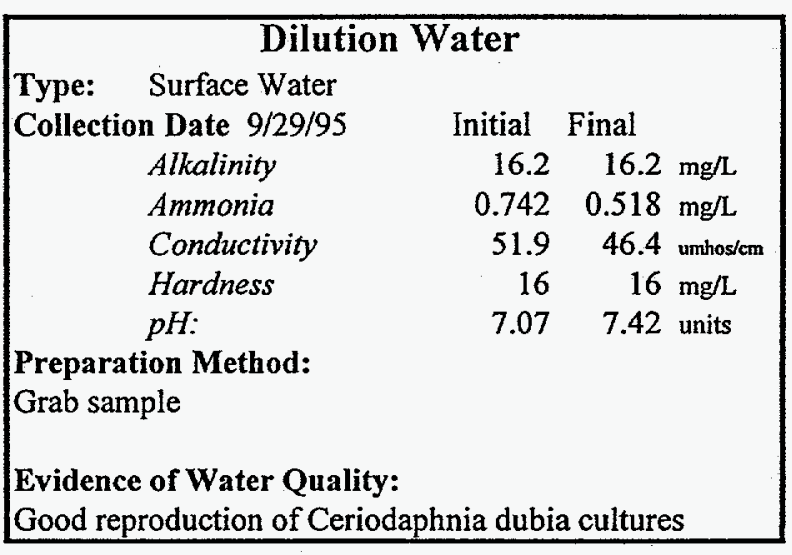

\begin{tabular}{|l|}
\hline \multicolumn{1}{|c|}{ Test Sediment } \\
Source: WSRC BG 002 \\
Collection Date $8 / 22 / 95 \quad$ Homogenized?: yes \\
Preservation: kept at $0-4^{\circ} \mathrm{C}$ in plastic containers \\
Date/Time Added to Test Chambers: $9 / 29 / 95$ \\
Observations: organic \\
Collection Method: grab \\
Shipment: Overnight at $4^{\circ} \mathrm{C}$ \\
\hline
\end{tabular}

\begin{tabular}{|l|}
\multicolumn{1}{c|}{ Test Chambers } \\
Type: Plastic $500 \mathrm{~mL}$ beakers \\
Sediment Volume: $\quad 100 \mathrm{~mL}$ \\
Sediment Depth $4 \mathrm{~cm}$ \\
$\begin{array}{l}\text { Overlying Water Volume: } \quad 175 \mathrm{~mL} \\
\text { Overlying Water Depth: } \quad 5 \mathrm{~cm} \\
\text { Aeration: none }\end{array}$ \\
\hline
\end{tabular}




\section{DAY AMPHIPOD SEDIMENT TOXICITY TEST}

\begin{tabular}{|c|c|c|c|c|}
\hline \multirow{2}{*}{$\begin{array}{l}\text { Lab ID\# } \\
\text { T4442 }\end{array}$} & \multicolumn{2}{|c|}{ Client: $\quad$ Westinghouse Savannah River Company } & Start Date: & $9-30-95$ \\
\hline & Sample Identification: & WSRC BG 002 & End Date: & $10-10-95$ \\
\hline
\end{tabular}

\section{WATER CHEMISTRY TEST METHODS}

Temperature: SM 18th Ed. 2550

Dissolved Oxygen: SM 18th Ed. 4500-O

pH: SM 18th Ed. 4500-H+
Alkalinity: SM 18th Ed. 2320

Hardness: SM 18th Ed. 2340

Conductivity: SM 18th Ed. 2510

WATER CHEMISTRY TEST DATA - OVERLYING WATER

CONTROL TREATMENT

\begin{tabular}{|c|r|r|r|r|}
\hline Test Day & $\begin{array}{c}\text { Temp. } \\
\text { C }\end{array}$ & $\begin{array}{c}\mathrm{pH} \\
\text { units }\end{array}$ & $\begin{array}{c}\text { D.O. } \\
\mathrm{mg} / \mathrm{L}\end{array}$ & $\begin{array}{c}\text { D.O. } \\
\% \text { sat. }\end{array}$ \\
\hline Start & 22.5 & 6.67 & 7.0 & $80 \%$ \\
\hline 1 & 23.2 & & 6.4 & $74 \%$ \\
\hline 2 & 22.6 & & 6.8 & $78 \%$ \\
\hline 3 & 22.7 & & 5.7 & $66 \%$ \\
\hline 4 & 22.9 & & 5.5 & $63 \%$ \\
\hline 5 & 23.1 & & 5.6 & $65 \%$ \\
\hline 6 & 22.2 & & 5.6 & $64 \%$ \\
\hline 7 & 22.3 & & 8.3 & $95 \%$ \\
\hline 8 & 23.5 & & 5.4 & $63 \%$ \\
\hline 9 & 23.7 & & 7.2 & $84 \%$ \\
\hline 10 & 22.0 & 6.38 & 6.2 & $71 \%$ \\
\hline
\end{tabular}

SAMPLE TREATMENT

\begin{tabular}{|c|c|c|c|c|c|c|c|c|}
\hline $\begin{array}{c}\text { Test } \\
\text { Day }\end{array}$ & $\begin{array}{c}\text { Cond. } \\
\text { umhos/cm }\end{array}$ & $\begin{array}{c}\text { NH3 } \\
\mathrm{mg} / \mathrm{L}\end{array}$ & $\begin{array}{c}\text { Temp. } \\
\mathrm{C}\end{array}$ & $\begin{array}{c}\mathrm{pH} \\
\text { units }\end{array}$ & $\begin{array}{c}\text { D.O. } \\
\mathrm{mg} / \mathrm{L}\end{array}$ & $\begin{array}{c}\text { D.O. } \\
\% \text { sat. }\end{array}$ & $\begin{array}{c}\text { Alk. } \\
\mathrm{mg} / \mathrm{L}\end{array}$ & $\begin{array}{c}\text { Hard. } \\
\mathrm{mg} / \mathrm{L}\end{array}$ \\
\hline Start & 39.2 & 0.742 & 22.3 & 5.38 & 6.4 & $74 \%$ & 4.1 & 50.0 \\
\hline 1 & & & 23.4 & & 5.2 & $60 \%$ & & \\
\hline 2 & & & 22.2 & & 7.0 & $80 \%$ & & \\
\hline 3 & & & 22.5 & & 6.5 & $75 \%$ & & \\
\hline 4 & & & 22.9 & & 6.2 & $71 \%$ & & \\
\hline 5 & & & 23.0 & & 5.9 & $69 \%$ & & \\
\hline 6 & & & 22.2 & & 6.0 & $69 \%$ & & \\
\hline 7 & & & 22.1 & & 6.1 & $70 \%$ & & \\
\hline 8 & & & 23.5 & & 6.4 & $74 \%$ & & \\
\hline 9 & & & 23.1 & & 6.8 & $79 \%$ & & \\
\hline 10 & 26 & 0.725 & 22.1 & 5.76 & 6.5 & $75 \%$ & 4.1 & 30.0 \\
\hline
\end{tabular}

SURVIVAL AND GROWTH RESULTS

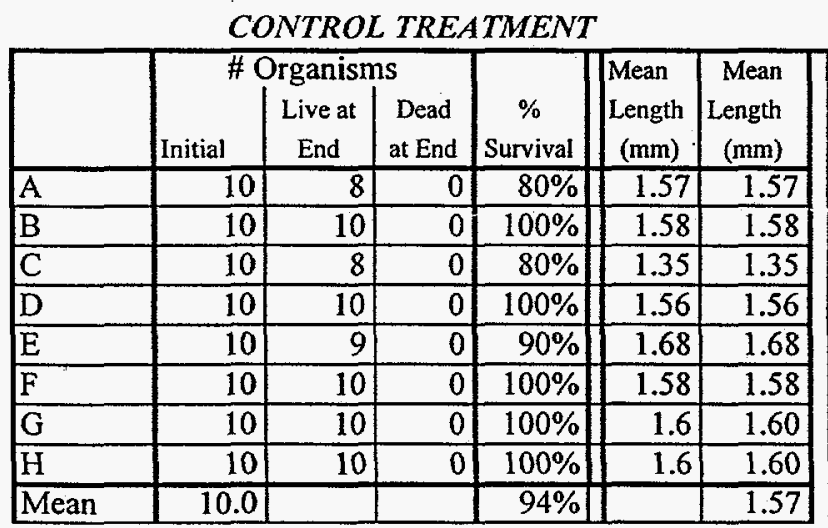

\begin{tabular}{|c|c|c|c|c|c|c|}
\hline & \multicolumn{6}{|c|}{ SAMPLE TREATMENT } \\
\hline & \multicolumn{3}{|c|}{ \# Organisms } & \multirow[b]{2}{*}{$\begin{array}{c}\% \\
\text { Survival } \\
\end{array}$} & \multirow{2}{*}{$\begin{array}{l}\text { Mean } \\
\text { Length } \\
(\mathrm{mm})\end{array}$} & \multirow{2}{*}{$\begin{array}{r}\text { Mean } \\
\text { Length } \\
(\mathrm{mm})\end{array}$} \\
\hline & Initial & $\begin{array}{c}\text { Live at } \\
\text { End }\end{array}$ & $\begin{array}{c}\text { Dead } \\
\text { at End }\end{array}$ & & & \\
\hline $\mathrm{A}$ & 10 & 0 & 0 & $0 \%$ & NA & NA \\
\hline $\mathrm{B}$ & 10 & 0 & 0 & $0 \%$ & $\mathrm{NA}$ & NA \\
\hline $\mathrm{C}$ & 10 & 2 & 2 & $20 \%$ & 1.64 & 1.64 \\
\hline D & 10 & 0 & 0 & $0 \%$ & $\mathrm{NA}$ & NA \\
\hline $\mathrm{E}$ & 10 & 1 & 1 & $10 \%$ & 1.73 & 1.73 \\
\hline $\mathrm{F}$ & 10 & 0 & 2 & $0 \%$ & NA & NA \\
\hline $\mathrm{G}$ & 10 & 0 & 1 & $0 \%$ & $\mathrm{NA}$ & $\mathrm{NA}$ \\
\hline $\mathrm{H}$ & 10 & 0 & 0 & $0 \%$ & NA & $\mathrm{NA}$ \\
\hline Mean & 10.0 & & & $4 \%$ & & 1.69 \\
\hline
\end{tabular}




\section{DAY AMPHIPOD SEDIMENT TOXICITY TEST}

\begin{tabular}{|c|c|c|c|c|}
\hline Lab ID\# & Client: & Nestinghouse Savannah River Company & Start Date: & $9-30-95$ \\
\hline $\mathrm{T} 4442$ & Sample Identification: & WSRC BG 002 & End Date: & $10-10-95$ \\
\hline
\end{tabular}

\section{RESULTS}

\begin{tabular}{|l|r|}
\hline \multicolumn{2}{|c|}{$\begin{array}{c}\text { SURVIVAL DATA } \\
\text { Mean of Eight Replicates }\end{array}$} \\
\cline { 2 - 2 } & Mean \% \\
\hline Control & $93.8 \%$ \\
\hline Sample & $3.8 \%$ \\
\hline
\end{tabular}

\begin{tabular}{|l|c|}
\hline \multicolumn{2}{|c|}{$\begin{array}{c}\text { GROWTH DATA } \\
\text { Mean Length (mm) }\end{array}$} \\
\cline { 2 - 2 } & Mean \\
\hline Control & 1.57 \\
\hline Sample & 1.69 \\
\hline
\end{tabular}

\section{STATISTICAL ANALYSES}

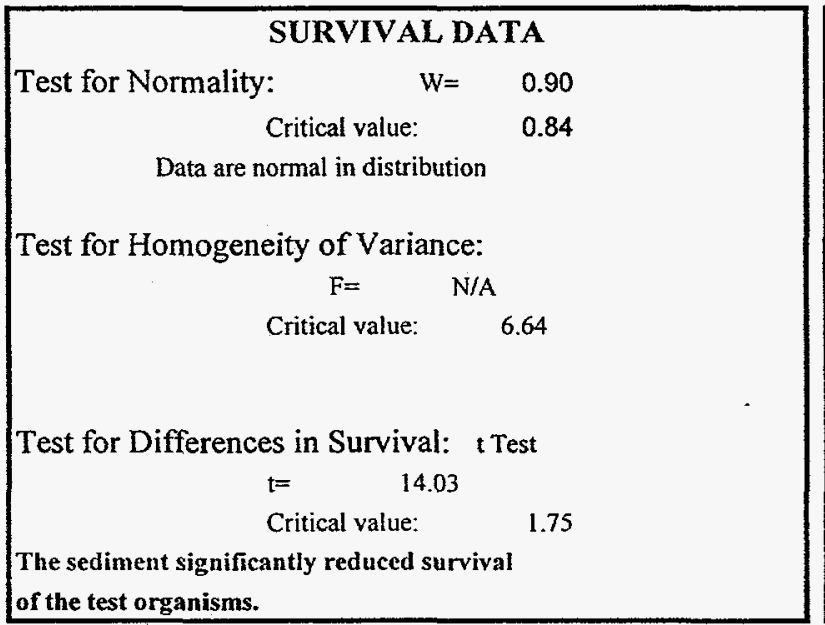

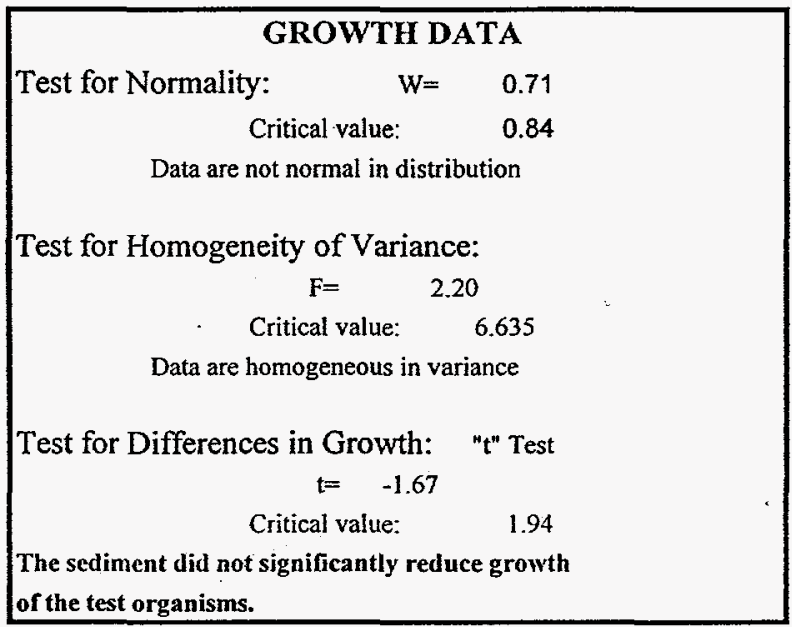

\section{COMMENTS}

\begin{tabular}{|c|c|c|}
\hline $\begin{array}{l}\text { Principal Analyst: } \\
\text { Report Reviewed By: }\end{array}$ & $\begin{array}{l}\text { Aimee Arnold } \\
\text { Robert W. Kelley, Ph.D. }\end{array}$ & 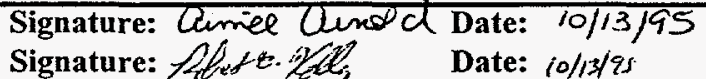 \\
\hline
\end{tabular}

\section{CONCLUSIONS}

The test sediment does adversely affect the survival but not the growth of freshwater amphipods. 


\title{
10 DAY AMPHIPOD SEDIMENT TOXICITY TEST
}

\author{
EPA/600/R-94/024 Method 100.1
}

Test Organism: Hyallela azteca

Client: Westinghouse Savannah River Company Sample Identification: $\quad$ WSRC BG003

Test Start Date:

$8 / 29 / 95$ 


\section{DAY AMPHIPOD SEDIMENT TOXICITY TEST}

\begin{tabular}{|c|c|c|c|c|}
\hline \multirow{2}{*}{$\begin{array}{l}\text { Lab ID } \# \\
\text { T4443 }\end{array}$} & \multicolumn{2}{|c|}{ Westinghouse Savannah River Company } & Start Date: & $8-29-95$ \\
\hline & Sample Identification: & WSRC BG003 & End Date: & $9-8.95$ \\
\hline
\end{tabular}

Test System

EPA/600/R-94/024 Method 100.1

The test was set as a Pass/Fail test with a control treatment and one sample treatment. Each treatment replicate consisted of $100 \mathrm{~g}$ of sediment and $175 \mathrm{~mL}$ of dilution water. There were eight replicates for each treatment. Ten test organisms were placed in each replicate. Sediment from a local creek was used in the control treatment. Test organisms were fed with YCT food. The test was conducted at a temperature of $23^{\circ} \mathrm{C}$ with ambient laboratory light conditions. Test vessels were $500 \mathrm{~mL}$ plastic beakers placed in a constant temperature incubator room.

Test Organisms
Order: Amphipoda
Species: Hyallela azteca
Source: Aquatic Biosystems, CO
Life Stage: $\quad 2-3 \mathrm{~mm} \quad 0 \%$
\% Mortality During $48 \mathrm{Hr}$ Prior to Test::
$\begin{aligned} & \text { Taxonomic Verification: RWK } 8 / 28 / 95 \\ & \text { Culturing: } \quad \text { as received }\end{aligned}$
$\begin{aligned} & \text { Observations: } \quad \text { Cultures healthy } \\ & \text { Acclimation: } \quad \text { None }\end{aligned}$

\section{Control Sediment}

Source: Resurrection Creek (Greer S C)

Collection Date: $8-28-95$

Preservation: kept at $0-4^{\circ} \mathrm{C}$ in plastic containers DaterTime Added to Test Chambers: $\quad 8-28-95$ Observations: organic

Collection Method: grab

- Disposal:

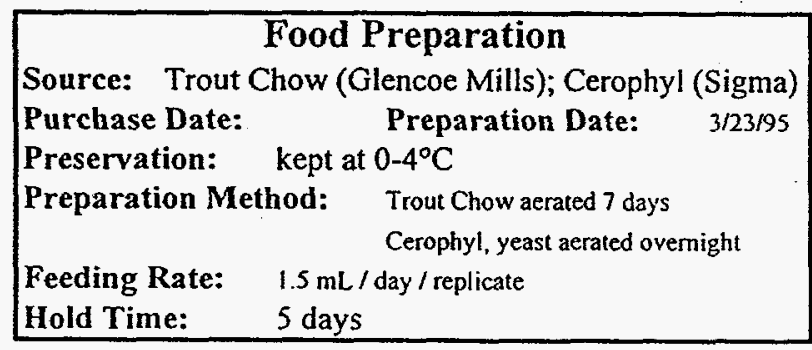

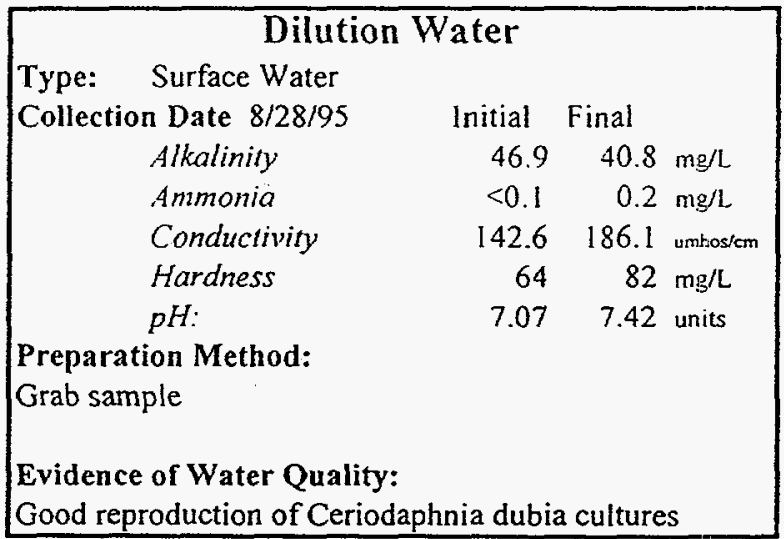

\section{Test Sediment}

Source: WSRC BG003

Collection Date $8 / 22 / 95 \quad$ Homogenized?: yes

Preservation: kept at $0-4^{\circ} \mathrm{C}$ in plastic containers

Date/Time Added to Test Chambers: $8 / 28 / 95$

Observations: organic

Collection Method: grab

Shipment: $\quad$ Overnight at $4^{\circ} \mathrm{C}$

\section{Test Chambers}

Type: Plastic $500 \mathrm{~mL}$ beakers

Sediment Volume: $\quad 100 \mathrm{~mL}$

Sediment Depth $4 \mathrm{~cm}$

Overlying Water Volume: $175 \mathrm{~mL}$

Overlying Water Depth: $\quad 5 \mathrm{~cm}$

Aeration: none 


\section{DAY AMPHIPOD SEDIMENT TOXICITY TEST}

\begin{tabular}{|c|c|c|c|c|}
\hline \multirow{2}{*}{$\begin{array}{l}\text { Lab IDH } \\
\text { T4443 }\end{array}$} & Client: & Vestinghouse Savannah River Company & Start Date: & $8-29-95$ \\
\hline & Sample Identification: & WSRC BG003 & End Date: & $9-8-95$ \\
\hline
\end{tabular}

\section{WATER CHEMISTRY TEST METHODS}

\begin{tabular}{|l|l|}
\hline Temperature: SM 18th Ed. 2550 & Alkalinity: SM 18th Ed. 2320 \\
Dissolved Oxygen: SM 18th Ed. 4500-O & Hardness: SM 18th Ed. 2340 \\
pH: SM 18th Ed. 4500-H+ & Conductivity: SM 18th Ed. 2510 \\
\hline
\end{tabular}

\section{WATER CHEMISTRY TEST DATA - OVERLYING WATER}

CONTROL TREATMENT

\begin{tabular}{|c|r|r|r|r|}
\hline Test Day & $\begin{array}{c}\text { Temp. } \\
\text { C }\end{array}$ & $\begin{array}{c}\mathrm{pH} \\
\text { units }\end{array}$ & $\begin{array}{c}\text { D.O. } \\
\text { mg/L }\end{array}$ & $\begin{array}{c}\text { D.O. } \\
\% \text { sat. }\end{array}$ \\
\hline Start & 23.3 & 7.07 & 7.8 & $91 \%$ \\
\hline 1 & 22.1 & & 7.0 & $80 \%$ \\
\hline 2 & 21.9 & & 6.0 & $67 \%$ \\
\hline 3 & 21.8 & & 5.6 & $63 \%$ \\
\hline 4 & 22.0 & & 5.3 & $61 \%$ \\
\hline 5 & 23.0 & & 5.8 & $67 \%$ \\
\hline 6 & 22.5 & & 5.5 & $63 \%$ \\
\hline 7 & 22.3 & & 5.6 & $64 \%$ \\
\hline 8 & 22.0 & & 5.3 & $61 \%$ \\
\hline 9 & 22.0 & & 5.2 & $60 \%$ \\
\hline 10 & 22.1 & 7.42 & 4.7 & $54 \%$ \\
\hline
\end{tabular}

SAMPLE TREATMENT

\begin{tabular}{|c|c|c|c|c|c|c|c|c|}
\hline $\begin{array}{c}\text { Test } \\
\text { Day }\end{array}$ & $\begin{array}{c}\text { Cond. } \\
\text { umhos/cm }\end{array}$ & $\begin{array}{c}\text { NH3 } \\
\text { mg/L }\end{array}$ & $\begin{array}{c}\text { Temp. } \\
\mathrm{C}\end{array}$ & $\begin{array}{c}\mathrm{pH} \\
\text { units }\end{array}$ & $\begin{array}{c}\text { D.O. } \\
\mathrm{mg} / \mathrm{L}\end{array}$ & $\begin{array}{c}\text { D.O. } \\
\% \text { sat. }\end{array}$ & $\begin{array}{c}\text { Alk. } \\
\mathrm{mg} / \mathrm{L}\end{array}$ & $\begin{array}{c}\text { Hard. } \\
\mathrm{mg} / \mathrm{L} .\end{array}$ \\
\hline Start & 80.5 & 0.586 & 23.1 & 6.43 & 6.2 & $72 \%$ & 16.3 & 280 \\
\hline 1 & & & 22.1 & & 5.9 & $68 \%$ & & \\
\hline 2 & & & 22.0 & & 5.6 & $64 \%$ & & \\
\hline 3 & & & 21.9 & & 5.4 & $61 \%$ & & \\
\hline 4 & & & 22.1 & & 5.6 & $64 \%$ & & \\
\hline 5 & & & 22.6 & & 5.2 & $60 \%$ & & \\
\hline 6 & & & 22.3 & & 5.2 & $60 \%$ & & \\
\hline 7 & & & 22.1 & & 5.0 & $57 \%$ & & \\
\hline 8 & & & 22.1 & & 5.2 & $60 \%$ & & \\
\hline 9 & & & 22.0 & & 5.1 & $59 \%$ & & \\
\hline 10 & 134 & $<0.1$ & 22.2 & 6.94 & 4.9 & $56 \%$ & 42.8 & 54.0 \\
\hline
\end{tabular}

\section{SURVIVAL AND GROWTH RESULTS}

CONTROL TREATMENT

SAMPLE TREATMENT

\begin{tabular}{|c|c|c|c|c|c|c|}
\hline & \multicolumn{3}{|c|}{ \# Organisms } & \multirow[b]{2}{*}{$\begin{array}{c}\% \\
\text { Survival }\end{array}$} & \multirow{2}{*}{\begin{tabular}{|l|} 
Mean \\
Length \\
$(\mathrm{mm})$
\end{tabular}} & \multirow{2}{*}{$\begin{array}{l}\text { Mean } \\
\text { Length } \\
(\mathrm{mm})\end{array}$} \\
\hline & Initial & $\begin{array}{l}\text { Live at } \\
\text { End }\end{array}$ & $\begin{array}{l}\text { Dead } \\
\text { at End }\end{array}$ & & & \\
\hline$A$ & 10 & $\overline{6}$ & 1 & $60 \%$ & 2.4 & 2.40 \\
\hline B & 10 & $\overline{N A}$ & $\mathrm{NA}$ & $\mathrm{NA}$ & $\mathrm{NA}$ & $\mathrm{NA}$ \\
\hline C & 10 & 7 & 0 & $70 \%$ & 2.18 & 2.18 \\
\hline$D$ & 10 & 7 & 0 & $70 \%$ & 2.18 & 2.18 \\
\hline$E$ & 10 & 10 & 0 & $100 \%$ & 2.17 & 2.17 \\
\hline $\mathrm{F}$ & 10 & 8 & 2 & $80 \%$ & 2.12 & 2.12 \\
\hline$G$ & 10 & 9 & 0 & $90 \%$ & 2.13 & 2.13 \\
\hline $\mathrm{H}$ & 10 & 9 & 0 & $90 \%$ & 2.2 & 2.15 \\
\hline Mean & 10.0 & & & $80 \%$ & & 2.19 \\
\hline
\end{tabular}

\begin{tabular}{|c|c|c|c|c|c|c|}
\hline & \multicolumn{3}{|c|}{ \# Organisms } & \multirow[b]{2}{*}{$\begin{array}{c}\% \\
\text { Survival }\end{array}$} & \multirow{2}{*}{\begin{tabular}{|l|} 
Mean \\
Length \\
$(\mathrm{mm})$
\end{tabular}} & \multirow{2}{*}{$\begin{array}{r}\text { Mean } \\
\text { Length } \\
(\mathrm{mm})\end{array}$} \\
\hline & Initial & $\begin{array}{c}\text { Live at } \\
\text { End }\end{array}$ & $\begin{array}{l}\text { Dead } \\
\text { at End }\end{array}$ & & & \\
\hline $\mathrm{A}$ & 10 & 8 & 0 & $80 \%$ & 2.23 & 2.23 \\
\hline $\bar{B}$ & 10 & 8 & 1 & $80 \%$ & 2.39 & 2.39 \\
\hline C & 10 & 8 & 2 & $80 \%$ & 2.26 & 2.26 \\
\hline D & 10 & $\overline{6}$ & 0 & $60 \%$ & 2.51 & 2.51 \\
\hline $\mathrm{E}$ & 10 & 7 & 0 & $70 \%$ & 2.27 & 2.27 \\
\hline $\mathrm{F}$ & 10 & 6 & 0 & $60 \%$ & 2.33 & 2.33 \\
\hline $\mathrm{G}$ & 10 & 6 & 0 & $60 \%$ & 2.33 & 2.33 \\
\hline $\mathrm{H}$ & 10 & 5 & 0 & $50 \%$ & 2.4 & 2.37 \\
\hline Mean & 10.0 & & & $68 \%$ & & 2.34 \\
\hline
\end{tabular}




\section{DAY AMPHIPOD SEDIMENT TOXICITY TEST}

\begin{tabular}{|l|l|l|l|}
\hline $\begin{array}{l}\text { Lab IDH } \\
\text { T4443 }\end{array}$ & Client: $\quad$ Westinghouse Savannah River Company & Start Date: & $8-29-95$ \\
\hline
\end{tabular}

\section{RESULTS}

\begin{tabular}{|l|r|}
\hline \multicolumn{2}{|c|}{$\begin{array}{c}\text { SURVIVAL DATA } \\
\text { Mean of Eight Replicates }\end{array}$} \\
\cline { 2 - 2 } & Mean $\%$ \\
\hline Control & $80.0 \%$ \\
\hline Sample & $67.5 \%$ \\
\hline
\end{tabular}

\begin{tabular}{|l|r|}
\hline \multicolumn{1}{|c|}{$\begin{array}{r}\text { GROWTH DATA } \\
\text { Mean Length }(\mathrm{mm})\end{array}$} \\
\hline Control & Mean \\
\hline Sample & 2.19 \\
\hline
\end{tabular}

\section{STATISTICAL ANALYSES}

SURVIVAL DATA
$\begin{gathered}\text { Test for Normality: } \quad W=\quad 0.95 \\ \text { Critical value. }\end{gathered} \quad 0.84$
Data are normal in distribution
Test for Homogeneity of Variance:
\[ F \quad \text { Critical value: } \quad 6.64 \]
Test for Differences in Survival: $\quad$ T Test
$\quad$ Critical value:
The sediment significantly reduced survival
of the test organisms.

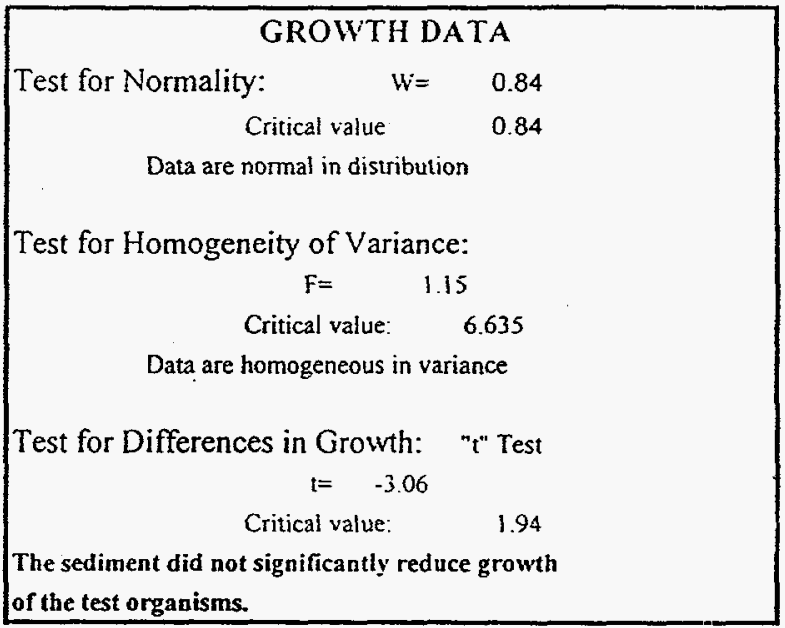

\section{COMMENTS}

\begin{tabular}{|c|c|c|c|}
\hline $\begin{array}{l}\text { Principal Analyst: } \\
\text { Report Reviewed By: }\end{array}$ & $\begin{array}{l}\text { Aimee Arnold } \\
\text { Robert W. Kelley, Ph.D. }\end{array}$ & 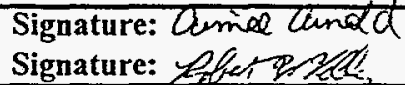 & $\begin{array}{l}\text { Date: } 10 / 13 / 95 \\
\text { Date: } 10 / 13 / 7 ;\end{array}$ \\
\hline
\end{tabular}

\section{CONCLUSIONS}

The test sediment does adversely affect the survival but not the growth of freshwater amphipods. 
(803) 877-6942 - FAX (803) 877-6938

P.O. Box 16414, Greenville, SC 29606 - 4 Craftsman Court, Greer, SC 29650

\title{
10 DAY AMPHIPOD SEDIMENT TOXICITY TEST
}

\author{
EPA/600/R-94/024 Method 100.1 \\ Test Organism: Hyallela azteca
}

Client: Westinghouse Savannah River Company

Sample Identification: WSRC FHB 006

Test Start Date: $\quad 9-30-95$ 


\section{DAY AMPHIPOD SEDIMENT TOXICITY TEST}

\begin{tabular}{|l|l|ll|}
\hline Lab ID\# & Client: $\quad$ Westinghouse Savannah River Company & Start Date: & $9-30-95$ \\
\cline { 2 - 4 } T4451 & Sample Identification: $\quad$ WSRC FHB 006 & End Date: & $10-10-95$ \\
\hline
\end{tabular}

\section{Test System}

EPA/600/R-94/024 Method 100.1

The test was set as a Pass/Fail test with a control treatment and one sample treatment. Each treatment replicate consisted of $100 \mathrm{~g}$ of sediment and $175 \mathrm{~mL}$ of dilution water. There were eight replicates for each treatment. Ten test organisms were placed in each replicate. Sediment from a local river was used in the control treatment. Test organisms were fed with YCT food. The test was conducted at a temperature of $23^{\circ} \mathrm{C}$ with ambient laboratory light conditions. Test vessels were $500 \mathrm{~mL}$ plastic beakers placed in a constant temperature incubator room.

\begin{tabular}{|c|c|c|}
\hline \multicolumn{3}{|c|}{ Test Organisms } \\
\hline \multirow{3}{*}{\multicolumn{3}{|c|}{$\begin{array}{ll}\text { Order: } & \text { Amphipoda } \\
\text { Species: } & \text { Hyallela azteca } \\
\text { Source: } & \text { Aquatic Research Organisms, NH }\end{array}$}} \\
\hline & & \\
\hline & & \\
\hline \multirow{2}{*}{\multicolumn{2}{|c|}{$\begin{array}{l}\text { Life Stage: } \quad 2-3 \mathrm{~mm} \\
\% \text { Mortality During } 48 \text { Hr Prior to Test:: } \\
\text { Taxonomic Verification: RWK 9/29/95 }\end{array}$}} & \\
\hline & & $0 \%$ \\
\hline Culturing: & as received & \\
\hline Observations: & Cultures healthy & \\
\hline Acclimation: & None & \\
\hline
\end{tabular}

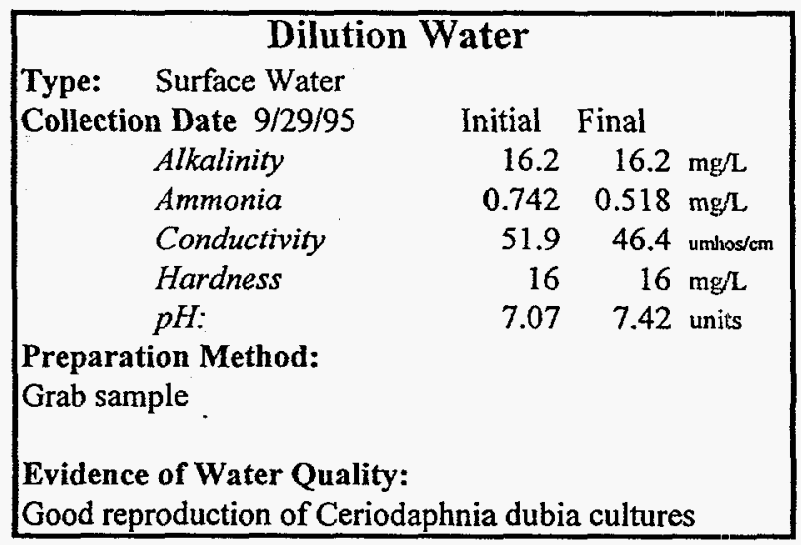

\section{Control Sediment}

Source: South Tyger river@ Hwy. 14

Collection Date: 9-19-95

Preservation: kept at $0-4^{\circ} \mathrm{C}$ in plastic containers

Date/Time Added to Test Chambers: $\quad 9-29-95$

Observations: silt

Collection Method: grab

Disposal:

\section{Test Sediment}

Source: WSRC FHB 006

Collection Date 8/22/95 Homogenized?: yes

Preservation: kept at $0-4^{\circ} \mathrm{C}$ in plastic containers

Date/Time Added to Test Chambers: 9/29/95

Observations: organic

Collection Method: grab

Shipment: $\quad$ Overnight at $4^{\circ} \mathrm{C}$

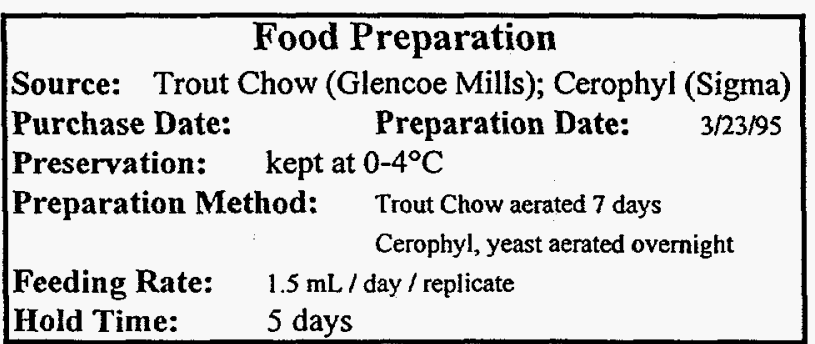

\section{Test Chambers}

Type: Plastic $500 \mathrm{~mL}$ beakers

Sediment Volume: $\quad 100 \mathrm{~mL}$

Sediment Depth $4 \mathrm{~cm}$

Overlying Water Volume: $175 \mathrm{~mL}$

Overlying Water Depth: $\quad 5 \mathrm{~cm}$

Aeration: none 


\section{DAY AMPHIPOD SEDIMENT TOXICITY TEST}

\begin{tabular}{|l|l|ll|}
\hline \multirow{2}{*}{$\begin{array}{l}\text { Tab ID\# } \\
\text { T4451 }\end{array}$} & Client: $\quad$ Westinghouse Savannah River Company & Start Date: & 9-30-95 \\
\cline { 2 - 4 } & Sample Identification: $\quad$ WSRC FHB 006 & End Date: & $10-10-95$ \\
\hline
\end{tabular}

\section{WATER CHEMISTRY TEST METHODS}

\begin{tabular}{|l|l|}
\hline Temperature: SM 18th Ed. 2550 \\
Dissolved Oxygen: SM 18th Ed. 4500-O & Alkalinity: SM 18th Ed. 2320 \\
pH: SM 18th Ed. 4500-H+ & Hardness: SM 18th Ed. 2340 \\
\hline
\end{tabular}

WATER CHEMISTRY TEST DATA - OVERLYING WATER

CONTROL TREATMENT

\begin{tabular}{|c|c|c|c|c|c|c|c|c|c|c|c|c|c|}
\hline Test Day & $\begin{array}{c}\text { Temp. } \\
\text { C } \\
\end{array}$ & $\begin{array}{c}\mathrm{pH} \\
\text { units }\end{array}$ & $\begin{array}{l}\text { D.o. } \\
\mathrm{mg} / \mathrm{L}\end{array}$ & $\begin{array}{c}\text { D.o. } \\
\text { \% sat. }\end{array}$ & $\begin{array}{l}\text { Test } \\
\text { Day } \\
\end{array}$ & $\begin{array}{c}\text { Cond. } \\
\text { umhos } / \mathrm{cm}\end{array}$ & $\begin{array}{l}\mathrm{NH3} \\
\mathrm{mg} / \mathrm{L}\end{array}$ & $\begin{array}{c}\text { Temp. } \\
\text { C } \\
\end{array}$ & $\begin{array}{c}\mathrm{pH} \\
\text { units } \\
\end{array}$ & $\begin{array}{l}\text { D.O. } \\
\mathrm{mg} / \mathrm{L}\end{array}$ & $\begin{array}{l}\text { D.o. } \\
\text { \% sat. }\end{array}$ & $\begin{array}{c}\text { Alk. } \\
\mathrm{mg} / \mathrm{L}\end{array}$ & $\begin{array}{l}\text { Hard. } \\
\mathrm{mg} / \mathrm{L}\end{array}$ \\
\hline Start & 22.5 & 6.67 & 7.0 & $80 \%$ & Start & 28 & 0.378 & $\overline{22.2}$ & 6.63 & 7.6 & $87 \%$ & 4.1 & 46.0 \\
\hline 1 & 23.2 & & 6.4 & $74 \%$ & 1 & & & 22.3 & & 7.8 & $90 \%$ & & \\
\hline 2 & 22.6 & & 6.8 & $78 \%$ & 2 & & & 23.0 & & 6.4 & $74 \%$ & & \\
\hline 3 & 22.7 & & 5.7 & $66 \%$ & 3 & & & 22.3 & & 5.2 & $60 \%$ & & \\
\hline 4 & 22.9 & & 5.5 & $63 \%$ & 4 & & & 22.9 & & 6.2 & $71 \%$ & & \\
\hline 5 & 23.1 & & 5.6 & $65 \%$ & 5 & & & 22.9 & & 5.8 & $67 \%$ & & \\
\hline 6 & 22.2 & & 5.6 & $64 \%$ & 6 & & & 22.1 & & 6.7 & $77 \%$ & & \\
\hline 7 & 22.3 & & 8.3 & $95 \%$ & 7 & & & 22.2 & & 6.5 & $75 \%$ & & \\
\hline 8 & 23.5 & & 5.4 & $63 \%$ & 8 & & & 23.3 & & 6.7 & $78 \%$ & & \\
\hline 9 & 23.7 & & 7.2 & $84 \%$ & 9 & & & 23.1 & & 7.2 & $84 \%$ & & \\
\hline 10 & 22.0 & 6.38 & 6.2 & $71 \%$ & 10 & 23.9 & 0.641 & 22.1 & 6.08 & 6.7 & $77 \%$ & 6.1 & 50.0 \\
\hline
\end{tabular}

SURVIVAL AND GROWTH RESULTS

CONTROL TREATMENT

SAMPLE TREATMENT

\begin{tabular}{|c|c|c|c|c|c|c|}
\hline & \multicolumn{3}{|c|}{ \# Organisms } & \multirow[b]{2}{*}{$\begin{array}{c}\% \\
\text { Survival }\end{array}$} & \multirow{2}{*}{$\begin{array}{l}\text { Mean } \\
\text { Length } \\
\text { (mm) }\end{array}$} & \multirow{2}{*}{$\begin{array}{l}\text { Mean } \\
\text { Length } \\
(\mathrm{mm})\end{array}$} \\
\hline & Initial & $\begin{array}{l}\text { Live at } \\
\text { End }\end{array}$ & $\begin{array}{l}\text { Dead } \\
\text { at End }\end{array}$ & & & \\
\hline $\bar{A}$ & 10 & 8 & $\overline{0}$ & $80 \%$ & 1.57 & 1.57 \\
\hline$B$ & 10 & 10 & 0 & $100 \%$ & 1.58 & 1.58 \\
\hline IC & 10 & 8 & $\overline{0}$ & $80 \%$ & 1.35 & 1.35 \\
\hline $\bar{D}$ & 10 & 10 & $\overline{0}$ & $100 \%$ & 1.56 & 1.56 \\
\hline$E$ & 10 & 9 & $\overline{0}$ & $90 \%$ & 1.68 & 1.68 \\
\hline$F$ & 10 & 10 & 0 & $100 \%$ & 1.58 & 1.58 \\
\hline$G$ & 10 & 10 & 0 & $100 \%$ & 1.6 & 1.60 \\
\hline$\overline{\mathrm{H}}$ & 10 & 10 & 0 & $100 \%$ & 1.6 & 1.60 \\
\hline Mean & 10.0 & & & $94 \%$ & & 1.57 \\
\hline
\end{tabular}

\begin{tabular}{|c|c|c|c|c|c|c|}
\hline & \multicolumn{3}{|c|}{ \# Organisms } & \multirow[b]{2}{*}{$\begin{array}{c}\% \\
\text { Survival }\end{array}$} & \multirow{2}{*}{\begin{tabular}{|l|} 
Mean \\
Length \\
$(\mathrm{mm})$
\end{tabular}} & \multirow{2}{*}{\begin{tabular}{|} 
Mean \\
Length \\
$(\mathrm{mm})$
\end{tabular}} \\
\hline & Initial & $\begin{array}{c}\text { Live at } \\
\text { End }\end{array}$ & $\begin{array}{l}\text { Dead } \\
\text { at End }\end{array}$ & & & \\
\hline $\mathrm{A}$ & 10 & 0 & 0 & $0 \%$ & 1.62 & 1.62 \\
\hline $\mathrm{B}$ & 10 & 1 & 1 & $10 \%$ & 1.51 & 1.51 \\
\hline $\mathrm{C}$ & 10 & 0 & 1 & $0 \%$ & 1.51 & 1.51 \\
\hline $\bar{D}$ & 10 & 1 & 0 & $10 \%$ & 1.59 & 1.59 \\
\hline $\mathrm{E}$ & 10 & 0 & 1 & $0 \%$ & 1.58 & 1.58 \\
\hline$F$ & 10 & 0 & 1 & $0 \%$ & 1.59 & 1.59 \\
\hline$G$ & 10 & 0 & 0 & $0 \%$ & 1.67 & 1.67 \\
\hline $\mathrm{H}$ & 10 & 0 & 5 & $0 \%$ & 1.29 & 1.29 \\
\hline Mea & 10.0 & & & $3 \%$ & & 1.55 \\
\hline
\end{tabular}




\section{DAY AMPHIPOD SEDIMENT TOXICITY TEST}

\begin{tabular}{|c|c|c|c|c|}
\hline \multirow{2}{*}{$\begin{array}{l}\text { Lab ID\# } \\
\text { T4451 }\end{array}$} & Client: & ghouse Savannah River Company & Start Date: & $9-30-95$ \\
\hline & Sample Identification: & WSRC FHB 006 & End Date: & $10-10-95$ \\
\hline
\end{tabular}

\section{RESULTS}

\begin{tabular}{|l|r|}
\hline \multicolumn{2}{|c|}{$\begin{array}{c}\text { SURVIVAL DATA } \\
\text { Mean of Eight Replicates }\end{array}$} \\
\cline { 2 - 2 } & Mean \% \\
\hline Control & $93.8 \%$ \\
\hline Sample & $2.5 \%$ \\
\hline
\end{tabular}

\begin{tabular}{|c|c|c|}
\hline & \multicolumn{2}{|c|}{ GROWTH DATA } \\
\hline & & Mean \\
\hline Control & & 1.57 \\
\hline Sample & & 1.55 \\
\hline
\end{tabular}

\section{STATISTICAL ANALYSES}

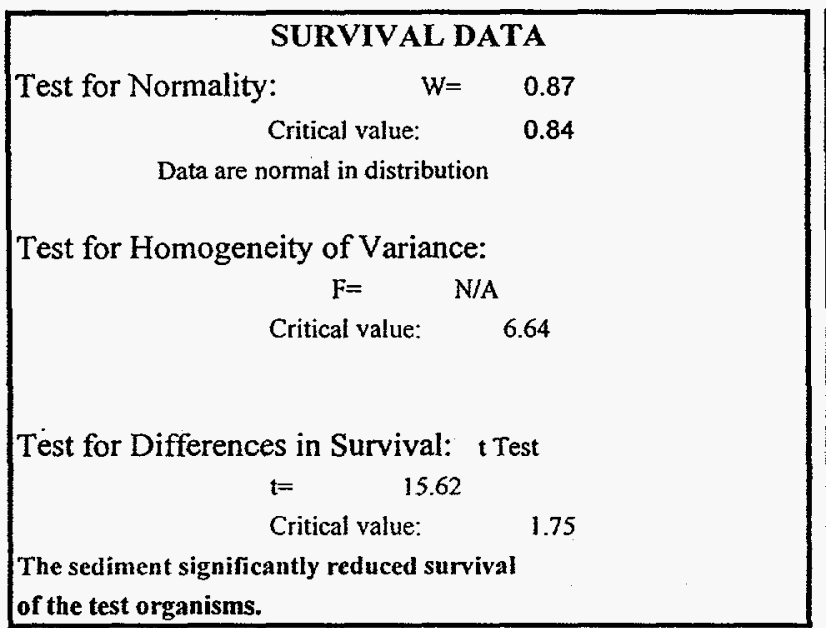

\begin{tabular}{|c|c|}
\hline \multicolumn{2}{|c|}{ GROWTH DATA } \\
\hline Test for Normality: & 0.81 \\
\hline Critical value: & 0.84 \\
\hline \multicolumn{2}{|c|}{ Data are not normal in distribution } \\
\hline \multicolumn{2}{|l|}{ Test for Homogeneity of Variance: } \\
\hline \multicolumn{2}{|c|}{1.51} \\
\hline \multirow{2}{*}{\multicolumn{2}{|c|}{$\begin{array}{l}\text { Critical value: } \\
\text { Data are homogeneous in variance }\end{array}$}} \\
\hline & \\
\hline Test for Differences in Growth: & $t=0.38$ \\
\hline Critical value: & 1.94 \\
\hline $\begin{array}{l}\text { The sediment did not significantly red } \\
\text { of the test organisms. }\end{array}$ & growth \\
\hline
\end{tabular}

\section{COMMENTS}

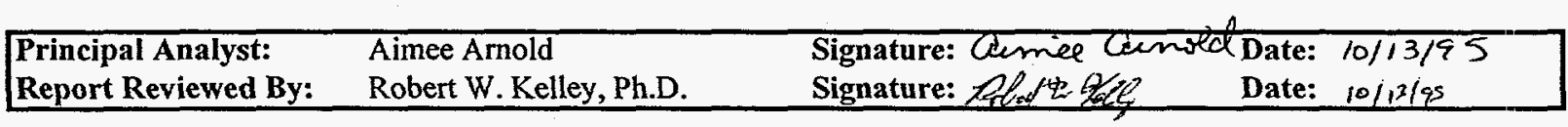

\section{CONCLUSIONS}

The test sediment does adversely affect the survival but not the growth of freshwater amphipods. 


\title{
10 DAY AMPHIPOD SEDIMENT TOXICITY TEST
}

\author{
EPA/600/R-94/024 Method 100.1 \\ Test Organism: Hyallela azteca
}

Client: Westinghouse Savannah River Company Sample Identification: WSRC FHB 012

Test Start Date: $\quad 9-19-95$ 


\section{DAY AMPHIPOD SEDIMENT TOXICITY TEST}

\begin{tabular}{|l|l|ll|}
\hline Lab ID & Client: & Start Date: & $9-19-95$ \\
\cline { 2 - 5 } T4503 & Sample Identification: $\quad$ WSRC FHB 012 & End Date: & $9-29-95$ \\
\hline
\end{tabular}

\section{Test System \\ EPA/600/R-94/024 Method 100.1}

The test was set as a Pass/Fail test with a control treatment and one sample treatment. Each treatment replicate consisted of $100 \mathrm{~g}$ of sediment and $175 \mathrm{~mL}$ of dilution water. There were eight replicates for each treatment. Ten test organisms were placed in each replicate. Sediment from a local creek was used in the control treatment. Test. organisms were fed with YCT food. The test was conducted at a temperature of $23^{\circ} \mathrm{C}$ with ambient laboratory light conditions. Test vessels were $500 \mathrm{~mL}$ plastic beakers placed in a constant temperature incubator room.

Order: Amphipoda

\section{Test Organisms}

Species: Hyallela azteca

Source: Aquatic Biosystems, $\mathrm{CO}$

Life Stage: $\quad 2-3 \mathrm{~mm}$

$\%$ Mortality During $48 \mathrm{Hr}$ Prior to Test::

Taxonomic Verification: RWK 9/19/95

Culturing: as received

Observations: Cultures healthy

Acclimation: None

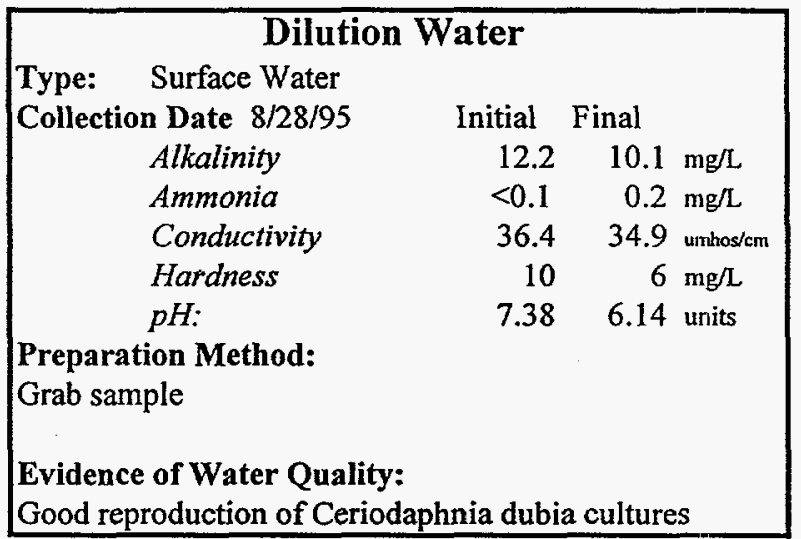

\section{Control Sediment}

Source: S. Tyger River at Hwy. 14

Collection Date: 9-19-95

Preservation: kept at $0-4^{\circ} \mathrm{C}$ in plastic containers

Date/Time Added to Test Chambers: $\quad$ 9-19-95

Observations: organic

Collection Method: grab

Disposal:

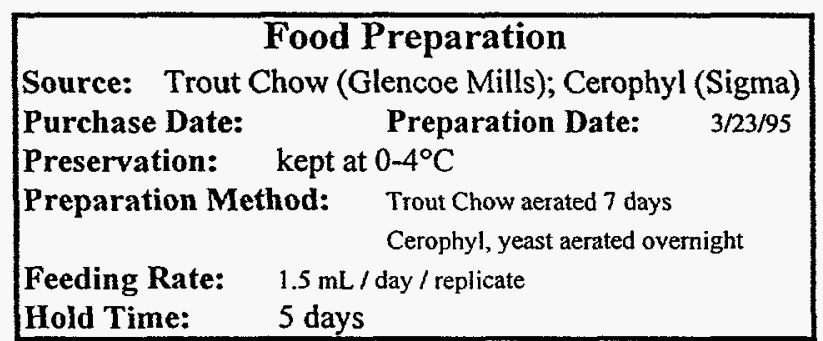

\section{Test Sediment}

Source: WSRC FHB 012

Collection Date 9/11/95 Homogenized?: yes

Preservation: kept at $0-4^{\circ} \mathrm{C}$ in plastic containers

Date/Time Added to Test Chambers: 9/18/95

Observations: organic

Collection Method: grab

Shipment: $\quad$ Overnight at $4^{\circ} \mathrm{C}$

\section{Test Chambers}

Type: Plastic $500 \mathrm{~mL}$ beakers

Sediment Volume: $\quad 100 \mathrm{~mL}$

Sediment Depth $4 \mathrm{~cm}$

Overlying Water Volume: $\quad 175 \mathrm{~mL}$

Overlying Water Depth: $\quad 5 \mathrm{~cm}$

Aeration: . none 


\section{DAY AMPHIPOD SEDIMENT TOXICITY TEST}

\begin{tabular}{|l|l|ll|}
\hline Lab ID\# & Client: $\quad$ Westinghouse Savannah River Company & Start Date: & 9-19-95 \\
\cline { 2 - 5 } T4503 & Sample Identification: WSRC FHB 012 & End Date: & $9-29-95$ \\
\hline
\end{tabular}

\section{WATER CHEMISTRY TEST METHODS}

\begin{tabular}{|l|l|}
\hline Temperature: SM 18th Ed. 2550 & Alkalinity: SM 18th Ed. 2320 \\
Dissolved Oxygen: SM 18th Ed. 4500-O & Hardness: SM 18th Ed. 2340 \\
pH: SM 18th Ed. 4500-H+ & Conductivity: SM 18th Ed. 2510 \\
\hline
\end{tabular}

\section{WATER CHEMISTRY TEST DATA - OVERLYING WATER}

CONTROL TREATMENT

\begin{tabular}{|c|r|r|r|r|}
\hline Test Day & $\begin{array}{c}\text { Temp. } \\
\text { C }\end{array}$ & $\begin{array}{c}\mathrm{pH} \\
\text { units }\end{array}$ & $\begin{array}{c}\text { D.O. } \\
\mathrm{mg} / \mathrm{L}\end{array}$ & $\begin{array}{c}\text { D.O. } \\
\% \text { sat. }\end{array}$ \\
\hline Start & 22.8 & 7.38 & 7.8 & $90 \%$ \\
\hline 1 & 22.5 & & 6.9 & $79 \%$ \\
\hline 2 & 22.7 & & 6.6 & $76 \%$ \\
\hline 3 & 22.4 & & 7.2 & $83 \%$ \\
\hline 4 & 22.1 & & .8 .1 & $93 \%$ \\
\hline 5 & 23.2 & & 8.0 & $93 \%$ \\
\hline 6 & 22.2 & & 6.8 & $78 \%$ \\
\hline 7 & 23.9 & & 5.9 & $69 \%$ \\
\hline 8 & 22.0 & & 6.4 & $74 \%$ \\
\hline 9 & 22.2 & & 6.2 & $71 \%$ \\
\hline 10 & 22.1 & 6.14 & 6.2 & $71 \%$ \\
\hline
\end{tabular}

SAMPLE TREATMENT

\begin{tabular}{|c|c|c|c|c|c|c|c|c|}
\hline $\begin{array}{c}\text { Test } \\
\text { Day }\end{array}$ & $\begin{array}{c}\text { Cond. } \\
\text { umhos/cm }\end{array}$ & $\begin{array}{c}\text { NH3 } \\
\mathrm{mg} / \mathrm{L}\end{array}$ & $\begin{array}{c}\text { Temp. } \\
\mathrm{C}\end{array}$ & $\begin{array}{c}\mathrm{pH} \\
\text { units }\end{array}$ & $\begin{array}{c}\text { D.O. } \\
\mathrm{mg} / \mathrm{L}\end{array}$ & $\begin{array}{c}\text { D.O. } \\
\% \text { sat. }\end{array}$ & $\begin{array}{c}\text { Alk. } \\
\mathrm{mg} / \mathrm{L}\end{array}$ & $\begin{array}{c}\text { Hard. } \\
\mathrm{mg} / \mathrm{L}\end{array}$ \\
\hline Start & 30.4 & 0.675 & 22.2 & 6.31 & 8.4 & $97 \%$ & 6.1 & 4.0 \\
\hline 1 & & & 22.4 & & 8.4 & $97 \%$ & & \\
\hline 2 & & & 22.6 & & 7.2 & $83 \%$ & & \\
\hline 3 & & & 22.5 & & 7.4 & $85 \%$ & & \\
\hline 4 & & & 22.3 & & 7.9 & $91 \%$ & & \\
\hline 5 & & & 23.2 & & 7.6 & $88 \%$ & & \\
\hline 6 & & & 22.3 & & 7.2 & $83 \%$ & & \\
\hline 7 & & & 23.9 & & 6.4 & $74 \%$ & & \\
\hline 8 & & & 22.0 & & 7.0 & $80 \%$ & & \\
\hline 9 & & & 22.4 & & 7.2 & $83 \%$ & & \\
\hline 10 & 37 & 0.166 & 22.1 & 6.10 & 6.4 & $74 \%$ & 12.9 & 8.0 \\
\hline
\end{tabular}

\section{SURVIVAL AND GROWTH RESULTS}

\begin{tabular}{|l|r|r|r|r|r|r|}
\hline & \multicolumn{7}{|c|}{ \# Organisms } & & Mean & $\begin{array}{r}\text { Mean } \\
\text { Length } \\
\text { Length } \\
\text { Initial }\end{array}$ & $\begin{array}{r}\text { Live at } \\
\text { End }\end{array}$ & $\begin{array}{c}\text { Dead } \\
\text { at End }\end{array}$ & $\begin{array}{c}\% \\
\text { Survival }\end{array}$ & $\begin{array}{r}\text { (mm) } \\
(\mathrm{mm})\end{array}$ \\
\hline $\mathrm{A}$ & 10 & 9 & 0 & $90 \%$ & 2.1 & 2.10 \\
\hline $\mathrm{B}$ & 10 & 10 & 0 & $100 \%$ & 2.04 & 2.04 \\
\hline $\mathrm{C}$ & 10 & 9 & 0 & $90 \%$ & 2.04 & 2.04 \\
\hline $\mathrm{D}$ & 10 & 10 & 0 & $100 \%$ & 2.02 & 2.02 \\
\hline $\mathrm{E}$ & 10 & 9 & 0 & $90 \%$ & 2.04 & 2.04 \\
\hline $\mathrm{F}$ & 10 & 10 & 0 & $100 \%$ & 2 & 2.00 \\
\hline $\mathrm{G}$ & 10 & 10 & 0 & $100 \%$ & 2.04 & 2.04 \\
\hline H & 10 & 10 & 0 & $100 \%$ & 2.1 & 2.09 \\
\hline Mean & 10.0 & & & $96 \%$ & & 2.05 \\
\hline
\end{tabular}

\begin{tabular}{|l|r|r|r|r|r|r|}
\hline & \multicolumn{7}{|c|}{ SAMPLE TREATMENT } \\
\hline & \multicolumn{2}{|c|}{$\begin{array}{c}\text { Organisms } \\
\text { End }\end{array}$} & $\begin{array}{c}\text { Dead } \\
\text { at End }\end{array}$ & $\begin{array}{c}\text { \% } \\
\text { Survival }\end{array}$ & $\begin{array}{r}\text { Leangth } \\
\text { (mm) }\end{array}$ & $\begin{array}{r}\text { Mean } \\
\text { Length } \\
\text { (mm) }\end{array}$ \\
\hline A & 10 & 10 & 0 & $100 \%$ & 2.32 & 2.32 \\
\hline B & 10 & 10 & 0 & $100 \%$ & 2.17 & 2.17 \\
\hline C & 10 & 9 & 0 & $90 \%$ & 2.22 & 2.22 \\
\hline D & 10 & 10 & 0 & $100 \%$ & 2.3 & 2.30 \\
\hline E & 10 & 8 & 0 & $80 \%$ & 2.23 & 2.23 \\
\hline F & 10 & 10 & 0 & $100 \%$ & 1.96 & 1.96 \\
\hline G & 10 & 9 & 0 & $90 \%$ & 1.82 & 1.82 \\
\hline H & 10 & 10 & 0 & $100 \%$ & 2.0 & 1.98 \\
\hline Mean & 10.0 & & & $95 \%$ & & 2.13 \\
\hline
\end{tabular}


Test Organism: Hyallela azteca

Client: Westinghouse Savannah River Company Sample Identification: WSRC FHB 013

Test Start Date: 9-19-95 


\section{DAY AMPHIPOD SEDIMENT TOXICITY TEST}

\begin{tabular}{|c|c|c|c|c|}
\hline \multirow{2}{*}{$\begin{array}{l}\text { Lab ID\# } \\
\text { T4504 }\end{array}$} & Client: & ighouse Savannah River Company & Start Date: & $9-19-95$ \\
\hline & Sample Identification: & WSRC FHB 013 & End Date: & $9-29-95$ \\
\hline
\end{tabular}

Test System

EPA/600/R-94/024 Method 100.1

The test was set as a Pass/Fail test with a control treatment and one sample treatment. Each treatment replicate consisted of $100 \mathrm{~g}$ of sediment and $175 \mathrm{~mL}$ of dilution water. There were eight replicates for each treatment. Ten. test organisms were placed in each replicate. Sediment from a local creek was used in the control treatment. Test organisms were fed with YCT food. The test was conducted at a temperature of $23^{\circ} \mathrm{C}$ with ambient laboratory light conditions. Test vessels were $500 \mathrm{~mL}$ plastic beakers placed in a constant temperature incubator room.

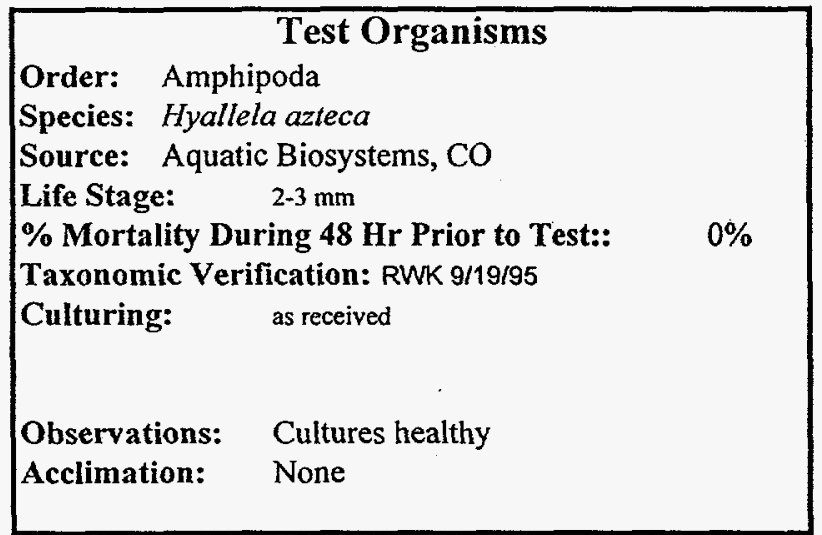

\section{Control Sediment}

Source: S. Tyger River at Hwy. 14

Collection Date: 9-19-95

Preservation: kept at $0-4^{\circ} \mathrm{C}$ in plastic containers

Date/Time Added to Test Chambers: $\quad$ 9-19-95

Observations: organic

Collection Method: grab

Disposal:

\begin{tabular}{|c|c|}
\hline \multicolumn{2}{|c|}{ Food Preparation } \\
\hline $\begin{array}{l}\text { Purchase Date: } \\
\text { Preservation: kept }\end{array}$ & $\begin{array}{l}\text { Preparation Date: } \\
0-4^{\circ} \mathrm{C}\end{array}$ \\
\hline Preparation Method: & $\begin{array}{l}\text { Trout Chow aerated } 7 \text { days } \\
\text { Cerophyl, yeast aerated overnight }\end{array}$ \\
\hline $\begin{array}{l}\text { Feeding Rate: } \\
\text { Hold Time: }\end{array}$ & day / replicate \\
\hline
\end{tabular}

\section{Dilution Water}

Type: Surface Water

Collection Date $8 / 28 / 95$

Alkalinity

Ammonia

Conductivity

Hardness

pH:

Preparation Method:

Grab sample

Evidence of Water Quality:

Good reproduction of Ceriodaphnia dubia cultures

$\begin{array}{rrl}\text { Initial } & \text { Final } & \\ 12.2 & 10.1 \mathrm{mg} / \mathrm{L} \\ <0.1 & 0.2 \mathrm{mg} / \mathrm{L} \\ 36.4 & 34.9 \mathrm{unhos} / \mathrm{cm} \\ 10 & 6 \mathrm{mg} / \mathrm{L} \\ 7.38 & 6.14 \text { units }\end{array}$

\section{Test Sediment}

Source: WSRC FHB 013

Collection Date 9/11/95 Homogenized?: yes

Preservation: kept at $0-4^{\circ} \mathrm{C}$ in plastic containers

Date/Time Added to Test Chambers: 9/18/95

Observations: organic

Collection Method: grab

Shipment: $\quad$ Overnight at $4^{\circ} \mathrm{C}$

\section{Test Chambers}

Type: Plastic $500 \mathrm{~mL}$ beakers

Sediment Volume: $\quad 100 \mathrm{~mL}$

Sediment Depth $4 \mathrm{~cm}$

Overlying Water Volume: $175 \mathrm{~mL}$

Overlying Water Depth: $\quad 5 \mathrm{~cm}$

Aeration: none 


\section{DAY AMPHIPOD SEDIMENT TOXICITY TEST}

\begin{tabular}{|l|l|l|ll|}
\hline \multirow{2}{*}{$\begin{array}{l}\text { Lab ID } \# \\
\text { T4504 }\end{array}$} & Client: $\quad$ Westinghouse Savannah River Company & Start Date: & $9-19-95$ \\
\cline { 2 - 5 } & Sample Identification: $\quad$ WSRC FHB 013 & End Date: & $9-29-95$ \\
\hline
\end{tabular}

\section{WATER CHEMISTRY TEST METHODS}

\begin{tabular}{|l|l|}
\hline Temperature: SM 18th Ed. 2550 & Alkalinity: SM 18th Ed. 2320 \\
Dissolved Oxygen: SM 18th Ed. 4500-O & Hardness: SM 18th Ed. 2340 \\
pH: SM 18th Ed. 4500-H+ & Conductivity: SM 18th Ed. 2510 \\
\hline
\end{tabular}

\section{WATER CHEMISTRY TEST DATA - OVERLYING WATER}

CONTROL TREATMENT

\begin{tabular}{|c|r|r|r|r|}
\hline Test Day & $\begin{array}{c}\text { Temp. } \\
\text { C }\end{array}$ & $\begin{array}{c}\mathrm{pH} \\
\text { units }\end{array}$ & $\begin{array}{c}\text { D.O. } \\
\mathrm{mg} / \mathrm{L}\end{array}$ & $\begin{array}{c}\text { D.O. } \\
\% \text { sat. }\end{array}$ \\
\hline Start & 22.8 & 7.38 & 7.8 & $90 \%$ \\
\hline 1 & 22.5 & & 6.9 & $79 \%$ \\
\hline 2 & 22.7 & & 6.6 & $76 \%$ \\
\hline 3 & 22.4 & & 7.2 & $83 \%$ \\
\hline 4 & 22.1 & & 8.1 & $93 \%$ \\
\hline 5 & 23.2 & & 8.0 & $93 \%$ \\
\hline 6 & 22.2 & & 6.8 & $78 \%$ \\
\hline 7 & 23.9 & & 5.9 & $69 \%$ \\
\hline 8 & 22.0 & & 6.4 & $74 \%$ \\
\hline 9 & 22.2 & & 6.2 & $71 \%$ \\
\hline 10 & 22.1 & 6.14 & 6.2 & $71 \%$ \\
\hline
\end{tabular}

SAMPLE TREATMENT

\begin{tabular}{|c|c|c|c|c|c|c|c|c|}
\hline $\begin{array}{c}\text { Test } \\
\text { Day }\end{array}$ & $\begin{array}{c}\text { Cond. } \\
\text { umhos/cm }\end{array}$ & $\begin{array}{c}\mathrm{NH} 3 \\
\mathrm{mg} / \mathrm{L}\end{array}$ & $\begin{array}{c}\text { Temp. } \\
\mathrm{C}\end{array}$ & $\begin{array}{c}\mathrm{pH} \\
\text { units }\end{array}$ & $\begin{array}{c}\text { D.O. } \\
\mathrm{mg} / \mathrm{L}\end{array}$ & $\begin{array}{c}\text { D.O. } \\
\% \text { sat. }\end{array}$ & $\begin{array}{c}\text { Alk. } \\
\mathrm{mg} / \mathrm{L}\end{array}$ & $\begin{array}{c}\text { Hard. } \\
\mathrm{mg} / \mathrm{L}\end{array}$ \\
\hline Start & 36.4 & 0.949 & 22.3 & 5.94 & 7.0 & $80 \%$ & 2.0 & 6.0 \\
\hline 1 & & & 22.4 & & 8.2 & $94 \%$ & & \\
\hline 2 & & & 22.7 & & 6.4 & $74 \%$ & & \\
\hline 3 & & & 22.5 & & 6.8 & $78 \%$ & & \\
\hline 4 & & & 22.2 & & 8.3 & $95 \%$ & & \\
\hline 5 & & & 23.3 & & 8.1 & $94 \%$ & & \\
\hline 6 & & & 22.2 & & 7.1 & $82 \%$ & & \\
\hline 7 & & & 23.9 & & 5.7 & $66 \%$ & & \\
\hline 8 & & & 22.0 & & 6.4 & $74 \%$ & & \\
\hline 9 & & & 22.4 & & 7.3 & $84 \%$ & & \\
\hline 10 & 35 & 0.434 & 22.0 & 6.07 & 5.5 & $63 \%$ & 6.1 & 4.0 \\
\hline
\end{tabular}

\section{SURVIVAL AND GROWTH RESULTS}

\begin{tabular}{|l|r|r|r|r|r|r||}
\hline & \multicolumn{7}{|c|}{$\begin{array}{c}\text { \# Organisms } \\
\text { Initial }\end{array}$} & $\begin{array}{r}\text { Live at } \\
\text { End }\end{array}$ & $\begin{array}{c}\text { Dead } \\
\text { at End }\end{array}$ & $\begin{array}{c}\% \\
\text { Survival }\end{array}$ & $\begin{array}{r}\text { Leangth } \\
(\mathrm{mm})\end{array}$ & $\begin{array}{r}\text { Mean } \\
\text { Length } \\
(\mathrm{mm})\end{array}$ \\
\hline A & 10 & 9 & 0 & $90 \%$ & 2.1 & 2.10 \\
\hline B & 10 & 10 & 0 & $100 \%$ & 2.04 & 2.04 \\
\hline C & 10 & 9 & 0 & $90 \%$ & 2.04 & 2.04 \\
\hline D & 10 & 10 & 0 & $100 \%$ & 2.02 & 2.02 \\
\hline E & 10 & 9 & 0 & $90 \%$ & 2.04 & 2.04 \\
\hline F & 10 & 10 & 0 & $100 \%$ & 2 & 2.00 \\
\hline G & 10 & 10 & 0 & $100 \%$ & 2.04 & 2.04 \\
\hline H & 10 & 10 & 0 & $100 \%$ & 2.1 & 2.09 \\
\hline Mean & 10.0 & & & $96 \%$ & & 2.05 \\
\hline
\end{tabular}

\begin{tabular}{|c|c|c|c|c|c|c|}
\hline & \multicolumn{6}{|c|}{ SAMPLE TREATMENT } \\
\hline & \multicolumn{3}{|c|}{ \# Organisms } & \multirow[b]{2}{*}{$\begin{array}{c}\% \\
\text { Survival }\end{array}$} & \multirow{2}{*}{$\begin{array}{l}\text { Mean } \\
\text { Length } \\
(\mathrm{mm})\end{array}$} & \multirow{2}{*}{$\begin{array}{r}\text { Mean } \\
\text { Length } \\
(\mathrm{mm})\end{array}$} \\
\hline & Initial & $\begin{array}{l}\text { Live at } \\
\text { End }\end{array}$ & $\begin{array}{l}\text { Dead } \\
\text { at End }\end{array}$ & & & \\
\hline $\mathrm{A}$ & 10 & 4 & 0 & $40 \%$ & 1.94 & 1.94 \\
\hline $\mathrm{B}^{*}$ & 10 & 6 & 0 & $60 \%$ & 2.19 & 2.19 \\
\hline $\mathrm{C}^{*}$ & 10 & 5 & 0 & $50 \%$ & 2.11 & 2.11 \\
\hline D & 10 & 7 & 0 & $70 \%$ & 2.06 & 2.06 \\
\hline$E$ & .10 & 7 & 0 & $70 \%$ & 2.15 & 2.15 \\
\hline $\mathrm{F}$ & 10 & 5 & 0 & $50 \%$ & 2.02 & 2.02 \\
\hline$G$ & 10 & 5 & 0 & $50 \%$ & 2.11 & 2.11 \\
\hline $\mathrm{H}$ & 10 & 5 & 0 & $50 \%$ & 2.2 & 2.19 \\
\hline Mean & 10.0 & & & $55 \%$ & & 2.10 \\
\hline
\end{tabular}

* Predators observed in sample. 


\section{DAY AMPHIPOD SEDIMENT TOXICITY TEST}

\begin{tabular}{|c|c|c|c|c|}
\hline \multirow{2}{*}{\begin{tabular}{|l} 
Lab ID\# \\
T4504
\end{tabular}} & Client: & Nestinghouse Savannah River Company & Start Date: & $9-19-95$ \\
\hline & Sample Identification & WSRC FHB 013 & End Date: & $9-29-95$ \\
\hline
\end{tabular}

\section{RESULTS}

\begin{tabular}{|l|r|}
\hline \multicolumn{2}{|c|}{$\begin{array}{c}\text { SURVIVAL DATA } \\
\text { Mean of Eight Replicates }\end{array}$} \\
\cline { 2 - 2 } & Mean \% \\
\hline Control & $96.3 \%$ \\
\hline Sample & $55.0 \%$ \\
\hline
\end{tabular}

\begin{tabular}{|c|c|c|}
\hline \multicolumn{3}{|c|}{ GROWTH DATA } \\
\hline & & Mean \\
\hline Control & & 2.05 \\
\hline Sample & & 2.10 \\
\hline
\end{tabular}

\section{STATISTICAL ANALYSES}

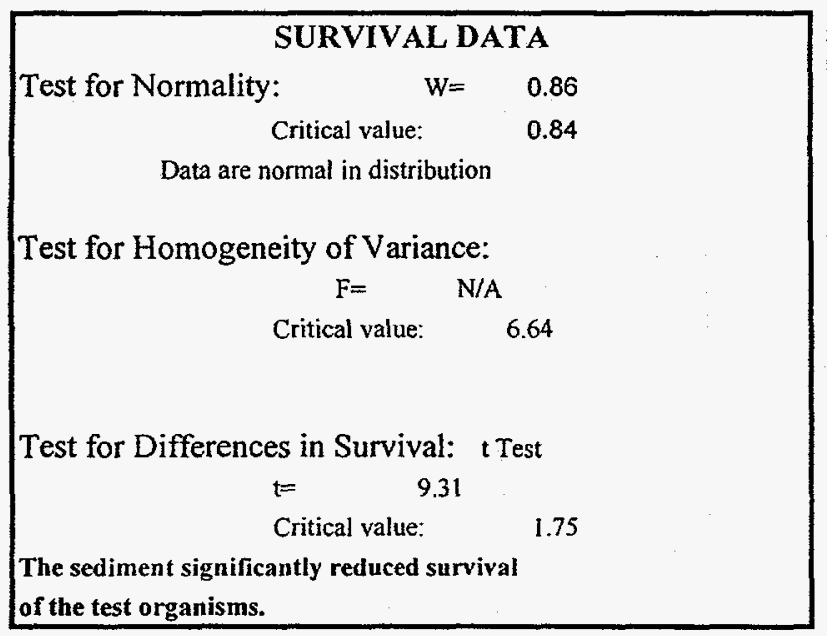

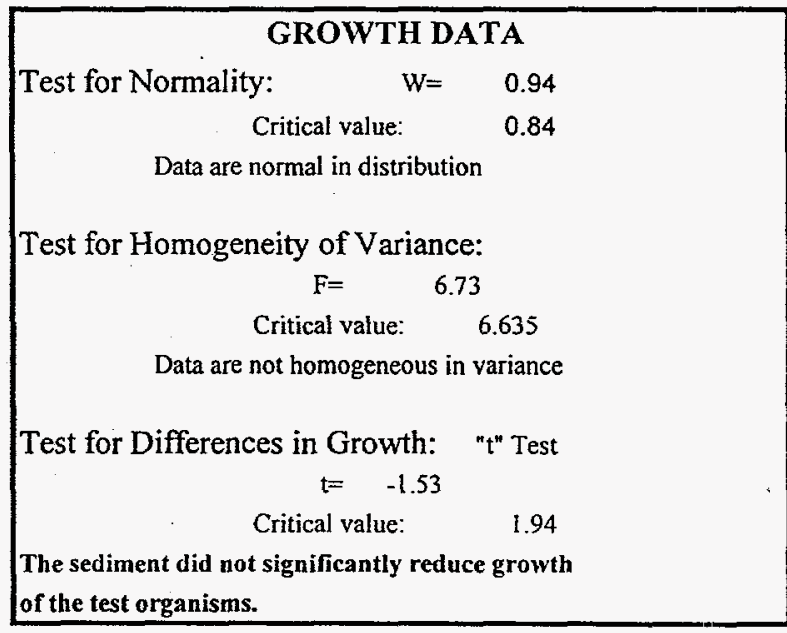

\section{COMMENTS}

\begin{tabular}{|c|c|c|c|}
\hline $\begin{array}{l}\text { Principal Analyst: } \\
\text { Report Reviewed By: }\end{array}$ & $\begin{array}{l}\text { Aimee Arnold } \\
\text { Robert W. Kelley, Ph.D. }\end{array}$ & 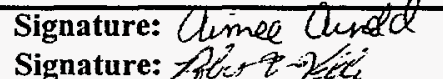 & $\begin{array}{l}\text { Date: } 10 / 13 / 95 \\
\text { Date: } 10 / 13 / 45\end{array}$ \\
\hline
\end{tabular}

\section{CONCLUSIONS}

The test sediment does adversely affect the survival but not the growth of freshwater amphipods. 
(803) 877-6942 - FAX (803) 877-6938

P.O. Box 16414, Greenville, SC 29606 - 4 Craftsman Court, Greer, SC 29650

\title{
10 DAY AMPHIPOD SEDIMENT TOXICITY TEST
}

\section{EPA/600/R-94/024 Method 100.1}

Test Organism: Hyallela azteca

\author{
Client: Westinghouse Savannah River Company \\ Sample Identification: WSRC FHB014
}

Test Start Date:

$8 / 29 / 95$ 


\section{DAY AMPHIPOD SEDIMENT TOXICITY TEST}

\begin{tabular}{|c|c|c|c|c|}
\hline \multirow{2}{*}{\begin{tabular}{|l} 
Lab ID\# \\
T4444
\end{tabular}} & \multicolumn{2}{|c|}{ Client: $\quad$ Westinghouse Savannah River Corporation } & \multicolumn{2}{|c|}{ Start Date: $8-29-95$} \\
\hline & Sample Identification: & WSRC FHB014 & End Date: & $9-8-95$ \\
\hline
\end{tabular}

\section{WATER CHEMISTRY TEST METHODS}

\begin{tabular}{|l|l|}
\hline Temperature: SM 18th Ed. 2550 & Alkalinity: SM 18th Ed. 2320 \\
Dissolved Oxygen: SM 18th Ed. 4500-O & Hardness: SM 18th Ed. 2340 \\
pH: SM 18th Ed. 4500-H+ & Conductivity: SM 18th Ed. 2510 \\
\hline
\end{tabular}

WATER CHEMISTRY TEST DATA - OVERLYING WATER

CONTROL TREATMENT
\begin{tabular}{|c|r|r|r|r|}
\hline Test Day & $\begin{array}{c}\text { Temp. } \\
\text { C }\end{array}$ & $\begin{array}{c}\text { pH } \\
\text { units }\end{array}$ & $\begin{array}{c}\text { D.O } \\
\mathrm{mg} / \mathrm{L}\end{array}$ & $\begin{array}{c}\text { D.O. } \\
\% \text { sat. }\end{array}$ \\
\hline Start & 23.3 & 7.07 & 7.8 & $91 \%$ \\
\hline 1 & 22.1 & & 7.0 & $80 \%$ \\
\hline 2 & 21.9 & & 6.0 & $67 \%$ \\
\hline 3 & 21.8 & & 5.6 & $63 \%$ \\
\hline 4 & 22.0 & & 5.3 & $61 \%$ \\
\hline 5 & 23.0 & & 5.8 & $67 \%$ \\
\hline 6 & 22.5 & & 5.5 & $63 \%$ \\
\hline 7 & 22.3 & & 5.6 & $64 \%$ \\
\hline 8 & 22.0 & & 5.3 & $61 \%$ \\
\hline 9 & 22.0 & & 5.2 & $60 \%$ \\
\hline 10 & 22.1 & 7.42 & 4.7 & $54 \%$ \\
\hline
\end{tabular}

SAMPLE TREATMENT

\begin{tabular}{|c|c|c|c|c|c|c|c|c|}
\hline $\begin{array}{c}\text { Test } \\
\text { Day }\end{array}$ & $\begin{array}{c}\text { Cond. } \\
\text { umhosicm }\end{array}$ & $\begin{array}{c}\text { NH3 } \\
\mathrm{mg} / \mathrm{L}\end{array}$ & $\begin{array}{c}\text { Temp. } \\
\mathrm{C}\end{array}$ & $\begin{array}{c}\mathrm{pH} \\
\text { units }\end{array}$ & $\begin{array}{c}\text { D.O. } \\
\mathrm{mg} / \mathrm{L}\end{array}$ & $\begin{array}{c}\text { D.O. } \\
\% \text { sat. }\end{array}$ & $\begin{array}{c}\text { Alk } \\
\mathrm{mg} / \mathrm{L}\end{array}$ & $\begin{array}{c}\text { Hard } \\
\mathrm{mg} / \mathrm{L}\end{array}$ \\
\hline Stan & 91.7 & 0.16 & 22.8 & 6.26 & 6.2 & $71 \%$ & 20.4 & 28.0 \\
\hline 1 & & & 22.0 & & 5.0 & $57 \%$ & & \\
\hline 2 & & & 22.0 & & 5.3 & $61 \%$ & & \\
\hline 3 & & & 21.9 & & 5.0 & $56 \%$ & & \\
\hline 4 & & & 21.9 & & 5.4 & $61 \%$ & & \\
\hline 5 & & & 22.5 & & 4.7 & $54 \%$ & & \\
\hline 6 & & & 22.2 & & 4.4 & $51 \%$ & & \\
\hline 7 & & & 22.1 & & 5.3 & $61 \%$ & & \\
\hline 8 & & & 22.0 & & 4.8 & $55 \%$ & & \\
\hline 9 & & & 22.0 & & 4.7 & $54 \%$ & & \\
\hline 10 & 137 & 0.3 & 22.2 & 6.98 & 4.6 & $53 \%$ & 51.0 & 56.0 \\
\hline
\end{tabular}

\section{SURVIVAL AND GROWTH RESULTS}

CONTROL TREATMENT

\begin{tabular}{|c|c|c|c|c|c|c|}
\hline & \multicolumn{3}{|c|}{ \# Organisms } & \multirow[b]{2}{*}{$\begin{array}{c}\% \\
\text { Survival } \\
\end{array}$} & \multirow{2}{*}{$\begin{array}{l}\text { Mean } \\
\text { Length } \\
(\mathrm{mm})\end{array}$} & \multirow{2}{*}{$\begin{array}{r}\text { Mean } \\
\text { Length } \\
(\mathrm{mm})\end{array}$} \\
\hline & Initial & $\begin{array}{c}\text { Live at } \\
\text { End }\end{array}$ & $\begin{array}{l}\text { Dead } \\
\text { at End }\end{array}$ & & & \\
\hline $\bar{A}$ & 10 & 6 & 1 & $60 \%$ & 2.4 & 2.40 \\
\hline $\mathrm{B}$ & 10 & $\overline{N A}$ & $\mathrm{NA}$ & $\mathrm{NA}$ & $\longdiv { \mathrm { NA } }$ & NA \\
\hline C & 10 & 7 & 0 & $70 \%$ & 2.18 & 2.18 \\
\hline D & 10 & 7 & 0 & $70 \%$ & 2.18 & 2.18 \\
\hline$E$ & 10 & 10 & 0 & $100 \%$ & 2.17 & 2.17 \\
\hline $\mathrm{F}$ & 10 & 8 & 2 & $80 \%$ & 2.12 & 2.12 \\
\hline $\mathrm{G}$ & 10 & 9 & 0 & $90 \%$ & 2.13 & 2.13 \\
\hline $\mathrm{H}$ & 10 & 9 & 0 & $90 \%$ & 2.2 & 2.15 \\
\hline Mean & 10.0 & & & $80 \%$ & & 2.19 \\
\hline
\end{tabular}

SAMPLE TREATMENT

\begin{tabular}{|c|c|c|c|c|c|c|}
\hline & \multicolumn{3}{|c|}{ \# Organisms } & \multirow[b]{2}{*}{$\begin{array}{c}\% \\
\text { Survival }\end{array}$} & \multirow{2}{*}{$\begin{array}{l}\text { Mean } \\
\text { Length } \\
(\mathrm{mm})\end{array}$} & \multirow{2}{*}{$\begin{array}{r}\begin{array}{r}\text { Mean } \\
\text { Length } \\
(\mathrm{mm})\end{array} \\
\end{array}$} \\
\hline & Initial & $\begin{array}{c}\text { Liye at } \\
\text { End }\end{array}$ & $\begin{array}{l}\text { Dead } \\
\text { at End }\end{array}$ & & & \\
\hline $\bar{A}$ & 10 & 6 & 3 & $60 \%$ & 2.22 & 2.22 \\
\hline$B$ & 10 & 8 & 0 & $80 \%$ & 2.37 & 2.37 \\
\hline $\mathrm{C}$ & 10 & 10 & 0 & $100 \%$ & 2.37 & 2.37 \\
\hline D & 10 & 9 & 0 & $90 \%$ & 2.29 & 2.29 \\
\hline$E$ & 10 & 8 & 0 & $80 \%$ & 2.18 & 2.18 \\
\hline $\mathrm{F}$ & 10 & 8 & 1 & $80 \%$ & 2.27 & 2.27 \\
\hline $\mathrm{G}$ & 10 & 8 & 0 & $80 \%$ & 2.26 & 2.26 \\
\hline $\mathrm{H}$ & 10 & 9 & 0 & $90 \%$ & 2.3 & 2.25 \\
\hline Mean & 10.0 & & & $83 \%$ & & 2.28 \\
\hline
\end{tabular}




\section{DAY AMPHIPOD SEDIMENT TOXICITY TEST}

\begin{tabular}{|c|c|c|c|c|}
\hline \multirow{2}{*}{\begin{tabular}{|l} 
Lab ID\# \\
T4444 \\
\end{tabular}} & Client: & Vestinghouse Savannah River Corporation & Start Date: & $8-29-95$ \\
\hline & Sample Identification & WSRC FHBO 14 & End Date: & $9-8-95$ \\
\hline
\end{tabular}

\section{RESULTS}

\begin{tabular}{|l|r|}
\hline \multicolumn{2}{|c|}{$\begin{array}{c}\text { SURVIVAL DATA } \\
\text { Mean of Eight Replicates }\end{array}$} \\
\cline { 2 - 2 } & Mean \% \\
\hline Control & $80.0 \%$ \\
\hline Sample & $82.5 \%$ \\
\hline
\end{tabular}

\begin{tabular}{|l|r|}
\hline \multicolumn{2}{|c|}{ GROWTH DATA } \\
& Mean Length $(\mathrm{mm})$ \\
\hline Control & Mean \\
\hline Sample & 2.19 \\
\hline
\end{tabular}

\section{STATISTICAL ANALYSES}

\begin{tabular}{|c|c|c|}
\hline \multicolumn{3}{|c|}{ SURVIVAL DATA } \\
\hline Test for Normality: & $w=$ & 0.93 \\
\hline & & 0.84 \\
\hline \multicolumn{3}{|c|}{ Data are normal in distribution } \\
\hline \multicolumn{3}{|c|}{ Test for Homogeneity of Variance: } \\
\hline & \multicolumn{2}{|c|}{ N/A } \\
\hline & & 6.64 \\
\hline \multicolumn{3}{|c|}{ Test for Differences in Survival: 1 Test } \\
\hline & \multicolumn{2}{|c|}{-0.24} \\
\hline & 1e: & 1.75 \\
\hline
\end{tabular}

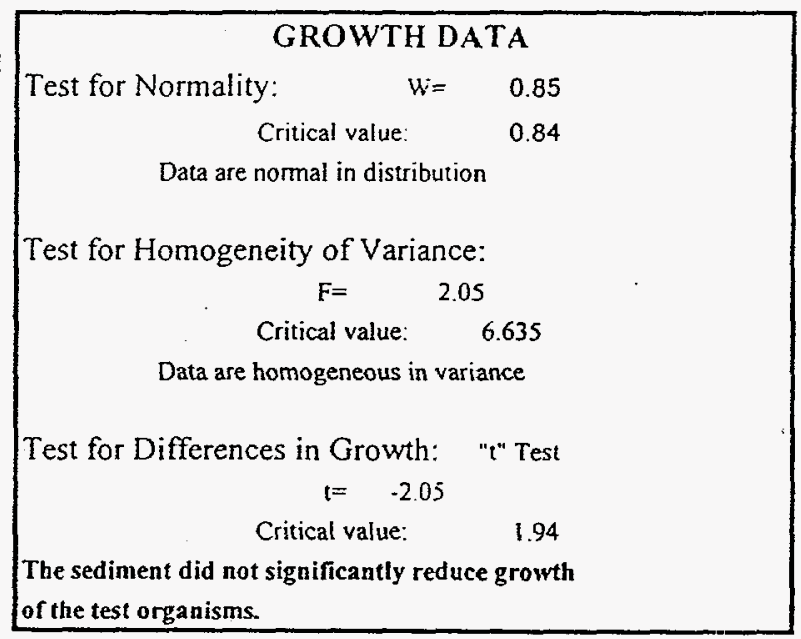

\section{COMMENTS}

\begin{tabular}{|c|c|c|c|c|}
\hline $\begin{array}{l}\text { Principal Analyst: } \\
\text { Report Reviewed By: }\end{array}$ & $\begin{array}{l}\text { Aimee Arnold } \\
\text { Robert W. Kelley, Ph.D. }\end{array}$ & $\begin{array}{l}\text { Signature: Aumee curete } \\
\text { Signature: thet }\end{array}$ & $\begin{array}{l}\text { Date: } \\
\text { Date: }\end{array}$ & 7011195 \\
\hline
\end{tabular}

\section{CONCLUSIONS}

The test sediment does not adversely affect the survival and growth of freshwater amphipods. 


\title{
10 DAY AMPHIPOD SEDIMENT TOXICITY TEST
}

\author{
EPA/600/R-94/024 Method 100.1
}

Test Organism: Hyallela azteca

Client: Westinghouse Savannah River Company Sample Identification: WSRC FHB 018

Test Start Date: $\quad 9-19-95$ 


\section{DAY AMPHIPOD SEDIMENT TOXICITY TEST}

\begin{tabular}{|c|c|c|c|c|}
\hline \multirow{2}{*}{$\begin{array}{l}\text { Lab ID\# } \\
\text { T4505 }\end{array}$} & Client: & Vestinghouse Savannah River Company & Start Date: & $9-19-95$ \\
\hline & Sample Identification & WSRC FHB 018 & End Date: & $9-29-95$ \\
\hline
\end{tabular}

\section{Test System}

\section{EPA/600/R-94/024 Method 100.1}

The test was set as a Pass/Fail test with a control treatment and one sample treatment. Each treatment replicate consisted of $100 \mathrm{~g}$ of sediment and $175 \mathrm{~mL}$ of dilution water. There were eight replicates for each treatment. Ten test organisms were placed in each replicate. Sediment from a local creek was used in the control treatment. Test: organisms were fed with YCT food. The test was conducted at a temperature of $23^{\circ} \mathrm{C}$ with ambient laboratory light conditions. Test vessels were $500 \mathrm{~mL}$ plastic beakers placed in a constant temperature incubator room.

Order: Amphipoda

\section{Test Organisms}

Species: Hyallela azteca

Source: Aquatic Biosystems, CO

Life Stage: $\quad 2-3 \mathrm{~mm}$

\% Mortality During 48 Hr Prior to Test:: $\quad 0 \%$

Taxonomic Verification: RWK 9/19/95

Observations: Cultures healthy

Acclimation: None
Culturing: as received

\begin{tabular}{|c|c|c|}
\hline \multicolumn{3}{|c|}{ Dilution Water } \\
\hline $\begin{array}{l}\text { Type: Surface Water } \\
\text { Collection Date } 8 / 28 / 95\end{array}$ & Initial & Final \\
\hline Alkalinity & 12.2 & $10.1 \mathrm{mg} / \mathrm{L}$ \\
\hline Ammonia & $<0.1$ & $0.2 \mathrm{mg} / \mathrm{L}$ \\
\hline Conductivity & 36.4 & 34.9 urrhos $/ \mathrm{cm}$ \\
\hline Hardness & 10 & $6 \mathrm{mg} / \mathrm{L}$ \\
\hline$p H:$ & 7.38 & 6.14 units \\
\hline \multicolumn{3}{|l|}{$\begin{array}{l}\text { Preparation Method: } \\
\text { Grab sample }\end{array}$} \\
\hline \multicolumn{3}{|c|}{ Evidence of Water Quality: } \\
\hline
\end{tabular}

\section{Control Sediment}

Source: S. Tyger River at Hwy. 14

Collection Date: 9-19-95

Preservation: $\quad$ kept at $0-4^{\circ} \mathrm{C}$ in plastic containers

Date/Time Added to Test Chambers: $\quad$ 9-19-95

Observations: silt

Collection Method: grab

Disposal:

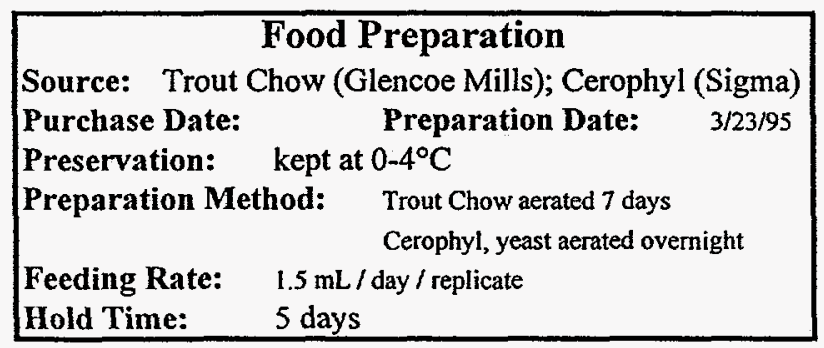

\section{Test Sediment}

Source: WSRC FHB 018

Collection Date 9/11/95 Homogenized?: yes

Preservation: kept at $0-4^{\circ} \mathrm{C}$ in plastic containers

Date/Time Added to Test Chambers: $9 / 18 / 95$

Observations: organic

Collection Method: grab

Shipment: $\quad$ Overnight at $4^{\circ} \mathrm{C}$

\begin{tabular}{|l|}
\multicolumn{1}{c|}{ Test Chambers } \\
Type: Plastic $500 \mathrm{~mL}$ beakers \\
Sediment Volume: $\quad 100 \mathrm{~mL}$ \\
Sediment Depth $4 \mathrm{~cm}$ \\
$\begin{array}{l}\text { Overlying Water Volume: } \quad 175 \mathrm{~mL} \\
\text { Overlying Water Depth: } \quad 5 \mathrm{~cm} \\
\text { Aeration: none }\end{array}$ \\
\hline
\end{tabular}




\section{DAY AMPHIPOD SEDIMENT TOXICITY TEST}

\begin{tabular}{|c|c|c|c|c|}
\hline Lab ID\# & Client: & ghouse Savannah River Company & Start Date: & $9-19-95$ \\
\hline T4505 & Sample Identification & WSRC FHB 018 & End Date: & $9-29-95$ \\
\hline
\end{tabular}

WATER CHEMISTRY TEST METHODS

\begin{tabular}{|l|l|}
\hline \begin{tabular}{l|l} 
Temperature: SM 18th Ed. 2550 \\
Dissolved Oxygen: SM 18th Ed. 4500-O \\
pH: SM 18th Ed. 4500-H+
\end{tabular} & $\begin{array}{l}\text { Alkalinity: SM 18th Ed. 2320 } \\
\text { Hardness: SM 18th Ed. 2340 } \\
\text { Conductivity: SM 18th Ed. 2510 }\end{array}$ \\
\hline
\end{tabular}

WATER CHEMISTRY TEST DATA - OVERLYING WATER

CONTROL TREATMENT

\begin{tabular}{|c|r|r|r|r|}
\hline Test Day & $\begin{array}{c}\text { Temp. } \\
\text { C }\end{array}$ & $\begin{array}{c}\mathrm{pH} \\
\text { units }\end{array}$ & $\begin{array}{c}\text { D.O. } \\
\mathrm{mg} / \mathrm{L}\end{array}$ & $\begin{array}{r}\text { D.O. } \\
\% \text { sat. }\end{array}$ \\
\hline Start & 22.8 & 7.38 & 7.8 & $90 \%$ \\
\hline 1 & 22.5 & & 6.9 & $79 \%$ \\
\hline 2 & 22.7 & & 6.6 & $76 \%$ \\
\hline 3 & 22.4 & & 7.2 & $83 \%$ \\
\hline 4 & 22.1 & & 8.1 & $93 \%$ \\
\hline 5 & 23.2 & & 8.0 & $93 \%$ \\
\hline 6 & 22.2 & & 6.8 & $78 \%$ \\
\hline 7 & 23.9 & & 5.9 & $69 \%$ \\
\hline 8 & 22.0 & & 6.4 & $74 \%$ \\
\hline 9 & 22.2 & & 6.2 & $71 \%$ \\
\hline 10 & 22.1 & 6.14 & 6.2 & $71 \%$ \\
\hline
\end{tabular}

SAMPLE TREATMENT

\begin{tabular}{|c|c|c|r|r|r|r|r|c|}
\hline $\begin{array}{c}\text { Test } \\
\text { Day }\end{array}$ & $\begin{array}{c}\text { Cond. } \\
\text { umhos/cm }\end{array}$ & $\begin{array}{c}\mathrm{NH} 3 \\
\mathrm{mg} / \mathrm{L}\end{array}$ & $\begin{array}{c}\text { Temp. } \\
\mathrm{C}\end{array}$ & $\begin{array}{c}\mathrm{pH} \\
\text { units }\end{array}$ & $\begin{array}{c}\text { D.O. } \\
\mathrm{mg} / \mathrm{L}\end{array}$ & $\begin{array}{c}\text { D.O. } \\
\% \text { sat. }\end{array}$ & $\begin{array}{c}\text { Alk. } \\
\mathrm{mg} / \mathrm{L}\end{array}$ & $\begin{array}{c}\text { Hard. } \\
\mathrm{mg} / \mathrm{L}\end{array}$ \\
\hline Start & 28.2 & 0.882 & 22.3 & 6.18 & 6.3 & $72 \%$ & 8.1 & 6.0 \\
\hline 1 & & & 22.4 & & 7.7 & $89 \%$ & & \\
\hline 2 & & & 22.7 & & 6.8 & $78 \%$ & & \\
\hline 3 & & & 22.4 & & 7.2 & $83 \%$ & & \\
\hline 4 & & & 22.3 & & 8.4 & $97 \%$ & & \\
\hline 5 & & & 23.3 & & 7.4 & $86 \%$ & & \\
\hline 6 & & & 22.1 & & 6.6 & $76 \%$ & & \\
\hline 7 & & & 23.9 & & 6.4 & $74 \%$ & & \\
\hline 8 & & & 22.0 & & 6.6 & $76 \%$ & & \\
\hline 9 & & & 22.3 & & 6.4 & $74 \%$ & & \\
\hline 10 & 35 & 0.322 & 22.1 & 6.10 & 5.9 & $68 \%$ & 10.1 & 6.0 \\
\hline
\end{tabular}

\section{SURVIVAL AND GROWTH RESULTS}

CONTROL TREATMENT

\begin{tabular}{|c|c|c|c|c|c|c|}
\hline & \multicolumn{3}{|c|}{ \# Organisms } & \multirow[b]{2}{*}{$\begin{array}{c}\% \\
\text { Survival }\end{array}$} & \multirow{2}{*}{$\begin{array}{l}\text { Mean } \\
\text { Length } \\
(\mathrm{mm})\end{array}$} & \multirow{2}{*}{$\begin{array}{r}\begin{array}{r}\text { Mean } \\
\text { Length } \\
(\mathrm{mm})\end{array} \\
\end{array}$} \\
\hline & Initial & $\begin{array}{l}\text { Live at } \\
\text { End }\end{array}$ & $\begin{array}{l}\text { Dead } \\
\text { at End }\end{array}$ & & & \\
\hline $\mathrm{A}$ & 10 & 9 & 0 & $90 \%$ & 2.1 & 2.10 \\
\hline B & 10 & 10 & 0 & $100 \%$ & 2.04 & 2.04 \\
\hline C & 10 & 9 & 0 & $90 \%$ & 2.04 & 2.04 \\
\hline D & 10 & 10 & 0 & $100 \%$ & 2.02 & 2.02 \\
\hline$E$ & 10 & 9 & 0 & $90 \%$ & 2.04 & 2.04 \\
\hline $\mathrm{F}$ & 10 & 10 & 0 & $100 \%$ & 2 & 2.00 \\
\hline $\mathrm{G}$ & 10 & 10 & 0 & $100 \%$ & 2.04 & 2.04 \\
\hline $\mathrm{H}$ & 10 & 10 & 0 & $100 \%$ & 2.1 & 2.09 \\
\hline Mean & 10.0 & & & $96 \%$ & & 2.05 \\
\hline
\end{tabular}

SAMPLE TREATMENT

\begin{tabular}{|c|c|c|c|c|c|c|}
\hline & \multicolumn{3}{|c|}{ \# Organisms } & \multirow[b]{2}{*}{$\begin{array}{c}\% \\
\text { Survival }\end{array}$} & \multirow{2}{*}{$\begin{array}{l}\text { Mean } \\
\text { Length } \\
(\mathrm{mm})\end{array}$} & \multirow{2}{*}{$\begin{array}{r}\text { Mean } \\
\text { Length } \\
(\mathrm{mm}) \\
\end{array}$} \\
\hline & Initial & $\begin{array}{l}\text { Live at } \\
\text { End }\end{array}$ & $\begin{array}{c}\text { Dead } \\
\text { at End }\end{array}$ & & & \\
\hline A & 10 & 5 & 0 & $50 \%$ & 2.45 & 2.45 \\
\hline $\mathrm{B}$ & 10 & 8 & 0 & $80 \%$ & 2.1 & 2.10 \\
\hline C & 10 & 6 & 0 & $60 \%$ & 2.01 & 2.01 \\
\hline D & 10 & 3 & 0 & $30 \%$ & 2.37 & 2.37 \\
\hline $\mathrm{E}$ & 10 & 7 & 0 & $70 \%$ & 2.21 & 2.21 \\
\hline $\mathrm{F}$ & 10 & 4 & 0 & $40 \%$ & 2.42 & 2.42 \\
\hline $\mathrm{G}$ & 10 & 5 & 0 & $50 \%$ & 2.49 & 2.49 \\
\hline $\mathrm{H}$ & 10 & 2 & 0 & $20 \%$ & 2.5 & 2.47 \\
\hline Mean & 10.0 & & & $50 \%$ & & 2.32 \\
\hline
\end{tabular}




\section{DAY AMPHIPOD SEDIMENT TOXICITY TEST}

\begin{tabular}{|c|c|c|c|c|}
\hline \multirow{2}{*}{$\begin{array}{l}\text { Lab ID\# } \\
\text { T4505 }\end{array}$} & Client: & Vestinghouse Savannah River Company & Start Date: & $9-19-95$ \\
\hline & Sample Identification: & WSRC FHB 018 & End Date: & $9-29-95$ \\
\hline
\end{tabular}

\section{RESULTS}

\begin{tabular}{|c|c|c|}
\hline \multicolumn{3}{|c|}{ SURVIVAL DATA } \\
\hline & & Mean $\%$ \\
\hline Control & & $96.3 \%$ \\
\hline Sample & & $50.0 \%$ \\
\hline
\end{tabular}

\begin{tabular}{|c|c|c|}
\hline \multicolumn{3}{|c|}{ GROWTH DATA } \\
\hline \multicolumn{3}{|c|}{ Mean Length (mm) } \\
\hline & & Mean \\
\hline Control & & 2.05 \\
\hline Sample & & 2.32 \\
\hline
\end{tabular}

\section{STATISTICAL ANALYSES}

\begin{tabular}{|c|c|c|}
\hline \multicolumn{3}{|c|}{ SURVIVAL DATA } \\
\hline Test for Normality: & $W=$ & 0.94 \\
\hline \multicolumn{2}{|c|}{ Critical value: } & 0.84 \\
\hline \multicolumn{3}{|c|}{ Data are normal in distribution } \\
\hline \multicolumn{3}{|c|}{ Test for Homogeneity of Variance: } \\
\hline \multicolumn{3}{|c|}{ N/A } \\
\hline \multicolumn{2}{|c|}{ Critical value: } & 6.64 \\
\hline \multicolumn{3}{|c|}{ Test for Differences in Survival: $t$ Test } \\
\hline \multicolumn{3}{|c|}{$t=\quad 7.07$} \\
\hline \multicolumn{3}{|c|}{ Critical value: $\quad 1.75$} \\
\hline \multicolumn{3}{|c|}{$\begin{array}{l}\text { The sediment significantly reduced survival } \\
\text { of the test organisms. }\end{array}$} \\
\hline
\end{tabular}

Test for Normality: $\quad$ W $\quad 0.92$
Critical value: $\quad 0.84$
Data are normal in distribution
Test for Homogeneity of Variance:
$\mathrm{F}=\quad 30.46$
Critical value: $\quad 6.635$
Data are not homogeneous in variance
Test for Differences in Growth: $\quad$ " $\mathrm{t}$ " Test
$\mathrm{t}=\quad-4.06$
Critical value:
The sediment did not significantly reduce growth
of the test organisms.

\section{COMMENTS}

\begin{tabular}{|c|c|c|c|}
\hline $\begin{array}{l}\text { Principal Analyst: } \\
\text { Report Reviewed By: }\end{array}$ & $\begin{array}{l}\text { Aimee Arnold } \\
\text { Robert W. Kelley, Ph.D. }\end{array}$ & 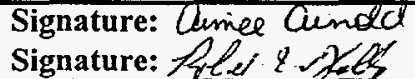 & $\begin{array}{l}\text { Date: } 10 / 13 / 95 \\
\text { Date: } 10 / 13 / 99\end{array}$ \\
\hline
\end{tabular}

\section{CONCLUSIONS}

The test sediment does adversely affect the survival but not the growth of freshwater amphipods. 


\title{
10 DAY AMPHIPOD SEDIMENT TOXICITY TEST
}

\author{
EPA/600/R-94/024 Method 100.1 \\ Test Organism: Hyallela azteca
}

Client: Westinghouse Savannah River Company

Sample Identification:

WSRC FHB020

Test Start Date: $\quad 8 / 29 / 95$ 


\section{DAY AMPHIPOD SEDIMENT TOXICITY TEST}

\begin{tabular}{|c|c|c|c|c|}
\hline \multirow{2}{*}{\begin{tabular}{|l} 
Lab ID\# \\
T4445
\end{tabular}} & Client: & ghouse Savannah River Company & Start Date: & $8-29-95$ \\
\hline & Sample Identification: & WSRC FHB020 & End Date: & $9-8-\overline{95}$ \\
\hline
\end{tabular}

\section{Test System}

EPA/600/R-94/024 Method 100.1

The test was set as a Pass/Fail test with a control treatment and one sample treatment. Each treatment replicate consisted of $100 \mathrm{~g}$ of sediment and $175 \mathrm{~mL}$ of dilution water. There were eight replicates for each treatment. Ten test organisms were placed in each replicate. Sediment from a local creek was used in the control treatment. Test organisms were fed with YCT food. The test was conducted at a temperature of $23^{\circ} \mathrm{C}$ with ambient laboratory light conditions. Test vessels were $500 \mathrm{~mL}$ plastic beakers placed in a constant temperature incubator room.

Test Organisms
Order: Amphipoda
Species: Hyallela azleca
Source: Aquatic Biosystems, CO
Life Stage: $\quad 2-3 \mathrm{~mm}$
\% Mortality During $48 \mathrm{Hr}$ Prior to Test:: $\quad 0 \%$
Taxonomic Verification: RWK 8/28/95
Culturing: $\quad$ as received

$\begin{aligned} & \text { Observations: } \quad \text { Cultures healthy } \\ & \text { Acclimation: } \quad \text { None }\end{aligned}$

Control Sediment
Source: Resurrection Creek (Greer S C)
Collection Date: $8-28-95$
Preservation: kept at $0-4^{\circ} \mathrm{C}$ in plastic containers
Date/Time Added to Test Chambers: 8-28-95
Observations: organic
Collection Method: petite ponar grab
Disposal:

\begin{tabular}{|l|}
\hline \multicolumn{2}{|c|}{ Food Preparation } \\
Source: Trout Chow (Glencoe Mills); Cerophyl (Sigma) \\
Purchase Date: $\quad$ Preparation Date: 3/23/95 \\
Preservation: $\quad$ kept at $0-4^{\circ} \mathrm{C}$ \\
$\begin{array}{ll}\text { Preparation Method: } \quad \text { Trout Chow aerated } 7 \text { days } \\
\text { Feeding Rate: } \quad 1.5 \mathrm{~mL} / \text { day/ replicate } \\
\text { Hold Time: } \quad 5 \text { days }\end{array}$ \\
\hline
\end{tabular}

\begin{tabular}{|c|c|c|}
\hline \multicolumn{3}{|c|}{ Dilution Water } \\
\hline Surface Water & & \\
\hline \multicolumn{3}{|l|}{ Collection Date $8 / 28 / 95$} \\
\hline Alkalinity & 46.9 & $40.8 \mathrm{mg} / \mathrm{L}$. \\
\hline Amimonia & $<0.1$ & $0.2 \mathrm{mg} / \mathrm{L}$ \\
\hline Conductivity & 142.6 & 186.1 urrhros/cm \\
\hline Hardness & 64 & $82 \mathrm{mg} / \mathrm{L}$ \\
\hline pH: & 7.07 & 7.42 units \\
\hline $\begin{array}{l}\text { Preparation Method: } \\
\text { Grab sample }\end{array}$ & & \\
\hline $\begin{array}{l}\text { Evidence of Water Quali } \\
\text { Good reproduction of Ceri }\end{array}$ & mia dut & cultures \\
\hline
\end{tabular}

\begin{tabular}{|l|}
\hline \multicolumn{1}{|c|}{ Test Sediment } \\
Source: WSRC FHB 020 \\
Collection Date $8 / 22 / 95 \quad$ Homogenized?: yes \\
Preservation: kept at $0-4^{\circ} \mathrm{C}$ in plastic containers \\
Date/Time Added to Test Chambers: $8 / 28 / 95$ \\
Observations: organic \\
Collection Method: petite ponar grab \\
Shipment: Overnight at $4^{\circ} \mathrm{C}$ \\
\hline
\end{tabular}

\begin{tabular}{l} 
Test Chambers \\
Type: Plastic $500 \mathrm{~mL}$ beakers \\
Sediment Volume: $\quad 100 \mathrm{~mL}$ \\
Sediment Depth $4 \mathrm{~cm}$ \\
Overlying Water Volume: $\quad 175 \mathrm{~mL}$ \\
Overlying Water Depth: $\quad 5 \mathrm{~cm}$ \\
Aeration: none \\
\hline
\end{tabular}




\section{DAY AMPHIPOD SEDIMENT TOXICITY TEST}

\begin{tabular}{|l|l|ll|}
\hline Lab ID $\#$ & Client: $\quad$ Westinghouse Savannah River Corporation & Start Date: & $8-29-95$ \\
\cline { 2 - 5 } T4445 & Sample Identification: $\quad$ WSRC FHB020 & End Date: & $9-8-95$ \\
\hline
\end{tabular}

WATER CHEMISTRY TEST METHODS

\begin{tabular}{|l|l|}
\hline Temperature: SM 18th Ed. 2550 & Alkalinity: SM 18th Ed. 2320 \\
Dissolved Oxygen: SM 18th Ed. 4500-O \\
pll: SM 18th Ed. 4500-H+
\end{tabular}$\quad \begin{aligned} & \text { Hardness: SM 18th Ed. 2340 } \\
& \text { Conductivity: SM 18th Ed. 2510 }\end{aligned}$

\section{WATER CHEMISTRY TEST DATA- OVERLYING WATER}

CONTROL TREATMENT

\begin{tabular}{|c|r|r|r|r|}
\hline Test Day & $\begin{array}{c}\text { Temp } \\
\text { C }\end{array}$ & $\begin{array}{c}\mathrm{pH} \\
\text { units }\end{array}$ & $\begin{array}{c}\text { D.O. } \\
\text { mg/L }\end{array}$ & $\begin{array}{c}\text { D.O. } \\
\% \text { sat. }\end{array}$ \\
\hline Start & 23.3 & 7.07 & 7.8 & $91 \%$ \\
\hline 1 & 22.1 & & 7.0 & $80 \%$ \\
\hline 2 & 21.9 & & 6.0 & $67 \%$ \\
\hline 3 & 21.8 & & 5.6 & $63 \%$ \\
\hline 4 & 22.0 & & 5.3 & $61 \%$ \\
\hline 5 & 23.0 & & 5.8 & $67 \%$ \\
\hline 6 & 22.5 & & 5.5 & $63 \%$ \\
\hline 7 & 22.3 & & 5.6 & $64 \%$ \\
\hline 8 & 22.0 & & 5.3 & $61 \%$ \\
\hline 9 & 22.0 & & 5.2 & $60 \%$ \\
\hline 10 & 22.1 & 7.42 & 4.7 & $54 \%$ \\
\hline
\end{tabular}

SAMPLE TREATMENT

\begin{tabular}{|c|c|c|c|c|c|c|c|c|}
\hline $\begin{array}{c}\text { Test } \\
\text { Day }\end{array}$ & $\begin{array}{c}\text { Cond. } \\
\text { umhos/cm }\end{array}$ & $\begin{array}{c}\mathrm{NH} 3 \\
\mathrm{mg} / \mathrm{L}\end{array}$ & $\begin{array}{c}\text { Temp. } \\
\mathrm{C}\end{array}$ & $\begin{array}{c}\mathrm{pH} \\
\text { units }\end{array}$ & $\begin{array}{c}\text { D.O. } \\
\mathrm{mg} / \mathrm{L}\end{array}$ & $\begin{array}{c}\text { D.O. } \\
\% \text { sat. }\end{array}$ & $\begin{array}{c}\text { Alk. } \\
\mathrm{mg} / \mathrm{L}\end{array}$ & $\begin{array}{c}\text { Hard. } \\
\mathrm{mg} / \mathrm{L}\end{array}$ \\
\hline Start & 90 & 0.83 & 22.9 & 6.15 & 6.6 & $76 \%$ & 22.4 & 38.0 \\
\hline 1 & & & 22.1 & & 5.8 & $67 \%$ & & \\
\hline 2 & & & 22.0 & & 5.2 & $60 \%$ & & \\
\hline 3 & & & 21.9 & & 5.3 & $60 \%$ & & \\
\hline 4 & & & 22.0 & & 5.2 & $60 \%$ & & \\
\hline 5 & & & 22.8 & & 5.2 & $60 \%$ & & \\
\hline 6 & & & 22.4 & & 5.7 & $66 \%$ & & \\
\hline 7 & & & 22.1 & & 5.6 & $64 \%$ & & \\
\hline 8 & & & 22.0 & & 5.4 & $62 \%$ & & \\
\hline 9 & & & 21.9 & & 5.3 & $60 \%$ & & \\
\hline 10 & 104 & 0.19 & 22.1 & 6.86 & 4.8 & $55 \%$ & 30.6 & 38.0 \\
\hline
\end{tabular}

\section{SURVIVAL AND GROWTH RESULTS}

\begin{tabular}{|l|r|r|r|r|r|r|}
\hline & \multicolumn{7}{|c|}{$\begin{array}{c}\text { \# Organisms } \\
\text { Live at } \\
\text { End }\end{array}$} & $\begin{array}{c}\text { Dead } \\
\text { at End }\end{array}$ & $\begin{array}{c}\% \\
\text { Survival }\end{array}$ & $\begin{array}{r}\text { Mean } \\
\text { Lenth } \\
\text { (mm) }\end{array}$ & $\begin{array}{r}\text { Mean } \\
\text { Length } \\
\text { (mm) }\end{array}$ \\
\hline A & 10 & 6 & 1 & $60 \%$ & 2.4 & 2.40 \\
\hline B & 10 & NA & NA & NA & NA & NA \\
\hline C & 10 & 7 & 0 & $70 \%$ & 2.18 & 2.18 \\
\hline D & 10 & 7 & 0 & $70 \%$ & 2.18 & 2.18 \\
\hline E & 10 & 10 & 0 & $100 \%$ & 2.17 & 2.17 \\
\hline F & 10 & 8 & 2 & $80 \%$ & 2.12 & 2.12 \\
\hline G & 10 & 9 & 0 & $90 \%$ & 2.13 & 2.13 \\
\hline H & 10 & 9 & 0 & $90 \%$ & 2.2 & 2.15 \\
\hline Mean & 10.0 & & & $80 \%$ & & 2.19 \\
\hline
\end{tabular}

\begin{tabular}{|l|r|r|r|r|r|r|}
\hline & \multicolumn{2}{|c|}{ \# Organisms } & Mean & $\begin{array}{r}\text { Mean } \\
\text { Length } \\
\end{array}$ \\
& Initial & $\begin{array}{r}\text { Live at } \\
\text { End }\end{array}$ & $\begin{array}{c}\text { Dead } \\
\text { at End }\end{array}$ & $\begin{array}{c}\% \\
\text { Survival }\end{array}$ & $\begin{array}{r}\text { Length } \\
(\mathrm{mm})\end{array}$ & $\begin{array}{r}\text { Length } \\
(\mathrm{mm})\end{array}$ \\
\hline $\mathrm{A}$ & 10 & 7 & 2 & $70 \%$ & 2.15 & 2.15 \\
\hline $\mathrm{B}$ & 10 & 8 & 1 & $80 \%$ & 2.18 & 2.18 \\
\hline $\mathrm{C}$ & 10 & 7 & 0 & $70 \%$ & 2.21 & 2.21 \\
\hline $\mathrm{D}$ & 10 & 9 & 1 & $90 \%$ & 2.22 & 2.22 \\
\hline $\mathrm{E}$ & 10 & 9 & 1 & $90 \%$ & 2.25 & 2.25 \\
\hline F & 10 & 7 & 0 & $70 \%$ & 2.24 & 2.24 \\
\hline G & 10 & 8 & 0 & $80 \%$ & 2.15 & 2.15 \\
\hline H & 10 & 10 & 0 & $100 \%$ & 2.1 & 2.11 \\
\hline Mean & 10.0 & & & $81 \%$ & & 2.19 \\
\hline
\end{tabular}




\section{DAY AMPHIPOD SEDIMENT TOXICITY TEST}

\begin{tabular}{|c|c|c|c|c|}
\hline \multirow{2}{*}{$\begin{array}{l}\text { Lab ID\# } \\
\text { T4445 }\end{array}$} & \multicolumn{2}{|r|}{ Westinghouse Savannah River Company } & Start Date: & $8-29-95$ \\
\hline & Sample Identification & WS: WSC FHB020 & End Date: & $9-8-95$ \\
\hline
\end{tabular}

\section{RESULTS}

\begin{tabular}{|l|r|}
\hline \multicolumn{2}{|c|}{$\begin{array}{l}\text { SURVIVAL DATA } \\
\text { Mean of Eight Replicates }\end{array}$} \\
\cline { 2 - 2 } & Mean $\%$ \\
\hline Control & $80.0 \%$ \\
\hline Sample & $81.3 \%$ \\
\hline
\end{tabular}

\begin{tabular}{|l|r|}
\hline \multicolumn{2}{|c|}{$\begin{array}{l}\text { GROWTH DATA } \\
\text { Mean Length }(\mathrm{mm})\end{array}$} \\
\hline Control & Mean \\
\hline Sample & 2.19 \\
\hline
\end{tabular}

\section{STATISTICAL ANALYSES}

\begin{tabular}{|c|c|c|c|}
\hline \multicolumn{4}{|c|}{ SURVIVAL DATA } \\
\hline Test for Normality: & & 0.89 & \\
\hline \multicolumn{3}{|c|}{ Critical value: } & Data are normal in distribution \\
\hline \multicolumn{4}{|c|}{ Test for Homogeneity of Variance: } \\
\hline & \multicolumn{2}{|c|}{ N/A } & \\
\hline & & \multicolumn{2}{|l|}{6.64} \\
\hline \multicolumn{4}{|c|}{ Test for Differences in Survival: $t$ Test } \\
\hline$t=$ & \multicolumn{3}{|c|}{-0.09} \\
\hline & & 1.75 & \\
\hline \multicolumn{4}{|c|}{$\begin{array}{l}\text { The sediment did not significantly reduce survival } \\
\text { of the test organisms. }\end{array}$} \\
\hline
\end{tabular}

\begin{tabular}{|c|c|c|}
\hline \multicolumn{3}{|c|}{ GROWTH DATA } \\
\hline Test for Normality: & $W=$ & 0.83 \\
\hline \multicolumn{3}{|c|}{ Critical value: } \\
\hline \multicolumn{3}{|c|}{ Data are not normal in distribution } \\
\hline \multicolumn{3}{|c|}{ Test for Homogeneity of Variance: } \\
\hline \multicolumn{3}{|c|}{3.74} \\
\hline \multicolumn{3}{|c|}{ Critical value: } \\
\hline \multicolumn{3}{|c|}{ Data are homogeneous in variance } \\
\hline \multicolumn{3}{|c|}{ Test for Differences in Growth: " $t$ "Test } \\
\hline \multicolumn{3}{|c|}{ Critical value: $\quad 1.94$} \\
\hline \multicolumn{3}{|c|}{$\begin{array}{l}\text { The scdiment did not significantly reduce growth } \\
\text { of the test organisms. }\end{array}$} \\
\hline
\end{tabular}

\section{COMMENTS}

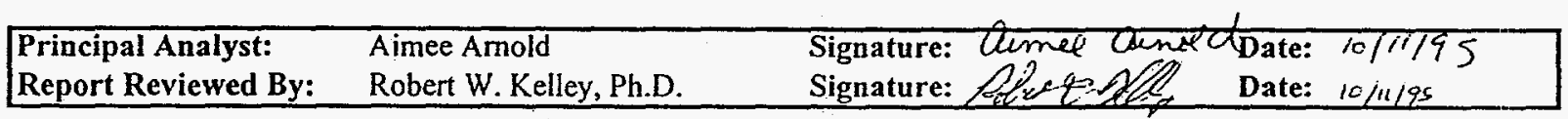

\section{CONCLUSIONS}

The test sediment does not adversely affect the survival and the growth of freshwater amphipods. 


\title{
10 DAY AMPHIPOD SEDIMENT TOXICITY TEST
}

\author{
EPA/600/R-94/024 Method 100.1
}

Test Organism: Hyallela azteca

Client: Westinghouse Savannah River Company Sample Identification: WSRC FHB 021

Test Start Date: $\quad 9-19-95$ 


\section{DAY AMPHIPOD SEDIMENT TOXICITY TEST}

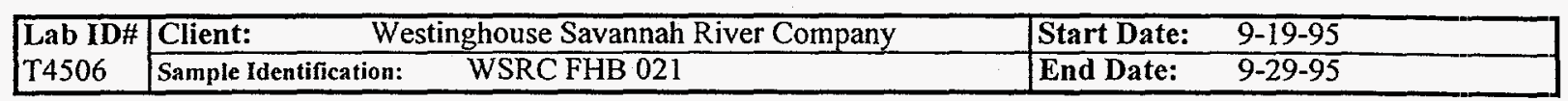

\section{Test System}

EPA/600/R-94/024 Method 100.1

The test was set as a Pass/Fail test with a control treatment and one sample treatment. Each treatment replicate consisted of $100 \mathrm{~g}$ of sediment and $175 \mathrm{~mL}$ of dilution water. There were eight replicates for each treatment. Ten test organisms were placed in each replicate. Sediment from a local creek was used in the control treatment. Test organisms were fed with YCT food. The test was conducted at a temperature of $23^{\circ} \mathrm{C}$ with ambient laboratory light conditions. Test vessels were $500 \mathrm{~mL}$ plastic beakers placed in a constant temperature incubator room.

Order: Amphipoda

Species: Hyallela azteca

Source: Aquatic Biosystems, $\mathrm{CO}$

Life Stage: $\quad .2-3 \mathrm{~mm}$

\% Mortality During $48 \mathrm{Hr}$ Prior to Test:: $\quad$ 0\%

Taxonomic Verification: RWK 9/19/95

Culturing: as received

Observations: Cultures healthy

Acclimation: None

\section{Control Sediment}

Source: S. Tyger River at Hwy. 14

Collection Date: 9-19-95

Preservation: kept at $0-4^{\circ} \mathrm{C}$ in plastic containers

Date/Time Added to Test Chambers: 9-19-95

Observations: silt

Collection Method: grab

Disposal:

\begin{tabular}{|l|}
\hline \multicolumn{3}{|c|}{ Food Preparation } \\
Source: Trout Chow (Glencoe Mills); Cerophyl (Sigma) \\
\begin{tabular}{ll} 
Purchase Date: & \multicolumn{1}{c|}{ Preparation Date: $3 / 23 / 95$} \\
Preservation: $\quad$ kept at $0-4^{\circ} \mathrm{C}$ & \\
Preparation Method: $\quad$ Trout Chow aerated 7 days \\
\end{tabular} \\
$\begin{array}{ll}\text { Feeding Rate: } & \text { Cerophyl, yeast aerated overnight } \\
\text { Hold Time: } & 5 \mathrm{~mL} / \text { day } / \text { replicate }\end{array}$ \\
\hline
\end{tabular}

\section{Dilution Water}

Type: Surface Water

Collection Date $8 / 28 / 95$ Initial Final

Alkalinity

Ammonia

Conductivity

Hardness

pH:

Preparation Method:

Grab sample

Evidence of Water Quality:

Good reproduction of Ceriodaphnia dubia cultures

\section{Test Sediment}

Source: WSRC FHB 021

Collection Date 9/11/95 Homogenized?: yes

Preservation: kept at $0-4^{\circ} \mathrm{C}$ in plastic containers

Date/Time Added to Test Chambers: $\quad 9 / 18 / 95$

Observations: organic

Collection Method: grab

Shipment: Overnight at $4^{\circ} \mathrm{C}$

\begin{tabular}{|l|}
\multicolumn{1}{c|}{ Test Chambers } \\
Type: Plastic $500 \mathrm{~mL}$ beakers \\
Sediment Volume: $\quad 100 \mathrm{~mL}$ \\
Sediment Depth $4 \mathrm{~cm}$ \\
$\begin{array}{l}\text { Overlying Water Volume: } \quad 175 \mathrm{~mL} \\
\text { Overlying Water Depth: } \quad 5 \mathrm{~cm} \\
\text { Aeration: none }\end{array}$ \\
\hline
\end{tabular}




\section{DAY AMPHIPOD SEDIMENT TOXICITY TEST}

\begin{tabular}{|l|l|l|ll|}
\hline Lab ID\# & Client: $\quad$ Westinghouse Savannah River Company & Start Date: & 9-19-95 \\
\cline { 2 - 5 } T4506 & Sample Identification: $\quad$ WSRC FHB 021 & End Date: & $9-29-95$ \\
\hline
\end{tabular}

\section{WATER CHEMISTRY TEST METHODS}

\begin{tabular}{|l|l|}
\hline Temperature: SM 18th Ed. 2550 & Alkalinity: SM 18th Ed. 2320 \\
Dissolved Oxygen: SM 18th Ed. 4500-O & Hardness: SM 18th Ed. 2340 \\
pH: SM 18th Ed. 4500-H+ & Conductivity: SM 18th Ed. 2510 \\
\hline
\end{tabular}

\section{WATER CHEMISTRY TEST DATA - OVERLYING WATER}

CONTROL TREATMENT

\begin{tabular}{|c|r|r|r|r|}
\hline Test Day & $\begin{array}{c}\text { Temp. } \\
\text { C }\end{array}$ & $\begin{array}{c}\mathrm{pH} \\
\text { units }\end{array}$ & $\begin{array}{c}\text { D.O. } \\
\mathrm{mg} / \mathrm{L}\end{array}$ & $\begin{array}{c}\text { D.O. } \\
\% \text { sat. }\end{array}$ \\
\hline Start & 22.8 & 7.38 & 7.8 & $90 \%$ \\
\hline 1 & 22.5 & & 6.9 & $79 \%$ \\
\hline 2 & 22.7 & & 6.6 & $76 \%$ \\
\hline 3 & 22.4 & & 7.2 & $83 \%$ \\
\hline 4 & 22.1 & & 8.1 & $93 \%$ \\
\hline 5 & 23.2 & & 8.0 & $93 \%$ \\
\hline 6 & 22.2 & & 6.8 & $78 \%$ \\
\hline 7 & 23.9 & & 5.9 & $69 \%$ \\
\hline 8 & 22.0 & & 6.4 & $74 \%$ \\
\hline 9 & 22.2 & & 6.2 & $71 \%$ \\
\hline 10 & 22.1 & 6.14 & 6.2 & $71 \%$ \\
\hline
\end{tabular}

SAMPLE TREATMENT

\begin{tabular}{|c|c|c|c|c|r|r|c|c|}
\hline $\begin{array}{c}\text { Test } \\
\text { Day }\end{array}$ & $\begin{array}{c}\text { Cond. } \\
\text { unhosicm }\end{array}$ & $\begin{array}{c}\mathrm{NH} 3 \\
\mathrm{mg} / \mathrm{L}\end{array}$ & $\begin{array}{c}\text { Temp. } \\
\mathrm{C}\end{array}$ & $\begin{array}{c}\mathrm{pH} \\
\text { units }\end{array}$ & $\begin{array}{c}\text { D.O. } \\
\mathrm{mg} / \mathrm{L}\end{array}$ & $\begin{array}{c}\text { D.O. } \\
\% \text { sat. }\end{array}$ & $\begin{array}{c}\text { Alk. } \\
\mathrm{mg} / \mathrm{L}\end{array}$ & $\begin{array}{c}\text { Hard. } \\
\mathrm{mg} / \mathrm{L}\end{array}$ \\
\hline Start & 32.4 & 0.586 & 22.4 & 6.16 & 5.8 & $67 \%$ & 6.1 & 4.0 \\
\hline 1 & & & 22.5 & & 7.0 & $80 \%$ & & \\
\hline 2 & & & 22.7 & & 7.2 & $83 \%$ & & \\
\hline 3 & & & 22.5 & & 7.6 & $87 \%$ & & \\
\hline 4 & & & 22.3 & & 8.2 & $94 \%$ & & \\
\hline 5 & & & 23.4 & & 7.8 & $91 \%$ & & \\
\hline 6 & & & 22.1 & & 8.0 & $92 \%$ & & \\
\hline 7 & & & 23.9 & & 6.1 & $71 \%$ & & \\
\hline 8 & & & 22.0 & & 7.6 & $87 \%$ & & \\
\hline 9 & & & 22.3 & & 7.0 & $80 \%$ & & \\
\hline 10 & 32 & 0.216 & 22.1 & 5.92 & 6.0 & $69 \%$ & 8.1 & 6.0 \\
\hline
\end{tabular}

\section{SURVIVAL AND GROWTH RESULTS}

CONTROL TREATMENT

SAMPLE TREATMENT

\begin{tabular}{|c|c|c|c|c|c|c|}
\hline & \multicolumn{3}{|c|}{ \# Organisms } & \multirow[b]{2}{*}{$\begin{array}{c}\% \\
\text { Survival }\end{array}$} & \multirow{2}{*}{$\begin{array}{l}\text { Mean } \\
\text { Length } \\
(\mathrm{mm})\end{array}$} & \multirow{2}{*}{$\begin{array}{r}\text { Mean } \\
\text { Length } \\
(\mathrm{mm})\end{array}$} \\
\hline & Initial & $\begin{array}{l}\text { Live at } \\
\text { End }\end{array}$ & $\begin{array}{l}\text { Dead } \\
\text { at End }\end{array}$ & & & \\
\hline $\bar{A}$ & 10 & 9 & 0 & $90 \%$ & 2.1 & 2.10 \\
\hline B & 10 & 10 & 0 & $100 \%$ & 2.04 & 2.04 \\
\hline $\mathrm{C}$ & 10 & 9 & 0 & $90 \%$ & 2.04 & 2.04 \\
\hline$\overline{\mathrm{D}}$ & 10 & 10 & 0 & $100 \%$ & 2.02 & 2.02 \\
\hline $\mathrm{E}$ & 10 & 9 & 0 & $90 \%$ & 2.04 & 2.04 \\
\hline $\mathrm{F}$ & 10 & 10 & 0 & $100 \%$ & 2 & 2.00 \\
\hline$G$ & 10 & 10 & 0 & $100 \%$ & 2.04 & 2.04 \\
\hline $\mathrm{H}$ & 10 & 10 & 0 & $100 \%$ & 2.1 & 2.09 \\
\hline Mean & 10.0 & & & $96 \%$ & & 2.05 \\
\hline
\end{tabular}

\begin{tabular}{|c|c|c|c|c|c|c|}
\hline & \multicolumn{3}{|c|}{ \# Organisms } & \multirow[b]{2}{*}{$\begin{array}{c}\% \\
\text { Survival }\end{array}$} & \multirow{2}{*}{$\begin{array}{l}\text { Mean } \\
\text { Length } \\
(\mathrm{mm})\end{array}$} & \multirow{2}{*}{$\begin{array}{r}\text { Mean } \\
\text { Length } \\
(\mathrm{mm})\end{array}$} \\
\hline & Initial & $\begin{array}{l}\text { Live at } \\
\text { End }\end{array}$ & $\begin{array}{l}\text { Dead } \\
\text { at End }\end{array}$ & & & \\
\hline$A$ & 10 & 9 & 0 & $90 \%$ & 2.25 & 2.25 \\
\hline $\bar{B}$ & $\overline{10}$ & 7 & 0 & $70 \%$ & 2.15 & 2.15 \\
\hline $\bar{C}$ & 10 & 7 & 0 & $70 \%$ & 2.01 & 2.01 \\
\hline D & 10 & NA & $\overline{N A}$ & $\mathrm{NA}$ & NA & $\mathrm{NA}$ \\
\hline $\mathrm{E}$ & 10 & 6 & 0 & $60 \%$ & 2.01 & 2.01 \\
\hline$\overline{\mathrm{F}}$ & 10 & 8 & 0 & $80 \%$ & 1.75 & 1.75 \\
\hline $\mathrm{G}$ & 10 & 6 & 0 & $60 \%$ & 2.01 & 2.01 \\
\hline $\mathrm{H}$ & 10 & 8 & 0 & $80 \%$ & 2.1 & 2.12 \\
\hline Mean & 10.0 & & & $73 \%$ & & 2.04 \\
\hline
\end{tabular}




\section{DAY AMPHIPOD SEDIMENT TOXICITY TEST}

\begin{tabular}{|l|ll|ll|}
\hline Lab ID\# & Client: & \multicolumn{1}{|c|}{ Westinghouse Savannah River Company } & Start Date: & $9-19-95$ \\
\cline { 2 - 5 } T4506 & Sample Identification: & WSRC FHB 021 & End Date: & $9-29-95$ \\
\hline
\end{tabular}

\section{RESULTS}

\begin{tabular}{|l|r|}
\hline \multicolumn{2}{|c|}{$\begin{array}{l}\text { SURVIVAL DATA } \\
\text { Mean of Eight Replicates }\end{array}$} \\
\cline { 2 - 2 } & Mean \% \\
\hline Control & $96.3 \%$ \\
\hline Sample & $72.9 \%$ \\
\hline
\end{tabular}

\begin{tabular}{|c|c|c|}
\hline \multicolumn{3}{|c|}{ GROWTH DATA } \\
\hline & & Mean \\
\hline Control & & 2.05 \\
\hline Sample & & 2.04 \\
\hline
\end{tabular}

\section{STATISTICAL ANALYSES}

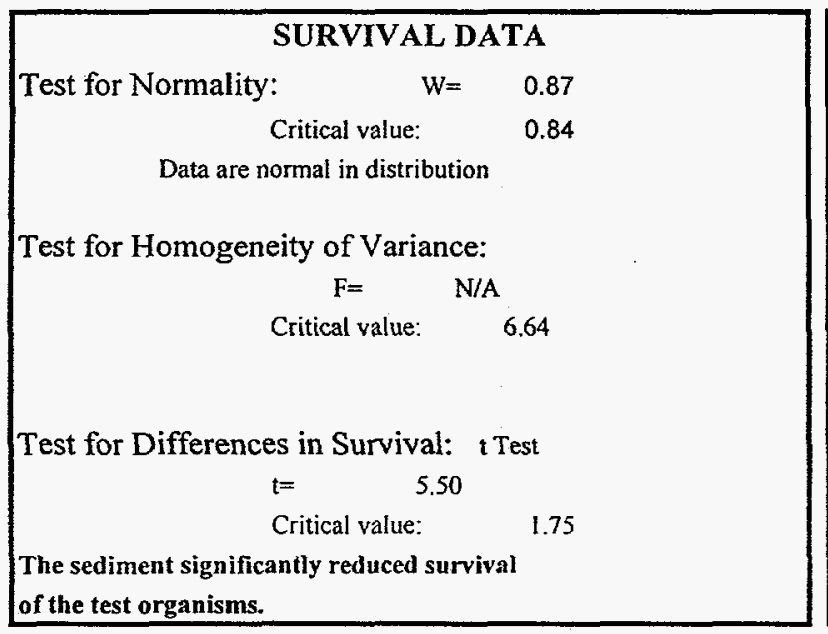

\begin{tabular}{|c|c|}
\hline \multicolumn{2}{|c|}{ GROWTH DATA } \\
\hline Test for Normality: & 0.83 \\
\hline Critical value: & 0.84 \\
\hline \multicolumn{2}{|c|}{ Data are not normal in distribution } \\
\hline \multicolumn{2}{|l|}{ Test for Homogeneity of Variance: } \\
\hline \multicolumn{2}{|c|}{$F=\quad 22,37$} \\
\hline \multicolumn{2}{|c|}{ Critical value: $\quad 6.635$} \\
\hline \multicolumn{2}{|c|}{ Data are not homogeneous in variance } \\
\hline \multirow{2}{*}{$\begin{array}{l}\text { Test for Differences in Growth: } \\
\qquad \begin{array}{r}t=0.06 \\
\text { Critical value: }\end{array}\end{array}$} & \\
\hline & 1.94 \\
\hline \multicolumn{2}{|c|}{$\begin{array}{l}\text { The sediment did not significantly reduce growth } \\
\text { of the test organisms. }\end{array}$} \\
\hline
\end{tabular}

\section{COMMENTS}

\begin{tabular}{|llll|}
\hline $\begin{array}{l}\text { Principal Analyst: } \\
\text { Report Reviewed By: }\end{array}$ & Aimee Arnold & Robert W. Kelley, Ph.D. & Signature: Uninee Uhold Date: $10 / 1395$ \\
\hline
\end{tabular}

\section{CONCLUSIONS}

The test sediment does adversely affect the survival but not the growth of freshwater amphipods. 


\title{
10 DAY AMPHIPOD SEDIMENT TOXICITY TEST
}

\author{
EPA/600/R-94/024 Method 100.1 \\ Test Organism: Hyallela azteca
}

Client: Westinghouse Savannah River Company Sample Identification: WSRC FHB 022

Test Start Date: $\quad 9-30-95$ 


\section{DAY AMPHIPOD SEDIMENT TOXICITY TEST}

\begin{tabular}{|c|c|c|c|}
\hline \multirow{2}{*}{$\begin{array}{l}\text { Lab ID\# } \\
\text { T4450 }\end{array}$} & Client: & Vestinghouse Savannah River Company & Start Date: \\
\hline & Sample Identification & WSRC FHB 022 & End Date: \\
\hline
\end{tabular}

\section{Test System}

EPA/600/R-94/024 Method 100.1

The test was set as a Pass/Fail test with a control treatment and one sample treatment. Each treatment replicate consisted of $100 \mathrm{~g}$ of sediment and $175 \mathrm{~mL}$ of dilution water. There were eight replicates for each treatment. Ten test organisms were placed in each replicate. Sediment from a local river was used in the control treatment. Test organisms were fed with YCT food. The test was conducted at a temperature of $23^{\circ} \mathrm{C}$ with ambient laboratory light conditions. Test vessels were $500 \mathrm{~mL}$ plastic beakers placed in a constant temperature incubator room.

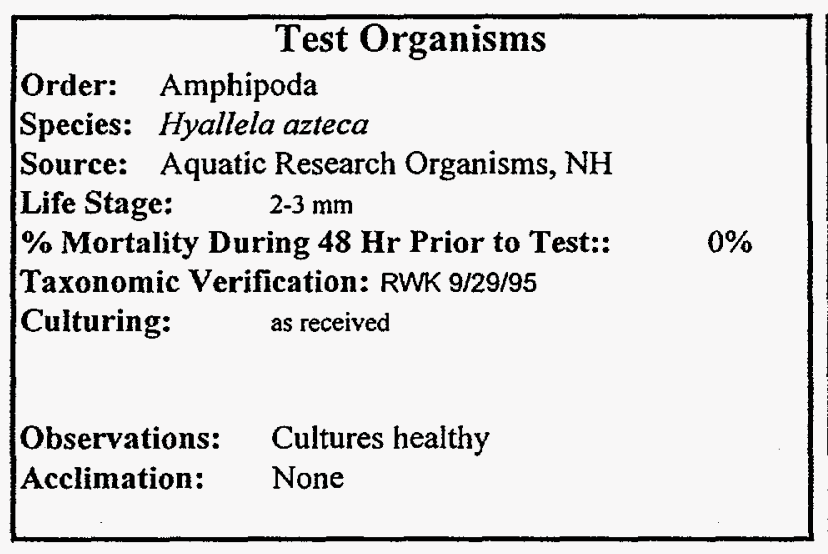

Control Sediment
Source: South Tyger river @ Hwy. 14
Collection Date: $9-19-95$
Preservation: kept at $0-4^{\circ} \mathrm{C}$ in plastic containers
Date/Time Added to Test Chambers: 9-29-95
Observations: silt
Collection Method: grab
Disposal:

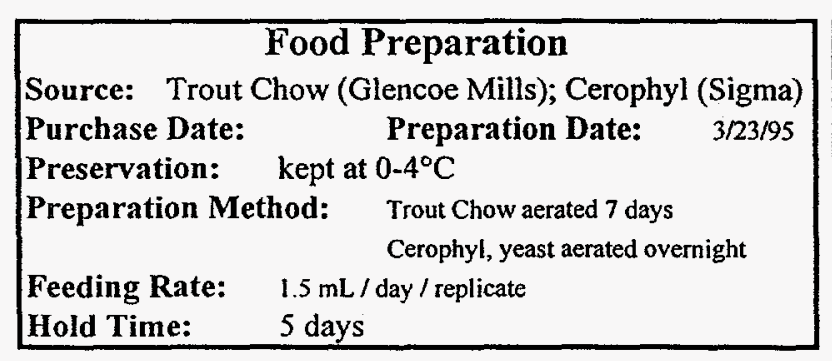

\begin{tabular}{|c|c|c|}
\hline \multicolumn{3}{|c|}{ Dilution Water } \\
\hline Surface Water & & \\
\hline \multicolumn{3}{|l|}{ Collection Date $9 / 29 / 95$} \\
\hline Alkalinity & 16.2 & $16.2 \mathrm{mg} / \mathrm{L}$ \\
\hline Ammonia & 0.742 & $0.518 \mathrm{mg} / \mathrm{L}$ \\
\hline Conductivity & 51.9 & 46.4 umhos/cm \\
\hline Hardness & 16 & $16 \mathrm{mg} / \mathrm{L}$ \\
\hline$p H:$ & 7.07 & 7.42 units \\
\hline \multicolumn{3}{|l|}{$\begin{array}{l}\text { Preparation Method: } \\
\text { Grab sample }\end{array}$} \\
\hline \multicolumn{3}{|c|}{$\begin{array}{l}\text { Evidence of Water Quality: } \\
\text { Good reproduction of Ceriodaphnia dubia cultures }\end{array}$} \\
\hline
\end{tabular}

\begin{tabular}{l} 
Test Sediment \\
Source: WSRC FHB 022 \\
Collection Date $8 / 22 / 95 \quad$ Homogenized?: yes \\
Preservation: kept at $0-4^{\circ} \mathrm{C}$ in plastic containers \\
Date/Time Added to Test Chambers: $9 / 29 / 95$ \\
Observations: organic \\
Collection Method: grab \\
Shipment: Overnight at $4^{\circ} \mathrm{C}$ \\
\hline
\end{tabular}

\begin{tabular}{|l|}
\multicolumn{1}{c|}{ Test Chambers } \\
Type: Plastic $500 \mathrm{~mL}$ beakers \\
Sediment Volume: $\quad 100 \mathrm{~mL}$ \\
Sediment Depth $4 \mathrm{~cm}$ \\
$\begin{array}{l}\text { Overlying Water Volume: } \quad 175 \mathrm{~mL} \\
\text { Overlying Water Depth: }\end{array} \quad 5 \mathrm{~cm}$ \\
Aeration: none
\end{tabular}




\section{DAY AMPHIPOD SEDIMENT TOXICITY TEST}

\begin{tabular}{|l|ll|ll|}
\hline Lab ID\# & Client: & \multicolumn{1}{|c|}{ Westinghouse Savannah River Company } & Start Date: & $9-30-95$ \\
\cline { 2 - 5 } T4450 & Sample Identification: & WSRC FHB 022 & End Date: & $10-10-95$ \\
\hline
\end{tabular}

\section{WATER CHEMISTRY TEST METHODS}

\begin{tabular}{|l|l|}
\hline Temperature: SM 18th Ed. 2550 & Alkalinity: SM 18th Ed. 2320 \\
Dissolved Oxygen: SM 18th Ed. 4500-O & Hardness: SM 18th Ed. 2340 \\
pH: SM 18th Ed. 4500-H+ & Conductivity: SM 18th Ed. 2510 \\
\hline
\end{tabular}

\section{WATER CHEMISTRY TEST DATA - OVERLYING WATER}

CONTROL TREATMENT

\begin{tabular}{|c|r|r|r|r|}
\hline Test Day & $\begin{array}{c}\text { Temp. } \\
\text { C }\end{array}$ & $\begin{array}{c}\text { pH } \\
\text { units }\end{array}$ & $\begin{array}{c}\text { D.O. } \\
\mathrm{mg} / \mathrm{L}\end{array}$ & $\begin{array}{c}\text { D.O. } \\
\% \text { sat. }\end{array}$ \\
\hline Start & 22.5 & 6.67 & 7.0 & $80 \%$ \\
\hline 1 & 23.2 & & 6.4 & $74 \%$ \\
\hline 2 & 22.6 & & 6.8 & $78 \%$ \\
\hline 3 & 22.7 & & 5.7 & $66 \%$ \\
\hline 4 & 22.9 & & 5.5 & $63 \%$ \\
\hline 5 & 23.1 & & 5.6 & $65 \%$ \\
\hline 6 & 22.2 & & 5.6 & $64 \%$ \\
\hline 7 & 22.3 & & 8.3 & $95 \%$ \\
\hline 8 & 23.5 & & 5.4 & $63 \%$ \\
\hline 9 & 23.7 & & 7.2 & $84 \%$ \\
\hline 10 & 22.0 & 6.38 & 6.2 & $71 \%$ \\
\hline
\end{tabular}

SAMPLE TREATMENT

\begin{tabular}{|c|c|c|c|c|r|r|c|c|}
\hline $\begin{array}{c}\text { Test } \\
\text { Day }\end{array}$ & $\begin{array}{c}\text { Cond. } \\
\text { umbos/cm }\end{array}$ & $\begin{array}{c}\text { NH3 } \\
\mathrm{mg} / \mathrm{L}\end{array}$ & $\begin{array}{c}\text { Temp. } \\
\mathrm{C}\end{array}$ & $\begin{array}{c}\mathrm{pH} \\
\text { units }\end{array}$ & $\begin{array}{c}\text { D.O. } \\
\mathrm{mg} / \mathrm{L}\end{array}$ & $\begin{array}{c}\text { D.O. } \\
\% \text { sat. }\end{array}$ & $\begin{array}{c}\text { Alk. } \\
\mathrm{mg} / \mathrm{L}\end{array}$ & $\begin{array}{c}\text { Hard. } \\
\mathrm{mg} / \mathrm{L}\end{array}$ \\
\hline Start & 81.6 & 1.89 & 22.2 & 6.20 & 5.9 & $68 \%$ & 6.1 & 46.0 \\
\hline 1 & & & 22.7 & & 5.2 & $60 \%$ & & \\
\hline 2 & & & 22.2 & & 7.2 & $83 \%$ & & \\
\hline 3 & & & 22.3 & & 5.2 & $60 \%$ & & \\
\hline 4 & & & 22.8 & & 5.0 & $57 \%$ & & \\
\hline 5 & & & 22.9 & & 5.4 & $62 \%$ & & \\
\hline 6 & & & 22.2 & & 6.1 & $70 \%$ & & \\
\hline 7 & & & 22.2 & & 6.1 & $70 \%$ & & \\
\hline 8 & & & 23.0 & & 5.8 & $67 \%$ & & \\
\hline 9 & & & 23.1 & & 6.0 & $70 \%$ & & \\
\hline 10 & 49.3 & 0.871 & 22.1 & 5.15 & 5.8 & $67 \%$ & 14.2 & 40.0 \\
\hline
\end{tabular}

\section{SURVIVAL AND GROWTH RESULTS}

CONTROL TREATMENT

SAMPLE TREATMENT

\begin{tabular}{|c|c|c|c|c|c|c|c|c|c|c|c|c|c|}
\hline & \multicolumn{3}{|c|}{ \# Organisms } & \multirow[b]{2}{*}{$\begin{array}{c}\% \\
\text { Survival }\end{array}$} & \multirow{2}{*}{$\begin{array}{c}\text { Mean } \\
\text { Length } \\
(\mathrm{mm})\end{array}$} & \multirow{2}{*}{$\begin{array}{r}\text { Mean } \\
\text { Length } \\
(\mathrm{mm})\end{array}$} & & \multicolumn{3}{|c|}{ \# Organisms } & \multirow[b]{2}{*}{$\begin{array}{c}\% \\
\text { Survival }\end{array}$} & \multirow{2}{*}{$\begin{array}{l}\text { Mean } \\
\text { Length } \\
(\mathrm{mm})\end{array}$} & \multirow{2}{*}{$\begin{array}{r}\text { Mean } \\
\text { Length } \\
(\mathrm{mm})\end{array}$} \\
\hline & Initial & $\begin{array}{c}\text { Live at } \\
\text { End }\end{array}$ & $\begin{array}{l}\text { Dead } \\
\text { at End }\end{array}$ & & & & & Initial & $\begin{array}{c}\text { Live at } \\
\text { End }\end{array}$ & $\begin{array}{l}\text { Dead } \\
\text { at End }\end{array}$ & & & \\
\hline $\bar{A}$ & 10 & 8 & 0 & $80 \%$ & 1.57 & 1.57 & $\bar{A}$ & 10 & 0 & 0 & $0 \%$ & $\mathrm{NA}$ & NA \\
\hline $\mathrm{B}$ & 10 & 10 & 0 & $100 \%$ & 1.58 & 1.58 & B & 10 & 1 & 1 & $10 \%$ & 1.93 & 1.93 \\
\hline $\bar{C}$ & 10 & 8 & 0 & $80 \%$ & 1.35 & 1.35 & C & 10 & 0 & 1 & $0 \%$ & NA & NA \\
\hline $\mathrm{D}$ & 10 & 10 & 0 & $100 \%$ & 1.56 & 1.56 & $D$ & 10 & 1 & 0 & $10 \%$ & 1.72 & 1.72 \\
\hline $\mathrm{E}$ & 10 & 9 & 0 & $90 \%$ & 1.68 & 1.68 & $\bar{E}$ & 10 & 0 & 1 & $0 \%$ & NA & $\overline{\mathrm{NA}}$ \\
\hline $\mathrm{F}$ & 10 & 10 & 0 & $100 \%$ & 1.58 & 1.58 & $F$ & 10 & 0 & 1 & $0 \%$ & $\overline{\mathrm{NA}}$ & $\overline{\mathrm{NA}}$ \\
\hline $\mathrm{G}$ & 10 & 10 & 0 & $100 \%$ & 1.6 & 1.60 & $G$ & 10 & 0 & 0 & $0 \%$ & $\overline{\mathrm{NA}}$ & $\mathrm{NA}$ \\
\hline $\mathrm{H}$ & 10 & 10 & 0 & $100 \%$ & 1.6 & 1.60 & $\mathrm{H}$ & 10 & 0 & 5 & $0 \%$ & $\mathrm{NA}$ & $\mathrm{NA}$ \\
\hline Mean & 10.0 & & & $94 \%$ & & 1.57 & Mean & 10.0 & & & $3 \%$ & & 1.83 \\
\hline
\end{tabular}




\section{DAY AMPHIPOD SEDIMENT TOXICITY TEST}

\begin{tabular}{|c|c|c|c|c|}
\hline \multirow{2}{*}{$\begin{array}{l}\text { Lab ID\# } \\
\text { T4450 }\end{array}$} & Client: & Jestinghouse Savannah River Company & Start Date: & $9-30-95$ \\
\hline & Sample Identification: & WSRC FHB 022 & End Date: & $10-10-95$ \\
\hline
\end{tabular}

\section{RESULTS}

\begin{tabular}{|c|c|c|c|c|}
\hline \multicolumn{2}{|c|}{$\begin{array}{l}\text { SURVIVAL DATA } \\
\text { Mean of Eight Replicates }\end{array}$} & \multirow{2}{*}{\multicolumn{2}{|c|}{$\begin{array}{l}\text { GROWTH DATA } \\
\text { Mean Length }(\mathrm{mm})\end{array}$}} & \\
\hline & Mean $\%$ & & & Mean \\
\hline Control & $93.8 \%$ & Control & & 1.57 \\
\hline Sample & $2.5 \%$ & Sample & & 1.83 \\
\hline
\end{tabular}

\section{STATISTICAL ANALYSES}

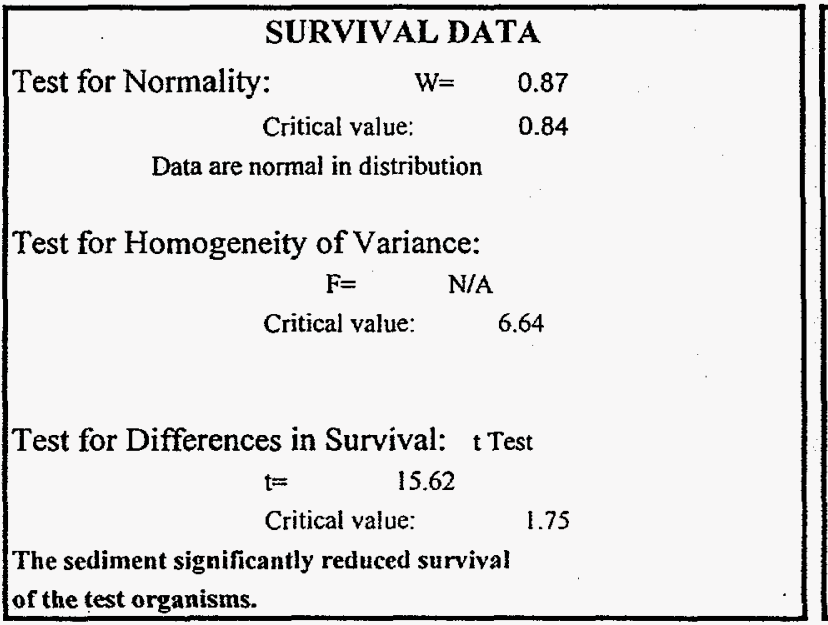

\begin{tabular}{|c|c|c|}
\hline \multicolumn{3}{|c|}{ GROWTH DATA } \\
\hline Test for Normality: & $W=$ & 0.78 \\
\hline \multicolumn{2}{|c|}{ Critical value: } & 0.84 \\
\hline \multicolumn{3}{|c|}{ Data are not normal in distribution } \\
\hline \multicolumn{3}{|c|}{ Test for Homogeneity of Variance: } \\
\hline \multicolumn{3}{|c|}{2.47} \\
\hline \multirow{2}{*}{\multicolumn{2}{|c|}{ Critical value: }} & 6.635 \\
\hline & & Data are homogeneous in variance \\
\hline \multicolumn{2}{|c|}{ Test for Differences in Growth: } & $" \mathrm{t}^{\mathrm{n}}$ Test \\
\hline \multicolumn{2}{|c|}{ Critical value: } & 1.94 \\
\hline
\end{tabular}

\section{COMMENTS}

\begin{tabular}{|c|c|c|c|}
\hline $\begin{array}{l}\text { Principal Analyst: } \\
\text { Report Reviewed By: }\end{array}$ & $\begin{array}{l}\text { Aimee Arnold } \\
\text { Robert W. Kelley, Ph.D. }\end{array}$ & $\begin{array}{l}\text { Signature: Aumiee Qunod } \\
\text { Signature: } A l d \text { to }\end{array}$ & $\begin{array}{l}\text { Date: } 10 / 13 / 95 \\
\text { Date: } 10 / 13 / 45\end{array}$ \\
\hline
\end{tabular}

\section{CONCLUSIONS}

The test sediment does adversely affect the survival but not the growth of freshwater amphipods. 


\title{
10 DAY AMPHIPOD SEDIMENT TOXICITY TEST
}

\author{
EPA/600/R-94/024 Method 100.1 \\ Test Organism: Hyallela azteca
}

Client: Westinghouse Savannah River Company Sample Identification: WSRC FHB 023

Test Start Date: $\quad 9-30-95$ 


\section{DAY AMPHIPOD SEDIMENT TOXICITY TEST}

\begin{tabular}{|c|c|c|c|}
\hline \multirow{2}{*}{$\begin{array}{l}\text { Lab ID\# } \\
\text { T4452 }\end{array}$} & Client: & Vestinghouse Savannah River Company & Start Date: \\
\hline & Sample Identification: & WSRC FHB 023 & End Date: \\
\hline
\end{tabular}

\section{Test System}

\section{EPA/600/R-94/024 Method 100.1}

The test was set as a Pass/Fail test with a control treatment and one sample treatment. Each treatment replicate consisted of $100 \mathrm{~g}$ of sediment and $175 \mathrm{~mL}$ of dilution water. There were eight replicates for each treatment. Ten test organisms were placed in each replicate. Sediment from a local river was used in the control treatment. Test: organisms were fed with YCT food. The test was conducted at a temperature of $23^{\circ} \mathrm{C}$ with ambient laboratory light conditions. Test vessels were $500 \mathrm{~mL}$ plastic beakers placed in a constant temperature incubator room.

\section{Order: Amphipoda}

\section{Test Organisms}

Species: Hyallela azteca

Source: Aquatic Research Organisms, NH

Life Stage: $\quad 2-3 \mathrm{~mm}$

\% Mortality During 48 Hr Prior to Test:: . 0\%

Taxonomic Verification: RWK 9/29/95

Culturing: as received

Observations: Cultures healthy

Acclimation: None

\section{Control Sediment}

Source: South Tyger river (a) Hwy. 14

Collection Date: 9-19-95

Preservation: kept at $0-4^{\circ} \mathrm{C}$ in plastic containers

Date/Time Added to Test Chambers: $\quad 9-29-95$

Observations: silt

Collection Method: grab

Disposal:

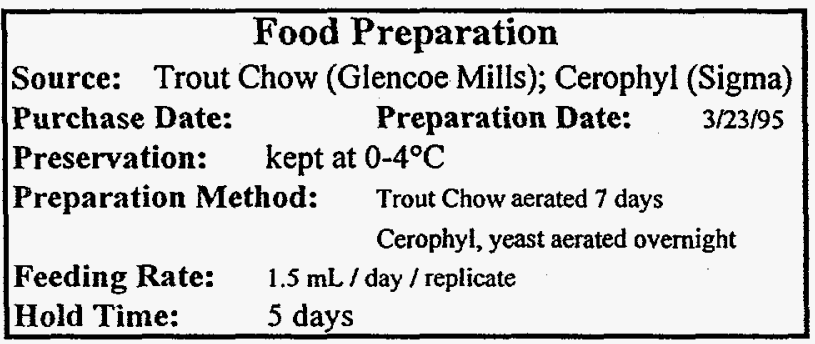

\section{Dilution Water}

Type: Surface Water Collection Date $9 / 29 / 95$

$$
\text { Alkalinity }
$$

Ammonia

Conductivity

Hardness

pH:

Preparation Method:

Grab sample

Evidence of Water Quality:

Good reproduction of Ceriodaphnia dubia cultures

Initial Final

$16.2 \quad 16.2 \mathrm{mg} / \mathrm{L}$

$0.7420 .518 \mathrm{mg} / \mathrm{L}$

$51.9 \quad 46.4$ unhos/cm

$16 \quad 16 \mathrm{mg} / \mathrm{L}$

$7.07 \quad 7.42$ units

\section{Test Sediment}

Source: WSRC FHB 023

Collection Date $8 / 22 / 95$ Homogenized?: yes

Preservation: kept at $0-4^{\circ} \mathrm{C}$ in plastic containers

Date/Time Added to Test Chambers: 9/29/95

Observations: organic

Collection Method: grab

Shipment: $\quad$ Overnight at $4^{\circ} \mathrm{C}$

\begin{tabular}{|c|c|}
\hline $\begin{array}{l}\text { Test Ch } \\
\text { Type: Plastic } 500 \mathrm{~mL} \text { be } \\
\text { Sediment Volume: } \\
\text { Sediment Depth } 4 \mathrm{~cm} \\
\text { Overlying Water Volume: } \\
\text { Overlying Water Depth: } \\
\text { Aeration: none }\end{array}$ & $\begin{array}{l}\text { lbers } \\
\text { rs } \\
\text { L } \\
175 \mathrm{~mL} \\
5 \mathrm{~cm}\end{array}$ \\
\hline
\end{tabular}




\section{DAY AMPHIPOD SEDIMENT TOXICITY TEST}

\begin{tabular}{|l|l|l|l|}
\hline Lab ID\# & Client: $\quad$ Westinghouse Savannah River Company & Start Date: & $9-30-95$ \\
\cline { 2 - 4 } T4452 & Sample Identification: $\quad$ WSRC FHB 023 & End Date: & $10-10-95$ \\
\hline
\end{tabular}

\section{WATER CHEMISTRY TEST METHODS}

Temperature: SM 18th Ed. 2550

Dissolved Oxygen: SM 18th Ed. 4500-O

pH: SM 18th Ed. 4500-H+
Alkalinity: SM 18th Ed. 2320

Hardness: SM 18th Ed. 2340

Conductivity: SM 18th Ed. 2510

WATER CHEMISTRY TEST DATA - OVERLYING WATER

CONTROL TREATMENT

\begin{tabular}{|c|r|r|r|r|}
\hline Test Day & $\begin{array}{c}\text { Temp. } \\
\text { C }\end{array}$ & $\begin{array}{c}\mathrm{pH} \\
\text { units }\end{array}$ & $\begin{array}{c}\text { D.O. } \\
\mathrm{mg} / \mathrm{L}\end{array}$ & $\begin{array}{c}\text { D.O. } \\
\% \text { sat. }\end{array}$ \\
\hline Start & 22.5 & 6.67 & 7.0 & $80 \%$ \\
\hline 1 & 23.2 & & 6.4 & $74 \%$ \\
\hline 2 & 22.6 & & 6.8 & $78 \%$ \\
\hline 3 & 22.7 & & 5.7 & $66 \%$ \\
\hline 4 & 22.9 & & 5.5 & $63 \%$ \\
\hline 5 & 23.1 & & 5.6 & $65 \%$ \\
\hline 6 & 22.2 & & 5.6 & $64 \%$ \\
\hline 7 & 22.3 & & 8.3 & $95 \%$ \\
\hline 8 & 23.5 & & 5.4 & $63 \%$ \\
\hline 9 & 23.7 & & 7.2 & $84 \%$ \\
\hline 10 & 22.0 & 6.38 & 6.2 & $71 \%$ \\
\hline
\end{tabular}

SAMPLE TREATMENT

\begin{tabular}{|c|c|c|c|c|r|r|c|c|}
\hline $\begin{array}{c}\text { Test } \\
\text { Day }\end{array}$ & $\begin{array}{c}\text { Cond. } \\
\text { umhos } / \mathrm{cm}\end{array}$ & $\begin{array}{c}\mathrm{NH} 3 \\
\mathrm{mg} / \mathrm{L}\end{array}$ & $\begin{array}{c}\text { Temp. } \\
\mathrm{C}\end{array}$ & $\begin{array}{c}\mathrm{pH} \\
\text { units }\end{array}$ & $\begin{array}{c}\text { D.O. } \\
\mathrm{mg} / \mathrm{L}\end{array}$ & $\begin{array}{c}\text { D.O. } \\
\% \text { sat. }\end{array}$ & $\begin{array}{c}\text { Alk. } \\
\mathrm{mg} / \mathrm{L}\end{array}$ & $\begin{array}{c}\text { Hard. } \\
\mathrm{mg} / \mathrm{L}\end{array}$ \\
\hline Start & 25.5 & 0.518 & 22.2 & 6.72 & 7.3 & $84 \%$ & 2.0 & 26.0 \\
\hline 1 & & & 23.3 & & 5.6 & $65 \%$ & & \\
\hline 2 & & & 22.2 & & 7.2 & $83 \%$ & & \\
\hline 3 & & & 22.4 & & 6.1 & $70 \%$ & & \\
\hline 4 & & & 22.8 & & 6.0 & $69 \%$ & & \\
\hline 5 & & & 23.0 & & 6.1 & $71 \%$ & & \\
\hline 6 & & & 22.1 & & 6.2 & $71 \%$ & & \\
\hline 7 & & & 22.1 & & 6.2 & $71 \%$ & & \\
\hline 8 & & & 23.4 & & 6.4 & $74 \%$ & & \\
\hline 9 & & & 23.4 & & 6.9 & $80 \%$ & & \\
\hline 10 & 23.6 & 0.653 & 22.1 & 6.23 & 6.8 & $78 \%$ & 4.1 & 32.0 \\
\hline
\end{tabular}

SURVIVAL AND GROWTH RESULTS

CONTROL TREATMENT

\begin{tabular}{|c|c|c|c|c|c|c|}
\hline & \multicolumn{3}{|c|}{ \# Organisms } & \multirow[b]{2}{*}{$\begin{array}{c}\% \\
\text { Survival }\end{array}$} & \multirow{2}{*}{$\begin{array}{l}\text { Mean } \\
\text { Length } \\
(\mathrm{mm})\end{array}$} & \multirow{2}{*}{$\begin{array}{l}\text { Mean } \\
\text { Length } \\
(\mathrm{mm})\end{array}$} \\
\hline & Initial & $\begin{array}{l}\text { Live at } \\
\text { End }\end{array}$ & $\begin{array}{c}\text { Dead } \\
\text { at End }\end{array}$ & & & \\
\hline $\mathrm{A}$ & 10 & 8 & 0 & $80 \%$ & 1.57 & 1.57 \\
\hline $\mathrm{B}$ & 10 & 10 & 0 & $100 \%$ & 1.58 & 1.58 \\
\hline C & 10 & 8 & 0 & $80 \%$ & 1.35 & 1.35 \\
\hline $\mathrm{D}$ & 10 & 10 & 0 & $100 \%$ & 1.56 & 1.56 \\
\hline $\mathrm{E}$ & 10 & 9 & 0 & $90 \%$ & 1.68 & 1.68 \\
\hline $\mathrm{F}$ & 10 & 10 & 0 & $100 \%$ & 1.58 & 1.58 \\
\hline $\bar{G}$ & 10 & 10 & 0 & $100 \%$ & 1.6 & 1.60 \\
\hline $\mathrm{H}$ & 10 & 10 & 0 & $100 \%$ & 1.6 & 1.60 \\
\hline Mean & 10.0 & & & $94 \%$ & & 1.57 \\
\hline
\end{tabular}

\begin{tabular}{|c|c|c|c|c|c|c|}
\hline & & rganis & & & Mean & Mean \\
\hline & Initial & $\begin{array}{l}\text { Live at } \\
\text { End }\end{array}$ & $\begin{array}{l}\text { Dead } \\
\text { at End }\end{array}$ & $\begin{array}{c}\% \\
\text { Survival }\end{array}$ & $\begin{array}{l}\text { Length } \\
(\mathrm{mm})\end{array}$ & $\begin{array}{r}\text { Length } \\
(\mathrm{mm})\end{array}$ \\
\hline A & 10 & 2 & 0 & $20 \%$ & 1.51 & 1.51 \\
\hline B & 10 & 0 & 0 & $0 \%$ & NA & $\mathrm{NA}$ \\
\hline C & 10 & 1 & 0 & $10 \%$ & 1.29 & 1.29 \\
\hline D & 10 & 5 & 0 & $50 \%$ & 1.56 & 1.56 \\
\hline $\mathrm{E}$ & 10 & 1 & 0 & $10 \%$ & 1.29 & 1.29 \\
\hline $\mathrm{F}$ & 10 & 1 & 1 & $10 \%$ & 1.55 & 1.55 \\
\hline $\mathrm{G}$ & 10 & 3 & 1 & $30 \%$ & 1.22 & 1.22 \\
\hline $\mathrm{H}$ & 10 & 3 & 0 & $30 \%$ & 1.58 & 1.58 \\
\hline Mean & 10.0 & & & $20 \%$ & & 1.43 \\
\hline
\end{tabular}




\section{DAY AMPHIPOD SEDIMENT TOXICITY TEST}

\begin{tabular}{|l|l|ll|}
\hline Lab ID $\#$ & Client: $\quad$ Westinghouse Savannah River Company & Start Date: & $9-30-95$ \\
\cline { 2 - 5 } T4452 & Sample Identification: $\quad$ WSRC FHB 023 & End Date: & $10-10-95$ \\
\hline
\end{tabular}

\section{RESULTS}

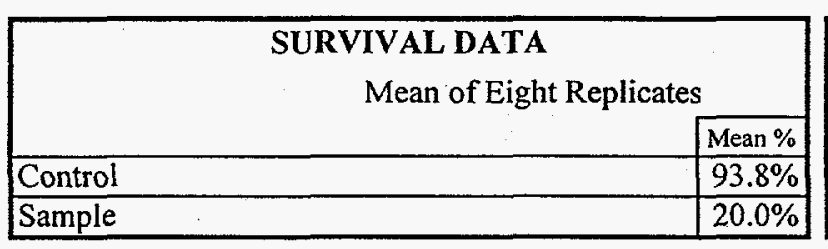

\begin{tabular}{|c|c|c|}
\hline \multicolumn{3}{|c|}{ GROWTH DATA } \\
\hline & & Mean \\
\hline Control & & 1.57 \\
\hline Sample & & 1.43 \\
\hline
\end{tabular}

\section{STATISTICAL ANALYSES}

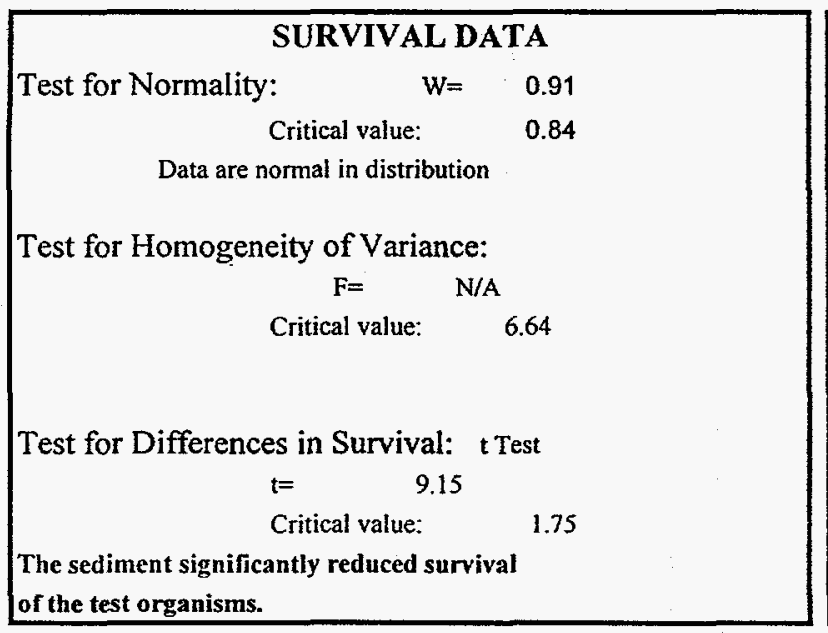

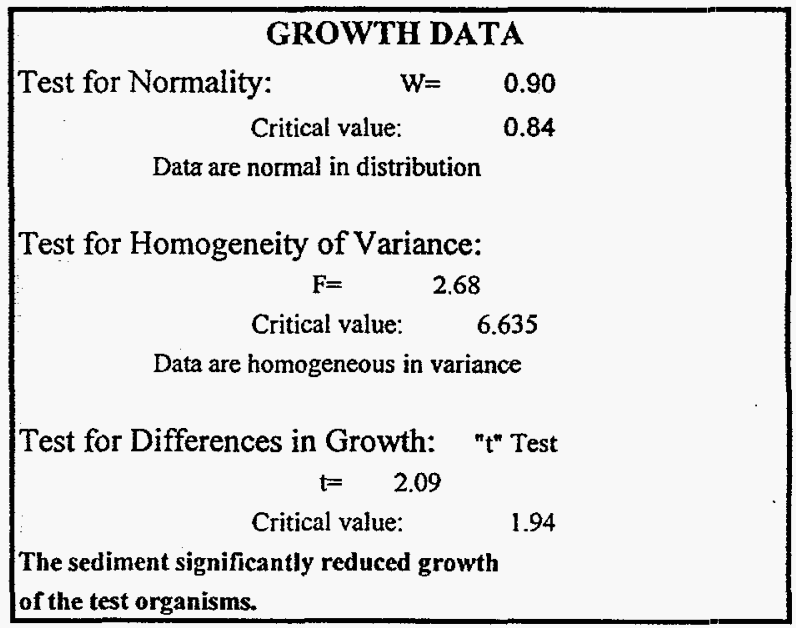

\section{COMMENTS}

\begin{tabular}{|c|c|c|c|}
\hline $\begin{array}{l}\text { Principal Analyst: } \\
\text { Report Reviewed By: }\end{array}$ & $\begin{array}{l}\text { Aimee Arnold } \\
\text { Robert W. Kelley, Ph.D. }\end{array}$ & 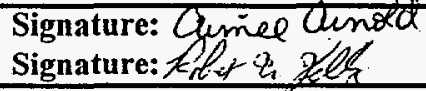 & $\begin{array}{l}\text { Date: } 10 / 13 / 95 \\
\text { Date: } 10 / 13 / 95\end{array}$ \\
\hline
\end{tabular}

\section{CONCLUSIONS}

The test sediment does adversely affect the survival and the growth of freshwater amphipods. 


\title{
10 DAY AMPHIPOD SEDIMENT TOXICITY TEST
}

\author{
EPA/600/R-94/024 Method 100.1 \\ Test Organism: Hyallela azteca
}

Client: Westinghouse Savannah River Company

Sample Identification: WSRC HSP 064

Test Start Date: $\quad 9-30-95$ 


\section{DAY AMPHIPOD SEDIMENT TOXICITY TEST}

\begin{tabular}{|l|l|l|l|}
\hline Lab ID\# & Client: $\quad$ Westinghouse Savannah River Company & Start Date: & 9-30-95 \\
\cline { 2 - 4 } T4449 & Sample Identification: $\quad$ WSRC HSP 064 & End Date: & 10-10-95 \\
\hline
\end{tabular}

\section{WATER CHEMISTRY TEST METHODS}

Temperature: SM 18th Ed. 2550

Dissolved Oxygen: SM 18th Ed. 4500-O

pH: SM 18th Ed. 4500-H+
Alkalinity: SM 18th Ed. 2320

Hardness: SM 18th Ed. 2340

Conductivity: SM 18th Ed. 2510

\section{WATER CHEMISTRY TEST DATA - OVERLYING WATER}

CONTROL TREATMENT

\begin{tabular}{|c|r|r|r|r|}
\hline Test Day & $\begin{array}{c}\text { Temp. } \\
\mathrm{C}\end{array}$ & $\begin{array}{c}\mathrm{pH} \\
\text { units }\end{array}$ & $\begin{array}{c}\text { D.O. } \\
\mathrm{mg} / \mathrm{L}\end{array}$ & $\begin{array}{c}\text { D.O. } \\
\% \text { sat. }\end{array}$ \\
\hline Start & 22.5 & 6.67 & 7.0 & $80 \%$ \\
\hline 1 & 23.2 & & 6.4 & $74 \%$ \\
\hline 2 & 22.6 & & 6.8 & $78 \%$ \\
\hline 3 & 22.7 & & 5.7 & $66 \%$ \\
\hline 4 & 22.9 & & 5.5 & $63 \%$ \\
\hline 5 & 23.1 & & 5.6 & $65 \%$ \\
\hline 6 & 22.2 & & 5.6 & $64 \%$ \\
\hline 7 & 22.3 & & 8.3 & $95 \%$ \\
\hline 8 & 23.5 & & 5.4 & $63 \%$ \\
\hline 9 & 23.7 & & 7.2 & $84 \%$ \\
\hline 10 & 22.0 & 6.38 & 6.2 & $71 \%$ \\
\hline
\end{tabular}

SAMPLE TREATMENT

\begin{tabular}{|c|c|c|c|c|c|c|c|c|}
\hline $\begin{array}{c}\text { Test } \\
\text { Day }\end{array}$ & $\begin{array}{c}\text { Cond. } \\
\text { umhos/cm }\end{array}$ & $\begin{array}{c}\mathrm{NH3} \\
\mathrm{mg} / \mathrm{L}\end{array}$ & $\begin{array}{c}\text { Temp. } \\
\mathrm{C}\end{array}$ & $\begin{array}{c}\mathrm{pH} \\
\text { units }\end{array}$ & $\begin{array}{c}\text { D.O. } \\
\mathrm{mg} / \mathrm{L}\end{array}$ & $\begin{array}{c}\text { D.O. } \\
\% \text { sat. }\end{array}$ & $\begin{array}{c}\text { Alk. } \\
\mathrm{mg} / \mathrm{L}\end{array}$ & $\begin{array}{c}\text { Hard. } \\
\mathrm{mg} / \mathrm{L}\end{array}$ \\
\hline Start & 37.5 & 0.21 & 22.2 & 6.49 & 7.8 & $90 \%$ & 4.1 & 42.0 \\
\hline 1 & & & 23.0 & & 4.2 & $49 \%$ & & \\
\hline 2 & & & 22.2 & & 5.9 & $68 \%$ & & \\
\hline 3 & & & 22.4 & & 6.3 & $72 \%$ & & \\
\hline 4 & & & 22.8 & & 6.0 & $69 \%$ & & \\
\hline 5 & & & 22.9 & & 6.0 & $69 \%$ & & \\
\hline 6 & & & 22.1 & & 6.2 & $71 \%$ & & \\
\hline 7 & & & 22.2 & & 6.3 & $72 \%$ & & \\
\hline 8 & & & 23.4 & & 6.2 & $72 \%$ & & \\
\hline 9 & & & 23.1 & & 6.5 & $76 \%$ & & \\
\hline 10 & 36 & 1.7 & 22.0 & 6.01 & 6.8 & $78 \%$ & 8.1 & 50.0 \\
\hline
\end{tabular}

\section{SURVIVAL AND GROWTH RESULTS}

CONTROL TREATMENT

\begin{tabular}{|c|c|c|c|c|c|c|}
\hline & \multicolumn{3}{|c|}{ \# Organisms } & \multirow[b]{2}{*}{$\begin{array}{c}\% \\
\text { Survival } \\
\end{array}$} & \multirow{2}{*}{\begin{tabular}{|l|} 
Mean \\
Length \\
$(\mathrm{mm})$
\end{tabular}} & \multirow{2}{*}{$\begin{array}{r}\text { Mean } \\
\text { Length } \\
(\mathrm{mm})\end{array}$} \\
\hline & Initial & $\begin{array}{l}\text { Live at } \\
\text { End }\end{array}$ & $\begin{array}{l}\text { Dead } \\
\text { at End }\end{array}$ & & & \\
\hline $\mathrm{A}$ & 10 & 8 & 0 & $80 \%$ & 1.57 & 1.57 \\
\hline B & 10 & 10 & 0 & $100 \%$ & 1.58 & 1.58 \\
\hline C & 10 & 8 & 0 & $80 \%$ & 1.35 & 1.35 \\
\hline $\mathrm{D}$ & 10 & 10 & 0 & $100 \%$ & 1.56 & 1.56 \\
\hline$E$ & 10 & 9 & 0 & $90 \%$ & 1.68 & 1.68 \\
\hline$F$ & 10 & 10 & 0 & $100 \%$ & 1.58 & 1.58 \\
\hline $\mathrm{G}$ & 10 & 10 & 0 & $100 \%$ & 1.6 & 1.60 \\
\hline $\mathrm{H}$ & 10 & 10 & 0 & $100 \%$ & 1.6 & 1.60 \\
\hline Mean & 10.0 & & & $94 \%$ & & 1.57 \\
\hline
\end{tabular}

SAMPLE TREATMENT

\begin{tabular}{|c|c|c|c|c|c|c|}
\hline & \multicolumn{3}{|c|}{ \# Organisms } & \multirow[b]{2}{*}{$\begin{array}{c}\% \\
\text { Survival }\end{array}$} & \multirow{2}{*}{$\begin{array}{l}\text { Mean } \\
\text { Length } \\
(\mathrm{mm})\end{array}$} & \multirow{2}{*}{$\begin{array}{r}\text { Mean } \\
\text { Length } \\
(\mathrm{mm})\end{array}$} \\
\hline & Initial & $\begin{array}{l}\text { Live at } \\
\text { End }\end{array}$ & $\begin{array}{l}\text { Dead } \\
\text { at End }\end{array}$ & & & \\
\hline $\bar{A}$ & 10 & 6 & 0 & $60 \%$ & 1.67 & 1.67 \\
\hline $\mathrm{B}$ & 10 & 8 & 0 & $80 \%$ & 1.53 & 1.53 \\
\hline C & 10 & 6 & 0 & $60 \%$ & 1.41 & 1.41 \\
\hline $\bar{D}$ & 10 & 5 & 0 & $50 \%$ & 1.59 & 1.59 \\
\hline $\mathrm{E}$ & 10 & NA & NA & $\overline{N A}$ & NA & $\mathrm{NA}$ \\
\hline $\mathbf{F}$ & 10 & 6 & 0 & $60 \%$ & 1.54 & 1.54 \\
\hline $\bar{G}$ & 10 & 8 & 0 & $80 \%$ & 1.49 & 1.49 \\
\hline $\mathrm{H}$ & 10 & 9 & 0 & $90 \%$ & 1.5 & 1.54 \\
\hline Mean & 10.0 & & & $69 \%$ & & 1.54 \\
\hline
\end{tabular}




\section{DAY AMPHIPOD SEDIMENT TOXICITY TEST}

\begin{tabular}{|l|ll|ll|}
\hline Lab ID\# & Client: & Westinghouse Savannah River Company & Start Date: & 9-30-95 \\
\cline { 2 - 5 } T4449 & Sample Identification: & WSRC HSP 064 & End Date: & 10-10-95 \\
\hline
\end{tabular}

\section{RESULTS}

\begin{tabular}{|l|l|}
\hline \multicolumn{2}{|c|}{$\begin{array}{c}\text { SURVIVAL DATA } \\
\text { Mean of Eight Replicates }\end{array}$} \\
\cline { 2 - 2 } & Mean \% \\
\hline Control & $93.8 \%$ \\
\hline Sample & $68.6 \%$ \\
\hline
\end{tabular}

\begin{tabular}{|c|c|c|}
\hline \multicolumn{3}{|c|}{ GROWTH DATA } \\
\hline \multicolumn{3}{|c|}{ Mean Length (mm) } \\
\hline & & Mean \\
\hline Control & & 1.57 \\
\hline Sample & & 1.54 \\
\hline
\end{tabular}

\section{STATISTICAL ANALYSES}

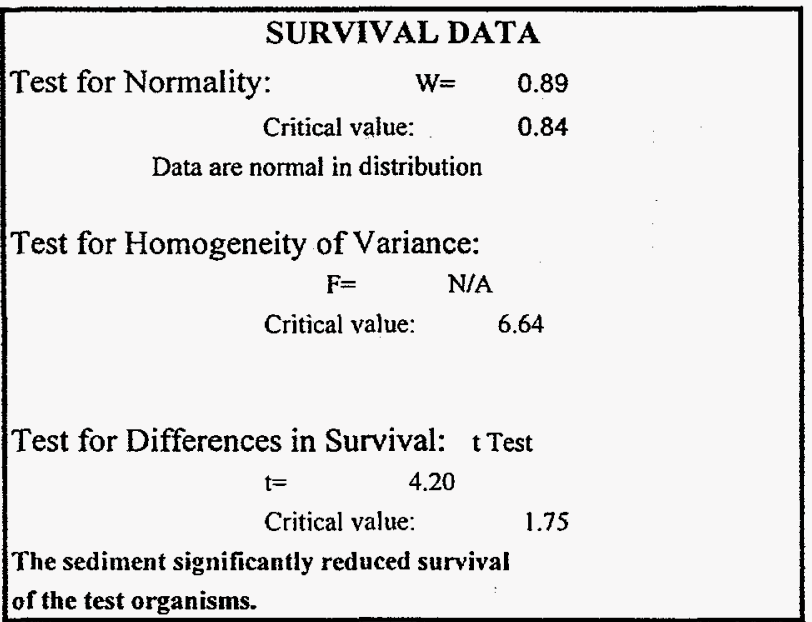

\begin{tabular}{|c|c|}
\hline \multicolumn{2}{|c|}{ GROWTH DATA } \\
\hline Test for Normality: & 0.87 \\
\hline \multicolumn{2}{|c|}{ Data are normal in distribution } \\
\hline \multicolumn{2}{|l|}{ Test for Homogeneity of Variance: } \\
\hline \multicolumn{2}{|c|}{1.38} \\
\hline \multicolumn{2}{|l|}{ Critical value: } \\
\hline \multicolumn{2}{|c|}{ Data are homogeneous in variance } \\
\hline $\begin{array}{l}\text { Test for Differences in Growth: } \\
\qquad t=0.58\end{array}$ & Test for Differences in Growth: $\quad t^{n}$ Test \\
\hline Critical value: & 1.94 \\
\hline $\begin{array}{l}\text { The sediment did not significantly red } \\
\text { of the test organisms. }\end{array}$ & growth \\
\hline
\end{tabular}

\section{COMMENTS}

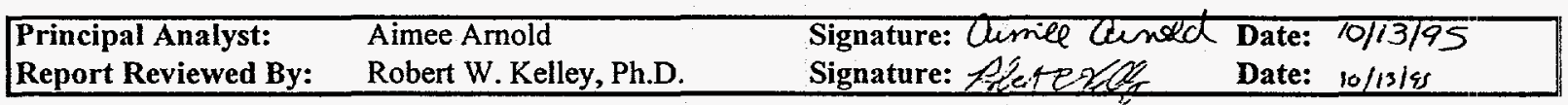

\section{CONCLUSIONS}

The test sediment does adversely affect the survival but not the growth of freshwater amphipods. 


\section{DAY AMPHIPOD SEDIMENT TOXICITY TEST}

EPA/600/R-94/024 Method 100.1

Test Organism: Hyallela azteca

Client: Westinghouse Savannah River Company Sample Identification: WSRC FMC 002H

Test Start Date: $\quad 9-30-95$ 


\section{DAY AMPHIPOD SEDIMENT TOXICITY TEST}

\begin{tabular}{|c|c|c|c|c|}
\hline \multirow{2}{*}{$\begin{array}{l}\text { Lab ID\# } \\
\text { T4453 }\end{array}$} & Client: & Jestinghouse Savannah River Company & Start Date & $9-30-95$ \\
\hline & Sample Identification: & WSRC FMC 002H & End Date: & $10-10-95$ \\
\hline
\end{tabular}

\section{Test System}

EPA/600/R-94/024 Method 100.1

The test was set as a Pass/Fail test with a control treatment and one sample treatment. Each treatment replicate consisted of $100 \mathrm{~g}$ of sediment and $175 \mathrm{~mL}$ of dilution water. There were eight replicates for each treatment. Ten test organisms were placed in each replicate. Sediment from a local river was used in the control treatment. Test organisms were fed with $\mathrm{YCT}$ food. The test was conducted at a temperature of $23^{\circ} \mathrm{C}$ with ambient laboratory light conditions. Test vessels were $500 \mathrm{~mL}$ plastic beakers placed in a constant temperature incubator room.

Order: Amphipoda

\section{Test Organisms}

Species: Hyallela azteca

Source: Aquatic Research Organisms, NH

Life Stage: $\quad 2-3 \mathrm{~mm}$

\% Mortality During 48 Hr Prior to Test::

Taxonomic Verification: RWK 9/29/95

Culturing: as received

Observations: Cultures healthy

Acclimation: None

\section{Control Sediment}

Source: South Tyger river@Hwy. 14

Collection Date: 9-19-95

Preservation: kept at $0-4^{\circ} \mathrm{C}$ in plastic containers

Date/Time Added to Test Chambers: $\quad$ 9-29-95

Observations: silt

Collection Method: grab

Disposal:

\begin{tabular}{|c|c|c|c|}
\hline \multicolumn{4}{|c|}{ Food Preparation } \\
\hline \multicolumn{4}{|c|}{ Source: Trout Chow (Glencoe Mills); Cerophyl (Sigma) } \\
\hline \multicolumn{4}{|c|}{$\begin{array}{ll}\text { Purchase Date: } & \text { Preparation Date: } \\
\text { Preservation: } & \text { kept at } 0-4^{\circ} \mathrm{C}\end{array}$} \\
\hline \multirow{2}{*}{\multicolumn{2}{|c|}{ Preparation Method: }} & Trout Chow aerated 7 day & \\
\hline & & Cerophyl, yeast aerated ov & \\
\hline Feeding Rate: & $1.5 \mathrm{~m}$ & y & \\
\hline Hold Time: & 5 da & & \\
\hline
\end{tabular}

\section{Dilution Water}

Type: Surface Water

Collection Date 9/29/95 Initial Final

$\begin{array}{lrrl}\text { Alkalinity } & 16.2 & 16.2 \mathrm{mg} / \mathrm{L} \\ \text { Ammonia } & 0.742 & 0.518 \mathrm{mg} / \mathrm{L} \\ \text { Conductivity } & 51.9 & 46.4 \mathrm{urnhos} / \mathrm{cm} \\ \text { Hardness } & 16 & 16 \mathrm{mg} / \\ \text { pH: } & 7.07 & 7.42 \text { units }\end{array}$

Preparation Method:

Grab sample

Evidence of Water Quality:

Good reproduction of Ceriodaphnia dubia cultures

\section{Test Sediment}

Source: WSRC FMC 002H

Collection Date 8/22/95 Homogenized?: yes

Preservation: kept at $0-4^{\circ} \mathrm{C}$ in plastic containers

Date/Time Added to Test Chambers: 9/29/95

Observations: organic

Collection Method: grab

Shipment: $\quad$ Overnight at $4^{\circ} \mathrm{C}$

\section{Test Chambers}

Type: Plastic $500 \mathrm{~mL}$ beakers

Sediment Volume: $\quad 100 \mathrm{~mL}$

Sediment Depth $4 \mathrm{~cm}$

Overlying Water Volume: $\quad 175 \mathrm{~mL}$

Overlying Water Depth: $\quad 5 \mathrm{~cm}$

Aeration: $\quad$ starting at Day 3 


\section{DAY AMPHIPOD SEDIMENT TOXICITY TEST}

\begin{tabular}{|l|l|ll|}
\hline Lab ID\# & Client: $\quad$ Westinghouse Savannah River Company & Start Date: & $9-30-95$ \\
\cline { 2 - 5 } T4453 & Sample Identification: $\quad$ WSRC FMC 002H & End Date: & $10-10-95$ \\
\hline
\end{tabular}

\section{WATER CHEMISTRY TEST METHODS}

\begin{tabular}{|l|l|}
\hline Temperature: SM 18th Ed. 2550 & Alkalinity: SM 18th Ed. 2320 \\
Dissolved Oxygen: SM 18th Ed. 4500-O & Hardness: SM 18th Ed. 2340 \\
pH: SM 18th Ed. 4500-H+ & Conductivity: SM 18th Ed. 2510 \\
\hline
\end{tabular}

\section{WATER CHEMISTRY TEST DATA - OVERLYING WATER}

CONTROL TREATMENT

\begin{tabular}{|c|r|r|r|r|}
\hline Test Day & $\begin{array}{c}\text { Temp. } \\
\text { C }\end{array}$ & $\begin{array}{c}\mathrm{pH} \\
\text { units }\end{array}$ & $\begin{array}{c}\text { D.O. } \\
\mathrm{mg} / \mathrm{L}\end{array}$ & $\begin{array}{c}\text { D.O. } \\
\% \text { sat. }\end{array}$ \\
\hline Start & 22.5 & 6.67 & 7.0 & $80 \%$ \\
\hline 1 & 23.2 & & 6.4 & $74 \%$ \\
\hline 2 & 22.6 & & 6.8 & $78 \%$ \\
\hline 3 & 22.7 & & 5.7 & $66 \%$ \\
\hline 4 & 22.9 & & 5.5 & $63 \%$ \\
\hline 5 & 23.1 & & 5.6 & $65 \%$ \\
\hline 6 & 22.2 & & 5.6 & $64 \%$ \\
\hline 7 & 22.3 & & 8.3 & $95 \%$ \\
\hline 8 & 23.5 & & 5.4 & $63 \%$ \\
\hline 9 & 23.7 & & 7.2 & $84 \%$ \\
\hline 10 & 22.0 & 6.38 & 6.2 & $71 \%$ \\
\hline
\end{tabular}

SAMPLE TREATMENT

\begin{tabular}{|c|c|c|c|c|c|c|c|c|}
\hline $\begin{array}{c}\text { Test } \\
\text { Day }\end{array}$ & $\begin{array}{c}\text { Cond. } \\
\text { unhos/cm }\end{array}$ & $\begin{array}{c}\mathrm{NH} 3 \\
\mathrm{mg} / \mathrm{L}\end{array}$ & $\begin{array}{c}\text { Temp. } \\
\mathrm{C}\end{array}$ & $\begin{array}{c}\mathrm{pH} \\
\text { units }\end{array}$ & $\begin{array}{c}\text { D.O. } \\
\mathrm{mg} / \mathrm{L}\end{array}$ & $\begin{array}{c}\text { D.O. } \\
\% \text { sat. }\end{array}$ & $\begin{array}{c}\text { Alk. } \\
\mathrm{mg} / \mathrm{L}\end{array}$ & $\begin{array}{c}\text { Hard. } \\
\mathrm{mg} / \mathrm{L}\end{array}$ \\
\hline Start & 44.3 & 1.11 & 22.3 & 6.25 & 4.4 & $51 \%$ & 10.1 & 40.0 \\
\hline 1 & & & 23.0 & & 4.2 & $49 \%$ & & \\
\hline 2 & & & 22.2 & & 5.9 & $68 \%$ & & \\
\hline 3 & & & 22.4 & & 4.1 & $47 \%$ & & \\
\hline 4 & & & 22.9 & & 8.0 & $92 \%$ & & \\
\hline 5 & & & 22.0 & & 8.5 & $98 \%$ & & \\
\hline 6 & & & 22.0 & & 8.2 & $94 \%$ & & \\
\hline 7 & & & 22.0 & & 6.0 & $69 \%$ & & \\
\hline 8 & & & 22.8 & & 8.2 & $94 \%$ & & \\
\hline 9 & & & 22.4 & & 7.9 & $91 \%$ & & \\
\hline 10 & 32.4 & 3.04 & 22.0 & 5.77 & 7.7 & $89 \%$ & 4.1 & 28.0 \\
\hline
\end{tabular}

\section{SURVIVAL AND GROWTH RESULTS}

\begin{tabular}{|l|r|r|r|r||r|r|}
\hline & \multicolumn{7}{|c|}{ \# Organisms } & Mean & $\begin{array}{r}\text { Mean } \\
\text { (nitial }\end{array}$ & $\begin{array}{c}\text { Live at } \\
\text { End }\end{array}$ & $\begin{array}{c}\text { Dead } \\
\text { at End }\end{array}$ & $\begin{array}{c}\% \\
\text { Survival }\end{array}$ & $\begin{array}{r}\text { Length } \\
(\mathrm{mm})\end{array}$ & $\begin{array}{r}\text { Length } \\
(\mathrm{mm})\end{array}$ \\
\hline $\mathrm{A}$ & 10 & 8 & 0 & $80 \%$ & 1.57 & 1.57 \\
\hline $\mathrm{B}$ & 10 & 10 & 0 & $100 \%$ & 1.58 & 1.58 \\
\hline $\mathrm{C}$ & 10 & 8 & 0 & $80 \%$ & 1.35 & 1.35 \\
\hline $\mathrm{D}$ & 10 & 10 & 0 & $100 \%$ & 1.56 & 1.56 \\
\hline $\mathrm{E}$ & 10 & 9 & 0 & $90 \%$ & 1.68 & 1.68 \\
\hline $\mathrm{F}$ & 10 & 10 & 0 & $100 \%$ & 1.58 & 1.58 \\
\hline $\mathrm{G}$ & 10 & 10 & 0 & $100 \%$ & 1.6 & 1.60 \\
\hline $\mathrm{H}$ & 10 & 10 & 0 & $100 \%$ & 1.6 & 1.60 \\
\hline Mean & 10.0 & & & $94 \%$ & & 1.57 \\
\hline
\end{tabular}

\begin{tabular}{|l|r|r|r|r|l|l|}
\hline & \multicolumn{2}{|c|}{ \# Organisms } & Mean & $\begin{array}{c}\text { Mean } \\
\text { Live at } \\
\end{array}$ \\
& Initial & End & $\begin{array}{c}\text { Dead } \\
\text { at End }\end{array}$ & $\begin{array}{c}\% \\
\text { Survival }\end{array}$ & $\begin{array}{c}\text { Length } \\
\text { (mm) }\end{array}$ & $\begin{array}{c}\text { Length } \\
(\mathrm{mm})\end{array}$ \\
\hline A & 10 & 0 & 0 & $0 \%$ & NA & NA \\
\hline B & 10 & 0 & 0 & $0 \%$ & NA & NA \\
\hline C & 10 & 0 & 0 & $0 \%$ & NA & NA \\
\hline D & 10 & 0 & 0 & $0 \%$ & NA & NA \\
\hline E & 10 & 0 & 0 & $0 \%$ & NA & NA \\
\hline F & 10 & 0 & 0 & $0 \%$ & NA & NA \\
\hline G & 10 & 0 & 0 & $0 \%$ & NA & NA \\
\hline H & 10 & 0 & 0 & $0 \%$ & NA & NA \\
\hline Mean & 10.0 & & & $0 \%$ & & NA \\
\hline
\end{tabular}




\section{DAY AMPHIPOD SEDIMENT TOXICITY TEST}

\begin{tabular}{|c|c|c|c|}
\hline \multirow{2}{*}{\begin{tabular}{|l|} 
Lab ID\# \\
T4453
\end{tabular}} & Client: & Vestinghouse Savannah River Company & Start Date: \\
\hline & Sample Identification & WSRC FMC 002H & End Date: \\
\hline
\end{tabular}

\section{RESULTS}

\begin{tabular}{|l|r|}
\hline \multicolumn{2}{|c|}{$\begin{array}{c}\text { SURVIVAL DATA } \\
\text { Mean of Eight Replicates }\end{array}$} \\
\cline { 2 - 2 } & Mean \% \\
\hline Control & $93.8 \%$ \\
\hline Sample & $0.0 \%$ \\
\hline
\end{tabular}

\begin{tabular}{|lr|}
\hline \multicolumn{2}{|c|}{$\begin{array}{r}\text { GROWTH DATA } \\
\text { Mean Length (mm) } \\
\end{array}$} \\
\hline Control & Mean \\
\hline Sample & 1.57 \\
\hline
\end{tabular}

\section{STATISTICAL ANALYSES}

\begin{tabular}{|c|c|}
\hline \multicolumn{2}{|c|}{ SURVIVAL DATA } \\
\hline Test for Normality: & 0.80 \\
\hline Critical value: & 0.84 \\
\hline \multicolumn{2}{|c|}{ Data are not normal in distribution } \\
\hline \multicolumn{2}{|l|}{ Test for Homogeneity of Variance: } \\
\hline \multicolumn{2}{|r|}{ N/A } \\
\hline \multicolumn{2}{|l|}{ Critical value: } \\
\hline \multirow{2}{*}{\multicolumn{2}{|c|}{$\begin{array}{l}\text { Test for Differences in Survival: Wilcoxon Test } \\
\qquad \text { Sum }=\end{array}$}} \\
\hline & 82 \\
\hline $\begin{array}{l}\text { The sediment significantly reduced su } \\
\text { of the test organisms. }\end{array}$ & Irvival \\
\hline
\end{tabular}

\begin{tabular}{|c|c|c|}
\hline \multicolumn{3}{|c|}{ GROWTH DATA } \\
\hline Test for Normality: & $=N / A$ & \\
\hline Critical value: & 0.84 & \\
\hline \multicolumn{2}{|l|}{ Test for Homogeneity of Variance: } & \\
\hline$F=$ & N/A & \\
\hline Critical value: & 6.635 & \\
\hline $\begin{array}{l}\text { Test for Differences in Growth: } \\
\qquad t=\text { N/A }\end{array}$ & "t" Test & \\
\hline Critical value: & 1.94 & \\
\hline Test organism growth effects cannot b & be determined & \\
\hline
\end{tabular}

\section{COMMENTS}

All test organisms exposed to the test sediment died during the test.

\begin{tabular}{|c|c|c|c|}
\hline $\begin{array}{l}\text { Principal Analyst: } \\
\text { Report Reviewed By: }\end{array}$ & $\begin{array}{l}\text { Aimee Arnold } \\
\text { Robert W. Kelley, Ph.D. }\end{array}$ & 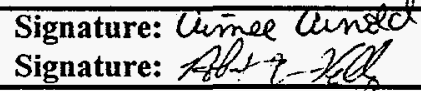 & $\begin{array}{l}\text { Date: } 10 / 13 / 95 \\
\text { Date: } 10 / 13 / 95\end{array}$ \\
\hline
\end{tabular}

\section{CONCLUSIONS}

The test sediment does adversely affect the survival and the growth of freshwater amphipods. 